MÉMOIRES DE LA SMF 101/102

\title{
STRICHARTZ ESTIMATES FOR SCHRÖDINGER EQUATIONS WITH VARIABLE COEFFICIENTS
}

\author{
Luc Robbiano
}

Claude Zuily

Société Mathématique de France 2005

Publié avec le concours du Centre National de la Recherche Scientifique 
L. Robbiano

Université de Versailles, UMR 8100, Bât. Fermat, 45, Avenue des États-Unis, 78035 Versailles.

C. Zuily

Université Paris Sud, UMR 8628, Département de Mathématiques, Bât. 425, 91406 Orsay Cedex.

2000 Mathematics Subject Classification. - 35A17, 35A22, 35Q40, 35Q55.

Key words and phrases. - Strichartz estimates, Schrödinger equations, dispersive estimates, FBI transform, Sjöstrand's theory. 


\title{
STRICHARTZ ESTIMATES FOR SCHRÖDINGER EQUATIONS WITH VARIABLE COEFFICIENTS
}

\author{
Luc Robbiano, Claude Zuily
}

\begin{abstract}
We prove the (local in time) Strichartz estimates (for the full range of parameters given by the scaling unless the end point) for asymptotically flat and non trapping perturbations of the flat Laplacian in $\mathbb{R}^{n}, n \geqslant 2$. The main point of the proof, namely the dispersion estimate, is obtained in constructing a parametrix. The main tool for this construction is the use of the FBI transform.

Résumé (Inégalités de Strichartz pour l'équation de Schrödinger à coefficients variables)

On démontre les inégalités de Strichartz (locales en temps) pour l'ensemble des indices donnés par l'invariance d'échelle (sauf le point final) pour des perturbations asymptotiquement plates et non captantes du laplacien usuel de $\mathbb{R}^{n}, n \geqslant 2$. Le point principal de la preuve, à savoir l'estimation de dispersion, est obtenu en construisant une paramétrixe. L'outil principal de cette construction est la théorie de la transformation de FBI construite par Sjöstrand.
\end{abstract}





\section{CONTENTS}

1. Introduction and statement of the result $\ldots \ldots \ldots \ldots \ldots \ldots \ldots \ldots \ldots$

2. Preliminaries and reduction to the case of a small perturbation of

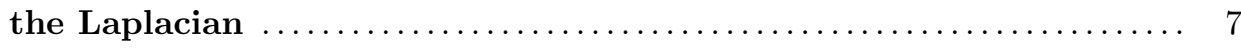

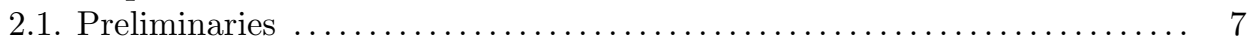

2.2. Reduction to a small perturbation $\ldots \ldots \ldots \ldots \ldots \ldots \ldots \ldots \ldots \ldots \ldots \ldots$

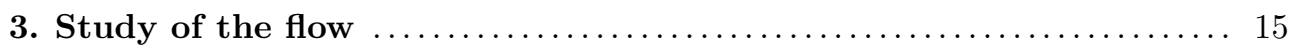

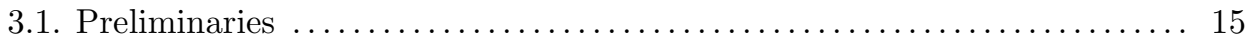

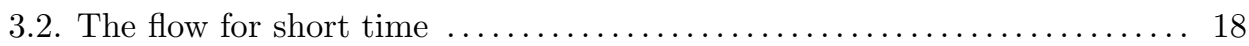

3.3. The forward flow from points in $\mathcal{S}_{+}$and backward from $\mathcal{S}_{-} \ldots \ldots \ldots \ldots 19$

3.4. Precisions on the flow in the general case $\ldots \ldots \ldots \ldots \ldots \ldots \ldots \ldots \ldots \ldots \ldots \ldots$

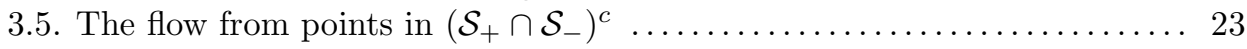

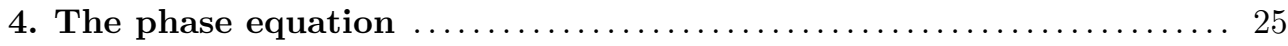

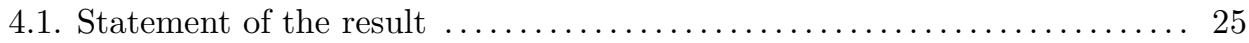

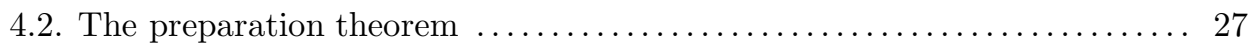

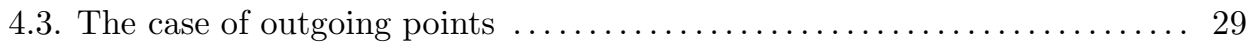

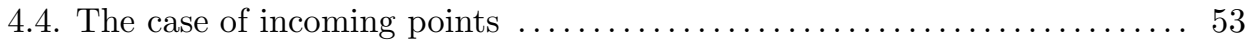

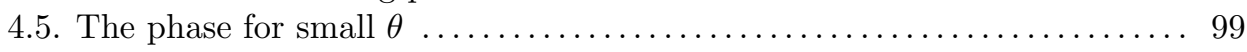

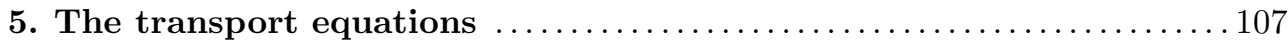

5.1. Statement of the result and preliminaries $\ldots \ldots \ldots \ldots \ldots \ldots \ldots \ldots \ldots \ldots \ldots \ldots$

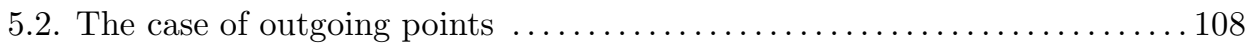

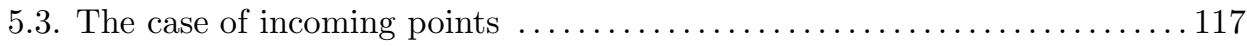

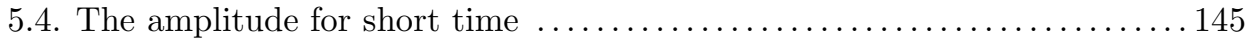

6. Microlocal localizations and the use of the FBI transform ......... 149

6.1. Preliminaries ............................................ 149

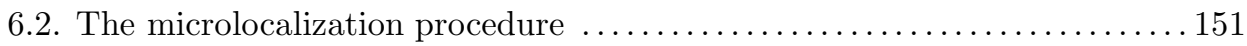

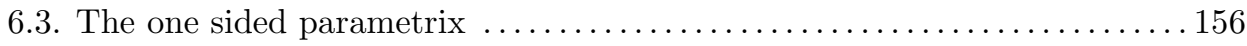

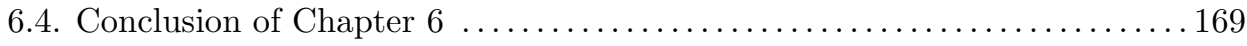


7. The dispersion estimate and the end of the proof of Theorem 1.0.1 171 7.1. The dispersion estimate for the operators $K_{ \pm}(t) \ldots \ldots \ldots \ldots \ldots \ldots \ldots 1$

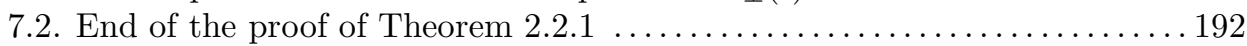

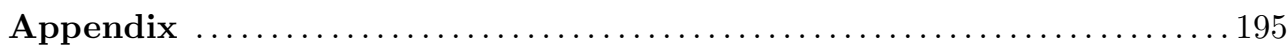

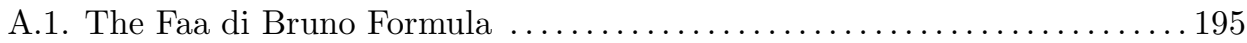

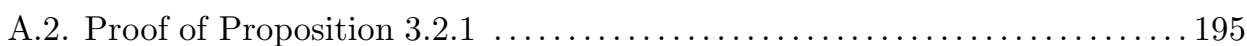

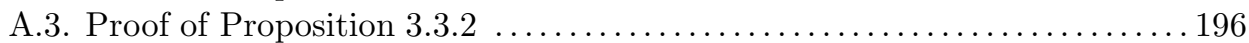

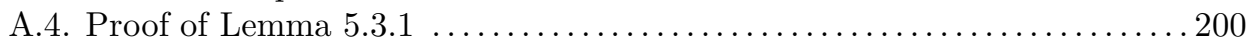

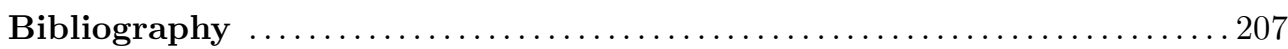




\section{CHAPTER 1}

\section{INTRODUCTION AND STATEMENT OF THE RESULT}

The purpose of this work is to provide a proof of the full (local in time) Strichartz estimates for the Schrödinger operator related to a non trapping asymptotically flat perturbation of the usual Laplacian in $\mathbb{R}^{n}$.

Let $\sigma_{0}$ be in $] 0,1\left[\right.$. We introduce a space of symbols which decay like $\langle x\rangle^{-1-\sigma_{0}}$ where $\langle x\rangle=\left(1+|x|^{2}\right)^{1 / 2}$. More precisely we set

$$
\mathcal{B}_{\sigma_{0}}=\left\{a \in C^{\infty}\left(\mathbb{R}^{n}\right): \forall \alpha \in \mathbb{N}^{n}, \exists C_{\alpha}>0:\left|\partial^{\alpha} a(x)\right| \leqslant \frac{C_{\alpha}}{\langle x\rangle^{1+|\alpha|+\sigma_{0}}}, \forall x \in \mathbb{R}^{n}\right\}
$$

Let $P$ be a second order differential operator,

$$
P=\sum_{j, k=1}^{n} D_{j}\left(g^{j k}(x) D_{k}\right)+\sum_{j=1}^{n}\left(D_{j} b_{j}(x)+b_{j}(x) D_{j}\right)+V(x), D_{j}=\frac{1}{i} \frac{\partial}{\partial x_{j}},
$$

with principal symbol $p(x, \xi)=\sum_{j, k=1}^{n} g^{j k}(x) \xi_{j} \xi_{k}$. (Here $\left.g^{j k}=g^{k j}\right)$.

We shall make the following assumptions.

$$
\left\{\begin{array}{l}
\text { (i) The coefficients } g^{j k}, b_{j}, V \text { are real valued, } 1 \leqslant j \leqslant k \leqslant n . \\
\text { (ii) There exists } \sigma_{0}>0 \text { such that } g^{j k}-\delta_{j k} \in \mathcal{B}_{\sigma_{0}}, b_{j} \in \mathcal{B}_{\sigma_{0}} . \\
\\
\text { Here } \delta_{j k} \text { is the Kronecker symbol. } \\
\text { (iii) } V \in L^{\infty}\left(\mathbb{R}^{n}\right) .
\end{array}\right.
$$

(1.0.4) There exists $\nu>0$ such that for every $(x, \xi)$ in $\mathbb{R}^{n} \times \mathbb{R}^{n}, p(x, \xi) \geqslant \nu|\xi|^{2}$.

Then $P$ has a self-adjoint extension with domain $H^{2}\left(\mathbb{R}^{n}\right)$.

Now we associate to the symbol $p$ the bicharacteristic flow given by the following equations for $j=1, \ldots, n$,

$$
\left\{\begin{array}{l}
\dot{x}_{j}(t)=\frac{\partial p}{\partial \xi_{j}}(x(t), \xi(t)), \quad x_{j}(0)=x_{j}, \\
\dot{\xi}_{j}(t)=-\frac{\partial p}{\partial x_{j}}(x(t), \xi(t)), \quad \xi_{j}(0)=\xi_{j} .
\end{array}\right.
$$

We shall denote by $(x(t, x, \xi), \xi(t, x, \xi))$ the solution, whenever it exists, of the system (1.0.5). In fact it is an easy consequence of (1.0.3) and (1.0.4) that this flow exists for 
all $t$ in $\mathbb{R}$. Indeed by (1.0.4) we have

$$
\nu|\xi(t)|^{2} \leqslant p(x(t), \xi(t))=p(x, \xi),
$$

and it follows from (1.0.4) that

$$
\left|\dot{x}_{j}(t)\right| \leqslant 2 \sum_{k=1}^{n}\left|g^{j k}(x) \xi_{k}(t)\right| \leqslant C|\xi(t)| \leqslant C \nu^{-1 / 2} p(x, \xi)^{1 / 2} .
$$

Our last assumption will be the following.

$$
\text { For all }(x, \xi) \text { in } T^{*} \mathbb{R}^{n} \backslash\{0\} \text { we have } \lim _{t \rightarrow \pm \infty}|x(t, x, \xi)|=+\infty \text {. }
$$

This means that the flow is not trapped backward nor forward. Now let us denote by $e^{-i t P}$ the solution of the following initial value problem

$$
\left\{\begin{array}{l}
i \frac{\partial u}{\partial t}-P u=0 \\
u(0, \cdot)=u_{0}
\end{array}\right.
$$

Then the main result of this work is the following.

THEOREM 1.0.1. - Assume that the operator $P$ satisfies the conditions (1.0.3), (1.0.4), (1.0.6). Let $T>0$ and $(q, r)$ be a couple of real numbers such that $q>2$ and $\frac{2}{q}=\frac{n}{2}-\frac{n}{r}$. Then there exists a positive constant $C$ such that

$$
\left\|e^{-i t P} u_{0}\right\|_{L^{q}\left([-T, T], L^{r}\left(\mathbb{R}^{n}\right)\right)} \leqslant C\left\|u_{0}\right\|_{L^{2}\left(\mathbb{R}^{n}\right)}
$$

for all $u_{0}$ in $L^{2}\left(\mathbb{R}^{n}\right)$.

Such estimates are known in the literature under the name of Strichartz estimates. They have been proved for the flat Laplacian by Strichartz $[\mathbf{S t r}]$ when $p=q=\frac{2 n+4}{n}$ and extended to the full range of $(p, q)$ given by the scaling by Ginibre-Velo [GV] and Yajima $[\mathbf{Y}]$. The limit case $q=2$ (the end point) when $n \geqslant 3$ is due to Keel-Tao $[\mathbf{K T}]$. These estimates have been a key tool in the study of non linear equations. Very recently several works appeared showing a new interest for such estimates in the case of variable coefficients. Staffilani-Tataru $[\mathbf{S T}]$ proved Theorem 1.0.1 under conditions (1.0.4) and (1.0.6) for compactly supported perturbations of the flat Laplacian. In [B] Burq gave an alternative proof of this result using the work of Burq-Gérard-Tzvetkov [BGT]. In the same work Burq announced without proof that if you accept to replace in the right hand side of (1.0.8) the $L^{2}$ norm by an $H^{\varepsilon}$ norm, for any small $\varepsilon>0$, then you can weaken the decay hypotheses on the coefficients of $P$ in the sense that you may replace in the definition (1.0.1) of $\mathcal{B}_{\sigma_{0}}$ the power $|\alpha|+1+\sigma_{0}$ by $|\alpha|+\sigma_{0}$. We have also to mention a recent work of Hassell-Tao-Wunsch [HTW1] who proved in dimension $n=3$ a weaker form of our result corresponding to the case where $q=4, r=3$, under conditions similar to ours. Still more recently these three authors announced the same result as ours under hypotheses on the coefficients similar to ours (see $[$ HTW2]).

MÉMOIRES DE LA SMF 101/102 
It is also worthwhile to mention the work of Burq-Gérard-Tzvetkov who investigate the Strichartz estimates on compact Riemannian manifolds. In that case they show that such estimates hold with the $L^{2}$ norm replaced by the $H^{1 / q}$ norm. In the same paper these authors show that the same result holds on $\mathbb{R}^{n}$ when the coefficients of their Laplacian (and its derivatives) are merely bounded. Let us note also that these estimates concern also the wave equation and many works have been devoted to this case. However we would like to emphasize that, due to the finite speed of propagation, the extension to the variable coefficients case appear to be much less technical (see $[\mathbf{S S}])$.

Let us now give some ideas on the proof. It is by now well known that a proof of the Strichartz estimates can be done using a dispersion result, duality arguments and the Hardy-Littlewood-Sobolev lemma. This has been formulated as an abstract result in the paper $[\mathbf{K T}]$ as follows. Assume that for every $t \in \mathbb{R}$ we have an operator $U(t)$ which maps $L^{2}\left(\mathbb{R}^{n}\right)$ to $L^{2}\left(\mathbb{R}^{n}\right)$ and satisfies,

$$
\left\{\begin{array}{l}
\text { (i) }\|U(t) f\|_{L^{2}\left(\mathbb{R}^{n}\right)} \leqslant C\|f\|_{L^{2}\left(\mathbb{R}^{n}\right)}, \quad \forall t \in \mathbb{R}, C \text { independent of } t, \\
\text { (ii) }\left\|U(s)(U(t))^{*} g\right\|_{L^{\infty}\left(\mathbb{R}^{n}\right)} \leqslant C|t-s|^{-n / 2}\|g\|_{L^{1}\left(\mathbb{R}^{n}\right)}, \quad t \neq s,
\end{array}\right.
$$

then the Strichartz estimates (1.0.5) hold for $U(t)$. It is not difficult to see that the serious estimate to be proved is (ii). In the case when $U(t)=e^{i t \Delta_{0}}$ (the flat Laplacian) this estimate is obtained by the explicit formula giving the solution in term of the data $u_{0}$. In the variable coefficients case such a formula is of course out of hope and the better we can have is a parametrix. However due to strong technical difficulties (which we try to explain below) which seem to be serious we are not able to write a parametrix for $e^{-i t P}$ so we have to explain what we do instead. First of all let $\varphi_{0} \in C_{0}^{\infty}\left(\mathbb{R}^{n}\right)$ be such that $\varphi_{0}(x)=1$ if $|x| \leqslant \frac{3}{2}$ and $\operatorname{supp} \varphi_{0} \subset[-1,1]$. With a large $R>0$ we write

$$
e^{-i t P} u_{0}(x)=\varphi_{0}\left(\frac{x}{R}\right) e^{-i t P} u_{0}(x)+\left(1-\varphi_{0}\left(\frac{x}{R}\right)\right) e^{-i t P} u_{0}(x)=v+w .
$$

It is not difficult to see that the Strichartz estimates for $v$ will be ensured by the result of Staffilani-Tataru $[\mathbf{S T}]$ while the same estimate for $w$ leads to consider an operator which is a small perturbation of the Laplacian (see Chapter 2).

Now it is not a surprise that microlocal analysis is strongly needed in our proof. So let $\xi_{0} \in \mathbb{R}^{n},\left|\xi_{0}\right|=1$ be a fixed direction. Let $\chi_{0} \in C^{\infty}(\mathbb{R}), \chi_{0}(s)=1$ if $s \leqslant \frac{3}{4}$, $\chi_{0}(s)=0$ if $s \geqslant 1,0 \leqslant \chi_{0} \leqslant 1$ and let us set $\chi_{+}(x)=\chi_{0}\left(-x \cdot \xi_{0} / \delta_{1}\right), \chi_{-}(x)=$ $\chi_{0}\left(x \cdot \xi_{0} / \delta_{1}\right), \delta_{1}>0$. We set $U_{+}(t)=\chi_{+} e^{-i t P}, U_{-}(t)=\chi_{-} e^{-i t P}$. Now since $\chi_{+}(x)+\chi_{-}(x) \geqslant 1$ for all $x$ in $\mathbb{R}^{n}$ then Strichartz estimates separately for $U_{+}(t)$ and $U_{-}(t)$ will give the result. It is therefore sufficient to prove the estimate (ii) above for $U_{+}(s)\left(U_{+}(t)\right)^{*}=\chi_{+} e^{i(s-t) P} \chi_{+}$(and for $\left.U_{-}(s)\left(U_{-}(t)\right)^{*}\right)$. In our proof we shall construct a parametrix for these operators. 
Our construction relies heavily on the theory of FBI transform (see Sjöstrand $[\mathbf{S j}$ ] and Melin-Sjöstrand [MS]) viewed as a Fourier integral operator with complex phase. One of the reason of our choice is that in our former works on the analytic smoothing effect [RZ2] we have already done similar constructions (but only near the outgoing points: see below). Let us explain very roughly the main ideas. The standard FBI transform is given by

$$
T v(\alpha, \lambda)=c_{n} \lambda^{3 n / 4} \int_{\mathbb{R}^{n}} e^{i \lambda\left(y-\alpha_{x}\right) \cdot \alpha_{\xi}-\frac{\lambda}{2}\left|y-\alpha_{x}\right|^{2}+\frac{\lambda}{2}\left|\alpha_{\xi}\right|^{2}} v(y) d y
$$

where $\alpha=\left(\alpha_{x}, \alpha_{\xi}\right) \in \mathbb{R}^{n} \times \mathbb{R}^{n}$ and $c_{n}$ is a positive constant.

Let us note that the phase can be written $i \lambda \varphi_{0}$ where $\varphi_{0}(y, \alpha)=\frac{i}{2}\left(y-\left(\alpha_{x}+i \alpha_{\xi}\right)\right)^{2}$. Then $T$ maps $L^{2}\left(\mathbb{R}^{n}\right)$ into the space $L^{2}\left(\mathbb{R}^{2 n}, e^{-\lambda\left|\alpha_{\xi}\right|^{2}} d \alpha\right)$. The adjoint $T^{*}$ of $T$ is given by a similar formula (see (6.1.2)) and we have,

$T^{*} T$ is the identity operator on $L^{2}\left(\mathbb{R}^{n}\right)$.

We embed the transform $T$ into a continuous family of FBI transform

$$
\left\{\begin{array}{l}
T_{\theta} v(\alpha, \lambda)=\lambda^{3 n / 4} \int_{\mathbb{R}^{n}} e^{i \lambda \varphi(\theta, y, \alpha)} a(\theta, y, \alpha) v(y) d y \text { with } \\
\varphi(0, y, \alpha)=\frac{1}{2}\left(y-\left(\alpha_{x}+i \alpha_{\xi}\right)\right)^{2}, a(0, y, \alpha)=c_{n}
\end{array}\right.
$$

Let us set $U(\theta, t, \alpha, \lambda)=T_{\theta}\left[K_{ \pm}(t) u_{0}\right](\alpha, \lambda)$, where $K_{ \pm}(t)=\chi_{ \pm} e^{-i t P} \chi_{ \pm}$. Then it is shown that if $\varphi$ satisfies the eikonal equation,

$$
\left[\frac{\partial \varphi}{\partial \theta}+p\left(x, \frac{\partial \varphi}{\partial x}\right)\right](\theta, x, \alpha)=0
$$

and if the symbol $a$ satisfies appropriate transport equations then $U$ is a solution of the following equation

$$
\left(\frac{\partial U}{\partial t}+\lambda \frac{\partial U}{\partial \theta}\right)(\theta, t, \alpha, \lambda) \sim 0
$$

It follows that essentially we have, $U(\theta, t, \alpha, \lambda)=V(\theta-\lambda t, \alpha, \lambda)$. In particular this shows that $U(0, t, \alpha, \lambda)=U(-\lambda t, 0, \alpha, \lambda)$. Written in terms of the transformations $T_{\theta}$ this reads

$$
T\left[K_{ \pm}(t) u_{0}\right](\alpha, \lambda)=T_{-\lambda t}\left[\chi_{ \pm}^{2} u_{0}\right](\alpha, \lambda) .
$$

Applying $T^{*}$ to both members and using (1.0.10) we obtain

$$
K_{ \pm}(t) u_{0}(x)=T^{*}\left\{T_{-\lambda t}\left[\chi_{ \pm}^{2} u_{0}\right](\cdot, \lambda)\right\}(t, x) .
$$

Thus we have expressed the solution in terms of the data through a Fourier integral operator with complex phase.

This short discussion shows that as usual the main point of the proof is to solve the eikonal and transport equations. Let us point out the main difficulties which occur in solving these equations. They are of three types: the bad behavior of the flow from incoming points and for large time, the global (in $\theta, x$ ) character of all our constructions and the mixing of $C^{\infty}$ coefficients and complex variables (coming from the non real character of our phase). Let us discuss each of them. First of all 
whatever the method you use to solve an eikonal equation (symplectic geometry or another one) a precise description of the flow of the symbol $p$ is needed. Let us recall (see (1.0.5)) that our flow $(x(t, x, \xi), \xi(t, x, \xi))$, issued from the point $(x, \xi) \in T^{*} \mathbb{R}^{n} \backslash 0$, is defined for all $t \in \mathbb{R}$. In the case of the flat Laplacian we have $\xi(t, x, \xi)=\xi$ and $x(t, x, \xi)=x+2 t \xi$. Let now $(x, \xi) \in T^{*} \mathbb{R}^{n} \backslash\{0\}$ and assume that $x \cdot \xi \geqslant 0$. Then it is easy to see that $|x(t, x, \xi)|^{2} \geqslant|x|^{2}+4 t^{2}|\xi|^{2}$ for $t \geqslant 0$ so that $|x(t, x, \xi)|$ becomes larger and larger while $x(t, x, \xi)$ may vanish for a large $t<0$. Such a point is called "outgoing for $t \geqslant 0$ " and "incoming for $t<0$ ". In the case of a perturbed Laplacian this distinction between the directions is very important. Indeed although the flow from outgoing points for $t \geqslant 0$ is very well described for $t \geqslant 0$ and has very similar properties to the flat case, it has a bad behavior for $t<0$ in what concerns its derivatives with respect to $(x, \xi)$. For instance $\frac{\partial x_{j}}{\partial \xi_{k}}(t, x, \xi)$ does not behave at all as $2 t \delta_{j k}$. This is of great importance and causes some trouble in the proof. However still when $t<0$, the flow behaves correctly as long as the point $(x(t, x, \xi), \xi(t, x, \xi))$ is outgoing for $t \geqslant 0$. Roughly speaking that is the reason why we are not able to construct a parametrix for $e^{-i t P}$ while it is possible for the operator $\chi_{ \pm} e^{-i t P} \chi_{ \pm}$. The Chapter 3 is entirely devoted to a careful study of the flow. Let us now describe our method of resolution of the eikonal equation. The classical method uses the ideas of symplectic geometry. Roughly speaking the manifold constructed from the flow is a Lagrangian manifold on which the symbol $\tau+p(x, \xi)$ is constant. If it projects (globally) and clearly on the basis then it is a graph of some function $\varphi$ which is the desired phase. However this general method leads immediately to a difficulty in our case. Indeed since we want that for $\theta=0$ the phase $\varphi$ coincides with the phase $\varphi_{0}$ of the FBI transform (see (1.0.9)) which is non real, we should take, in solving the flow, data which are non real, so the flow itself would be non real; but our symbol has merely $C^{\infty}$ coefficients. To circumvent this difficulty a method has been proposed by MelinSjöstrand [MS] which uses the almost analytic machinery. Another method, different in spirit, that the one described above and known under the name of "Lagrangian ideals", has been introduced by Hörmander $[\mathbf{H}]$. Here the initial data in the flow are kept real. Let us set $u_{j}(x, \xi)=\xi_{j}-\frac{\partial \varphi_{0}}{\partial x_{j}}(x, \xi)=\xi_{j}-\alpha_{\xi}^{j}-i\left(x_{j}-\alpha_{x}^{j}\right)$. Then obviously we have $\left\{u_{j}, u_{k}\right\}=0$ if $j \neq k$ (where $\{$,$\} denotes the Poisson bracket). Now let us$ set $v_{j}(\theta, x, \xi)=u_{j}(x(-\theta, x, \xi), \xi(-\theta, x, \xi)), j=1, \ldots, n$. Then for every $\theta$ in $\mathbb{R}$ the Poisson bracket of $v_{j}$ and $v_{k}$ still vanishes if $j \neq k$. Thus the ideal generated by the $v_{j}$ 's is closed under the Poisson bracket. The main step in Hörmander's method is to show that this ideal is generated by functions of the form $\xi_{j}-\Phi_{j}(\theta, x, \alpha)$. This will imply that one can find a function $\varphi=\varphi(\theta, x, \alpha)$ such that $\frac{\partial \varphi}{\partial x_{j}}(\theta, x, \alpha)=\Phi_{j}(\theta, x, \alpha)$ and it turns out that $\varphi$ is the desired phase. To achieve its main step, Hörmander uses a precise version of the Malgrange preparation theorem which is discussed in $[\mathbf{H}]$, tome 1. This is the way we chose to use in our case. It occupies all Chapter 4 of the paper. The proof is made separately for outgoing and incoming points. Since the $v_{j}$ 's are defined by mean of the backward flow, in both cases we encounter the difficulty 
caused by the bad behavior of the flow from incoming points. As it can be seen many technical difficulties arise in the procedure.

The next step in the proof is the resolution of the transport equations. Here also the cases of outgoing and incoming points have to be separated. We have also to be careful since these are first order equations with non real $C^{\infty}$ coefficients. The first case is easier. Indeed due to the good behavior of the flow and the decay of the perturbation one can cut the Taylor expansion of the coefficients of the vector field to some order and thus reduce ourselves to the case of polynomial coefficients. Then by classical holomorphic methods one can solve the equations modulo flat terms which will be enough for our purpose. In the second case there is no more such an asymptotic and the situation is much more intricate. So we use the classical idea which consists in straightening the vector field. This forces us to enter in the almost analytic machinery of Melin-Sjöstrand [MS] (see Chapter 5). Of course all the constructions made above are done microlocally and in a neighborhood of the bicharacteristic. Therefore to define the general FBI transform $T_{\theta}$ (see (1.0.11)) as well as to pass from the standard $T$ to $T_{-\lambda t}$ we have to insert many microlocal cut-off. Of course we have to check at each microlocalization that the remainder leads to an acceptable error. This is the goal of Chapter 6. At this stage of the proof the operator $K_{ \pm}(t)=\chi_{ \pm} e^{-i t P} \chi_{ \pm}$is written as

$$
K_{ \pm}(t) u_{0}(x)=\int k_{ \pm}(t, x, y) u_{0}(y) d y
$$

where

$$
k_{ \pm}(t, x, y)=\int e^{i \lambda F(-\lambda t, x, y, \alpha)} a(\lambda t, x, y, \alpha) d \alpha .
$$

Thus the dispersion estimate would follow from the bound

$$
\left|k_{ \pm}(t, x, y)\right| \leqslant \frac{C}{|t|^{n / 2}}
$$

for $0<|t| \leqslant T$.

Here we have two regimes according to the fact that $|\lambda t| \geqslant 1$ or $|\lambda t| \leqslant 1$. In the first case on the support of $a(\lambda t, x, y, \alpha)$ we could be very far from the critical point of $F$. Fortunately the phase $F$ has enough convexity to produce the desired bound of $k_{ \pm}$. In the second regime we are close to the critical point of $F$ so we expect a stationary phase method to work. However since the phase $F$ is non real and since the determinant of its Hessian in $\alpha$ degenerates in some direction when $|\lambda t| \rightarrow 0$ we cannot apply the standard results as they appear in $[\mathbf{H}]$. Instead, after a careful study of the phase $F$ we use merely an integration by part method with an appropriate vector field to conclude. This is done in Chapter 7. The rest of this part is devoted, using the Littlewood-Paley theory, to the end of the proof of our main Theorem.

Finally an Appendix gathers the proofs of some technical results used in the paper. Acknowledgments. - We would like to thank Nicolas Burq for useful discussions at an earlier stage of the work. 


\section{CHAPTER 2}

\section{PRELIMINARIES AND REDUCTION TO THE CASE OF A SMALL PERTURBATION OF THE LAPLACIAN}

\subsection{Preliminaries}

We begin by recalling several earlier results which will be used in the sequel.

The first result concerns the case of compactly supported perturbations of the Laplacian.

Theorem 2.1.1 (Staffilani-Tataru $[\mathbf{S T}]$ ). - Let $P$ be defined by (1.0.2). Assume that $P$ satisfies (1.0.4), (1.0.6) and

$$
\text { for } j, k=1, \ldots, n, g^{j k}-\delta_{j k}, b_{j}, V \text { are compactly supported. }
$$

Then the Strichartz estimates (1.0.8) hold.

The second result which we recall is the extension to the variable coefficients case by Doï $[\mathbf{D}]$ of the Kato smoothing effect. Let us introduce the following space. We set for $s, \mu$ in $\mathbb{R}$

$$
H_{\mu}^{s}\left(\mathbb{R}^{n}\right)=\left\{u \in \mathcal{S}^{\prime}:\langle x\rangle^{\mu}(I-\Delta)^{s / 2} u \in L^{2}\left(\mathbb{R}^{n}\right)\right\}
$$

with its standard norm.

Theorem 2.1.2 (Doï $[\mathbf{D}])$. - Let $P$ be defined by (1.0.2) and assume it satisfies the conditions (1.0.3), (1.0.4), (1.0.6). Then for all $T>0$ and all $\sigma>\frac{1}{2}$ one can find a constant $C \geqslant 0$ such that,

$$
\left\|e^{-i t P} u_{0}\right\|_{L^{2}\left([-T, T], H_{-\sigma}^{1 / 2}\left(\mathbb{R}^{n}\right)\right)} \leqslant C\left\|u_{0}\right\|_{L^{2}\left(\mathbb{R}^{n}\right)},
$$

for all $u_{0}$ in $L^{2}\left(\mathbb{R}^{n}\right)$.

We shall also use the following result.

Lemma 2.1.3 (Keel-Tao $[\mathbf{K T}])$. — Let $(X, d x)$ be a measure space, $H$ a Hilbert space and $T>0$. Suppose that for each time $t \in[-T, T]$ we have an operator $U(t): H \rightarrow$ $L^{2}(X)$ which satisfies the following estimates. 
(i) There exists $C_{1} \geqslant 0$ such that for all $t \in[-T, T]$ and all $f \in H$,

$$
\|U(t) f\|_{L^{2}(X)} \leqslant C_{1}\|f\|_{H} .
$$

(ii) There exists $C_{2} \geqslant 0$ such that for all $t, s \in[-T, T], t \neq s$ and all $g \in L^{1}(X)$,

$$
\left\|U(t)(U(s))^{*} g\right\|_{L^{\infty}(X)} \leqslant C_{2}|t-s|^{-n / 2}\|g\|_{L^{1}(X)} .
$$

Let $(q, r)$ be a couple of real numbers such that $q \geqslant 2, r<+\infty$ and $\frac{2}{q}=\frac{n}{2}-\frac{n}{r}$. Then there exists $C \geqslant 0$ such that for all $f$ in $H$

$$
\|U(t) f\|_{L^{q}\left([-T, T], L^{r}(X)\right)} \leqslant C\|f\|_{H} .
$$

This result will be used in the sequel with $H=L^{2}\left(\mathbb{R}^{n}\right), X=\mathbb{R}^{n}$.

Finally let's recall the following technical lemma.

Lemma 2.1.4 (Christ-Kiselev [CK]). — Let $X, Y$ be two Banach spaces and $K(t, s)$ be a continuous function taking its values in $B(X, Y)$, the space of bounded linear mappings from $X$ to $Y$. Let $-\infty \leqslant a<b \leqslant+\infty$ and set

$$
\begin{aligned}
S f(t) & =\int_{a}^{b} K(t, s) f(s) d s \\
W f(t) & =\int_{a}^{t} K(t, s) f(s) d s .
\end{aligned}
$$

Let $1 \leqslant p<q \leqslant+\infty$. Then if we can find a constant $C>0$ such that

$$
\|S f\|_{L^{q}((a, b), Y)} \leqslant C\|f\|_{L^{p}((a, b), X)}
$$

it follows that

$$
\|W f\|_{L^{q}((a, b), Y)} \leqslant \frac{2^{-2\left(\frac{1}{p}-\frac{1}{q}\right)} \cdot 2 C}{1-2^{-\left(\frac{1}{p}-\frac{1}{q}\right)}}\|f\|_{L^{p}((a, b), X)} .
$$

Using these results we shall see that Theorem 1.0.1 will be a consequence of the following Theorem.

THEOREM 2.1.5. - Let us set $\Delta_{g}=\sum_{j, k=1}^{n} \frac{\partial}{\partial x_{j}}\left(g^{j k} \frac{\partial}{\partial x_{k}}\right)$ and assume that the conditions (1.0.3), (1.0.4), (1.0.6) are satisfied by $\Delta_{g}$. Let $T>0$ and $(q, r)$ be a couple of real numbers such that $q>2$ and $\frac{2}{q}=\frac{n}{2}-\frac{n}{r}$. Then there exists a positive constant $C$ such that

$$
\left\|e^{i t \Delta_{g}} u_{0}\right\|_{L^{q}\left([-T, T], L^{r}\left(\mathbb{R}^{n}\right)\right)} \leqslant C\left\|u_{0}\right\|_{L^{2}\left(\mathbb{R}^{n}\right)}
$$

for all $u_{0}$ in $L^{2}\left(\mathbb{R}^{n}\right)$.

Let us show how Theorem 2.1.5 implies Theorem 1.0.1.

Let us set $I=[0, T]$. (The case $I=[-T, 0]$ is symmetric). Using (1.0.2) we can write

$$
i \partial_{t} u+\Delta_{g} u=-\left(\sum_{j=1}^{n}\left(D_{j} b_{j}\right)+V\right) u-2 \sum_{j=1}^{n} b_{j} D_{j} u=: F=F_{1}+F_{2}
$$


It follows from Duhamel formula that

$$
e^{-i t P} u_{0}=e^{i t \Delta_{g}} u_{0}+i \int_{0}^{t} e^{i(t-s) \Delta_{g}}[F(s, \cdot)] d s .
$$

Using Theorem 2.1.5 we obtain

$$
\left\|e^{i t \Delta_{g}} u_{0}\right\|_{L^{q}\left(I, L^{r}\left(\mathbb{R}^{n}\right)\right)} \leqslant C\left\|u_{0}\right\|_{L^{2}\left(\mathbb{R}^{n}\right)} .
$$

Let us set now

$$
S f(t)=\int_{0}^{T} e^{i(t-s) \Delta_{g}}[f(s, \cdot)] d s .
$$

Since $S f(t)=e^{i t \Delta_{g}} \int_{0}^{T} e^{-i s \Delta_{g}}[f(s, \cdot)] d s$ we can use Theorem 2.1.5 to write

$$
\begin{aligned}
\|S f(t)\|_{L^{q}\left(I, L^{r}\left(\mathbb{R}^{n}\right)\right)} & \leqslant C\left\|\int_{0}^{T} e^{-i s \Delta_{g}}[f(s, \cdot)] d s\right\|_{L^{2}\left(\mathbb{R}^{n}\right)} \\
& \leqslant C \int_{0}^{T}\left\|e^{-i s \Delta_{g}}[f(s, \cdot)]\right\|_{L^{2}\left(\mathbb{R}^{n}\right)} d s \\
& \leqslant C \int_{0}^{T}\|f(s, \cdot)\|_{L^{2}\left(\mathbb{R}^{n}\right)} d s=C\|f\|_{L^{1}\left(I, L^{2}\left(\mathbb{R}^{n}\right)\right)} .
\end{aligned}
$$

Using Lemma 2.1.4 with $p=1, q>2, Y=L^{r}\left(\mathbb{R}^{n}\right), X=L^{2}\left(\mathbb{R}^{n}\right)$ we deduce that

$$
\left\|\int_{0}^{t} e^{i(t-s) \Delta_{g}}\left[F_{1}(s, \cdot)\right] d s\right\|_{L^{q}\left(I, L^{r}\left(\mathbb{R}^{n}\right)\right)} \leqslant C\left\|F_{1}\right\|_{L^{1}\left(I, L^{2}\left(\mathbb{R}^{n}\right)\right)}
$$

where $F_{1}=-\left(\sum_{j=1}^{n}\left(D_{j} b_{j}\right)+V\right) u$. Since $\sum_{j=1}^{n}\left|D_{j} b_{j}\right|+|V|$ is bounded (by condition (1.0.3)) we have

$$
\left\|F_{1}\right\|_{L^{1}\left(I, L^{2}\left(\mathbb{R}^{n}\right)\right)} \leqslant C \int_{0}^{T}\|u(s, \cdot)\|_{L^{2}\left(\mathbb{R}^{n}\right)} d s \leqslant C^{\prime} T\left\|u_{0}\right\|_{L^{2}} .
$$

Therefore we have

$$
\left\|\int_{0}^{t} e^{i(t-s) \Delta_{g}}\left[F_{1}(s, \cdot)\right] d s\right\| \leqslant C(T)\left\|u_{0}\right\|_{L^{2}\left(\mathbb{R}^{n}\right)} .
$$

Let us look to the term corresponding to $F_{2}$ in (2.1.3), (2.1.4). Let us fix $\sigma=$ $\frac{1}{2}+\frac{1}{2} \sigma_{0}$. Then by Theorem 2.1.2 the operator $e^{i t \Delta_{g}}$ is continuous from $L^{2}\left(\mathbb{R}^{n}\right)$ to $L^{2}\left(I, H_{-\sigma}^{1 / 2}\left(\mathbb{R}^{n}\right)\right)$. Its adjoint is defined by

$$
\left(\left(e^{i t \Delta_{g}} u_{0}, f\right)\right)=\left(u_{0}, U^{*} f\right)_{L^{2}\left(\mathbb{R}^{n}\right)}
$$

where $(()$,$) denotes the duality between L^{2}\left(I, H_{-\sigma}^{1 / 2}\right)$ and $L^{2}\left(I, H_{\sigma}^{-1 / 2}\right)$. It satisfies the estimate

$$
\left\|U^{*} f\right\|_{L^{2}\left(\mathbb{R}^{n}\right)} \leqslant C\|f\|_{L^{2}\left(I, H_{\sigma}^{-1 / 2}\left(\mathbb{R}^{n}\right)\right)} .
$$

A straightforward computation shows that

$$
U^{*} f(x)=\int_{0}^{T} e^{-i s \Delta_{g}}[f(s, \cdot)] d s .
$$


Using Theorem 2.1.5 for $\Delta_{g}$ we see that the operator $S$ introduced in (2.1.6) satisfies the estimate

$$
\|S f(t)\|_{L^{q}\left(I, L^{r}\left(\mathbb{R}^{n}\right)\right)} \leqslant C\|f\|_{L^{2}\left(I, H_{\sigma}^{-1 / 2}\left(\mathbb{R}^{n}\right)\right)} .
$$

Using Lemma 2.1.4 with $p=2, q>2, Y=L^{r}\left(\mathbb{R}^{n}\right), X=H_{\sigma}^{-1 / 2}\left(\mathbb{R}^{n}\right)$ we see that

$$
\left\|\int_{0}^{t} e^{i(t-s) \Delta_{g}}\left[F_{2}(s, \cdot)\right] d s\right\|_{L^{q}\left(I, L^{r}\left(\mathbb{R}^{n}\right)\right)} \leqslant C\left\|F_{2}\right\|_{L^{2}\left(I, H_{\sigma}^{-1 / 2}\left(\mathbb{R}^{n}\right)\right)}
$$

where $F_{2}=-2 \sum_{j=1}^{n} b_{j} D_{j} u$. If we set, with $\Delta=\sum_{j=1}^{n} \frac{\partial^{2}}{\partial x_{j}^{2}}$,

$$
A=\langle x\rangle^{\sigma}(I-\Delta)^{-1 / 4} \sum_{j=1}^{n} b_{j} D_{j}(I-\Delta)^{-1 / 4}\langle x\rangle^{\sigma}
$$

then we can write

$$
\left\|F_{2}\right\|_{L^{2}\left(I, H_{\sigma}^{-1 / 2}\left(\mathbb{R}^{n}\right)\right)}^{2}=4 \int_{0}^{T}\left\|A\langle x\rangle^{-\sigma}(I-\Delta)^{1 / 4} u(s, \cdot)\right\|_{L^{2}\left(\mathbb{R}^{n}\right)}^{2} d s .
$$

Let us consider the metric on the cotangent space

$$
G=\frac{d x^{2}}{\langle x\rangle^{2}}+\frac{d \xi^{2}}{\langle\xi\rangle^{2}}
$$

It is a Hörmander's metric and we have $\langle x\rangle^{\sigma} \in \mathrm{Op} S\left(\langle x\rangle^{\sigma}, G\right),(I-\Delta)^{-1 / 4} \in$ Op $S\left(\langle\xi\rangle^{-1 / 2}, G\right), b_{j} \in \mathrm{Op} S\left(\langle x\rangle^{-2 \sigma}, G\right), D_{j} \in \mathrm{Op} S(\langle\xi\rangle, G)$. It follows that the operator $A$ introduced in (2.1.9) belongs to $\mathrm{Op} S(1, G)$ and therefore is $L^{2}$ continuous. It follows then from (2.1.8), (2.1.10) that

$$
\left\|\int_{0}^{t} e^{i(t-s) \Delta_{g}}\left[F_{2}(s, \cdot)\right] d s\right\|_{L^{q}\left(I, L^{r}\left(\mathbb{R}^{n}\right)\right)} \leqslant C\left[\int_{0}^{T}\|u(s, \cdot)\|_{H_{-\sigma}^{1 / 2}\left(\mathbb{R}^{n}\right)}^{2} d s\right]^{1 / 2} .
$$

Using Theorem 2.1.2 for $P$ we deduce that

$$
\left\|\int_{0}^{t} e^{i(t-s) \Delta_{g}}\left[F_{2}(s, \cdot)\right] d s\right\|_{L^{q}\left(I, L^{r}\left(\mathbb{R}^{n}\right)\right)} \leqslant C^{\prime}\left\|u_{0}\right\|_{L^{2}\left(\mathbb{R}^{n}\right)} .
$$

Gathering the informations given by (2.1.4), (2.1.5), (2.1.7) and (2.1.11) we obtain the conclusion of Theorem 1.0.1. So we are left with the proof of Theorem 2.1.5.

\subsection{Reduction to a small perturbation}

The purpose of this Section is to show that, using the result of 2.1 one can reduce the proof of Theorem 2.1.5 to the case of a small perturbation of the flat Laplacian.

Let $\varphi$ be in $C_{0}^{\infty}\left(\mathbb{R}^{n}\right)$. We write $e^{i t \Delta_{g}} u_{0}=u$ and

$$
u=\varphi u+(1-\varphi) u=v+w
$$


(i) Estimate of $v$. - Since $v=\varphi u$ it follows from (1.0.7) that $\left(i \partial_{t}+\Delta_{g}\right) v=\left[\Delta_{g}, \varphi\right] u$. Let $\varphi_{1} \in C_{0}^{\infty}\left(\mathbb{R}^{n}\right)$ be such $\varphi_{1}=1$ on the support of $\varphi$ then setting $\Delta=\sum_{j=1}^{n} \frac{\partial^{2}}{\partial x_{j}^{2}}$ one can write

$$
\left(i \partial_{t}+\Delta_{g}\right) v=\left(i \partial_{t}+\Delta+\varphi_{1}\left(\Delta_{g}-\Delta\right) \varphi_{1}\right) v=\left[\Delta_{g}, \varphi\right] u
$$

and $\varphi_{1}\left(\Delta_{g}-\Delta\right)$ is a compactly supported perturbation of the flat Laplacian. Let us set $\widetilde{P}=-\Delta-\varphi_{1}\left(\Delta_{g}-\Delta\right) \varphi_{1}$. We have, from $(2.2 .2)$

$$
v=e^{-i t \widetilde{P}} \varphi u_{0}+\int_{0}^{t} e^{-i(t-s) \widetilde{P}}[f(s, \cdot)] d s
$$

where $f=\left[\Delta_{g}, \varphi\right] u$.

It follows from Theorem 2.1.1 that

$$
\left\|e^{-i t \widetilde{P}} \varphi u_{0}\right\|_{L^{q}\left([-T, T], L^{r}\left(\mathbb{R}^{n}\right)\right)} \leqslant C\left\|u_{0}\right\|_{L^{2}\left(\mathbb{R}^{n}\right)} .
$$

To estimate the second term in the right-hand side of (2.2.3) we shall use Lemma 2.1.4 with $a=-T, b=T, Y=L^{r}\left(\mathbb{R}^{n}\right), p=2, X=H^{-1 / 2}\left(\mathbb{R}^{n}\right)$. For this one first remark that if $U=e^{-i t \widetilde{P}}$ then Theorem 2.1.2 shows that $U$ is continuous from $L^{2}\left(\mathbb{R}^{n}\right)$ to $L^{2}\left([-T, T], H_{\text {loc }}^{1 / 2}\left(\mathbb{R}^{n}\right)\right)$. Then it is easy to see that $U^{*}: L^{2}\left([-T, T], H_{c}^{-1 / 2}\left(\mathbb{R}^{n}\right)\right) \rightarrow$ $L^{2}\left(\mathbb{R}^{n}\right)$ is continuous and is given by $U^{*} f(x)=\int_{0}^{T} e^{-i s \widetilde{P}}[f(s, \cdot)] d s$. It follows that

$$
\left\|\int_{0}^{T} e^{-i(t-s) \widetilde{P}}[f(s, \cdot)] d s\right\|_{L^{q}\left([-T, T], L^{r}\left(\mathbb{R}^{n}\right)\right)}=\left\|U U^{*} f\right\|_{L^{q}\left([-T, T], L^{r}\left(\mathbb{R}^{n}\right)\right)} .
$$

Then, using again Theorem 2.1.1 and the above continuity of $U^{*}$ we get

$$
\left\|U U^{*} f\right\|_{L^{q}\left([-T, T], L^{r}\left(\mathbb{R}^{n}\right)\right)} \leqslant C\left\|U^{*} f\right\|_{L^{2}\left(\mathbb{R}^{n}\right)} \leqslant C^{\prime}\|f\|_{L^{2}\left([-T, T], H^{-1 / 2}\left(\mathbb{R}^{n}\right)\right)}
$$

since $f=\left[\Delta_{g}, \varphi\right] u$ has compact support in $x$.

Now we use Lemma 2.1.4 to deduce that

$$
\left\|\int_{0}^{t} e^{-i(t-s) \widetilde{P}}[f(s, \cdot)] d s\right\|_{L^{q}\left([-T, T], L^{r}\left(\mathbb{R}^{n}\right)\right)} \leqslant C^{\prime \prime}\|f\|_{L^{2}\left([-T, T], H^{-1 / 2}\left(\mathbb{R}^{n}\right)\right)}
$$

since $f(s, \cdot)$ has compact support in $x$ and $q>2$.

Moreover since $\left[\Delta_{g}, \varphi\right]$ is first order we have, using again Theorem 2.1.2,

$$
\|f\|_{L^{2}\left([-T, T], H^{-1 / 2}\left(\mathbb{R}^{n}\right)\right)} \leqslant C\|\psi u\|_{L^{2}\left([-T, T], H^{1 / 2}\left(\mathbb{R}^{n}\right)\right)} \leqslant C^{\prime}\left\|u_{0}\right\|_{L^{2}\left(\mathbb{R}^{n}\right)}
$$

where $\psi \in C_{0}^{\infty}\left(\mathbb{R}^{n}\right), \psi=1$ on the support of $\varphi$. This gives the estimate of the second term in the right hand side of (2.2.3) which, together with (2.2.4) shows that $v$ satisfies the Strichartz estimate.

(ii) Estimate of $w$. - We shall take the function $\varphi$, introduced above, of the following form. Let $R>0$ (which will be chosen large enough) and $\varphi_{0} \in C_{0}^{\infty}\left(\mathbb{R}^{n}\right)$ such that $\varphi_{0}(x)=1$ if $|x| \leqslant \frac{3}{2}, \operatorname{supp} \varphi_{0} \subset[-2,2]$. We shall take $\varphi(x)=\varphi_{R}(x)=\varphi_{0}(x / R)$.

Let $\widetilde{\varphi}_{0} \in C_{0}^{\infty}\left(\mathbb{R}^{n}\right)$ be such that $\widetilde{\varphi}_{0}(x)=1$ if $|x| \leqslant \frac{1}{2}$, supp $\widetilde{\varphi}_{0} \subset[-1,1]$ and let us set $\widetilde{\varphi}_{R}(x)=\widetilde{\varphi}_{0}\left(\frac{x}{R}\right)$. 
Let $w=\left(1-\varphi_{R}\right) u$ be the second term in the right hand side of (2.2.1). Since $1-\widetilde{\varphi}_{R}=1$ on the support of $1-\varphi_{R}$ we have according to (1.0.2)

$$
\left(i \partial_{t}+\Delta_{g}\right) w=\left(i \partial_{t}+\Delta+\sum_{j, k=1}^{n} \frac{\partial}{\partial x_{j}}\left[\left(1-\widetilde{\varphi}_{R}\right) b_{j k} \frac{\partial}{\partial x_{k}}\right]\right) w=-\left[\Delta_{g}, \varphi_{R}\right] u
$$

where $b_{j k}=g^{j k}-\delta_{j k}$.

Now if we denote by $f$ one of the coefficients $b_{j k}$ we claim that we have

$$
\left|\partial_{x}^{\alpha}\left[\left(1-\widetilde{\varphi}_{R}\right) f\right](x)\right| \leqslant \frac{1}{R^{\sigma_{0} / 2}} \frac{C_{\alpha}}{\langle x\rangle^{|\alpha|+1+\frac{\sigma_{0}}{2}}}, \quad \forall x \in \mathbb{R}^{n} .
$$

Using (1.0.1) and denoting by $A$ the left hand side of (2.2.6) we see that

$$
\begin{aligned}
& A \leqslant\left(1-\widetilde{\varphi}\left(\frac{x}{R}\right)\right)\left|\partial_{x}^{\alpha} f(x)\right|+\sum_{0<\beta \leqslant \alpha}\left(\begin{array}{c}
\alpha \\
\beta
\end{array}\right) \frac{1}{R^{|\beta|}}\left|\left(\partial_{x}^{\beta} \widetilde{\varphi}\right)\left(\frac{x}{R}\right)\right|\left|\partial_{x}^{\alpha-\beta} f(x)\right| \\
& A \leqslant\left(1-\widetilde{\varphi}\left(\frac{x}{R}\right)\right) \frac{C_{\alpha}}{\langle x\rangle^{|\alpha|+1+\sigma_{0}}}+\sum_{0<\beta \leqslant \alpha} \frac{C_{\alpha \beta}^{\prime}}{R^{|\beta|}}\left|\left(\partial_{x}^{\beta} \widetilde{\varphi}\right)\left(\frac{x}{R}\right)\right| \frac{1}{\langle x\rangle^{|\alpha|-|\beta|+1+\sigma_{0}}} .
\end{aligned}
$$

Now, on the support of $1-\widetilde{\varphi}(x / R)$ we have $\langle x\rangle>|x| \geqslant \frac{3}{2} R$ so the first term is bounded by

$$
\frac{C_{\alpha}^{\prime}}{R^{\sigma_{0} / 2}} \frac{1}{\langle x\rangle^{|\alpha|+1+\frac{\sigma_{0}}{2}}}
$$

On the support of $\partial^{\beta} \widetilde{\varphi}\left(\frac{x}{R}\right)$, with $\beta \neq 0$, we have $\frac{1}{2} R \leqslant|x| \leqslant R$ so $\langle x\rangle \leqslant \sqrt{2} R$ if $R>1$. Therefore the second term is bounded by

$$
\frac{1}{R^{\sigma_{0} / 2}} \sum_{0<\beta \leqslant \alpha} C_{\alpha \beta}^{\prime \prime} \frac{1}{\langle x\rangle^{|\beta|-\frac{\sigma_{0}}{2}+|\alpha|-|\beta|+1+\sigma_{0}}} \leqslant \frac{1}{R^{\sigma_{0} / 2}} \frac{C_{\alpha}^{\prime \prime}}{\langle x\rangle^{|\alpha|+1+\frac{\sigma_{0}}{2}}} .
$$

It follows from (2.2.6) that we can work in the rest of the paper with a non negative self adjoint operator $P$ such that

$$
\left\{\begin{array}{l}
P=-\Delta+\varepsilon Q, \text { where } Q=\sum_{|\beta| \leqslant 2} a_{\beta}^{\varepsilon} D^{\beta}, \\
\varepsilon \text { is a small constant and }\left|D_{x}^{\alpha} a_{\beta}^{\varepsilon}(x)\right| \leqslant C_{\alpha} /\langle x\rangle^{|\alpha|+1+\sigma_{0} / 2}, \forall \alpha \in \mathbb{N}^{n}, \\
\text { uniformly for } x \in \mathbb{R}^{n} \text { with } C_{\alpha} \text { independent of } \varepsilon .
\end{array}\right.
$$

Since the estimates on the coefficients are uniform in $\varepsilon$ we shall write $a_{\beta}$ instead of $a_{\beta}^{\varepsilon}$. The principal symbol $p$ of $P$ will be written as

$$
p(x, \xi)=|\xi|^{2}+\varepsilon q(x, \xi), \quad q(x, \xi)=\sum_{j, k=1}^{n} b_{j k}(x) \xi_{j} \xi_{k}
$$

and we shall take $\varepsilon$ so small that

$$
\frac{9}{10}|\xi|^{2} \leqslant p(x, \xi) \leqslant \frac{11}{10}|\xi|^{2} .
$$

Finally without loss of generality we shall take $\sigma_{0}$ instead of $\frac{\sigma_{0}}{2}$ in (2.2.7).

We assume that $P$ satisfies the condition (1.0.3) and (1.0.6). Let $T>0$. 
TheOrem 2.2.1. - Let $(q, r)$ be such $q \geqslant 2$ and $\frac{2}{q}=\frac{n}{2}-\frac{n}{r}$. If $\varepsilon$ is small enough then there exists $C>0$ such that

$$
\left\|e^{-i t P} v_{0}\right\|_{L^{q}\left([-T, T], L^{r}\left(\mathbb{R}^{n}\right)\right)} \leqslant C\left\|v_{0}\right\|_{L^{2}\left(\mathbb{R}^{n}\right)}
$$

for all $v_{0} \in L^{2}\left(\mathbb{R}^{n}\right)$.

Let us assume that we have proved this result. Then we can applied it to the operator occurring in (2.2.5) with $R$ large enough. We have,

$$
w=e^{-i t P}\left(1-\varphi_{R}\right) u_{0}+\int_{0}^{t} e^{-i(t-s) P}\left[f_{R}(s, \cdot)\right] d s .
$$

It follows from Theorem 2.2.1 that

$$
\left\|e^{-i t P}\left(1-\varphi_{R}\right) u_{0}\right\|_{L^{q}\left([-T, T], L^{r}\left(\mathbb{R}^{n}\right)\right)} \leqslant C\left\|u_{0}\right\|_{L^{2}\left(\mathbb{R}^{n}\right)}
$$

and the same argument as used in the estimate of $v$, namely the use of Theorem 2.2.1, and Lemma 2.1.4 shows that

$$
\left\|\int_{0}^{t} e^{-i(t-s) P}\left[f_{R}(s, \cdot)\right] d s\right\|_{L^{q}\left([-T, T], L^{r}\left(\mathbb{R}^{n}\right)\right)} \leqslant C(R)\left\|u_{0}\right\|_{L^{2}\left(\mathbb{R}^{n}\right)} .
$$

Then using (2.2.8) we see that the second term $w$ in the right hand side of (2.2.1) satisfies the Strichartz estimate which completes the proof of Theorem 2.1.5.

Our goal now is to prove Theorem 2.2.1. The first step is to make a careful study of the flow. 



\section{CHAPTER 3}

\section{STUDY OF THE FLOW}

\subsection{Preliminaries}

Let $p(x, \xi)=|\xi|^{2}+\varepsilon q(x, \xi), q(x, \xi)=\sum_{j, k=1}^{n} b_{j k}(x) \xi_{j} \xi_{k}$ where,

$$
\left\{\begin{array}{l}
\text { there exists } \sigma_{0}>0 \text { such that for every } \ell \in \mathbb{N} \text { one can find } A_{\ell}>0 \\
\text { such that } \sum_{|\alpha|=\ell} \sum_{j, k=1}^{n}\left|\partial_{x}^{\alpha} b_{j k}(x)\right| \leqslant A_{\ell} /\langle x\rangle^{1+\ell+\sigma_{0}} \text { for all } x \text { in } \mathbb{R}^{n} .
\end{array}\right.
$$

We introduce the equations of the bicharacteristic flow issued from a point $(x, \xi)$ in $T^{*} \mathbb{R}^{n} \backslash\{0\}$. They are given for $j=1, \ldots, n$, by

$$
\left\{\begin{array}{l}
\dot{x}_{j}(t)=\frac{\partial p}{\partial \xi_{j}}(x(t), \xi(t)), \quad x_{j}(0)=x_{j}, \\
\dot{\xi}_{j}(t)=-\frac{\partial p}{\partial x_{j}}(x(t), \xi(t)), \xi_{j}(0)=\xi_{j},
\end{array}\right.
$$

and we denote by $(x(t, x, \xi), \xi(t, x, \xi))$ the solution of (3.1.2) whenever it exists (or $(x(t), \xi(t))$ for short if no confusion is possible).

Let us remark that when $p(x, \xi)=|\xi|^{2}$ then

$$
\left\{\begin{array}{l}
x(t, x, \xi)=x+2 t \xi \\
\xi(t, x, \xi)=\xi
\end{array}\right.
$$

In general case assuming $\varepsilon$ so small that $\varepsilon A_{0} \leqslant \frac{1}{10}$ we see that $\frac{9}{10}|\xi|^{2} \leqslant p(x, \xi) \leqslant$ $\frac{11}{10}|\xi|^{2}$. It follows that

$$
\frac{9}{10}|\xi(t, x, \xi)|^{2} \leqslant p(x(t), \xi(t))=p(x, \xi) \leqslant \frac{11}{10}|\xi|^{2},
$$

so that

$$
|\xi(t, x, \xi)| \leqslant 2|\xi| .
$$

Using the first equation of (3.1.2) we see then, that the solution of (3.1.2) exists for all $t$ in $\mathbb{R}$ and is a $C^{\infty}$ function with respect to $(x, \xi)$. Moreover we have the following lemma. 
LEMmA 3.1.1. - For all $t$ in $\mathbb{R}$ we have

$$
x(t, x, \xi) \cdot \xi(t, x, \xi)=x \cdot \xi+2 t p(x, \xi)+f(t, x, \xi)
$$

where

$$
|f(t, x, \xi)| \leqslant 4 \varepsilon A_{1}|\xi|^{2}\left|\int_{0}^{t} \frac{d s}{\langle x(s)\rangle^{1+\sigma_{0}}}\right| \leqslant 4 \varepsilon A_{1}|t||\xi|^{2} .
$$

Proof. — We have by (3.1.2)

$$
\frac{d}{d t}[x(t) \cdot \xi(t)]=\xi(t) \cdot \frac{\partial p}{\partial \xi}(x(t), \xi(t))-\varepsilon x(t) \cdot \frac{\partial q}{\partial x}(x(t), \xi(t)) .
$$

Using Euler's identity we obtain

$$
\xi(t) \cdot \frac{\partial p}{\partial \xi}(x(t), \xi(t))=2 p(x(t), \xi(t))=2 p(x, \xi) .
$$

We set $f(t, x, \xi)=-\varepsilon \int_{0}^{t} x(s) \cdot \frac{\partial q}{\partial x}(x(s), \xi(s)) d s$. Now since

$$
\left|\frac{\partial q}{\partial x}(x(s), \xi(s))\right| \leqslant \frac{A_{1}}{\langle x(s)\rangle^{2+\sigma_{0}}}|\xi(s)|^{2},
$$

it follows from (3.1.4) that

$$
|f(t, x, \xi)| \leqslant 4 \varepsilon A_{1}|\xi|^{2}\left|\int_{0}^{t} \frac{d s}{\langle x(s)\rangle^{1+\sigma_{0}}}\right| \leqslant 4 \varepsilon A_{1}|t||\xi|^{2} .
$$

We shall use later on the result given by the following lemma.

For $t \in \mathbb{R}$ and $(x, \xi) \in T^{*} \mathbb{R}^{n} \backslash\{0\}$ let us set,

$$
\rho(t, x, \xi)=(x(t, x, \xi), \xi(t, x, \xi))
$$

LEMMA 3.1.2. - We have the following identities for $j, k=1, \ldots n$,

$$
\begin{aligned}
& \frac{\partial x_{j}}{\partial x_{k}}(t, x, \xi)=\frac{\partial \xi_{k}}{\partial \xi_{j}}(-t, \rho(t, x, \xi)) \\
& \frac{\partial x_{j}}{\partial \xi_{k}}(t, x, \xi)=-\frac{\partial x_{k}}{\partial \xi_{j}}(-t, \rho(t, x, \xi)) \\
& \frac{\partial \xi_{j}}{\partial x_{k}}(t, x, \xi)=-\frac{\partial \xi_{k}}{\partial x_{j}}(-t, \rho(t, x, \xi)) \\
& \frac{\partial \xi_{j}}{\partial \xi_{k}}(t, x, \xi)=\frac{\partial x_{k}}{\partial x_{j}}(-t, \rho(t, x, \xi)) .
\end{aligned}
$$

Proof. - For $j=1, \ldots, n$ we have

$$
\left\{\begin{array}{l}
x_{j}(-t ; \rho(t ; x, \xi))=x_{j} \\
\xi_{j}(-t ; \rho(t, x, \xi))=\xi_{j}
\end{array}\right.
$$

MÉMOIRES DE LA SMF 101/102 
Differentiating both sides with respect to $x_{k}$ and $\xi_{k}$ we obtain

$$
\begin{aligned}
& \sum_{\ell=1}^{n} \frac{\partial x_{j}}{\partial x_{\ell}}(-t ; \rho(t ; x, \xi)) \frac{\partial x_{\ell}}{\partial x_{k}}(t ; x, \xi)+\sum_{\ell=1}^{n} \frac{\partial x_{j}}{\partial \xi_{\ell}}(-t ; \rho(t ; x, \xi)) \frac{\partial \xi_{\ell}}{\partial x_{k}}(t ; x, \xi)=\delta_{j k} \\
& \sum_{\ell=1}^{n} \frac{\partial x_{j}}{\partial x_{\ell}}(-t ; \rho(t ; x, \xi)) \frac{\partial x_{\ell}}{\partial \xi_{k}}(t ; x, \xi)+\sum_{\ell=1}^{n} \frac{\partial x_{j}}{\partial \xi_{\ell}}(-t ; \rho(t ; x, \xi)) \frac{\partial \xi_{\ell}}{\partial \xi_{k}}(t ; x, \xi)=0 \\
& \sum_{\ell=1}^{n} \frac{\partial \xi_{j}}{\partial x_{\ell}}(-t ; \rho(t ; x, \xi)) \frac{\partial x_{\ell}}{\partial x_{k}}(t ; x, \xi)+\sum_{\ell=1}^{n} \frac{\partial \xi_{j}}{\partial \xi_{\ell}}(-t ; \rho(t ; x, \xi)) \frac{\partial \xi_{\ell}}{\partial x_{k}}(t ; x, \xi)=0 \\
& \sum_{\ell=1}^{n} \frac{\partial \xi_{j}}{\partial x_{\ell}}(-t ; \rho(t ; x, \xi)) \frac{\partial x_{\ell}}{\partial \xi_{k}}(t ; x, \xi)+\sum_{\ell=1}^{n} \frac{\partial \xi_{j}}{\partial \xi_{\ell}}(-t ; \rho(t ; x, \xi)) \frac{\partial \xi_{\ell}}{\partial \xi_{k}}(t ; x, \xi)=\delta_{j k}
\end{aligned}
$$

where $\delta_{j k}$ is the Kronecker symbol.

If we set

$$
M(t ; \rho)=\left(\begin{array}{ll}
\left(\partial x_{j} / \partial x_{k}\right)(t ; \rho) & \left(\partial x_{j} / \partial \xi_{k}\right)(t ; \rho) \\
\left(\partial \xi_{j} / \partial x_{k}\right)(t ; \rho) & \left(\partial \xi_{j} / \partial \xi_{k}\right)(t ; \rho)
\end{array}\right)
$$

then the above relations can be written

$$
M(-t ; \rho(t ; x, \xi)) \cdot M(t ; x, \xi)=\left(\begin{array}{cc}
I_{n} & 0 \\
0 & I_{n}
\end{array}\right)
$$

where $I_{n}$ denotes the $n \times n$ identity matrix.

Let us introduce for $s \in \mathbb{R}$ the following matrix.

$$
A(s ; \rho)=\left(\begin{array}{cc}
{ }^{t}\left(\partial \xi_{j} / \partial \xi_{k}\right)(s ; \rho) & -{ }^{t}\left(\partial x_{j} / \partial \xi_{k}\right)(s ; \rho) \\
-{ }^{t}\left(\partial \xi_{j} / \partial x_{k}\right)(s ; \rho) & { }^{t}\left(\partial x_{j} / \partial x_{k}\right)(s ; \rho)
\end{array}\right) .
$$

We claim that for $s \in \mathbb{R}$ and $\rho \in T^{*} \mathbb{R}^{n}$ we have

$$
A(s ; \rho) M(s ; \rho)=I_{2 n}
$$

where $I_{2 n}$ is the $2 n \times 2 n$ identity matrix.

Indeed let us set $A(s ; \rho) \cdot M(s ; \rho)=\left(C_{\alpha \beta}\right)_{1 \leqslant \alpha, \beta \leqslant 2 n}$. We have for $j, k=1 \ldots n$,

$$
\left\{\begin{aligned}
C_{j, k} & =\sum_{\ell=1}^{n}\left(\frac{\partial \xi_{\ell}}{\partial \xi_{j}} \frac{\partial x_{\ell}}{\partial x_{k}}-\frac{\partial x_{\ell}}{\partial \xi_{j}} \frac{\partial \xi_{\ell}}{\partial x_{k}}\right)(s ; \rho) \\
C_{j, k+n} & =\sum_{\ell=1}^{n}\left(\frac{\partial \xi_{\ell}}{\partial \xi_{j}} \frac{\partial x_{\ell}}{\partial \xi_{k}}-\frac{\partial x_{\ell}}{\partial \xi_{j}} \frac{\partial \xi_{\ell}}{\partial \xi_{k}}\right)(s ; \rho) \\
C_{j+n, k} & =\sum_{\ell=1}^{n}\left(\frac{\partial x_{\ell}}{\partial x_{j}} \frac{\partial \xi_{\ell}}{\partial x_{k}}-\frac{\partial x_{\ell}}{\partial x_{k}} \frac{\partial \xi_{\ell}}{\partial x_{j}}\right)(s ; \rho) \\
C_{j+n, k+n} & =\sum_{\ell=1}^{n}\left(\frac{\partial x_{\ell}}{\partial x_{j}} \frac{\partial \xi_{\ell}}{\partial \xi_{k}}-\frac{\partial \xi_{\ell}}{\partial x_{j}} \frac{\partial x_{\ell}}{\partial \xi_{k}}\right)(s ; \rho) .
\end{aligned}\right.
$$

Let us remark that $C_{j+n, k+n}=C_{k, j}$. 
Now we recall that for every $s \in \mathbb{R}$ the map $(x, \xi) \mapsto \rho(s ; x, \xi)$ is symplectic which means that

$$
\sum_{\ell=1}^{n} d\left(\xi_{\ell}(s ; x, \xi)\right) \wedge d\left(x_{\ell}(s ; x, \xi)\right)=\sum_{j=1}^{n} d \xi_{j} \wedge d x_{j} .
$$

Writing $u(s)=u(s ; x, \xi)$ for short we have

$$
\begin{aligned}
(1)=\sum_{\ell=1}^{n} d\left(\xi_{\ell}(s)\right) \wedge d\left(x_{\ell}(s)\right)= & \sum_{\ell=1}^{n}\left(\sum_{j=1}^{n}\left(\frac{\partial \xi_{\ell}}{\partial x_{j}}(s) d x_{j}+\frac{\partial \xi_{\ell}}{\partial \xi_{j}}(s) d \xi_{j}\right)\right. \\
& \left.\wedge \sum_{k=1}^{n}\left(\frac{\partial x_{\ell}}{\partial x_{k}}(s) d x_{k}+\frac{\partial x_{\ell}}{\partial \xi_{k}}(s) d \xi_{k}\right)\right)
\end{aligned}
$$

It follows that

$$
\begin{aligned}
(1)= & \sum_{j \leqslant k}\left(\sum_{\ell=1}^{n}\left(\frac{\partial \xi_{\ell}}{\partial x_{j}}(s) \frac{\partial x_{\ell}}{\partial x_{k}}(s)-\frac{\partial \xi_{\ell}}{\partial x_{k}}(s) \frac{\partial x_{\ell}}{\partial x_{j}}(s)\right)\right) d x_{j} \wedge d x_{k}+ \\
& \sum_{j, k=1}^{n}\left(\sum_{\ell=1}^{n}\left(\frac{\partial \xi_{\ell}}{\partial \xi_{j}}(s) \frac{\partial x_{\ell}}{\partial x_{k}}(s)-\frac{\partial \xi_{\ell}}{\partial x_{k}}(s) \frac{\partial x_{\ell}}{\partial \xi_{j}}(s)\right)\right) d \xi_{j} \wedge d x_{k}+ \\
& \sum_{j \leqslant k}\left(\sum_{\ell=1}^{n}\left(\frac{\partial \xi_{\ell}}{\partial \xi_{j}}(s) \frac{\partial x_{\ell}}{\partial \xi_{k}}(s)-\frac{\partial \xi_{\ell}}{\partial \xi_{k}}(s) \frac{\partial x_{\ell}}{\partial \xi_{j}}(s)\right)\right) d \xi_{j} \wedge d \xi_{k} .
\end{aligned}
$$

Using (3.1.9) and (3.1.10) we see easily that

$$
C_{j, k}=\delta_{j k}, \quad C_{j, k+n}=C_{j+n, k}=0, \quad C_{j+n, k+n}=C_{k, j}=\delta_{j k} .
$$

This proves (3.1.8).

It follows from (3.1.6) and (3.1.8) that

$$
M(t ; x, \xi)=A(-t ; \rho(t ; x, \xi))
$$

which by (3.1.7) proves the Lemma 3.1.2.

\subsection{The flow for short time}

Here is a description of the flow for short time.

Proposition 3.2.1. - Let us set

$$
\left\{\begin{array}{l}
r(t, x, \xi)=x(t, x, \xi)-(x+2 t \xi) \\
\zeta(t, x, \xi)=\xi(t, x, \xi)-\xi
\end{array}\right.
$$

Let $T>0$. Then for all $A, B$ in $\mathbb{N}^{n}$ one can find $C_{A, B}>0$ such that

$$
\left\{\begin{array}{l}
\text { (i) }\left|\partial_{x}^{A} \partial_{\xi}^{B} Z(t, x, \xi)\right| \leqslant C_{A, B} \varepsilon|t| \\
\text { (ii) }\left|\partial_{t} \partial_{x}^{A} \partial_{\xi}^{B} Z(t, x, \xi)\right| \leqslant C_{A, B} \varepsilon
\end{array}\right.
$$

if $Z=r$ or $\zeta$, for all $|t| \leqslant T$ and all $(x, \xi) \in T^{*} \mathbb{R}^{n}$ with $|\xi| \leqslant 3$. 
Proof. - See Appendix, Paragraph A.2.

We introduce now the following definition which distinguish microlocally the points in the cotangent bundle.

Definition 3.2.2. - Let

$$
\begin{aligned}
& \mathcal{S}_{+}=\left\{(x, \xi) \in T^{*} \mathbb{R}^{n} \backslash\{0\}: x \cdot \xi \geqslant-\frac{1}{4}\langle x\rangle|\xi|\right\} \\
& \mathcal{S}_{-}=\left\{(x, \xi) \in T^{*} \mathbb{R}^{n} \backslash\{0\}: x \cdot \xi \leqslant \frac{1}{4}\langle x\rangle|\xi|\right\} .
\end{aligned}
$$

Then $\mathcal{S}_{+}$(resp. $\mathcal{S}_{-}$) is called the set of outgoing points for $t \geqslant 0$ (resp. $t \leqslant 0$ ).

Of course the constant $\frac{1}{4}$ in the above definition is unimportant and could be replaced by any fixed small constant. The reason for this definition is the following. If $(x, \xi) \in \mathcal{S}_{+}$then, for $t \geqslant 0$

$$
1+|x+2 t \xi|^{2} \geqslant \frac{1}{2}\left(\langle x\rangle^{2}+t^{2}|\xi|^{2}\right) .
$$

Since $x+2 t \xi$ will be an approximation of $x(t ; x, \xi)$, then $\mathcal{S}_{+}$, will be the set of points $(x, \xi)$ for which the projection of the bicharacteristic goes to $+\infty$ when $t \rightarrow+\infty$ in staying away from the origin.

\subsection{The forward flow from points in $\mathcal{S}_{+}$and backward from $\mathcal{S}_{-}$}

Our goal is to obtain for these points a nice global representation of the flow together with precise estimates of its derivatives with respect to $x$ and $\xi$.

Proposition 3.3.1. - There exists $\varepsilon_{0}>0$ depending on the constants $A_{0}, A_{1}$ in (3.1.1) such that for $\varepsilon$ in $] 0, \varepsilon_{0}\left[\right.$ the solution of (3.1.2) with $(x, \xi)$ in $\mathcal{S}_{+}$(resp. $\mathcal{S}_{-}$) and $\frac{1}{2} \leqslant|\xi| \leqslant 2$ can be written for all $t \geqslant 0$ (resp. $t \leqslant 0$ )

$$
\left\{\begin{array}{l}
x(t ; x, \xi)=x+2 t \xi(t ; x, \xi)+z(t ; x, \xi) \\
\xi(t ; x, \xi)=\xi+\zeta(t ; x, \xi)
\end{array}\right.
$$

with

$$
\left|z_{j}(t ; x, \xi)\right| \leqslant \frac{2 \cdot 10^{2}}{\sigma_{0}} \varepsilon \max \left(A_{0}, A_{1}\right), \quad\left|\zeta_{j}(t ; x, \xi)\right| \leqslant \frac{2 \cdot 10^{2}}{\sigma_{0}} \varepsilon \max \left(A_{0}, A_{1}\right),
$$

where $A_{0} A_{1}$ are the constants arising in (3.1.1) and $j=1, \ldots, n$.

Moreover for all $t \geqslant 0$ (resp. $t \leqslant 0$ ) we have

$$
\frac{1}{3} \leqslant \frac{1+|x(t ; x, \xi)|^{2}}{1+|x|^{2}+t^{2}} \leqslant 40
$$

Proof. - Let

$$
\begin{array}{r}
I=\left\{T>0:\left|z_{j}(t)\right| \leqslant \frac{2 \cdot 10^{2}}{\sigma_{0}} \varepsilon \max \left(A_{0}, A_{1}\right),\left|\zeta_{j}(t)\right| \leqslant \frac{2 \cdot 10^{2}}{\sigma_{0}} \varepsilon \max \left(A_{0}, A_{1}\right)\right. \\
\text { for } j=1, \ldots, n \text { and all } t \in[0, T]\} .
\end{array}
$$


Then $I$ is an interval which is non empty by the local Cauchy-Lipschitz Theorem. Let $T^{*}=\sup I$. If $T^{*}=+\infty$ we are done. Otherwise let $T<T^{*}$. Since $\frac{1}{2} \leqslant|\xi| \leqslant 2$ we have for $t \in[0, T], \frac{1}{3} \leqslant|\xi(t)| \leqslant 3$ if $\varepsilon \max \left(A_{0}, A_{1}\right)$ is small enough. Indeed we have

$$
\begin{gathered}
\left(1-\varepsilon A_{0}\right)|\xi|^{2} \leqslant p(x, \xi)=p(x(t), \xi(t)) \leqslant\left(1+\varepsilon A_{0}\right)|\xi(t)|^{2} \\
\left(1-\varepsilon A_{0}\right)|\xi(t)|^{2} \leqslant p(x(t), \xi(t))=p(x, \xi) \leqslant\left(1+\varepsilon A_{0}\right)|\xi|^{2} .
\end{gathered}
$$

Now, for $t$ in $[0, T]$ we have

$$
\begin{aligned}
1+|x(t)|^{2}=\langle x\rangle^{2}+ & 4 t^{2}|\xi|^{2}+4 t^{2}|\zeta(t)|^{2}+|z(t)|^{2}+\underbrace{4 t x \cdot \xi}_{(1)}+\underbrace{4 t x \cdot \zeta(t)}_{(2)} \\
& +\underbrace{2 x \cdot z(t)}_{(3)}+\underbrace{8 t^{2} \xi \cdot \zeta(t)}_{(4)}+\underbrace{4 t \xi \cdot z(t)}_{(5)}+\underbrace{4 t \zeta(t) \cdot z(t)}_{(6)} .
\end{aligned}
$$

Since $(x, \xi) \in \mathcal{S}_{+}$we have for $t \geqslant 0,(1) \geqslant-\frac{1}{2}\left(\langle x\rangle^{2}+t^{2}|\xi|^{2}\right)$. Now, by the definition of $I$ we have on $[0, T]$ if $\varepsilon \max \left(A_{0}, A_{1}\right)$ is small enough.

$$
\begin{aligned}
& |(2)| \leqslant C_{1}(n) t|x| \varepsilon \max \left(A_{0}, A_{1}\right) \leqslant 10^{-2}\left(|x|^{2}+t^{2}\right) \\
& |(3)| \leqslant C_{2}(n)|x| \varepsilon \max \left(A_{0}, A_{1}\right) \leqslant 10^{-2}\langle x\rangle^{2} \\
& |(4)| \leqslant C_{3}(n) t^{2} \varepsilon \max \left(A_{0}, A_{1}\right) \leqslant 10^{-2} t^{2} \\
& |(5)| \leqslant C_{4}(n) t \varepsilon \max \left(A_{0}, A_{1}\right) \leqslant 10^{-2}\left(1+t^{2}\right) \\
& |(6)| \leqslant C_{5}(n) t\left(\varepsilon \max \left(A_{0}, A_{1}\right)\right)^{2} \leqslant 10^{-2}\left(1+t^{2}\right) .
\end{aligned}
$$

It follows that

$$
\langle x(t)\rangle^{2} \geqslant \frac{1}{2}\left(\langle x\rangle^{2}+t^{2}\right)-4 \cdot 10^{-2}\left(\langle x\rangle^{2}+t^{2}\right) \geqslant \frac{1}{3}\left(\langle x\rangle^{2}+t^{2}\right) .
$$

The same computation shows that $\langle x(t)\rangle^{2} \leqslant 40\left(\langle x\rangle^{2}+t^{2}\right)$. It follows that on $[0, T]$ we have

$$
\frac{1}{\sqrt{6}}(1+t) \leqslant \frac{1}{\sqrt{3}}\left(\langle x\rangle^{2}+t^{2}\right)^{1 / 2} \leqslant\langle x(t)\rangle \leqslant 7\left(\langle x\rangle^{2}+t^{2}\right)^{1 / 2} .
$$

Now it follows from (3.1.2) that $(z(t), \zeta(t))$ satisfy the equations

$$
\left\{\begin{array}{l}
\dot{z}_{j}(t)=-\varepsilon \frac{\partial q}{\partial \xi_{j}}(x(t), \xi(t))+2 t \varepsilon \frac{\partial q}{\partial x_{j}}(x(t), \xi(t)) \\
\dot{\zeta}_{j}(t)=-\varepsilon \frac{\partial q}{\partial x_{j}}(x(t), \xi(t))
\end{array}\right.
$$

with $z_{j}(0)=\zeta_{j}(0)=0$.

We deduce from (3.1.1), (3.3.4) and the bounds $\frac{1}{3} \leqslant|\xi(t)| \leqslant 3$ that

$$
\begin{aligned}
& \left|\frac{\partial q}{\partial \xi_{j}}(x(t), \xi(t))\right| \leqslant \frac{3 A_{0}}{\langle x(t)\rangle^{1+\sigma_{0}}} \leqslant \frac{3(\sqrt{6})^{1+\sigma_{0}} A_{0}}{(1+t)^{1+\sigma_{0}}} \leqslant \frac{12 A_{0}}{(1+t)^{1+\sigma_{0}}}, \\
& \left|\frac{\partial q}{\partial x_{j}}(x(t), \xi(t))\right| \leqslant \frac{9 A_{1}}{\langle x(t)\rangle^{2+\sigma_{0}}} \leqslant \frac{9(\sqrt{6})^{1+\sigma_{0}} \sqrt{3} A_{1}}{(1+t)^{1+\sigma_{0}}\langle t\rangle} \leqslant \frac{60 A_{1}}{(1+t)^{1+\sigma_{0}}\langle t\rangle},
\end{aligned}
$$


(since we may assume that $1+\sigma_{0}<\frac{3}{2}$ and $(\sqrt{6})^{1+\sigma_{0}}<4$ ). It follows from (3.3.5) that

$$
\left|\dot{z}_{j}(t)\right| \leqslant \frac{132 \varepsilon}{(1+t)^{1+\sigma_{0}}} \max \left(A_{0}, A_{1}\right), \quad\left|\dot{\zeta}_{j}(t)\right| \leqslant \frac{60 \varepsilon}{(1+t)^{1+\sigma_{0}}} \max \left(A_{0}, A_{1}\right) .
$$

Therefore we have on $[0, T]$

$$
\left|z_{j}(t)\right| \leqslant \frac{132}{\sigma_{0}} \varepsilon \max \left(A_{0}, A_{1}\right), \quad\left|\zeta_{j}(t)\right| \leqslant \frac{60}{\sigma_{0}} \varepsilon \max \left(A_{0}, A_{1}\right) .
$$

Since $z(t)$ and $\zeta(t)$ exist for all $t \geqslant 0$ and are smooth we still have the above estimates on $\left[0, T^{*}\right]$. By continuity it will exist $\eta>0$ such that $\left|z_{j}(t)\right| \leqslant \frac{2 \cdot 10^{2}}{\sigma_{0}} \varepsilon \max \left(A_{0}, A_{1}\right)$ and $\left|\zeta_{j}(t)\right| \leqslant \frac{2 \cdot 10^{2}}{\sigma_{0}} \varepsilon \max \left(A_{0}, A_{1}\right)$ on $\left[0, T^{*}+\eta\right]$. This contradicts the maximality of $T^{*}$ and proves that $T^{*}=+\infty$.

Now we estimate the derivatives of the flow with respect to $(x, \xi)$.

Proposition 3.3.2. - With the notations of Proposition 3.3.1, for every integer $k$ one can find a positive constant $M_{k}$ such that for all $(A, B) \in \mathbb{N}^{n} \times \mathbb{N}^{n}$ such that $|A|+|B| \leqslant k$, all $t \geqslant 0$ (resp. $t \leqslant 0)$ and $(x, \xi)$ in $\mathcal{S}_{+}$(resp. $\left.\mathcal{S}_{-}\right)$we have,

$$
\left\{\begin{array}{l}
\left|\partial_{x}^{A} \partial_{\xi}^{B} z(t, x, \xi)\right| \leqslant \frac{\varepsilon M_{k}}{\langle x\rangle^{|A|+\sigma_{0}}}, \\
\left|\partial_{x}^{A} \partial_{\xi}^{B} \zeta(t, x, \xi)\right| \leqslant \frac{\varepsilon M_{k}}{\langle x\rangle^{1+|A|+\sigma_{0}}} .
\end{array}\right.
$$

Proof. - See Appendix A.3.

Corollary 3.3.3. - Keeping the notations of Proposition 3.3.1 we have, for all $t \geqslant 0$ (resp. $t \leqslant 0)$ and all $(x, \xi) \in \mathcal{S}_{+}$(resp. $\left.\mathcal{S}_{-}\right)$

$$
\begin{gathered}
\frac{\partial x_{j}}{\partial \xi_{k}}(t, x, \xi)=2 t \delta_{j k}+\mathcal{O}(\varepsilon\langle t\rangle), \quad \frac{\partial x_{j}}{\partial x_{k}}(t, x, \xi)=\delta_{j k}+\mathcal{O}(\varepsilon\langle t\rangle) \\
\frac{\partial \xi_{j}}{\partial \xi_{k}}(t, x, \xi)=\delta_{j k}+\mathcal{O}(\varepsilon), \quad \frac{\partial \xi_{j}}{\partial x_{k}}(t, x, \xi)=\mathcal{O}(\varepsilon), \quad j, k=1, \ldots, n,
\end{gathered}
$$

where $\delta_{j k}$ is the Kronecker symbol and $\mathcal{O}(\varepsilon)$ means "bounded by $C \varepsilon$ where $C$ is independent of $(x, \xi)$ ". In particular we have

$$
\frac{\partial \xi_{j}}{\partial \xi_{k}}(t, x, \xi)-i \frac{\partial x_{j}}{\partial \xi_{k}}(t, x, \xi)=(1-2 i t) \delta_{j k}+\mathcal{O}(\varepsilon\langle t\rangle), \quad j, k=1, \ldots, n
$$

\subsection{Precisions on the flow in the general case}

The results obtained above allow us to give a rough form of the flow through any point in $T^{*} \mathbb{R}^{n} \backslash\{0\}$ for $t \in \mathbb{R}$.

Proposition 3.4.1. - Let $(x, \xi) \in T^{*} \mathbb{R}^{n} \backslash\{0\}$ with $|\xi| \leqslant 2$. Then,

(i) the function $s \mapsto\langle x(s, x, \xi)\rangle^{-\left(1+\sigma_{0}\right)}$ belongs to $L^{1}(\mathbb{R})$, 
(ii) for $t \in \mathbb{R}$ we have,

$$
\left\{\begin{array}{l}
x(t, x, \xi)=x+2 t \xi+r(t, x, \xi), \\
\xi(t, x, \xi)=\xi+\zeta(t, x, \xi)
\end{array}\right.
$$

where $|r(t, x, \xi)| \leqslant C \varepsilon\langle t\rangle,|\zeta(t, x, \xi)| \leqslant C \varepsilon$ with $C$ independent of $(x, \xi)$.

Before going into the proof let us note that in general we do not have good estimates on the derivatives of $r$ with respect to $(x, \xi)$ (in the spirit of those given in Proposition 3.3.1 for instance). In particular we do not have a good control of $\frac{\partial x_{j}}{\partial \xi_{k}}(t, x, \xi)$. This occurs for instance for points $(x, \xi)$ such that $|x|$ is very large and the bicharacteristic crosses back a neighborhood of the origin. That's why we used the term rough for this description.

Proof of Proposition 3.4.1. - If $|x \cdot \xi| \leqslant-\frac{1}{4}\langle x\rangle|\xi|$ then Proposition 3.3.1 gives the claimed description of the flow for $t \geqslant 0$ and $t \leqslant 0$. If $x \cdot \xi \leqslant-\frac{1}{4}\langle x\rangle|\xi|$ the same Proposition applies for $t \leqslant 0$ so we are left with the case $t \geqslant 0$. (The case $x \cdot \xi \geqslant$ $-\frac{1}{4}\langle x\rangle|\xi|$ is symmetric). It follows from Lemma 3.1.1 that, if $\varepsilon A_{1}$ is small enough, we have $\lim _{t \rightarrow+\infty} x(t) \cdot \xi(t)=+\infty$. Since $x \cdot \xi \leqslant 0$ one can find $t^{*}>0$ such that $x\left(t^{*}, x, \xi\right) \cdot \xi\left(t^{*}, x, \xi\right)=0$. If we set $x^{*}=x\left(t^{*}, x, \xi\right) \xi^{*}=\xi\left(t^{*}, x, \xi\right)$ then, according to Definition 3.2.2, we have $\left(x^{*}, \xi^{*}\right) \in \mathcal{S}_{+} \cap \mathcal{S}_{-}$so we can use Proposition 3.3.1 for $t \in \mathbb{R}$. Now we have by the flow property for $t \geqslant 0$,

$$
x(t, x, \xi)=x\left(t-t^{*}, x^{*}, \xi^{*}\right) .
$$

Using Proposition 3.3.1 we deduce the following lower bound

$$
\langle x(t, x, \xi)\rangle=\left\langle x\left(t-t^{*}, x^{*}, \xi^{*}\right)\right\rangle \geqslant \frac{1}{\sqrt{3}}\left\langle t-t^{*}\right\rangle .
$$

This proves the part (i) in Proposition 3.4.1. To prove part (ii) we use the formulas (3.1.2) for the flow. Then we see that for $t \geqslant 0$,

$$
\xi_{\ell}(t, x, \xi)=\xi_{\ell}+\zeta_{\ell}(t, x, \xi), \quad \zeta_{\ell}(t, x, \xi)=-\varepsilon \int_{0}^{t} \sum_{j k=1}^{n} \frac{\partial b_{j k}}{\partial x_{\ell}}(x(s)) \xi_{j}(s) \xi_{k}(s) d s .
$$

Then using (3.1.1), (3.4.1), (3.1.4) and the fact that $|\xi| \leqslant 2$ we see that $\left|\zeta_{\ell}(t, x, \xi)\right| \leqslant$ $C \varepsilon$, where $C$ depends only on $A_{1}$.

On the other hand we have

$$
\dot{x}_{j}(t, x, \xi)=2 \xi_{j}+2 \zeta_{j}(t, x, \xi)+2 \varepsilon \sum_{k=1}^{n} b_{j k}(x(t, x, \xi)) \xi_{k}(t, x, \xi) .
$$

Integrating between 0 and $t$ and using the above estimates we obtain the claimed description of $x(t, x, \xi)$. 


\subsection{The flow from points in $\left(\mathcal{S}_{+} \cap \mathcal{S}_{-}\right)^{c}$}

We study now, more carefully the flow from points $(x, \xi) \in T^{*} \mathbb{R}^{n} \backslash\{0\}$, such that,

$$
|x \cdot \xi|>c_{0}\langle x\rangle|\xi| \text { and } \frac{1}{2} \leqslant|\xi| \leqslant 2 .
$$

Even if, as we said before, we do not have a nice representation of the flow for all $t$ in $\mathbb{R}$ we shall see that such a representation is available for limited values of $t$.

Since the description is symmetric, we shall assume that

$$
x \cdot \xi \leqslant-c_{0}\langle x\rangle|\xi| .
$$

Then $(x, \xi) \in \mathcal{S}_{-}$and Proposition 3.3.1 give a good description of the flow for $t \leqslant 0$.

Definition 3.5.1. - Let $(x, \xi)$ satisfying 3.5.2. We set

$$
I_{+}=\left\{t \geqslant 0: x(t, x, \xi) \cdot \xi(t, x, \xi) \leqslant \frac{1}{4}\langle x(t, x, \xi)\rangle|\xi(t, x, \xi)|\right\} .
$$

In other words $I_{+}$is the set of $t \geqslant 0$ such that $(x(t, x, \xi), \xi(t, x, \xi))$ belongs to $\mathcal{S}_{-}$.

The main result of this Section is the following description of the flow on $I_{+}$.

Proposition 3.5.2. - Let $(x, \xi)$ satisfying 3.5.2. Then for $t$ in $I_{+}$we have

$$
\begin{aligned}
& x(t, x, \xi)=x+2 t \xi-z(-t, x(t, x, \xi), \xi(t, x, \xi)), \\
& \xi(t, x, \xi)=\xi-\zeta(-t, x(t, x, \xi), \xi(t, x, \xi)),
\end{aligned}
$$

where $z$ and $\zeta$ have been defined in Proposition 3.3.1. Moreover for $j, k=1, \ldots, n$ we have,

$$
\begin{array}{ll}
\frac{\partial x_{j}}{\partial x_{k}}(t, x, \xi)=\delta_{j k}+\mathcal{O}(\varepsilon), & \frac{\partial x_{j}}{\partial \xi_{k}}(t, x, \xi)=2 t \delta_{j k}+\mathcal{O}(\varepsilon\langle t\rangle) \\
\frac{\partial \xi_{j}}{\partial \xi_{k}}(t, x, \xi)=\delta_{j k}+\mathcal{O}(\varepsilon\langle t\rangle), & \frac{\partial \xi_{j}}{\partial x_{k}}(t, x, \xi)=\mathcal{O}(\varepsilon)
\end{array}
$$

where $\delta_{j k}$ is the Kronecker symbol and $\mathcal{O}(A)$ means "bounded by $C A$ " with $C$ independent of $(x, \xi)$. In particular we have

$$
\frac{\partial \xi_{j}}{\partial \xi_{k}}(t, x, \xi)-i \frac{\partial x_{j}}{\partial \xi_{k}}(t, x, \xi)=(1-2 i t) \delta_{j k}+\mathcal{O}(\varepsilon\langle t\rangle) .
$$

Proof. - As said before, for $t \in I_{+}$the point $\rho(t, x, \xi)=(x(t, x, \xi), \xi(t, x, \xi))$ belongs to $\mathcal{S}_{-}$. Therefore we can apply Proposition 3.3.1 for $\theta \leqslant 0$. We get

$$
\begin{aligned}
& x(\theta, \rho(t, x, \xi))=x(t, x, \xi)+2 \theta \xi(\theta, \rho(t, x, \xi))+z(\theta, \rho(t, x, \xi)) \\
& \xi(\theta, \rho(t, x, \xi))=\xi(t, x, \xi)+\zeta(\theta, \rho(t, x, \xi)) .
\end{aligned}
$$

Taking $\theta=-t$ with $t \geqslant 0$ we obtain

$$
\begin{aligned}
& x=x(t, x, \xi)-2 t \xi+z(-t, \rho(t, x, \xi)), \\
& \xi=\xi(t, x, \xi)+\zeta(-t, \rho(t, x, \xi)) .
\end{aligned}
$$

This proves the first part of Proposition 3.5.2. To prove the claim on the derivatives we use Lemma 3.1.2 and Corollary 3.3.3. 
Remark 3.5.3. - Since the points $(x, \xi)$ satisfying 3.5 .2 belong to $\mathcal{S}_{-}$, Propositions 3.3.1 and 3.5.2 provide a description of the flow on $(-\infty, 0) \cup I_{+}$. 


\section{CHAPTER 4}

\section{THE PHASE EQUATION}

The goal of this section is to solve approximatively the phase equation

$$
\frac{\partial \varphi}{\partial \theta}+p\left(x, \frac{\partial \varphi}{\partial x}\right)=0, \quad \varphi(0, x, \alpha)=\varphi_{0}(x, \alpha)
$$

(see Theorem 4.1.2). In the case of the flat Laplacian this problem can be solved exactly and we have

$$
\varphi(\theta, x, \alpha)=\frac{\left(x-\alpha_{x}\right) \alpha_{\xi}+\frac{i}{2}\left|x-\alpha_{x}\right|^{2}+\frac{1}{2 i}\left|\alpha_{\xi}\right|^{2}}{1+2 i \theta}
$$

In the general case the classical method using the symplectic geometry leads to a major difficulty. Indeed the symbol $p$ has $\mathcal{C}^{\infty}$ coefficients but since $\varphi_{0}$ has to be non real we must deal with a non real flow. Instead we use here a method introduced by Hörmander $[\mathbf{H}]$ called method of "Lagrangian ideals" which keeps real the data of the flow. It is briefly described in the Introduction (section 1 ).

The main result of this section is Theorem 4.1.2 whose proof is fairly long and could be skipped in a first lecture. One of the reasons for the length of the proof is that we have to consider separately the cases of outgoing and incoming points and then to match them. Moreover in the case of incoming points the flow behaves badly for large time which leads to serious difficulties.

\subsection{Statement of the result}

Let $p(x, \xi)=|\xi|^{2}+\varepsilon q(x, \xi), q(x, \xi)=\sum_{j, k=1}^{n} b_{j k}(x) \xi_{j} \xi_{k}$ where the coefficients $b_{j k}$ satisfy the condition (3.1.1).

In this Section $\alpha=\left(\alpha_{x}, \alpha_{\xi}\right)$ will be a fixed point in $T^{*} \mathbb{R}^{n}$ such that $\frac{1}{2} \leqslant\left|\alpha_{\xi}\right| \leqslant 2$. Let us recall that $(x(t, \alpha), \xi(t, \alpha))$ denotes the flow of $p$ starting for $t=0$ at the point $\alpha$.

We introduce now several sets.

Definition 4.1.1. - Let $\delta>0, c_{0}>0, c_{1}>0$ be small constants (chosen later on). 
(i) If $\left|\alpha_{x} \cdot \alpha_{\xi}\right| \leqslant c_{0}\left\langle\alpha_{x}\right\rangle\left|\alpha_{\xi}\right|$ we set,

$$
\Omega_{\delta}=\left\{(\theta, x) \in \mathbb{R} \times \mathbb{R}^{n}:|x-x(\theta, \alpha)| \leqslant \delta\langle\theta\rangle\right\} .
$$

(ii) If $\alpha_{x} \cdot \alpha_{\xi}>c_{0}\left\langle\alpha_{x}\right\rangle\left|\alpha_{\xi}\right|$ we set,

$$
\Omega_{\delta}=\left\{(\theta, x) \in \mathbb{R} \times \mathbb{R}^{n}:|x-x(\theta, \alpha)| \leqslant \delta\langle\theta\rangle, x \cdot \alpha_{\xi} \geqslant-c_{1}\langle x\rangle\left|\alpha_{\xi}\right|\right\} .
$$

(iii) If $\alpha_{x} \cdot \alpha_{\xi}<-c_{0}\left\langle\alpha_{x}\right\rangle\left|\alpha_{\xi}\right|$ we set,

$$
\Omega_{\delta}=\left\{(\theta, x) \in \mathbb{R} \times \mathbb{R}^{n}:|x-x(\theta, \alpha)| \leqslant \delta\langle\theta\rangle, x \cdot \alpha_{\xi} \leqslant c_{1}\langle x\rangle\left|\alpha_{\xi}\right|\right\} .
$$

Let us give some explanations on this Definition.

Taking $c_{0}$ and $c_{1}$ small with respect to $\frac{1}{4}$ we see from Definition 3.2.2 that the case (i) corresponds to points $\left(\alpha_{x}, \alpha_{\xi}\right)$ which are outgoing for $\theta \geqslant 0$ and $\theta \leqslant 0$. Then $\Omega_{\delta}$ is simply a conic neighborhood of the projection of the bicharacteristic. In the case (ii) the point $\left(\alpha_{x} \alpha_{\xi}\right)$ is outgoing for $\theta \geqslant 0$ and $\Omega_{\delta}$ can be written as follows.

$$
\begin{array}{r}
\Omega_{\delta}=\left\{(\theta, x) \in(0,+\infty) \times \mathbb{R}^{n}:|x-x(\theta, \alpha)| \leqslant \delta\langle\theta\rangle\right\} \cup\left\{(\theta, x) \in(-\infty, 0) \times \mathbb{R}^{n}:\right. \\
\left.|x-x(\theta, \alpha)| \leqslant \delta\langle\theta\rangle \text { and } x \cdot \alpha_{\xi} \geqslant-c_{1}\langle x\rangle\left|\alpha_{\xi}\right|\right\} .
\end{array}
$$

Indeed if $|x-x(\theta, \alpha)| \leqslant \delta\langle\theta\rangle$ and $\theta \geqslant 0$ we have by Proposition 3.4.1, $x \cdot \alpha_{\xi}=$ $(x-x(\theta, \alpha)) \cdot \alpha_{\xi}+\alpha_{x} \cdot \alpha_{\xi}+2 \theta\left|\alpha_{\xi}\right|^{2}+\mathcal{O}(\varepsilon\langle\theta\rangle)$. Since $\left|\alpha_{\xi}\right| \geqslant \frac{1}{2}$ and we are in case (ii) we deduce that $x \cdot \alpha_{\xi} \geqslant c_{0}\left\langle\alpha_{x}\right\rangle\left|\alpha_{\xi}\right|+\frac{1}{2} \theta-C(\varepsilon+\delta)\langle\theta\rangle \geqslant 0$ if $\varepsilon+\delta$ is small enough. Therefore when $\theta \geqslant 0$ the condition $x \cdot \alpha_{\xi} \geqslant-c_{1}\langle x\rangle\left|\alpha_{\xi}\right|$ is automatically satisfied.

In the case (iii) we have the same discussion changing $\theta \geqslant 0$ to $\theta \leqslant 0$.

The purpose of this Section is to prove the following result.

THEOREM 4.1.2. - There exist $\delta>0, c_{0}>0, c_{1}>0$ such that for any $\alpha \in T^{*} \mathbb{R}^{n}$ with $\frac{1}{2} \leqslant\left|\alpha_{\xi}\right| \leqslant 2$ one can find a function $\varphi=\varphi(\theta, x, \alpha)$ on $\Omega_{\delta}$ which is $C^{\infty}$ and satisfies the following.

(i) $\varphi(0, x, \alpha)=\left(x-\alpha_{x}\right) \cdot \alpha_{\xi}+\frac{i}{2}\left|x-\alpha_{x}\right|^{2}+\frac{1}{2 i}\left|\alpha_{\xi}\right|^{2}+g(x, \alpha)$

where $|g(x, \alpha)| \leqslant C_{N}\left|x-\alpha_{x}\right|^{N}$ for all $N \in \mathbb{N}$.

(ii) For any $N \in \mathbb{N}$ there exists $C_{N} \geqslant 0$ such that

$$
\left|\frac{\partial \varphi}{\partial \theta}(\theta, x, \alpha)+p\left(x, \frac{\partial \varphi}{\partial x}(\theta, x, \alpha)\right)\right| \leqslant C_{N}\left(\frac{|x-x(\theta, \alpha)|}{\langle\theta\rangle}\right)^{N}
$$

for all $(\theta, x)$ in $\Omega_{\delta}$.

Moreover for $(\theta, x)$ in $\Omega_{\delta}$ we have

(iii) $\left|\frac{\partial \varphi}{\partial x}(\theta, x, \alpha)-\alpha_{\xi}\right| \leqslant C(\varepsilon+\sqrt{\delta})$.

(iv) $\left.\left|\operatorname{Im} \varphi(\theta, x, \alpha)-\frac{1}{2} \frac{|x-x(\theta, \alpha)|^{2}}{1+4 \theta^{2}}+\frac{1}{2}\right| \alpha_{\xi}\right|^{2} \mid \leqslant C(\varepsilon+\sqrt{\delta}) \frac{|x-x(\theta, \alpha)|^{2}}{\langle\theta\rangle^{2}}$.

(v) $\left|\partial_{x}^{A} \varphi(\theta, x, \alpha)\right| \leqslant C_{A}$, for every $A$ in $\mathbb{N}^{n} \backslash\{0\}$

where $C, C_{N}$ and $C_{A}$ are independent of $(\theta, x, \alpha)$. 
The proof of this result is based on the theory of Lagrangian ideals of L. Hörmander ([H], vol 4, chap. XXV). It will require several steps. The first one is a slight extension of Theorem 7.5.4 in $[\mathbf{H}]$, vol. 1 to the case of higher dimensions.

\subsection{The preparation theorem}

The aim of this Section is to prove the following result.

LEMmA 4.2.1. - Let $g \in \mathcal{S}\left(\mathbb{R}_{\xi}^{n}\right)$ and $z \in \mathbb{C}^{n}$. Then there exist functions $q_{j}(\xi, z, g)$, $j=1, \ldots, n, r(z, g)$ which are $C^{\infty}$ with respect to $\xi$ and $z$, which depend linearly on $g$ such that

$$
\begin{gathered}
g(\xi)=\sum_{j=1}^{n} q_{j}(\xi, z, g)\left(\xi_{j}+z_{j}\right)+r(z, g) \\
\left\{\begin{array}{l}
\left|\partial_{\xi}^{\alpha} \partial_{z}^{\beta} q_{j}(\xi, z, g)\right| \leqslant C_{\alpha \beta} \sum_{|\gamma| \leqslant|\alpha|+|\beta|+4 n} \int\left|\partial_{\eta}^{\gamma} g(\eta)\right| d \eta \\
\left|\partial_{z}^{\beta} r(z, g)\right| \leqslant C_{\beta} \sum_{|\gamma| \leqslant|\beta|+3 n} \int^{\gamma} g(\eta) \mid d \eta .
\end{array}\right.
\end{gathered}
$$

Proof. - We proceed by induction on the dimension $n$. If $n=1$ this follows from Theorem 7.5 .4 of $[\mathbf{H}]$. Let $n \geqslant 2$ and let us set $\xi^{\prime}=\left(\xi_{1}, \ldots, \xi_{n-1}\right)$. For fixed $\xi^{\prime} \in \mathbb{R}^{n-1}$ we apply Theorem 7.5 .4 of $[\mathbf{H}]$ to the function $\xi_{n} \mapsto g\left(\xi^{\prime}, \xi_{n}\right)$. We get

$$
g\left(\xi^{\prime}, \xi_{n}\right)=q\left(\xi_{n}, z_{n}, g\left(\xi^{\prime}, \cdot\right)\right)\left(\xi_{n}+z_{n}\right)+r\left(z_{n}, g\left(\xi^{\prime}, \cdot\right)\right) .
$$

Let us set $Q_{n}\left(\xi, z_{n}, g\right)=q\left(\xi_{n}, z_{n}, g\left(\xi^{\prime}, \cdot\right)\right)$ and $\widetilde{r}\left(z_{n}, \xi^{\prime}, g\right)=r\left(z_{n}, g\left(\xi^{\prime}, \cdot\right)\right)$. Since $r$ is linear in $g$ we have $\partial_{\xi^{\prime}}^{\alpha} \widetilde{r}\left(z_{n}, \xi^{\prime}, g\right)=r\left(z_{n}, \partial_{\xi^{\prime}}^{\alpha} g\left(\xi^{\prime}, \cdot\right)\right)$ and the estimates (4.2.2) for $n=1$ show that $\xi^{\prime} \mapsto \widetilde{r}\left(z_{n}, \xi^{\prime}, g\right)$ is in $\mathcal{S}\left(\mathbb{R}^{n-1}\right)$. Therefore we can apply, by the induction, the Lemma to the function $\xi^{\prime} \mapsto \widetilde{r}\left(z_{n}, \xi^{\prime}, g\right)$ and to $z^{\prime}=\left(z_{1}, \ldots, z_{n-1}\right)$. We obtain the existence of $q_{j}, j=1, \ldots, n-1$ and $R$ satisfying the estimates (4.2.2) such that

$$
\widetilde{r}\left(z_{n}, \xi^{\prime}, g\right)=\sum_{j=1}^{n-1} q_{j}\left(\xi^{\prime}, z^{\prime}, \widetilde{r}\left(z_{n}, \cdot, g\right)\left(\xi_{j}+z_{j}\right)+R\left(z^{\prime}, \widetilde{r}\left(z_{n}, \cdot, g\right)\right) .\right.
$$

Using (4.2.3) we obtain therefore

$$
g(\xi)=Q_{n}\left(\xi, z_{n}, g\right)\left(\xi_{n}+z_{n}\right)+\sum_{j=1}^{n} q_{j}\left(\xi^{\prime}, z^{\prime}, \widetilde{r}\left(z_{n}, \cdot, g\right)\right)\left(\xi_{j}+z_{j}\right)+R\left(z^{\prime}, \widetilde{r}\left(z_{n}, \cdot, g\right)\right) .
$$

If we set

$$
\left\{\begin{array}{l}
Q_{j}(\xi, z, g)=q_{j}\left(\xi^{\prime}, z^{\prime}, \widetilde{r}\left(z_{n}, \cdot, g\right)\right), \quad j=1, \ldots, n-1 \\
r(z, g)=R\left(z^{\prime}, \widetilde{r}\left(z_{n}, \cdot, g\right)\right)
\end{array}\right.
$$


we obtain (4.2.1) at the level $n$. Moreover $Q_{j}$ and $r$ are linear in $g$ since $q_{j}, R$ are linear in $\widetilde{r}\left(z_{n}, \cdot, g\right)$ and $\widetilde{r}$ is linear in $g$. Let us look to the estimate (4.2.2) for $r$. We have

$$
\partial_{z^{\prime}}^{\beta^{\prime}} \partial_{z_{n}}^{\beta_{n}} r(z, g)=\partial_{z^{\prime}}^{\beta^{\prime}} R\left(z^{\prime}, \partial_{z_{n}}^{\beta_{n}} \widetilde{r}\left(z_{n}, \cdot, g\right)\right)
$$

so,

$$
\left|\partial_{z}^{\beta} r(z, g)\right| \leqslant C \sum_{\left|\gamma^{\prime}\right| \leqslant\left|\beta^{\prime}\right|+3(n-1)} \int\left|\partial_{\xi^{\prime}}^{\gamma^{\prime}} \partial_{z_{n}}^{\beta_{n}} \widetilde{r}\left(z_{n}, \xi^{\prime}, g\right)\right| d \xi^{\prime} .
$$

Now $\mid \partial_{\xi^{\prime}}^{\gamma^{\prime}} \partial_{z_{n}}^{\beta_{n}} \widetilde{r}\left(z_{n}, \xi^{\prime}, g\right)=\partial_{z_{n}}^{\beta_{n}} r\left(z_{n}, \partial_{\xi^{\prime}}^{\gamma^{\prime}} g\left(\xi^{\prime}, \cdot\right)\right)$ and from the case $n=1$ we have

$$
\left|\partial_{z_{n}}^{\beta_{n}} r\left(z_{n}, \partial_{\xi^{\prime}}^{\gamma^{\prime}} g\left(\xi^{\prime}, \cdot\right)\right)\right| \leqslant C \sum_{\left|\gamma_{n}\right| \leqslant \beta_{n}+3} \int\left|\partial_{\xi_{n}}^{\gamma^{\prime}} \partial_{\xi^{\prime}}^{\gamma^{\prime}} g\left(z_{n}, \xi^{\prime}, \xi_{n}\right)\right| d \xi_{n} .
$$

It follows that

$$
\left|\partial_{z}^{\beta} r(z, g)\right| \leqslant \sum_{\left|\gamma^{\prime}\right| \leqslant\left|\beta^{\prime}\right|+3(n-1)} \sum_{\left|\gamma_{n}\right| \leqslant \beta_{n}+3} \int\left|\partial_{\xi^{\prime}}^{\gamma^{\prime}} \partial_{\xi_{n}}^{\gamma_{n}} g\left(\xi^{\prime}, \xi_{n}\right)\right| d \xi^{\prime} d \xi_{n} .
$$

The proof of the estimates for the $q_{j}^{\prime} s$ is the same.

REMARK 4.2.2. - Let us set $z=a+i b$ and let us write $r(z, g)=r(a, b, g)$ and $q_{j}(\xi, z, g)=q_{j}(\xi, a, b, g)$. If we take in (4.2.1) $b=0, \xi=-a$ we obtain

$$
r(a, 0, g)=g(-a) .
$$

If we differentiate (4.2.1) with respect to $b_{k}$ and then take $z=a \in \mathbb{R}^{n}, \xi=-a$, we get

$$
\frac{\partial r}{\partial b_{k}}(a, 0, g)=-i q_{k}(-a, a, 0, g), \quad k=1, \ldots, n .
$$

Finally if we differentiate (4.2.1) with respect to $\xi_{\ell}$ and then take $z=a \in \mathbb{R}^{n}, \xi=-a$ we obtain

$$
\frac{\partial g}{\partial \xi_{\ell}}(-a)=q_{\ell}(-a, a, 0,), \quad \ell=1, \ldots, n .
$$

We introduce now the following notations which will be used in the next sections.

Notation 4.2.3. - Let $\alpha=\left(\alpha_{x}, \alpha_{\xi}\right) \in T^{*} \mathbb{R}^{n} \backslash 0$. We introduce

$$
\left\{\begin{array}{l}
\varphi_{0}(x, \alpha)=\left(x-\alpha_{x}\right) \alpha_{\xi}+\frac{i}{2}\left(x-\alpha_{x}\right)^{2}+\frac{1}{2 i} \alpha_{\xi}^{2}, \\
u_{j}(x, \xi, \alpha)=\xi_{j}-\frac{\partial \varphi_{0}}{\partial x_{j}}(x, \alpha)=\xi_{j}-\alpha_{\xi}^{j}-i\left(x_{j}-\alpha_{x}^{j}\right) .
\end{array}\right.
$$

Let $p(x, \xi)=|\xi|^{2}+\varepsilon q(x, \xi)$; we denote by $H_{p}$ its hamiltonian and we introduce the pull-back by the backward flow of the function $u_{j}$. We set

$$
\left\{\begin{aligned}
v_{j}(\theta ; x, \xi, \alpha) & =u_{j}\left(\exp \left(-\theta H_{p}\right)(x, \xi)\right) \\
& =\xi_{j}(-\theta ; x, \xi)-\alpha_{\xi}^{j}-i\left(x_{j}(-\theta ; x, \xi)-\alpha_{x}^{j}\right)
\end{aligned}\right.
$$

where $(x, \xi)$ is close to $(x(\theta ; \alpha), \xi(\theta, \alpha))$. 
In the flat case we find

$$
v_{j}=(1+2 i \theta)\left[\xi_{j}-\frac{\alpha_{\xi}^{j}-i\left(x_{j}-\alpha_{x}^{j}\right)}{1+2 i \theta}\right]
$$

We split the proof of Theorem 4.1.2 according to the different values of $\alpha$ described in Definition 4.1.1.

\subsection{The case of outgoing points}

Let us set

$$
\mathcal{S}=\left\{\alpha \in \mathbb{R}^{2 n}: \frac{1}{2} \leqslant\left|\alpha_{\xi}\right| \leqslant 2,\left|\alpha_{x} \cdot \alpha_{\xi}\right| \leqslant c_{0}\left\langle\alpha_{x}\right\rangle\left|\alpha_{\xi}\right|\right\} .
$$

We shall use the following notations

$$
\left\{\begin{array}{l}
\widetilde{\Omega}_{\delta}=\left\{(\theta, y) \in \mathbb{R} \times \mathbb{R}^{n}:|y| \leqslant \delta\langle\theta\rangle\right\} \\
\operatorname{sgn} \theta=1(\text { resp. }-1) \text { if } \theta>0 \text { (resp. } \theta<0) .
\end{array}\right.
$$

Let now $\chi_{0} \in C_{0}^{\infty}\left(\mathbb{R}^{n}\right), \chi_{1} \in C_{0}^{\infty}(\mathbb{R})$ be such that,

$$
\begin{gathered}
\chi_{0}(t)=1 \text { if }|t| \leqslant 1, \quad \chi_{0}(t)=0 \text { if }|t| \geqslant 2 \text { and } 0 \leqslant \chi_{0} \leqslant 1, \\
\chi_{1}(\theta)=1 \text { if }|\theta| \leqslant 1, \quad \chi_{1}(\theta)=0 \text { if }|\theta| \geqslant 2 \text { and } 0 \leqslant \chi_{1} \leqslant 1 .
\end{gathered}
$$

Then we can state the following result.

THEOREM 4.3.1. - There exist small positive constants $\mu_{0}, \delta$ such that if we set for $\alpha \in \mathcal{S}, \theta \in \mathbb{R}, y \in \mathbb{R}^{n}, \eta \in \mathbb{R}^{n}, j=1, \ldots, n$,

$g_{j}(\eta)=\chi_{0}\left(\frac{1}{\mu_{0}} \eta\right) v_{j}\left(\theta, y+x(\theta, \alpha), \eta \chi_{1}(\theta)+\left(1-\chi_{1}(\theta)\right)\left[\frac{\eta}{\langle\theta\rangle}+\frac{1}{2} \frac{\operatorname{sgn} \theta}{\langle\theta\rangle} y\right]+\xi(\theta, \alpha), \alpha\right)$

there exist smooth functions $a_{j}=a_{j}(\theta, y, \alpha), b_{j}=b_{j}(\theta, y, \alpha)$ defined on $\widetilde{\Omega}_{\delta}$ such that, with $a=\left(a_{j}\right)_{j=1, \ldots, n}, b=\left(b_{j}\right)_{j=1, \ldots, n}$ we have for $\eta$ in $\mathbb{R}^{n}$ and $(\theta, y) \in \widetilde{\Omega}_{\delta}$,

(i) $g_{j}(\eta)=\sum_{k=1}^{n} q_{k}\left(\eta, a, b, g_{j}\right)\left(\eta_{k}+a_{k}(\theta, y, \alpha)+i b_{k}(\theta, y, \alpha)\right)$

where the $q_{k}^{\prime} s$ have been introduced in Lemma 4.2.1.

Moreover in the set $\widetilde{\Omega}_{\delta}$ we have

(ii) $|a(\theta, y, \alpha)| \leqslant 10 \frac{|y|}{\langle\theta\rangle}, \quad\left|b(\theta, y, \alpha)+\frac{K(\theta)}{1+4 \theta^{2}} y\right| \leqslant \sqrt{\delta} \frac{|y|}{\langle\theta\rangle}$,

where $K(\theta)=\frac{\langle\theta\rangle}{\langle\theta\rangle \chi_{1}(\theta)+1-\chi_{1}(\theta)}, \frac{1}{\sqrt{5}}\langle\theta\rangle \leqslant K(\theta) \leqslant\langle\theta\rangle$.

On the other hand we have, uniformly with respect to $(\theta, y) \in \widetilde{\Omega}_{\delta}$ and $\alpha \in \mathcal{S}$,

(iii) $\left|\partial_{y}^{A} a(\theta, y, \alpha)\right|+\left|\partial_{y}^{\alpha} b(\theta, y, \alpha)\right| \leqslant \frac{C_{A}}{\langle\theta\rangle^{|A|}}, \quad A \in \mathbb{N}^{n}$.

Moreover for $j=1, \ldots, n, k=1, \ldots, n$,

(iv) $\left|q_{k}\left(\eta, a, b, g_{j}\right)-(1+2 i \theta) \frac{k(\theta)}{\langle\theta\rangle} \delta_{j k}\right| \leqslant C(\varepsilon+\delta)$, if $|\eta| \leqslant \delta$

where $k(\theta)=\langle\theta\rangle \chi_{1}(\theta)+1-\chi_{1}(\theta)$.

(v) $\left|\partial_{(a, b)}^{A} \partial_{\eta}^{B} q_{k}\left(\eta, a, b, g_{j}\right)\right| \leqslant C\left(\mu_{0}\right)$, if $|A|+|B| \geqslant 1,|\eta| \leqslant \mu_{0}, 1 \leqslant j, k \leqslant n$. 
Let us make some comments about the above Theorem. First of all we have skipped for convenience the dependence of $g_{j}$ with respect to $(\theta, y, \alpha)$ which are considered here as parameters. The main object to be considered is $v_{j}(\theta, x, \xi)$. Since $x$ has to be close to $x(\theta, \alpha)$ we have set $x=y+x(\theta, \alpha)$. For small $|\theta|\left(\chi_{1} \equiv 1\right) \xi$ has to be close to $\xi(\theta, \alpha)$ that's why we have set $\xi=\eta+\xi(\theta, \alpha)$. For large $|\theta|$ we have $\chi_{1} \equiv 0$. In this region for technical reasons we need to renormalize the variable $\eta$ and to isolate the first term of $a$. This is the reason for the expression $\frac{\eta}{\langle\theta\rangle}+\frac{1}{2} \frac{\operatorname{sgn} \theta}{\langle\theta\rangle} y$. Finally the two regimes $|\theta|$ small and $|\theta|$ large have to be matched. This is the reason for introducing $\chi_{1}$.

Proof. - According to Definition 3.2.2 we have $\mathcal{S} \subset \mathcal{S}_{+} \cap \mathcal{S}_{-}$. Moreover

$$
B\left(\alpha, c_{0}\right):=\left\{\widetilde{\alpha} \in T^{*} \mathbb{R}^{n}:|\alpha-\widetilde{\alpha}| \leqslant c_{0}\right\} \subset \mathcal{S}_{+} \cap \mathcal{S}_{-} .
$$

We start with the following Lemma.

Lemma 4.3.2. - There exists a small positive constant $\mu_{0}$ such that for all $(\theta, y)$ with $\theta \in \mathbb{R},|y| \leqslant \mu_{0}\langle\theta\rangle$ and all $\eta$ in $\mathbb{R}^{n}$ such that $|\eta| \leqslant 2 \mu_{0}$ there exists a unique $\beta=\beta(\theta, y, \alpha, \eta)$ in $B\left(\alpha, c_{0}\right)$ such that

$$
\left\{\begin{array}{l}
x(\theta, \beta)=y+x(\theta, \alpha) \\
\xi(\theta, \beta)=\chi_{1}(\theta) \eta+\left(1-\chi_{1}(\theta)\right)\left[\frac{\eta}{\langle\theta\rangle}+\frac{1}{2} \frac{\operatorname{sgn} \theta}{\langle\theta\rangle} y\right]+\xi(\theta, \alpha) .
\end{array}\right.
$$

Moreover we have

$$
\left\{\begin{array}{r}
\beta_{x}=\alpha_{x}+\left[1-\frac{|\theta|}{\langle\theta\rangle}\left(1-\chi_{1}(\theta)\right)\right] y-\frac{2 \theta}{\langle\theta\rangle} \eta\left(\chi_{1}(\theta)\langle\theta\rangle+1-\chi_{1}(\theta)\right) \\
+z(\theta, \alpha)-z(\theta, \beta) \\
\beta_{\xi}=\alpha_{\xi}+\frac{\eta}{\langle\theta\rangle}\left[\chi_{1}(\theta)\langle\theta\rangle+\left(1-\chi_{1}(\theta)\right)\right]+\frac{1}{2} \frac{1-\chi_{1}(\theta)}{\langle\theta\rangle} \operatorname{sgn} \theta y \\
+\zeta(\theta, \alpha)-\zeta(\theta, \beta)
\end{array}\right.
$$

where $z$ and $\zeta$ have been introduced in Proposition 3.3 .1 and

$$
\left\{\begin{array}{l}
\text { (i) }|\beta-\alpha| \leqslant 10\left(|\eta|+\frac{|y|}{\langle\theta\rangle}\right) \\
\text { (ii) }\left|\frac{\partial \beta}{\partial \eta}(\theta, y, \alpha, \eta)\right| \leqslant C \\
\text { (iii) }\left|\left(\frac{\partial \beta_{\xi}^{j}}{\partial \eta_{k}}-i \frac{\partial \beta_{x}^{j}}{\partial \eta_{k}}\right)(\theta, y, \alpha, \eta)-\frac{1+2 i \theta}{\langle\theta\rangle}\left(\langle\theta\rangle \chi_{1}(\theta)+\left(1-\chi_{1}(\theta)\right)\right) \delta_{j k}\right| \\
\text { (iv) }\left|\partial_{\eta}^{\alpha} \partial_{y}^{B} \beta(\theta, y, \alpha, \eta)\right| \leqslant C_{A B} \varepsilon, \text { if }|A|+|B| \geqslant 2 .
\end{array}\right.
$$


Proof. - The system (4.3.4) with $\beta \in B\left(\alpha, c_{0}\right) \subset \mathcal{S}_{+} \cap \mathcal{S}_{-}$is equivalent by Proposition 3.3.1 to the following

$$
\left\{\begin{array}{l}
\beta_{x}+2 \theta \xi(\theta, \beta)+z(\theta, \beta)=y+\alpha_{x}+2 \theta \xi(\theta, \alpha)+z(\theta, \alpha) \\
\beta_{\xi}+\zeta(\theta, \beta)=\chi_{1}(\theta) \eta+\frac{1-\chi_{1}(\theta)}{\langle\theta\rangle}\left[\eta+\frac{1}{2} \operatorname{sgn} \theta y\right]+\alpha_{\xi}+\zeta(\theta, \alpha) .
\end{array}\right.
$$

Using again (4.3.4) the left hand side of the first line of (4.3.7) can be written

$$
\beta_{x}+2 \theta \chi_{1}(\theta) \eta+\frac{2 \theta}{\langle\theta\rangle}\left(1-\chi_{1}(\theta)\right) \eta+\frac{|\theta|}{\langle\theta\rangle}\left(1-\chi_{1}(\theta)\right) y+2 \theta \xi(\theta, \alpha)+z(\theta, \beta) .
$$

Finally (4.3.7) is equivalent to

$$
\left\{\begin{array}{r}
\beta_{x}=\alpha_{x}+\left[1-\frac{|\theta|}{\langle\theta\rangle}\left(1-\chi_{1}(\theta)\right)\right] y-\frac{2 \theta}{\langle\theta\rangle} \eta\left(\chi_{1}(\theta)\langle\theta\rangle+1-\chi_{1}(\theta)\right) \\
+z(\theta, \alpha)-z(\theta, \beta) \\
\beta_{\xi}=\alpha_{\xi}+\frac{\eta}{\langle\theta\rangle}\left[\chi_{1}(\theta)\langle\theta\rangle+\left(1-\chi_{1}(\theta)\right)\right]+\frac{1}{2} \frac{1-\chi_{1}(\theta)}{\langle\theta\rangle} \operatorname{sgn} \theta y \\
+\zeta(\theta, \alpha)-\zeta(\theta, \beta) .
\end{array}\right.
$$

Writing this system $\beta_{x}=\Phi_{x}(\beta) \beta_{\xi}=\Phi_{\xi}(\beta)$ and setting $\Phi(\beta)=\left(\Phi_{x}(\beta), \Phi_{\xi}(\beta)\right)$ we are going to solve it using the fixed point theorem in $B\left(\alpha, c_{0}\right)$.

(i) $\Phi$ maps $B\left(\alpha, c_{0}\right)$ in itself.

We have $0<1-\frac{|\theta|}{\langle\theta\rangle} \leqslant \frac{1}{\langle\theta\rangle^{2}},|\theta| \chi_{1}(\theta) \leqslant 2, \chi_{1}(\theta)\langle\theta\rangle+1-\chi_{1}(\theta) \leqslant \sqrt{5},|y| \leqslant \mu_{0}\langle\theta\rangle$, $|\eta| \leqslant 2 \mu_{0},|z|+|\zeta| \leqslant C \varepsilon$ by Proposition 3.3.2. It follows that $|\Phi(\beta)-\alpha| \leqslant 20 \mu_{0}+C \varepsilon \leqslant$ $c_{0}$ if $\mu_{0}$ and $\varepsilon$ are small enough.

(ii) Let $\beta, \beta^{\prime}$ be in $B\left(\alpha, c_{0}\right)$. Then $t \beta+(1-t) \beta^{\prime} \in B\left(\alpha, c_{0}\right) \subset \mathcal{S}_{+} \cap \mathcal{S}_{-}$for all $t$ in $(0,1)$. It follows that

$$
\left|\Phi(\beta)-\Phi\left(\beta^{\prime}\right)\right| \leqslant\left|z(\theta, \beta)-z\left(\theta, \beta^{\prime}\right)\right|+\left|\zeta(\theta, \beta)-\zeta\left(\theta, \beta^{\prime}\right)\right| \leqslant C \varepsilon\left|\beta-\beta^{\prime}\right|
$$

by Proposition 3.3.2. Here $C$ depends only on the constants $A_{0}, A_{1}$ in (3.1.1). Taking $\varepsilon$ so small that $C \varepsilon<1$ we see that we can apply the fixed point theorem in $B\left(\alpha, c_{0}\right)$. This proves the existence of $\beta$ satisfying (4.3.4) and (4.3.5) by (4.3.8). Now (i) in (4.3.6) follows from (4.3.5) taking $\varepsilon$ small enough since $|Z(\theta, \alpha)-Z(\theta, \beta)| \leqslant C \varepsilon|\alpha-\beta|$ if $Z=z$ or $\zeta$. The claim (ii) is obtained by differentiating the equations (4.3.5) with respect to $\eta_{k}$ and using Proposition 3.3.2. Then (iii) follows easily from (4.3.5) and (ii). Finally (iv) is obtained by an induction on $|A|+|B|$.

From now on we fix the constant $\mu_{0}$ occurring in Lemma 4.3.2.

Now for $j=1, \ldots, n$ let $g_{j}$ be the function introduced in the statement of Theorem 4.3.1. Then, according to Lemma 4.3.2 and (4.2.10) we have for $|y| \leqslant \mu_{0}\langle\theta\rangle$ and $\eta \in \mathbb{R}$

$$
g_{j}(\eta)=\chi_{0}\left(\frac{\eta}{\mu_{0}}\right)\left[\beta_{\xi}^{j}(\theta, y, \alpha, \eta)-\alpha_{\xi}^{j}-i\left(\beta_{x}^{j}(\theta, y, \alpha, \eta)-\alpha_{x}^{j}\right)\right]
$$


since $f(-\theta, x(\theta, \beta), \xi(\theta, \beta))=\beta_{f}$ for $f=x$ and $\xi$. It follows from Lemma 4.2.1 that the existence of $a_{j}, b_{j}$ in Theorem 4.3 .1 will be proved if we can solve the equations

$$
r\left(a, b, g_{j}(\cdot)\right)=0, \quad j=1, \ldots, n .
$$

Let us now take $(\theta, y) \in \widetilde{\Omega}_{\delta}$, that is $\theta \in \mathbb{R},|y| \leqslant \delta\langle\theta\rangle$ where $0<\delta<\frac{1}{2} \mu_{0}$ is to be chosen. We look for a solution $(a, b)$ of the system (4.3.10) in the set

$$
\left\{\begin{array}{l}
E=\left\{(a, b) \in \mathbb{R}^{n} \times \mathbb{R}^{n}:|a| \leqslant \frac{10|y|}{\langle\theta\rangle},\left|b+\frac{K(\theta)}{1+4 \theta^{2}} y\right| \leqslant \sqrt{\delta} \frac{|y|}{\langle\theta\rangle}\right\} \\
\text { where } K(\theta)=\frac{\langle\theta\rangle}{\langle\theta\rangle \chi_{1}(\theta)+\left(1-\chi_{1}(\theta)\right)}, \frac{1}{\sqrt{5}}\langle\theta\rangle \leqslant K(\theta) \leqslant\langle\theta\rangle .
\end{array}\right.
$$

We shall first give equivalent equations to (4.3.10) in the set $E$. We write,

$$
r\left(a, b, g_{j}\right)=r\left(a, 0, g_{j}\right)+\sum_{k=1}^{n} \frac{\partial r}{\partial b_{k}}\left(a, 0, g_{j}\right) b_{k}+\sum_{p, q=1}^{n} H_{p, q}^{j}(\theta, y, \alpha, a, b) b_{p} b_{q}
$$

where

$$
H_{p, q}^{j}(\theta, y, \alpha, a, b)=\int_{0}^{1}(1-t) \frac{\partial^{2} r}{\partial b_{p} \partial b_{q}}\left(a, t b, g_{j}(\cdot)\right) d t .
$$

It follows from (4.2.6), (4.2.7) and (4.2.8) that

$$
r\left(a, b, g_{j}(\cdot)\right)=g_{j}(-a)-i \sum_{k=1}^{n} \frac{\partial g_{j}}{\partial \eta_{k}}(-a) b_{k}+\sum_{p, q=1}^{n} H_{p, q}^{j}(\theta, y, \alpha, a, b) b_{p} b_{q} .
$$

Now if $(a, b) \in E$ we have $|a| \leqslant \frac{12|y|}{\langle\theta\rangle} \leqslant 12 \delta \leqslant \mu_{0}$. Therefore $\chi_{0}\left(-a / \mu_{0}\right)=1$, $\chi_{0}^{\prime}\left(-a / \mu_{0}\right)=0$. Then by (4.3.9) and (4.3.5) we obtain,

$$
\begin{aligned}
& g_{j}(-a)=\frac{1}{2} \frac{1-\chi_{1}(\theta)}{\langle\theta\rangle} \operatorname{sgn} \theta y_{j}-\frac{a_{j}}{\langle\theta\rangle}\left[\chi_{1}(\theta)\langle\theta\rangle+1-\chi_{1}(\theta)\right] \\
& +\zeta_{j}(\theta, \alpha)-\zeta_{j}(\theta, \beta)-i\left(1-\frac{|\theta|}{\langle\theta\rangle}\left(1-\chi_{1}(\theta)\right)\right) y_{j} \\
& \quad-\frac{2 i \theta}{\langle\theta\rangle} a_{j}\left[\chi_{1}(\theta)\langle\theta\rangle+1-\chi_{1}(\theta)\right]-i\left(z_{j}(\theta, \alpha)-z_{j}(\theta, \beta)\right. \\
& \begin{aligned}
\frac{\partial g_{j}}{\partial \eta_{k}}(-a)= & \frac{1+2 i \theta}{\langle\theta\rangle}\left[\chi_{1}(\theta)\langle\theta\rangle+\left(1-\chi_{1}(\theta)\right)\right] \\
& -\partial \zeta_{j}(\theta, \beta(\theta, y, \alpha,-a)) \frac{\partial \beta}{\partial \eta_{k}}(\theta, y, \alpha,-a) \\
& +i \partial z_{j}(\theta, \beta(\theta, y, \alpha,-a)) \cdot \frac{\partial \beta}{\partial \eta_{k}}(\theta, y, \alpha,-a)
\end{aligned}
\end{aligned}
$$

where $\partial=\left(\partial_{x}, \partial_{\xi}\right)$ and $\beta=\left(\beta_{x}, \beta_{\xi}\right)$. 
On the other hand we deduce from (4.3.12) and (4.2.2) that

$$
\left|\partial_{(a, b)}^{A} \partial_{y}^{B} H_{p q}^{j}(\theta, y, \alpha, a, b)\right| \leqslant C_{A B} \sum_{|\gamma| \leqslant|A|+3 n+2} \int\left|\partial_{\eta}^{\gamma} \partial_{y}^{B} g_{j}(\eta)\right| d \eta
$$

Using (4.3.6) and (4.3.9) we obtain

$$
\left|\partial_{(a, b)}^{A} \partial_{y}^{B} H_{p q}^{j}(\theta, y, \alpha, a, b)\right| \leqslant C_{A B}^{\prime}\left(\mu_{0}\right) .
$$

It follows from (4.3.13), (4.3.14), (4.3.15) that (4.3.10) is equivalent to

$$
\begin{array}{r}
-\frac{a_{j}}{\langle\theta\rangle}\left[\chi_{1}(\theta)\langle\theta\rangle+1-\chi_{1}(\theta)\right]-\frac{2 i \theta}{\langle\theta\rangle} a_{j}\left[\chi_{1}(\theta)\langle\theta\rangle+1-\chi_{1}(\theta)\right]+\frac{1}{2} \frac{1-\chi_{1}(\theta)}{\langle\theta\rangle} \operatorname{sgn} \theta y_{j} \\
-i\left(1-\frac{|\theta|}{\langle\theta\rangle}\left(1-\chi_{1}(\theta)\right)\right) y_{j}+\zeta_{j}(\theta, \alpha)-\zeta_{j}(\theta, \beta)-i\left(z_{j}(\theta, \alpha)-z_{j}(\theta, \beta)\right) \\
-i \frac{1+2 i \theta}{\langle\theta\rangle}\left[\chi_{1}(\theta)\langle\theta\rangle+1-\chi_{1}(\theta)\right] b_{j}+F_{1}^{j}(\theta, y, \alpha, a) \cdot b+i F_{2}^{j}(\theta, y, \alpha, a) \cdot b \\
+H_{1}^{j}(\theta, y, \alpha, a, b) b \cdot b+i H_{2}^{j}(\theta, y, \alpha, a, b) b \cdot b=0
\end{array}
$$

where

$$
\left\{\begin{array}{l}
\beta=\beta(\theta, y, \alpha,-a), \\
F_{1}^{j}(\theta, y, \alpha, a)=\partial z_{j}(\theta, \beta) \cdot \frac{\partial \beta}{\partial \eta}(\theta, y, \alpha,-a), \\
F_{2}^{j}(\theta, y, \alpha, a)=\partial \zeta_{j}(\theta, \beta) \cdot \frac{\partial \beta}{\partial \eta}(\theta, y, \alpha,-a), \\
H^{j}=\left(H_{p q}^{j}\right)=H_{1}^{j}+i H_{2}^{j} .
\end{array}\right.
$$

Taking the real and the imaginary parts we are led to the system

$$
\begin{aligned}
& a_{j}-2 \theta b_{j}=K(\theta)\left[\frac{1}{2} \frac{1-\chi_{1}(\theta)}{\langle\theta\rangle} \operatorname{sgn} \theta y_{j}+\zeta_{j}(\theta, \alpha)-\zeta_{j}(\theta, \beta)+F_{1}^{j} b+H_{1}^{j} b \cdot b\right] \\
& 2 \theta a_{j}+b_{j}=K(\theta)\left[-\left(1-\frac{|\theta|}{\langle\theta\rangle}\left(1-\chi_{1}(\theta)\right)\right) y_{j}-\left(z_{j}(\theta, \alpha)-z_{j}(\theta, \beta)\right)+F_{2}^{j} b+H_{2}^{j} b \cdot b\right]
\end{aligned}
$$

where $K(\theta)=\frac{\langle\theta\rangle}{\chi_{1}(\theta)\langle\theta\rangle+1-\chi_{1}(\theta)}$.

Inverting this system we are led to solve

$$
\left\{\begin{array}{c}
a_{j}=\frac{K(\theta)}{1+4 \theta^{2}}\left(\frac{1}{2} \frac{1-\chi_{1}(\theta)}{\langle\theta\rangle} \operatorname{sgn} \theta-2 \theta\left(1-\frac{|\theta|}{\langle\theta\rangle}\left(1-\chi_{1}(\theta)\right)\right)\right) y_{j} \\
\quad+Z_{1}^{j}(\theta, \alpha)-Z_{1}^{j}(\theta, \beta)+F_{3}^{j} b+H_{3}^{j} b \cdot b=: \Phi_{1}^{j}(a, b) \\
b_{j}=-\frac{K(\theta)}{1+4 \theta^{2}} y_{j}+Z_{2}^{j}(\theta, \alpha)-Z_{2}^{j}(\theta, \beta)+F_{4}^{j} b+H_{4}^{j} b \cdot b=: \Phi_{2}^{j}(a, b)
\end{array}\right.
$$


where

$$
\left\{\begin{array}{l}
Z_{1}^{j}(\theta, \cdot)=\frac{K(\theta)}{1+4 \theta^{2}}\left(\zeta_{j}(\theta, \cdot)-2 \theta z_{j}(\theta, \cdot)\right) \\
Z_{2}^{j}(\theta, \cdot)=-\frac{K(\theta)}{1+4 \theta^{2}}\left(2 \theta \zeta_{j}(\theta, \cdot)+z_{j}(\theta, \cdot)\right) \\
F_{3}^{j}=\frac{K(\theta)}{1+4 \theta^{2}}\left(F_{1}^{j}+2 \theta F_{2}^{j}\right), \quad F_{4}^{j}=\frac{K(\theta)}{1+4 \theta^{2}}\left(-2 \theta F_{1}^{j}+F_{2}^{j}\right) \\
H_{3}^{j}=\frac{K(\theta)}{1+4 \theta^{2}}\left(H_{1}^{j}+2 \theta H_{2}^{j}\right), \quad H_{4}^{j}=\frac{K(\theta)}{1+4 \theta^{2}}\left(-2 \theta H_{1}^{j}+H_{2}^{j}\right) .
\end{array}\right.
$$

Let us set $\Phi^{j}=\left(\Phi_{1}^{j}, \Phi_{2}^{j}\right)$ (see (4.3.19)) and $\Phi=\left(\Phi^{j}\right)_{j=1, \ldots, n}$. We have shown that our initial system (4.3.10) is equivalent in $E$ to the equation $\Phi(a, b)=(a, b)$. We are going to show that this equation has a unique solution in $E$ by using the fixed point theorem.

(i) $\Phi(E) \subset E$.

We have $2|\theta|\left(1-\frac{|\theta|}{\langle\theta\rangle}\right) \leqslant 1 /\langle\theta\rangle, 2 \frac{|\theta|^{2}}{\langle\theta\rangle} \chi_{1}(\theta) \leqslant 8 /\langle\theta\rangle$ and by (4.3.20), (4.3.18), (4.3.6) we see that $\left|F_{3}^{j}\right|+\left|F_{4}^{j}\right| \leqslant C \varepsilon$. Moreover we deduce from (4.3.17) and (4.3.20) that $\left|H_{3}^{j}\right|+\left|H_{4}^{j}\right| \leqslant C\left(\mu_{0}\right)$. Finally in $E$ we have $|b| \leqslant \frac{2|y|}{\langle\theta\rangle} \leqslant 2 \delta$. It follows then from (4.3.19) that

$$
\left|\Phi_{1}(a, b)\right| \leqslant \frac{19}{2} \frac{|y|}{1+4 \theta^{2}}+C \varepsilon|\alpha-\beta|+C^{\prime} \varepsilon \frac{|y|}{\langle\theta\rangle}+C\left(\mu_{0}\right) \delta \frac{|y|}{\langle\theta\rangle} .
$$

Now using (4.3.6) (i) we see that when $(a, b)$ belongs to $E$ we have

$$
|\beta(\theta, y, \alpha,-a)-\alpha| \leqslant 10\left(|a|+\frac{|y|}{\langle\theta\rangle}\right) \leqslant 110 \frac{|y|}{\langle\theta\rangle} .
$$

Therefore

$$
\left|\Phi_{1}(a, b)\right| \leqslant\left(\frac{19}{2}+110 C \varepsilon+C^{\prime} \varepsilon+C\left(\mu_{0}\right) \delta\right) \frac{|y|}{\langle\theta\rangle} \leqslant \frac{10|y|}{\langle\theta\rangle},
$$

if $\varepsilon$ and $\delta$ are small enough.

By the same estimates we obtain,

$$
\left|\Phi_{2}(a, b)+\frac{K(\theta)}{1+4 \theta^{2}} y\right| \leqslant C \varepsilon|\beta-\alpha|+C \varepsilon \frac{|y|}{\langle\theta\rangle}+C\left(\mu_{0}\right) \delta \frac{|y|}{\langle\theta\rangle}
$$

so if $\varepsilon$ and $\delta$ are small enough we can bound the right hand side by $\sqrt{\delta}|y| /\langle\theta\rangle$. This shows that $\Phi$ maps $E$ to $E$.

(ii) $\Phi$ is a contraction. 
Let now $\left(a_{1}, b_{1}\right),\left(a_{2}, b_{2}\right)$ be two points in $E$. Then

$$
\begin{aligned}
\left|\Phi\left(a_{1}, b_{1}\right)-\Phi\left(a_{2}, b_{2}\right)\right| \leqslant & \sum_{j=1}^{n} \sum_{\ell=1}^{2}|\underbrace{Z_{\ell}^{j}\left(\theta, \beta\left(\theta, y, \alpha,-a_{1}\right)\right)-Z_{\ell}^{j}\left(\theta, \beta\left(\theta, y, \alpha,-a_{2}\right)\right)}_{(1)}| \\
& +\sum_{j=1}^{n} \sum_{\ell=3}^{4}\{\underbrace{\left|F_{\ell}^{j}\left(\theta, y, \alpha, a_{1}\right) \cdot b_{1}-F_{\ell}^{j}\left(\theta, y, \alpha, a_{2}\right) \cdot b_{2}\right|}_{(2)} \\
& +\mid \underbrace{H_{\ell}^{j}\left(\theta, y, \alpha, a_{1}, b_{1}\right) b_{1} \cdot b_{1}-H_{\ell}^{j}\left(\theta, y, \alpha, a_{2}, b_{2}\right) b_{2} \cdot b_{2} \mid}_{(3)}\} .
\end{aligned}
$$

Using (4.3.20) and Proposition 3.3.2 we can write

$$
(1) \leqslant C \varepsilon\left|\beta\left(\theta, y, \alpha,-a_{1}\right)-\beta\left(\theta, y, \alpha,-a_{2}\right)\right| .
$$

Then (4.3.6) gives $(1) \leqslant C^{\prime} \varepsilon\left|a_{1}-a_{2}\right|$.

To handle the therm (2) we use (4.3.18), (4.3.20), (4.3.6) and Proposition 3.3.2. We obtain

$$
(2) \leqslant C \varepsilon\left(\left|a_{1}-a_{2}\right|+\left|b_{1}-b_{2}\right|\right) .
$$

Finally using (4.3.17), (4.3.18), (4.3.20) and the fact that in $E$ we have $|b| \leqslant 2 \frac{|y|}{\langle\theta\rangle} \leqslant 2 \delta$ we see easily that

$$
(3) \leqslant C(\varepsilon+\delta)\left(\left|a_{1}-a_{2}\right|+\left|b_{1}-b_{2}\right|\right)
$$

It follows then that

$$
\left|\Phi\left(a_{1}, b_{1}\right)-\Phi\left(a_{2}, b_{2}\right)\right| \leqslant C(\varepsilon+\delta)\left(\left|a_{1}-b_{1}\right|+\left|a_{2}-b_{2}\right|\right)
$$

where $C$ is an absolute constant depending only on the dimension and a finite number of $A_{\ell}$ appearing in (3.1.1). Thus we can take $\varepsilon$ and $\delta$ so small that $C(\varepsilon+\delta)<1$.

Therefore we can apply the fixed point theorem to solve (4.3.19) which is equivalent to (4.3.10). This proves the claims (i) and (ii) in Theorem 4.3.1.

Let us now prove the point (iii). We state a Lemma.

Lemma 4.3.3. - There exists $C_{0}>0$ such that for every $A \in \mathbb{N}^{n}$ there exist $C_{A} \geqslant 0$, $C_{A}^{\prime} \geqslant 0$ such that with $\beta$ defined in Lemma 4.3.2,

$\begin{array}{ll}\text { a) } & \left|\partial_{y}^{A}[\beta(\theta, y, \alpha,-a(\theta, y, \alpha))]\right| \leqslant C_{0}\left|\partial_{y}^{A} a(\theta, y, \alpha)\right|+\frac{C_{A}}{\langle\theta\rangle|A|}, \\ \text { b) } & \left|\partial_{y}^{A} a(\theta, y, \alpha)\right|+\left|\partial_{y}^{A} b(\theta, y, \alpha)\right| \leqslant C_{A}^{\prime} /\langle\theta\rangle^{|A|} \text {, for all }(\theta, y) \text { in } \widetilde{\Omega}_{\delta} .\end{array}$

Proof. - We shall use an induction on $|A|$, starting with the formulas (4.3.19). But before we need some intermediate results. We introduce the following space of functions.

Let $f=f(\theta, y, \alpha)$ be a function from $\widetilde{\Omega}_{\delta} \times \mathbb{R}^{2 n}$ to $\mathbb{C}$. We shall say that $f \in \mathcal{G}_{ \pm}$if we can write

$$
f(\theta, y, \alpha)=G(\theta, \beta(\theta, y, \alpha,-a(\theta, y, \alpha)))
$$


where $G: \mathbb{R}_{\theta}^{ \pm} \times \mathbb{R}_{X}^{2 n} \rightarrow \mathbb{C}$ is smooth in $X$ and satisfies

$$
\sup _{\mathbb{R}^{ \pm} \times \mathbb{R}^{2 n}}\left|\partial_{X}^{\gamma} G(\theta, X)\right| \leqslant C_{\gamma} \varepsilon, \quad \forall \gamma \in \mathbb{N}^{n} .
$$

For example Proposition 3.3.2 shows that the functions

$$
(\theta, y, \alpha) \longmapsto z_{j}(\theta, \beta(\theta, y, \alpha,-a(\theta, y, \alpha))
$$

(and $\zeta_{j}$ ) belong to $\mathcal{G}_{ \pm}$if $\alpha \in \mathcal{S}_{ \pm}$. (Here we have the sign + if $\alpha \in \mathcal{S}_{+}$and - if $\alpha \in \mathcal{S}_{-}$).

Then we have the following claim.

Claim 1. - For all $\nu \in \mathbb{N}^{n},|\nu| \geqslant 1, j=1, \ldots, n$ we have

$$
\partial_{\eta}^{\nu} \beta_{x}^{j}(\theta, y, \alpha, \eta)=G_{\nu}^{j}(\theta, \beta(\theta, y, \alpha, \eta))
$$

where $G_{\nu}^{j}$ has all derivatives uniformly bounded on $\mathbb{R}^{ \pm} \times \mathbb{R}^{2 n}$. The same is true for $\partial_{\eta}^{\nu} \beta_{\xi}^{j}$.

Proof of the claim. - We proceed by induction on $|\nu|$ beginning with $|\nu|=1$. Let us set $k(\theta)=\langle\theta\rangle \chi_{1}(\theta)+1-\chi_{1}(\theta)$. Then $1 \leqslant k(\theta) \leqslant \sqrt{5}$ since $|\theta| \leqslant 2$ on $\operatorname{supp} \chi_{1}$. It follows from (4.3.5) that for fixed $k$ in $\{1,2, \ldots, n\}$ we have

$$
\begin{aligned}
\frac{\partial \beta_{x}^{j}}{\partial \eta_{k}}(\theta, y, \alpha, \eta)= & -\frac{2 \theta}{\langle\theta\rangle} k(\theta) \delta_{j k}-\sum_{\ell=1}^{n}\left(\frac{\partial z_{j}}{\partial x_{\ell}}(\theta, \beta(\theta, y, \alpha, \eta)) \frac{\partial \beta_{x}^{\ell}}{\partial \eta_{k}}(\theta, y, \alpha, \eta)\right. \\
& \left.+\frac{\partial z_{j}}{\partial \xi_{\ell}}(\theta, \beta(\theta, y, \alpha, \eta)) \frac{\partial \beta_{\xi}^{j}}{\partial \eta_{k}}(\theta, y, \alpha, \eta)\right) \\
\frac{\partial \beta_{\xi}^{j}}{\partial \eta_{k}}(\theta, y, \alpha, \eta)= & \frac{k(\theta)}{\langle\theta\rangle} \delta_{j k}-\sum_{\ell=1}^{n}\left(\frac{\partial \zeta_{j}}{\partial x_{\ell}}(\theta, \beta(\theta, y, \alpha, \eta)) \frac{\partial \beta_{x}^{\ell}}{\partial \eta_{k}}(\theta, y, \alpha, \eta)\right. \\
& \left.+\frac{\partial \zeta_{j}}{\partial \xi_{\ell}}(\theta, \beta(\theta, y, \alpha, \eta)) \frac{\partial \beta_{\xi}^{\ell}}{\partial \eta_{k}}(\theta, y, \alpha, \eta)\right) .
\end{aligned}
$$

Let us set

$$
\begin{gathered}
X_{j}=\frac{\partial \beta_{x}^{j}}{\partial \eta_{k}}, \quad \Xi_{j}=\frac{\partial \beta_{\xi}^{j}}{\partial \eta_{k}}, \quad U=\left(\begin{array}{c}
X_{1} \\
\Xi_{1} \\
\vdots \\
X_{n} \\
\Xi_{n}
\end{array}\right), \quad M_{j}=\left(\begin{array}{cccc}
\frac{\partial z_{j}}{\partial x_{1}} & \frac{\partial z_{j}}{\partial \xi_{1}} \cdots & \frac{\partial z_{j}}{\partial x_{n}} & \frac{\partial z_{j}}{\partial \xi_{n}} \\
\frac{\partial \zeta_{j}}{\partial x_{1}} & \frac{\partial \zeta_{j}}{\partial \xi_{1}} & \frac{\partial \zeta_{j}}{\partial x_{n}} & \frac{\partial \zeta_{j}}{\partial \xi_{n}}
\end{array}\right), \\
M=\left(\begin{array}{c}
M_{1} \\
M_{2} \\
\vdots \\
M_{n}
\end{array}\right) \quad \text { and } F(\theta)=\frac{k(\theta)}{\langle\theta\rangle}\left(\begin{array}{c}
-2 \theta \delta_{1 k} \\
\delta_{1 k} \\
\vdots \\
-2 \theta \delta_{n k} \\
\delta_{n k}
\end{array}\right)
\end{gathered}
$$

Then the above equation imply that

$$
U(\theta, y, \alpha, \eta)=F(\theta)-M(\theta, \beta(\theta, y, \alpha, \eta)) U(\theta, y, \alpha, \eta) .
$$


Now by Proposition 3.3.2 the entries of the matrix $M$ are $\mathcal{O}(\varepsilon)$. It follows that the matrix $I+M$ is invertible. Therefore we obtain

$$
U(\theta, y, \alpha, \eta)=(I+M(\theta, \beta(\theta, y, \alpha, \eta)))^{-1} F(\theta) .
$$

This proves the case $|\nu|=1$.

Assume now that our claim is proved for $|\nu| \leqslant N-1$. Then

$$
\partial_{\eta}^{\nu} \beta_{x}^{j}(\theta, y, \alpha, \eta)=G_{\nu}^{j}(\theta, \beta(\theta, y, \alpha, \eta))
$$

Then for $k=1, \ldots, n$

$$
\begin{aligned}
\frac{\partial}{\partial \eta_{k}} \partial_{\eta}^{\nu} \beta_{x}^{j}(\theta, y, \alpha, \eta)=\sum_{\ell=1}^{n} \frac{\partial G_{\nu}^{j}}{\partial X_{\ell}}(\theta, \beta(\theta, y, \alpha, \eta)) \frac{\partial \beta_{x}^{\ell}}{\partial \eta_{k}}(\theta, y, \alpha, \eta) & \\
& +\sum_{\ell=n+1}^{2 n} \frac{\partial G_{\nu}^{j}}{\partial X_{\ell}}(\theta, \beta(\theta, y, \alpha, \eta)) \frac{\partial \beta_{\xi}^{\ell}}{\partial \eta_{k}}(\theta, y, \alpha, \eta) .
\end{aligned}
$$

Using (4.3.23) with $|\nu|=1$ we obtain the claim up to the order $|\nu|=N$.

Consequence 4.3.4. - With the notations of (4.3.18), (4.3.20) and (4.3.21) The functions $(\theta, y, \alpha) \mapsto Z_{\ell}^{j}\left(\theta, \beta(\theta, y, \alpha,-a(\theta, y, \alpha)), \ell=1,2, F_{p}^{j}, p=3,4\right.$ belong to $\mathcal{G}_{+}$.

Let us now go back to the proof of Lemma 4.3.3. We begin by the case $|A|=1$. For convenience we shall set

$$
f(y)=\beta(\theta, y, \alpha,-a(\theta, y, \alpha)) .
$$

It follows from (4.3.5) that

$$
\begin{gathered}
\frac{\partial f_{x}^{j}}{\partial y_{k}}(y)=\left(1-\frac{|\theta|}{\langle\theta\rangle}\left(1-\chi_{1}(\theta)\right)\right) \delta_{j k}+\frac{2 \theta}{\langle\theta\rangle} k(\theta) \frac{\partial a_{j}}{\partial y_{k}}-\sum_{\ell=1}^{n}\left(\frac{\partial z_{j}}{\partial x_{\ell}}(\theta, f(y)) \frac{\partial f_{x}^{j}}{\partial y_{k}}(y)\right. \\
\left.-\frac{\partial z_{j}}{\partial \xi_{\ell}}(\theta, f(y)) \frac{\partial f_{\xi}^{j}}{\partial y_{k}}(y)\right) \\
\frac{\partial f_{\xi}^{j}}{\partial y_{k}}(y)=\frac{1}{2}\left(1-\chi_{1}(\theta)\right) \frac{\operatorname{sgn} \theta}{\langle\theta\rangle} \delta_{j k}-\frac{1}{\langle\theta\rangle} k(\theta) \frac{\partial a_{j}}{\partial y_{k}}-\sum_{\ell=1}^{n}\left(\frac{\partial \zeta_{j}}{\partial x_{\ell}}(\theta, f(y)) \frac{\partial f_{x}^{j}}{\partial y_{k}}(y)\right. \\
\left.-\frac{\partial \zeta_{j}}{\partial \xi_{\ell}}(\theta, f(y)) \frac{\partial f_{\xi}^{j}}{\partial y_{k}}(y)\right) .
\end{gathered}
$$

It follows from Proposition 3.3.2 that,

$$
\left|\frac{\partial f}{\partial y_{k}}(y)\right| \leqslant C\left(\frac{1}{\langle\theta\rangle}+\left|\frac{\partial a}{\partial y_{k}}(\theta, y, \alpha)\right|+\varepsilon\left|\frac{\partial f}{\partial y_{k}}(y)\right|\right)
$$

where $C$ depends only on the constants $A_{0}, A_{1}$ appearing in (3.1.1). Therefore taking $\varepsilon$ so small that $C \varepsilon \leqslant \frac{1}{2}$ we obtain the point a) in Lemma 4.3 .3 for $|A|=1$. Let us 
prove the point $\mathrm{b}$ ). First of all we deduce from a) and the Consequence 4.3.4 that

$$
\left\{\begin{array}{l}
\frac{\partial}{\partial y_{k}}\left[Z_{\ell}^{j}(\theta, f(y))\right], \quad \ell=1,2, \quad \frac{\partial}{\partial y_{k}}\left[F_{p}^{j}\right], \quad p=3,4 \\
\text { are bounded by } C \varepsilon\left(\frac{1}{\langle\theta\rangle}+\left|\frac{\partial a}{\partial y_{k}}(\theta, y, \alpha)\right|\right) .
\end{array}\right.
$$

Now we claim that for $p=3,4$,

$$
\left|\frac{\partial}{\partial y_{k}}\left[H_{p}^{j}(\theta, y, \alpha, a, b)\right]\right| \leqslant C\left(\left|\frac{\partial a}{\partial y_{k}}(\theta, y, \alpha)\right|+\left|\frac{\partial b}{\partial y_{k}}(\theta, y, \alpha)\right|+\frac{1}{\langle\theta\rangle}\right) .
$$

Indeed the left hand side of (4.3.26) can be written

$$
\frac{\partial H_{p}^{j}}{\partial a} \cdot \frac{\partial a}{\partial y_{k}}+\frac{\partial H_{p}^{j}}{\partial b} \cdot \frac{\partial b}{\partial y_{k}}+\frac{\partial H_{p}^{j}}{\partial y_{k}}=(1)+(2)+(3) .
$$

Now using (4.3.20), (4.3.18) and (4.3.17) we see that (1) and (2) can be bounded by the right hand side of (4.3.26). To handle the term (3) we use (4.3.16) with $A=0$, $|B|=1$. We obtain

$$
|(3)| \leqslant C \sum_{|\gamma| \leqslant 3 n+2} \int\left|\frac{\partial}{\partial y_{k}} \partial_{\eta}^{\gamma} g_{j}(\eta)\right| d \eta .
$$

Now we use (4.3.9) and (4.3.23). We obtain

$$
\begin{aligned}
\partial_{\eta}^{\gamma} g_{j}(\eta)=\partial_{\eta}^{\gamma}\left[\chi_{0}\left(\frac{1}{\mu_{0}} \eta\right)\right]\left[\left(\beta_{\xi}^{j}-i \beta_{x}^{j}\right)\right. & \left.(\theta, y, \alpha, \gamma)-\left(\alpha_{\xi}^{j}+i \alpha_{x}^{j}\right)\right] \\
& +\sum_{\left|\gamma^{\prime}\right|<|\gamma|} \partial_{\eta}^{\gamma^{\prime}}\left[\chi_{0}\left(\frac{1}{\mu_{0}} \eta\right)\right] G_{\gamma^{\prime}}^{j}(\theta, \beta(\theta, y, \alpha, \eta))
\end{aligned}
$$

where $G_{\gamma^{\prime}}^{j}$ satisfy (4.3.22). Since by (4.3.5) we have

$$
\left|\frac{\partial \beta}{\partial y_{k}}(\theta, y, \alpha, \eta)\right| \leqslant \frac{C}{\langle\theta\rangle} .
$$

We obtain

which proves (4.3.26).

$$
|(3)| \leqslant \frac{C}{\langle\theta\rangle}
$$

Now we use (4.3.19), (4.3.25), (4.3.26) and the fact that $|b| \leqslant \frac{2|y|}{\langle\theta\rangle} \leqslant 2 \delta$. We obtain with $a_{j}=a_{j}(\theta, y, \alpha), b_{j}=b_{j}(\theta, y, \alpha)$,

$$
\left|\frac{\partial a_{j}}{\partial y_{k}}\right|+\left|\frac{\partial b_{j}}{\partial y_{k}}\right| \leqslant \frac{12}{\langle\theta\rangle}+C(\varepsilon+\delta)\left(\frac{1}{\langle\theta\rangle}+\left|\frac{\partial a}{\partial y_{k}}\right|+\left|\frac{\partial b}{\partial y_{k}}\right|\right) .
$$

Taking $\varepsilon$ and $\delta$ small enough we obtain the point b) in Lemma 4.3.3 when $|A|=1$.

Assume now that a) and b) in Lemma 4.3 .3 are true when $|A| \leqslant N$ and let $|A|=$ $N+1$. It follows from the induction that

$$
\left|\partial_{y}^{B}[\beta(\theta, y, \alpha,-a(\theta, y, \alpha))]\right| \leqslant \frac{C_{B}}{\langle\theta\rangle^{|B|}}, \text { if }|B| \leqslant N .
$$


Claim 1. - If $|A|=N+1 \geqslant 2$,

$$
\left|\partial_{y}^{A}[\beta(\theta, y, \alpha,-a(\theta, y, \alpha))]\right| \leqslant C_{0}\left|\partial_{y}^{A} a(\theta, y, \alpha)\right|+\frac{C_{A}}{\langle\theta\rangle^{|A|}} .
$$

Indeed, according to (4.3.5) we have, setting for short $f(y)=\beta(\theta, y, \alpha,-a(\theta, y, \alpha))$ and $k(\theta)=\langle\theta\rangle \chi_{1}(\theta)+1-\chi_{1}(\theta)$,

$$
\left\{\begin{array}{l}
f_{x}(y)=\alpha_{x}+\left(1-\frac{|\theta|}{\langle\theta\rangle}\left(1-\chi_{1}(\theta)\right)\right) y+\frac{2 \theta}{\langle\theta\rangle} k(\theta) a(\theta, y, \alpha)+z(\theta, \alpha)-z(\theta, f(y)) \\
f_{\xi}(y)=\alpha_{\xi}+\frac{1}{2}\left(1-\chi_{1}(\theta)\right) \frac{\operatorname{sgn} \theta}{\langle\theta\rangle} y-\frac{k(\theta)}{\langle\theta\rangle} a(\theta, y, \alpha)+\zeta(\theta, \alpha)-\zeta(\theta, f(y)) .
\end{array}\right.
$$

Differentiating both side $A$ times with respect to $y$ we obtain since $|A| \geqslant 2$,

$$
\left|\partial_{y}^{A} f(y)\right| \leqslant 5\left|\partial_{y}^{A} a(\theta, y, \alpha)\right|+\left|\partial_{y}^{A}[z(\theta, f(y))]\right|+\left|\partial_{y}^{A}[\zeta(\theta, f(y))]\right| .
$$

We use now the Faa di Bruno formula (see Appendix A.1). Let $Z$ be $z$ or $\zeta$ then

$$
\partial_{y}^{A}[Z(\theta, f(y))]=\sum_{j=1}^{n}\{\underbrace{\frac{\partial Z}{\partial x_{j}}(\theta, f(y)) \partial_{y}^{A} f_{x}^{j}(y)+\frac{\partial Z}{\partial \xi_{j}}(\theta, f(y)) \partial_{y}^{A} f_{\xi}^{j}(y)}_{(1)}\}+(2)
$$

where (2) is a finite sum of terms of the form

$$
\left(\partial_{X}^{\nu} Z\right)(\theta, f(y)) \prod_{j=1}^{s}\left(\partial_{y}^{\ell_{j}} f(y)\right)^{k_{j}}
$$

where $X=(x, \xi), 2 \leqslant|\nu| \leqslant|A|,\left|\ell_{j}\right| \geqslant 1,\left|k_{j}\right| \geqslant 1$ and

$$
\sum_{j=1}^{s} k_{j}=\nu, \quad \sum_{j=1}^{s}\left|k_{j}\right| \ell_{j}=A
$$

The term (1) can be bounded by $C_{0} \varepsilon\left|\partial_{y}^{A} f(y)\right|$ (where $C_{0}$ depends on $A_{0}, A_{1}$ in (3.1.1)). Since $|\nu| \geqslant 2$ it is easy to see that $\left|\ell_{j}\right| \leqslant|A|-1$. We can therefore use (4.3.27) and Proposition 3.3.2 to write

$$
|(2)| \leqslant C_{A} \varepsilon \prod_{j=1}^{s} \frac{1}{\langle\theta\rangle^{\left|\ell_{j}\right|\left|k_{j}\right|}} \leqslant \frac{C_{A}}{\langle\theta\rangle^{|A|}} .
$$

Thus (4.3.28) is proved which implies the part a) of Lemma 4.3.3 when $|A|=N+1$.

Claim 2. - If $F \in \mathcal{G}_{ \pm}$(see (4.3.21)) and $|A|=N+1$ we have

$$
\left|\partial_{y}^{A} F(\theta, y, \alpha)\right| \leqslant \varepsilon\left(C_{0}\left|\partial_{y}^{A} a(\theta, y, \alpha)\right|+\frac{C_{A}}{\langle\theta\rangle^{|A|}}\right) .
$$

Let us set for convenience as in (4.3.24),

$$
f(y)=\beta(\theta, y, \alpha,-a(\theta, y, \alpha)) .
$$


We know by assumption that $F(\theta, y, \alpha)=G(\theta, f(y))$ where $G$ satisfies (4.3.22). By the Faa di Bruno formula we have

$$
\partial_{y}^{A} F(\theta, y, \alpha)=\sum_{i=1}^{2 n} \underbrace{\frac{\partial G}{\partial X_{i}}(\theta, f(y)) \partial_{y}^{A} f(y)}_{(1)}+(2)
$$

where (2) is a finite sum of terms of the form

$$
\left(\partial_{X}^{\nu} G\right)(\theta, f(y)) \prod_{j=1}^{s}\left(\partial_{y}^{\ell_{j}} f(y)\right)^{k_{j}}
$$

where $2 \leqslant|\nu| \leqslant|A|,\left|\ell_{j}\right| \geqslant 1,\left|k_{j}\right| \geqslant 1,1 \leqslant s \leqslant|A|$ and

$$
\sum_{j=1}^{s} k_{j}=\nu, \quad \sum_{j=1}^{s}\left|k_{j}\right| \ell_{j}=A .
$$

Now by the Claim 1 we have

$$
|(1)| \leqslant \varepsilon\left(C_{0}\left|\partial_{y}^{A} a(\theta, y, \alpha)\right|+\frac{C_{A}}{\langle\theta\rangle^{|A|}}\right) .
$$

On the other hand in the term (2) it is easy to see that $\left|\ell_{j}\right| \leqslant|A|-1$. Indeed if we had a $j_{0}$ such that $\left|\ell_{j_{0}}\right|=|A|$ it would follow from (4.3.31) that $j_{0}=1, s=1$ and $\left|k_{1}\right|=1$; but then $\left|k_{1}\right|=1=|\nu|$ which is in contradiction with $|\nu| \geqslant 2$. Therefore we can use (4.3.27), (4.3.31) to write,

$$
|(2)| \leqslant C_{A} \varepsilon \prod_{j=1}^{s}\left(\frac{1}{\langle\theta\rangle^{\left|\ell_{j}\right|}}\right)^{\left|k_{j}\right|}=C_{A} \varepsilon \frac{1}{\langle\theta\rangle^{|A|}} .
$$

Then (4.3.29) follows from (4.3.30) and (4.3.32).

Claim 3. - If $|A|=N+1, j=1, \ldots, n, \ell=3,4$ we have,

$$
\left|\partial_{y}^{A}\left(H_{\ell}^{j}(\theta, y, \alpha, a, b)\right)\right| \leqslant C_{0}\left(\left|\partial_{y}^{A} a(\theta, y, \alpha)\right|+\left|\partial_{y}^{A} b(\theta, y, \alpha)\right|\right)+\frac{C_{A}}{\langle\theta\rangle^{|A|}},
$$

where $H_{\ell}^{j}$ is defined in (4.3.20), (4.3.18), (4.3.13).

The proof is exactly the same as in the Claim 2. We use the Faa di Bruno formula, the estimates on $a, b$ given by the induction, the estimate (4.3.16) to obtain (4.3.33). We are ready now to prove the part b) of Lemma 4.3 .3 when $|A|=N+1$.

We use the equations (4.3.19), (4.3.20) which we differentiate $|A|$ times with respect to $y$. Since $Z_{\ell}^{j}(\theta, \beta)$ and $F_{k}^{j}$ belong to $\mathcal{G}_{ \pm}$we use the Claim 2 to estimate them ; the term $H_{\ell}^{j} b \cdot b$ is handled by (4.3.33), the Leibniz formula and the induction hypothesis. Finally we obtain since $|b| \leqslant \frac{2|y|}{\langle\theta\rangle} \leqslant 2 \delta$,

$$
\left|\partial_{y}^{A} a_{j}\right| \leqslant(\varepsilon+\delta) C_{0}\left(\left|\partial_{y}^{A} a\right|+\left|\partial_{y}^{A} b\right|\right)+\frac{C_{A}}{\langle\theta\rangle^{|A|}} .
$$

Taking $\varepsilon$ and $\delta$ small enough we obtain the part b) of Lemma 4.3.3. 
So far we have proved the points (i), (ii), (iii) in Theorem 4.3.1.

Let us prove (iv). It follows from (4.2.1), (4.3.6) and (4.3.9) that

$$
\mid \frac{\partial q_{k}}{\partial \eta_{\ell}}\left(\eta, a, b, g_{j}|+| \frac{\partial q_{k}}{\partial b_{\ell}}\left(\eta, a, b, g_{j}\right) \mid \leqslant C\right.
$$

where $C$ is an absolute constant.

Since $|\eta| \leqslant \delta,|a| \leqslant \frac{10|y|}{\langle\theta\rangle} \leqslant 10 \delta,|b| \leqslant 2 \frac{|y|}{\langle\theta\rangle} \leqslant 2 \delta$ we can write

$$
\left|q_{k}\left(\eta, a, b, g_{j}\right)-q_{k}\left(-a, a, 0, g_{j}\right)\right| \leqslant C^{\prime} \delta .
$$

Now (4.2.8) gives $q_{k}\left(-a, a, 0, g_{j}\right)=\frac{\partial g_{j}}{\partial \eta_{k}}(-a)$. It follows then from (4.3.15), (4.3.6) and Proposition 3.3.2 that

$$
\left|q_{k}\left(-a, a, 0, g_{j}\right)-(1+2 i \theta) \frac{k(\theta)}{\langle\theta\rangle} \delta_{j k}\right| \leqslant C \varepsilon
$$

which combined with (4.3.34) gives the point (iv).

Finally (v) follows easily from (4.2.2), (4.3.9) and (4.3.6). This ends the proof of Theorem 4.3.1.

Corollary 4.3.5. - Let us set $k(\theta)=\langle\theta\rangle \chi_{1}(\theta)+\left(1-\chi_{1}(\theta)\right)$ and for $j=1, \ldots, n$,

$$
\widetilde{g}_{j}(\eta)=\chi_{0}\left(\frac{\eta-\frac{1}{2}\left(1-\chi_{1}(\theta)\right)(\operatorname{sgn} \theta) y}{\mu_{0} k(\theta)}\right) v_{j}\left(\theta, y+x(\theta, \alpha), \frac{\eta}{\langle\theta\rangle}+\xi(\theta, \alpha), \alpha\right) .
$$

Then we can write

$$
\widetilde{g}_{j}(\eta)=\sum_{\ell=1}^{n} \widetilde{q}_{\ell}\left(\eta, \widetilde{a}, \widetilde{b}, \widetilde{g}_{j}\right)\left(\eta_{\ell}-\frac{1}{2}\left(1-\chi_{1}(\theta)\right)(\operatorname{sgn} \theta) y+\left(\widetilde{a}_{\ell}+i \widetilde{b}_{\ell}\right)(\theta, y, \alpha)\right)
$$

where

$$
\widetilde{q}_{\ell}\left(\eta, \widetilde{a}, \widetilde{b}, \widetilde{g}_{j}\right)=\frac{1}{k(\theta)} q_{\ell}\left(\frac{\eta-\frac{1}{2}\left(1-\chi_{1}(\theta)\right)(\operatorname{sgn} \theta) y}{k(\theta)}, \frac{\widetilde{a}}{k(\theta)}, \frac{\widetilde{b}}{k(\theta)}, \widetilde{g}_{j}\right)
$$

and $\widetilde{q}_{\ell}, \widetilde{a}_{\ell}, \widetilde{b}_{\ell}$ satisfy

(i) $|\widetilde{a}(\theta, y, \alpha)| \leqslant 10 \sqrt{5} \frac{|y|}{\langle\theta\rangle}, \quad\left|\widetilde{b}(\theta, y, \alpha)+\frac{\langle\theta\rangle}{1+4 \theta^{2}} y\right| \leqslant \sqrt{5 \delta} \frac{|y|}{\langle\theta\rangle}$.

(ii) $\left|\partial_{y}^{A} \widetilde{a}(\theta, y, \alpha)\right|+\left|\partial_{y}^{A} \widetilde{b}(\theta, y, \alpha)\right| \leqslant \frac{C_{A}}{\langle\theta\rangle^{|A|}}, A \in \mathbb{N}^{n}$.

(iii) $\left|\widetilde{q}_{\ell}\left(\eta, \widetilde{a}, \widetilde{b}, \widetilde{g}_{j}\right)-\frac{(1+2 i \theta)}{\langle\theta\rangle} \delta_{j k}\right| \leqslant C(\varepsilon+\delta)$ if $|\eta| \leqslant \delta$.

(iv) $\left|\partial_{(a, b)}^{A} \partial_{\eta}^{B} \widetilde{q}_{\ell}\left(\eta, \widetilde{a}, \widetilde{b}, \widetilde{g}_{j}\right)\right| \leqslant C\left(\mu_{0}\right)$ if $|A|+|B| \geqslant 1,|\eta| \leqslant \mu_{0}, 1 \leqslant j, \ell \leqslant n$ uniformly with respect to $(\theta, y) \in \widetilde{\Omega}_{\delta}$ and $\alpha \in \mathcal{S}$.

Proof. - We have

$$
\chi_{1}(\theta) \eta+\left(1-\chi_{1}(\theta)\right)\left[\frac{\eta}{\langle\theta\rangle}+\frac{1}{2} \frac{1-\chi_{1}(\theta)}{\langle\theta\rangle}(\operatorname{sgn} \theta) y\right]=\frac{k(\theta)}{\langle\theta\rangle} \eta+\frac{1}{2} \frac{1-\chi_{1}(\theta)}{\langle\theta\rangle}(\operatorname{sgn} \theta) y .
$$


So let us set in the statement of Theorem 4.3.1 $\widetilde{\eta}=k(\theta) \eta+\frac{1}{2}\left(1-\chi_{1}(\theta)\right)\left(\underset{\sigma_{l}}{\operatorname{sgn}} \theta\right) y$; then we obtain the decomposition of $\widetilde{g}_{j}$ in Corollary 4.3 .5 with $\widetilde{a}_{\ell}=k(\theta) a_{\ell}, \widetilde{b}_{\ell}=k(\theta) b_{\ell}$ and the estimates on $\widetilde{q}_{\ell}, \widetilde{a}_{\ell}, \widetilde{b}_{\ell}$ follow easily from the correspondent one for $q_{\ell}, a_{\ell}, b_{\ell}$ stated in Theorem 4.3.1.

Lagrangian ideals and the phase equation. - We pursue here the proof of Theorem 4.1.2. Let us set

$$
\mathcal{O}=\left\{(\theta, y, \eta) \in \mathbb{R} \times \mathbb{R}^{n} \times \mathbb{R}^{n}:|y|<\delta\langle\theta\rangle, \frac{\left|\eta-\frac{1}{2}\left(1-\chi_{1}(\theta)\right)(\operatorname{sgn} \theta) y\right|}{k(\theta)}<\delta\right\}
$$

We introduce now a space of families $(f(\cdot, \alpha))_{\alpha \in \mathcal{S}}$ of functions on $\mathcal{O}$.

Definition 4.3.6. - We say that $(f(\cdot, \alpha))_{\alpha \in \mathcal{S}}$ belongs to $\mathcal{H}$ if

(i) For all $\alpha$ in $\mathcal{S},(\theta, y, \eta) \mapsto f(\theta, y, \eta, \alpha)$ belongs to $C^{\infty}(\mathcal{O})$.

(ii) For every $A, B$ in $\mathbb{N}^{n}$ there exists $C_{A B}>0$ independent of $\alpha$ such that $\sup _{(\theta, y, \eta) \in \mathcal{O}}\left|\partial_{y}^{A} \partial_{\eta}^{B} f(\theta, y, \eta, \alpha)\right| \leqslant C_{A B}$, for all $\alpha \in \mathcal{S}$.

\section{REMARK 4.3 .7}

1) $\mathcal{H}$ is closed under multiplication and derivation with respect to $(y, \eta)$.

2) If we set, with notation (4.2.10)

$$
f(\theta, y, \eta, \alpha)=v_{j}\left(\theta, y+x(\theta, \alpha), \frac{\eta}{\langle\theta\rangle}+\xi(\theta, \alpha), \alpha\right)
$$

then $(f(\cdot, \alpha))_{\alpha \in \mathcal{S}}$ belongs to $\mathcal{H}$. This follows from (4.3.6).

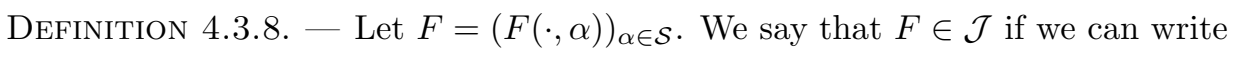

$$
F(\theta, y, \eta, \alpha)=\sum_{j=1}^{n} f_{j}(\theta, y, \eta, \alpha) v_{j}\left(\theta, y+x(\theta, \alpha), \frac{\eta}{\langle\theta\rangle}+\xi(\theta, \alpha), \alpha\right)
$$

for all $(\theta, y, \eta, \alpha)$ in $\mathcal{O} \times \mathcal{S}$ with $(f(\cdot, \alpha))_{\alpha \in \mathcal{S}} \in \mathcal{H}$.

EXAMPLE 4.3.9. - Let us set $F=(F(\cdot, \alpha))_{\alpha \in \mathcal{S}}$ where

$$
F(\theta, y, \eta, \alpha)=\eta_{k}-\frac{1}{2}\left(1-\chi_{1}(\theta)(\operatorname{sgn} \theta) y_{k}+\left(\widetilde{a}_{k}+i \widetilde{b}_{k}\right)(\theta, y, \alpha), \quad k \in\{1,2, \ldots, n\} .\right.
$$

Then $F \in \mathcal{J}$.

This follows from Corollary 4.3.5. Indeed the matrix $\left(\widetilde{q}_{\ell}\left(\eta, \widetilde{a}, \widetilde{b}, \widetilde{g}_{j}\right)\right)_{j, \ell}$ is invertible if $\varepsilon+\delta$ is small enough and according to the estimate (v) if we set $\left(d_{\ell j}\right)=(\widetilde{q}(\cdots))^{-1}$ then $\left(d_{j k}(\cdot, \alpha)\right)_{\alpha \in \mathcal{S}}$ belongs to $\mathcal{H}$ so our claim follows from Remark 4.3.7.

Now if $F=(F(\cdot, \alpha))_{\alpha \in \mathcal{S}}, G=(G(\cdot, \alpha))_{\alpha \in \mathcal{S}}$ we set

$$
\{F, G\}=\left(\sum_{j=1}^{n}\left(\frac{\partial F}{\partial \eta_{j}} \frac{\partial G}{\partial y_{j}}-\frac{\partial F}{\partial y_{j}} \frac{\partial G}{\partial \eta_{j}}\right)(\cdot, \alpha)\right)_{\alpha \in \mathcal{S}} .
$$

Then we have the following result. 
Lemma 4.3.10. $-\mathcal{J}$ is closed under the Poisson bracket (4.3.37).

Proof. - Recall (see (4.2.10)) that $v_{j}(\theta, x, \xi, \alpha)=u_{j} \circ \chi_{-\theta}(x, \xi)$ where $u_{j}(x, \xi, \alpha)=$ $\xi_{j}-\alpha_{\xi}^{j}-i\left(x_{j}-\alpha_{x}^{j}\right)$ and $\chi_{-\theta}(x, \xi)=(x(-\theta ; x, \xi), \xi(-\theta ; x, \xi))$ is the symplectic map defined by the flow. Since $\left\{u_{j}, u_{k}\right\}=0$ we have, denoting by $\{$,$\} the Poisson bracket$ in $(x, \xi),\left\{v_{j}, v_{k}\right\}=\left\{u_{j} \circ \chi_{-\theta}, u_{k} \circ \chi_{-\theta}\right\}=\left\{u_{j}, u_{k}\right\} \circ \chi_{-\theta}=0$. It follows that, in the coordinates $(y, \eta)$,

$$
\left\{v_{j}\left(\theta ; y+x(\theta ; \alpha), \frac{\eta}{\langle\theta\rangle}+\xi(\theta ; \alpha)\right), v_{k}\left(\theta ; y+x(\theta ; \alpha), \frac{\eta}{\langle\theta\rangle}+\xi(\theta ; \alpha)\right)\right\}=0 .
$$

Let $F=\left(\sum_{j} f_{j} v_{j}(\cdot, \alpha)\right)_{\alpha \in \mathcal{S}}, G=\left(\sum_{k} g_{k} v_{k}(\cdot, \alpha)\right)_{\alpha \in \mathcal{S}}$ two elements of $\mathcal{J}$. Then if $\{$,$\} is the Poisson bracket in (y, \eta)$, we have,

$$
\begin{aligned}
& \left\{\sum_{j} f_{j} v_{j}, \sum_{k} g_{k} v_{k}\right\} \\
& \quad=\sum_{k}\left(\sum_{j} f_{j}\left\{v_{j}, g_{k}\right\}\right) v_{k}+\sum_{k}\left(\sum_{j}\left\{f_{j}, g_{k}\right\} v_{j}\right) v_{k}+\sum_{j}\left(\sum_{k}\left\{f_{j}, v_{k}\right\} g_{k}\right) v_{j} .
\end{aligned}
$$

Since $f_{j}, g_{k}, v_{j}$ belong to $\mathcal{H}$ it follows from Remark 4.3.7 (i) and Definition 4.3.8 that $\{F, G\} \in \mathcal{J}$.

Here is an important lemma which is a generalization to our context of Lemma 7.5.10 of $[\mathbf{H}]$.

According to Corollary 4.3.5, we shall set

$$
\psi_{k}(\theta, y, \alpha)=\frac{1}{2}\left(1-\chi_{1}(\theta)\right)(\operatorname{sgn} \theta) y_{k}-\left(\widetilde{a}_{k}(\theta ; y, \alpha)+i \widetilde{b}_{k}(\theta ; y, \alpha)\right), \quad k=1, \ldots, n .
$$

Lemma 4.3.11. - Let $R=(R(\cdot, \alpha))_{\alpha \in \mathcal{S}} \in \mathcal{J}$ where $R(\theta, y, \alpha)$ is independent of $\eta$. Then for every $N \in \mathbb{N}$ one can find $C_{N}>0$ such that for every $(\theta, y)$ in $\widetilde{\Omega}_{\delta}$ (see (4.3.2)) and $\alpha$ in $\mathcal{S}$ we have

$$
|R(\theta, y, \alpha)| \leqslant C_{N}|\operatorname{Im} \psi(\theta, y, \alpha)|^{N} .
$$

Proof. - We are going to show by induction on $N \in \mathbb{N}^{*}$ that we can write for $(\theta, y, \alpha)$ in $\widetilde{\Omega}_{\delta} \times \mathcal{S}$,

$$
R(\theta, y, \alpha)=\sum_{0<|\gamma|<N} h_{\gamma}(\theta, y, \alpha)(\eta-\psi)^{\gamma}+\sum_{|\gamma|=N} w_{\gamma}(\theta, y, \eta, \alpha)(\eta-\psi)^{\gamma},
$$

where $\left(h_{\gamma}(\cdot, \alpha)\right)_{\alpha \in \mathcal{S}}$ and $\left(w_{\gamma}(\cdot, \alpha)\right)_{\alpha \in \mathcal{S}}$ belong to $\mathcal{H}$.

For $N=1$ the first sum in (4.3.39) is empty and by assumption we have

$$
R(\theta, y, \alpha)=\sum_{j=1}^{n} f_{j}(\theta, y, \eta, \alpha) v_{j}\left(\theta ; y+x(\theta ; \alpha), \frac{\eta}{\langle\theta\rangle}+\xi(\theta ; \alpha)\right)
$$


where $\left(f_{j}(\cdot, \alpha)\right)_{\alpha \in \mathcal{H}}$. Now we use Corollary 4.3.5. Since $\chi_{0}\left(\frac{\eta-\frac{1}{2}\left(1-\chi_{1}(\theta)\right)(\operatorname{sgn} \theta) y}{k(\theta) \mu_{0}}\right)=1$ if $(\theta, y, \eta) \in \mathcal{O}$ we can write

$$
R(\theta, y, \alpha)=\sum_{k=1}^{n} r_{k}(\theta, y, \eta, \alpha)\left(\eta_{k}-\psi_{k}(\theta, y, \alpha)\right)
$$

where

$$
r_{k}(\theta, y, \eta, \alpha)=\sum_{j=1}^{n} f_{j}(\theta, y, \eta, \alpha) \widetilde{q}_{k}\left(\eta, \widetilde{a}, \widetilde{b}, \widetilde{g}_{j}\right) .
$$

Now it follows from Corollary 4.3.5 and Remark 4.3.7 that $\left(r_{k}(\cdot, \alpha)\right)_{\alpha \in \mathcal{S}}$ belongs to $\mathcal{H}$. Therefore (4.3.39) holds when $N=1$. Assume now that (4.3.39) is true at the level $N$. Then apply Lemma 4.2.1 to the function

$$
g_{\gamma}(\theta, y, \eta, \alpha)=\chi_{0}\left(\frac{\eta-\frac{1}{2}\left(1-\chi_{1}(\theta)\right)(\operatorname{sgn} \theta) y}{\mu_{0} k(\theta)}\right) w_{\gamma}(\theta, y, \eta, \alpha), \quad|\gamma|=N,
$$

with $z=-\psi(\theta, y, \alpha)$. It follows then that

$$
g_{\gamma}(\theta, y, \eta, \alpha)=\sum_{k=1}^{n} q_{k}\left(\eta, a, b, g_{\gamma}\right)\left(\eta_{k}-\psi_{k}(\theta, y, \alpha)\right)+r\left(a, b, g_{\gamma}\right)
$$

For the $q_{k}^{\prime} s$ and $r$ we have the estimates (4.2.2). If we set

$$
\left\{\begin{array}{l}
h_{\gamma}(\theta, y, \alpha)=r\left(a(\theta, y \alpha), b(\theta, y, \alpha), g_{\gamma}(\theta, y, \cdot, \alpha)\right) \\
w_{\gamma j}(\theta, y, \eta, \alpha)=q_{j}\left(\eta, a(\theta, y, \alpha), b(\theta, y, \alpha), g_{\gamma}(\theta, y, \cdot, \alpha)\right) .
\end{array}\right.
$$

We deduce from (4.2.2) and Corollary 4.3.5 that $\left(h_{\gamma}(\cdot, \alpha)\right)_{\alpha \in \mathcal{S}}$ and $\left(w_{\gamma j}(\cdot, \alpha)\right)_{\alpha \in \mathcal{S}}$ belong to $\mathcal{H}$. Then using (4.3.39) at the level $N$ and (4.3.40), (4.3.41) we obtain (4.3.39) at the level $N+1$. Let us take now in (4.3.39) $\eta=\operatorname{Re} \psi(\theta, y, \alpha)+s \operatorname{Im} \psi(\theta, y, \alpha)$ where $s \in[0,1]$. We obtain

$$
\left|R(\theta, y, \alpha)-\sum_{0<|\gamma|<N} h_{\gamma}(\theta, y, \alpha)(\operatorname{Im} \psi(\theta, y, \alpha))^{\gamma}(s-i)^{\gamma}\right| \leqslant C_{N}|\operatorname{Im} \psi(\theta, y, \alpha)|^{N}
$$

where $C_{N}$ is independent of $(\theta, y, \alpha) \in \widetilde{\Omega}_{\delta} \times \mathcal{S}$.

Using an interpolation formula we deduce that the coefficients of the polynomial in $(s-i)$ in the left hand side of (4.3.42) satisfy the same estimate which proves that $R$ has the claimed bound.

Corollary 4.3.12. - For every $N \in \mathbb{N}$ there exists a constant $C_{N}>0$ such that

$$
\left|\left(\frac{\partial \psi_{j}}{\partial y_{k}}-\frac{\partial \psi_{k}}{\partial y_{j}}\right)(\theta, y, \alpha)\right| \leqslant C_{N}|\operatorname{Im} \psi(\theta, y, \alpha)|^{N}
$$

for every $(\theta, y)$ in $\widetilde{\Omega}_{\delta}$ and $\alpha$ in $\mathcal{S}$. 
Proof. - According to (4.3.38) and Example 4.3.9 we have $\eta_{k}-\psi_{k}(\theta, y, \alpha) \in \mathcal{J}$. It follows from Lemma 4.3.10 that

$$
R_{j k}(\theta, y, \alpha):=\left(\frac{\partial \psi_{j}}{\partial y_{k}}-\frac{\partial \psi_{k}}{\partial y_{j}}\right)(\theta, y, \alpha)=\left\{\eta_{j}-\psi_{j}, \eta_{k}-\psi_{k}\right\}(\theta, y, \alpha)
$$

defines an element of $\mathcal{J}$. Since $R_{j k}$ does not depend on $\eta$ we can apply Lemma 4.3.11 and the conclusion follows.

So far we have worked in the coordinates $(y, \eta)$. Let us go back to the original coordinates $(x, \xi)$ and let us summarize the results already obtained.

We set $x=y+x(\theta, \alpha), \xi=\frac{\eta}{\langle\theta\rangle}+\xi(\theta, \alpha)$. Then $(\theta, x) \in \Omega_{\delta}$ (see (4.3.1)). Let us recall that,

$$
v_{j}(\theta, x, \xi, \alpha)=\xi_{j}(-\theta, x, \xi)-\alpha_{\xi}^{j}-i\left(x_{j}(-\theta, x, \xi)-\alpha_{x}^{j}\right),
$$

(see (4.2.10)).

Now let us introduce

$$
\begin{aligned}
& \Phi_{k}(\theta, x, \alpha)=\xi_{k}(\theta, \alpha) \\
& +\frac{\frac{1}{2}(\operatorname{sgn} \theta)\left(1-\chi_{1}(\theta)\right)(x-x(\theta, \alpha))-\left(\widetilde{a}_{k}+i \widetilde{b}_{k}\right)(\theta, x-x(\theta, \alpha), \alpha)}{\langle\theta\rangle}
\end{aligned}
$$

where $\widetilde{a}_{k}, \widetilde{b}_{k}$ have been introduced in Corollary 4.3.5. Then we have,

THEOREM 4.3.13. - We can write

(i) $\xi_{k}-\Phi_{k}(\theta, x, \alpha)=\sum_{j=1}^{n} d_{k}^{j}(\theta, x, \xi, \alpha) v_{j}(\theta, x, \xi, \alpha)$

where $d_{k}^{j}$ are smooth functions defined for $(\theta, x) \in \Omega_{\delta}$ and

$$
\left|\xi-\xi(\theta, \alpha)-\frac{1}{2}\left(1-\chi_{1}(\theta)\right)(\operatorname{sgn} \theta) \frac{x-x(\theta, \alpha)}{\langle\theta\rangle}\right| \leqslant \frac{\delta}{\langle\theta\rangle} .
$$

Moreover we have in this set,

(ii) $\left|\partial_{x}^{A} d_{k}^{j}(\theta, x, \xi, \alpha)\right| \leqslant \frac{C_{A}}{\langle\theta\rangle}, \quad A \in \mathbb{N}^{n}$,

(iii) $\left|\Phi_{k}(\theta, x, \alpha)-\alpha_{\xi}\right| \leqslant C(\varepsilon+\delta)$,

(iv) $\left|\operatorname{Im} \Phi_{k}(\theta, x, \alpha)-\frac{x_{k}-x_{k}(\theta, \alpha)}{1+4 \theta^{2}}\right| \leqslant \sqrt{5 \delta} \frac{|x-x(\theta, \alpha)|}{\langle\theta\rangle^{2}}$.

(v) $\left|\partial_{x}^{A} \Phi_{k}(\theta, x, \alpha)\right| \leqslant\left\{\begin{array}{lll}C_{A}\langle\theta\rangle^{-|A|} & \text { if }|A| \leqslant 1 \\ C_{A}\langle\theta\rangle^{-|A|-1} & \text { if }|A| \geqslant 2\end{array}\right.$.

(vi) $\Phi_{k}(\theta, x(\theta, \alpha), \alpha)=\xi(\theta, \alpha)$.

(vii) $\left|\left(\frac{\partial \Phi_{j}}{\partial x_{k}}-\frac{\partial \Phi_{k}}{\partial x_{j}}\right)(\theta, x, \alpha)\right| \leqslant \frac{C_{N}}{\langle\theta\rangle}\left(\frac{|x-x(\theta, \alpha)|}{\langle\theta\rangle}\right)^{N}, \quad \forall N \in \mathbb{N}$

where the constants $C_{A}, C_{0}, C_{N}$ are independent of $(\theta, x, \xi, \alpha)$. 
Proof. - It follows from Corollary 4.3.5 that when

$$
\left|\xi-\xi(\theta, \alpha)-\frac{1}{2}(\operatorname{sgn} \theta)\left(1-\chi_{1}(\theta)\right) \frac{x-x(\theta, \alpha)}{\langle\theta\rangle}\right| \leqslant \frac{\delta}{\langle\theta\rangle}
$$

then

with

$$
v_{j}(\theta, x, \xi, \alpha)=\sum_{k=1}^{n} c_{j k}(\theta, x, \xi, \alpha)\left(\xi_{k}-\Phi_{k}(\theta, x, \alpha)\right)
$$

$$
c_{j k}(\theta, x, \xi, \alpha)=\langle\theta\rangle q_{k}\left(\langle\theta\rangle(\xi-\xi(\theta, \alpha))-\frac{1}{2}(\operatorname{sgn} \theta)\left(1-\chi_{1}(\theta)\right)(x-x(\theta, \alpha)), \widetilde{a}, \widetilde{b}, \widetilde{g}_{j}\right)
$$

where $\widetilde{q}_{k}$ is defined in Corollary 4.3.5 (i). By (v) of the same result we have,

$$
\left|c_{j k}(\theta, x, \xi)-(1+2 i \theta) \delta_{j k}\right| \leqslant C(\varepsilon+\delta)\langle\theta\rangle .
$$

It follows then that the matrix $\left(c_{j k}\right)$ is uniformly invertible. Let us set $\left(d_{k}^{j}(\theta, x, \xi)\right)=$ $\left(c_{j k}(\theta, x, \xi)\right)^{-1}$. Then we obtain (i) in Theorem 4.3.13. The estimate (ii) follows then from Corollary 4.3.5 (v) and (4.3.44).

Let us now prove the claimed properties of $\Phi_{k}$. First of all since $\xi(\theta, \alpha)=\alpha_{\xi}+\mathcal{O}(\varepsilon)$, $\frac{|x-x(\theta, \alpha)|}{\langle\theta\rangle} \leqslant \delta,\left|\widetilde{a}_{k}\right|+\left|\widetilde{b}_{k}\right| \leqslant C \delta$. We deduce easily from (4.3.43) that $\left|\Phi_{k}(\theta, x, \xi)-\alpha_{\xi}\right| \leqslant$ $C(\varepsilon+\delta)$. On the other hand it follows from (4.3.43) and Corollary 4.3.5 (i)

$$
\operatorname{Im} \Phi_{k}(\theta, x, \xi)=-\frac{1}{\langle\theta\rangle} \widetilde{b}_{k}(\theta, x-x(\theta, \alpha), \alpha)=\frac{x_{k}-x_{k}(\theta, \alpha)}{1+4 \theta^{2}}+R
$$

where $|R| \leqslant|x-x(\theta, \alpha)| /\langle\theta\rangle^{2}$.

The point (v) in Theorem 4.3.13 follows easily from (4.3.43) and Corollary 4.3.5 (ii) ; the point (vi) is obvious since $\widetilde{a}_{k}(\theta, 0, \alpha)=\widetilde{b}_{k}(\theta, 0, \alpha)=0$. Finally for the point (vii) we remark that according to (4.3.38) and (4.3.43) we have

$$
(1)=\left(\frac{\partial \Phi_{j}}{\partial x_{k}}-\frac{\partial \Phi_{k}}{\partial x_{j}}\right)(\theta, x, \alpha)=\frac{1}{\langle\theta\rangle}\left(\frac{\partial \psi_{j}}{\partial y_{k}}-\frac{\partial \psi_{k}}{\partial y_{j}}\right)(\theta, x-x(\theta, \alpha), \alpha) .
$$

Using Corollary 4.3.12 and the point (iv) we obtain

$$
\begin{aligned}
& |(1)| \leqslant \frac{C_{N}}{\langle\theta\rangle}|\operatorname{Im} \psi(\theta, x-x(\theta, \alpha), \alpha)|^{N}=\frac{C_{N}}{\langle\theta\rangle}(\langle\theta\rangle|\operatorname{Im} \Phi(\theta, x, \alpha)|)^{N} \\
& |(1)| \leqslant \frac{C_{N}}{\langle\theta\rangle}\left(\frac{|x-x(\theta, \alpha)|}{\langle\theta\rangle}\right)^{N}, \text { for all } N \in \mathbb{N} .
\end{aligned}
$$

We need now to introduce the definition of Lagrangian ideals in the coordinates $(x, \xi)$.

Let $(F(\cdot, \alpha))_{\alpha \in \mathcal{S}}$ a family of functions $F(\theta, x, \xi, \alpha)$ defined for $(\theta, x)$ in $\Omega_{\delta}$ and $\left|\xi-\xi(\theta, \alpha)-\frac{1}{2}(\operatorname{sgn} \theta) \frac{(x-x(\theta, \alpha))}{\langle\theta\rangle}\right|<\delta /\langle\theta\rangle$.

Definition 4.3.14. - We shall say that the family $(F(\cdot, \alpha))_{\alpha \in \mathcal{S}}$ belongs to $\mathcal{J}_{(x, \xi)}$ if

$$
\left(\langle\theta\rangle F\left(\theta, y+x(\theta, \alpha), \frac{\eta}{\langle\theta\rangle}+\xi(\theta, \alpha), \alpha\right)\right)_{\alpha \in \mathcal{S}} \in \mathcal{J}
$$

where $\mathcal{J}$ has been introduced in Definition 4.3.8. 
For example, $\left(\xi_{j}-\Phi_{j}(\theta, x, \alpha)\right)_{\alpha \in \mathcal{S}} \in \mathcal{J}_{(x, \xi)}$. As a consequence of Lemma 4.3 .11 we have the following result.

Lemma 4.3.15. - Let $\left(R(\cdot, \alpha)_{\alpha \in \mathcal{S}}\right.$ be in $\mathcal{J}_{(x, \xi)}$ and assume that $R$ is independent of $\xi$ then for every $N \in \mathbb{N}$ there exists $C_{N}>0$ such that,

$$
|R(\theta, x, \alpha)| \leqslant \frac{C_{N}}{\langle\theta\rangle}\left(\frac{|x-x(\theta, \alpha)|}{\langle\theta\rangle}\right)^{N}
$$

We can now pursue the proof of the existence of a phase as described in Theorem 4.1.2.

Lemma 4.3.16. - With $\Phi$ defined in (4.3.43) we have

$$
\left(-\frac{\partial p}{\partial x_{k}}(x, \xi)-\frac{\partial \Phi_{k}}{\partial \theta}(\theta, x, \alpha)-\sum_{j=1}^{n} \frac{\partial \Phi_{k}}{\partial x_{j}}(\theta, x, \alpha) \frac{\partial p}{\partial \xi_{j}}(x, \xi)\right)_{\alpha \in \mathcal{S}} \in \mathcal{J}_{(x, \xi)} .
$$

Proof. - We know from (4.3.43), (4.3.38) and Example 4.3.9, with $\xi=\frac{\eta}{\langle\theta\rangle}+\xi(\theta, \alpha)$ that,

$$
\begin{aligned}
\xi_{k}-\Phi_{k}(\theta, x, \alpha) & =\frac{1}{\langle\theta\rangle}\left(\eta_{k}-\psi_{k}(\theta, x-x(\theta, \alpha), \alpha)\right) \\
& =\frac{1}{\langle\theta\rangle} \sum_{j=1}^{n} f_{j k}(\theta, x-x(\theta, \alpha),\langle\theta\rangle(\xi-\xi(\theta, \alpha)), \alpha) v_{j}(\theta, x, \xi, \alpha)
\end{aligned}
$$

where $\left(f_{j k}(\cdot, \alpha)\right)_{\alpha \in \mathcal{S}}=\left(\left(q_{k}\left(\eta-\frac{1}{2}(\operatorname{sgn} \theta)\left(1-\chi_{1}(\theta)\right) y, \widetilde{a}, \widetilde{b}, g_{j}\right)\right)^{-1}\right)_{\alpha \in \mathcal{S}} \in \mathcal{H}$ (see Definition 4.3.6).

Recall now that $v_{j}(\theta, x, \xi, \alpha)=u_{j} \circ \chi_{-\theta}(x, \xi)$ where $u_{j}(X, \Xi)=\Xi_{j}-\alpha_{\xi}^{j}-i\left(X_{j}-\alpha_{x}^{j}\right)$ (see (IV.2.9)). Let us set

$$
\chi_{-\theta}(x, \xi)=(X, \Xi) \Longleftrightarrow x(\theta, X, \Xi)=x, \quad \xi(\theta, X, \Xi)=\xi .
$$

It follows that

$$
\begin{aligned}
& \xi_{k}(\theta, X, \Xi)-\Phi_{k}(\theta, x(\theta, X, \Xi), \alpha) \\
= & \frac{1}{\langle\theta\rangle} \sum_{j=1}^{n} f_{j k}(\theta, x(\theta, X, \Xi)-x(\theta, \alpha),\langle\theta\rangle(\xi(\theta, X, \Xi)-\xi(\theta, \alpha)), \alpha) u_{j}(X, \Xi) .
\end{aligned}
$$

Let us set

$$
\left\{\begin{array}{l}
M(\theta, X, \Xi, \alpha)=(\theta, x(\theta, X, \Xi)-x(\theta, \alpha),\langle\theta\rangle(\xi(\theta, X, \Xi)-\xi(\theta, \alpha))), \\
\rho(\theta, X, \Xi)=(x(\theta, X, \Xi), \xi(\theta, X, \Xi)), \\
\rho(\theta, \alpha)=(x(\theta, \alpha), \xi(\theta, \alpha)) .
\end{array}\right.
$$


Now we differentiate (4.3.47) with respect to $\theta$ using the equation of the flow given in (3.1.2). We obtain

$$
\begin{aligned}
-\frac{\partial p}{\partial x_{k}}(\rho(\theta, X, \Xi))-\frac{\partial \Phi_{k}}{\partial \theta}(\theta, x(\theta, X, \Xi), \alpha)-\sum_{j=1}^{n} \frac{\partial \Phi_{k}}{\partial x_{j}}(\theta, x(\theta, X, \Xi), \alpha) \cdot \frac{\partial p}{\partial \xi_{j}}(\rho(\theta, X, \Xi)) \\
=\sum_{j=1}^{n}\left[-\frac{\theta}{\langle\theta\rangle^{3}} f_{j k}(M(\theta, X, \Xi, \alpha))+\frac{1}{\langle\theta\rangle} \frac{\partial f_{j k}}{\partial \theta}(M(\theta, X, \Xi, \alpha))\right. \\
+\frac{1}{\langle\theta\rangle} \sum_{\ell=1}^{n}\left[\left(\frac{\partial p}{\partial \xi_{\ell}}(\rho(\theta, X, \Xi))-\frac{\partial p}{\partial \xi_{\ell}}(\rho(\theta, \alpha))\right) \frac{\partial f_{j k}}{\partial y_{\ell}}(M(\theta, X, \Xi, \alpha))\right. \\
+\left\{\frac{\theta}{\langle\theta\rangle}\left(\xi_{\ell}(\theta, X, \Xi)-\xi_{\ell}(\theta, \alpha)\right)-\langle\theta\rangle\left(\frac{\partial p}{\partial x_{\ell}}\left(\rho(\theta, X, \Xi)-\frac{\partial p}{\partial x_{\ell}}(\rho, \alpha)\right)\right\}\right. \\
\left.\left.\frac{\partial f_{j k}}{\partial \eta_{\ell}}(M(\theta, X, \Xi, \alpha))\right]\right] u_{j}(X, \Xi)
\end{aligned}
$$

We can now go back to the coordinates $(x, \xi)=\rho(\theta, X, \Xi)$ and then to $(y, \eta)$ where $y=x-x(\theta, \alpha), \xi-\xi(\theta, \alpha)=\frac{\eta}{\langle\theta\rangle}$. We obtain

$$
\begin{aligned}
& -\left[\frac{\partial p}{\partial x_{k}}-\frac{\partial \Phi_{k}}{\partial \theta}-\sum_{j=1}^{n} \frac{\partial \Phi_{k}}{\partial x_{j}} \frac{\partial p}{\partial \xi_{j}}\right]\left(\theta, y+x(\theta, \alpha), \frac{\eta}{\langle\theta\rangle}+\xi(\theta, \alpha)\right) \\
& =\sum_{j=1}^{n}\left[\frac{-\theta}{\langle\theta\rangle^{3}} f_{j k}(\theta, y, \eta, \alpha)+\frac{1}{\langle\theta\rangle} \frac{\partial f_{j k}}{\partial \theta}(\theta, y, \eta, \alpha)\right. \\
& +\frac{1}{\langle\theta\rangle} \sum_{\ell=1}^{n}\left(\frac{\partial p}{\partial \xi_{\ell}}\left(y+x(\theta, \alpha), \frac{\eta}{\langle\theta\rangle}+\xi(\theta, \alpha)\right)-\frac{\partial p}{\partial \xi_{\ell}}(\rho(\theta, \alpha)) \frac{\partial f_{j k}}{\partial y_{\ell}}(\theta, y, \eta, \alpha)\right. \\
& \left.+\sum_{\ell=1}^{n}\left(\frac{\theta}{\langle\theta\rangle^{3}} \eta_{\ell}-\left\{\frac{\partial p}{\partial x_{\ell}}\left(y+x(\theta, \alpha) \frac{\eta}{\langle\theta\rangle}+\xi(\theta, \alpha)\right)-\frac{\partial p}{\partial x_{\ell}}(\rho(\theta, \alpha))\right\}\right) \frac{\partial f_{j k}}{\partial \eta_{\ell}}(\theta, y, \eta, \alpha)\right] \\
& v_{j}\left(\theta, y+x(\theta, \alpha), \frac{\eta}{\langle\theta\rangle}+\xi(\theta, \alpha)\right)=: \sum_{j=1}^{n} G_{j}(\theta, y, \eta, \alpha) v_{j}\left(\theta, y+x(\theta, \alpha) \frac{\eta}{\langle\theta\rangle}+\xi(\theta, \alpha)\right) .
\end{aligned}
$$

According to Definition 4.3.14 the Lemma will be proved if we show that $\left(\langle\theta\rangle G_{j}(\theta, y, \eta, \alpha)\right)_{\alpha \in \mathcal{S}}$ belongs to $\mathcal{H}$ that is all the derivatives with respect to $(y, \eta)$ are uniformly bounded when $|y| \leqslant \delta\langle\theta\rangle$ and $\left|\eta-\frac{1}{2} \operatorname{sgn} \theta\left(1-\chi_{1}(\theta)\right) y\right| \leqslant \delta$. Recall that $\left(f_{j k}\right)=\left(\left(q_{k}\left(\eta-\frac{1}{2} \operatorname{sgn} \theta\left(1-\chi_{1}(\theta)\right) y, \widetilde{a}, \widetilde{b}, g_{j}\right)\right)^{-1}\right)$. Using (4.3.5) we see that $\frac{\partial \beta}{\partial \theta} \in \mathcal{H}$. Then differentiating (4.3.19) with respect to $\theta$ we see that $\frac{\partial \alpha}{\partial \theta}, \frac{\partial b}{\partial \theta} \in \mathcal{H}$. It follows from (4.3.9) that $\frac{\partial g_{j}}{\partial \theta} \in \mathcal{H}$. Then we deduce from the estimates (4.2.2) that $\frac{\partial}{\partial \theta}\left[q_{k}\left(\eta-\frac{1}{2} \operatorname{sgn} \theta\left(1-\chi_{1}(\theta)\right) y, \widetilde{a}, \widetilde{b}, g_{j}\right)\right]$ belongs to $\mathcal{H}$ and we deduce from Corollary 4.3 .5 (iii) that $\frac{\partial}{\partial \theta}\left[\left(q_{k}\left(\eta-\frac{1}{2} \operatorname{sgn} \theta\left(1-\chi_{1}(\theta)\right) y, \widetilde{a}, \widetilde{b}, g_{j}\right)\right)^{-1}\right] \in \mathcal{H}$. Thus $\left(\frac{\partial f_{j k}}{\partial \theta}\right) \in \mathcal{H}$. Since $f_{j k}, \frac{\partial f_{j k}}{\partial \theta}, \frac{\partial f_{j k}}{\partial y_{\ell}}, \frac{\partial f_{j k}}{\partial \eta_{\ell}}$ belong to $\mathcal{H}$ and since $\mathcal{H}$ is closed under multiplication it remains to prove that the functions $h(\theta, y, \eta, \alpha)=\frac{\partial p}{\partial \xi_{\ell}}\left(y+x(\theta, \alpha), \frac{\eta}{\langle\theta\rangle}+\xi(\theta, \alpha)\right)$ or 
$\langle\theta\rangle \frac{\partial p}{\partial x_{\ell}}\left(y+x(\theta, \alpha), \frac{\eta}{\langle\theta\rangle}+\xi(\theta, \alpha)\right)$ belong to $\mathcal{H}$. Since $\frac{\eta_{\ell}}{\langle\theta\rangle}+\xi_{\ell}(\theta, \alpha)$ has all its derivatives in $\eta$ uniformly bounded, we are led to prove that if $g^{j k}(x)$ are the coefficients of $p(x, \xi)$ then $\partial_{x}^{A} g^{j k}(y+x(\theta, \alpha))$ for $A \in \mathbb{N}^{n}$ and $\langle\theta\rangle \partial_{x}^{B} g^{j k}(y+x(\theta, \alpha))$ for $B \in \mathbb{N}^{n}$, $|B| \geqslant 1$ are uniformly bounded when $|y| \leqslant \delta\langle\theta\rangle$. This is obvious if $A=0$ and if $|B| \geqslant 1$ condition (3.1.1) shows that

$$
\begin{aligned}
\left|\partial_{x}^{B} g^{j k}(y+x(\theta, \alpha))\right| & \leqslant \frac{C_{B}}{\langle y+x(\theta, \alpha)\rangle^{|B|+1+\sigma_{0}}}=\frac{C_{B}}{\langle x(\theta, \beta)\rangle^{|B|+1+\sigma_{0}}} \\
& \leqslant \frac{C_{B}}{\langle\theta\rangle^{|B|+1+\sigma_{0}}}
\end{aligned}
$$

since $\beta \in \mathcal{S} \subset \mathcal{S}_{+} \cap \mathcal{S}_{-}($see $(3.3 .3))$.

Corollary 4.3.17. - With $\Phi$ defined in (4.3.43) we have for $k=1, \ldots, n$,

$$
\left(-\frac{\partial p}{\partial x_{k}}(x, \Phi(\theta, x, \alpha))-\frac{\partial \Phi_{k}}{\partial \theta}(\theta, x, \alpha)-\sum_{j=1}^{n} \frac{\partial \Phi_{k}}{\partial x_{j}}(\theta, x, \alpha) \frac{\partial p}{\partial \xi_{j}}(x, \Phi(\theta, x, \alpha))\right)_{\alpha \in \mathcal{S}} \in \mathcal{J}_{(x, \xi)} .
$$

Proof. - First of all we show that

$$
(1)=\left(\frac{\partial p}{\partial x_{k}}(x, \Phi(\theta, x, \alpha))-\frac{\partial p}{\partial x_{k}}(x, \xi)\right)_{\alpha \in \mathcal{S}} \in \mathcal{J}_{(x, \xi)} .
$$

Denoting by $g^{i j}$ the coefficients of $p$ we can write

$$
\frac{\partial g^{i j}}{\partial x_{k}}(x) \Phi_{i} \Phi_{j}-\frac{\partial g^{i j}}{\partial x_{k}}(x) \xi_{i} \xi_{j}=\underbrace{\frac{\partial g^{i j}}{\partial x_{k}}(x)\left(\Phi_{i}-\xi_{i}\right) \xi_{j}}_{(a)}-\underbrace{\frac{\partial g^{i j}}{\partial x_{k}}(x) \Phi_{i}\left(\xi_{j}-\Phi_{j}\right)}_{(b)} .
$$

To see that this belongs to $\mathcal{J}_{(x, \xi)}$ we use (4.3.45). In the coordinates $(y, \eta)$ we have

$$
\langle\theta\rangle(a)=-\frac{\partial g^{i j}}{\partial x_{k}}(y+x(\theta, \alpha))\left(\frac{\eta_{j}}{\langle\theta\rangle}+\xi_{j}(\theta, \alpha)\right)\left(\eta_{i}-\psi_{i}(\theta, y, \alpha)\right) .
$$

Since $\eta_{i}-\psi_{i}(\theta, y, \alpha) \in \mathcal{J}$ (see Example 4.3 .9 and $\left.(4.3 .38)\right)$ and $\left(\frac{\eta_{j}}{\langle\theta\rangle}+\xi_{j}(\theta, \alpha)\right)$. $\frac{\partial g^{i j}}{\partial x_{k}}(y+x(\theta, \alpha))$ belongs to $\mathcal{H}$ we have $(a) \in \mathcal{J}_{(x, \xi)}$.

A similar argument shows that (b) belongs to $\mathcal{J}_{(x, \xi)}$.

We show now that

$$
(2)=\left(\frac{\partial \Phi_{k}}{\partial x_{j}}(\theta, x, \alpha)\left[\frac{\partial p}{\partial \xi_{j}}(x, \Phi(\theta, x, \alpha))-\frac{\partial p}{\partial \xi_{j}}(x, \xi)\right]\right)_{\alpha \in \mathcal{S}} \in \mathcal{J}_{(x, \xi)} .
$$

The coefficients of $p$ can be written $g^{i j}(x)=\delta_{i j}+c_{i j}(x)$. It follows that

$$
(2)=\left(2 \frac{\partial \Phi_{k}}{\partial x_{j}}(\theta, x, \alpha)\left(\Phi_{j}(\theta, x, \alpha)-\xi_{j}+\sum_{\ell=1}^{n} c_{j \ell}(x)\left(\Phi_{\ell}(\theta, x, \alpha)-\xi_{\ell}\right)\right)\right) .
$$

Now $\frac{\partial \Phi_{k}}{\partial x_{j}}=\frac{1}{\langle\theta\rangle} \frac{\partial \psi_{k}}{\partial y_{\ell}} \in \mathcal{H}$ and $c_{j \ell}(y+x(\theta, \alpha)) \in \mathcal{H}$. It follows that $(2) \in \mathcal{J}_{(x, \xi)}$ and the Corollary follows from Lemma 4.3.16. 
Corollary 4.3.18. - For every $N \in \mathbb{N}$ there exists $C_{N}>0$ such that

$$
\begin{aligned}
\mid-\frac{\partial p}{\partial x_{k}}(x, \Phi(\theta, x, \alpha))-\frac{\partial \Phi_{k}}{\partial \theta}(\theta, x, \alpha)-\frac{\partial \Phi_{k}}{\partial x}(\theta, x, \alpha) \cdot & \frac{\partial p}{\partial \xi}(x, \Phi(\theta, x, \alpha)) \mid \\
& \leqslant \frac{C_{N}}{\langle\theta\rangle}\left(\frac{|x-x(\theta, \alpha)|}{\langle\theta\rangle}\right)^{N} .
\end{aligned}
$$

Proof. - This follows from Lemma 4.3.15 and Corollary 4.3.17 since the left hand side does not depend of $\xi$.

Proposition 4.3.19. - Let $\alpha \in \mathcal{S}$ (see (4.3.1)). Let us set for $(\theta, x) \in \Omega_{\delta}$,

$$
\varphi(\theta, x, \alpha)=\int_{0}^{1}(x-x(\theta, \alpha)) \cdot \Phi(\theta, s x+(1-s) x(\theta, \alpha), \alpha) d s+\theta p(\alpha)+\frac{1}{2 i}\left|\alpha_{\xi}\right|^{2} .
$$

Then we have

(i) $\varphi(0, x, \alpha)=\left(x-\alpha_{x}\right) \cdot \alpha_{\xi}+\frac{i}{2}\left(x-\alpha_{x}\right)^{2}+\frac{1}{2 i}\left|\alpha_{\xi}\right|^{2}+\mathcal{O}\left(\left|x-\alpha_{x}\right|^{N}\right)$.

For every $N \in \mathbb{N}$ there exists $C_{N}^{2}>0$ such that

(ii) $\left|\frac{\partial \varphi}{\partial x}(\theta, x, \alpha)-\Phi(\theta, x, \alpha)\right| \leqslant C_{N}\left(\frac{|x-x(\theta, \alpha)|}{\langle\theta\rangle}\right)^{N}$.

(iii) $\left|\frac{\partial \varphi}{\partial \theta}(\theta, x, \alpha)+p\left(x, \frac{\partial \varphi}{\partial x}(\theta, x, \alpha)\right)\right| \leqslant C_{N}\left(\frac{|x-x(\theta, \alpha)|}{\langle\theta\rangle}\right)^{N}$

uniformly with respect to $(\theta, x) \in \Omega_{\delta}$ and $\alpha \in \mathcal{S}$.

Moreover, uniformly with respect to $(\theta, x, \alpha) \in \Omega_{\delta} \times \mathcal{S}$, we have

(iv) $\left|\frac{\partial \varphi}{\partial x}(\theta, x, \alpha)-\alpha_{\xi}\right| \leqslant C(\varepsilon+\sqrt{\delta})$.

(v) $\left|\partial_{x}^{A} \varphi(\theta, x, \alpha)\right| \leqslant C_{A}, \forall A \in \mathbb{N}^{n}$.

(vi) $\left.\left|\operatorname{Im} \varphi(\theta, x, \alpha)-\frac{1}{2} \frac{|x-x(\theta, \alpha)|^{2}}{1+4 \theta^{2}}+\frac{1}{2}\right| \alpha_{\xi}\right|^{2} \mid \leqslant C(\varepsilon+\sqrt{\delta}) \frac{|x-x(\theta, \alpha)|^{2}}{\langle\theta\rangle^{2}}$.

Proof. - If we can prove that for $j=1, \ldots, n$,

$$
\Phi_{j}\left(0, s x+(1-s) \alpha_{x}, \alpha\right)=\alpha_{\xi}^{j}+i s\left(x_{j}-\alpha_{x}^{j}\right)+\mathcal{O}\left(s^{N}\left|x-\alpha_{x}\right|^{N}\right)
$$

then (i) will follow according to (4.3.48). By (4.3.43), Corollary 4.3.5 and Theorem 4.3.1 we have

$$
\Phi_{j}(0, x, \alpha)=\alpha_{\xi}^{j}-\left(\widetilde{a}_{j}+i \widetilde{b}_{j}\right)\left(0, x-\alpha_{x}, \alpha\right)=\alpha_{\xi}^{j}-\left(a_{k}+i b_{k}\right)\left(0, x-\alpha_{x}, \alpha\right) .
$$

Now Example 4.3 .9 (for $\theta=0$ ) shows that $\eta_{j}+a_{j}(0, y, \alpha)+i b_{j}(0, y, \alpha)$ belongs to the ideal $\mathcal{J}$ introduced in Definition 4.3.8. On the other hand, since by (4.2.10) for $\theta=0 g_{j}(\eta)=\chi_{0}\left(\frac{1}{\mu_{0}} \eta\right)\left(\eta_{j}-i y_{j}\right)$ it follows that $\eta_{j}-i y_{j}$ belongs also to $\mathcal{J}$. Thus the difference $a_{j}(0, y, \alpha)+i b_{j}(0, y, \alpha)+i y_{j}$ belongs also to $\mathcal{J}$ and does not depend on $\eta$. It follows from Lemma 4.3.11 that for all $N \in \mathbb{N}$,

$$
\begin{aligned}
a_{j}(0, y, \alpha) & =\mathcal{O}\left(|\operatorname{Im} \psi(0, y, \alpha)|^{N}\right) \\
b_{j}(0, y, \alpha)+y_{j} & =\mathcal{O}\left(|\operatorname{Im} \psi(0, y, \alpha)|^{N}\right) .
\end{aligned}
$$


Now (4.3.38) and Theorem 4.3.1 (ii) show that $\left|\operatorname{Im} \psi_{j}(0, y, \alpha)\right|=\left|b_{j}(0, y, \alpha)\right| \leqslant C|y|$. Thus for all $N \in \mathbb{N}$,

$$
a_{j}(0, y, \alpha)=\mathcal{O}\left(|y|^{N}\right), \quad b_{j}(0, y, \alpha)=-y_{j}+\mathcal{O}\left(|y|^{N}\right) .
$$

It follows that for all $N \in \mathbb{N}$,

$$
\Phi_{j}(0, y, \alpha)=\alpha_{\xi}^{j}+i\left(x-\alpha_{x}^{j}\right)+\mathcal{O}\left(\left|x-\alpha_{x}\right|^{N}\right)
$$

which proves our claim.

Let us prove (ii). We have, by (4.3.48),

$$
\begin{array}{r}
\frac{\partial \varphi}{\partial x_{j}}(\theta, x, \alpha)=\int_{0}^{1} \Phi_{j}(\theta, s x+(1-s) x(\theta, \alpha), \alpha) d s+\sum_{k=1}^{n} \int_{0}^{1} s\left(x_{k}-x_{k}(\theta, \alpha)\right) \\
\cdot \frac{\partial \Phi_{k}}{\partial x_{j}}(s x+(1-s) x(\theta, \alpha), \alpha) d s
\end{array}
$$

Now we use Theorem 4.3.13 (vii) and the fact that $|x-x(\theta, \alpha)| \leqslant \delta\langle\theta\rangle$. We deduce that

$$
\begin{gathered}
\left|\frac{\partial \varphi}{\partial x_{j}}(\theta, x, \alpha)-\int_{0}^{1}\left[\Phi_{j}+s \sum_{k=1}^{n}\left(x_{k}-x_{k}(\theta, \alpha)\right) \frac{\partial \Phi_{j}}{\partial x_{k}}\right](\theta, s x+(1-s) x(\theta, \alpha), \alpha) d s\right| \\
\leqslant C_{N}\left(\frac{|x-x(\theta, \alpha)|}{\langle\theta\rangle}\right)^{N}
\end{gathered}
$$

where $C_{N}$ is independent of $(\theta, x, \alpha)$.

It follows that

$$
\begin{aligned}
\mid \frac{\partial \varphi}{\partial x_{j}}(\theta, x, \alpha) & -\int_{0}^{1} \Phi_{j}(\theta, s x+(1-s) x(\theta, \alpha), \alpha) d s \\
& -\int_{0}^{1} s \frac{d}{d s}\left[\Phi_{j}(\theta, s x+(1-s) x(\theta, \alpha), \alpha)\right] d s \mid \leqslant C_{N}\left(\frac{|x-x(\theta, \alpha)|}{\langle\theta\rangle}\right)^{N} .
\end{aligned}
$$

Integrating by parts in the second integral above, we obtain (ii). As a consequence of (ii) we have the estimate

$$
\left|p(x, \Phi(\theta, x, \alpha))-p\left(x, \frac{\partial \varphi}{\partial x}(\theta, x, \alpha)\right)\right| \leqslant C_{N}\left(\frac{|x-x(\theta, \alpha)|}{\langle\theta\rangle}\right)^{N} .
$$

Let us prove (iii). We deduce from (4.3.48) that

$$
\frac{\partial \varphi}{\partial \theta}(\theta, x, \alpha)=(1)+(2)+(3)+(4)
$$


where

$$
\begin{aligned}
& (1)=-\sum_{k=1}^{n} \int_{0}^{1} \dot{x}_{k}(\theta, \alpha) \Phi_{k}(\theta, s x+(1-s) x(\theta, \alpha), \alpha) d s \\
& (2)=\sum_{k=1}^{n} \int_{0}^{1}\left(x_{k}-x_{k}(\theta, \alpha)\right) \frac{\partial \Phi_{k}}{\partial \theta}(\theta, s x+(1-s) x(\theta, \alpha), \alpha) d s \\
& (3)=\sum_{k=1}^{n} \int_{0}^{1}\left(x_{k}-x_{k}(\theta, \alpha)\right) \sum_{j=1}^{n} \frac{\partial \Phi_{k}}{\partial x_{j}}(\theta, s x+(1-s) x(\theta, \alpha), \alpha)(1-s) \dot{x}_{j}(\theta, \alpha) d s \\
& (4)=p(\alpha) .
\end{aligned}
$$

Let us consider the term (2). We use Corollary 4.3 .18 to get

$$
\begin{aligned}
(2)=\sum_{k=1}^{n} \int_{0}^{1}\left(x_{k}-x_{k}(\theta, \alpha)\right)\left[-\frac{\partial p}{\partial x_{k}}(s x+(1-s) x(\theta, \alpha), \Phi(\theta, s x+(1-s) x(\theta, \alpha), \alpha))\right. \\
-\sum_{j=1}^{n} \frac{\partial \Phi_{k}}{\partial x_{j}}(\theta, s x+(1-s) x(\theta, \alpha), \alpha) \frac{\partial p}{\partial \xi_{j}}(s x+(1-s) x(\theta, \alpha), \\
\Phi(\theta, s x+(1-s) x(\theta, \alpha), \alpha))] d s \\
+\mathcal{O}\left(\left(\frac{|x-x(\theta, \alpha)|}{\langle\theta\rangle}\right)^{N}\right) .
\end{aligned}
$$

Now, by Theorem 4.3.13, (vii),

$$
\left|\left(\frac{\partial \Phi_{k}}{\partial x_{j}}-\frac{\partial \Phi_{j}}{\partial x_{k}}\right)(\theta, s x+(1-s) x(\theta, \alpha), \alpha)\right| \leqslant C_{N} \frac{s^{N}}{\langle\theta\rangle} \frac{|x-x(\theta, \alpha)|^{N}}{\langle\theta\rangle^{N}}
$$

and $s^{N} \leqslant 1$. Therefore,

$$
\begin{aligned}
(2)=-\int_{0}^{1} \frac{d}{d s}[p(s x+(1-s) x(\theta, \alpha), \Phi(\theta, s x+(1-s) x(\theta, \alpha), \alpha))] d s & \\
& +\mathcal{O}\left(\frac{|x-x(\theta, \alpha)|^{N}}{\langle\theta\rangle^{N}}\right) .
\end{aligned}
$$

Therefore we obtain

$$
|(2)+p(x, \Phi(\theta, x, \alpha))-p(x(\theta, \alpha), \Phi(\theta, x(\theta, \alpha), \alpha))| \leqslant C_{N}\left(\frac{|x-x(\theta, \alpha)|}{\langle\theta\rangle}\right)^{N} .
$$

Let us consider the term (3). Using again (4.3.51) we get

$$
(3)=\sum_{j=1}^{n} \int_{0}^{1}(1-s) \dot{x}_{j}(\theta, \alpha) \frac{d}{d s}\left(\Phi_{j}(\theta, s x+(1-s) x(\theta, \alpha), \alpha)\right)+\mathcal{O}\left(\frac{|x-x(\theta, \alpha)|^{N}}{\langle\theta\rangle^{N}}\right) .
$$

Integrating by part we obtain

$$
(3)=-\dot{x}(\theta, \alpha) \cdot \Phi(\theta, x(\theta, \alpha), \alpha)+\sum_{j=1}^{n} \int_{0}^{1} \dot{x}_{j}(\theta, \alpha) \Phi_{j}(\theta, s x+(1-s) x(\theta, \alpha), \alpha) d s .
$$


Comparing with the term (1) we obtain,

$$
|(1)+(3)+\dot{x}(\theta, \alpha) \Phi(\theta, x(\theta, \alpha), \alpha)| \leqslant C_{N}\left(\frac{|x-x(\theta, \alpha)|}{\langle\theta\rangle}\right)^{N} .
$$

Now by Theorem 4.3.13 (vi) and the Euler relation we have,

$$
\begin{aligned}
\dot{x}(\theta, \alpha) \cdot \Phi(\theta, x(\theta, \alpha), \alpha) & =\xi(\theta, \alpha) \frac{\partial p}{\partial \xi}(x(\theta, \alpha), \xi(\theta, \alpha)) \\
& =2 p(x(\theta, \alpha), \xi(\theta, \alpha))=2 p(\alpha) .
\end{aligned}
$$

It follows that

$$
|(1)+(3)+2 p(\alpha)| \leqslant C_{N}\left(\frac{|x-x(\theta, \alpha)|}{\langle\theta\rangle}\right)^{N} .
$$

Since in (4.3.52) we have $p(x(\theta, \alpha), \Phi(\theta, x(\theta, \alpha), \alpha))=p(x(\theta, \alpha), \xi(\theta, \alpha))=p(\alpha)$, we deduce from (4.3.50), (4.3.52) and (4.3.53) that

$$
\left|\frac{\partial \varphi}{\partial \theta}(\theta, x, \alpha)+p(x, \Phi(\theta, x, \alpha))\right| \leqslant C_{N}\left(\frac{|x-x(\theta, \alpha)|}{\langle\theta\rangle}\right)^{N} .
$$

Therefore (iii) follows from (4.3.54) and (4.3.49).

Finally (iv), (v), (vi) follow easily from Theorem 4.3.13.

Remark 4.3.20. - Assume that $\alpha$ is such that

$$
\frac{1}{2} \leqslant\left|\alpha_{\xi}\right| \leqslant 2 \text { and } \quad \alpha_{x} \cdot \alpha_{\xi} \leqslant c_{0}\left\langle\alpha_{x}\right\rangle\left|\alpha_{\xi}\right|
$$

(so $\alpha \in \mathcal{S}_{-}$instead of $\alpha \in \mathcal{S}$ ). Then Theorem 4.3.1, Corollary 4.3.5 and Proposition 4.3.19 are true for $\theta \leqslant 0$.

By the same way if $\frac{1}{2} \leqslant\left|\alpha_{\xi}\right| \leqslant 2$ and $\alpha_{x} \cdot \alpha_{\xi} \geqslant-c_{0}\left\langle\alpha_{x}\right\rangle\left|\alpha_{\xi}\right|$ (which imply that $\left.\alpha \in \mathcal{S}_{+}\right)$the above results hold for $\theta \geqslant 0$.

\subsection{The case of incoming points}

We are going to prove Theorem 4.1.2 when

$$
\left|\alpha_{x} \cdot \alpha_{\xi}\right|>c_{0}\left\langle\alpha_{x}\right\rangle\left|\alpha_{\xi}\right| \text { and } \frac{1}{2} \leqslant\left|\alpha_{\xi}\right| \leqslant 2 .
$$

Since the problem is entirely symmetric we can without loss of generality assume that

$$
\frac{1}{2} \leqslant\left|\alpha_{\xi}\right| \leqslant 2, \quad \alpha_{x} \cdot \alpha_{\xi}<-c_{0}\left\langle\alpha_{x}\right\rangle\left|\alpha_{\xi}\right| .
$$

It follows from Definition 4.1.1 and the discussion after, that

$$
\begin{array}{r}
\widetilde{\Omega}_{\delta}=\left\{(\theta, y) \in \mathbb{R} \times \mathbb{R}^{n}: \theta \leqslant 0,|y| \leqslant \delta\langle\theta\rangle\right\} \cup\left\{(\theta, y) \in \mathbb{R} \times \mathbb{R}^{n}: \theta \geqslant 0,|y| \leqslant \delta\langle\theta\rangle\right. \\
\text { and } \left.(y+x(\theta, \alpha)) \cdot \alpha_{\xi} \leqslant c_{1}\langle y+x(\theta, \alpha)\rangle\left|\alpha_{\xi}\right|\right\} .
\end{array}
$$


Let now $\chi_{0} \in C_{0}^{\infty}\left(\mathbb{R}^{n}\right), \chi_{1} \in C^{\infty}\left(\mathbb{R}^{n}\right)$ be such that,

$$
\begin{array}{ccc}
\chi_{0}(t)=1 \quad \text { if } \quad|t| \leqslant 1, \quad \chi_{0}(t)=0 \quad \text { if } \quad|t| \geqslant 2 \quad \text { and } \quad 0 \leqslant \chi_{0} \leqslant 1, \\
\chi_{1}(\theta)=1 \quad \text { if } \quad \theta \geqslant-1, \quad \chi_{1}(\theta)=0 \quad \text { if } \quad \theta \leqslant-2 \quad \text { and } \quad 0 \leqslant \chi_{1} \leqslant 1 .
\end{array}
$$

For $j=1, \ldots, n$ we introduce

$$
\begin{array}{r}
g_{j}(\eta)=\chi_{0}\left(\frac{1}{\mu_{0}} \eta\right) v_{j}\left(\theta, y+x(\theta, \alpha), \eta \chi_{1}(\theta)+\left(1-\chi_{1}(\theta)\right)\left[\frac{\eta}{\langle\theta\rangle}+\frac{1}{2} \frac{\operatorname{sgn} \theta}{\langle\theta\rangle} y\right]\right. \\
+\xi(\theta, \alpha), \alpha))
\end{array}
$$

where $\mu_{0}$ is a small constant to be chosen, $(\theta, y) \in \widetilde{\Omega}_{\delta}, \alpha$ satisfies (4.4.1) and $v_{j}$ has been introduced in (4.2.9).

\section{REMARK 4.4.1}

(i) According to Remark 4.3.20 since (4.4.1) implies (4.3.55) the phase has been already constructed when $(\theta, x)$ belongs to the first part of $\widetilde{\Omega}_{\delta}$ where $\theta \leqslant 0$. Therefore we are left with the case $\theta \geqslant 0$.

(ii) If $\alpha$ satisfies (4.4.1) and $(\theta, y) \in \widetilde{\Omega}_{\delta}, \theta \geqslant 0$, then the point $(y+x(\theta, \alpha), \eta+\xi(\theta, \alpha))$ belongs to $\mathcal{S}_{-}$. Indeed recall that $\frac{1}{2} \leqslant\left|\alpha_{\xi}\right| \leqslant 2$ implies $\frac{1}{4} \leqslant \frac{1}{2}\left|\alpha_{\xi}\right| \leqslant|\eta+\xi(\theta, \alpha)| \leqslant$ $2\left|\alpha_{\xi}\right|$ if $|\eta| \leqslant 2 \mu_{0}$ and $\mu_{0}, \varepsilon$ are small enough. Therefore setting $x=x(\theta, \alpha)$ we can write

$$
\begin{aligned}
x \cdot(\eta+\xi(\theta, \alpha)) & =x \cdot\left(\alpha_{\xi}+\eta+\zeta(\theta, \alpha)\right) \leqslant c_{1}\langle x\rangle\left|\alpha_{\xi}\right|+|x|\left(\mu_{0}+\varepsilon\right) \\
& \leqslant 2 c_{1}\langle x\rangle|\eta+\xi(\theta, \alpha)|+4\left(\mu_{0}+\varepsilon\right)\langle x\rangle|\eta+\xi(\theta, \alpha)| \\
& \leqslant \frac{1}{4}\langle x\rangle|\eta+\xi(\theta, \alpha)|
\end{aligned}
$$

if $c_{1}, \mu_{0}, \varepsilon$ are small enough.

Our first step will be the proof of the following result.

THEOREM 4.4.2. - There exist small positive constants $\mu_{0}, \delta$ and $C^{\infty}$ functions $a=$ $a(\theta, y, \alpha), b_{k}=b_{k}(\theta, y, \alpha), k=1, \ldots, n$, defined on $\widetilde{\Omega}_{\delta}$ with $\theta \geqslant 0$ such that, with $a=\left(a_{k}\right), b=\left(b_{k}\right)$ we have for $\eta \in \mathbb{R}^{n}$,

(i) $g_{j}(\eta)=\sum_{k=1}^{n} q_{k}\left(\eta, a, b, g_{j}\right)\left(\eta_{k}+a_{k}(\theta, y, \alpha)+i b_{k}(\theta, y, \alpha)\right)$

where the $q_{k}^{\prime} s$ have been introduced in Lemma 4.2.1.

Moreover we have for $(\theta, y) \in \widetilde{\Omega}_{\delta}, \theta \geqslant 0$ and $k=1,2, \ldots, n$,

(ii) $\left|a_{k}(\theta, y, \alpha)+\frac{2 \theta y_{k}}{1+4 \theta^{2}}\right| \leqslant \sqrt{\delta} \inf (1,|y|)$,

$\left|b(\theta, y, \alpha)+\frac{y_{k}}{1+4 \theta^{2}}\right| \leqslant \sqrt{\delta} \frac{|y|}{\langle\theta\rangle^{2}}$.

(iii) If we set

$$
\widetilde{a}(\theta, y, \alpha)=a(\theta, y, \alpha)+\frac{2 \theta y}{1+4 \theta^{2}} \quad \text { and } \quad \widetilde{b}(\theta, y, \alpha)=\langle\theta\rangle\left(b(\theta, y, \alpha)+\frac{y}{1+4 \theta^{2}}\right)
$$


then for every $A \in \mathbb{N}^{n},|A| \geqslant 1$ one can find $C_{A} \geqslant 0$ such that with $x=y+x(\theta, \alpha)$, $\theta \geqslant 0$,

$$
\left|\partial_{y}^{A} \widetilde{a}(\theta, y, \alpha)\right|+\left|\partial_{y}^{A} \widetilde{b}(\theta, y, \alpha)\right| \leqslant C_{A}\left[\frac{\varepsilon}{\langle x\rangle^{\sigma_{0}}}\left(\frac{1}{\langle x\rangle}+\frac{1}{\langle\theta\rangle}\right)^{|A|+1}+\frac{\delta}{\langle\theta\rangle^{|A|+1}}\right]
$$

(iv) $\left|q_{k}\left(\eta, a, b, g_{j}\right)-(1+2 i \theta) \delta_{j k}\right| \leqslant C(\varepsilon+\sqrt{\delta})\langle\theta\rangle$ if $|\eta| \leqslant \sqrt{\delta}$.

(v) $\left|\partial_{(a, b)}^{B} \partial_{\eta}^{\gamma} q_{k}\left(\eta, a, b, g_{j}\right)\right| \leqslant C_{B, \gamma}\langle\theta\rangle, \quad$ if $\quad B \in \mathbb{N}^{n}, \quad \gamma \in \mathbb{N}^{n}, \quad 1 \leqslant k \leqslant n$.

Proof. - We use the same method as in Theorem 4.3.1. According to Lemma 4.2.1, the claim (i) is equivalent to solve the system of equations

$$
r\left(a, g_{j}(\theta, y, \alpha ; \cdot)=0, \quad j=1, \ldots, n .\right.
$$

We shall solve this system in the set

$$
E=\left\{(a, b) \in \mathbb{R}^{n} \times \mathbb{R}^{n}:\left|a+\frac{2 \theta y}{1+4 \theta^{2}}\right| \leqslant \sqrt{\delta} \inf (1,|y|),\left|b+\frac{y}{1+4 \theta^{2}}\right| \leqslant \sqrt{\delta} \frac{|y|}{\langle\theta\rangle^{2}}\right\}
$$

where $0<\delta \ll 1$.

First of all we give equivalent equations to (4.4.3) in the set $E$. We write as in (4.3.13)

$$
r\left(a, b, g_{j}\right)=g_{j}(-a)-i \sum_{k=1}^{n} \frac{\partial g_{j}}{\partial \xi_{k}}(-a) b_{k}+\sum_{p, q=1}^{n} H_{p q}^{j}(\theta, y, \alpha, a, b) b_{p} b_{q}
$$

where

$$
H_{p q}^{j}(\theta, y, \alpha, a, b)=\int_{0}^{1} \frac{\partial^{2} r}{\partial b_{p} \partial b_{q}}\left(a, t b, g_{j}(\theta, y, \alpha ; \cdot)\right)(1-t) d t .
$$

By (4.2.2) we have

$$
\left|\partial_{(a, b)}^{\alpha} \partial_{y}^{B} r\left(a, t b, g_{j}(\cdots)\right)\right| \leqslant C_{A B} \sum_{|\gamma| \leqslant|A|+3 n} \int\left|\partial_{\xi}^{\gamma} \partial_{y}^{B} g_{j}(\theta, y, \alpha, \xi)\right| d \xi .
$$

Since for $\theta \geqslant 0$ we have,

$$
g_{j}(\eta)=\chi\left(\frac{\eta}{\mu_{0}}\right)\left[\left(\xi_{j}-i x_{j}\right)(-\theta ; y+x(\theta ; \alpha), \eta+\xi(\theta ; \alpha))+i\left(\alpha_{x}+i \alpha_{\xi}\right)\right]
$$

we deduce from Propositions 3.3.1 and 3.3.2 that $\left|\partial_{\eta}^{\gamma} \partial_{y}^{B} g_{j}(\theta, y, \alpha, \eta)\right|$ is bounded on the support of $\chi$, by

$$
\left\{\begin{array}{lll}
C\langle\theta\rangle & \text { if } \quad B=0 \\
C\left(1+\frac{\varepsilon\langle\theta\rangle}{\langle x\rangle^{2+\sigma_{0}}}\right) & \text { if } \quad|B|=1 \\
\frac{C_{B} \varepsilon}{\langle x\rangle^{B \mid+\sigma_{0}}}\left(1+\frac{\langle\theta\rangle}{\langle x\rangle}\right) & \text { if } & |B| \geqslant 2
\end{array}\right.
$$


It follows that

$$
\left|\partial_{(a, b)}^{A} \partial_{y}^{B} H_{p, q}^{j}(\theta, y, \alpha, a, b)\right| \leqslant \begin{cases}C\langle\theta\rangle & \text { if }|B|=0 \\ C\left(1+\frac{\varepsilon\langle\theta\rangle}{\langle x\rangle^{2+\sigma_{0}}}\right) & \text { if }|B|=1 \\ \frac{C_{B} \varepsilon}{\langle x\rangle^{|B|+\sigma_{0}}}\left(1+\frac{\langle\theta\rangle}{\langle x\rangle}\right) & \text { if }|B| \geqslant 2\end{cases}
$$

Since $\chi(-a)=1$ and $\chi^{\prime}(-a)=0$ we see that (4.4.3) is equivalent in $E$ to the vectorial equation,

$$
\begin{aligned}
& {\left[\xi-i x-i \sum_{k=1}^{n}\left(\frac{\partial \xi}{\partial \xi_{k}}-i \frac{\partial x}{\partial \xi_{k}}\right) b_{k}\right](-\theta ; y+x(\theta ; \alpha), \xi(\theta ; \alpha)-a)} \\
& +i\left(\alpha_{x}+i \alpha_{\xi}\right)+\sum_{p, q=1}^{n} H_{p q}(\theta, y, \alpha, a, b) b_{p} b_{q}=0
\end{aligned}
$$

To shorten the notations we shall set

$$
\left\{\begin{array}{l}
\rho(\theta ; \alpha)=(x(\theta ; \alpha), \xi(\theta ; \alpha)) \\
\rho_{y}(\theta ; \alpha)=(y+x(\theta ; \alpha), \xi(\theta ; \alpha)) \\
\rho_{y, a}(\theta ; \alpha)=(y+x(\theta ; \alpha), \xi(\theta ; \alpha)-a) .
\end{array}\right.
$$

Since, by assumption, the point $\rho_{y, a}(\theta ; \alpha)$ belongs to $\mathcal{S}_{-}$(the outgoing set for $\theta \leqslant 0$ ) we can use the Proposition 3.3.1 to write

$$
\begin{aligned}
& x\left(-\theta ; \rho_{y, a}(\theta ; \alpha)\right)=y+x(\theta ; \alpha)-2 \theta \xi\left(-\theta ; \rho_{y, a}(\theta ; \alpha)\right)+z\left(-\theta ; \rho_{y, a}(\theta ; \alpha)\right) \\
& \xi\left(-\theta ; \rho_{y, a}(\theta ; \alpha)\right)=-a+\xi(\theta ; \alpha)+\zeta\left(-\theta ; \rho_{y, a}(\theta ; \alpha)\right) .
\end{aligned}
$$

It follows that (4.4.9) is equivalent to

$$
\begin{aligned}
& (1+2 i \theta) \xi\left(-\theta ; \rho_{y, a}(\theta ; \alpha)\right)-i y-i x(\theta ; \alpha)-i z\left(-\theta ; \rho_{y, a}(\theta ; \alpha)\right) \\
& -i\left[(1+2 i \theta) \sum_{k=1}^{n} \frac{\partial \xi}{\partial \xi_{k}}\left(-\theta ; \rho_{y, a}(\theta ; \alpha)\right) b_{k}-i \sum_{k=1}^{n} \frac{\partial z}{\partial \xi_{k}}\left(-\theta ; \rho_{y, a}(\theta ; \alpha)\right) b_{k}\right] \\
& +i\left(\alpha_{x}+i \alpha_{\xi}\right)+\sum_{p, q=1}^{n} H_{p q}(\cdots) b_{p} b_{q}=0 .
\end{aligned}
$$

Taking the real and the imaginary parts, we are led to the $2 n$ real equations

$$
\begin{aligned}
& \xi_{j}\left(-\theta ; \rho_{y, a}(\theta ; \alpha)\right)+2 \theta b_{j}+2 \theta \frac{\partial \zeta_{j}}{\partial \xi}\left(-\theta ; \rho_{y, a}(\theta ; \alpha)\right) \cdot b \\
&-\frac{\partial z_{j}}{\partial \xi}\left(-\theta ; \rho_{y, a}(\theta ; \alpha)\right) \cdot b-\alpha_{\xi}^{j}+H_{1}^{j} b \cdot b=0 \\
&-2 \theta \xi_{j}\left(-\theta ; \rho_{y, a}(\theta ; \alpha)\right)+b_{j}+y_{j}+x_{j}(\theta ; \alpha)-\alpha_{x}^{j}+z_{j}\left(-\theta ; \rho_{y, a}(\theta ; \alpha)\right) \\
&+\frac{\partial \zeta_{j}}{\partial \xi}\left(-\theta ; \rho_{y, a}(\theta ; \alpha)\right) \cdot b+H_{2}^{j} b \cdot b=0
\end{aligned}
$$

MÉMOIRES DE LA SMF 101/102 
where

$$
\frac{\partial \zeta_{j}}{\partial \xi} \cdot b=\sum_{k=1}^{n} \frac{\partial \zeta_{j}}{\partial \xi_{k}} \cdot b_{k}, \quad H^{j} b \cdot b=\sum_{p, q=1}^{n} H_{p, q}^{j} b_{p} b_{q}, \quad H^{j}=H_{1}^{j}+i H_{2}^{j} .
$$

Setting $X=\left(\begin{array}{c}\xi_{j}\left(-\theta ; \rho_{y, a}(\theta ; \alpha)\right) \\ b_{j}\end{array}\right), A=\left(\begin{array}{cc}1 & 2 \theta \\ -2 \theta & 1\end{array}\right)$, our system can be written $A X=F$. Since $A^{-1}=\frac{1}{1+4 \theta^{2}}\left(\begin{array}{cc}1 & -2 \theta \\ 2 \theta & 1\end{array}\right)$, it is equivalent to the following system.

$$
\begin{aligned}
& \xi\left(-\theta ; \rho_{y, a}(\theta ; \alpha)\right)=\frac{2 \theta y}{1+4 \theta^{2}}+ \frac{1}{1+4 \theta^{2}}\left[2 \theta\left(x(\theta ; \alpha)-\alpha_{x}\right)+2 \theta z\left(-\theta ; \rho_{y, a}(\theta ; \alpha)\right)\right. \\
&\left.+\alpha_{\xi}+\frac{\partial z}{\partial \xi}\left(-\theta ; \rho_{y, a}(\theta ; \alpha)\right) \cdot b-\left(H_{1}-2 \theta H_{2}\right) b \cdot b\right] . \\
& b=\frac{-y}{1+4 \theta^{2}}-\frac{1}{1+4 \theta^{2}}\left[x(\theta ; \alpha)-\alpha_{x}-2 \theta \alpha_{\xi}+z\left(-\theta ; \rho_{y, a}(\theta ; \alpha)\right)+\left(2 \theta H_{1}+H_{2}\right) b \cdot b\right] \\
&-\frac{\partial \zeta}{\partial \xi}\left(-\theta ; \rho_{y, a}(\theta ; \alpha)\right) \cdot b+\frac{2 \theta}{1+4 \theta^{2}} \frac{\partial z}{\partial \xi}\left(-\theta ; \rho_{y, a}(\theta ; \alpha)\right) \cdot b .
\end{aligned}
$$

Finally, since $\xi\left(-\theta ; \rho_{y, a}(\theta ; \alpha)\right)=-a+\xi(\theta ; \alpha)+\zeta\left(-\theta ; \rho_{y, a}(\theta ; \alpha)\right)$ the system (4.4.3) is equivalent to

$$
\begin{gathered}
a=\frac{-2 \theta y}{1+4 \theta^{2}}+\xi(\theta ; \alpha)-\frac{1}{1+4 \theta^{2}}\left[\alpha_{\xi}+2 \theta\left(x(\theta ; \alpha)-\alpha_{x}\right)+2 \theta z\left(-\theta ; \rho_{y, a}(\theta ; \alpha)\right)\right. \\
\left.+\frac{\partial z}{\partial \xi}\left(-\theta ; \rho_{y, a}(\theta ; \alpha)\right) \cdot b\right]+\zeta\left(-\theta ; \rho_{y, a}(\theta ; \alpha)\right)+H_{3} b \cdot b \\
b=\frac{-y}{1+4 \theta^{2}}-\frac{1}{1+4 \theta^{2}}\left[x(\theta ; \alpha)-\alpha_{x}-2 \theta \alpha_{\xi}+z\left(-\theta ; \rho_{y, a}(\theta ; \alpha)\right)\right] \\
-\frac{\partial \zeta}{\partial \xi}\left(-\theta ; \rho_{y, a}(\theta ; \alpha)\right) \cdot b+\frac{2 \theta}{1+4 \theta^{2}} \frac{\partial z}{\partial \xi}\left(-\theta ; \rho_{y, a}(\theta ; \alpha)\right) \cdot b+H_{4} b \cdot b
\end{gathered}
$$

where according to (4.4.8) $H_{\ell}, \ell=3,4$, are two matrices which entries satisfy the following estimates

$$
\left|\partial_{(a, b)}^{A} \partial_{y}^{B} H_{\ell, p, q}^{j}(\theta, y, \alpha, a, b)\right| \leqslant \begin{cases}C, & \text { if }|B|=0 \\ \frac{C}{\langle\theta\rangle}\left(1+\frac{\varepsilon\langle\theta\rangle}{\langle x\rangle^{2+\sigma_{0}}}\right), & \text { if }|B|=1 \\ \frac{C_{B} \varepsilon}{\langle\theta\rangle\langle x\rangle^{|\alpha|+1+\sigma_{0}}}\left(1+\frac{\langle\theta\rangle}{\langle x\rangle}\right), & \text { if }|B| \geqslant 2 .\end{cases}
$$

Let us set

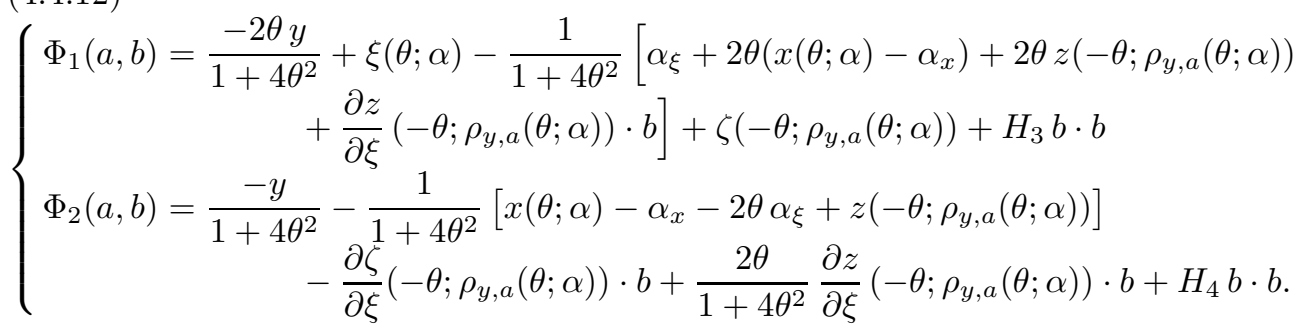


Then the system (4.4.3) to be solved is equivalent in $E$ to the equation

$$
\left(\Phi_{1}(a, b), \Phi_{2}(a, b)\right)=(a, b) .
$$

Let us set $\Phi(a, b)=\left(\Phi_{1}(a, b), \Phi_{2}(a, b)\right)$. We shall use the fixed point theorem in $E$ (see (4.4.4)).

(i) $\Phi(E) \subset E$.

Let us recall that $(y+x(\theta ; \alpha)) \cdot \alpha_{\xi} \leqslant c_{0}\langle y+x(\theta ; \alpha)\rangle\left|\alpha_{\xi}\right|$.

Case 1. - Assume that

$$
x(\theta ; \alpha) \cdot \alpha_{\xi} \geqslant 2 c_{0}\langle x(\theta ; \alpha)\rangle\left|\alpha_{\xi}\right|
$$

It follows that

$$
|y| \geqslant \frac{c_{0}}{2}\langle x(\theta ; \alpha)\rangle \geqslant \frac{c_{0}}{2}
$$

Indeed one can write

$$
\begin{aligned}
2 c_{0}\langle x(\theta ; \alpha)\rangle\left|\alpha_{\xi}\right| & \leqslant(x(\theta ; \alpha)+y) \cdot \alpha_{\xi}-y \cdot \alpha_{\xi} \\
& \leqslant c_{0}\langle y+x(\theta ; \alpha)\rangle\left|\alpha_{\xi}\right|+|y| \cdot\left|\alpha_{\xi}\right| \\
& \leqslant c_{0}\langle x(\theta ; \alpha)\rangle\left|\alpha_{\xi}\right|+c_{0}|y| \cdot\left|\alpha_{\xi}\right|+|y| \cdot\left|\alpha_{\xi}\right| .
\end{aligned}
$$

Therefore $c_{0}\langle x(\theta ; \alpha)\rangle \leqslant 2|y|$. Here we have used the inequality $\langle a+b\rangle \leqslant\langle a\rangle+|b|$.

Let $\theta^{*} \geqslant 0$ be such that $x\left(\theta^{*} ; \alpha\right) \cdot \alpha_{\xi}=0$ (this is possible since $\alpha_{x} \cdot \alpha_{\xi} \leqslant 0$ and $x(\theta ; \alpha) \cdot \alpha_{\xi} \geqslant \alpha_{x} \cdot \alpha_{\xi}+\theta\left|\alpha_{\xi}\right|^{2} \rightarrow+\infty$ if $\left.\theta \rightarrow+\infty\right)$. Then the point $\left(x\left(\theta^{*} ; \alpha\right), \alpha_{\xi}\right)$ is outgoing for $\theta \geqslant 0$ and $\theta \leqslant 0$.

We can write by Proposition 3.3.1,

$$
\begin{aligned}
x(\theta ; \alpha) & =x\left(\theta-\theta^{*} ; x\left(\theta^{*} ; \alpha\right), \xi\left(\theta^{*} ; \alpha\right)\right) \\
& =x\left(\theta^{*} ; \alpha\right)+2\left(\theta-\theta^{*}\right) \xi\left(\theta-\theta^{*} ; x\left(\theta^{*} ; \alpha\right), \xi\left(\theta^{*} ; \alpha\right)\right)+z\left(\theta-\theta^{*}, \cdots\right) \\
& =x\left(\theta^{*} ; \alpha\right)+2\left(\theta-\theta^{*}\right) \xi(\theta ; \alpha)+z\left(\theta-\theta^{*} ; x\left(\theta^{*} ; \alpha\right), \xi\left(\theta^{*} ; \alpha\right)\right) .
\end{aligned}
$$

It follows that

$$
x(\theta ; \alpha) \cdot \alpha_{\xi}=2\left(\theta-\theta^{*}\right)\left|\alpha_{\xi}\right|^{2}+\mathcal{O}(\varepsilon)\left|\theta-\theta^{*}\right|+z\left(\theta-\theta^{*} ; x\left(\theta^{*}, \alpha\right), \xi\left(\theta^{*} ; \alpha\right)\right) \cdot \alpha_{\xi} .
$$

Since $\left|z\left(\theta-\theta^{*}, \cdots\right)\right| \leqslant C \varepsilon\left|\theta-\theta^{*}\right|$ we deduce the estimate,

$$
2\left|\theta-\theta^{*}\right|\left|\alpha_{\xi}\right|^{2} \leqslant|x(\theta, \alpha)| \cdot\left|\alpha_{\xi}\right|+C^{\prime} \varepsilon\left|\theta-\theta^{*}\right| .
$$

Therefore if $\varepsilon$ is small enough we obtain

$$
\left|\theta-\theta^{*}\right| \leqslant 5|x(\theta, \alpha)| .
$$

Now let us introduce

$$
u(\theta)=x(\theta ; \alpha)-\alpha_{x}-2 \theta \alpha_{\xi} .
$$

We claim that

$$
|u(\theta)| \leqslant C \varepsilon\left\langle\theta-\theta^{*}\right\rangle .
$$


Indeed we have $u(0)=0$ and for all $\theta$ in $\mathbb{R}$,

$$
\dot{u}(\theta)=\dot{x}(\theta ; \alpha)-2 \alpha_{\xi}=2\left(\xi(\theta ; \alpha)-\alpha_{\xi}\right)+2 \varepsilon b(x(\theta ; \alpha)) \cdot \xi(\theta ; \alpha) .
$$

It follows from Proposition 3.4.1 that

$$
|\dot{u}(\theta)| \leqslant C \varepsilon .
$$

Now since $x\left(\theta^{*} ; \alpha\right) \cdot \alpha_{\xi}=0$ it follows from Proposition 3.5.2 that

$$
x\left(\theta^{*} ; \alpha\right)=\alpha_{x}+2 \theta^{*} \alpha_{\xi}-z\left(-\theta^{*} ; x\left(\theta^{*} ; \alpha\right), \xi\left(\theta^{*} ; \alpha\right)\right) .
$$

This implies that $\left|u\left(\theta^{*}\right)\right| \leqslant C_{1} \varepsilon$. Now we write

$$
\left|u(\theta)-u\left(\theta^{*}\right)\right| \leqslant\left|\int_{\theta^{*}}^{\theta}\right| \dot{u}(s)|d s| \leqslant C_{2} \varepsilon\left|\theta-\theta^{*}\right|
$$

and

$$
|u(\theta)| \leqslant C_{1} \varepsilon+C_{2} \varepsilon\left|\theta-\theta^{*}\right| \leqslant C_{3} \varepsilon\left\langle\theta-\theta^{*}\right\rangle .
$$

It follows then from (4.4.14) to (4.4.17) that,

$$
\begin{aligned}
\left|x(\theta ; \alpha)-\alpha_{x}-2 \theta \alpha_{\xi}\right| & \leqslant C_{3} \varepsilon\left\langle\theta-\theta^{*}\right\rangle \leqslant 5 C_{3} \varepsilon\langle x(\theta ; \alpha)\rangle \\
& \leqslant \frac{10 C_{3}}{c_{0}} \varepsilon|y| .
\end{aligned}
$$

Using (4.4.12) we see that

$$
\begin{aligned}
\left|\Phi_{2}(a, b)+\frac{y}{1+4 \theta^{2}}\right| & \leqslant \underbrace{\frac{\left|x(\theta ; \alpha)-\alpha_{x}-2 \theta \alpha_{\xi}\right|}{1+4 \theta^{2}}}_{(1)}+\underbrace{\frac{\left|z\left(-\theta ; \rho_{y, a}\right)\right|}{1+4 \theta^{2}}}_{(2)} \\
& +\underbrace{\left|\frac{\partial \zeta}{\partial \xi}\left(-\theta, \rho_{y, a}\right)\right|}_{(3)} \cdot|b|+\frac{1}{\langle 2 \theta\rangle} \underbrace{\frac{\partial z}{\partial \xi}\left(-\theta ; \rho_{y, a}\right) \mid}_{(4)} \cdot|b|+\underbrace{\left\|H_{4}\right\| \cdot|b|^{2}}_{(5)} .
\end{aligned}
$$

We have (1) $\leqslant C_{4} \varepsilon|y| /\langle\theta\rangle^{2},(2) \leqslant C \varepsilon /\langle\theta\rangle^{2} \leqslant C^{\prime} \varepsilon|y| /\langle\theta\rangle^{2}$ since by (4.4.14) $|y| \geqslant c_{0} / 2$. Moreover

$$
(3) \leqslant C \varepsilon|b| \leqslant \frac{C^{\prime} \varepsilon|y|}{\langle\theta\rangle^{2}}, \quad(4) \leqslant \frac{C \varepsilon}{\langle 2 \theta\rangle}|b| \leqslant \frac{C^{\prime} \varepsilon|y|}{\langle\theta\rangle^{2}}
$$

and, by (4.4.11), $(5) \leqslant C \frac{|y|}{\langle\theta\rangle^{2}} \frac{|y|}{\langle\theta\rangle^{2}}$. Since $|y| \leqslant \delta\langle\theta\rangle$ it follows that $(5) \leqslant C \delta|y| /\langle\theta\rangle^{2}$.

Summing up we obtain

$$
\left|\Phi_{2}(a, b)+\frac{y}{1+4 \theta^{2}}\right| \leqslant C(\varepsilon+\delta) \frac{|y|}{1+4 \theta^{2}}
$$

so we take $\varepsilon, \delta$ so small that $C(\varepsilon+\delta) \leqslant \sqrt{\delta}$.

Let us look to the term

$$
(I I)=\left|\Phi_{1}(a, b)+\frac{2 \theta y}{1+4 \theta^{2}}\right| .
$$


We have

$$
\begin{aligned}
\Phi_{1}(a, b) & +\frac{2 \theta y}{1+4 \theta^{2}}=\underbrace{\xi(\theta ; \alpha)-\alpha_{\xi}}_{(1)}-\underbrace{\frac{2 \theta}{1+4 \theta^{2}}\left[x(\theta ; \alpha)-\alpha_{x}-2 \theta \alpha_{\xi}\right]}_{(2)} \\
& -\underbrace{\frac{2 \theta}{1+4 \theta^{2}} z\left(-\theta ; \rho_{y, a}\right)}_{(3)}-\underbrace{\frac{1}{1+4 \theta^{2}} \frac{\partial z}{\partial \xi}\left(-\theta ; \rho_{y, a}\right) \cdot b}_{(4)}+\underbrace{\zeta\left(-\theta ; \rho_{y, a}\right)}_{(5)}+\underbrace{H_{3} b \cdot b}_{(6)} .
\end{aligned}
$$

We have $|(1)| \leqslant C \varepsilon,|(2)| \leqslant \frac{C \varepsilon \theta\left\langle\theta-\theta^{*}\right\rangle}{1+4 \theta^{2}} \leqslant C^{\prime} \varepsilon$ (see (4.4.17)), $|(3)| \leqslant \frac{C \varepsilon}{\langle\theta\rangle}$ (by Proposition 3.3.2), $|(4)| \leqslant \frac{C \varepsilon}{\langle\theta\rangle^{2}}|b| \leqslant C^{\prime \prime} \varepsilon,|(5)| \leqslant C \varepsilon,|(6)| \leqslant C \delta^{2}$. It follows that if $\varepsilon$ and $\delta$ are small enough we have,

$$
(I I) \leqslant C\left(\varepsilon+\delta^{2}\right) \leqslant C\left(\varepsilon+\delta^{2}\right) \frac{2}{c_{0}} \inf (1,|y|) \leqslant \sqrt{\delta} \inf (1,|y|)
$$

since $|y| \geqslant c_{0} / 2$. It follows from (4.4.18) and (4.4.19) that $\Phi$ maps $E$ into $E$.

We show now that one can find a constant $k \in] 0,1[$ such that

$$
\left|\Phi(a, b)-\Phi\left(a^{\prime}, b^{\prime}\right)\right| \leqslant k\left|(a, b)-\left(a^{\prime}, b^{\prime}\right)\right|, \quad \forall(a, b), \quad\left(a^{\prime}, b^{\prime}\right) \in E .
$$

We have

$$
\begin{aligned}
\mid \Phi_{1}(a, b)- & \Phi_{1}\left(a^{\prime}, b^{\prime}\right) \mid \leqslant \underbrace{\frac{1}{1+4 \theta^{2}}\left|z\left(-\theta ; \rho_{y, a}(\theta ; \alpha)\right)-z\left(-\theta ; \rho_{y, a^{\prime}}(\theta, \alpha)\right)\right|}_{(1)} \\
& +\underbrace{\frac{1}{1+4 \theta^{2}}\left|\frac{\partial z}{\partial \xi}\left(-\theta ; \rho_{y, a}(\theta ; \alpha)\right) \cdot b-\frac{\partial z}{\partial \xi}\left(-\theta ; \rho_{y, a^{\prime}}(\theta ; \alpha)\right) \cdot b^{\prime}\right|}_{(2)} \\
+ & \underbrace{\zeta \zeta\left(-\theta ; \rho_{y, a}(\theta ; \alpha)\right)-\zeta\left(-\theta ; \rho_{y, a^{\prime}}(\theta ; \alpha)\right)}_{(3)}|+| \underbrace{H_{3} b \cdot b-H_{3}^{\prime} b^{\prime} \cdot b^{\prime}}_{(4)} \mid .
\end{aligned}
$$

Since the point $\left(y+x(\theta ; \alpha), \alpha_{\xi}\right)$ is outgoing we can use Proposition 3.3.2, (4.4.11) and the fact that $|b| \leqslant \frac{2|y|}{\langle\theta\rangle^{2}} \leqslant 2 \delta$, to write

$$
\begin{array}{ll}
(1) \leqslant \frac{C \theta}{1+4 \theta^{2}} \varepsilon\left|a-a^{\prime}\right|, & (2) \leqslant \frac{C \theta \varepsilon}{1+4 \theta^{2}}\left(\left|b-b^{\prime}\right|+\left|a-a^{\prime}\right|\right) \\
(3) \leqslant C \varepsilon\left|a-a^{\prime}\right|, & (4) \leqslant C \delta\left(\left|a-a^{\prime}\right|+\left|b-b^{\prime}\right|\right) .
\end{array}
$$

It follows that

$$
\left|\Phi_{1}(a, b)-\Phi_{1}\left(a^{\prime}, b^{\prime}\right)\right| \leqslant C(\varepsilon+\delta)\left(\left|a-a^{\prime}\right|+\left|b-b^{\prime}\right|\right) .
$$


Now we have

$$
\begin{aligned}
\mid \Phi_{2}(a, b) & -\Phi_{2}\left(a^{\prime}, b^{\prime}\right)|\leqslant| \frac{\partial \zeta}{\partial \xi}\left(-\theta ; \rho_{y, a}(\theta ; \alpha)\right) \cdot b-\frac{\partial \zeta}{\partial \xi}\left(-\theta ; \rho_{y, a^{\prime}}(\theta ; \alpha)\right) \cdot b^{\prime} \mid \\
& +\frac{2 \theta}{1+4 \theta^{2}}\left|\frac{\partial z}{\partial \xi}\left(-\theta ; \rho_{y, a}(\theta ; \alpha)\right) \cdot b-\frac{\partial z}{\partial \xi}\left(-\theta ; \rho_{y, a^{\prime}}(\theta ; \alpha)\right) \cdot b^{\prime}\right| \\
& +\left|H_{4} b \cdot b-H_{4}^{\prime} b^{\prime} \cdot b^{\prime}\right|+\frac{1}{1+4 \theta^{2}} \mid z\left(\left(-\theta ; \rho_{y, a}(\theta ; \alpha)\right)-z\left(-\theta ; \rho_{y, a^{\prime}}(\theta ; \alpha)\right) \mid .\right.
\end{aligned}
$$

The same estimates as those used in the first case show that

$$
\left|\Phi_{2}(a, b)-\Phi_{2}\left(a^{\prime}, b^{\prime}\right)\right| \leqslant C(\varepsilon+\delta)\left(\left|a-a^{\prime}\right|+\left|b-b^{\prime}\right|\right) .
$$

Thus (4.4.20) is proved if $(\varepsilon+\delta)$ is small enough. Then the fixed point theorem shows that the system (4.4.3) has a unique solution in $E$.

Case 2. - Assume that

$$
x(\theta ; \alpha) \cdot \alpha_{\xi} \leqslant 2 c_{0}\langle x(\theta ; \alpha)\rangle\left|\alpha_{\xi}\right| .
$$

It follows that we can apply Proposition 3.5.2 with $(y, \eta)=\alpha$ which allows us to write

$$
\left\{\begin{array}{l}
x(\theta ; \alpha)=\alpha_{x}+2 \theta \alpha_{\xi}-z(-\theta ; \rho(\theta ; \alpha)) \\
\xi(\theta ; \alpha)=\alpha_{\xi}-\zeta(-\theta ; \rho(\theta ; \alpha))
\end{array}\right.
$$

where $\rho(\theta ; \alpha)=(x(\theta ; \alpha), \xi(\theta ; \alpha))$.

Using (4.4.12) we obtain the following expressions of $\Phi_{1}, \Phi_{2}$.

$$
\begin{aligned}
\Phi_{1}(a, b)= & \frac{-2 \theta y}{1+4 \theta^{2}}-\underbrace{\frac{2 \theta}{1+4 \theta^{2}}\left[z \left(\left(-\theta ; \rho_{y, a}(\theta ; \alpha)\right)-z(-\theta ; \rho(\theta ; \alpha))\right.\right.}_{(1)}] \\
- & \underbrace{\frac{1}{1+4 \theta^{2}} \frac{\partial z}{\partial \xi}\left(-\theta ; \rho_{y, a}(\theta ; \alpha)\right) \cdot b}_{(2)}+\underbrace{\zeta\left(-\theta ; \rho_{y, a}(\theta ; \alpha)\right)-\zeta(-\theta ; \rho(\theta ; \alpha))}_{(3)}+\underbrace{H_{3} b \cdot b}_{(4)} . \\
\Phi_{2}(a, b)= & -\frac{y}{1+4 \theta^{2}}-\underbrace{\frac{1}{1+4 \theta^{2}}\left[z\left(-\theta ; \rho_{y, a}(\theta ; \alpha)\right)-z(-\theta ; \rho(\theta ; \alpha))\right.}_{(5)}] \\
& -\underbrace{\frac{\partial \zeta}{\partial \xi}\left(-\theta ; \rho_{y, a}(\theta ; \alpha)\right) \cdot b}_{(6)}+\underbrace{\frac{2 \theta}{1+4 \theta^{2}} \frac{\partial z}{\partial \xi}\left(-\theta ; \rho_{y, a}(\theta ; \alpha)\right)}_{(7)} \cdot b+\underbrace{H_{4} b \cdot b}_{(8)} .
\end{aligned}
$$

Let us show that

$$
\left\{\begin{array}{l}
\left|\Phi_{1}(a, b)+\frac{2 \theta y}{1+4 \theta^{2}}\right| \leqslant \sqrt{\delta} \inf (1,|y|) \\
\left|\Phi_{2}(a, b)+\frac{y}{1+4 \theta^{2}}\right| \leqslant \sqrt{\delta} \frac{|y|}{\langle\theta\rangle^{2}} .
\end{array}\right.
$$


If $|y| \geqslant c_{0}$ then $\inf (1,|y|) \geqslant c_{0}$. It follows that

$$
\begin{aligned}
& |(1)| \leqslant \frac{2 \theta}{1+4 \theta^{2}} C \varepsilon \leqslant \frac{C \varepsilon}{c_{0}} \inf (1,|y|), \\
& |(2)| \leqslant C \varepsilon \leqslant \frac{C \varepsilon}{c_{0}} \inf (1,|y|), \\
& |(3)| \leqslant C \varepsilon|b| \leqslant \frac{2 C \varepsilon|y|}{\langle\theta\rangle^{2}} \leqslant 2 C \varepsilon \delta \leqslant \frac{2 C \varepsilon \delta}{c_{0}} \inf (1,|y|), \\
& |(4)| \leqslant \frac{C \delta}{c_{0}} \inf (1,|y|), \\
& |(5)| \leqslant \frac{C \varepsilon}{\langle\theta\rangle^{2}} \leqslant \frac{C \varepsilon}{c_{0}} \frac{|y|}{\langle\theta\rangle^{2}}, \\
& |(6)| \leqslant C \varepsilon|b| \leqslant C^{\prime} \varepsilon \frac{|y|}{\langle\theta\rangle^{2}}, \\
& |(7)| \leqslant C \varepsilon \frac{|y|}{\langle\theta\rangle^{2}}, \\
& |(8)| \leqslant C \delta \frac{|y|}{\langle\theta\rangle^{2}} .
\end{aligned}
$$

These estimates imply (4.4.23).

Assume now that $|y| \leqslant c_{0}$. It follows that for every $t$ in $[0,1]$ the point $(t y+$ $x(\theta ; \alpha), \xi(\theta ; \alpha)-t a)$ is outgoing for $\theta \leqslant 0$ (i.e. belongs to $\mathcal{S}_{-}$). Indeed we have

$$
\begin{gathered}
\xi(\theta ; \alpha)-t a=\alpha_{\xi}+\mathcal{O}(\varepsilon+\delta) \\
|x(\theta ; \alpha)| \leqslant|y|+|y+x(\theta ; \alpha)| \leqslant 1+|y+x(\theta ; \alpha)|
\end{gathered}
$$

so

$$
\begin{aligned}
(t y+x(\theta ; \alpha)) \cdot \alpha_{\xi} & \leqslant|y| \cdot\left|\alpha_{\xi}\right|+2 c_{0}\langle x(\theta ; \alpha)\rangle\left|\alpha_{\xi}\right| \\
& \leqslant 3 c_{0}\langle x(\theta ; \alpha)\rangle\left|\alpha_{\xi}\right| \\
& \leqslant 6 c_{0}\langle y+x(\theta ; \alpha)\rangle\left|\alpha_{\xi}\right| .
\end{aligned}
$$

Then we have the following estimates.

$$
\begin{aligned}
& |(1)| \leqslant \int_{0}^{1}\left[|y|\left|\frac{\partial z}{\partial y}\right|+|a|\left|\frac{\partial z}{\partial \xi}\right|\right](-\theta, t y+x(\theta ; \alpha), \xi(\theta ; \alpha)-t a) d t \\
& |(1)| \leqslant C \varepsilon|y| \text { since }|a| \leqslant C^{\prime}|y| .
\end{aligned}
$$

By the same way we have $|(3)| \leqslant C \varepsilon|y|$. Moreover $|(2)| \leqslant C \varepsilon|y|,|(4)| \leqslant C \delta|y|$ since $|b| \leqslant \frac{2|y|}{\langle\theta\rangle^{2}} \leqslant 2 \delta$.

On the other hand we have

$$
\begin{array}{ll}
|(5)| \leqslant \frac{C \varepsilon|y|}{\langle\theta\rangle^{2}}, & |(6)| \leqslant C \varepsilon|b| \leqslant \frac{C^{\prime} \varepsilon|y|}{\langle\theta\rangle^{2}} \\
|(7 \mid) \leqslant C \varepsilon| b \mid \leqslant C^{\prime} \varepsilon \frac{|y|}{\langle\theta\rangle^{2}}, & |(8)| \leqslant C \delta \frac{|y|}{\langle\theta\rangle^{2}} .
\end{array}
$$


These estimates imply (4.4.23) since in this case $|y|=\inf (1,|y|)$. Summing up we have proved that $\Phi$ maps $E$ into itself.

We show now the estimate (4.4.22). But it is easy to see that the proof given in Case 1 works also in Case 2. Using again the fixed point theorem we see that the system (4.4.3) has a unique solution in $E$. This proves the points (i) and (ii) of Theorem 4.4.2.

To prove (iii) we use an induction on $|A|$ starting with $|A|=1$. Let us set for fixed $(\theta, \alpha)$

$$
\gamma_{y, \widetilde{a}}=\left(y+x(\theta, \alpha), \xi(\theta, \alpha)-\widetilde{a}(\theta, y, \alpha)+\frac{2 \theta y}{1+4 \theta^{2}}\right) .
$$

Using (4.4.12) we see that $(\widetilde{a}, \widetilde{b})$ satisfy the system

$$
\begin{aligned}
\widetilde{a}=\xi(\theta, \alpha) & -\frac{\alpha_{\xi}+2 \theta\left(x(\theta, \alpha)-\alpha_{x}\right)}{1+4 \theta^{2}} \\
- & \underbrace{\frac{2 \theta}{1+4 \theta^{2}} z\left(-\theta, \gamma_{y, \widetilde{a}}\right)}_{(1)}-\underbrace{\frac{1}{1+4 \theta^{2}} \frac{\partial z}{\partial \xi}\left(-\theta, \gamma_{y, \widetilde{a}} \frac{1}{\langle\theta\rangle} \widetilde{b}\right.}_{(2)} \\
& +\underbrace{\frac{1}{1+4 \theta^{2}} \frac{\partial z}{\partial \xi}\left(-\theta, \gamma_{y}, \widetilde{a}\right) \frac{y}{1+4 \theta^{2}}}_{(3)}+\underbrace{\zeta\left(-\theta, \gamma_{y, \widetilde{a}}\right)}_{(4)} \\
& \underbrace{\frac{1}{\langle\theta\rangle^{2}} \widetilde{H}_{3} \widetilde{b} \cdot \widetilde{b}}_{(5)}+\underbrace{\frac{2}{\langle\theta\rangle} \widetilde{H}_{3}, \widetilde{b} \frac{y}{1+4 \theta^{2}}}_{(6)}+\underbrace{\widetilde{H}_{3} \frac{y}{1+4 \theta^{2}} \frac{y}{1+4 \theta^{2}}}_{(7)} .
\end{aligned}
$$

$$
\begin{aligned}
& \widetilde{b}=-\frac{\langle\theta\rangle\left(x(\theta, \alpha)-\alpha_{x}-2 \theta \alpha_{\xi}\right)}{1+4 \theta^{2}}-\underbrace{\frac{\langle\theta\rangle}{1+4 \theta^{2}} z\left(-\theta, \gamma_{y, \widetilde{a}}\right)}_{(8)} \\
& -\underbrace{\frac{\partial \zeta}{\partial \xi}\left(-\theta, \gamma_{y}, \widetilde{a}\right)}_{(9)} \tilde{b}+\underbrace{\frac{\partial \zeta}{\partial \xi}\left(-\theta, \gamma_{y}, \widetilde{a}\right) \frac{y\langle\theta\rangle}{1+4 \theta^{2}}}_{(10)}+\underbrace{\frac{1}{\langle\theta\rangle} \widetilde{H}_{4} \widetilde{b} \cdot \widetilde{b}}_{(11)}+\underbrace{2 \widetilde{H}_{4} \widetilde{b} \frac{y}{1+4 \theta^{2}}}_{(12)}
\end{aligned}
$$

$$
+\underbrace{\langle\theta\rangle \widetilde{H}_{4} \frac{y}{1+4 \theta^{2}} \cdot \frac{y}{1+4 \theta^{2}}}_{(13)}+\underbrace{\frac{2 \theta}{1+4 \theta^{2}} \frac{\partial z}{\partial \xi}\left(-\theta, \gamma_{y}, \tilde{a}\right)}_{(14)} \tilde{b}-\underbrace{\frac{2 \theta}{1+4 \theta^{2}} \frac{\partial z}{\partial \xi}\left(-\theta, \gamma_{y}, \tilde{a}\right) \frac{y\langle\theta\rangle}{1+4 \theta^{2}}}_{(15)}
$$

where for $j=3,4 \widetilde{H}_{j}=H_{j}\left(\theta, y, \alpha, \widetilde{a}-\frac{2 \theta y}{1+4 \theta^{2}},\langle\theta\rangle \widetilde{b}-\frac{y}{1+4 \theta^{2}}\right)$ and $H_{j}$ satisfies (4.4.11). We claim that, for $j=3,4$,

$$
\left|\partial_{y}\left[\widetilde{H}_{j}\right]\right| \leqslant C\left(\left|\partial_{y} \widetilde{a}\right|+\langle\theta\rangle\left|\partial_{y} \widetilde{b}\right|+\frac{1}{\langle\theta\rangle}+\frac{\varepsilon}{\langle x\rangle^{2+\sigma_{0}}}\right) .
$$


Indeed, skipping the index $j$ for convenience, we have

$$
\frac{\partial}{\partial y_{k}}[\widetilde{H}]=\frac{\partial H}{\partial y_{k}}+\frac{\partial H}{\partial a} \frac{\partial \widetilde{a}}{\partial y_{k}}-\frac{2 \theta}{1+4 \theta^{2}} \frac{\partial H}{\partial a_{k}}+\langle\theta\rangle \frac{\partial H}{\partial \widetilde{b}} \frac{\partial \widetilde{b}}{\partial y_{k}}-\frac{1}{1+4 \theta^{2}} \frac{\partial H}{\partial b_{k}} .
$$

Now we use (4.4.11). The first term in the right hand side is bounded by $\frac{C}{\langle\theta\rangle}+\frac{C \varepsilon}{\langle x\rangle^{\sigma} 0^{+2}}$, the second by $C|\nabla \widetilde{a}|$, the third by $\frac{C}{\langle\theta\rangle}$, the fourth by $C\langle\theta\rangle\left|\nabla_{y} \widetilde{b}\right|$ and the last one by $\langle\theta\rangle^{-2}$.

For $\ell \in \mathbb{N}$ let us introduce the following space

$$
\mathcal{F}_{\ell}=\left\{F \in C^{\infty}\left(\mathbb{R}^{n} \times \mathbb{R}^{n}\right):\left|\partial_{x}^{A} \partial_{\xi}^{B} F(x, \xi)\right| \leqslant \frac{C_{A B} \varepsilon}{\langle x\rangle^{|A|+\ell+\sigma_{0}}}, \quad \forall(x, \xi) \in \mathbb{R}^{n} \times \mathbb{R}^{n}\right\} .
$$

For example $\zeta, \frac{\partial \zeta}{\partial \xi} \in \mathcal{F}_{1}, z, \frac{\partial z}{\partial \xi} \in \mathcal{F}_{0}$ according to Proposition 3.3.2.

Let us set now,

$$
\left\{\begin{array}{l}
g(y)=y+x(\theta ; \alpha) \\
h(y)=\xi(\theta ; \alpha)-\widetilde{a}(\theta, y, \alpha)+\frac{2 \theta y}{1+4 \theta^{2}} .
\end{array}\right.
$$

Then for $F \in \mathcal{F}_{\ell}$ and $k=1, \ldots, n$ we have,

$$
\left|\frac{\partial}{\partial y_{k}}[F(g(y), h(y))]\right| \leqslant C \varepsilon\left(\frac{1}{\langle x\rangle^{\ell+1+\sigma_{0}}}+\frac{1}{\langle x\rangle^{\ell+\sigma_{0}}\langle\theta\rangle}+\left|\nabla_{y} \widetilde{a}\right|\right)
$$

where $x=y+x(\theta ; \alpha)$.

Let us prove (iii) for $|A|=1$. We differentiate the equations (4.4.25), (4.4.26) with respect to $y_{k}$ and we use (4.4.27), (4.4.30) and the fact that $|\widetilde{b}| \leqslant \frac{3|y|}{\langle\theta\rangle} \leqslant 3 \delta \leqslant 1$. We have, with the notations in (4.4.25), (4.4.26),

$$
\begin{aligned}
& \left|\partial_{y_{k}}(1)\right|+\left|\partial_{y_{k}}(8)\right| \leqslant C \varepsilon\left|\nabla_{y} \widetilde{a}\right|+C \varepsilon \frac{1}{\langle x\rangle^{\sigma_{0}}} \frac{1}{\langle\theta\rangle}\left(\frac{1}{\langle x\rangle}+\frac{1}{\langle\theta\rangle}\right) \\
& \left|\partial_{y_{k}}(2)\right| \leqslant C \varepsilon\left(\left|\nabla_{y} \widetilde{a}\right|+\left|\nabla_{y} \widetilde{b}\right|\right)+\frac{C \varepsilon}{\langle x\rangle^{\sigma_{0}}\langle\theta\rangle^{3}}\left(\frac{1}{\langle x\rangle}+\frac{1}{\langle\theta\rangle}\right) \\
& \left|\partial_{y_{k}}(3)\right| \leqslant C \varepsilon\left|\nabla_{y} \widetilde{a}\right|+\frac{C \varepsilon}{\langle x\rangle^{\sigma} 0\langle\theta\rangle^{3}}\left(\frac{1}{\langle x\rangle}+\frac{1}{\langle\theta\rangle}\right) \\
& \left|\partial_{y_{k}}(4)\right| \leqslant C \varepsilon\left|\nabla_{y} \widetilde{a}\right|+\frac{C \varepsilon}{\langle x\rangle^{\sigma_{0}+1}}\left(\frac{1}{\langle x\rangle}+\frac{1}{\langle\theta\rangle}\right) \\
& \left|\partial_{y_{k}}(5)\right|+\left|\partial_{y_{k}}(6)\right|+\left|\partial_{y_{k}}(7)\right| \leqslant C \delta(|\nabla \widetilde{a}|+|\nabla \widetilde{b}|)+\frac{C \varepsilon}{\langle x\rangle^{2+\sigma_{0}\langle\theta\rangle^{2}}+\frac{C \delta}{\langle\theta\rangle^{3}}} \\
& \left|\partial_{y_{k}}(9)\right| \leqslant C \varepsilon(|\nabla \widetilde{a}|+|\nabla \widetilde{b}|)+\frac{C \varepsilon}{\langle x\rangle^{\sigma_{0}+1}}\left(\frac{1}{\langle x\rangle}+\frac{1}{\langle\theta\rangle}\right) \\
& \left|\partial_{y_{k}}(10)\right| \leqslant C \varepsilon|\nabla \widetilde{a}|+\frac{C \varepsilon}{\langle x\rangle^{\sigma_{0}}}\left(\frac{1}{\langle x\rangle}+\frac{1}{\langle\theta\rangle}\right)^{2} \\
& \left|\partial_{y_{k}}(11)\right|+\left|\partial_{y_{k}}(12)\right|+\left|\partial_{y_{k}}(13)\right| \leqslant C \delta(|\nabla \widetilde{a}|+|\nabla \widetilde{b}|)+\frac{C \varepsilon}{\langle x\rangle^{2+\sigma_{0}}\langle\theta\rangle}+\frac{C \delta}{\langle\theta\rangle^{2}} \\
& \left|\partial_{y_{k}}(14)\right|+\left|\partial_{y_{k}}(15)\right| \leqslant C(\varepsilon+\delta)\left(\left|\nabla_{y} \widetilde{a}\right|+|\nabla \widetilde{b}|\right)+\frac{C \varepsilon}{\langle x\rangle^{\sigma_{0}}}\left(\frac{1}{\langle x\rangle}+\frac{1}{\langle\theta\rangle}\right)^{2} .
\end{aligned}
$$

It follows from (4.4.25), (4.4.26), that

$$
\left|\nabla_{y} \widetilde{a}\right|+\left|\nabla_{y} \widetilde{b}\right| \leqslant C(\varepsilon+\delta)(|\nabla \widetilde{a}|+|\nabla \widetilde{b}|)+\frac{C \varepsilon}{\langle x\rangle^{\sigma_{0}}}\left(\frac{1}{\langle x\rangle}+\frac{1}{\langle\theta\rangle}\right)^{2}+\frac{C \delta}{\langle\theta\rangle^{2}}
$$

which is (iii) for $|A|=1$. 
Let us assume that (iii) is true for $1 \leqslant|A| \leqslant k$ and let $|A|=k+1 \geqslant 2$.

We claim that for $F \in \mathcal{F}_{\ell}$ and $|B| \leqslant k$ we have

$$
\left|\partial_{y}^{B}[F(g(y), h(y))]\right| \leqslant \frac{C_{B} \varepsilon}{\langle x\rangle^{\sigma_{0}}}\left(\frac{1}{\langle x\rangle}+\frac{1}{\langle\theta\rangle}\right)^{|B|+\ell} .
$$

Indeed the term we want to estimate is a finite sum of terms of the following form (see Section A.1).

$$
R_{\beta \gamma s}=\left(\partial_{x}^{\beta} \partial_{\xi}^{\gamma} F\right)(g(y), h(y)) \prod_{j=1}^{s}\left(\partial_{y}^{\ell_{j}} g\right)^{k_{j}^{\prime}}\left(\partial_{y}^{\ell_{j}} h\right)^{k_{j}}
$$

where $1 \leqslant s \leqslant|B|, 1 \leqslant|\beta|+|\gamma| \leqslant|B|, \sum_{j=1}^{s} k_{j}^{\prime}=\beta, \sum_{j=1}^{s} k_{j}=\gamma, \sum_{j=1}^{s}\left(\left|k_{j}\right|+\right.$ $\left.\left|k_{j}^{\prime}\right|\right) \ell_{j}=B, \ell_{j} \neq 0,\left(k_{j}^{\prime}, k_{j}\right) \neq 0, j=1, \ldots, s$.

Let us write $\{1, \ldots, s\}=I_{1} \cup I_{2}$ where

$$
I_{1}=\left\{j:\left|\ell_{j}\right|=1\right\}, \quad\left|I_{2}\right|=\left\{j:\left|\ell_{j}\right| \geqslant 2\right\} .
$$

For $j \in I_{1}$ we have $\partial_{y}^{\ell_{j}} g_{k}=\mathcal{O}(1), \partial_{y}^{\ell_{j}} h_{k}=-\partial_{y}^{\ell_{j}} \widetilde{a}+\mathcal{O}\left(\frac{1}{\langle\theta\rangle}\right)$. For $j \in I_{2}$ we have $\partial_{y}^{\ell_{j}} g_{k} \equiv 0$. Therefore the only terms which are present are those for which $k_{j}^{\prime}=0$. It follows that $\sum_{j=1}^{s} k_{j}^{\prime}=\sum_{j \in I_{1}} k_{j}^{\prime}=\beta$. Moreover $\partial_{y}^{\ell_{j}} h=-\partial_{y}^{\ell_{j}} \widetilde{a}$. It follows from these facts and the definition of $\mathcal{F}_{\ell}$ that

$\left|R_{\beta \gamma s}\right| \leqslant \frac{C_{\beta \gamma} \varepsilon}{\langle x\rangle^{|\beta|+\ell+\sigma_{0}}}\left[\frac{(C \varepsilon)^{\sum_{j=1}^{s}\left|k_{j}\right|}}{\langle x\rangle^{\sigma_{0} \sum_{j=1}^{s}\left|k_{j}\right|}}\left(\frac{1}{\langle x\rangle}+\frac{1}{\langle\theta\rangle}\right)^{\sum_{j=1}^{s}\left|k_{j}\right|\left(\left|\ell_{j}\right|+1\right)}+\frac{(C \delta)^{\sum_{j=1}^{s}\left|k_{j}\right|}}{\langle\theta\rangle^{\sum_{j=1}^{s}\left|k_{j}\right|\left(\left|\ell_{j}\right|+1\right)}}\right]$.

Now $|\beta|=\sum_{j=1}^{s}\left|k_{j}^{\prime}\right|=\sum_{j=1}^{s}\left|k_{j}^{\prime}\right|\left|\ell_{j}\right|$ since $\left|\ell_{j}\right|=1$ in $I_{1}$ and $k_{j}^{\prime}=0$ in $I_{2}$. It follows that

$$
\left|R_{\beta \gamma s}\right| \leqslant \frac{C \varepsilon}{\langle x\rangle^{\sigma_{0}}}\left(\frac{1}{\langle x\rangle}+\frac{1}{\langle\theta\rangle}\right)^{\sum_{j=1}^{s}\left|k_{j}^{\prime}\right|\left|\ell_{j}^{\prime}\right|+\ell}\left(\frac{1}{\langle x\rangle}+\frac{1}{\langle\theta\rangle}\right)^{\sum_{j=1}^{s}\left|k_{j}\right|\left|\ell_{j}\right|} .
$$

The result follows then, since $\sum_{j=1}^{s}\left(\left|k_{j}\right|+\left|k_{j}^{\prime}\right|\right)\left|\ell_{j}\right|=|B|$.

On the other hand, for $F \in \mathcal{F}_{\ell}$ and $|A|=k+1 \geqslant 2$, we have

$$
\mid \partial_{y}^{A}\left(F(g(y), h(y))\left|\leqslant C_{0} \varepsilon\right| \partial_{y}^{A} \widetilde{a} \mid+\frac{C_{A} \varepsilon}{\langle x\rangle^{\sigma_{0}}}\left(\frac{1}{\langle x\rangle}+\frac{1}{\langle\theta\rangle}\right)^{|A|+\ell} .\right.
$$

Indeed

$$
\partial_{y}^{A}\left(F(g(y), h(y))-\sum_{k=1}^{n}\left(\frac{\partial F}{\partial x_{k}}(g(y), h(y)) \partial_{y}^{A} g_{k}+\frac{\partial F}{\partial \xi_{k}}(g(y), h(y)) \partial_{y}^{A} h_{k}\right)\right.
$$

is a finite sum of terms $R_{\beta \gamma s}$ given by (4.4.32) where $1 \leqslant s \leqslant|A|, 2 \leqslant|\beta|+|\gamma| \leqslant|A|$, $\sum_{j=1}^{s} k_{j}^{\prime}=\beta, \sum_{j=1}^{s} k_{j}=\gamma, \sum_{j=1}^{s}\left(\left|k_{j}\right|+\left|k_{j}^{\prime}\right|\right) \ell_{j}=A,\left|\ell_{j}\right| \geqslant 1,\left|k_{j}\right|+\left|k_{j}^{\prime}\right| \geqslant 1$, $j=1, \ldots, s$. Since $|\beta|+|\gamma| \geqslant 2$ we have $\left|\ell_{j}\right| \leqslant|A|-1$ so the term $R_{\beta \gamma s}$ is bounded, using the induction, by

$$
\frac{C_{A} \varepsilon}{\langle x\rangle^{\sigma_{0}}}\left(\frac{1}{\langle x\rangle}+\frac{1}{\langle\theta\rangle}\right)^{|A|+\ell}
$$

On the other hand, since $|A| \geqslant 2$ we have $\partial_{y}^{A} g_{k}=0$ and $\partial_{y}^{A} h_{k}=-\partial_{y}^{A} \widetilde{a}_{k}$. By (4.4.28) we have $\left|\frac{\partial F}{\partial \xi_{k}}(g(y), h(y))\right| \leqslant C \varepsilon$ so (4.4.33) is proved. Note that $C$ is independent of $A$. 
Let us now prove the last step of the induction. We apply $\partial_{y}^{A}$ to both members of (4.4.25), (4.4.26). Then we estimate each term of the right hand side using (4.4.31), (4.4.33). We obtain

$$
\begin{aligned}
& \left|\partial_{y}^{A}(1)\right|+\left|\partial_{\gamma}^{A}(8)\right| \leqslant C_{0} \varepsilon\left|\partial_{y}^{A} \widetilde{a}\right|+\frac{C_{A} \varepsilon}{\langle x\rangle^{\sigma_{0}}} \frac{1}{\langle\theta\rangle}\left(\frac{1}{\langle x\rangle}+\frac{1}{\langle\theta\rangle}\right)^{|A|} . \\
& \left|\partial_{y}^{A}(4)\right| \leqslant C_{0} \varepsilon\left|\partial_{y}^{A} \widetilde{a}\right|+\frac{C_{A} \varepsilon}{\langle x\rangle^{\sigma_{0}}}\left(\frac{1}{\langle x\rangle}+\frac{1}{\langle\theta\rangle}\right)^{|A|+1} . \\
& \left|\partial_{y}^{\alpha}(2)\right|+\left|\partial_{y}^{A}(3)\right| \leqslant C_{0} \varepsilon\left(\left|\partial_{y}^{A} \widetilde{a}\right|+\left|\partial_{y}^{A} \widetilde{b}\right|\right)+\frac{C_{A} \varepsilon}{\langle x\rangle^{\sigma_{0}}} \frac{1}{\langle\theta\rangle^{3}}\left(\frac{1}{\langle x\rangle}+\frac{1}{\langle\theta\rangle}\right)^{|A|} . \\
& \left|\partial_{y}^{A}(9)\right|+\left|\partial_{y}^{A}(10)\right| \leqslant C_{0} \varepsilon\left(\left(\left|\partial_{y}^{A} \widetilde{a}\right|+\left|\partial_{y}^{A} \widetilde{b}\right|\right)+\frac{C_{A} \varepsilon}{\langle x\rangle^{\sigma_{0}}}\left(\frac{1}{\langle x\rangle}+\frac{1}{\langle\theta\rangle}\right)^{|A|+1} .\right.
\end{aligned}
$$

Finally

$$
\begin{aligned}
\left|\partial_{y}^{A}(5)\right|+\left|\partial_{y}^{A}(6)\right| & +\left|\partial_{y}^{A}(7)\right|+\left|\partial_{y}^{A}(11)\right|+\left|\partial_{y}^{A}(12)\right|+\left|\partial_{y}^{A}(13)\right| \\
& \leqslant C_{0} \delta\left(\left|\partial_{y}^{A}, \widetilde{a}\right|+\left|\partial_{y}^{A} \widetilde{b}\right|\right)+\frac{C_{A} \varepsilon}{\langle x\rangle^{\sigma_{0}}}\left(\frac{1}{\langle x\rangle}+\frac{1}{\langle\theta\rangle}\right)^{|A|+1}+\frac{C_{A} \delta}{\langle\theta\rangle^{|A|+1}} .
\end{aligned}
$$

If $\varepsilon+\delta$ is small enough (compared with a finite number of fixed constants) we can absorb the term $C_{0}(\varepsilon+\delta)\left(\left|\partial_{y}^{A} \widetilde{a}\right|+\left|\partial_{y}^{A} \widetilde{b}\right|\right)$ by the left hand side and we obtain the estimate given in (iii).

Let us now prove (iv). First of all since the point $(y+x(\theta, \alpha), \eta+\xi(\theta, \alpha))$ belongs to $\mathcal{S}_{-}$and $-\theta \leqslant 0$ we deduce from Proposition 3.3.2 that

$$
\left|\partial_{\xi}^{\gamma} \xi(-\theta, y+x(\theta, \alpha), \eta+\xi(\theta, \alpha))\right|+\mid \partial_{\xi}^{\gamma} x\left(-\theta, y+x(\theta, \alpha), \eta+\xi(\theta, \alpha) \mid \leqslant C_{\gamma}\langle\theta\rangle .\right.
$$

It follows then, from (4.4.2) and Lemma 4.2.1 that

$$
\left|\frac{\partial q_{k}}{\partial \xi_{j}}\left(\eta, a, b, g_{j}\right)\right|+\left|\frac{\partial q_{k}}{\partial b_{\ell}}\left(\eta, a, b, g_{j}\right)\right| \leqslant C\langle\theta\rangle .
$$

Therefore we will have,

$$
\left|q_{k}\left(\eta, a, b, g_{j}\right)-q_{k}\left(-a, a, 0, g_{j}\right)\right| \leqslant C\langle\theta\rangle(|\eta+a|+|b|) .
$$

Now if $|\eta| \leqslant \sqrt{\delta}$ we will have $|\eta+a|+|b| \leqslant 5 \sqrt{\delta}$. On the other hand (4.2.7) shows that, when $|\eta| \leqslant \sqrt{\delta}<\frac{1}{2} c_{0}$,

$$
q_{k}\left(-a, a, 0, g_{j}\right)=\frac{\partial g_{j}}{\partial \xi_{k}}(-a)=\left(\frac{\partial \xi_{j}}{\partial \xi_{k}}-i \frac{\partial x_{j}}{\partial x_{k}}\right)(-\theta, y+x(\theta, \alpha),-a+\xi(\theta, \alpha)) .
$$

By Corollary 3.3.3 we have

$$
q_{k}\left(-a, a, 0, g_{j}\right)=(1+2 i \theta) \delta_{j k}+\mathcal{O}(\varepsilon\langle\theta\rangle) .
$$

Finally we obtain,

$$
\left|q_{k}\left(\eta, a, b, g_{j}\right)-(1+2 i \theta) \delta_{j k}\right| \leqslant C(\varepsilon+\sqrt{\delta})\langle\theta\rangle,
$$

which is precisely the claim of point (iv).

The last point (v) can be easily deduced from Proposition 3.3.2 and Lemma 4.2.1. This ends the proof of Theorem 4.4.2. 
We continue the proof of Theorem 4.1.2 in the case (4.4.1). We follow basically the same method as in Section 4.3 with small changes. For the convenience of the reader we give some details.

Let us set,

$$
\left\{\begin{aligned}
& \mathcal{O}=\left\{(\theta,(y, \eta)) \in \mathbb{R}_{+} \times \mathbb{R}^{2 n}:\right. \\
&\left.|y|<\delta\langle\theta\rangle,(y+x(\theta, \alpha)) \cdot \alpha_{\xi} \leqslant c_{1}\langle y+x(\theta \alpha)\rangle\left|\alpha_{\xi}\right| ;|\eta| \leqslant \sqrt{\delta}\right\} \\
& \widetilde{\Lambda}=\left\{\alpha \in T^{*} \mathbb{R}^{n}: \frac{1}{2} \leqslant\left|\alpha_{\xi}\right| \leqslant 2, \alpha_{x} \cdot \alpha_{\xi} \leqslant-c_{0}\left\langle\alpha_{x}\right\rangle\left|\alpha_{\xi}\right|\right\}
\end{aligned}\right.
$$

We consider families $(f(\cdot, \alpha))_{\alpha \in \widetilde{\Lambda}}$ of function on $\mathcal{O}$.

Definition 4.4.3. - We say that $(f(\cdot, \alpha))_{\alpha \in \widetilde{\Lambda}}$ belongs to $\mathcal{H}$ if

(i) for all $\alpha \in \widetilde{\Lambda},(\theta, y, \eta) \mapsto f(\theta, y, \eta, \alpha)$ is $C^{\infty}$ on $\mathcal{O}$.

(ii) For every $A, B$ in $\mathbb{N}^{n}$ there exists $C_{A B}>0$ such that

$$
\sup _{(\theta, y, \alpha) \in \mathcal{O} \times \widetilde{\Lambda}}\left|\partial_{y}^{A} \partial_{\eta}^{B} f(\theta, y, \eta, \alpha)\right| \leqslant C_{A B} .
$$

REMARK 4.4 .4

1) $\mathcal{H}$ is closed under multiplication and derivation with respect to $(y, \eta)$.

2) If we set, with the notation (4.2.10),

$$
\begin{aligned}
f(\theta, y, \eta, \alpha)= & \frac{1}{\langle\theta\rangle} v_{j}(\theta, y+x(\theta, \alpha), \eta+\xi(\theta, \alpha)) \\
=\frac{1}{\langle\theta\rangle}\left[\xi_{j}(-\theta, y+x(\theta, \alpha), \eta+\xi(\theta, \alpha))-\alpha_{\xi}^{j}\right. & \left.\quad-i\left(x(-\theta, y+x(\theta, \alpha), \eta+\xi(\theta, \alpha))-\alpha_{x}^{j}\right)\right]
\end{aligned}
$$

then $(f(\cdot, \alpha))_{\alpha \in \widetilde{\Lambda}} \in \mathcal{H}$. This is a consequence of Proposition 3.3.2.

Definition 4.4.5 (Lagrangian ideals). - The Lagrangian ideal $\mathcal{J}$ is defined as the set of families $F=(F(\cdot, \alpha))_{\alpha \in \widetilde{\Lambda}}$ which can be written as

$$
F(\theta, y, \eta, \alpha)=\sum_{j=1}^{n} f_{j}(\theta, y, \eta, \alpha) \frac{1}{\langle\theta\rangle} v_{j}(\theta, y+x(\theta, \alpha), \eta+\xi(\theta, \alpha))
$$

for all $(\theta, y, \eta)$ in $\mathcal{O}$ and $\alpha$ in $\widetilde{\Lambda}$, where $(f(\cdot, \alpha))_{\alpha \in \widetilde{\Lambda}} \in \mathcal{H}$.

EXAMPLE 4.4.6. - Let us set

$$
F(\theta, y, \eta, \alpha)=\eta_{k}-\psi_{k}(\theta, y, \alpha)
$$

with

$$
\psi_{k}(\theta, y, \alpha)=-\left(a_{k}+i b_{k}\right)(\theta, y, \alpha),
$$

where $a_{k}, b_{k}$ are those given in Theorem 4.4.2. Then $\left(F((\cdot, \alpha))_{\alpha \in \Lambda} \in \mathcal{J}\right.$. 
Indeed if $|\eta| \leqslant \sqrt{\delta}<\frac{1}{2} c_{0}$ then $\chi_{0}(\eta)=1$ so it follows from (4.4.2) and Theorem 4.4.2 that we have

$$
v_{j}(\theta, y+x(\theta, \alpha), \xi(\theta, \alpha)+\eta)=\sum_{k=1}^{n} q_{k}\left(\eta, a, b, g_{j}\right)\left(\eta_{k}-\psi_{k}(\theta, y, \alpha)\right) .
$$

Since $q_{k}\left(\eta, a, b, g_{j}\right)=(1+2 i \theta) \delta_{j k}+\mathcal{O}((\varepsilon+\sqrt{\delta})\langle\theta\rangle)$ (by $\left.(\mathrm{v})\right)$ it follows that the matrix $\left(q_{k}\left(\eta, a, b, g_{j}\right)\right)^{-1}=\left(d_{j k}(\theta, y, \eta, \alpha)\right)_{1 \leqslant j, k \leqslant n}$ exists. Moreover $\left(\langle\theta\rangle d_{j k}(\cdot, \alpha)\right)_{\alpha \in \widetilde{\Lambda}} \in \mathcal{H}$. Now we have

$$
\eta_{k}-\psi_{k}(\theta, y, \alpha)=\sum_{j=1}^{n}\langle\theta\rangle d_{j k}(\theta, y, \alpha) \cdot \frac{1}{\langle\theta\rangle} v_{j}(\theta, y+x(\theta, \alpha), \eta+\xi(\theta, \alpha)) .
$$

This proves our claim.

Lemma 4.4.7. - For $F$ and $G$ in $\mathcal{J}$ let us define

$$
\{F, G\}(\theta, y, \eta, \alpha)=\sum_{j=1}^{n}\left(\frac{\partial F}{\partial \eta_{j}} \frac{\partial G}{\partial y_{j}}-\frac{\partial F}{\partial y_{j}} \frac{\partial G}{\partial \eta_{j}}\right)(\theta, y, \eta, \alpha) .
$$

Then $\{F, G\} \in \mathcal{J}$.

Proof. - Since $v_{j}\left(\theta, y+x(\theta, \alpha), \eta+\xi(\theta, \alpha)=u_{j} \circ \chi_{-\theta}(y, \eta)\right.$ where $u_{j}(x, \xi, \alpha)=\xi_{j}-$ $\alpha_{\xi}^{j}-i\left(x_{j}-\alpha_{x}^{j}\right)$ and $\chi_{-\theta}(y, \eta)=(x(-\theta, y, \eta), \xi(-\theta, y, \theta))$ is the symplectic map defined by the flow we have

$$
\left\{v_{j}, v_{k}\right\}(\theta, y, \eta, \alpha)=\left\{u_{j}, u_{k}\right\}\left(\chi_{-\theta}(y, \eta)\right)=0
$$

because $\left\{u_{j}, u_{k}\right\} \equiv 0$.

Let $F=\sum f_{j} \frac{1}{\langle\theta\rangle} v_{j}, G=\sum g_{k} \frac{1}{\langle\theta\rangle} v_{k}$ be two elements of $\mathcal{J}$ with $f_{j} \in \mathcal{H}, g_{k} \in \mathcal{H}$. Then a straightforward computation and the Remark 4.4.4 give the conclusion (see the proof of Lemma 4.3.10).

Lemma 4.4.8. - Let $R=(R(\cdot, \alpha))_{\alpha \in \tilde{\Lambda}} \in \mathcal{J}$ and assume that $R(\cdot, \alpha)$ does not depend on $\eta$. Then for every $N \in \mathbb{N}$ one can find $C_{N}>0$ such that for every $(\theta, y)$ in $\widetilde{\Omega}_{\delta}$ and $\alpha$ in $\widetilde{\Lambda}$ we have

$$
|R(\theta, y, \alpha)| \leqslant C_{N}|\operatorname{Im} \psi(\theta, y, \alpha)|^{N} .
$$

Proof. - We are going to show by induction on $N \geqslant 1$ that we can write

$$
R(\theta, y, \alpha)=\sum_{0<|\gamma|<N} h_{\gamma}(\theta, y, \alpha)(\eta-\psi)^{\gamma}+\sum_{|\gamma|=N} g_{\gamma}(\theta, y, \alpha, \eta)(\eta-\psi)^{\gamma}
$$

where $\left(h_{\gamma}(\cdot, \alpha)\right)_{\alpha \in \widetilde{\Lambda}}$ and $\left.\left(g_{\gamma}(\cdot, \alpha)\right)_{\alpha \in \widetilde{\Lambda}}\right)$ belong to $\mathcal{H}$.

For $N=1$ the first sum in the right hand side of (4.4.36) is empty and by assumption we have

$$
R(\theta, y, \alpha)=\sum_{j=1}^{n} f_{j}(\theta, y, \eta, \alpha) \frac{1}{\langle\theta\rangle} v_{j}(\theta, y+x(\theta, \alpha), \eta+\xi(\theta, \alpha)) .
$$


Using Theorem 4.4.2 we obtain since $\chi_{0}(\eta)=1$ when $|\eta| \leqslant \sqrt{\delta}$,

$$
R(\theta, y, \alpha)=\sum_{k=1}^{n}\left(\sum_{j=1}^{n} \frac{1}{\langle\theta\rangle} f_{j}(\theta, y, \eta, \alpha) q_{k}\left(\eta, a, b, g_{j}\right)\right)\left(\eta_{k}-\psi_{k}(\theta, y, \alpha)\right) .
$$

Since $f_{j}$ and $\frac{1}{\langle\theta\rangle} q_{k}$ belong to $\mathcal{H}$ this shows that (4.4.36) is true when $N=1$. Assume now it is true up to the order $N$. We can apply Lemma 4.2.1 to the function

$$
\tilde{g}_{\gamma}(\theta, y, \eta, \alpha)=\chi_{0}(\eta) g_{\gamma}(\theta, y, \eta, \alpha), \quad|\gamma|=N
$$

with $z_{j}=-\psi_{j}(\theta, y, \alpha)$. It follows that

$$
\widetilde{g}_{\gamma}(\theta, y, \eta, \alpha)=\sum_{k=1}^{n} q_{k}\left(\eta, a, b, \widetilde{g}_{\gamma}\right)\left(\eta_{k}-\psi_{k}(\theta, y, \alpha)\right)+r\left(a, b, \widetilde{g}_{\gamma}\right) .
$$

For the $q_{k}^{\prime} s$ and $r$ we have the estimates (4.2.2). Let us set

$$
\left\{\begin{array}{l}
h_{\gamma}(\theta, y, \alpha)=r\left(a(\theta, y, \alpha), b(\theta, y, \alpha), \widetilde{g}_{\gamma}(h, y, \cdot, \alpha)\right) \\
g_{\gamma}(\theta, y, \eta, \alpha)=q_{k}\left(\eta, a(\theta, y, \alpha), b(\theta, y, \alpha), \widetilde{g}_{\gamma}(\theta, y, \cdot, \alpha)\right) .
\end{array}\right.
$$

It follows from (4.2.2) and Theorem 4.4.2 that $\left(h_{\gamma}(\cdot, \alpha)\right)_{\alpha}$ and $\left(g_{\gamma k}(\cdot, \alpha)\right)_{\alpha}$ belong to $\mathcal{H}$. Using (4.4.36) at the level $N$ and (4.4.37), (4.4.38) we deduce that (4.4.36) holds at the level $N+1$.

Now let us take in $(4.4 .36) \eta=(\operatorname{Re} \psi+s \operatorname{Im} \psi)(\theta, y, \alpha)$ when $s \in[0,1]$. Then the same argument as in the end of the proof of Lemma 4.3.11 gives the result.

Corollary 4.4.9. - For every $N \in \mathbb{N}$ there exists a constant $C_{N}>0$ such that

$$
\left|\left(\frac{\partial \psi_{j}}{\partial y_{k}}-\frac{\partial \psi_{k}}{\partial y_{j}}\right)(\theta, y, \alpha)\right| \leqslant C_{N}|\operatorname{Im} \psi(\theta, y, \alpha)|^{N}
$$

for every $(\theta, y)$ in $\widetilde{\Omega}_{\delta}$ and $\alpha$ in $\widetilde{\Lambda}$.

Proof. - Identical to the proof of Corollary 4.3.12.

Now we go back to the original coordinates

$$
x=y+x(\theta, \alpha), \quad \xi=\eta+\xi(\theta, \alpha)
$$

and we set for $k=1, \ldots, n$,

$$
\Phi_{k}(\theta, x, \alpha)=\psi_{k}(\theta, x-x(\theta, \alpha), \alpha)=\xi_{k}(\theta, \alpha)-\left(a_{k}+i b_{k}\right)(\theta, x-x(\theta, \alpha), \alpha)
$$

where $a_{k}, b_{k}$ have been described in Theorem 4.4.2.

Then we can state the following result.

TheOREM 4.4.10. - We can write for $(\theta, x) \in \Omega_{\delta}$ and $|\xi-\xi(\theta, \alpha)| \leqslant \sqrt{\delta}$,

(i) $\xi_{k}-\Phi_{k}(\theta, x, \alpha)=\sum_{j=1}^{n} e_{j k}(\theta, x, \xi, \alpha) v_{j}(\theta, x, \xi)$

where $e_{j k}$ are smooth functions which satisfy 
(ii) $\left|\partial_{\theta}^{\ell} \partial_{x}^{A} e_{j k}(\theta, x, \xi, \alpha)\right| \leqslant \frac{C_{A}}{\langle\theta\rangle}$ for all $\alpha \in \mathbb{N}$ and $\ell=0,1$.

Moreover we have for $(\theta, x)$ in $\Omega_{\delta}$ and $|\xi-\xi(\theta, \alpha)|<\sqrt{\delta}$,

(iii) $\left|\Phi_{k}(\theta, x, \alpha)-\alpha_{\xi}\right| \leqslant C_{0}(\varepsilon+\sqrt{\delta})$.

(iv) $\left|\operatorname{Im} \Phi_{k}(\theta, x, \alpha)-\frac{x_{k}-x_{k}(\theta, \alpha)}{1+4 \theta^{2}}\right| \leqslant \sqrt{\delta} \frac{|x-x(\theta, \alpha)|}{\langle\theta\rangle^{2}}$.

(v) $|\Phi(\theta, x, \alpha)| \leqslant C_{0}$,

$\left|\partial_{x}^{A} \Phi(\theta, x, \alpha)\right| \leqslant C_{A}\left(\frac{1}{\langle\theta\rangle^{|A|+1}}+\frac{1}{\langle x\rangle^{|A|+1+\sigma_{0}}}\right)$ if $A \in \mathbb{N}^{n},|A| \geqslant 1$.

(vi) $\Phi_{k}(\theta, x(\theta, \alpha), \alpha)=\xi_{k}(\theta, \alpha)$.

(vii) $\left|\left(\frac{\partial \Phi_{k}}{\partial x_{j}}-\frac{\partial \Phi_{j}}{\partial x_{k}}\right)(\theta, x, \alpha)\right| \leqslant C_{N}\left(\frac{1}{\langle x\rangle^{3 / 2}}+\frac{1}{\langle\theta\rangle^{3 / 2}}\right) \frac{|x-x(\theta, \alpha)|^{N}}{\langle\theta\rangle^{2 N}}, \quad N \in \mathbb{N}$

where the constants $C_{A}, C_{0}, C_{N}$ are independent of $(\theta, x, \xi, \alpha)$.

Proof. - (i) follows immediately from the computations made in Example 4.4.6 as well as (ii). The point (iii) is obvious since $\xi(\theta, \alpha)=\alpha_{\xi}+O(\varepsilon)$ and $\left|a_{k}\right|+\left|b_{k}\right|=\mathcal{O}(\sqrt{\delta})$ by (4.4.4). Then (iv) follows from Theorem 4.4.2 (ii) as well as (v). The point (vi) is obvious since $a_{k}=b_{k}=0$ when $y=0$. To prove (vii) we use Corollary 4.4.9, (4.4.35), (4.4.39) and (iv) of Theorem 4.4.10. We obtain

$$
\left|\left(\frac{\partial \Phi_{k}}{\partial x_{j}}-\frac{\partial \Phi_{j}}{\partial x_{k}}\right)(\theta, x, \alpha)\right| \leqslant C_{N} \frac{|x-x(\theta, \alpha)|^{N}}{\langle\theta\rangle^{2 N}} .
$$

Now by (v) of the present theorem we have,

$$
\left|\partial_{j} \Phi(\theta, x, \alpha)\right| \leqslant C\left(\frac{1}{\langle\theta\rangle^{2}}+\frac{1}{\langle x\rangle^{2}}\right) .
$$

Writing $|a|=|a|^{3 / 4}|a|^{1 / 4}$ and using the above estimates we obtain (vii).

Definition 4.4.11. - Let $(F(\cdot, \alpha))_{\alpha \in \widetilde{\Lambda}}$ be a family of $C^{\infty}$ functions for $(\theta, x)$ in $\Omega_{\delta}$ and $|\xi-\xi(\theta, \alpha)|<\sqrt{\delta}$. We shall say that $F \in \mathcal{J}_{(x, \xi)}$ if we can write

$$
F(\theta, x, \xi, \alpha)=\sum_{j=1}^{n} f_{j}(\theta, x, \xi, \alpha) \frac{1}{\langle\theta\rangle} v_{j}(\theta, x, \xi, \alpha)
$$

where

$$
\left|\partial_{x}^{A} \partial_{\xi}^{B} f_{j}(\theta, x, \xi, \alpha)\right| \leqslant C_{A B}
$$

for all $(\theta, x)$ in $\Omega_{\delta},|\xi-\xi(\theta, \alpha)|<\sqrt{\delta}, \alpha \in \widetilde{\Lambda}$ where $C_{A B}$ is independent of $(\theta, x, \xi, \alpha)$.

Then exactly as in Lemma 4.4.7 $\mathcal{J}_{(x, \xi)}$ is closed under the Poisson bracket in $(x, \xi)$ and we have the analogue of Lemma 4.4.8. In fact $\mathcal{J}_{(x, \xi)}$ is just the image of $\mathcal{J}$ under the diffeomorphism $x=y+x(\theta, \alpha), \xi=\eta+\xi(\theta, \alpha)$. Then we have

TheOrem 4.4.12. - With $\Phi$ defined in (4.4.39) we have for $k=1, \ldots, n$,

$$
\left(-\frac{\partial p}{\partial x_{k}}(x, \Phi(\theta, x, \alpha))-\frac{\partial \Phi_{k}}{\partial \theta}(\theta, x, \alpha)-\frac{\partial \Phi_{k}}{\partial x}(\theta, x, \alpha) \frac{\partial p}{\partial \xi}(x, \Phi(\theta, x, \alpha))\right)_{\alpha} \in \mathcal{J}_{(x, \xi)} .
$$


Proof. - We follow word by word the proof of Lemma 4.3.16 and Corollary 4.3.17. Let us just sketch the proof.

We begin by the proof of the theorem when $\Phi(\theta, x, \alpha)$ is replaced by $\xi$. By Theorem 4.4.10 (i) we have

$$
\xi_{k}-\Phi_{k}(\theta, x, \alpha)=\sum e_{j k}(\theta, x, \xi, \alpha) v_{j}(\theta, x, \xi) .
$$

Then we set $x(-\theta, x, \xi)=X, \xi(-\theta, x, \xi)=\Xi$ that is $x(\theta, X, \Xi)=x, \xi(\theta, X, \Xi)=\xi$. The above identity reads

$$
\xi_{k}(\theta, X, \Xi)-\Phi_{k}(\theta, x(\theta, X, \Xi), \alpha)=\sum_{k=1}^{n} e_{j k}(\theta, x(\theta, X, \Xi), \xi(\theta, X, \Xi), \alpha) u_{j}(X, \Xi) .
$$

We differentiate this equality with respect to $\theta$ using the equations of the flow given by (3.1.2). Then we use Theorem 4.4.10 (ii) and we come back to the original coordinates $(x, \xi)$. Finally we write $\xi=\xi-\Phi(\theta, x, \alpha)+\Phi(\theta, x, \alpha)$ as in the proof of Corollary 4.3.17. Details are left to the reader.

Corollary 4.4.13. - For every $N \in \mathbb{N}$ one can find $C_{N}>0$ such that

$$
\begin{aligned}
\mid-\frac{\partial p}{\partial x_{k}}(x, \Phi(\theta, x, \alpha))-\frac{\partial \Phi_{k}}{\partial \theta}(\theta, x, \alpha)-\frac{\partial \Phi_{k}}{\partial x}(\theta, x, \alpha) \frac{\partial p}{\partial \xi} & (x, \Phi(\theta, x, \alpha)) \mid \\
& \leqslant C_{N}\left(\frac{|x-x(\theta, \alpha)|}{\langle\theta\rangle^{2}}\right)^{N} .
\end{aligned}
$$

Proof. - Use Theorem 4.4.12 and Lemma 4.4.8 in the coordinates $(x, \xi)$.

We are ready now to define the phase $\varphi$, as we did in Proposition 4.3.19 for the outgoing points, but we find here a slight problem. Indeed if we look to formula (4.3.48) we see that $\varphi$ is defined by mean of $\Phi(\theta, s x+(1-s) x(\theta, \alpha)), s \in[0,1]$. In the present case when $\theta \geqslant 0, \Phi(\theta, z, \alpha)$ is defined for $z \cdot \alpha_{\xi} \leqslant c_{0}\langle z\rangle\left|\alpha_{\xi}\right|$ and $|z-x(\theta, \alpha)| \leqslant \delta\langle\theta\rangle$ and it is easily seen that the point $z=s x+(1-s) x(\theta, \alpha)$ does not satisfy these conditions. Therefore we have to modify the expression of $\varphi$ in (4.3.48) to take care of this problem. We split the discussion into several cases giving in each of them a different expression of $\varphi$. Our purpose is to prove the following result.

Let us set,

$$
\mathcal{O}_{\delta}=\left\{(\theta, x) \in \mathbb{R}_{+} \times \mathbb{R}^{n}: x \cdot \alpha_{\xi} \leqslant \frac{c_{0}}{10}\langle x\rangle\left|\alpha_{\xi}\right|,|x-x(\theta, \alpha)| \leqslant \frac{\delta}{10}\langle\theta\rangle\right\} .
$$

Proposition 4.4.14. - There exists a smooth function $\varphi=\varphi(\theta, x, \alpha)$ defined on $\mathcal{O}_{\delta}$ such that,

(i) $\varphi(0, x, \alpha)=\left(x-\alpha_{x}\right) \alpha_{\xi}+\frac{i}{2}\left(x-\alpha_{x}\right)^{2}+\frac{1}{2 i} \alpha_{\xi}^{2}+\mathcal{O}\left(\left|x-\alpha_{x}\right|^{N}\right), \forall N \in \mathbb{N}$.

For every $N \in \mathbb{N}$ there exists $C_{N}>0$ such that

(ii) $\left|\frac{\partial \varphi}{\partial x}(\theta, x, \alpha)-\Phi(\theta, x, \alpha)\right| \leqslant C_{N}\left(\frac{|x-x(\theta, \alpha)|}{\langle\theta\rangle}\right)^{N}$ 
(iii) $\left|\frac{\partial \varphi}{\partial \theta}(\theta, x, \alpha)+p\left(x, \frac{\partial \varphi}{\partial x}(\theta, x, \alpha)\right)\right| \leqslant C_{N}\left(\frac{|x-x(\theta, \alpha)|}{\langle\theta\rangle}\right)^{N}$ uniformly in $(\theta, x, \alpha)$.

Moreover

(iv) $\left|\frac{\partial \varphi}{\partial x}(\theta, x, \alpha)-\alpha_{\xi}\right| \leqslant C(\varepsilon+\sqrt{\delta})$.

(v) $\left|\partial_{x}^{A} \varphi(\theta, x, \alpha)\right| \leqslant C_{A}, \forall A \in \mathbb{N}^{n},|A| \geqslant 1$.

(vi) $\left|\operatorname{Im} \varphi(\theta, x, \alpha)-\frac{1}{2} \frac{|x-x(\theta, \alpha)|^{2}}{1+4 \theta^{2}}+\frac{1}{2} \alpha_{\xi}^{2}\right| \leqslant C(\varepsilon+\sqrt{\delta}) \frac{|x-x(\theta, \alpha)|^{2}}{\langle\theta\rangle^{2}}$.

We split the proof into several cases which are summarized in the following figures. Case 1 (see Figure 1). - Let $(\theta, x) \in \mathcal{O}_{\delta}$ be such that

$$
x(\theta, \alpha) \cdot \alpha_{\xi} \leqslant \frac{c_{0}}{3}\langle x(\theta, \alpha)\rangle\left|\alpha_{\xi}\right| \text { and }|x-x(\theta, \alpha)| \leqslant\langle x(\theta, \alpha)\rangle .
$$

Lemma 4.4.15. - In the case 1 we have $s x+(1-s) x(\theta, \alpha) \in \Omega_{\delta}$, for $s \in[0,1]$, that is

$$
(s x+(1-s) x(\theta, \alpha)) \cdot \alpha_{\xi} \leqslant c_{0}\langle s x+(1-s) x(\theta, \alpha)\rangle\left|\alpha_{\xi}\right|
$$

and

$$
|s x+(1-s) x(\theta, \alpha)-x(\theta, \alpha)| \leqslant \delta\langle\theta\rangle .
$$

Proof. - We use the following elementary lemma.

Lemma 4.4.16. - Let $a, b \in \mathbb{R}^{n}$ be such that $|a-b| \leqslant\langle a\rangle$. Then for all $s$ in $[0,1]$

$$
(1-s)|a|+s|b| \leqslant \sqrt{2}\langle(1-s) a+s b\rangle .
$$

Proof. - Since $|a-b|^{2} \leqslant|a|^{2}+1$ we have $2 a \cdot b \geqslant-1$, it follows that

$$
\begin{aligned}
|(1-s) a+s b|^{2}=(1-s)^{2}|a|^{2} & +2 s(1-s) a \cdot b+s^{2}|b|^{2} \geqslant(1-s)^{2}|a|^{2} \\
& \left.+s^{2}|b|^{2}-s(1-s) \geqslant \frac{1}{2}((1-s)|a|+s|b|)\right)^{2}-\frac{1}{2} .
\end{aligned}
$$

Therefore $\langle(1-s) a+s b\rangle^{2} \geqslant \frac{1}{2}((1-s)|a|+s|b|)^{2}$.

Let us now apply Lemma 4.4.16 to $a=x(\theta, \alpha), b=x$. Using our hypotheses we obtain

$$
\begin{aligned}
(s x+(1-s) x(\theta, \alpha)) \cdot \alpha_{\xi} & \leqslant \frac{c_{0}}{3}(s\langle x\rangle+(1-s)\langle x(\theta, \alpha)\rangle)\left|\alpha_{\xi}\right| \\
& \leqslant \frac{c_{0}}{3}(s+s|x|+(1-s)+(1-s)|x(\theta, \alpha)|)\left|\alpha_{\xi}\right| \\
& \leqslant \frac{c_{0}}{3}(1+\sqrt{2}\langle s x+(1-s) x(\theta, \alpha)\rangle) \cdot\left|\alpha_{\xi}\right| \\
& \leqslant c_{0}\langle s x+(1-s) x(\theta, \alpha)\rangle\left|\alpha_{\xi}\right|,
\end{aligned}
$$

On the other hand $|s x+(1-s) x(\theta, \alpha)-x(\theta, \alpha)|=s|x-x(\theta, \alpha)| \leqslant \delta\langle\theta\rangle$. 


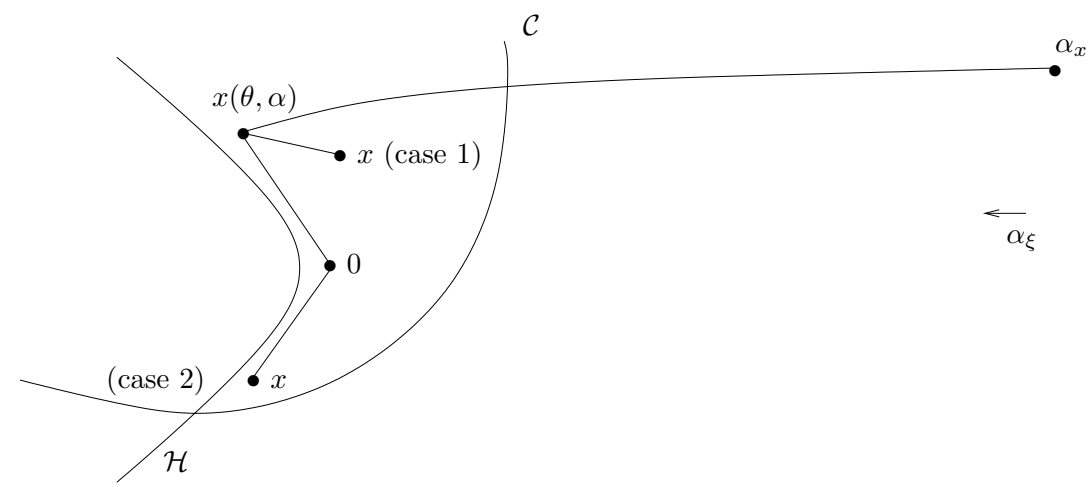

Figure 1. $\mathcal{C}=\{y,|y-x(\theta, \alpha)|=\delta\langle\theta\rangle\}$ et $\mathcal{H}=\left\{y, y \cdot \alpha_{\xi}=c_{0}\langle y\rangle\left|\alpha_{\xi}\right|\right\}$

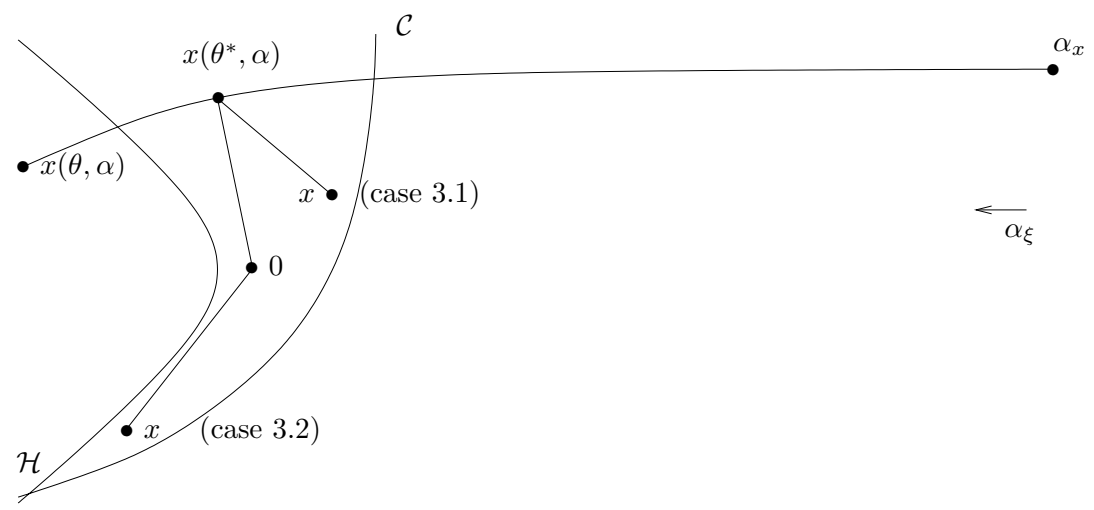

Figure 2. $\mathcal{C}=\{y,|y-x(\theta, \alpha)|=\delta\langle\theta\rangle\}$ et $\mathcal{H}=\left\{y, y . \alpha_{\xi}=c_{0}\langle y\rangle\left|\alpha_{\xi}\right|\right\}$

In the set defined in case 1 we can therefore define $\varphi$ by the same formula as in Proposition 4.3.19. We set

(4.4.41) $\varphi(\theta, x, \alpha)=\int_{0}^{1}(x-x(\theta, \alpha)) \cdot \Phi(\theta, s x+(1-s) x(\theta, \alpha), \alpha) d s+\theta p(\alpha)+\frac{1}{2 i} \alpha_{\xi}^{2}$.

The proof of the points (i) to (vi) is exactly the same as the corresponding points in Proposition 4.3.19 using Theorem 4.4.10.

Case 2. - Let $(\theta, x) \in \mathcal{O}_{\delta}$ be such that

$$
x(\theta, \alpha) \cdot \alpha_{\xi} \leqslant \frac{c_{0}}{3}\langle x(\theta, \alpha)\rangle\left|\alpha_{\xi}\right| \text { and }|x-x(\theta, \alpha)| \geqslant \frac{1}{2}|x(\theta, \alpha)| .
$$

(See Figure 1). 
In this set we have

$$
\left\{\begin{array}{l}
|x(\theta, \alpha)| \leqslant 2|x-x(\theta, \alpha)| \leqslant \delta\langle\theta\rangle \\
|x| \leqslant 3|x-x(\theta, \alpha)| \leqslant \delta\langle\theta\rangle .
\end{array}\right.
$$

Moreover for $y \in[0, x] \cup[0, x(\theta, \alpha)]$ the point $(\theta, y)$ belongs to the set $\Omega_{\delta}$ on which $\Phi$ is defined. Indeed if $s \in[0,1]$ we have $s x \cdot \alpha_{\xi} \leqslant s \frac{c_{0}}{2}\langle x\rangle\left|\alpha_{\xi}\right|$ and $|s x-x(\theta, \alpha)| \leqslant$ $s|x-x(\theta, \alpha)|+(1-s)|x(\theta, \alpha)| \leqslant 2|x-x(\theta, \alpha)| \leqslant \delta\langle\theta\rangle$ by (4.4.42). On the other hand, $s x(\theta, \alpha) \cdot \alpha_{\xi} \leqslant s \frac{c_{0}}{3}\langle x(\theta, \alpha)\rangle\left|\alpha_{\xi}\right| \leqslant c_{0}\langle x(\theta, \alpha)\rangle\left|\alpha_{\xi}\right|$ and $|s x(\theta, \alpha)-x(\theta, \alpha)|=$ $(1-s)|x(\theta, \alpha)| \leqslant \delta\langle\theta\rangle$. Therefore we can define the phase $\varphi$ by the following formula.

$$
\begin{aligned}
& \varphi(\theta, x, \alpha) \\
= & \int_{0}^{1} x \cdot \Phi(\theta, s x, \alpha) d s-\int_{0}^{1} x(\theta, \alpha) \cdot \Phi(\theta, s x(\theta, \alpha), \alpha) d s+\theta p(\alpha)+\frac{1}{2 i} \alpha_{\xi}^{2} .
\end{aligned}
$$

Let us show that $\varphi$ satisfies the conditions of Proposition 4.4.14. It follows from Theorem 4.4.2 and (4.4.39) that

$$
\Phi(0, z, \alpha)=\alpha_{\xi}+i\left(z-\alpha_{x}\right)+\mathcal{O}\left(\left|z-\alpha_{x}\right|^{N}\right) .
$$

Therefore

$$
\begin{aligned}
\varphi(0, x, \alpha)= & \int_{0}^{1}\left(x \cdot \alpha_{\xi}+i x\left(s x-\alpha_{x}\right)-\alpha_{x} \alpha_{\xi}-\right. \\
+ & \left.+\left|\alpha_{x}\right| \mathcal{O}\left(\left|s x-\alpha_{x}\right|^{N}\right)\right) d s+\frac{1}{2 i} \alpha_{\xi}^{2} \\
= & \left(x-\alpha_{x}\right) \cdot \alpha_{\xi}+\frac{i}{2}\left(x-\alpha_{x}\right)^{2}+\frac{1}{2 i} \alpha_{\xi}^{2}+\mathcal{O}\left(\left|x-\alpha_{x}\right|^{N}\right)
\end{aligned}
$$

because $|x| \leqslant\left|x-\alpha_{x}\right|+\left|\alpha_{x}\right|,\left|s x-\alpha_{x}\right| \leqslant\left|x-\alpha_{x}\right|$ and $\left|\alpha_{x}\right| \leqslant\left|x-\alpha_{x}\right|$. Thus (i) is proved. Now

$$
\frac{\partial \varphi}{\partial x_{j}}(\theta, x, \alpha)=\int_{0}^{1} \Phi_{j}(\theta, s x, \alpha) d s+\sum_{k=1}^{n} \int_{0}^{1} s x_{k} \frac{\partial \Phi_{k}}{\partial x_{j}}(\theta, s x, \alpha) d s .
$$

Using Theorem 4.4.10, (vii) we obtain

$$
\begin{aligned}
& \mid \frac{\partial \varphi}{\partial x_{j}}(\theta, x, \alpha)-\int_{0}^{1} \Phi_{j}(\theta, s x, \alpha) d s-\int_{0}^{1} s \frac{d}{d s}\left(\Phi_{j}(\theta, s x, \alpha)\right) d s \mid \\
& \leqslant C_{N} \int_{0}^{1} s|x|\left(\frac{1}{\langle s x\rangle^{3 / 2}}+\frac{1}{\langle\theta\rangle^{3 / 2}}\right) \frac{|s x-x(\theta, \alpha)|^{N}}{\langle\theta\rangle^{2 N}} d s .
\end{aligned}
$$

Now $s|x| \leqslant\langle s x\rangle^{3 / 2}$ and by (4.4.42), $s|x| \leqslant \delta\langle\theta\rangle,|s x-x(\theta, \alpha)| \leqslant s|x-x(\theta, \alpha)|+$ $(1-s)|x(\theta, \alpha)| \leqslant 2|x-x(\theta, \alpha)|$. Therefore the right hand side of the above inequality is bounded by $C_{N}|x-x(\theta, \alpha)|^{N} /\langle\theta\rangle^{2 N}$. Integrating by parts in the second integral of the left hand side we obtain (ii). 
Now we have

$$
\begin{aligned}
\frac{\partial \varphi}{\partial \theta}=\underbrace{\int_{0}^{1} x \frac{\partial \Phi}{\partial \theta}(\theta, s x, \alpha) d s}_{(1)} & -\underbrace{\int_{0}^{1} \dot{x}(\theta, \alpha) \cdot \Phi(\theta, s x(\theta, \alpha), \alpha) d s}_{(2)} \\
& -\underbrace{\int_{0}^{1} x(\theta, \alpha) \cdot \frac{\partial \Phi}{\partial \theta}(\theta, s x(\theta, \alpha), \alpha) d s}_{(3)} \\
& -\sum_{k, \ell=1}^{\int_{0}^{n} x_{k}^{1}(\theta, \alpha) \frac{\partial \Phi_{k}}{\partial x_{\ell}}(\theta, s x(\theta, \alpha), \alpha) s \dot{x}_{\ell}(\theta, \alpha) d s}+p(\alpha) .
\end{aligned}
$$

We use Corollary 4.4.13 to write

$$
\begin{aligned}
(1)=\sum_{k=1}^{n} \int_{0}^{1} x_{k} \frac{\partial p}{\partial x_{k}} & (s x, \Phi(\theta, s x, \alpha)) d s \\
& \quad-\sum_{k, \ell=1}^{n} \int_{0}^{1} x_{k} \frac{\partial \Phi_{k}}{\partial x_{\ell}}(\theta, s x, \alpha) \cdot \frac{\partial p}{\partial \xi_{\ell}}(s x, \Phi(\theta, s x, \alpha)) d s+R_{0}
\end{aligned}
$$

with

$$
\left|R_{0}\right| \leqslant \int_{0}^{1}|x| \frac{|s x-x(\theta, \alpha)|^{N}}{\langle\theta\rangle^{2 N}} .
$$

By (4.4.42) we have $|x| \leqslant \delta\langle\theta\rangle$; since $\langle\theta\rangle^{2 N-1} \geqslant\langle\theta\rangle^{N}$ if $N \geqslant 1$, and

$$
|s x-x(\theta, \alpha)| \leqslant s|x-x(\theta, \alpha)|+(1-s)|x(\theta, \alpha)| \leqslant 2|x-x(\theta, \alpha)|,
$$

we obtain $\left|R_{0}\right| \leqslant C_{N}|x-x(\theta, \alpha)|^{N} /\langle\theta\rangle^{N}$ if $N \geqslant 1$. Now $|x-x(\theta, \alpha)| \leqslant \delta\langle\theta\rangle$ so the same estimate is valid for $N=0$. Finally

$$
\left|R_{0}\right| \leqslant C_{N} \frac{|x-x(\theta, \alpha)|^{N}}{\langle\theta\rangle^{N}}, \quad \forall N \geqslant 0 .
$$

Using Theorem 4.4 .10 (vii) we obtain

$$
(1)=-\int_{0}^{1} \frac{d}{d s}(p(s x, \Phi(\theta, s x, \alpha))) d s+R_{1}
$$

where $R$ satisfies (4.4.44). Therefore

$$
\mid(1)+p\left(\Phi(\theta, x, \alpha)-p(0, \Phi(\theta, 0, \alpha)) \mid \leqslant C_{N} \frac{|x-x(\theta, \alpha)|^{N}}{\langle\theta\rangle^{N}} .\right.
$$

Let us look the term (4); we use Theorem 4.4 .10 (vii) again and we obtain

$$
(4)=\sum_{\ell=1}^{n} \int_{0}^{1} s \dot{x}_{\ell}(\theta, \alpha) \frac{d}{d s}\left(\Phi_{\ell}(\theta, s x(\theta, \alpha), \alpha)\right) d s+R_{2},
$$


with

$$
\left|R_{2}\right| \leqslant \int_{0}^{1} s|x(\theta, \alpha)|\left(\frac{1}{\langle s x(\theta, \alpha)\rangle^{3 / 2}}+\frac{1}{\langle\theta\rangle^{3 / 2}}\right)\left(\frac{|x(\theta, \alpha)|^{N}}{\langle\theta\rangle^{2 N}}\right)(s-1)^{N} d s .
$$

It follows from (4.4.42) that $R_{2}$ satisfies (4.4.44). Therefore integrating by parts in the above integral we obtain

$$
\left.(4)=-\int_{0}^{1} \dot{x}(\theta, \alpha) \cdot \Phi(\theta, s x(\theta, \alpha), \alpha)\right) d s+\dot{x}(\theta, \alpha) \Phi(\theta, x(\theta, \alpha), \alpha)+\mathcal{O}\left(\frac{|x-x(\theta, \alpha)|^{N}}{\langle\theta\rangle^{N}}\right) .
$$

Using Theorem 4.4.10, (vi) and the Euler identity we can write

$$
\dot{x}(\theta, \alpha) \Phi(\theta, x(\theta, \alpha), \alpha)=\xi(\theta, \alpha) \cdot \frac{\partial p}{\partial \xi}(x(\theta, \alpha), \xi(\theta, \alpha))=2 p(\alpha) .
$$

It follows that

$$
|(2)+(4)-2 p(\alpha)| \leqslant C_{N} \frac{|x-x(\theta, \alpha)|^{N}}{\langle\theta\rangle^{N}} .
$$

Now, by Corollary 4.4.13 we have

$$
\begin{aligned}
(3)= & -\int_{0}^{1} x(\theta, \alpha) \cdot \frac{\partial p}{\partial x}(s x(\theta, \alpha), \Phi(\theta, s x(\theta, \alpha), \alpha)) d s \\
& -\sum_{\ell, k=1}^{n} \int_{0}^{1} x_{k}(\theta, \alpha) \cdot \frac{\partial \Phi_{k}}{\partial x_{\ell}}(\theta, s x(\theta, \alpha), \alpha) \frac{\partial p}{\partial \xi_{\ell}}\left(s x(\theta, \alpha), \Phi(\theta, s x(\theta, \alpha), \alpha) d s+R_{3}\right.
\end{aligned}
$$

where

$$
\left|R_{3}\right| \leqslant C_{N}|x(\theta, \alpha)| \int_{0}^{1} \frac{|x(\theta, \alpha)|^{N}|s-1|^{N}}{\langle\theta\rangle^{2 N}} d s .
$$

If $N \geqslant 1$ we have $\frac{|x(\theta, \alpha)|}{\langle\theta\rangle^{2 N}} \leqslant \frac{1}{\langle\theta\rangle^{N}}$ so $R_{3}$ satisfies (4.4.44) using (4.4.42).

Using again Theorem 4.4 .10 (vii) we obtain

$$
(3)=-\int_{0}^{1} \frac{d}{d s}\left[p(s x(\theta, \alpha), \Phi(\theta, s x(\theta, \alpha), \alpha)] d s+\mathcal{O}\left(\frac{|x-x(\theta, \alpha)|^{N}}{\langle\theta\rangle^{N}}\right)\right.
$$

So

$$
\mid(3)+p\left(x(\theta, \alpha), \Phi(\theta, x(\theta, \alpha), \alpha)-p\left(0, \Phi(\theta, 0, \alpha) \mid \leqslant C_{N} \frac{|x-x(\theta, \alpha)|^{N}}{\langle\theta\rangle^{N}} .\right.\right.
$$

Finally we obtain

$$
|(3)+p(\alpha)-p(0, \Phi(\theta, 0, \alpha))| \leqslant C_{N} \frac{|x-x(\theta, \alpha)|^{N}}{\langle\theta\rangle^{N}} .
$$

Since $\frac{\partial \varphi}{\partial \theta}(\theta, x, \alpha)=(1)-(2)-(3)-(4)+p(\alpha)$ we deduce from (4.4.45) to (4.4.47) that

$$
\left|\frac{\partial \varphi}{\partial \theta}(\theta, x, \alpha)+p(x, \Phi(\theta, x, \alpha))\right| \leqslant C_{N} \frac{|x-x(\theta, \alpha)|^{N}}{\langle\theta\rangle^{N}} .
$$

Using the point (ii) already proved in Proposition 4.4.14 we obtain the point (iii).

The point (iv) follows easily from (ii) since by (4.4.39) and Theorem 4.4.10 we have $\Phi(\theta, x, \alpha)=\alpha_{\xi}+\mathcal{O}(\varepsilon+\sqrt{\delta})$. 
Let us prove (v). To bound $\partial_{x}^{A} \varphi$ when $|A| \geqslant 1$ we have to bound, according to (4.4.43) the quantities (1) $=\int_{0}^{1} s^{\left|A^{\prime}\right|}\left(\partial_{x}^{A^{\prime}} \Phi\right)(\theta, s x, \alpha) d s,\left|A^{\prime}\right| \leqslant|A|-1$ and (2) $=\int_{0}^{1}|x|\left|\partial_{x}^{A} \Phi(\theta, s x, \alpha)\right| d s$. Using Theorem 4.4.10, (v), we see easily that (1) is uniformly bounded and

$$
|(2)| \leqslant C_{A}|x| \frac{1}{\langle\theta\rangle^{|A|}}+C_{A}|x| \int_{0}^{1} \frac{d s}{\langle s x\rangle^{|A|+1+\sigma_{0}}} .
$$

By (4.4.42) we have $|x| \leqslant 2 \delta\langle\theta\rangle \leqslant 2 \delta\langle\theta\rangle^{|A|}$ since $|A| \geqslant 1$ and setting $t=|x| s$ in the integral above we see that (2) is uniformly bounded in $(\theta, x, \alpha)$. This shows $(\mathrm{v})$. Finally by (4.4.43),

$$
\operatorname{Im} \varphi(\theta, x, \alpha)=\int_{0}^{1} x \cdot \operatorname{Im} \Phi(\theta, s x, \alpha) d s-\int_{0}^{1} x(\theta, \alpha) \cdot \operatorname{Im} \Phi(\theta, s x(\theta, \alpha), \alpha) d s-\frac{1}{2} \alpha_{\xi}^{2} .
$$

Using Theorem 4.4.10, (iv) and (4.4.42) we obtain (vi). This completes the proof of Proposition 4.4.14 in the case 2.

Case 3. - We consider here the case where

$$
(\theta, x) \in \mathcal{O}_{\delta} \text { and } x(\theta, \alpha) \cdot \alpha_{\xi}>\frac{c_{0}}{3}\langle x(\theta, \alpha)\rangle\left|\alpha_{\xi}\right| \cdot
$$

(See Figure 2).

Let us recall that we are dealing in this Section 4.4 with the case where $\alpha_{x} \cdot \alpha_{\xi} \leqslant-c_{0}\left\langle\alpha_{x}\right\rangle\left|\alpha_{\xi}\right|$, (see (4.4.1)).

(1) The continuous function $t \mapsto x(t, \alpha) \cdot \alpha_{\xi}$ is then strictly negative for $t=0$ and strictly positive for $t=\theta$. It follows that

(4.4.49) there exists $\left.\theta^{*} \in\right] 0, \theta\left[\right.$ depending only on $\alpha$ such that $x\left(\theta^{*}, \alpha\right) \cdot \alpha_{\xi}=0$.

Then we have the following Lemma.

LEMMA 4.4.17

(i) $\frac{3}{2}\left|\theta-\theta^{*}\right|\left|\alpha_{\xi}\right| \leqslant\left|x(\theta, \alpha)-x\left(\theta^{*}, \alpha\right)\right| \leqslant 3\left|\theta-\theta^{*}\right|\left|\alpha_{\xi}\right|$,

(ii) $\left|\theta-\theta^{*}\right| \geqslant \frac{c_{0}}{50}$,

(iii) $|x-x(\theta, \alpha)| \leqslant\left|x-x\left(\theta^{*}, \alpha\right)\right|+\left|x\left(\theta^{*}, \alpha\right)-x(\theta, \alpha)\right| \leqslant 5|x-x(\theta, \alpha)|$,

(iv) $K_{1}\langle\theta\rangle \leqslant\left\langle\theta^{*}\right\rangle \leqslant K_{2}\langle\theta\rangle$.

Proof. - It follows from (4.4.48) and Definition 3.2.2 that the point

$$
\rho^{*}=\left(x\left(\theta^{*}, \alpha\right), \xi\left(\theta^{*}, \alpha\right)\right)
$$

belongs to $\mathcal{S}_{+} \cap \mathcal{S}_{-}$. By the group property and Proposition 3.3.1 we have

$$
x(\theta, \alpha)=x\left(\theta-\theta^{*}, \rho^{*}\right)=x\left(\theta^{*}, \alpha\right)+2\left(\theta-\theta^{*}\right) \alpha_{\xi}+\mathcal{O}\left(\varepsilon\left|\theta-\theta^{*}\right|\right)+\mathcal{O}(\varepsilon) .
$$

It follows that

$$
x(\theta, \alpha)-x\left(\theta^{*}, \alpha\right)=2\left(\theta-\theta^{*}\right) \alpha_{\xi}+\mathcal{O}\left(\varepsilon\left|\theta-\theta^{*}\right|\right)+\mathcal{O}(\varepsilon) .
$$


Now we deduce from (4.4.48) and (4.4.49) that

$$
\left(x(\theta, \alpha)-x\left(\theta^{*}, \alpha\right)\right) \cdot \alpha_{\xi}>\frac{c_{0}}{3}\langle x(\theta, \alpha)\rangle\left|\alpha_{\xi}\right| \geqslant \frac{c_{0}}{3}\left|\alpha_{\xi}\right| \geqslant \frac{c_{0}}{6}
$$

since $\frac{1}{2} \leqslant\left|\alpha_{\xi}\right| \leqslant 2$. So by (4.4.50),

$$
2\left(\theta-\theta^{*}\right)\left|\alpha_{\xi}\right|^{2} \geqslant \frac{c_{0}}{6}-C_{1} \varepsilon\left|\theta-\theta^{*}\right|-C_{2} \varepsilon .
$$

Taking $\varepsilon$ small compared to $c_{0}$ and $C_{1}$ we obtain (ii). Then (i) follows easily from (4.4.50) if $\varepsilon \ll c_{0}$. Now the first inequality in (iii) being obvious, let us prove the second one. We write

$$
\left\{\begin{array}{l}
|x-x(\theta, \alpha)|^{2}=(1)+(2) \\
(1)=\left|x-x\left(\theta^{*}, \alpha\right)\right|^{2}+\left|x\left(\theta^{*}, \alpha\right)-x(\theta, \alpha)\right|^{2} \\
(2)=2\left(x-x\left(\theta^{*}, \alpha\right)\right)\left(x\left(\theta^{*}, \alpha\right)-x(\theta, \alpha)\right) .
\end{array}\right.
$$

If we use (4.4.50), (i) and (ii) we obtain,

$$
(2)=-4\left(\theta-\theta^{*}\right)\left(x-x\left(\theta^{*}, \alpha\right)\right) \cdot \alpha_{\xi}+\mathcal{O}(\varepsilon(1)),
$$

so by (4.4.49),

$$
(2)=-4\left(\theta-\theta^{*}\right) x \cdot \alpha_{\xi}+\mathcal{O}(\varepsilon(1)) .
$$

Now since $(\theta, x)$ belongs to $\mathcal{O}_{\delta}$ (see (4.4.40)) we have

$$
x \cdot \alpha_{\xi} \leqslant \frac{c_{0}}{10}\langle x\rangle\left|\alpha_{\xi}\right| \leqslant \frac{c_{0}}{10}\langle x(\theta, \alpha)\rangle\left|\alpha_{\xi}\right|+\frac{c_{0}}{10}|x-x(\theta, \alpha)|\left|\alpha_{\xi}\right| .
$$

It follows from (4.4.48) that

$$
x \cdot \alpha_{\xi} \leqslant \frac{3}{10} x(\theta, \alpha) \cdot \alpha_{\xi}+\frac{c_{0}}{10}|x-x(\theta, \alpha)|\left|\alpha_{\xi}\right|,
$$

and we deduce from (4.4.49) that

$$
\begin{aligned}
x \cdot \alpha_{\xi} & \leqslant \frac{3}{10}\left(x(\theta, \alpha)-x\left(\theta^{*}, \alpha\right)\right) \cdot \alpha_{\xi}+\frac{c_{0}}{10}|x-x(\theta, \alpha)|\left|\alpha_{\xi}\right|, \\
x \cdot \alpha_{\xi} & \leqslant \frac{3}{10}\left|x(\theta, \alpha)-x\left(\theta^{*}, \alpha\right)\right|\left|\alpha_{\xi}\right|+\frac{c_{0}}{10}|x-x(\theta, \alpha)|\left|\alpha_{\xi}\right|, \\
x \cdot \alpha_{\xi} & \leqslant\left(\frac{3}{10}+\frac{c_{0}}{10}\right)\left|x(\theta, \alpha)-x\left(\theta^{*}, \alpha\right)\right|\left|\alpha_{\xi}\right|+\frac{c_{0}}{10}\left|x-x\left(\theta^{*}, \alpha\right)\right|\left|\alpha_{\xi}\right|, \\
(2) & \geqslant-4\left(\frac{3}{10}+\frac{c_{0}}{10}\right)\left|x(\theta, \alpha)-x\left(\theta^{*}, \alpha\right)\right|\left|\theta-\theta^{*}\right|\left|\alpha_{\xi}\right| \\
& -\frac{2 c_{0}}{5}\left|x-x\left(\theta^{*}, \alpha\right)\right|\left|\theta-\theta^{*}\right|\left|\alpha_{\xi}\right|+\mathcal{O}(\varepsilon(1)) .
\end{aligned}
$$

Using the first inequality in (i) we obtain

$(2) \geqslant-\left(\frac{4}{5}+\frac{4 c_{0}}{15}\right)\left|x(\theta, \alpha)-x\left(\theta^{*}, \alpha\right)\right|^{2}-\frac{4 c_{0}}{15}\left|x-x\left(\theta^{*}, \alpha\right)\right|\left|x(\theta, \alpha)-x\left(\theta^{*}, \alpha\right)\right|+\mathcal{O}(\varepsilon(1))$.

Finally

$$
(2) \geqslant-\left(\frac{4}{5}+\frac{12 c_{0}}{15}+C \varepsilon\right)(1)
$$


If $c_{0}$ and $\varepsilon$ are small enough we find (1) $+(2) \geqslant \frac{1}{6}$ (1) so (iii) is proved using (4.4.51). Finally (iv) follows from (i) and (iii) taking $\delta$ small enough.

Then we split the case 3 in two subcases.

Case 3.1. - $(\theta, x) \in \mathcal{O}_{\delta}, x(\theta, \alpha) \cdot \alpha_{\xi}>\frac{c_{0}}{3}\langle x(\theta, \alpha)\rangle\left|\alpha_{\xi}\right|$ and $\left|x-x\left(\theta^{*}, \alpha\right)\right| \leqslant\left\langle x\left(\theta^{*}, \alpha\right)\right\rangle$. It follows then that

$$
s x+(1-s) x\left(\theta^{*}, \alpha\right) \in \Omega_{\delta} \text { for all } s \in[0,1] .
$$

Indeed, using Lemma 4.4.16 with $a=x\left(\theta^{*}, \alpha\right), b=x$ we obtain

$$
s|x| \leqslant \sqrt{2}\left\langle s x+(1-s) x\left(\theta^{*}, \alpha\right)\right\rangle
$$

so if $(\theta, x) \in \mathcal{O}_{\delta}$ we get

$$
\left(s x+(1-s) x\left(\theta^{*}, \alpha\right)\right) \cdot \alpha_{\xi}=s x \cdot \alpha_{\xi} \leqslant \frac{c_{0}}{10} s\langle x\rangle\left|\alpha_{\xi}\right| \leqslant c_{0}\left\langle s x+(1-s) x\left(\theta^{*}, \alpha\right)\right\rangle\left|\alpha_{\xi}\right| .
$$

Moreover by Lemma 4.4.17,

$$
\begin{aligned}
\left|s x+(1-s) x\left(\theta^{*}, \alpha\right)-x(\theta, \alpha)\right| & \leqslant s|x-x(\theta, \alpha)|+(1-s)\left|x\left(\theta^{*}, \alpha\right)-x(\theta, \alpha)\right| \\
& \leqslant s|x-x(\theta, \alpha)|+(1-s) 5|x-x(\theta, \alpha)| \\
& \leqslant 5 \mid x-x((\theta, \alpha) \mid \leqslant \delta\langle\theta\rangle
\end{aligned}
$$

since in $\mathcal{O}_{\delta},|x-x(\theta, \alpha)| \leqslant \frac{\delta}{10}\langle\theta\rangle$.

Therefore we can define $\varphi$ on this part of $\mathcal{O}_{\delta}$ by the following formula.

$$
\begin{aligned}
\varphi(\theta, x, \alpha)=\int_{0}^{1} & \left(x-x\left(\theta^{*}, \alpha\right)\right) \cdot \Phi\left(\theta, s x+(1-s) x\left(\theta^{*}, \alpha\right), \alpha\right) d s \\
& -\int_{\theta^{*}}^{\theta} p\left(x\left(\theta^{*}, \alpha\right), \Phi\left(s, x\left(\theta^{*}, \alpha\right), \alpha\right) d s+\theta^{*} p(\alpha)+\frac{1}{2 i}\left|\alpha_{\xi}\right|^{2} .\right.
\end{aligned}
$$

Our goal now is to show that $\varphi$ satisfies the claims (i) to (vi) of Proposition 4.4.14.

The point $\theta=0$ does not belong, by (4.4.48), to this part of $\mathcal{O}_{\delta}$. Thus the claim (i) is empty. Let us check (ii). We have

$$
\begin{aligned}
\frac{\partial \varphi}{\partial x_{k}}(\theta, x, \alpha)=\int_{0}^{1} & \Phi_{k}\left(\theta, s x+(1-s) x\left(\theta^{*}, \alpha\right), \alpha\right) d s \\
& +\sum_{\ell=1}^{n} \int_{0}^{1} s\left(x_{\ell}-x_{\ell}\left(\theta^{*}, \alpha\right)\right) \frac{\partial \Phi_{\ell}}{\partial x_{k}}\left(\theta, s x+(1-s) x\left(\theta^{*}, \alpha\right), \alpha\right) d s .
\end{aligned}
$$

Using Theorem 4.4.10, (vii) we see easily that

$$
\begin{aligned}
\frac{\partial \varphi}{\partial x_{k}}(\theta, x, \alpha)=\int_{0}^{1} \Phi_{k}\left(\theta, s x+(1-s) x\left(\theta^{*}, \alpha\right), \alpha\right) d s & \\
& +\int_{0}^{1} s \frac{d}{d s}\left[\Phi_{k}\left(\theta, s x+(1-s) x\left(\theta^{*}, \alpha\right), \alpha\right)\right] d s+R
\end{aligned}
$$


with

$$
|R| \leqslant C_{N}\left|x-x\left(\theta^{*}, \alpha\right)\right| \int_{0}^{1} \frac{\left|s x+(1-s) x\left(\theta^{*}, \alpha\right)-x(\theta, \alpha)\right|^{N}}{\langle\theta\rangle^{2 N}} d s .
$$

It follows from Lemma 4.4.17, (iii) that

$$
|R| \leqslant C_{N} \frac{|x-x(\theta, \alpha)|^{N}}{\langle\theta\rangle^{N}}, \quad N \geqslant 0 .
$$

Integrating by parts in the second integral of the right hand side of (4.4.54) we obtain the claim (ii). Let us prove (iii). We have

$$
\frac{\partial \varphi}{\partial \theta}(\theta, x, \alpha)=\int_{0}^{1}\left(x-x\left(\theta^{*}, \alpha\right)\right) \cdot \frac{\partial \Phi}{\partial \theta}\left(\theta, X_{s}, \alpha\right) d s-p\left(x\left(\theta^{*}, \alpha\right), \Phi\left(\theta, x\left(\theta^{*}, \alpha\right), \alpha\right)\right.
$$

where $X_{s}=s x+(1-s) x\left(\theta^{*}, \alpha\right)$.

Using Corollary 4.4.13 we obtain

$$
\begin{aligned}
\frac{\partial \varphi}{\partial \theta}(\theta, x, \alpha) & =-\int_{0}^{1}\left(x-x\left(\theta^{*}, \alpha\right)\right) \cdot \frac{\partial p}{\partial x}\left(X_{s}, \Phi\left(\theta, X_{s}, \alpha\right)\right) d s \\
-\sum_{k, \ell=1}^{n} \int_{0}^{1}\left(x_{k}-x_{k}\left(\theta^{*}, \alpha\right)\right) \frac{\partial \Phi_{k}}{\partial x_{\ell}}\left(\theta, X_{s}, \alpha\right) \frac{\partial p}{\partial \xi_{\ell}}\left(X_{s}, \Phi\left(\theta, X_{s}, \alpha\right)\right) d s & -p\left(x\left(\theta^{*}, \alpha\right), \Phi\left(\theta, x\left(\theta^{*}, \alpha\right), \alpha\right)\right)+R
\end{aligned}
$$

where $R$ satisfies (4.4.55). We use again Theorem 4.4.10, (vii), and we obtain

$$
\frac{\partial \varphi}{\partial \theta}(\theta, x, \alpha)=-\int_{0}^{1} \frac{d}{d s}\left[p\left(X_{s}, \Phi\left(\theta, X_{s}, \alpha\right)\right)\right] d s-p\left(x\left(\theta^{*}, \alpha\right), \Phi\left(\theta, x\left(\theta^{*}, \alpha\right), \alpha\right)+R^{\prime}\right.
$$

where $R^{\prime}$ satisfies also (4.4.55). This implies immediately (iii).

The points (iv), (v) follow easily from Theorem 4.4.10. Let us check (vi). According to $(4.4 .53)$ we can write

$$
\left\{\begin{array}{l}
\varphi(\theta, x, \alpha)=A+B \text { with } \\
A=\int_{0}^{1}\left(x-x\left(\theta^{*}, \alpha\right)\right) \cdot \Phi\left(\theta, s x+(1-s) x\left(\theta^{*}, \alpha\right), \alpha\right) d s .
\end{array}\right.
$$

Using Theorem 4.4.10, (iv) and Lemma 4.4 .17 (iii) we see that

$$
\left\{\begin{array}{l}
\operatorname{Im} A=\frac{1}{2} \frac{1}{1+4 \theta^{2}}\left[\left(x-x\left(\theta^{*}, \alpha\right)\right)^{2}+2\left(x-x\left(\theta^{*}, \alpha\right)\right)\left(x\left(\theta^{*}, \alpha\right)-x(\theta, \alpha)\right)\right]+R \\
|R| \leqslant C \sqrt{\delta} \frac{|x-x(\theta, \alpha)|^{2}}{\langle\theta\rangle^{2}}
\end{array}\right.
$$

To check the term $B$ let us set $x(t)=x(t, \alpha)$ and

$$
F(t)=-\int_{t}^{\theta} p(x(t), \Phi(s, x(t), \alpha)) d s+t p(\alpha)
$$

MÉMOIRES DE LA SMF 101/102 
Then

$$
\begin{gathered}
F^{\prime}(t)=\underbrace{p(x(t), \Phi(t, x(t), \alpha))}_{(1)}-\underbrace{\int_{t}^{\theta} \sum_{k=1}^{n} \frac{\partial p}{\partial x_{j}}(x(t), \Phi(s, x(t), \alpha)) \dot{x}_{k}(t) d t}_{(2)} \\
-\underbrace{\int_{t}^{\theta} \sum_{k, \ell=1}^{n} \frac{\partial p}{\partial \xi_{\ell}}(x(t), \Phi(s, x(t), \alpha)) \frac{\partial \Phi_{\ell}}{\partial x_{k}}(s, x(t), \alpha) \dot{x}_{k}(t) d s+p(\alpha) .}_{(3)}
\end{gathered}
$$

By Theorem 4.4.10 (vi) we have,

$$
(1)=p(x(t), \xi(t))=p(\alpha) .
$$

By the point (vii) of the same theorem we have,

$$
(3)=\sum_{k, \ell=1}^{n} \int_{t}^{\theta} \frac{\partial p}{\partial \xi_{\ell}}(x(t), \Phi(s, x(t), \alpha)) \frac{\partial \Phi_{k}}{\partial x_{\ell}}(s, x(t), \alpha) \dot{x}_{k}(t) d s+R_{0}
$$

with

$$
\left|R_{0}\right| \leqslant C_{N} \int_{t}^{\theta} \frac{|x(t)-x(s)|^{N}}{\langle s\rangle^{2 N}} .
$$

Since $|x(t)-x(s)| \leqslant \int_{t}^{s}|\dot{x}(\sigma)| d \sigma \leqslant C(s-t)$ we obtain

$$
\left|R_{0}\right| \leqslant C_{N}^{\prime} \int_{t}^{\theta} \frac{(s-t)^{N}}{\langle s\rangle^{2 N}} d s, \quad \theta^{*} \leqslant t \leqslant \theta .
$$

Using Corollary 4.4.13 we obtain

$$
\begin{aligned}
(3)=-\sum_{k, \ell=1}^{n} \int_{t}^{\theta} \frac{\partial p}{\partial x_{k}}(x(t), \Phi(s, x(t), \alpha), \alpha) \dot{x}(t) d s & \\
& -\sum_{k=1}^{n} \int_{t}^{\theta} \frac{\partial \Phi_{k}}{\partial s}(s, x(t), \alpha) \dot{x}_{k}(t) d s+R_{1}
\end{aligned}
$$

where $R_{1}$ satisfies (4.4.58)

It follows that

$$
(3)=-(2)-\sum_{k=1}^{n} \dot{x}_{k}(t)\left(\Phi_{k}(\theta, x(t), \alpha)-\Phi_{k}(t, x(t), \alpha)\right)+R_{1} .
$$

Now

$$
\sum_{k=1}^{n} \dot{x}_{k}(t) \Phi_{k}(t, x(t), \alpha)=\sum_{k=1}^{n} \xi_{k}(t) \frac{\partial p}{\partial \xi_{k}}(x(t), \xi(t))=2 p(\alpha) .
$$

Therefore we obtain,

$$
F^{\prime}(t)=(1)-(2)-(3)+p(\alpha)=\sum_{k=1}^{n} \dot{x}_{k}(t) \Phi_{k}(\theta, x(t), \alpha)+R_{1} .
$$


Now by Theorem 4.4.10 (iv),

$$
\operatorname{Im} \Phi_{k}(\theta, x(t), \alpha)=\frac{x_{k}(t)-x_{k}(\theta)}{1+4 \theta^{2}}+\mathcal{O}(\sqrt{\delta}) \frac{\left|x_{k}(t)-x_{k}(\theta)\right|}{\langle\theta\rangle^{2}} .
$$

Since $\dot{x}(t)$ is uniformly bounded we deduce that

$$
\begin{aligned}
\operatorname{Im} F^{\prime}(t) & =\frac{1}{2} \frac{d}{d t} \frac{|x(t)-x(\theta)|^{2}}{1+4 \theta^{2}}+G(t) \text { with } \\
|G(t)| & \leqslant C \sqrt{\delta} \frac{\theta-t}{\langle\theta\rangle^{2}}+C_{N} \int_{t}^{\theta} \frac{(s-t)^{N}}{\langle s\rangle^{2 N}} d s .
\end{aligned}
$$

Integrating between $\theta^{*}$ and $\theta$ we obtain

$$
\begin{aligned}
& \left|\operatorname{Im} F\left(\theta^{*}\right)-\frac{1}{2} \frac{\left|x\left(\theta^{*}\right)-x(\theta)\right|^{2}}{1+4 \theta^{2}}\right| \\
& \qquad C^{\prime} \sqrt{\delta} \frac{\left(\theta-\theta^{*}\right)^{2}}{\langle\theta\rangle^{2}}+C_{N} \int_{\theta^{*}}^{\theta} \int_{t}^{\theta} \frac{(s-t)^{N}}{\langle s\rangle^{2 N}} d s d t .
\end{aligned}
$$

Let us call $I$ (resp. $I I$ ) the first (resp. the second) term in the right hand side of (4.4.59). By Lemma 4.4.17 we have

$$
|I| \leqslant C \sqrt{\delta} \frac{|x-x(\theta, \alpha)|^{2}}{\langle\theta\rangle^{2}}
$$

Now

$$
|I I| \leqslant C_{N}^{\prime} \int_{\theta^{*}}^{\theta}\left(\int_{\theta^{*}}^{s}(s-t)^{N} d t\right) \frac{d s}{(1+s)^{2 N}} \leqslant C_{N}^{\prime \prime} \int_{\theta^{*}}^{\theta} \frac{\left(s-\theta^{*}\right)^{N+1}}{(1+s)^{2 N}} d s .
$$

Now it follows from Lemma 4.4.17 and (4.4.40) that $\theta-\theta^{*} \leqslant 2 \delta\langle\theta\rangle \leqslant 2 \delta(1+\theta)$ which means that $(1-2 \delta) \theta \leqslant \theta^{*}+2 \delta$. Since $2 \delta \leqslant 1 / 2$ we have $\theta \leqslant 2 \theta^{*}+1$. It is then easy to see that the function $s \mapsto\left(s-\theta^{*}\right)^{N+1} /(1+s)^{2 N}$ is increasing on $\left(\theta^{*}, \theta\right)$. Therefore

$$
|I I| \leqslant C_{N} \frac{\left(\theta-\theta^{*}\right)^{N+2}}{\langle\theta\rangle^{2 N}} \leqslant C_{N}\left(\theta-\theta^{*}\right)^{2} \frac{(2 \delta)^{N}}{\langle\theta\rangle^{N}} .
$$

Taking $N=2$ and using Lemma 4.4 .17 (i) and (iii) we obtain

$$
|I I| \leqslant C \delta^{2} \frac{|x-x(\theta, \alpha)|^{2}}{\langle\theta\rangle^{2}}
$$

It follows from (4.4.59) to (4.4.61) and from (4.4.53), (4.4.56), (4.4.57) that

$$
\operatorname{Im} \varphi(\theta, x, \alpha)=\frac{1}{2} \frac{|x-x(\theta, \alpha)|^{2}}{1+4 \theta^{2}}-\frac{1}{2}\left|\alpha_{\xi}\right|^{2}+\mathcal{O}\left(\sqrt{\delta} \frac{|x-x(\theta, \alpha)|^{2}}{\langle\theta\rangle^{2}}\right)
$$

which is precisely the claim of point (vii) of Proposition 4.4.14. 
Case 3.2. - $(\theta, x) \in \mathcal{O}_{\delta}, x(\theta, \alpha) \cdot \alpha_{\xi}>\frac{c_{0}}{3}\langle x(\theta, \alpha)\rangle\left|\alpha_{\xi}\right|$ and $\left|x-x\left(\theta^{*}, \alpha\right)\right| \geqslant \frac{1}{2}\left|x\left(\theta^{*}, \alpha\right)\right|$. According to Lemma 4.4.17 (iii) we have

$$
\left\{\begin{array}{l}
\left|x\left(\theta^{*}, \alpha\right)\right| \leqslant 10|x-x(\theta, \alpha)| \leqslant 2 \delta\langle\theta\rangle \\
|x| \leqslant\left|x-x\left(\theta^{*}, \alpha\right)\right|+\left|x\left(\theta^{*}, \alpha\right)\right| \leqslant 15|x-x(\theta, \alpha)| \leqslant \frac{3}{2} \delta\langle\theta\rangle .
\end{array}\right.
$$

On the other hand if $y$ belongs to the union of the two segments $[0, x]$ and $\left[0, x\left(\theta^{*}, \alpha\right)\right]$ then $(y, \theta)$ belongs to $\Omega_{\delta}$, the set (defined in (4.1.4)) on which $\Phi$ is defined. Indeed, by (4.4.40), if $s \in(0,1)$ then $s x \cdot \alpha_{\xi} \leqslant s \cdot \frac{c_{0}}{10}\langle x\rangle\left|\alpha_{\xi}\right| \leqslant c_{0}\langle s x\rangle\left|\alpha_{\xi}\right|$. Moreover

$$
\begin{aligned}
|s x-x(\theta, \alpha)| & \leqslant\left|s x-x\left(\theta^{*}, \alpha\right)\right|+\left|x\left(\theta^{*}, \alpha\right)-x(\theta, \alpha)\right| \\
& \leqslant s\left|x-x\left(\theta^{*}, \alpha\right)\right|+(1-s)\left|x\left(\theta^{*}, \alpha\right)\right|+\left|x\left(\theta^{*}, \alpha\right)-x(\theta, \alpha)\right| .
\end{aligned}
$$

Since we are in case 3.2 we have by Lemma 4.4.17, $|s x-x(\theta, \alpha)| \leqslant 10|x-x(\theta, \alpha)| \leqslant$ $\delta\langle\theta\rangle$. On the other hand, if $s \in(0,1)$ we have, by $(4.4 .49), s x\left(\theta^{*}, \alpha\right) \cdot \alpha_{\xi}=0$. Moreover

$$
\begin{aligned}
\left|s x\left(\theta^{*}, \alpha\right)-x(\theta, \alpha)\right| & \leqslant\left|x\left(\theta^{*}, \alpha\right)-x(\theta, \alpha)\right|+(1-s)\left|x\left(\theta^{*}, \alpha\right)\right| \\
& \leqslant\left|x\left(\theta^{*}, \alpha\right)-x(\theta, \alpha)\right|+2(1-s)\left|x-x\left(\theta^{*}, \alpha\right)\right| \\
& \leqslant 10|x-x(\theta, \alpha)| \leqslant \delta\langle\theta\rangle,
\end{aligned}
$$

by Lemma 4.4.17, (iii).

Therefore in the present case we can set

$$
\begin{aligned}
\varphi(\theta, x, \alpha)=\int_{0}^{1} x & \cdot \Phi(\theta, s x, \alpha) d s-\int_{0}^{1} x\left(\theta^{*}, \alpha\right) \cdot \Phi\left(\theta, s x\left(\theta^{*}, \alpha\right), \alpha\right) d s \\
& -\int_{\theta^{*}}^{\theta} p\left(x\left(\theta^{*}, \alpha\right), \Phi\left(s, x\left(\theta^{*}, \alpha\right), \alpha\right)\right) d s+\theta^{*} p(\alpha)+\frac{1}{2 i} \alpha_{\xi}^{2} .
\end{aligned}
$$

Our goal is to show that $\varphi$ satisfies all the requirements of Proposition 4.4.14.

The point (i) is empty since $\theta=0$ does not belong to this part of $\mathcal{O}_{\delta}$. Let us check (ii). We have

$$
\frac{\partial \varphi}{\partial x_{j}}(\theta, x, \alpha)=\int_{0}^{1} \Phi_{j}(\theta, x, \alpha) d s+\int_{0}^{1} \sum_{k=1}^{n} s x_{k} \frac{\partial \Phi_{k}}{\partial x_{j}}(\theta, s x, \alpha) d s .
$$

By Theorem 4.4.10 and (4.4.62) we have

$$
\begin{aligned}
\left|\frac{\partial \Phi_{k}}{\partial x_{j}}(\theta, s x, \alpha)-\frac{\partial \Phi_{j}}{\partial x_{k}}(\theta, s x, \alpha)\right| & \leqslant C_{N} \frac{|s x-x(\theta, \alpha)|^{N}}{\langle\theta\rangle^{2 N}} \\
& \leqslant C_{N}^{\prime} \frac{|x-x(\theta, \alpha)|^{N}}{\langle\theta\rangle^{2 N}} .
\end{aligned}
$$

It follows that

$$
\frac{\partial \varphi}{\partial x_{j}}(\theta, x, \alpha)=\int_{0}^{1} \Phi_{j}(\theta, s x, \alpha) d s+\int_{0}^{1} s \frac{d}{d s}\left(\Phi_{j}(\theta, s x, \alpha)\right) d s+\mathcal{O}\left(\frac{|x-x(\theta, \alpha)|^{N+1}}{\langle\theta\rangle^{2 N}}\right) .
$$


Integrating by parts and using the bound $|x-x(\theta, \alpha)| \leqslant \delta\langle\theta\rangle$ we obtain

$$
\left|\frac{\partial \varphi}{\partial x_{j}}(\theta, x, \alpha)-\Phi_{j}(\theta, x, \alpha)\right| \leqslant C_{N} \frac{|x-x(\theta, \alpha)|^{N}}{\langle\theta\rangle^{N}}, \quad \forall N \in \mathbb{N} .
$$

Thus (ii) is proved. Let us prove (iii). We have

$$
\begin{array}{r}
\frac{\partial \varphi}{\partial \theta}(\theta, x, \alpha)=\underbrace{\int_{0}^{1} x \frac{\partial \Phi}{\partial \theta}(\theta, s x, \alpha) d s}_{(1)}-\underbrace{\int_{0}^{1} x\left(\theta^{*}, \alpha\right) \frac{\partial \Phi}{\partial \theta}\left(\theta, s x\left(\theta^{*}, \alpha\right), \alpha\right) d s}_{(2)} \\
-\underbrace{p\left(x\left(\theta^{*}, \alpha\right), \Phi\left(\theta, x\left(\theta^{*}, \alpha\right), \alpha\right)\right.}_{(3)} .
\end{array}
$$

Using Corollary 4.4.13 we can write

$$
\begin{aligned}
(1)=-\sum_{k=1}^{n} \int_{0}^{1} x_{k} & \frac{\partial p}{\partial x_{k}}(s x, \Phi(\theta, s x, \alpha)) d s \\
& +\sum_{k, \ell=1}^{n} \int_{0}^{1} x_{k}(\theta, s x, \alpha) \cdot \frac{\partial p}{\partial x_{\ell}}(s x, \Phi(\theta, s x, \alpha)) \frac{\partial \Phi_{k}}{\partial x_{k}}(\theta, s x, \alpha) d s .
\end{aligned}
$$

Using again (4.4.64) we obtain,

$$
(1)=-\int_{0}^{1} \frac{d}{d s}(p(s x, \Phi(\theta, s x, \alpha))) d s+\mathcal{O}\left(\frac{|x-x(\theta, \alpha)|^{N+1}}{\langle\theta\rangle^{2 N}}\right) .
$$

Finally, since $|x-x(\theta, \alpha)| \leqslant \delta\langle\theta\rangle$, we have

$$
(1)=p(0, \Phi(\theta, 0, \alpha))-p(x, \Phi(\theta, x, \alpha))+\mathcal{O}\left(\frac{|x-x(\theta, \alpha)|^{N}}{\langle\theta\rangle^{N}}\right) .
$$

By exactly the same computation (using (4.4.62)) we obtain

$$
(2)=p(0, \Phi(\theta, 0, \alpha))-p\left(x\left(\theta^{*}, \alpha\right), \Phi\left(\theta, x\left(\theta^{*}, \alpha\right), \alpha\right)+\mathcal{O}\left(\frac{|x-x(\theta, \alpha)|^{N}}{\langle\theta\rangle^{N}}\right) .\right.
$$

So using (4.4.65) to (4.4.67) we derive the point (iii). The last non trivial point to be proved is the point (vi).

Using the expression of $\operatorname{Im} \Phi$ given by Theorem 4.4.10, (4.4.62) and (4.4.59) to (4.4.61) which are valid also in the Case 3.2 , we can write

$$
\begin{aligned}
\operatorname{Im} \varphi(\theta, x, \alpha)=\frac{1}{2} & \frac{1}{1+4 \theta^{2}}\left(|x-x(\theta, \alpha)|^{2}-|x(\theta, \alpha)|^{2}-\left|x\left(\theta^{*}, \alpha\right)-x(\theta, \alpha)\right|^{2}\right. \\
& \left.+|x(\theta, \alpha)|^{2}+\left|x\left(\theta^{*}, \alpha\right)-x(\theta, \alpha)\right|^{2}\right)+\mathcal{O}\left(\sqrt{\delta} \frac{|x-x(\theta, \alpha)|^{2}}{\langle\theta\rangle^{2}}\right)
\end{aligned}
$$

which is exactly what is needed.

To finish the proof of Proposition 4.4.14 we must show that the phases $\varphi$ which have been constructed by the formulas (4.4.41), (4.4.43), (4.4.53), (4.4.63) in different regions can be matched in only one phase. We begin by a Lemma. 
Lemma 4.4.18. - Let $I=\left[\frac{c_{0}}{10}, \frac{c_{0}}{2}\right]$ and let us consider the function on $[0,+\infty[\times I$,

$$
g(s, c)=x(s, \alpha) \cdot \alpha_{\xi}-c\langle x(s, \alpha)\rangle\left|\alpha_{\xi}\right| .
$$

(i) For all $c$ in $I$ the function $[0,+\infty[\times \mathbb{R}, s \mapsto g(s, c)$ is strictly increasing.

(ii) For all $c$ in $I$ there exists a unique $\theta(c)>0$ such that

$$
g(\theta(c), c)=0 .
$$

(iii) The function $I \rightarrow[0,+\infty[, c \mapsto \theta(c)$ is strictly increasing.

Moreover we have the following estimates

(iv) $\frac{3}{2}\left|\theta(c)-\theta^{*}\right|\left|\alpha_{\xi}\right| \leqslant\left|x(\theta(c), \alpha)-x\left(\theta^{*}, \alpha\right)\right| \leqslant 3\left|\theta(c)-\theta^{*}\right|\left|\alpha_{\xi}\right|$.

(v) $\left|\theta(c)-\theta^{*}\right| \geqslant c_{0} / 120$.

(vi) For all $x$ in $\mathcal{O}_{\delta}$ and $c$ in $\left[\frac{c_{0}}{3} \frac{c_{0}}{2}\right]$,

$$
|x-x(\theta(c), \alpha)| \leqslant\left|x-x\left(\theta^{*}, \alpha\right)\right|+\left|x\left(\theta^{*}, \alpha\right)-x(\theta(c), \alpha)\right| \leqslant 4|x-x(\theta(c), \alpha)| .
$$

(vii) If $c \in\left[\frac{c_{0}}{3} \frac{c_{0}}{2}\right]$ we have

$$
\frac{c}{10}\left\langle x\left(\theta^{*}, \alpha\right)\right\rangle \leqslant\left|\theta(c)-\theta^{*}\right| \leqslant 4 c\left\langle x\left(\theta^{*}, \alpha\right)\right\rangle .
$$

Proof

(i) We have

$$
\frac{\partial g}{\partial s}(s, c)=\dot{x}(s, \alpha) \cdot \alpha_{\xi}-c \frac{x(s, \alpha) \cdot \dot{x}(s, \alpha)}{\langle x(s, \alpha)\rangle}\left|\alpha_{\xi}\right|=2\left|\alpha_{\xi}\right|^{2}+\mathcal{O}\left(\varepsilon+c_{0}\right) .
$$

Thus $\frac{\partial g}{\partial s}(s, c) \geqslant \frac{1}{10}$ if $\varepsilon$ and $c_{0}$ are small enough.

(ii) It follows from above that $g(s, c) \geqslant \frac{1}{10} s+g(0, c)$ so $g(s, c) \rightarrow+\infty$ if $s \rightarrow+\infty$. Moreover $g(0, c)=\alpha_{x} \cdot \alpha_{\xi}-c\left\langle\alpha_{x}\right\rangle\left|\alpha_{\xi}\right| \leqslant \alpha_{x} \cdot \alpha_{\xi} \leqslant-c_{0}\left\langle\alpha_{x}\right\rangle\left|\alpha_{\xi}\right|<0$. Therefore there exists a unique $\theta(c)$ such that $g(\theta(c), c)=0$ and $c \mapsto \theta(c)$ is $C^{\infty}$. Differentiating this equality with respect to $c$ we obtain

$$
\theta^{\prime}(c) \frac{\partial g}{\partial s}(\theta(c), c)+\frac{\partial g}{\partial c}(\theta(c), c)=0 .
$$

By the above computation of $\frac{\partial g}{\partial s}$ we can write

$$
\theta^{\prime}(c)=\frac{\langle x(\theta(c), \alpha)\rangle\left|\alpha_{\xi}\right|}{2\left|\alpha_{\xi}\right|^{2}+\mathcal{O}\left(\varepsilon+c_{0}\right)}
$$

which proves (iii). Now we have

$$
x(\theta(c), \alpha)-x\left(\theta^{*}, \alpha\right)=\int_{\theta^{*}}^{\theta(c)} x(s, \alpha) d s=2 \alpha_{\xi}\left(\theta(c)-\theta^{*}\right)+\mathcal{O}\left(\varepsilon\left|\theta(c)-\theta^{*}\right|\right)
$$

from which (iv) follows easily. Let us prove (v). By definition of $\theta(c)$ and $\theta^{*}$ we can write

$$
\left(x(\theta(c), \alpha)-x\left(\theta^{*}, \alpha\right)\right) \cdot \alpha_{\xi}=c\langle x(\theta(c), \alpha)\rangle\left|\alpha_{\xi}\right| \geqslant \frac{c_{0}}{20}
$$


so by (iv),

$$
\frac{c_{0}}{20} \leqslant 3\left|\theta(c)-\theta^{*}\right| \cdot\left|\alpha_{\xi}\right| \leqslant 6\left|\theta(c)-\theta^{*}\right| .
$$

The first inequality in (vi) being trivial let us prove the second one. We write

$$
\begin{aligned}
|x-x(\theta, \alpha)|^{2}=\underbrace{\left|x-x\left(\theta^{*}, \alpha\right)\right|^{2}+\left|x\left(\theta^{*}, \alpha\right)-x(\theta(c), \alpha)\right|^{2}}_{(1)} & +\underbrace{2\left(x-x\left(\theta^{*}, \alpha\right)\right) \cdot\left(x\left(\theta^{*}, \alpha\right)-x(\theta(c), \alpha)\right)}_{(2)} .
\end{aligned}
$$

We have

$$
(2)=2\left(x-x\left(\theta^{*}, \alpha\right)\right)\left[2\left(\theta^{*}-\theta(c)\right) \alpha_{\xi}+\mathcal{O}\left(\varepsilon\left|\theta^{*}-\theta(c)\right|\right] .\right.
$$

It follows from (iv) that

$$
(2)=-4\left(\theta(c)-\theta^{*}\right) x \cdot \alpha_{\xi}+\mathcal{O}(\varepsilon(1)), \quad \text { where } \theta(c)-\theta^{*} \geqslant 0 .
$$

Now in $\mathcal{O}_{\delta}$ we have $x \cdot \alpha_{\xi} \leqslant \frac{c_{0}}{10}\langle x\rangle\left|\alpha_{\xi}\right|$. It follows that

$$
x \cdot \alpha_{\xi} \leqslant \frac{c_{0}}{10}\langle x(\theta(c), \alpha)\rangle\left|\alpha_{\xi}\right|+\frac{c_{0}}{10}|x-x(\theta(c), \alpha)|\left|\alpha_{\xi}\right| .
$$

Using (4.4.68) we obtain

$$
x \cdot \alpha_{\xi} \leqslant \frac{c_{0}}{10 c}\left(x(\theta(c), \alpha)-x\left(\theta^{*}, \alpha\right)\right) \cdot \alpha_{\xi}+\frac{c_{0}}{10}|x-x(\theta(c), \alpha)|\left|\alpha_{\xi}\right|,
$$

so we will have

$$
\begin{aligned}
(2) \geqslant- & \frac{2 c_{0}}{5 c}\left|x(\theta(c), \alpha)-x\left(\theta^{*}, \alpha\right)\right|\left|\alpha_{\xi}\right|\left|\theta(c)-\theta^{*}\right| \\
& -\frac{2 c_{0}}{5}|x-x(\theta(c), \alpha)|\left|\alpha_{\xi}\right|\left|\theta(c)-\theta^{*}\right|-\mathcal{O}(\varepsilon(1)) \\
(2) \geqslant- & \frac{4 c_{0}}{15 c}\left|x(\theta(c), \alpha)-x\left(\theta^{*}, \alpha\right)\right|^{2} \\
& -\frac{8 c_{0}}{30}|x-x(\theta(c), \alpha)|\left|x(\theta(c), \alpha)-x\left(\theta^{*}, \alpha\right)\right|-\mathcal{O}(\varepsilon(1)) \\
(2) \geqslant( & \left.-\frac{4 c_{0}}{15 c}-\frac{4 c_{0}}{30}\right)\left|x(\theta(c), \alpha)-x\left(\theta^{*}, \alpha\right)\right|^{2} \\
& -\frac{2}{15} c_{0}|x-x(\theta(c), \alpha)|^{2}-\mathcal{O}(\varepsilon(1)) .
\end{aligned}
$$

If $c \geqslant c_{0} / 3$ then $4 c_{0} / 15 c \leqslant 4 / 5$ so we obtain

$$
(1)+(2) \geqslant \frac{1}{10}\left(\left|x(\theta(c), \alpha)-x\left(\theta^{*}, \alpha\right)\right|^{2}+|x-x(\theta, \alpha)|^{2}\right)
$$

if $c_{0}$ is small enough. This implies (vi). Let us prove (vii). We have

$$
x(\theta(c), \alpha)=x\left(\theta^{*}, \alpha\right)+2\left(\theta(c)-\theta^{*}\right) \cdot \alpha_{\xi}+\mathcal{O}(\varepsilon) .
$$

It follows that

$$
c\langle x(\theta(c), \alpha)\rangle\left|\alpha_{\xi}\right|=x(\theta(c), \alpha) \cdot \alpha_{\xi}=2\left(\theta(c)-\theta^{*}\right)\left|\alpha_{\xi}\right|^{2}+\mathcal{O}(\varepsilon)
$$


SO

$$
\frac{1}{2}\left|\theta(c)-\theta^{*}\right| \leqslant c\langle x(\theta(c), \alpha)\rangle \leqslant 5\left|\theta(c)-\theta^{*}\right| .
$$

Moreover we have

$$
\begin{aligned}
\langle x(\theta(c), \alpha)\rangle & \leqslant\left\langle x\left(\theta^{*}, \alpha\right)\right\rangle+\left|x\left(\theta^{*}, \alpha\right)-x(\theta(c), \alpha)\right| \\
& \leqslant\left\langle x\left(\theta^{*}, \alpha\right)\right\rangle+3\left|\theta(c)-\theta^{*}\right|\left|\alpha_{\xi}\right| \\
& \leqslant\left\langle x\left(\theta^{*}, \alpha\right)\right\rangle+6 c\langle x(\theta(c), \alpha)\rangle\left|\alpha_{\xi}\right| \\
& \leqslant\left\langle x\left(\theta^{*}, \alpha\right)\right\rangle+12 c\langle x(\theta(c), \alpha)\rangle
\end{aligned}
$$

which implies that $\langle x(\theta(c), \alpha)\rangle \leqslant 2\left\langle x\left(\theta^{*}, \alpha\right)\right\rangle$ if $c_{0}$ is small enough. By the same way $\left\langle x\left(\theta^{*}, \alpha\right)\right\rangle \leqslant 2\langle x(\theta(c), \alpha)\rangle$. Thus we obtain (vii).

Now let us set

$$
\left\{\begin{array}{l}
\widetilde{\mathcal{O}}_{\delta}(\theta)=\left\{x \in \mathbb{R}^{n}: x \cdot \alpha_{\xi} \leqslant \frac{c_{0}}{10}\langle x\rangle\left|\alpha_{\xi}\right|,|x-x(\theta, \alpha)| \leqslant \frac{\delta}{40}\langle\theta\rangle\right\} \\
\bar{\theta}=\theta\left(c_{0} / 2\right), \quad \theta^{\prime}=\theta\left(c_{0} / 10\right) .
\end{array}\right.
$$

In the beginning of this Section we have constructed the different $\varphi$ assuming $x \cdot \alpha_{\xi} \leqslant$ $\frac{c_{0}}{10}\langle x\rangle\left|\alpha_{\xi}\right|,|x-x(\theta, \alpha)| \leqslant \frac{\delta}{5}\langle\theta\rangle$.

In the proof of Proposition 4.4.14 we have constructed

$$
\begin{aligned}
& \varphi_{1} \text { when } \theta \in[0, \bar{\theta}] \text { and }|x-x(\theta, \alpha)| \leqslant\langle x(\theta, \alpha)\rangle, \quad \text { (case 1) } \\
& \varphi_{2} \text { when } \theta \in[0, \bar{\theta}] \text { and }|x-x(\theta, \alpha)| \geqslant \frac{1}{2}|x(\theta, \alpha)|, \quad \text { (case 2) } \\
& \varphi_{4} \text { when } \theta \in\left[\theta^{\prime}+\infty\left[\text { and }\left|x-x\left(\theta^{*}, \alpha\right)\right| \leqslant\left\langle x\left(\theta^{*}, \alpha\right)\right\rangle, \quad\right. \text { (case 3.1) }\right. \\
& \varphi_{5} \text { when } \theta \in\left[\theta^{\prime}+\infty\left[\text { and }\left|x-x\left(\theta^{*}, \alpha\right)\right| \geqslant \frac{1}{2}\left|x\left(\theta^{*}, \alpha\right)\right|, \quad\right. \text { (case 3.2). }\right.
\end{aligned}
$$

We are going first to match $\varphi_{1}$ and $\varphi_{2}, \varphi_{4}$ and $\varphi_{5}$. The matched phase will be defined on a smallest set than $\mathcal{O}_{\delta}$ defined in (4.4.40) namely for $(\theta, x)$ where $x \in \widetilde{\mathcal{O}}_{\delta}(\theta)$ (see $(4.4 .69))$. We show first that the point $(\theta, 0)$ belongs to the sets where $\varphi_{1}$ and $\varphi_{2}$ are defined. According to (4.4.40) and what we recalled above it will be the case if $|x(\theta, \alpha)| \leqslant \frac{\delta}{5}\langle\theta\rangle$. We may assume that the domain where $\varphi_{2}$ is defined contains points $(\theta, x)$ where $x \in \widetilde{\mathcal{O}}_{\delta}(\theta)$ otherwise we don't match $\varphi_{1}$ and $\varphi_{2}$ and we take only $\varphi_{1}$. So let $(\theta, x)$ be such $|x-x(\theta, \alpha)| \leqslant \frac{\delta}{40}\langle\theta\rangle$ and $|x(\theta, \alpha)| \leqslant 2|x-x(\theta, \alpha)|$. Then $|x(\theta, \alpha)| \leqslant \frac{\delta}{40}\langle\theta\rangle$ which implies our claim.

Now it follows from (4.4.41) and (4.4.43) that

$$
\varphi_{1}(\theta, 0, \alpha)=\varphi_{2}(\theta, 0, \alpha)
$$

By the same way we may assume that the domain where $\varphi_{5}$ is defined contains points $(\theta, x)$ where $x \in \widetilde{\mathcal{O}}_{\delta}(\theta)$. So let $x$ be such that $|x-x(\theta, \alpha)| \leqslant \frac{\delta}{40}\langle\theta\rangle$ and 
$\left|x-x\left(\theta^{*}, \alpha\right)\right| \geqslant \frac{1}{2}\left|x\left(\theta^{*}, \alpha\right)\right|$. Then we write, using Lemma 4.4.18,

$$
\begin{aligned}
& |x(\theta, \alpha)| \leqslant\left|x\left(\theta^{*}, \alpha\right)\right|+\left|x\left(\theta^{*}, \alpha\right)-x(\theta, \alpha)\right|, \\
& |x(\theta, \alpha)| \leqslant 2\left(\left|x-x\left(\theta^{*}, \alpha\right)\right|+\left|x\left(\theta^{*}, \alpha\right)-x(\theta, \alpha)\right|\right) \leqslant 8|x-x(\theta, \alpha)| \leqslant \frac{\delta}{5}\langle\theta\rangle .
\end{aligned}
$$

So the point $(\theta, 0)$ belongs also to the sets where $\varphi_{4}$ and $\varphi_{5}$ are defined and by (4.4.53), (4.4.63) we have

$$
\varphi_{4}(\theta, 0, \alpha)=\varphi_{5}(\theta, 0, \alpha) .
$$

Let us match $\varphi_{1}$ and $\varphi_{2}$. Let $x \in \widetilde{\mathcal{O}}_{\delta}, \theta \in[0, \bar{\theta}]$ be such that

$$
\frac{1}{2}|x(\theta, \alpha)| \leqslant|x-x(\theta, \alpha)| \leqslant\langle x(\theta, \alpha)\rangle .
$$

We are going to show then that

$$
\forall N \in \mathbb{N} \exists C_{N}>0:\left|\varphi_{1}(\theta, x, \alpha)-\varphi_{2}(\theta, x, \alpha)\right| \leqslant C_{N} \frac{|x-x(\theta, \alpha)|^{N+1}}{\langle\theta\rangle^{N}} .
$$

Indeed let $\gamma(\sigma, x)$ be a regular path such that

$$
\gamma(0, x)=0, \quad \gamma(1, x)=x
$$

and there exists $K \geqslant 0$ such that for all $\sigma$ in $[0,1]$,

$$
\begin{gathered}
\left|\frac{\partial \gamma}{\partial \sigma}(\sigma, x)\right| \leqslant K|x-x(\theta, \alpha)| \\
\gamma(\sigma, x) \cdot \alpha_{\xi} \leqslant \frac{c_{0}}{10}\langle\gamma(\sigma, x)\rangle\left|\alpha_{\xi}\right| \\
|\gamma(\sigma, x)-x(\theta, \alpha)| \leqslant \frac{\delta}{5}\langle\theta\rangle \\
\left\{\begin{array}{l}
\text { if }|x-x(\theta, \alpha)| \geqslant|x(\theta, \alpha)| \text { then, } \\
|x(\theta, \alpha)| \leqslant|\gamma(\sigma, x)-x(\theta, \alpha)| \leqslant|x-x(\theta, \alpha)|
\end{array}\right. \\
\left\{\begin{array}{l}
\text { if }|x-x(\theta, \alpha)| \leqslant|x(\theta, \alpha)| \text { then, } \\
|x-x(\theta, \alpha)| \leqslant|\gamma(\sigma, x)-x(\theta, \alpha)| \leqslant|x(\theta, \alpha)| .
\end{array}\right.
\end{gathered}
$$

The construction of this path will be made at the end of this Section.

It follows from (4.4.78) or (4.4.79) that

$$
\frac{1}{2}|x(\theta, \alpha)| \leqslant|\gamma(\sigma, x)-x(\theta, \alpha)| \leqslant\langle x(\theta, \alpha)\rangle .
$$

We write for $j=1,2$,

$$
\varphi_{j}(\theta, x, \alpha)=\varphi_{j}(\theta, 0, \alpha)+\int_{0}^{1} \frac{\partial \gamma}{\partial \sigma}(\sigma, x) \frac{\partial \varphi_{j}}{\partial x}(\theta, \gamma(\sigma, x), \alpha) d \sigma .
$$


Using Proposition 4.4.14 (ii) and (4.4.75) we obtain

$$
\left\{\begin{array}{l}
\varphi_{j}(\theta, x, \alpha)=\varphi_{j}(\theta, 0, \alpha)+\int_{0}^{1} \frac{\partial \gamma}{\partial \sigma}(\sigma, x) \Phi(\theta, \gamma(\sigma, x), \alpha) d \sigma+R_{j}, \\
\left|R_{j}\right| \leqslant C_{N} \int_{0}^{1}|x-x(\theta, \alpha)| \frac{|\gamma(\sigma, x)-x(\theta, \alpha)|^{N}}{\langle\theta\rangle^{N}} d \sigma .
\end{array}\right.
$$

Then by (4.4.72), (4.4.78) or (4.4.79) and (4.4.70) we obtain (4.4.73).

Now let $\chi_{0} \in C_{0}^{\infty}(\mathbb{R})$ be such that $0 \leqslant \chi_{0} \leqslant 1$ and $\chi_{0}(\sigma)=1$ if $|\sigma| \leqslant \frac{1}{2}, \chi_{0}(\sigma)=0$ if $|\sigma| \geqslant 1$. Let us set

$$
\chi_{1}(\theta, x)=\chi_{0}\left(\frac{x-x(\theta, \alpha)}{\langle x(\theta, \alpha)\rangle}\right) .
$$

Now for $x$ in $\widetilde{\mathcal{O}}_{\delta}(\theta)$ we set

$$
\varphi_{3}(\theta, x, \alpha)=\chi_{1}(\theta, x) \varphi_{1}(\theta, x, \alpha)+\left(1-\chi_{1}(\theta, x)\right) \varphi_{2}(\theta, x, \alpha) .
$$

On the support of $\chi_{1}$ we have $|x-x(\theta, \alpha)| \leqslant\langle x(\theta, \alpha)\rangle$ thus $\varphi_{1}$ is well defined. On the support of $1-\chi_{1}(\theta, x)$ we have $|x-x(\theta, \alpha)| \geqslant \frac{1}{2}\langle x(\theta, \alpha)\rangle \geqslant \frac{1}{2}|x(\theta, \alpha)|$ so $\varphi_{2}$ is well defined. Therefore $\varphi_{3}$ is well defined when $x \in \widetilde{\mathcal{O}}_{\delta}(\theta)$. We show now that $\varphi_{3}$ satisfies all the conditions in Proposition 4.4.14. We have

$$
\frac{\partial \varphi_{3}}{\partial x}(\theta, x, \alpha)=\left[\chi_{1} \frac{\partial \varphi_{1}}{\partial x}+\left(1-\chi_{1}\right) \frac{\partial \varphi_{2}}{\partial x}+\frac{\partial \chi_{1}}{\partial x}\left(\varphi_{1}-\varphi_{2}\right)\right](\theta, x, \alpha) .
$$

On the support of $\frac{\partial \chi_{1}}{\partial x}$ we have $\left|\left(\varphi_{1}-\varphi_{2}\right)(\theta, x, \alpha)\right| \leqslant C_{N} \frac{|x-x(\theta, \alpha)|^{N+1}}{\langle\theta\rangle^{N}}$ by (4.4.73). Moreover we have by $(4.4 .72)$

$$
\left|\frac{\partial \chi_{1}}{\partial x}(\theta, x)\right| \leqslant \frac{C}{\langle x(\theta, \alpha)\rangle} \leqslant \frac{C}{|x-x(\theta, \alpha)|}
$$

and

$$
\begin{aligned}
\left|\left[\chi_{1} \frac{\partial \varphi_{1}}{\partial x}+\left(1-\chi_{1}\right) \frac{\partial \varphi_{2}}{\partial x}-\Phi\right](\theta, x, \alpha)\right| \leqslant & \left|\chi_{1}\right|\left|\frac{\partial \varphi_{1}}{\partial x}(\theta, x, \alpha)-\Phi(\theta, x, \alpha)\right| \\
& +\left(1-\chi_{1}\right)\left|\frac{\partial \varphi_{2}}{\partial x}(\theta, x, \alpha)-\Phi(\theta, x, \alpha)\right| .
\end{aligned}
$$

It follows that the claim (ii) in Proposition 4.4.14 holds for $\varphi_{3}$. The point (iv) follows from (4.4.81) and (4.4.73) for $N=1$ which gives $\left|\left(\varphi_{1}-\varphi_{2}\right)(\theta, x, \alpha)\right| \leqslant C \delta$. The points (v) and (vi) are straightforward. Let us show (iii). We have

$$
\frac{\partial \chi_{1}}{\partial \theta}(\theta, x)=\left[-\frac{\dot{x}(\theta, \alpha)}{\langle x(\theta, \alpha)\rangle}-\frac{x(\theta, \alpha) \dot{x}(\theta, \alpha)}{\langle x(\theta, \alpha)\rangle^{3}}(x-x(\theta, \alpha))\right] \frac{\partial \chi_{0}}{\partial \sigma}(\cdots) .
$$

Since $\dot{x}(\theta, \alpha)$ is bounded we deduce from (4.4.73) that

$$
\left|\frac{\partial \chi_{1}}{\partial \theta}(\theta, x)\right| \leqslant \frac{C}{|x-x(\theta, \alpha)|} .
$$

It follows then that

$$
\left|\left(\frac{\partial \varphi_{3}}{\partial \theta}-\left(\chi_{1} \frac{\partial \varphi_{1}}{\partial \theta}+\left(1-\chi_{1}\right) \frac{\partial \varphi_{2}}{\partial \theta}\right)\right)(\theta, x, \alpha)\right| \leqslant C_{N} \frac{|x-x(\theta, \alpha)|^{N}}{\langle\theta\rangle^{N}} .
$$


Then (iii) follows easily.

Let us now take $\theta \in\left[\theta^{\prime},+\infty\left[\right.\right.$ and $x \in \widetilde{\mathcal{O}}_{\delta}(\theta)$. Assume that

$$
\frac{1}{2}\left|x\left(\theta^{*}, \alpha\right)\right| \leqslant\left|x-x\left(\theta^{*}, \alpha\right)\right| \leqslant\left\langle x\left(\theta^{*}, \alpha\right)\right\rangle .
$$

We shall show that

$$
\left|\left(\varphi_{4}-\varphi_{5}\right)(\theta, x, \alpha)\right| \leqslant C_{N} \frac{|x-x(\theta, \alpha)|^{N}}{\langle\theta\rangle^{N}}\left|x-x\left(\theta^{*}, \alpha\right)\right| .
$$

Let us take a path $\gamma$ satisfying (4.4.74), (4.4.76), (4.4.77) and

$$
\begin{aligned}
& \left\{\begin{array}{l}
\text { if }\left|x-x\left(\theta^{*}, \alpha\right)\right| \geqslant\left|x\left(\theta^{*}, \alpha\right)\right| \text { then } \\
\left|x\left(\theta^{*}, \alpha\right)\right| \leqslant\left|\gamma(\sigma, x)-x\left(\theta^{*}, \alpha\right)\right| \leqslant\left|x-x\left(\theta^{*}, \alpha\right)\right|,
\end{array}\right. \\
& \left\{\begin{array}{l}
\text { if }\left|x-x\left(\theta^{*}, \alpha\right)\right| \leqslant\left|x\left(\theta^{*}, \alpha\right)\right| \text { then } \\
\left|x-x\left(\theta^{*}, \alpha\right)\right| \leqslant\left|\gamma(\sigma, x)-x\left(\theta^{*}, \alpha\right)\right| \leqslant\left|x\left(\theta^{*}, \alpha\right)\right|,
\end{array}\right. \\
& \left|\frac{\partial \gamma}{\partial \sigma}(\sigma, x)\right| \leqslant K\left|x-x\left(\theta^{*}, \alpha\right)\right| .
\end{aligned}
$$

Let us remark that in the two cases (4.4.84) or (4.4.85) we have

$$
\left|\gamma(\sigma, x)-x\left(\theta^{*}, \alpha\right)\right| \leqslant 2\left|x-x\left(\theta^{*}, \alpha\right)\right| \leqslant 8|x-x(\theta, \alpha)|
$$

by Lemma 4.4.18.

Then using the same method as before we obtain easily (4.4.83). To match $\varphi_{4}$ and $\varphi_{5}$ we set

$$
\chi_{1}(x)=\chi_{0}\left(\frac{x-x\left(\theta^{*}, \alpha\right)}{\left\langle x\left(\theta^{*}, \alpha\right)\right\rangle}\right)
$$

and we deduce from (4.4.83) that $\left|\frac{\partial \chi_{1}}{\partial x}(x)\right| \leqslant \frac{C}{\mid x-x\left(\theta^{*}, \alpha\right)}$. Then we set

$$
\varphi_{6}(\theta, x, \alpha)=\left[\chi_{1} \varphi_{4}+\left(1-\chi_{1}\right) \varphi_{5}\right](\theta, x, \alpha) .
$$

It is then easy to see that $\varphi_{6}$ satisfies all the requirements of Proposition 4.4.14.

Our last step is to match $\varphi_{3}$ and $\varphi_{6}$. With the notation $\theta(c)$ introduced in Lemma 4.4.18 let us set

$$
\theta_{1}=\theta\left(\frac{11 c_{0}}{30}\right), \quad \theta_{2}=\theta\left(\frac{12 c_{0}}{30}\right), \quad \theta_{3}=\theta_{0}\left(\frac{14 c_{0}}{30}\right) .
$$

We have therefore according to (4.4.69)

$$
\theta^{\prime}<\theta_{1}<\theta_{2}<\theta_{3}<\bar{\theta} .
$$

Using (4.4.87), the fact that $\chi_{1}\left(x\left(\theta^{*}, \alpha\right)\right)=1$ and (4.4.53) we get

$$
\varphi_{6}\left(\theta_{2}, x\left(\theta^{*}, \alpha\right), \alpha\right)=-\int_{\theta^{*}}^{\theta_{2}} p\left(x\left(\theta^{*}, \alpha\right), \Phi\left(s, x\left(\theta^{*}, \alpha\right), \alpha\right) d s+\theta^{*} p(\alpha)+\frac{1}{2 i}\left|\alpha_{\xi}\right|^{2} .\right.
$$

On the other hand we have

$$
p\left(x\left(\theta^{*}, \alpha\right), \Phi\left(s, x\left(\theta^{*}, \alpha\right), \alpha\right)\right)=-\frac{\partial \varphi_{3}}{\partial s}\left(s, x\left(\theta^{*}, \alpha\right), \alpha\right)+A+B
$$


where

$$
\begin{aligned}
& A=p\left(x\left(\theta^{*}, \alpha\right), \Phi\left(s, x\left(\theta^{*}, \alpha\right), \alpha\right)\right)-p\left(x\left(\theta^{*}, \alpha\right), \frac{\partial \varphi_{3}}{\partial x}\left(s, x\left(\theta^{*}, \alpha\right), \alpha\right)\right) \\
& B=p\left(x\left(\theta^{*}, \alpha\right), \frac{\partial \varphi_{3}}{\partial x}\left(s, x\left(\theta^{*}, \alpha\right), \alpha\right)\right)+\frac{\partial \varphi_{3}}{\partial s}\left(s, x\left(\theta^{*}, \alpha\right), \alpha\right) .
\end{aligned}
$$

By the estimates proved in Proposition 4.4.14 we have

$$
|A|+|B| \leqslant C_{N} \frac{\left|x\left(\theta^{*}, \alpha\right)-x(s, \alpha)\right|^{N}}{\langle s\rangle^{N}} \leqslant C_{N} \frac{\left|x\left(\theta^{*}, \alpha\right)-x\left(\theta_{2}, \alpha\right)\right|^{N}}{\left\langle\theta^{*}\right\rangle^{N}} .
$$

Here we used the fact that for $s \in\left[\theta^{\prime}, \bar{\theta}\right]$ we have $\langle s\rangle \sim\left\langle\theta^{*}\right\rangle$ (see Lemma 4.4 .17 (ii)) and $\left|x\left(\theta^{*}, \alpha\right)-x(s, \alpha)\right| \sim\left|x\left(\theta^{*}, \alpha\right)-x\left(\theta_{2}, \alpha\right)\right|$ by Lemma 4.4.18.

It follows from (4.4.88) and (4.4.89) that

$\varphi_{6}\left(\theta_{2}, x\left(\theta^{*}, \alpha\right), \alpha\right)=\int_{\theta^{*}}^{\theta_{2}} \frac{\partial \varphi_{3}}{\partial s}\left(s, x\left(\theta^{*}, \alpha\right), \alpha\right) d s+\theta^{*} p(\alpha)+\frac{1}{2 i}\left|\alpha_{\xi}\right|^{2}+\int_{\theta^{*}}^{\theta_{2}}(|A|+|B|) d s$.

Therefore we obtain

$$
\begin{aligned}
\varphi_{6}\left(\theta_{2}, x\left(\theta^{*}, \alpha\right), \alpha\right)=\varphi_{3}\left(\theta_{2},\right. & \left.x\left(\theta^{*}, \alpha\right), \alpha\right) \\
& -\varphi_{3}\left(\theta^{*}, x\left(\theta^{*}, \alpha\right), \alpha\right)+\theta^{*} p(\alpha)+\frac{1}{2 i}\left|\alpha_{\xi}\right|^{2}+R
\end{aligned}
$$

where by $(4.4 .90)$

$$
|R| \leqslant C_{N} \frac{\left|x\left(\theta^{*}, \alpha\right)-x\left(\theta_{2}, \alpha\right)\right|^{N+1}}{\left\langle\theta^{*}\right\rangle^{N}}
$$

Now using (4.4.80) and (4.4.53) we have

$$
\varphi_{3}\left(\theta^{*}, x\left(\theta^{*}, \alpha\right), \alpha\right)=\varphi_{1}\left(\theta^{*}, x\left(\theta^{*}, \alpha\right), \alpha\right)=\theta^{*} p(\alpha)+\frac{1}{2 i}\left|\alpha_{\xi}\right|^{2}
$$

so we obtain

$$
\varphi_{6}\left(\theta_{2}, x\left(\theta^{*}, \alpha\right), \alpha\right)=\varphi_{3}\left(\theta_{2}, x\left(\theta^{*}, \alpha\right), \alpha\right)+R
$$

where $R$ satisfies (4.4.92).

Now let $\theta \in\left[\theta_{1}, \theta_{3}\right]$. We set

$$
\mathcal{O}_{\delta}^{\prime}(\theta)=\left\{x \in \mathbb{R}^{n}: x \cdot \alpha_{\xi} \leqslant \frac{c_{0}}{20}\langle x\rangle\left|\alpha_{\xi}\right|,|x-x(\theta, \alpha)| \leqslant \frac{\delta}{40}\langle\theta\rangle\right\} .
$$

Let $x \in \mathcal{O}_{\delta}^{\prime}(\theta)$. We can find a path $\gamma$ joining $x$ to $\left.x\left(\theta^{*}, \alpha\right)\right)$ such that $\gamma \subset \mathcal{O}_{\delta}^{\prime}(\theta)$ and there exists $K \geqslant 0$ such that

$$
\left|\frac{\partial \gamma}{\partial \sigma}(\sigma, x)\right| \leqslant K\left|x-x\left(\theta^{*}, \alpha\right)\right|,\left|\gamma(\sigma, s)-x\left(\theta_{2}, \alpha\right)\right| \leqslant K\left|x-x\left(\theta_{2}, \alpha\right)\right|
$$

Indeed if $\left|x-x\left(\theta^{*}, \alpha\right)\right| \leqslant\left|x\left(\theta^{*}, \alpha\right)\right|$ we set

$$
\gamma(\sigma, x)=\sigma x+(1-\sigma) x\left(\theta^{*}, \alpha\right)
$$


and Lemma 4.4.16 show that

$$
\begin{aligned}
\gamma(\sigma, x)) \cdot \alpha_{\xi}=\sigma x \cdot \alpha_{\xi} & \leqslant \frac{c_{0}}{20} \sigma(1+|x|)\left|\alpha_{\xi}\right| \\
& \left.\leqslant \frac{c_{0}}{20}\left(\sigma+\sigma|x|+(1-\sigma)\left|x\left(\theta^{*}, \alpha\right)\right|\right)\right)\left|\alpha_{\xi}\right| \\
& \leqslant \frac{c_{0}}{20} \sqrt{2}(1+|\gamma(\sigma, x)|)\left|\alpha_{\xi}\right| \leqslant \frac{c_{0}}{10}\langle\gamma(\sigma, x)\rangle\left|\alpha_{\xi}\right| .
\end{aligned}
$$

If $\left|x-x\left(\theta^{*}, \alpha\right)\right|>\left|x\left(\theta^{*}, \alpha\right)\right|$ we take $\gamma$ to be the union of the two segments joining $x$ and $x\left(\theta^{*}, \alpha\right)$ to 0 and we obtain with $y=x$ or $y=x\left(\theta^{*}, \alpha\right)$ for $t \in[0,1]$,

$$
t y \cdot \alpha_{\xi} \leqslant t \frac{c_{0}}{20}\langle y\rangle\left|\alpha_{\xi}\right| \leqslant \frac{c_{0}}{20}\langle t y\rangle\left|\alpha_{\xi}\right| .
$$

Since 0 belongs to $\mathcal{O}_{\delta}^{\prime}(\theta)$ these two segments are contained in $\mathcal{O}_{\delta}^{\prime}(\theta)$.

Let us prove the estimate on $\gamma$ given in (4.4.94).

If $\left|x-x\left(\theta^{*}, \alpha\right)\right| \leqslant\left|x\left(\theta^{*}, \alpha\right)\right|$ we have

$$
\begin{aligned}
\left|\sigma x+(1-\sigma) x\left(\theta^{*}, \alpha\right)-x\left(\theta_{2}, \alpha\right)\right| & \leqslant \sigma\left|x-x\left(\theta^{*}, \alpha\right)\right|+(1-\sigma)\left|x\left(\theta^{*}, \alpha\right)-x\left(\theta_{2}, \alpha\right)\right| \\
& \leqslant K\left|x-x\left(\theta_{2}, \alpha\right)\right| .
\end{aligned}
$$

If $\left|x-x\left(\theta^{*}, \alpha\right)\right|>\left|x\left(\theta^{*}, \alpha\right)\right|$ we have

$$
\begin{aligned}
\left|t x-x\left(\theta_{2}, \alpha\right)\right| & \leqslant t\left|x-x\left(\theta^{*}, \alpha\right)\right|+(1-t)\left|x\left(\theta^{*}, \alpha\right)\right|+\left|x\left(\theta^{*}, \alpha\right)-x\left(\theta_{2}, \alpha\right)\right| \\
& \leqslant\left|x-x\left(\theta^{*}, \alpha\right)\right|+\left|x\left(\theta^{*}, \alpha\right)-x\left(\theta_{2}, \alpha\right)\right| \\
& \leqslant K\left|x-x\left(\theta_{2}, \alpha\right)\right|
\end{aligned}
$$

again by Lemma 4.4.18. Moreover

$$
\begin{aligned}
\left|t x\left(\theta^{*}, \alpha\right)-x\left(\theta_{2}, \alpha\right)\right| & \leqslant\left|x\left(\theta_{2}, \alpha\right)-x\left(\theta^{*}, \alpha\right)\right|+(1-t)\left|x\left(\theta^{*}, \alpha\right)\right| \\
& \leqslant K\left|x-x\left(\theta_{2}, \alpha\right)\right| .
\end{aligned}
$$

Concerning the estimate on $\frac{\partial \gamma}{\partial \sigma}$, if $\left|x-x\left(\theta^{*}, \alpha\right)\right| \leqslant\left|x\left(\theta^{*}, \alpha\right)\right|$ it is straightforward by Lemma 4.4.18. If $\left|x\left(\theta^{*}, \alpha\right)\right| \leqslant\left|x-x\left(\theta^{*}, \alpha\right)\right|$ the same Lemma shows that $\left|x\left(\theta^{*}, \alpha\right)\right| \leqslant$ $K\left|\theta^{*}-\theta_{2}\right| \leqslant K\left|x\left(\theta^{*}, \alpha\right)-x\left(\theta_{2}, \alpha\right)\right|$ and $|x| \leqslant\left|x-x\left(\theta_{2}, \alpha\right)\right|+\left|x\left(\theta_{2}, \alpha\right)-x\left(\theta^{*}, \alpha\right)\right|+$ $\left|x\left(\theta^{*}, \alpha\right)\right| \leqslant K^{\prime}\left|x-x\left(\theta_{2}, \alpha\right)\right|$. Thus (4.4.94) is entirely proved.

Now for $j=3$ or 6 we can write

$$
\begin{aligned}
\varphi_{j}\left(\theta_{2}, x, \alpha\right) & =\varphi_{j}\left(\theta_{2}, x\left(\theta^{*}, \alpha\right), \alpha\right)+\int_{0}^{1} \frac{\partial \gamma}{\partial \sigma}(\sigma, x) \frac{\partial \varphi_{j}}{\partial x}\left(\theta_{2}, \gamma(\sigma, x), \alpha\right) d \sigma \\
& =\varphi_{j}\left(\theta_{2}, x\left(\theta^{*}, \alpha\right), \alpha\right)+\int_{0}^{1} \frac{\partial \gamma}{\partial \sigma}(\sigma, x) \Phi\left(\theta_{2}, \gamma(\sigma, x), \alpha\right) d \sigma+R_{j}
\end{aligned}
$$

where

$$
\left|R_{j}\right| \leqslant C_{N} \frac{\left|x-x\left(\theta_{2}, \alpha\right)\right|^{N+1}}{\left\langle\theta_{2}\right\rangle^{N}}
$$

by Proposition 4.4.14 and (4.4.94). By (4.4.91) and (4.4.92) we have

$$
\left|\left(\varphi_{3}-\varphi_{6}\right)\left(\theta_{2}, x, \alpha\right)\right| \leqslant C_{N} \frac{\left|x-x\left(\theta_{2}, \alpha\right)\right|^{N+1}}{\left\langle\theta_{2}\right\rangle^{N}} .
$$


Now for $\theta \in\left[\theta_{1}, \theta_{3}\right]$ and $j=3$ or 6 we can write

$$
\varphi_{j}(\theta, x, \alpha)=\varphi_{j}\left(\theta_{2}, x, \alpha\right)+\int_{0}^{1}\left(\theta-\theta_{2}\right) \frac{\partial \varphi_{j}}{\partial \theta}\left(\sigma \theta+(1-\sigma) \theta_{2}, x, \alpha\right) d \sigma
$$

so by Proposition 4.4.14 and Lemma 4.4.18 we have

$$
\varphi_{j}(\theta, x, \alpha)=\varphi_{j}\left(\theta_{2}, x, \alpha\right)-\int_{0}^{1}\left(\theta-\theta_{2}\right) p\left(x, \Phi\left(\sigma \theta+(1-\sigma) \theta_{2}, x, \alpha\right)\right) d \sigma+A
$$

where

$$
|A| \leqslant C_{N} \frac{\left|x-x\left(\sigma \theta+(1-\sigma) \theta_{2}, \alpha\right)\right|^{N}}{\left\langle\sigma \theta+(1-\sigma) \theta_{2}\right\rangle^{N}}
$$

Proposition 4.4.19

$$
\left\{\begin{array}{l}
\text { For all } \theta \text { in }\left[\theta_{1}, \theta_{3}\right] \text { and all } \sigma \text { in }[0,1] \text { we have } \\
(i)\left\langle\sigma \theta+(1-\sigma) \theta_{2}\right\rangle \geqslant K_{1}\langle\theta\rangle \\
\text { (ii) }\left|x-x\left(\sigma \theta+(1-\sigma) \theta_{2}, \alpha\right)\right| \leqslant K_{2}|x-x(\theta, \alpha)| .
\end{array}\right.
$$

Proof

Case 1: $\theta \in\left[\theta_{2}, \theta_{3}\right]$. - We write with $\theta^{\sigma}=\sigma \theta+(1-\sigma) \theta_{2}$,

$$
\left\{\begin{array}{c}
|x-x(\theta, \alpha)|^{2}=I+I I \quad \text { where } \\
I=\left|x-x\left(\theta^{\sigma}, \alpha\right)\right|^{2}+\left|x\left(\theta^{\sigma}, \alpha\right)-x(\theta, \alpha)\right|^{2} \\
I I=2\left(x-x\left(\theta^{\sigma}, \alpha\right)\right) \cdot\left(x\left(\theta^{\sigma}, \alpha\right)-x(\theta, \alpha)\right) .
\end{array}\right.
$$

Since $x(\theta, x)-x\left(\theta^{\sigma}, \alpha\right)=\int_{\theta^{\sigma}}^{\theta} \dot{x}(s, \alpha) d s=2\left(\theta-\theta^{\sigma}\right) \alpha_{\xi}+\mathcal{O}\left(\varepsilon\left|\theta-\theta^{\sigma}\right|\right)$ we have $I I=$ $-4\left(\theta-\theta^{\sigma}\right)\left(x-x\left(\theta^{\sigma}, \alpha\right)\right) \cdot \alpha_{\xi}+\mathcal{O}(\varepsilon I)$. Now in $\mathcal{O}_{\delta}^{\prime}$ we have $x \cdot \alpha_{\xi} \leqslant \frac{c_{0}}{20}\langle x\rangle\left|\alpha_{\xi}\right| ;$ moreover by Lemma 4.4.18 (i) we have $x\left(\theta^{\sigma}, \alpha\right) \cdot \alpha_{\xi} \geqslant \frac{12 c_{0}}{30}\left\langle x\left(\theta^{\sigma}, \alpha\right)\right\rangle\left|\alpha_{\xi}\right|$ since $\theta^{\sigma} \geqslant$ $\theta_{2}=\theta\left(\frac{12 c_{0}}{30}\right)$. It follows that $I I \geqslant-\frac{c_{0}}{5}\left(\theta-\theta^{\sigma}\right)\langle x\rangle\left|\alpha_{\xi}\right|+\frac{8 c_{0}}{5}\left\langle x\left(\theta^{\sigma}, \alpha\right)\right\rangle\left|\alpha_{\xi}\right|-\mathcal{O}(\varepsilon I)$. Therefore we obtain

$$
\begin{aligned}
I I \geqslant-\frac{c_{0}}{5}\left(\theta-\theta^{\sigma}\right) & \left\langle x\left(\theta^{\sigma}, \alpha\right)\right\rangle\left|\alpha_{\xi}\right| \\
& -\frac{c_{0}}{5}\left(\theta-\theta^{\sigma}\right)\left|x-x\left(\theta^{\sigma}, \alpha\right)\right|\left|\alpha_{\xi}\right|+\frac{8 c_{0}}{5}\left\langle x\left(\theta^{\sigma}, \alpha\right)\right\rangle\left|\alpha_{\xi}\right|-\mathcal{O}(\varepsilon I) .
\end{aligned}
$$

The second term in the right hand side can be bounded by $\frac{c_{0}}{10} I$. Using (4.4.98) we obtain

$$
|x-x(\theta, \alpha)|^{2}=I+I I \geqslant\left(1-\frac{c_{0}}{10}-\varepsilon K\right) I+\frac{7 c_{0}}{5}\left\langle x\left(\theta^{\sigma}, \alpha\right)\right\rangle\left|\alpha_{\xi}\right| .
$$

Taking $c_{0}$ and $\varepsilon$ small enough we obtain $I \leqslant 2|x-x(\theta, \alpha)|^{2}$ which implies since $\left|\theta-\theta^{\sigma}\right| \leqslant 2\left|x(\theta, \alpha)-x\left(\theta^{\sigma}, \alpha\right)\right| \leqslant 2 \sqrt{I}$, that $\left|\theta-\theta^{\sigma}\right| \leqslant 2|x-x(\theta, \alpha)| \leqslant 2 \delta\langle\theta\rangle$ so $\langle\theta\rangle \leqslant\left\langle\theta^{\sigma}\right\rangle+2 \delta\langle\theta\rangle$ and therefore $\left\langle\theta^{\sigma}\right\rangle \geqslant \frac{1}{2}\langle\theta\rangle$ since $\delta$ is small. This proves the claim (i) of (4.4.97).

To prove (ii) we just use the fact that

$$
\left|x(\theta, \alpha)-x\left(\theta^{\sigma}, \alpha\right)\right| \leqslant 3\left|\theta-\theta^{\sigma}\right| \leqslant 6|x-x(\theta, \alpha)| .
$$


Case 2: $\theta \in\left[\theta_{1}, \theta_{2}\right]$. - The point (i) in (4.4.97) is obvious in this case since $\sigma \theta+$ $(1-\sigma) \theta_{2} \geqslant \theta$.

By the same computation as above, since $\theta \geqslant \theta_{1}$, we will have

$$
\left\{\begin{array}{l}
|x-x(\theta, \alpha)| \geqslant \frac{1}{2}\left(\left|x-x\left(\theta_{1}, \alpha\right)\right|+\left|x\left(\theta_{1}, \alpha\right)-x(\theta, \alpha)\right|\right), \\
\frac{1}{2}\left|\theta-\theta_{1}\right| \leqslant\left|x(\theta, \alpha)-x\left(\theta_{1}, \alpha\right)\right| \leqslant 6\left|\theta-\theta_{1}\right| .
\end{array}\right.
$$

On the other hand we claim that we have

$$
\frac{9 c_{0}}{30}\langle x(\theta, \alpha)\rangle\left|\alpha_{\xi}\right| \leqslant 3|x-x(\theta, \alpha)| .
$$

Indeed we have

$$
(x-x(\theta, \alpha)) \cdot \alpha_{\xi}=x \cdot \alpha_{\xi}-x(\theta, \alpha) \cdot \alpha_{\xi} \leqslant \frac{c_{0}}{20}\langle x\rangle\left|\alpha_{\xi}\right|-\frac{11 c_{0}}{30}\langle x(\theta, \alpha)\rangle\left|\alpha_{\xi}\right|
$$

by Lemma 4.4.18 (i) since $\theta \geqslant \theta_{1}=\theta\left(\frac{11 c_{0}}{30}\right)$. Thus

$$
(x-x(\theta, \alpha)) \cdot \alpha_{\xi} \leqslant \frac{c_{0}}{20}\langle x(\theta, \alpha)\rangle\left|\alpha_{\xi}\right|+\frac{c_{0}}{20}|x-x(\theta, \alpha)|\left|\alpha_{\xi}\right|-\frac{11 c_{0}}{30}\langle x(\theta, \alpha)\rangle\left|\alpha_{\xi}\right| .
$$

It follows that

$$
\frac{9 c_{0}}{30}\langle x(\theta, \alpha)\rangle\left|\alpha_{\xi}\right| \leqslant \frac{c_{0}}{20}|x-x(\theta, \alpha)|\left|\alpha_{\xi}\right|-(x-x(\theta, \alpha)) \cdot \alpha_{\xi}
$$

from which (4.4.100) follows easily since $\left|\alpha_{\xi}\right| \leqslant 2$ and $\frac{c_{0}}{20}\left|\alpha_{\xi}\right| \leqslant 1$. Now $\theta^{\sigma}=\sigma \theta+$ $(1-\sigma) \theta_{2}$ belongs to $\left[\theta_{1}, \theta_{2}\right]$ for $\sigma \in[0,1]$. Since by Lemma 4.4 .18 (iii) the function $\theta(c)$ is strictly increasing there exists a unique $c_{\sigma} \in\left[\frac{11 c_{0}}{30}, \frac{12 c_{0}}{30}\right]$ such that $\theta^{\sigma}=\theta\left(c_{\sigma}\right)$. Now we have $\left|x-x\left(\theta^{\sigma}, \alpha\right)\right| \leqslant\left|x-x\left(\theta_{1}, \alpha\right)\right|+\left|x\left(\theta_{1}, \alpha\right)-x\left(\theta^{\sigma}, \alpha\right)\right|$ which implies

$$
\left|x-x\left(\theta^{\sigma}, \alpha\right)\right| \leqslant\left|x-x\left(\theta_{1}, \alpha\right)\right|+6\left|\theta\left(\frac{11 c_{0}}{30}\right)-\theta\left(c_{\sigma}\right)\right| .
$$

We claim that

$$
\left|\theta\left(\frac{11 c_{0}}{30}\right)-\theta\left(c_{\sigma}\right)\right| \leqslant \frac{c_{0}}{15} \sup _{s \in\left[\theta_{1}, \theta_{2}\right]}\langle x(s, \alpha)\rangle .
$$

To see this we compute $\theta^{\prime}(c)$. Recall (see Lemma 4.4.18) that $g(\theta(c), c)=0$ for $c \in\left[\frac{c_{0}}{10}, \frac{c_{0}}{2}\right]$.It follows that $\frac{\partial g}{\partial s}(\theta(c), c) \theta^{\prime}(c)+\frac{\partial g}{\partial c}(\theta(c), c)=0$. Now we have $\frac{\partial g}{\partial c}(s, c)=$ $-\langle x(s, \alpha)\rangle\left|\alpha_{\xi}\right|$ and $\frac{\partial g}{\partial s}(s, c)=\dot{x}(s, \alpha) \cdot \alpha_{\xi}-c \frac{x(s, \alpha) \cdot \dot{x}(s, \alpha)}{\langle x(s, \alpha)\rangle}\left|\alpha_{\xi}\right|$, which shows that $\frac{\partial g}{\partial s}(s, c)=2\left(\left|\alpha_{\xi}\right|^{2}+\mathcal{O}(\varepsilon+c)\right.$. Therefore we have $\left|\theta^{\prime}(c)\right| \leqslant 2\langle x(\theta(c), \alpha\rangle$ and we obtain (4.4.102). The last step consists in showing that

$$
\sup _{\theta \in\left[\theta_{1}, \theta_{2}\right]}\langle x(s, \alpha)\rangle \leqslant 2\langle x(\theta, \alpha)\rangle .
$$

To see this let us set $h(s)=\langle x(s, \alpha)\rangle$. Then $h^{\prime}(s)=\frac{x(s, \alpha) \cdot \dot{x}(s, \alpha)}{\langle x(s, \alpha)\rangle}$. Thus $h^{\prime}(s)=$ $\frac{2 x(s, \alpha) \cdot \alpha_{\xi}}{\langle x(s, \alpha)\rangle}+\mathcal{O}(\varepsilon)$. Now since $s \in\left[\theta_{1}, \theta_{2}\right]$ we have $\frac{11 c_{0}}{30}\langle x(s, \alpha)\rangle\left|\alpha_{\xi}\right| \leqslant x(s, \alpha)$. $\alpha_{\xi} \leqslant \frac{12 c_{0}}{30}\langle x(s, \alpha)\rangle\left|\alpha_{\xi}\right|$ so $0<h^{\prime}(s) \leqslant 2 c_{0}+\mathcal{O}(\varepsilon)$ and therefore if $s_{1}, s_{2} \in\left[\theta_{1}, \theta_{2}\right]$, 
$\left|h\left(s_{1}\right)-h\left(s_{2}\right)\right| \leqslant\left(2 c_{0}+\mathcal{O}(\varepsilon)\right)\left|s_{1}-s_{2}\right|$. Let us take $s_{1}=\theta(c), s_{2}=\theta\left(c^{\prime}\right)$ with $\frac{11 c_{0}}{30} \leqslant c$, $c^{\prime} \leqslant \frac{12 c_{0}}{30}$. Then

$$
\left|\langle x(\theta(c), \alpha)\rangle-\left\langle x\left(\theta\left(c^{\prime}\right), \alpha\right)\right\rangle\right| \leqslant\left(2 c_{0}+\mathcal{O}(\varepsilon)\right)\left|\theta(c)-\theta\left(c^{\prime}\right)\right| .
$$

On the other hand

$$
x(\theta(c), \alpha)-x\left(\theta\left(c^{\prime}\right), \alpha\right)=2\left(\theta(c)-\theta\left(c^{\prime}\right)\right) \cdot \alpha_{\xi}+\mathcal{O}\left(\varepsilon\left|\theta(c)-\theta\left(c^{\prime}\right)\right|\right)
$$

which implies that

$$
x(\theta(c), \alpha) \cdot \alpha_{\xi}-x\left(\theta\left(c^{\prime}\right), \alpha\right) \cdot \alpha_{\xi}=2\left(\theta(c)-\theta\left(c^{\prime}\right)\right)\left|\alpha_{\xi}\right|^{2}+\mathcal{O}\left(\varepsilon\left|\theta(c)-\theta\left(c^{\prime}\right)\right|\right.
$$

and, by definition of $\theta(c)$ (see Lemma 4.4.18)

$$
\left[c\left\langle x(\theta(c), \alpha\rangle-c^{\prime}\left\langle x\left(\theta\left(c^{\prime}\right), \alpha\right)\right\rangle\right]\left|\alpha_{\xi}\right|=2\left(\theta(c)-\theta\left(c^{\prime}\right)\right)\left|\alpha_{\xi}\right|^{2}+\mathcal{O}\left(\varepsilon\left|\theta(c)-\theta\left(c^{\prime}\right)\right|\right) .\right.
$$

Combining with (4.4.104) we obtain

$$
\begin{aligned}
\mid\langle x(\theta(c), \alpha)- & \left\langle x\left(\theta\left(c^{\prime}\right), \alpha\right)\right\rangle \mid \\
& \leqslant 2\left(2 c_{0}+\mathcal{O}(\varepsilon)\right)\left[c \mid\langle x(\theta(c), \alpha)\rangle-\left\langle x\left(\theta\left(c^{\prime}\right), \alpha\right\rangle|+| c-c^{\prime}\right|\left\langle x\left(\theta\left(c^{\prime}\right), \alpha\right\rangle\right] .\right.
\end{aligned}
$$

Since $c_{0}$ and $\varepsilon$ are small enough we obtain

$$
\left|\langle x(\theta(c), \alpha)\rangle-\left\langle x\left(\theta\left(c^{\prime}\right), \alpha\right)\right\rangle\right| \leqslant 2\left|c-c^{\prime}\right|\left\langle x\left(\theta\left(c^{\prime}\right), \alpha\right\rangle \leqslant \frac{c_{0}}{15}\left\langle x\left(\theta\left(c^{\prime}\right), \alpha\right)\right\rangle\right.
$$

which shows that all the $\langle x(\theta(c), \alpha)\rangle$ are equivalent in $\left[\theta_{1}, \theta_{2}\right]$, more precisely taking $s=\theta\left(c^{\prime}\right), \theta=\theta(c)$ we obtain

$$
\sup _{s \in\left[\theta_{1}, \theta_{2}\right]}\langle x(s, \alpha)\rangle \leqslant 2\langle x(\theta, \alpha)\rangle,
$$

which is (4.4.103).

Finally using (4.4.101), (4.4.99), (4.4.102), (4.4.103) and (4.4.100) we obtain

$$
\left|x-x\left(\theta^{\sigma}, \alpha\right)\right| \leqslant K|x-x(\theta, \alpha)|
$$

which is Proposition 4.4 .19 (iii) in the case 2.

Now using Proposition 4.4.19, (4.4.95), (4.4.96) we obtain

$$
\begin{gathered}
\left|\left(\varphi_{3}-\varphi_{6}\right)\left(\theta_{2}, x, \alpha\right)\right| \leqslant C_{N} \frac{|x-x(\theta, \alpha)|^{N+1}}{\langle\theta\rangle^{N}} \\
\left\{\begin{array}{l}
\varphi_{j}(\theta, x, \alpha)=\varphi_{j}\left(\theta_{2}, x, \alpha\right)-\int_{0}^{1} p\left(x, \Phi\left(\theta^{\sigma}, x, \alpha\right) d \sigma+A, \quad j=3,6,\right. \\
|A| \leqslant C_{N} \frac{|x-x(\theta, \alpha)|^{N+1}}{\langle\theta\rangle^{N}} .
\end{array}\right.
\end{gathered}
$$

Let now $\chi_{2} \in C^{\infty}(\mathbb{R})$ be such that $\chi_{2}(s)=1$ if $s \geqslant 1, \chi_{2}(s)=0$ if $s \leqslant 0$ and set $\chi_{3}(\theta)=\chi_{2}\left(\frac{\theta-\theta_{1}}{\theta_{3}-\theta_{1}}\right)$. Then let us set

$$
\varphi(\theta, x, \alpha)=\chi_{3}(\theta) \varphi_{3}(\theta, x, \alpha)+\left(1-\chi_{3}(\theta)\right) \varphi_{6}(\theta, x, \alpha) .
$$


We have

$\frac{\partial \varphi}{\partial \theta}(\theta, x, \alpha)=\chi_{3}(\theta) \frac{\partial \varphi_{3}}{\partial \theta}(\theta, x, \alpha)+\left(1-\chi_{3}(\theta)\right) \frac{\partial \varphi_{6}}{\partial \theta}(\theta, x, \alpha)+\frac{\partial \chi_{3}}{\partial \theta}(\theta)\left(\varphi_{3}-\varphi_{6}\right)(\theta, x, \alpha)$.

Now we deduce from (4.4.105) and (4.4.106) that on the support of $\frac{\partial \chi_{3}}{\partial \theta}$ we have

$$
\left|\left(\varphi_{3}-\varphi_{6}\right)(\theta, x, \alpha)\right| \leqslant C_{N} \frac{|x-x(\theta, \alpha)|^{N+1}}{\langle\theta\rangle^{N}} .
$$

By Proposition 4.4.14 for $\varphi_{3}$ and $\varphi_{6}$ we have for $j=3$ or 6 ,

$$
\left|\frac{\partial \varphi_{j}}{\partial x}(\theta, x, \alpha)-\Phi(\theta, x, \alpha)\right| \leqslant C_{N} \frac{|x-x(\theta, \alpha)|^{N}}{\langle\theta\rangle^{N}}
$$

therefore this is also true for $\varphi$ and since for $j=3$ or 6 ,

$$
\left|\frac{\partial \varphi_{j}}{\partial \theta}(\theta, x, \alpha)-p(x, \Phi(\theta, x, \alpha))\right| \leqslant C_{N} \frac{|x-x(\theta, \alpha)|^{N}}{\langle\theta\rangle^{N}},
$$

the function $\varphi$ defined in (4.4.107) satisfies all the requirements of Proposition 4.4.14.

The proof of Proposition 4.4.14 will be therefore complete when we will construct the path $\gamma(\sigma, x)$ satisfying (4.4.74) to (4.4.79).

Construction of $\gamma(\sigma, x)$. - Let us set $a=x(\theta, \alpha)$. We first show that we can join any point $x$ to a point $a-|x-a| \alpha_{\xi}$ by path remaining in the set

$$
\left\{y \in \mathbb{R}^{n}: y \cdot \alpha_{\xi} \leqslant \frac{c_{0}}{10}\langle y\rangle\left|\alpha_{\xi}\right|,|y-a|=|x-a|\right\} .
$$

Making rotations we may without loss of generality assume that $\frac{\alpha_{\xi}}{\left|\alpha_{\xi}\right|}=(-1,0, \ldots, 0)$, $a=\left(a_{1}, a_{2}, 0, \ldots, 0\right), x=\left(x_{1}, x_{2}, x_{3}, 0, \ldots, 0\right)$. Therefore it will be sufficient to restrict ourselves to the dimension three. We will construct our path on planes so we begin by the dimension two. Let us set with $D \in] 0,1[, k>0$,

$$
\begin{aligned}
\mathcal{C} & =\left\{y \in \mathbb{R}^{2}:|y-a|^{2}=|x-a|^{2}\right\}, \\
\mathcal{H} & =\left\{y \in \mathbb{R}^{2}:-y_{1}=D \sqrt{k^{2}+y_{2}^{2}}\right\}, \\
\mathcal{D} & =\left\{y \in \mathbb{R}^{2}:-y_{1} \leqslant D \sqrt{k^{2}+y_{2}^{2}}\right\} .
\end{aligned}
$$

Lemma 4.4.20. $-\mathcal{D}^{c}=\mathbb{R}^{2} \backslash \mathcal{D}$ is strictly convex.

Proof. - This follows easily from the strict convexity of the function $g(t)=\sqrt{k^{2}+t^{2}}$.

LEMma 4.4 .21

(i) Let $b \in \mathcal{D}$ and $u=(1, y)$ with $|y| \leqslant 1$. Then for all $t>0$ we have $b+t u \in \mathcal{D} \backslash \mathcal{H}$.

(ii) Let $b \in \overline{\mathcal{D}^{c}}$ and $v=(-1, y)$ with $|y| \leqslant 1$. Then for all $t>0$ we have $b+t v \in \mathcal{D}^{c}$. 
Proof

(i) Let $b=\left(b_{1}, b_{2}\right)$ and $h(t)=b_{1}+t+D \sqrt{k^{2}+\left(b_{2}+t y\right)}$. Then

$$
h(0)=b_{1}+D \sqrt{k^{2}+b_{2}^{2}} \geqslant 0 \quad \text { and } \quad h^{\prime}(t)=1+\frac{D\left(b_{2}+t y\right) y}{\sqrt{k^{2}+\left(b_{2}+t y\right)^{2}}} .
$$

Since $D|y|<1$ we have $D\left|b_{2}+t y\right||y| / \sqrt{k^{2}+\left(b_{2}+t y\right)^{2}}<1$. It follows that $h^{\prime}(t)>0$ so $h(t)>h(0) \geqslant 0$.

The proof of (ii) is the same.

Assume that $\mathcal{C} \cap \mathcal{H}$ contains at least two different points (otherwise $\mathcal{C} \backslash(\mathcal{C} \cap \mathcal{H})$ would be connected). Let us set

$$
\left\{\begin{array}{l}
M_{\theta}=a+|x-a|\left(\begin{array}{c}
\cos \theta \\
\sin \theta
\end{array}\right), \theta \in[0,2 \pi[, \\
\theta_{1}=\inf \left\{\theta \in \left[0,2 \pi\left[: M_{\theta} \in \mathcal{C} \cap \mathcal{H}\right\}\right.\right. \\
\theta_{2}=\sup \left\{\theta \in \left[0,2 \pi\left[: M_{\theta} \in \mathcal{C} \cap \mathcal{H}\right\} .\right.\right.
\end{array}\right.
$$

REMARK 4.4.22

(i) If $\theta \in\left[0, \frac{\pi}{4}\right] \cup\left[2 \pi-\frac{\pi}{4}, 2 \pi\right]$ we have $\overrightarrow{a M_{\theta}}=|x-a|\left(\begin{array}{c}\cos \theta \\ \sin \theta\end{array}\right)$ with $\cos \theta>0$ and $\left|\frac{\sin \theta}{\cos \theta}\right| \leqslant 1$. Since $a \in \mathcal{D}$ Lemma 4.4 .21 (i) implies that $M_{\theta} \in \mathcal{D} \backslash \mathcal{H}$. It follows that we have

$$
\frac{\pi}{4}<\theta_{1}<\theta_{2}<2 \pi-\frac{\pi}{4}
$$

(ii) We cannot have $\left.\left.\theta_{1} \in\right] \frac{\pi}{4}, \frac{\pi}{2}\right]$ and $\theta_{2} \in\left[\frac{3 \pi}{2}, 2 \pi-\frac{\pi}{4}[\right.$. Indeed if this was true then by Lemma 4.4 .20 the segment $] M_{\theta_{1}}, M_{\theta_{2}}$ [ would be in $\mathcal{D}^{c}$. But $\sin \theta_{1}>0$ and $\sin \theta_{2}<0$ so there exists $\left.t \in\right] 0,1\left[\operatorname{such}\right.$ that $t \sin \theta_{1}+(1-t) \sin \theta_{2}=0$; then

$$
N_{t}=t M_{\theta_{1}}+(1-t) M_{\theta_{2}}=a+|x-a|\left(\begin{array}{c}
t \cos \theta_{1}+(1-t) \cos \theta_{2} \\
0
\end{array}\right)=a+\alpha\left(\begin{array}{l}
1 \\
0
\end{array}\right)
$$

with $\alpha>0$ since $\cos \theta_{1}>0$ and $\cos \theta_{2}>0$. By Lemma 4.4.21 (i) $N_{t} \in \mathcal{D}$ since $a \in \mathcal{D}$ which is in contradiction with $\left.N_{t} \in\right] M_{\theta_{1}}, M_{\theta_{2}}\left[\subset \mathcal{D}^{c}\right.$.

(iii) If $\left.\left.\theta_{1} \in\right] \frac{\pi}{4}, \frac{\pi}{2}\right]$ then for all $\theta$ in $\left.] \theta_{1}, \pi\right]$ we have $M_{\theta} \in \mathcal{D}^{c}$ which implies $\theta_{2} \in$ ]$\left.\pi, \frac{3 \pi}{2}\right]$ by (ii). Indeed we have $\overrightarrow{M_{\theta_{1}} M_{\theta}}=|x-a|\left(\begin{array}{c}\cos \theta-\cos \theta_{1} \\ \sin \theta-\sin \theta_{1}\end{array}\right)$; since for $\theta \in[0, \pi]$, $\cos \theta$ is decreasing we have $\cos \theta-\cos \theta_{1}<0$ and

$$
\left|\frac{\sin \theta-\sin \theta_{1}}{\cos \theta-\cos \theta_{1}}\right|=\left|\operatorname{cotg}\left(\frac{\theta+\theta_{1}}{2}\right)\right| \leqslant 1
$$

since $\frac{\pi}{4} \leqslant \frac{\theta+\theta_{1}}{2} \leqslant \frac{3 \pi}{4}$. Then Lemma 4.4.21 (ii) implies that $M_{\theta} \in \mathcal{D}^{c}$.

It follows from Remark 4.4.22, (i), (ii), (iii) that we have else $\left.\theta_{1} \in\right] \frac{\pi}{2}, \pi[$ or $\left.\theta_{2} \in\right] \pi, \frac{3 \pi}{2}[$. By symmetry it is enough to consider one case. Therefore we shall assume in the sequel that $\left.\frac{\pi}{2}<\theta_{1}<\theta_{2}<2 \pi-\frac{\pi}{4}, \theta_{1} \in\right] \frac{\pi}{2}, \pi[$. We claim that

$$
\left.M_{\theta} \in \mathcal{D}^{c} \text { for all } \theta \text { in }\right] \theta_{1}, \theta_{2}[\text {. }
$$

We split the proof in two cases. 
Case 1: $\theta_{2} \leqslant \frac{3 \pi}{2}$. - Since $\sin \theta$ is decreasing on $\left[\frac{\pi}{2}, \frac{3 \pi}{2}\right]$ we have

- if $\frac{\pi}{2}<\theta_{1}<\theta_{2} \leqslant \frac{3 \pi}{2}, \sin \theta_{1}>\sin \theta>\sin \theta_{2}$

- if $\frac{\pi}{2}<\theta_{1}<\theta<3 \pi-\theta_{2} \leqslant \frac{3 \pi}{2}, \sin \theta_{1}>\sin \theta>\sin \left(3 \pi-\theta_{2}\right)=\sin \theta_{2}$.

Let us set

$$
N_{t}=t M_{\theta_{1}}+(1-t) M_{\theta_{2}}=a+|x-a|\left(\begin{array}{c}
t \cos \theta_{1}+(1-t) \cos \theta_{2} \\
t \sin \theta_{1}+(1-t) \sin \theta_{2}
\end{array}\right) .
$$

Now there exists $t \in] 0,1[$ such that

$$
t \sin \theta_{1}+(1-t) \sin \theta_{2}=\sin \theta
$$

and since $\theta \in\left[\frac{\pi}{2}, \frac{3 \pi}{2}\right]$ we have $\theta=\operatorname{Arcsin}\left(t \sin \theta_{1}+(1-t) \sin \theta_{2}\right)+\pi$. Then

$$
\begin{aligned}
& \cos \theta=-\cos \left(\operatorname{Arcsin}\left(t \sin \theta_{1}+(1-t) \sin \theta_{2}\right)\right)=-\sqrt{1-\left(t \sin \theta_{1}+(1-t) \sin \theta_{2}\right)^{2}}, \\
& \cos \theta<-t \sqrt{1-\sin ^{2} \theta_{1}}-\left(1-t \sqrt{1-\sin ^{2} \theta_{2}} \leqslant t \cos \theta_{1}+(1-t) \cos \theta_{2} .\right.
\end{aligned}
$$

Here we have used the strict convexity of the function $\sqrt{1-x^{2}}$. Since $N_{t} \in \mathcal{D}^{c}$ and $M_{\theta}=N_{t}+\alpha\left(\begin{array}{c}-1 \\ 0\end{array}\right)$ where $\alpha>0$ (see (4.4.108)) we deduce from Lemma 4.4.21 (ii) that $M_{\theta} \in \mathcal{D}^{c}$ which proves (4.4.109) in case 1.

Case 2: $\theta_{2}>\frac{3 \pi}{2}$ and $\theta<3 \pi-\theta_{2}$. - Since $\theta_{2}<2 \pi$ we have $\theta>\pi$. Now by (4.4.108),

$$
\overrightarrow{M_{\theta_{2}} M_{\theta}}=|x-a|\left(\begin{array}{c}
\cos \theta-\cos \theta_{2} \\
\sin \theta-\sin \theta_{2}
\end{array}\right) .
$$

Since $\cos \theta$ is increasing for $\theta \in[\pi, 2 \pi]$ we have $\cos \theta-\cos \theta_{2}<0$. Moreover, $\left|\frac{\sin \theta-\sin \theta_{2}}{\cos \theta-\cos \theta_{2}}\right|=\left|\operatorname{cotg} \frac{\theta+\theta_{2}}{2}\right| \leqslant 1$ since $\frac{\theta+\theta_{2}}{2} \geqslant \frac{3 \pi}{2}, \theta \leqslant 2 \pi-\frac{\pi}{4}, \theta_{2} \leqslant 2 \pi-\frac{\pi}{4}$ so $\frac{\theta+\theta_{2}}{2} \leqslant 2 \pi-\frac{\pi}{4}$. It follows from Lemma 4.4.21 (ii) that $M_{\theta} \in \mathcal{D}^{c}$ which proves (4.4.109) in case 2 .

We conclude that if $x \in \mathcal{C} \cup(\mathcal{D} \backslash \mathcal{H})$ then $x=a+|x-a|\left(\begin{array}{c}\cos \theta \\ \sin \theta\end{array}\right)$ with $\left.\theta \notin\right] \theta_{1}, \theta_{2}[$ and there exists a path joining the point $x$ to the point $a+|x-a|\left(\begin{array}{l}1 \\ 0\end{array}\right)$ with length less than $2 \pi|x-a|$.

Construction of the path in dimension 3. - We have $\alpha_{\xi}=(-1,0,0), a=\left(a_{1}, a_{2}, 0\right)$, $x=\left(x_{1}, x_{2}, x_{3}\right)$ and $-a \leqslant D_{0} \sqrt{1+|a|^{2}},-x_{1} \leqslant D_{0} \sqrt{1+|x|^{2}}$ with $D_{0}=\frac{c_{0}}{10}$. We first construct a path in the plane $y_{3}=x_{3}$. We set

$$
\begin{aligned}
\mathcal{D} & =\left\{\left(y_{1}, y_{2}, x_{3}\right):-y_{1} \leqslant D_{0} \sqrt{1+\left|x_{3}\right|^{2}+y_{1}^{2}+y_{2}^{2}}\right\} \\
& =\left\{\left(y_{1}, y_{2}, x_{3}\right):-y_{1} \leqslant \frac{D_{0}}{\sqrt{1-D_{0}^{2}}} \sqrt{1+\left|x_{3}\right|^{2}+y_{2}^{2}}\right\} .
\end{aligned}
$$

Since $c_{0}$ is small enough we have $\frac{D_{0}}{\sqrt{1-D_{0}^{2}}}<1$. By the same way we see that the point $a$ is such that $-a_{1} \leqslant \frac{D_{0}}{\sqrt{1-D_{0}^{2}}} \sqrt{1+a_{2}^{2}}$. Therefore $\widetilde{a}=\left(a_{1}, a_{2}, x_{3}\right) \in \mathcal{D}$. Since $x \in \mathcal{D}$, by the construction made in two dimensions there exists a path lying in the 
set $\left\{y: y_{3}=x_{3},|y-\widetilde{a}|=|x-\widetilde{a}|=\sqrt{\left(x_{1}-a_{1}\right)^{2}+\left(x_{2}-a_{2}\right)^{2}}\right\}$ joining $x$ to $z=$ $\left(a_{1}+\sqrt{\left(x_{1}-a_{1}\right)^{2}+\left(x_{2}-a_{2}\right)^{2}}, a_{2}, x_{3}\right)$ of length smaller than $2 \pi|x-\widetilde{a}| \leqslant 2 \pi|x-a|$.

Let us construct now a path in the plane $y_{2}=a_{2}$. Let us set

$$
\begin{aligned}
\mathcal{D} & =\left\{y=\left(y_{1}, a_{2}, y_{3}\right):-y_{1} \leqslant D_{0} \sqrt{1+y_{1}^{2}+a_{2}^{2}+y_{3}^{2}}\right\} \\
& =\left\{y=\left(y_{1}, a_{2}, y_{3}\right):-y_{1} \leqslant \frac{D_{0}}{\sqrt{1-D_{0}^{2}}} \sqrt{1+a_{2}^{2}+y_{3}^{2}}\right\} .
\end{aligned}
$$

We have $z \in \mathcal{D}, a \in \mathcal{D}$. There exists a path joining $z$ to $\left(a_{1}+|x-a|, a_{2}, 0\right)$ lying in the set $\left\{y=\left(y_{1}, y_{2}, y_{3}\right): y_{2}=a_{2},|y-a|=|x-a|\right\}$ with length smaller than $2 \pi|x-a|$.

Now to join 0 to $x$ we join 0 to $z_{1}=\left(a_{1}+|a|, a_{2}, 0\right)$ then $x$ to $z_{2}=\left(a_{1}+|x-a|, a_{2}, 0\right)$ and since the segment $\left[z_{1}, z_{2}\right]$ is included in $\mathcal{D}$ by Lemma 4.4 .21 (i), the path joins 0 to $x$ and its length is smaller than $C(|x-a|+|a|)$. Now by (4.4.78) we have $|a| \leqslant|x-a|$ or $|x-a| \leqslant|a| \leqslant 2|x-a|$, so the length of the path is smaller than $C|x-a|=c|x-x(\theta, \alpha)|$. Moreover $|\gamma(\sigma, x)-a| \leqslant 2|x-a| \leqslant \frac{\delta}{20}\langle\theta\rangle$ so (4.4.77) is satisfied. Finally (4.4.78) and (4.4.79) are obviously satisfied.

This ends the proof of Proposition 4.4.14.

\subsection{The phase for small $\theta$}

We shall need the following precision on the phase when $|\theta| \leqslant 1$.

Theorem 4.5.1. - Let $\varphi$ be the phase given by Theorem 4.1.2. Then one can find positive constants such that for $|\theta| \leqslant 1,|x-x(\theta, \alpha)| \leqslant \delta\langle\theta\rangle$ and $\left|\alpha_{\xi}\right| \leqslant 2$ one can write

$$
\varphi(\theta, x, \alpha)=\frac{\left(x-\alpha_{x}\right) \cdot \alpha_{\xi}-\theta\left|\alpha_{\xi}\right|^{2}+\frac{i}{2}\left|x-\alpha_{x}\right|^{2}}{1+2 i \theta}+\frac{1}{2 i}\left|\alpha_{\xi}\right|^{2}+R(\theta, x, \alpha) ;
$$

where

$$
\begin{aligned}
& \left|\frac{\partial R}{\partial \alpha_{x}}\right| \leqslant C(\varepsilon+\delta)\left(\left|x-\alpha_{x}\right|^{2}+|\theta|\right), \quad\left|\frac{\partial R}{\partial \alpha_{\xi}}\right| \leqslant C(\varepsilon+\delta)|\theta|, \\
& \left|\frac{\partial^{2} R}{\partial \alpha_{x}^{2}}\right| \leqslant C(\varepsilon+\delta)\left(\left|x-\alpha_{x}\right|^{2}+|\theta|\right), \quad\left|\frac{\partial^{2} R}{\partial \alpha_{x} \partial \alpha_{\xi}}\right| \leqslant C(\varepsilon+\delta)|\theta|, \\
& \left|\frac{\partial^{2} R}{\partial \alpha_{\xi}^{2}}\right| \leqslant C(\varepsilon+\delta)|\theta|,
\end{aligned}
$$

and

$$
\left|\partial_{\alpha_{x}}^{A_{1}} \partial_{\alpha_{\xi}}^{A_{2}} R(\theta, x, \alpha)\right| \leqslant\left\{\begin{array}{lll}
C_{A_{1}} & \text { if } & A_{2}=0 \\
C_{A_{1}, A_{2}}|\theta| & \text { if } & \left|A_{2}\right| \geqslant 1
\end{array}\right.
$$

Proof. - Let us introduce the following space of functions.

$$
\left\{\begin{array}{l}
\mathcal{E}=\left\{Z \in C^{\infty}\left(\mathbb{R} \times \mathbb{R}^{n} \times \mathbb{R}^{n}\right):\left|\partial_{t}^{\ell} \partial_{x}^{A_{1}} \partial_{\xi}^{A_{2}} Z(t, x, \xi)\right| \leqslant C_{\ell, A_{1}, A_{2}} \varepsilon|t|^{1-\ell},\right. \text { for all } \\
\left.A_{j} \in \mathbb{N}^{n}, \quad \ell=0,1,|t| \leqslant 1, \quad x \in \mathbb{R}^{n}, \quad \xi \in \mathbb{R}^{n} \text { with }|\xi| \leqslant 2 \text { and } Z(0, x, \xi)=0\right\}
\end{array}\right.
$$


Let us also recall that Proposition 3.2.1 gives the following description of the flow for $|t| \leqslant 1, x \in \mathbb{R}^{n}, \xi \in \mathbb{R}^{n}$ with $|\xi| \leqslant 3$.

$$
\left\{\begin{array}{l}
x(t, x, \xi)=x+2 t \xi+r(t, x, \xi) \\
\xi(t, x, \xi)=\xi+\zeta(t, x, \xi) \\
z, \zeta \in \mathcal{E}
\end{array}\right.
$$

It follows that, with $f=x$ or $\xi$, we have

$$
\left|\partial_{t}^{\ell} \partial_{x}^{A_{1}} \partial_{\xi}^{A_{2}} f(t, x, \xi)\right| \leqslant C_{\ell, A_{1}, A_{2}} \text { if } \ell+\left|A_{1}\right|+\left|A_{2}\right| \geqslant 1 .
$$

Let us set now

$$
g_{j}(\eta)=\chi_{0}\left(\frac{\eta}{\mu_{0}}\right)\left[\left(\xi_{j}-i x_{j}\right)(-\theta, y+x(\theta, \alpha), \eta+\xi(\theta, \alpha))-\left(\alpha_{\xi}^{j}-i \alpha_{x}^{j}\right)\right]
$$

where $\chi_{0} \in C_{0}^{\infty}\left(\mathbb{R}^{n}\right), \chi_{0}(\eta)=1$ if $|\eta| \leqslant \frac{1}{2}, \chi_{0}(\eta)=0$ if $|\eta| \geqslant 1$ and $|y| \leqslant \delta$. Setting $x=y+x(\theta, \alpha), \xi=\eta+\xi(\theta, \alpha)$ and using (4.5.2) we obtain

$$
\begin{aligned}
g_{j}(\eta)=\chi_{0}(\eta)\left[(1+2 i \theta) \eta_{j}-i y_{j}+\right. & (1+2 i \theta) \zeta_{j}(\theta, \alpha)-i r_{j}(\theta, \alpha) \\
& \left.+(1+2 i \theta) \zeta_{j}(-\theta, x, \xi)-i z_{j}(-\theta, x, \xi)\right]
\end{aligned}
$$

We claim that we have the following estimates for $\ell=0,1$,

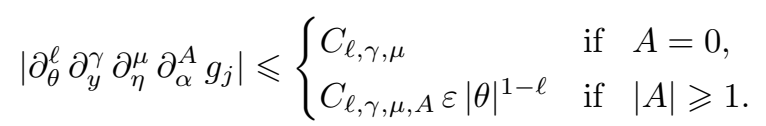

These estimates are obvious for the four first terms of $g_{j}$. So we are left with the estimate of

$$
(1)=\partial_{\theta}^{\ell} \partial_{y}^{\gamma} \partial_{\eta}^{\mu} \partial_{\alpha}^{A}[Z(-\theta, y+x(\theta, \alpha), \eta+\xi(\theta, \alpha))], \quad Z \in \mathcal{E} .
$$

To handle this term we shall make use of the Faa di Bruno formula given in Appendix A.1, with $F=Z, Y=(\theta, y, \eta, \alpha), U_{1}(Y)=-\theta, U_{1+j}(Y)=y_{j}+x_{j}(\theta, \alpha)$, $U_{1+n+j}(Y)=\eta_{j}+\xi_{j}(\theta, \alpha), j=1, \ldots, n$. Since $Z \in \mathcal{E}$ we find easily, using (4.5.3) that $(1) \leqslant C \varepsilon|\theta|^{1-\ell}$ which proves our claim.

Another property of $g_{j}$ which will be used in the sequel is the following.

$$
\text { For } \theta=0, g_{j}(\eta)=\chi_{0}(\eta)\left(\eta_{j}-i y_{j}\right) \text { is independent of } \alpha \text {. }
$$

Now according to our procedure we have solved the equations (see (4.3.14)),

$$
0=r\left(a, b, g_{j}\right)=g_{j}(-a)-i \sum_{k=1}^{n} \frac{\partial g_{j}}{\partial \eta_{k}}(-a) b_{k}+\sum_{p, q=1}^{n} H_{p q}^{j}(\theta, y, \alpha, a, b) b_{p} b_{q}
$$

in the set

$$
E=\left\{(a, b) \in \mathbb{R}^{n} \times \mathbb{R}^{n}:\left|a+\frac{2 \theta y}{1+4 \theta^{2}}\right| \leqslant \sqrt{\delta} \frac{|y|}{\langle\theta\rangle},\left|b+\frac{y}{1+4 \theta^{2}}\right| \leqslant \sqrt{\delta} \frac{|y|}{\langle\theta\rangle^{2}}\right\} .
$$


Let us recall that we have the following bounds on $H_{p q}^{j}$ (see (4.3.13) and (4.3.16))

$$
\left|\partial_{\theta}^{\ell} \partial_{y}^{\gamma} \partial_{\alpha}^{A} \partial_{(a, b)}^{M} H_{p, q}^{j}\right| \leqslant \sum_{|\mu| \leqslant|M|+3 n+2} \int\left|\partial_{\theta}^{\ell} \partial_{y}^{\gamma} \partial_{\alpha}^{A} \partial_{\eta}^{\mu} g_{j}(\eta)\right| d \eta, \quad \ell=0,1 .
$$

Here we have used the fact that $r\left(a, b, g_{j}\right)$ is linear with respect to $g_{j}$. It follows from (4.5.5), since $g_{j}$ has compact support in $\eta$, that

$$
\left|\partial_{\theta}^{\ell} \partial_{y}^{\gamma} \partial_{\alpha}^{A} \partial_{(a, b)}^{M} H_{p q}^{j}\right| \leqslant \begin{cases}C_{\ell, \gamma, M} & \text { if } A=0, \\ C_{\ell, \gamma, A, M} \varepsilon|\theta|^{1-\ell} & \text { if } \quad|A| \geqslant 1 .\end{cases}
$$

Using (4.5.4) we see easily that the equations (4.5.7) are equivalent to the following system

$$
\left\{\begin{aligned}
a_{j}=\frac{-2 \theta y_{j}}{1+4 \theta^{2}} & +Z_{j, 1}^{a}(\theta, \alpha)+Z_{j, 2}^{a}(-\theta, y+x(\theta, \alpha),-a+\xi(\theta, \alpha)) \\
& +Z_{j, 3}^{a}(-\theta, y+x(\theta, \alpha),-a+\xi(\theta, \alpha)) b+H_{j}^{a}(\theta, y, \alpha, a, b) b \cdot b \\
b_{j}=\frac{-y_{j}}{1+4 \theta^{2}} & +Z_{j, 1}^{b}(\theta, \alpha)+Z_{j, 2}^{b}(-\theta, y+x(\theta, \alpha),-a+\xi(\theta, \alpha)) \\
& +Z_{j, 3}^{b}(-\theta, y+x(\theta, \alpha),-a+\xi(\theta, \alpha)) b+H_{j}^{b}(\theta, y, \alpha, a, b) b \cdot b
\end{aligned}\right.
$$

where the $Z^{\prime} s$ belong to the space $\mathcal{E}$ defined in (4.5.1) and the $H_{j}^{\prime} s$ satisfy the estimates (4.5.8).

According to (4.5.6), (4.4.2), Theorem 4.4.2 and Theorem 4.3.1 for $\theta=0 a_{j}$ and $b_{j}$ do not depend on $\alpha$ and moreover we have,

$$
\left\{\begin{array}{l}
a_{j}(0, y, \alpha)=\widetilde{a}_{j}(y)=\mathcal{O}\left(|y|^{N}\right), \\
b_{j}(0, y, \alpha)=\widetilde{b}_{j}(y)=-y_{j}+\mathcal{O}\left(|y|^{N}\right),
\end{array}\right.
$$

for every $N \in \mathbb{N}$ and $|y| \leqslant \delta$.

Let us set

$$
G_{j}^{*}(\theta, y, \alpha)=H_{j}^{*}(\theta, y, \alpha, a(\theta, y, \alpha), b(\theta, y, \alpha)) \cdot b(\theta, y, \alpha) \cdot b(\theta, y, \alpha), *=a \text { or } b .
$$

Then, since the $Z_{j}^{\prime} s$ vanish for $\theta=0,(4.5 .10)$ implies that

$$
G_{j}^{*}(0, y, \alpha)=\widetilde{G}_{j}^{*}(y)=\mathcal{O}\left(|y|^{N}\right), \quad \forall N \in \mathbb{N} .
$$

Therefore we can write

$$
G_{j}^{*}(\theta, y, \alpha)=G_{j}^{*}(y)+\int_{0}^{\theta} \frac{d}{d \theta} G_{j}^{*}(\sigma, y, \alpha) d \sigma .
$$

We claim that we have the following estimates on $a_{j}, b_{j}$. Let us set for convenience $f_{j}=a_{j}$ or $b_{j}$.

$$
\left\{\begin{array}{l}
\left|\frac{\partial f_{j}}{\partial \theta}(\theta, y, \alpha)\right| \leqslant C(\varepsilon+\delta) \\
\left|\partial_{\theta}^{\ell} \partial_{y}^{\gamma} \partial_{\alpha}^{A} f_{j}(\theta, y, \alpha)\right| \leqslant \begin{cases}C_{\ell, \gamma} & \text { if } A=0, \\
C_{\ell, \gamma, A}(\varepsilon+\delta)|\theta|^{1-\ell} & \text { if } \quad|A| \geqslant 1, \quad \ell=1 .\end{cases}
\end{array}\right.
$$


To prove the first estimate we differentiate both sides of (4.5.9) with respect to $\theta$. Since the terms in $Z$ belong to $\mathcal{E}$ and using (4.5.8), the fact that $\dot{x}(\theta, \alpha), \dot{\xi}(\theta, \alpha)$ are bounded, we obtain

$$
\left|\frac{\partial a_{j}}{\partial \theta}\right|+\left|\frac{\partial b_{j}}{\partial \theta}\right| \leqslant C_{1}\left|y_{j}\right|+C_{2} \varepsilon+C_{3}(\varepsilon+\delta)\left(\left|\frac{\partial a}{\partial \theta}\right|+\left|\frac{\partial b}{\partial \theta}\right|\right) .
$$

Taking $\varepsilon+\delta$ small enough and since $|y| \leqslant \delta$ we obtain our first claim. To prove the second estimate we use the Faa di Bruno formula (see Appendix A.1) and an induction procedure.

Let us set $Y=(\theta, y, \alpha), \Lambda=(\ell, \gamma, A)$ and let us apply the operator $\partial_{Y}^{\Lambda}$ to both sides of (4.5.9). We have

$$
\begin{aligned}
& \partial_{Y}^{\Lambda} f_{0}=\left\{\begin{array}{ll}
0(1) & \text { if } A=0, \\
0 & \text { if }|A| \geqslant 1 .
\end{array}, f_{0}=\frac{-2 \theta y_{j}}{1+4 \theta^{2}} \quad \text { or } \frac{-y_{j}}{1+4 \theta^{2}}\right. \\
& \left.\left|\partial_{Y}^{\Lambda} Z(\theta, \alpha) \leqslant C \varepsilon\right| \theta\right|^{1-\ell} .
\end{aligned}
$$

Assume now that our estimate is true for $|\Lambda| \leqslant k$ and let $|\Lambda|=k+1$. Then

$$
\partial_{Y}^{\Lambda}[Z(-\theta, y+x(\theta, \alpha),-a+\xi(\theta, \alpha)) b(\theta, y, \alpha)]=(1)+(2)+(3)
$$

where

$$
\begin{aligned}
& (1)=Z(-\theta, \cdots) \partial_{Y}^{\Lambda} b(Y) \\
& (2)=\partial_{Y}^{\Lambda}[Z(-\theta, y+x(\theta, \alpha),-a(Y)+\xi(\theta, \alpha))] b(Y) \\
& (3)=\sum_{\substack{\Lambda_{1}+\Lambda_{2}=\Lambda \\
\Lambda_{j} \neq 0}}\left(\begin{array}{c}
\Lambda \\
\Lambda_{1}
\end{array}\right) \partial_{Y}^{\Lambda_{1}}[Z(-\theta, \cdots)] \partial_{Y}^{\Lambda_{2}} b .
\end{aligned}
$$

Using the Faa di Bruno formula in the terms (2) and (3) we see that

$$
\left|\partial_{Y}^{\Lambda}[Z(-\theta, \cdots)]\right| \leqslant C \varepsilon|\theta|\left(\left|\partial_{Y}^{\Lambda} a\right|+\left|\partial_{Y}^{\Lambda} b\right|\right)+\left\{\begin{array}{lll}
0(1) & \text { if } A=0 \\
0\left((\varepsilon+\delta)|\theta|^{1-\ell}\right) & \text { if } & |A| \neq 0
\end{array}\right.
$$

By the same way, using (4.5.8) we obtain

$$
\begin{aligned}
\mid \partial_{Y}^{\Lambda}[H(Y, a(Y), b(Y)) \cdot b(Y) \cdot b(Y) \mid] & \leqslant C(\varepsilon+\delta)|\theta|\left(\left|\partial_{Y}^{\Lambda} a(Y)\right|\right. \\
& \left.+\left|\partial_{Y}^{\Lambda} b(Y)\right|\right)+ \begin{cases}0(1) & \text { if } A=0 \\
0\left((\varepsilon+\delta)|\theta|^{1-\ell}\right) & \text { if } \quad|A| \geqslant 1 .\end{cases}
\end{aligned}
$$

Taking $\varepsilon+\delta$ small enough we obtain the second estimate of (4.5.14). 
Now it follows from (4.5.9), (4.5.11), (4.5.12) and (4.5.13) that we can write, with $Y=(\theta, y, \alpha)$,

$$
\left\{\begin{array}{l}
\left(a_{j}+i b_{j}\right)(Y)=-\frac{y_{j}}{2 \theta-i}+U_{j}(Y) \text { where } \\
U_{j}(Y)=Z_{j, 1}(\theta, \alpha)+Z_{j, 2}(-\theta, y+x(\theta, \alpha),-a(Y)+\xi(\theta, \alpha)) \\
\quad+Z_{j, 3}(-\theta, y+x(\theta, \alpha),-a(Y)+\xi(\theta, \alpha)) \cdot b(Y) \\
\quad+\widetilde{G}_{j}(y)+\int_{0}^{\theta}\left\{\left[\frac{\partial H}{\partial \theta}+\frac{\partial H}{\partial a} \cdot \frac{\partial a}{\partial \theta}+\frac{\partial H}{\partial b} \frac{\partial b}{\partial \theta}\right] b \cdot b+2 H b \cdot \frac{\partial b}{\partial \theta}\right\}(\sigma, y, \alpha) d \sigma \\
\text { with }\left|\partial_{y}^{\gamma} \widetilde{G}_{j}(y)\right| \leqslant C_{N}|y|^{N} \text { for all } N \in \mathbb{N} \text { and } \gamma \in \mathbb{N}^{n} .
\end{array}\right.
$$

Using (4.5.1), (4.5.3), (4.5.8), (4.5.14) we deduce the following estimates

$$
\left\{\begin{array}{l}
|U(Y)|+\left|\frac{\partial U}{\partial y}(Y)\right| \leqslant C_{N}|y|^{N}+C(\varepsilon+\delta)|\theta| \leqslant C^{\prime}(\varepsilon+\delta) \\
\left|\partial_{y}^{\gamma} U(Y)\right| \leqslant C_{N, \gamma}|y|^{N}+C_{\gamma}|\theta| \text { if }|\gamma| \geqslant 2 \\
\left|\partial_{y}^{\gamma} \partial_{\alpha}^{A} U(Y)\right| \leqslant C_{\gamma, A}(\varepsilon+\delta)|\theta| \text { if }|\gamma| \geqslant 0 \text { and }|A| \geqslant 1
\end{array}\right.
$$

Now recall that,

$$
\left\{\begin{array}{c}
\Phi_{j}(\theta, x, \alpha)=\xi_{j}(\theta, \alpha)-\left(a_{j}+i b_{j}\right)(\theta, x-x(\theta, \alpha), \alpha) \\
\varphi(\theta, x, \alpha)=\int_{0}^{1}(x-x(\theta, \alpha)) \cdot \Phi(\theta, s x+(1-s) x(\theta, \alpha), \alpha) d s \\
+\theta p(\alpha)+\frac{1}{2 i}\left|\alpha_{\xi}\right|^{2}
\end{array}\right.
$$

It follows that,

$$
\begin{aligned}
\varphi(\theta, x, \alpha)=\underbrace{(x-x(\theta, \alpha))}_{(1)} \cdot \xi(\theta, \alpha)+\underbrace{\frac{1}{2} \frac{|x-x(\theta, \alpha)|^{2}}{2 \theta-i}}_{(2)} \\
\quad-\int_{0}^{1}(x-x(\theta, \alpha)) \cdot U(\theta, s(x-x(\theta, \alpha)), \alpha) d s+\theta p(\alpha)+\frac{1}{2 i}\left|\alpha_{\xi}\right|^{2} .
\end{aligned}
$$

We have,

$$
\left\{\begin{array}{c}
(1)=\left(x-\alpha_{x}\right) \alpha_{\xi}-2 \theta\left|\alpha_{\xi}\right|^{2}-r(\theta, \alpha) \cdot \alpha_{\xi}+(x-x(\theta, \alpha)) \cdot \zeta(\theta, \alpha) \\
(2)=\frac{1}{2} \frac{1}{2 \theta-i}\left[\left(x-\alpha_{x}\right)^{2}-4 \theta\left(x-\alpha_{x}\right) \cdot \alpha_{\xi}+4 \theta^{2}\left|\alpha_{\xi}\right|^{2}\right. \\
\left.+2\left(x-\alpha_{x}-2 \theta \alpha_{\xi}\right) \cdot r(\theta, \alpha)+|r(\theta, \alpha)|^{2}\right]
\end{array}\right.
$$

Let us consider the term in $\varphi$ which does not contain any error term. It can be written as

$$
\left(x-\alpha_{x}\right) \cdot \alpha_{\xi}-2 \theta\left|\alpha_{\xi}\right|^{2}+\frac{\left(x-\alpha_{x}\right)^{2}-4 \theta\left(x-\alpha_{x}\right) \cdot \alpha_{\xi}+4 \theta^{2}\left|\alpha_{\xi}\right|^{2}}{2(2 \theta-i)}+\theta\left|\alpha_{\xi}\right|^{2}+\frac{1}{2 i}\left|\alpha_{\xi}\right|^{2}
$$

which is equal to

$$
\frac{\left(x-\alpha_{x}\right) \cdot \alpha_{\xi}+\frac{i}{2}\left(x-\alpha_{x}\right)^{2}-\theta\left|\alpha_{\xi}\right|^{2}}{1+2 i \theta}+\frac{1}{2 i}\left|\alpha_{\xi}\right|^{2} .
$$


It follows that

$$
\varphi(\theta, x, \alpha)=\frac{\left(x-\alpha_{x}\right) \cdot \alpha_{\xi}+\frac{i}{2}\left(x-\alpha_{x}\right)^{2}-\theta\left|\alpha_{\xi}\right|^{2}}{1+2 i \theta}+\frac{1}{2 i}\left|\alpha_{\xi}\right|^{2}+R(\theta, x, \alpha)
$$

where

$$
\begin{aligned}
& R(\theta, x, \alpha)= \underbrace{-r(\theta, \alpha) \cdot \alpha_{\xi}}_{(1)}+\underbrace{(x-x(\theta, \alpha)) \cdot \zeta(\theta, \alpha)}_{(2)} \\
& \underbrace{\frac{\left(x-\alpha_{x}-2 \theta \alpha_{\xi}\right) \cdot r(\theta, \alpha)+\frac{1}{2}|r(\theta, \alpha)|^{2}}{2 \theta-i}}_{(3)}+\underbrace{\theta\left(p(\alpha)-\left|\alpha_{\xi}\right|^{2}\right)}_{(4)} \\
&-\int_{0}^{1} \underbrace{(x-x(\theta, \alpha)) \cdot U(\theta, s(x-x(\theta, \alpha)), \alpha) d s}_{(5)} .
\end{aligned}
$$

We are ready now to show that the remainder $R$ satisfies the estimates given in Theorem 4.5.1.

First of all, since $r$ and $\zeta$ belong to $\mathcal{E}$ (see (4.5.1)), since the functions $x-x(\theta, \alpha)$ and $x-\alpha_{x}-2 \theta \alpha_{\xi}$ are bounded together with all their derivatives with respect to $\alpha$ and since $p(\alpha)=\left|\alpha_{\xi}\right|^{2}+\varepsilon \sum_{j, k=1}^{n} b_{j k}\left(\alpha_{x}\right) \alpha_{\xi}^{j} \alpha_{\xi}^{k}$ we have

$$
\left|\partial_{\alpha}^{A}(i)\right| \leqslant C_{A} \varepsilon|\theta| \text { for } i=1,2,3,4 \text {. }
$$

So we are left with the term (5). Let us note that if we set $f_{0}(\theta, x, \alpha)=x-x(\theta, \alpha)$ then,

$$
\left\{\begin{array}{l}
\left|f_{0}\right| \leqslant 2 \delta, \quad\left|f_{0}\right| \leqslant\left|x-\alpha_{x}\right|+C|\theta|, \quad\left|\frac{\partial f_{0}}{\partial \alpha_{x}}\right| \leqslant C, \quad\left|\frac{\partial f_{0}}{\partial \alpha_{\xi}}\right| \leqslant C|\theta| \\
\left|\partial_{\alpha}^{A} f_{0}\right| \leqslant C \varepsilon|\theta| \text { if }|A| \geqslant 2, \text { uniformly in }(x, \theta, \alpha) .
\end{array}\right.
$$

With this notation one has

$$
(5)=f_{0}(\theta, x, \alpha) \int_{0}^{1} U\left(\theta, s f_{0}(\theta, x, \alpha), \alpha\right) d s .
$$

Then, with $i=x$ or $\xi$,

$$
\frac{\partial}{\partial \alpha_{i}}(5)=\frac{\partial f_{0}}{\partial \alpha_{i}} \int_{0}^{1} U\left(\theta, s f_{0}, \alpha\right) d s+f_{0} \int_{0}^{1}\left(s \frac{\partial f_{0}}{\partial \alpha_{i}} \frac{\partial U}{\partial y}+\frac{\partial U}{\partial \alpha_{i}}\right)\left(\theta, s f_{0}, \alpha\right) d s .
$$

Now it follows from (4.5.18) and (4.5.20) that

$$
\left\{\begin{array}{l}
\left|\frac{\partial}{\partial \alpha_{x}}(5)\right| \leqslant C(\varepsilon+\delta)\left(\left|x-\alpha_{x}\right|^{2}+|\theta|\right), \\
\left.\left|\frac{\partial}{\partial \alpha_{\xi}}(5)\right| \leqslant C(\varepsilon+\delta)|\theta|\right)
\end{array}\right.
$$


since $\left|\frac{\partial f_{0}}{\partial \alpha_{\xi}}(5)\right| \leqslant C|\theta|$ and $\left|x-\alpha_{x}\right| \leqslant \delta$. Now, with $i, j=x$ or $\xi$, we have

$$
\begin{array}{r}
\frac{\partial^{2}}{\partial \alpha_{i} \partial \alpha_{j}}(5)=\frac{\partial^{2} f_{0}}{\partial \alpha_{i} \partial \alpha_{j}} \int_{0}^{1} U\left(\theta, s f_{0}, \alpha\right) d s+\frac{\partial f_{0}}{\partial \alpha_{i}} \int_{0}^{1}\left(s \frac{\partial f_{0}}{\partial \alpha_{j}} \frac{\partial U}{\partial y}+\frac{\partial U}{\partial \alpha_{j}}\right) d s \\
+\frac{\partial f_{0}}{\partial \alpha_{j}} \int_{0}^{1}\left(s \frac{\partial f_{0}}{\partial \alpha_{i}} \frac{\partial U}{\partial y}+\frac{\partial U}{\partial \alpha_{i}}\right) d s+f_{0} \int_{0}^{1}\left[s \frac{\partial^{2} f_{0}}{\partial \alpha_{i} \partial \alpha_{j}} \frac{\partial U}{\partial y}+s \frac{\partial f_{0}}{\partial \alpha_{i}}\left(\frac{\partial f_{0}}{\partial \alpha_{j}} \frac{\partial^{2} U}{\partial y^{2}}+\frac{\partial U}{\partial \alpha_{j}}\right)\right. \\
\left.+\frac{\partial^{2} U}{\partial \alpha_{i} \partial y} \frac{\partial f_{0}}{\partial \alpha_{j}}+\frac{\partial^{2} U}{\partial \alpha_{i} \partial \alpha_{j}}\right] d s .
\end{array}
$$

Using (4.5.16) and (4.5.20) we check easily that

$$
\left\{\begin{array}{l}
\left|\frac{\partial^{2}}{\partial \alpha_{x}^{2}}(5)\right| \leqslant C(\varepsilon+\delta)\left(\left|x-\alpha_{x}\right|^{2}+|\theta|\right) \\
\left|\frac{\partial^{2}}{\partial \alpha_{x} \partial \alpha_{\xi}}(5)\right| \leqslant C(\varepsilon+\delta)|\theta| \\
\left|\frac{\partial^{2}}{\partial \alpha_{\xi}^{2}}(5)\right| \leqslant C(\varepsilon+\delta)|\theta| .
\end{array}\right.
$$

Combining (4.5.19), (4.5.21) and (4.5.22) we obtain the claimed estimates on the two first derivatives of $R$.

Finally using again (4.5.18) and (4.5.20) we deduce the following estimates of the higher derivatives

$$
\left|\partial_{\alpha_{x}}^{A_{1}} \partial_{\alpha_{\xi}}^{A_{2}}(5)\right| \leqslant \begin{cases}C_{A_{1}} & \text { if } A_{2}=0, \\ C_{A_{1} A_{2}}|\theta| & \text { if }\left|A_{2}\right| \geqslant 1 .\end{cases}
$$

The gain of $|\theta|$ when $\left|A_{2}\right| \geqslant 1$ coming from the fact that a derivative of $x-x(\theta, \alpha)$ and $U$ with respect to $\alpha_{\xi}$ makes appear a $\theta$. Thus the proof of Theorem 4.5.1 is complete. 



\section{CHAPTER 5}

\section{THE TRANSPORT EQUATIONS}

\subsection{Statement of the result and preliminaries}

Let $P=\sum_{j, k=1}^{n} g^{j k}(x) D_{j} D_{k}$ be a second order differential operator of the form (2.2.7) We shall denote by ${ }^{t} P$ the transposed operator.

In Chapter 4 we have constructed a phase function for $P$. The purpose now is to construct an amplitude.

Recall that the set $\Omega_{\delta}$ have been introduced in Definition 4.1.1.

The main result of this Section is the following.

Theorem 5.1.1. - For every $\alpha \in T^{*} \mathbb{R}^{n}$ with $\frac{1}{2} \leqslant\left|\alpha_{\xi}\right| \leqslant 2$, every $N \in \mathbb{N}$ and every $\lambda \geqslant 1$ one can find an amplitude $e_{N}(\theta, y, \alpha, \lambda)$ which is $C^{\infty}$ on $\widetilde{\Omega}_{\delta}$ such that

(i) $e_{N}(0, y, \alpha, \lambda)=1$.

(ii) $\left(i \lambda \frac{\partial}{\partial \theta}-i \lambda \frac{n}{2} \frac{\theta}{1+\theta^{2}}-{ }^{t} P\right)\left(e^{i \lambda \varphi(\theta, x, \alpha)} e_{N}(\theta, x-x(\theta, \alpha), \alpha, \lambda)\right)$

$$
=R_{N}(\theta, x, \alpha, \lambda) e^{i \lambda \varphi(\theta, x, \alpha)} \text {, where }
$$

$$
\left|R_{N}(\theta, x, \alpha, \lambda)\right| \leqslant C_{N}\left(\lambda^{-N}+\lambda^{2} \frac{|x-x(\theta, \alpha)|^{N}}{\langle\theta\rangle^{N}}\right)
$$

for every $(\theta, x, \alpha)$ in $\Omega_{\delta}, \lambda \geqslant 1$ and $C_{N}$ is independent of $(\theta, x, \alpha, \lambda)$.

(iii) $\left|\partial_{x}^{A} e_{N}(\theta, x-x(\theta, \alpha), \alpha, \lambda)\right| \leqslant C_{N, A}$ uniformly with respect to $(\theta, x, \alpha, \lambda)$.

Corollary 5.1.2. - For every $\alpha \in T^{*} \mathbb{R}^{n}$ with $\frac{1}{2} \leqslant\left|\alpha_{\xi}\right| \leqslant 2$, every $N \in \mathbb{N}$ and every $\lambda \geqslant 1$ one can find an amplitude $a_{N}(\theta, x, \alpha, \lambda)$ which is $C^{\infty}$ on $\Omega_{\delta}$ such that

(i) $a_{N}(0, x, \alpha, \lambda)=1$,

(ii) $\left(i \lambda \frac{\partial}{\partial \theta}-{ }^{t} P\right)\left(e^{i \lambda \varphi(\theta, x, \alpha)} a_{N}(\theta, x, \alpha, \lambda)\right)=R_{N}^{\prime}(\theta, x, \alpha, \lambda) e^{i \lambda \varphi(\theta, x, \alpha)}$ where

$$
\left|R_{N}^{\prime}(\theta, x, \alpha, \lambda)\right| \leqslant C_{N}^{\prime}\left(\lambda^{-N}+\lambda^{2} \frac{|x-x(\theta, \alpha)|^{N}}{\langle\theta\rangle^{N}}\right)
$$

uniformly with respect to $(\theta, x, \alpha, \lambda)$.

(iii) $\left|\partial_{x}^{A} a_{N}(\theta, x, \alpha, \lambda)\right| \leqslant C_{N, A}\langle\theta\rangle^{-n / 2}$, uniformly with respect to $(\theta, x, \alpha, \lambda)$. 
In flat case $R_{N}$ and $R_{N}^{\prime}$ are bounded by $C_{N} \lambda^{-N}$, but no explicit formula for $e_{N}$ is available.

Proof. - We have just to set $a_{N}(\theta, x, \alpha, \lambda)=\langle\theta\rangle^{-n / 2} e_{N}(\theta, x-x(\theta, \alpha), \alpha, \lambda)$ where $e_{N}$ has been defined in Theorem 5.1.1.

Proof of Theorem 5.1.1. - We have ${ }^{t} P=\sum_{j, k=1}^{n} g^{j k}(x) D_{j} D_{k}+\sum_{j=1}^{n} g_{j}(x) D_{j}+$ $g_{0}(x)$, where $g^{j k}=\delta_{j k}+\varepsilon b_{j k}$ and $b_{j k} \in \mathcal{B}_{\sigma_{0}}^{1}, g_{j} \in \mathcal{B}_{\sigma_{0}}^{2}, 1 \leqslant j, k \leqslant n, g_{0} \in \mathcal{B}_{\sigma_{0}}^{3}$ where,

$$
\mathcal{B}_{\sigma_{0}}^{\ell}=\left\{g \in C^{\infty}\left(\mathbb{R}^{n}\right):\left|\partial_{x}^{A} g(x)\right| \leqslant \frac{C_{A}}{\langle x\rangle^{|A|+\ell+\sigma_{0}}}, \forall x \in \mathbb{R}^{n}, \forall A \in \mathbb{N}^{n}\right\} .
$$

A straightforward computation shows that

$$
\begin{aligned}
\left(i \lambda \frac{\partial}{\partial \theta}-i \lambda\right. & \left.\frac{n}{2} \frac{\theta}{1+\theta^{2}}-{ }^{t} P\right)\left(e^{i \lambda \varphi} f\right) \\
=e^{i \lambda \varphi}\left[-\lambda^{2}\left(\frac{\partial \varphi}{\partial \theta}+p\left(x, \frac{\partial \varphi}{\partial x}\right)\right) f+i \lambda\left(\frac{\partial f}{\partial \theta}+2 \sum_{j, k=1}^{n} g^{j k} \frac{\partial \varphi}{\partial x_{j}} \frac{\partial f}{\partial x_{k}}\right.\right. & \\
& \left.\left.\quad+\sum_{j, k=1}^{n} g^{j k} \frac{\partial^{2} \varphi}{\partial x_{j} \partial x_{k}} f-\frac{n}{2} \frac{\theta}{1+\theta^{2}} f+i \sum_{j=1}^{n} g_{j} \frac{\partial \varphi}{\partial x_{j}} f\right)-{ }^{t} P f\right]
\end{aligned}
$$

According to Theorem 4.1.2 the coefficient of $\lambda^{2}$ in the right hand side of (5.1.2) is bounded by $C_{N}\left(\frac{|x-x(\theta, \alpha)|}{\langle\theta\rangle}\right)^{N}$, for any $N$. Therefore if we set

$$
\left\{\begin{array}{l}
I=e^{-i \lambda \varphi}\left(i \lambda \frac{\partial}{\partial \theta}-i \lambda \frac{n}{2} \frac{\theta}{1+\theta^{2}}-{ }^{t} P\right)\left(e^{i \lambda \varphi} f\right) \\
X=\frac{\partial}{\partial \theta}+2 \sum_{j, k=1}^{n} g^{j k} \frac{\partial \varphi}{\partial x_{j}} \cdot \frac{\partial}{\partial x_{k}}
\end{array}\right.
$$

we obtain

$$
\begin{aligned}
\mid I-i \lambda X f-i \lambda\left(\sum_{j, k=1}^{n} g^{j k} \frac{\partial^{2} \varphi}{\partial x_{j} \partial x_{k}}-\frac{n}{2} \frac{\theta}{1+\theta^{2}}\right. & \left.+i \sum_{j=1}^{n} g_{j} \frac{\partial \varphi}{\partial x_{j}}\right) f-{ }^{t} P f \mid \\
& \leqslant C_{N} \lambda^{2}\left(\frac{|x-x(\theta, \alpha)|}{\langle\theta\rangle}\right)^{N} .
\end{aligned}
$$

To pursue the proof we consider separately the two cases.

\subsection{The case of outgoing points}

For convenience we shall set

$$
\begin{aligned}
& \widetilde{\mathcal{S}}_{+}=\left\{\alpha \in T^{*} \mathbb{R}^{n}: \frac{1}{2} \leqslant\left|\alpha_{\xi}\right| \leqslant 2, \alpha_{x} \cdot \alpha_{\xi} \geqslant-c_{0}\left\langle\alpha_{x}\right\rangle\left|\alpha_{\xi}\right|\right\} \\
& \widetilde{\mathcal{S}}_{-}=\left\{\alpha \in T^{*} \mathbb{R}^{n}: \frac{1}{2} \leqslant\left|\alpha_{\xi}\right| \leqslant 2, \alpha_{x} \cdot \alpha_{\xi} \leqslant c_{0}\left\langle\alpha_{x}\right\rangle\left|\alpha_{\xi}\right|\right\} .
\end{aligned}
$$


Here we assume $\alpha \in \widetilde{\mathcal{S}}_{ \pm}$. Let us set $R_{j}=\frac{\partial \varphi}{\partial x_{j}}-\Phi_{j}$. It follows from Theorem 4.3.13 (v) and (4.3.48) that $\left|\partial_{x}^{A} R_{j}(\theta, x, \alpha)\right| \leqslant C_{A} /\langle\theta\rangle^{|A|}$ for $A \in \mathbb{N}^{n}$ and from Proposition 4.3 .19 (ii) that $\left|R_{j}(\theta, x, \alpha)\right| \leqslant C_{N}\left(\frac{|x-x(\theta, \alpha)|}{\langle\theta\rangle}\right)^{N}$ for all $N \in \mathbb{N}$.

First of all, according to (4.3.43) and Taylor's formula, we have

$$
\left\{\begin{array}{c}
\frac{\partial \varphi}{\partial x_{j}}(\theta, x, \alpha)=\xi_{j}(\theta, \alpha) \\
+\frac{\frac{1}{2} \operatorname{sgn} \theta\left(1-\chi_{1}(\theta)\right)\left(x_{j}-x_{j}(\theta, \alpha)\right)-\left(a_{j}+i b_{j}\right)(\theta, x-x(\theta, \alpha), \alpha)}{\langle\theta\rangle}+R_{j}(\theta, x, \alpha) \\
\left|\partial_{x}^{A} R_{j}(\theta, x, \alpha)\right| \leqslant C_{A, N}\left(\frac{|x-x(\theta, \alpha)|}{\langle\theta\rangle}\right)^{N}
\end{array}\right.
$$

Using Theorem 4.3.1 (iii) we deduce,

$$
\left\{\begin{array}{l}
\frac{\partial^{2} \varphi}{\partial x_{j} \partial x_{k}}(\theta, x, \alpha)=\frac{1}{2} \frac{\operatorname{sgn} \theta}{\langle\theta\rangle}\left(1-\chi_{1}(\theta)\right) \delta_{j k}+d_{j k}(\theta, x-x(\theta, \alpha), \alpha) \\
+R_{j k}(\theta, x, \alpha), \\
\left|\partial_{x}^{A} d_{j k}(\theta, x-x(\theta, \alpha), \alpha)\right| \leqslant \frac{C_{A}}{\langle\theta\rangle^{|A|+2}}, \\
\left|\partial_{x}^{A} R_{j k}(\theta, x, \alpha)\right| \leqslant C_{A N}\left(\frac{|x-x(\theta, \alpha)|}{\langle\theta\rangle}\right)^{N}
\end{array}\right.
$$

uniformly with respect to $(\theta, x, \alpha)$.

Now if $g \in \mathcal{B}_{\sigma_{0}}$ (see (5.1.1)) and $(\theta, x) \in \Omega_{\delta}$ we can write

$$
\left|\partial_{x}^{A} g(x)\right|=\left|\left(\partial_{x}^{A} g\right)(y+x(\theta, \alpha))\right| \leqslant \frac{C_{A}}{\langle y+x(\theta, \alpha)\rangle^{|A|+1+\sigma_{0}}} .
$$

Since $|y| \leqslant \delta\langle\theta\rangle$ we can use Proposition 3.3.1 to write

$$
\langle y+x(\theta, \alpha)\rangle \geqslant\langle x(\theta, \alpha)\rangle-|y| \geqslant \frac{1}{\sqrt{3}}\langle\theta\rangle-\delta\langle\theta\rangle \geqslant \frac{1}{2}\langle\theta\rangle .
$$

It follows that

$$
\left|\partial_{x}^{A} g(x)\right| \leqslant \frac{C_{A}}{\langle\theta\rangle^{|A|+1+\sigma_{0}}} .
$$

This can be applied to the functions $g^{j k}-\delta_{j k}, g_{j}, g_{0}, 1 \leqslant j, k \leqslant n$. It follows from (5.2.2) that

$$
\sum_{j, k=1}^{n} g^{j k}(x) \frac{\partial^{2} \varphi}{\partial x_{j} \partial x_{k}}(\theta, x, \alpha)=\frac{n}{2} \frac{\operatorname{sgn} \theta}{\langle\theta\rangle}\left(1-\chi_{1}(\theta)\right)+d(\theta, x-x(\theta, \alpha), \alpha)+R(\theta, x, \alpha)
$$

where $d$ and $R$ satisfy the same estimates as in (5.2.2). Now we have

$$
\begin{aligned}
\frac{1}{2} \frac{\operatorname{sgn} \theta}{\langle\theta\rangle}\left(1-\chi_{1}(\theta)\right)-\frac{1}{2} \frac{\theta}{1+\theta^{2}} & =\frac{1}{2} \frac{\operatorname{sgn} \theta}{\langle\theta\rangle}\left(1-\chi_{1}(\theta)\right)\left(1-\frac{|\theta|}{\langle\theta\rangle}\right)-\frac{\theta \chi_{1}(\theta)}{2\langle\theta\rangle^{2}} \\
& =\frac{1}{2} \frac{\operatorname{sgn} \theta}{\langle\theta\rangle^{2}} \frac{1-\chi_{1}(\theta)}{\langle\theta\rangle+|\theta|}=\mathcal{O}\left(\frac{1}{\langle\theta\rangle^{3}}\right) .
\end{aligned}
$$


Summing up, we have proved

$$
\left\{\begin{array}{l}
\sum_{j, k=1}^{n} g^{j k}(x) \frac{\partial^{2} \varphi}{\partial x_{j} \partial x_{k}}(\theta, x, \alpha)-\frac{n}{2} \frac{\theta}{1+\theta^{2}}=D_{1}(\theta, x-x(\theta, \alpha), \alpha) \\
+R_{1}(\theta, x, \alpha), \\
\sum_{j=1}^{n} g_{j}(x) \frac{\partial \varphi}{\partial x_{j}}(\theta, x, \alpha)=D_{2}(\theta, x-x(\theta, \alpha), \alpha)+R_{2}(\theta, x, \alpha), \\
\left|\partial_{x}^{A} D_{j}(\theta, x-x(\theta, \alpha), \alpha)\right| \leqslant \frac{C_{A}}{\langle\theta\rangle^{|\alpha|+1+\sigma_{0}}, \quad j=1,2,} \\
\left|R_{j}(\theta, x, \alpha)\right| \leqslant C_{N}\left(\frac{|x-x(\theta, \alpha)|}{\langle\theta\rangle}\right)^{N}, \quad j=1,2, \quad \forall N \in \mathbb{N} .
\end{array}\right.
$$

We are going now to simplify the vector fields $X$ introduced in (5.1.3). Let us set

$$
\left\{\begin{array}{l}
s=\theta \\
y=x-x(\theta, \alpha) .
\end{array}\right.
$$

Since $\dot{x}_{k}(\theta, \alpha)=\frac{\partial p}{\partial \xi_{k}}(x(\theta, \alpha), \xi(\theta, \alpha))=2 \sum_{j=1}^{n} g^{j k}(x(\theta, \alpha)) \xi_{k}(\theta, \alpha)$, we obtain

$$
X=\frac{\partial}{\partial s}-2 \sum_{j, k=1}^{n}\left\{g^{j k}(x(s, \alpha)) \xi_{j}(s, \alpha)-g^{j k}(y+x(s, \alpha)) \frac{\partial \varphi}{\partial x_{j}}(s, y+x(s, \alpha), \alpha)\right\} \frac{\partial}{\partial y_{k}} .
$$

Now using (5.2.1) and $g^{j k}=\delta_{j k}+\varepsilon b_{j k}, b_{j k} \in \mathcal{B}_{\sigma_{0}}$ we can write

$$
\begin{aligned}
X= & \frac{\partial}{\partial s}+\frac{\operatorname{sgn} s}{\langle s\rangle}\left(1-\chi_{1}(s)\right) \sum_{j=1}^{n} y_{j} \frac{\partial}{\partial y_{j}} \\
& -2 \varepsilon \sum_{j, k=1}^{n}\left\{b_{j k}(x(s, \alpha))-b_{j k}(y+x(s, \alpha))\right\} \xi_{j}(s, \alpha) \frac{\partial}{\partial y_{k}} \\
& -2 \sum_{j=1}^{n} \frac{\left(a_{j}+i b_{j}\right)(s, y, \alpha)}{\langle s\rangle} \cdot \frac{\partial}{\partial y_{j}}+2 \varepsilon \sum_{j, k=1}^{n} b_{j k}(y+x(s, \alpha)) \frac{1}{\langle s\rangle}\left(\frac{1}{2} \operatorname{sgn} s y_{j}\right. \\
- & \left.\left(a_{j}+i b_{j}\right)(s, y, \alpha)\right) \frac{\partial}{\partial y_{k}}+2 \sum_{j, k=1}^{n} g^{j k}(y+x(s, \alpha)) R_{j}(s, y+x(s, \alpha), \alpha) \frac{\partial}{\partial y_{k}} .
\end{aligned}
$$

Definition 5.2.1. - We shall say that a function $f=f(s, y, \alpha)$ on $\widetilde{\Omega}_{\delta} \times \widetilde{\mathcal{S}}_{ \pm}$belongs to $\mathcal{E}$ if

$$
\left\{\begin{array}{l}
f(s, 0, \alpha)=0 \\
\left|\partial_{y}^{A} f(s, y, \alpha)\right| \leqslant \frac{C_{A}}{\langle s\rangle^{|A|+1}}, \quad A \in \mathbb{N}^{n}
\end{array}\right.
$$

uniformly when $(s, y) \in \widetilde{\Omega}_{\delta}$ and $\alpha \in \widetilde{\mathcal{S}}_{ \pm}$. 
According to (5.2.4), (5.2.3) and Theorem 4.3.1, (ii), (iii) we have,

$$
\left\{\begin{array}{l}
\left(1-\chi_{1}(s)\right) \frac{\operatorname{sgn} s}{\langle s\rangle} y_{j}-\frac{s}{1+s^{2}} y_{j} \in \mathcal{E} \\
\varepsilon\left(b_{j k}\left(x(s, \alpha)-b_{j k}(y+x(s, \alpha))\right)\right) \xi_{j}(s, \alpha) \in \mathcal{E} \\
\varepsilon b_{j k}(y+x(s, \alpha)) \frac{1}{\langle s\rangle}\left(\frac{1}{2} \cdot \operatorname{sgn} s \cdot y_{j}-\left(a_{j}+i b_{j}\right)(s, y, \alpha)\right) \in \mathcal{E} \\
\frac{1}{\langle s\rangle}\left(a_{j}+i b_{j}\right)(s, y, \alpha) \in \mathcal{E} .
\end{array}\right.
$$

Then we have,

$$
\left\{\begin{array}{l}
X=\frac{\partial}{\partial s}+\frac{s}{1+s^{2}} \sum_{j=1}^{n} y_{j} \frac{\partial}{\partial y_{j}}+\sum_{j=1}^{n} E_{j}(s, y, \alpha) \frac{\partial}{\partial y_{j}}+\sum_{j=1}^{n} R_{j}^{\prime}(s, y, \alpha) \frac{\partial}{\partial y_{j}} \\
\text { where } E_{j} \in \mathcal{E} \text { and }\left|R_{j}^{\prime}(s, y, \alpha)\right| \leqslant C_{N}\left(\frac{|y|}{\langle s\rangle}\right)^{N} .
\end{array}\right.
$$

Now we perform another change of variables. We set

$$
\left\{\begin{array}{l}
\theta=s \\
z=\frac{y}{\langle s\rangle} .
\end{array}\right.
$$

Then we have $\frac{\partial}{\partial \theta}=\frac{\partial}{\partial s}+\frac{s}{1+s^{2}} \sum_{j=1}^{n} y_{j} \frac{\partial}{\partial y_{j}}$. It follows that

$$
\begin{cases}\text { (i) } & X=\frac{\partial}{\partial \theta}+\sum_{j=1}^{n} h_{j}(\theta, z, \alpha) \frac{\partial}{\partial z_{j}}+\sum_{j=1}^{n} \widetilde{R}_{j}(\theta, z, \alpha) \frac{\partial}{\partial z_{j}}, \\ \text { (ii) } & h_{j}(\theta, 0, \alpha)=0, \\ \text { (iii) } & \left|\partial_{z}^{A} h_{j}(\theta, z, \alpha)\right| \leqslant \frac{C_{A}}{\langle\theta\rangle^{2}}, \quad A \in \mathbb{N}^{n}, \\ \text { (iv) } \quad & \left|\widetilde{R}_{j}(\theta, z, \alpha)\right| \leqslant \frac{C_{N}}{\langle\theta\rangle}|z|^{N}, \text { for all } N \text { in } \mathbb{N}, \text { uniformly when } \\ & \theta \geqslant 0 \text { (resp. } \theta \leqslant 0),|z| \leqslant \delta, \quad \alpha \in \Lambda_{ \pm}, j=1, \ldots, n .\end{cases}
$$

Moreover, since $\frac{\partial}{\partial y_{j}}=\frac{1}{\langle\theta\rangle} \frac{\partial}{\partial z_{j}}$ we have by (5.2.3),

$$
\left\{\begin{array}{l}
{ }^{t} P=\sum_{|\nu| \leqslant 2} k_{\nu}(\theta, z, \alpha) \partial_{z}^{\nu}, \\
\left|\partial_{z}^{\gamma} k_{\nu}(\theta, z, \alpha)\right| \leqslant \frac{C_{\gamma}}{\langle\theta\rangle^{1+\sigma_{0}}}, \quad \gamma \in \mathbb{N}^{n}
\end{array}\right.
$$

Let us set

$$
X_{0}=\frac{\partial}{\partial \theta}+\sum_{j=1}^{n} h_{j}(\theta, z, \alpha) \frac{\partial}{\partial z_{j}},
$$

where $h_{j}$ satisfies (5.2.11). 
It follows from (5.1.4), (5.2.5), (5.2.6), (5.2.10) and (5.2.11) (i) that

$$
\left\{\begin{array}{l}
\left|I-i \lambda\left(X_{0} f+d(\theta, z, \alpha) f-\frac{i}{\lambda} t P f\right)\right| \leqslant C_{N} \lambda^{2}|z|^{N}\left(|f|+\left|\nabla_{z} f\right|\right) \\
\left|\partial_{z}^{\gamma} d(\theta, z, \alpha)\right| \leqslant \frac{C_{\gamma}}{\langle\theta\rangle^{1+\sigma_{0}}}, \quad \gamma \in \mathbb{N}^{n}
\end{array}\right.
$$

Now let us fix an integer $N_{0}$ large enough depending only on the dimension $n$ (and chosen later on). For the coefficients $h_{j}, k_{\nu}, d$ in (5.2.13), (5.2.12) and (5.2.14) we write

$$
\left\{\begin{array}{l}
F(\theta, z, \alpha)=F^{N_{0}}(\theta, z, \alpha)+r^{N_{0}}(\theta, z, \alpha) \text { where } \\
F^{N_{0}}(\theta, z, \alpha)=\sum_{|\gamma| \leqslant N_{0}-1} \partial_{z}^{\gamma} F(\theta, 0, \alpha) \frac{z^{\gamma}}{\gamma !} \\
\left|r^{N_{0}}(\theta, z, \alpha)\right| \leqslant C_{N_{0}}|z|^{N_{0}}
\end{array}\right.
$$

Let us set

$$
\left\{\begin{array}{l}
L=\frac{\partial}{\partial \theta}+\sum_{j=1}^{n} h_{j}^{N_{0}}(\theta, z, \alpha) \frac{\partial}{\partial z_{j}}+d^{N_{0}}(\theta, z, \alpha), \\
Q=\sum_{|\nu| \leqslant 2} k_{\nu}^{N_{0}}(\theta, z, \alpha) \partial_{z}^{\nu} .
\end{array}\right.
$$

Using (5.2.14) to (5.2.16) we see that

$$
\left|I-i \lambda\left(L f-\frac{i}{\lambda} Q f\right)\right| \leqslant C_{N_{0}} \lambda^{2}|z|^{N_{0}} \sum_{|\gamma| \leqslant 2}\left|\partial_{z}^{\gamma} f(\theta, z, \alpha)\right| .
$$

Now we have the following result.

Lemma 5.2.2. - There exist functions $A_{\ell}=A_{\ell}(\theta, z, \alpha), \ell=0, \ldots, N_{0}+1$ which are $C^{\infty}$ in $(\theta, z)$ in the set $\mathcal{O}=\left\{(\theta, z): \theta \in \mathbb{R}^{ \pm},|z| \leqslant \delta\right\}$ such that

(i) $A_{0}(0, z, \alpha)=1, A_{\ell}(0, z, \alpha)=0, \ell=1, \ldots, N_{0}+1$,

(ii) $\left|\partial_{z}^{\gamma} A_{\ell}(\theta, z, \alpha)\right| \leqslant C_{\ell, \gamma}$, uniformly in $\mathcal{O} \times \widetilde{\mathcal{S}}_{ \pm},\left(\ell \in \mathbb{N}, \gamma \in \mathbb{N}^{n}\right)$,

(iii) $L A_{0}=0, L A_{\ell}=i Q A_{\ell-1}, \ell=1, \ldots, N_{0}+1$.

Let us assume for a moment this lemma proved. Let us set

$$
f=f_{N_{0}}=A_{0}+\frac{1}{\lambda} A_{1}+\cdots+\frac{1}{\lambda^{N_{0}+1}} A_{N_{0}+1} .
$$

Then Lemma 5.2.2 shows that

$$
\left\{\begin{array}{l}
f_{N_{0}}(0, z, \alpha, \lambda)=1 \\
\left|\partial_{z}^{\gamma} f_{N_{0}}(\theta, z, \alpha, \lambda)\right| \leqslant C_{\gamma, N_{0}} \text { if }(\theta, z) \in \mathcal{O}, \quad \alpha \in \widetilde{\mathcal{S}}_{ \pm}, \quad \lambda \geqslant 1 \\
\left|L f_{N_{0}}-\frac{i}{\lambda} Q f_{N_{0}}\right| \leqslant \lambda^{-N_{0}-2}\left|Q A_{N+1}\right| \leqslant C_{N_{0}} \lambda^{-N_{0}-2} .
\end{array}\right.
$$

It follows from (5.2.17) and (5.2.19) that

$$
|I| \leqslant C_{N_{0}}^{\prime} \lambda^{2}|z|^{N_{0}}+C_{N_{0}}^{\prime \prime} \lambda^{-N_{0}-1} .
$$


Coming back to the variables $(\theta, x)$ we set

$$
e_{N_{0}}(\theta, x-x(\theta, \alpha), \alpha, \lambda)=f_{N_{0}}\left(\theta, \frac{x-x(\theta, \alpha)}{\langle\theta\rangle}, \alpha, \lambda\right) .
$$

Then it follows from (5.2.20), (5.2.19) that $e_{N_{0}}$ satisfies the conditions (i), (ii), (iii) in Theorem 5.1.1.

So we are left with the proof of Lemma 5.2.2.

Proof of Lemma 5.2.2. - We are going to straighten the principal part of the operator $L$ given by (5.2.16). Recall that we have $L=L_{0}+d^{N_{0}}(\theta, z, \alpha)$ with

$$
L_{0}=\frac{\partial}{\partial \theta}+\sum_{j=1}^{n} h_{j}^{N_{0}}(\theta, z, \alpha) \frac{\partial}{\partial z_{j}} .
$$

Moreover, according to (5.2.11) and (5.2.15) we have

$$
\begin{cases}\text { (i) } & h_{j}^{N_{0}}(\theta, z, \alpha)=\sum_{k=1}^{n} \frac{\partial h_{j}}{\partial z_{k}}(\theta, 0, \alpha) z_{k}+g_{j}(\theta, z, \alpha), \\ \text { (ii) } \quad g_{j}(\theta, z, \alpha)=\sum_{2 \leqslant|\gamma| \leqslant N_{0}-1} \frac{1}{\gamma !} \partial_{z}^{\gamma} h_{j}(\theta, 0, \alpha) z^{\gamma}, \\ \text { (iii) } \quad \sum_{j=1}^{n}\left|\partial_{z}^{\gamma} h_{j}(\theta, 0, \alpha)\right| \leqslant \frac{C_{\gamma}}{\langle\theta\rangle^{2}}, \quad \forall \gamma \in \mathbb{N}^{n} .\end{cases}
$$

In that follows all the objects will depend on $\alpha \in \widetilde{\mathcal{S}}_{ \pm}$but all the estimates will be uniform with respect to $\alpha$.

Let us set

$$
H(\theta)=\left(\frac{\partial h_{j}}{\partial z_{k}}(\theta, 0, \alpha)\right)_{1 \leqslant j, k \leqslant n} .
$$

If $\theta_{0} \in \mathbb{R}^{ \pm}$we shall denote by $Y\left(\theta, \theta_{0}\right)$ the unique $n \times n$ matrix solution of the problem

$$
\left\{\begin{array}{l}
\dot{Y}\left(\theta, \theta_{0}\right)=H(\theta) Y\left(\theta, \theta_{0}\right), \theta \in \mathbb{R}^{ \pm}, \\
Y\left(\theta_{0}, \theta_{0}\right)=\operatorname{Id} .
\end{array}\right.
$$

Since by (5.2.21) (iii) the entries of the matrix $H(\theta)$ are bounded by $C /\langle\theta\rangle^{2}$, the Gronwall inequality shows that there exists $M_{0} \geqslant 1$ such that

$$
\left\|Y\left(\theta, \theta_{0}\right)\right\| \leqslant M_{0}, \text { for all } \theta, \theta_{0} \in \mathbb{R}^{ \pm} \text {and } \alpha \in \widetilde{\mathcal{S}}_{ \pm} .
$$

Moreover since $Y\left(\theta, \theta_{0}\right)^{-1}=Y\left(\theta_{0}, \theta\right)$ we have also,

$$
\left\|Y\left(\theta, \theta_{0}\right)^{-1}\right\| \leqslant M_{0}, \text { for all } \theta, \theta_{0} \in \mathbb{R}^{ \pm} \text {and } \alpha \in \widetilde{\mathcal{S}}_{ \pm} .
$$

Now using (5.2.21) we see that the problem

$$
\left\{\begin{array}{l}
\dot{z}_{j}(\theta)=h_{j}^{N_{0}}(\theta, z(\theta), \alpha), \quad \theta \in \mathbb{R}^{ \pm}, 1 \leqslant j \leqslant n, \\
z_{j}(0)=y_{j}
\end{array}\right.
$$


is equivalent, setting $z=\left(z_{j}\right)_{1 \leqslant j \leqslant n}, g=\left(g_{j}\right)_{1 \leqslant j \leqslant n}$, to

$$
z(\theta)=Y(\theta, 0) y+\int_{0}^{\theta} Y(\theta, t) g(t, z(t), \alpha) d t .
$$

Then we have the following Lemma.

Lemma 5.2.3. - One can find $\eta>0$ such that for all $y \in \mathbb{C}^{n}$ such that $|y| \leqslant \eta$, the problem (5.2.27) has a unique global solution $z$ such that $|z(\theta)| \leqslant 2 M_{0} \eta$ for all $\theta \in \mathbb{R}^{ \pm}$. This solution will be denoted by $z(\theta, y)$. Moreover one can find a constant $C\left(N_{0}, M_{0}\right) \geqslant 0$ such that

(i) $\left\|\left(\frac{\partial z_{j}}{\partial y_{k}}\right)(\theta, y)-Y(\theta, 0)\right\| \leqslant C\left(M_{0}, N_{0}\right) \eta$, and for every $\gamma \in \mathbb{N}^{n}$, one can find a constant $C_{\gamma} \geqslant 0$ such that

(ii) $\left|\partial_{y}^{\gamma} z(\theta, y)\right| \leqslant C_{\gamma}$, for all $\theta \geqslant 0$ and $|y| \leqslant \eta$.

Proof. - Let $\eta>0$ (to be chosen). Assume $|y| \leqslant \eta$ and set $A=\{T>0$ such that (5.2.27) has a solution for $\theta \in[0, T]$ satisfying $\left.|z(\theta)| \leqslant 2 M_{0} \eta\right\}$. Since (5.2.27) (which is equivalent to (5.2.26)) has a continuous solution for small $\theta$ and since $|z(0) \|=| y \mid \leqslant \eta$ there exists $\varepsilon_{0}>0$ such that $\varepsilon_{0} \in A$. Thus $A$ is non empty and it is obviously an interval. Let $T^{*}=\sup A$. If $T^{*}=+\infty$ we are done so assume $T^{*}<+\infty$. Let us take $T \in] 0, T^{*}[$. Then on $[0, T]$ we have

$$
|z(\theta)| \leqslant|Y(\theta, 0) y|+\int_{0}^{\theta}\|Y(\theta, t)\||g(t, z(t), \alpha)| d t .
$$

Now by (5.2.21) (ii) and (iii) we have

$$
|g(t, z(t), \alpha)| \leqslant \frac{K_{N_{0}}}{\langle t\rangle^{2}} 2 M_{0} \eta|z(t)|
$$

if $2 M_{0} \eta \leqslant 1$, where $K_{N_{0}}=\sum_{2 \leqslant|\gamma| \leqslant N_{0}-1} \frac{C_{\gamma}}{\gamma !}$.

It follows from (5.2.24) that,

$$
|z(\theta)| \leqslant M_{0} \eta+\int_{0}^{\theta} \frac{2 M_{0}^{2} K_{N_{0}} \eta}{\langle t\rangle^{2}}|z(t)| d t .
$$

Then the Gronwall inequality implies that

$$
|z(\theta)| \leqslant M_{0} \eta \exp \left[2 M_{0}^{2} K_{N_{0}} \eta \int_{0}^{+\infty} \frac{d t}{\langle t\rangle^{2}}\right]
$$

Therefore taking $\eta$ small (compared to $M_{0}$ and $K_{N_{0}}$ ) we can achieve that $|z(\theta)| \leqslant \frac{3}{2} M_{0} \eta$ for all $\theta \in[0, T]$. A classical argument shows that $z\left(T^{*}\right)$ can be defined and $\left|z\left(T^{*}\right)\right| \leqslant \frac{3}{2} M_{0} \eta$. Then solving again (5.2.26) with data $z\left(T^{*}\right)$ we see easily that this contradicts the definition of $T^{*}$ as the supremum of $A$. Therefore $T^{*}=+\infty$.

Now differentiating (5.2.27) with respect to $y_{k}$ yields

$$
\frac{\partial z}{\partial y_{k}}(\theta, y)-Y(\theta, 0) e_{k}+\int_{0}^{\theta} Y(\theta, t) \sum_{\ell=1}^{n} \frac{\partial g}{\partial z_{\ell}}(t, z(t), \alpha) \frac{\partial z_{\ell}}{\partial y_{k}}(t, y) d t .
$$


First of all (5.2.21) (ii) show that

$$
\sum_{\ell=1}^{n}\left|\frac{\partial g}{\partial z_{\ell}}(t, z(t), \alpha)\right| \leqslant \sum_{2 \leqslant|\gamma| \leqslant N_{0}-1} \frac{C_{\gamma}^{\prime}}{\langle t\rangle^{2}}|z(t, y)|^{|\gamma|-1} \leqslant \frac{C_{N_{0}} M_{0} \eta}{\langle t\rangle^{2}}
$$

if $2 M_{0} \eta \leqslant 1$. It follows that

$$
\left|\frac{\partial z}{\partial y_{k}}(\theta, y)\right| \leqslant M_{0}+\int_{0}^{\theta} \frac{C_{N_{0}}^{\prime} M_{0}^{2} \eta}{\langle t\rangle^{2}}\left|\frac{\partial z}{\partial y_{k}}(t, y)\right| d t .
$$

The Gronwall inequality shows that one can find $K=K\left(N_{0}, M_{0}\right)$ such that $\left|\frac{\partial z}{\partial y_{k}}(\theta, y)\right| \leqslant K$, for all $\theta \in \mathbb{R}^{ \pm}$and $|y| \leqslant \eta$.

Using again (5.2.28) and (5.2.29) we see that

$$
\left|\frac{\partial z}{\partial y_{k}}(\theta, y)-Y(\theta, 0) e_{k}\right| \leqslant \int_{0}^{\theta} \frac{C_{N_{0}} M_{0}^{2} \cdot K \eta}{\langle t\rangle^{2}} d t \leqslant C\left(M_{0}, N_{0}\right) \eta .
$$

Finally the estimate on $\partial_{y}^{\gamma} z$, which is true for $|\gamma|=0,1$ by the above results, can be easily obtained by induction on $|\gamma|$ using (5.2.27), (5.2.21) and the Gronwall Lemma.

In the sequel we shall take $\eta$ so small that $C\left(M_{0}, N_{0}\right) \eta \leqslant \frac{1}{2}$.

Let us now consider the map

$$
\left\{\begin{aligned}
\Phi: \mathbb{R}^{ \pm} \times\left\{y \in \mathbb{C}^{n}:|y| \leqslant \eta\right\} & \longrightarrow \mathbb{R}^{ \pm} \times \mathbb{C}^{n} \\
(\theta, y) & \longmapsto(\theta, z(\theta, y)) .
\end{aligned}\right.
$$

We claim that $\Phi$ is injective. Indeed for a fixed $\theta \in \mathbb{R}^{ \pm}$if we have $y_{j}, j=1,2$ such that $\left|y_{j}\right| \leqslant \eta$ and $z\left(\theta, y_{1}\right)=z\left(\theta, y_{2}\right)$ then

$$
0=\sum_{k=1}^{n} \int_{0}^{1} \frac{\partial z}{\partial y_{k}}\left(\theta, t y_{1}+(1-t) y_{2}\right)\left(y_{1}^{k}-y_{2}^{k}\right) d t
$$

Since $\left|t y_{1}+(1-t) y_{2}\right| \leqslant \eta$ when $t \in[0,1]$ we can use the estimate given in Lemma 5.2.3 to ensure that

$$
\left|Y(\theta, 0)\left(y_{1}-y_{2}\right)\right| \leqslant C^{\prime}\left(N_{0}, M_{0}\right) \eta\left|y_{1}-y_{2}\right| \text {. }
$$

According to (5.2.25) this implies that $y_{1}=y_{2}$ if $\eta$ is small enough.

It follows that $\Phi$ is bijective on its range. We show now that

$$
\left\{\begin{array}{l}
\text { If } \delta \text { is small enough we have } \\
\mathbb{R}^{ \pm} \times\left\{z \in \mathbb{C}^{n}:|z| \leqslant \delta\right\} \subset \Phi\left(\mathbb{R}^{ \pm} \times\left\{y \in \mathbb{C}^{n}:|y| \leqslant \eta\right\}\right) .
\end{array}\right.
$$

This equivalent to show that for fixed $\theta \in \mathbb{R}^{ \pm}$,

$$
\left\{\begin{array}{l}
\text { for all } z \in \mathbb{C}^{n} \text { such that }|z| \leqslant \delta \text { there exists } y \in \mathbb{C}^{n} \\
\text { such that }|y| \leqslant \eta \text { and } z(\theta, y)=z .
\end{array}\right.
$$

According to (5.2.27) the equation to solve is equivalent to the equation $y=F(y)$ where

$$
F(y)=Y(\theta, 0)^{-1} z-Y(\theta, 0)^{-1} \int_{0}^{\theta} Y(\theta, t) g(t, z(t, y), \alpha) d t
$$


Let $B=\left\{y \in \mathbb{C}^{n}:|y| \leqslant \eta\right\}$. We shall show that if $\delta$ and $\eta$ are small enough compared to $N_{0}, M_{0}$ then $F$ maps $B$ into $B$ and there exists $\varepsilon_{0}<1$ such that $\left|F\left(y_{1}\right)-F\left(y_{2}\right)\right| \leqslant \varepsilon_{0}\left|y_{1}-y_{2}\right|$ for all $y_{1}, y_{2}$ in $B$. Then (5.2.32) will follow from the fixed point Theorem. Since $Y(\theta, 0)^{-1}=Y(0, \theta)$ and $Y(0, \theta) Y(\theta, t)=Y(0, t)$ it follows from (5.2.24) and (5.2.21) that if $|z| \leqslant \delta$ we have

$$
|F(y)| \leqslant M_{0} \delta+\left|\int_{0}^{\theta} \frac{C\left(M_{0}\right) \eta^{2}}{\langle t\rangle^{2}} d t\right|
$$

since $|z(t, y)| \leqslant 2 M_{0} \eta$ by Lemma 5.2.3. Then $|F(y)| \leqslant \eta$ if $\eta$ is small enough in terms of $M_{0}$ and $M_{0} \delta \leqslant \frac{1}{2} \eta$.

Moreover if $y_{1}, y_{2}$ belong to $B$ we have

$$
\left|F\left(y_{1}\right)-F\left(y_{2}\right)\right| \leqslant\left|\int_{0}^{\theta} \frac{C\left(M_{0}, N_{0}\right)}{\langle t\rangle^{2}} \eta\right| z\left(t, y_{1}\right)-z\left(t, y_{2}\right)|d t| .
$$

Since by Lemma 5.2 .3 we have $\left|z\left(t, y_{1}\right)-z\left(t, y_{2}\right)\right| \leqslant C^{\prime}\left(M_{0}\right)\left|y_{1}-y_{2}\right|$ we obtain finally

$$
\left|F\left(y_{1}\right)-F\left(y_{2}\right)\right| \leqslant C^{\prime}\left(M_{0}, N_{0}\right) \eta\left|y_{1}-y_{2}\right| .
$$

Taking $\eta$ small enough we obtain (5.2.32).

We can now straighten the vector field $L_{0}$ which is the principal part of $L$ given in (5.2.16). Let us make the change of variables, $\left(\theta^{\prime}, y\right) \mapsto(\theta, z(\theta, y))$. Then we have, according to $(5.2 .26)$

$$
\frac{\partial}{\partial \theta^{\prime}}=\frac{\partial}{\partial \theta}+\sum_{j=1}^{n} \dot{z}_{j}(\theta, y) \frac{\partial}{\partial z_{j}}=\frac{\partial}{\partial \theta}+\sum_{j=1}^{n} h_{j}^{N_{0}}(\theta, z(\theta, y)) \frac{\partial}{\partial z_{j}}=L_{0} .
$$

In the new coordinates $\left(\theta^{\prime}, y\right)$ the operator $L$ has therefore the form

$$
L=\frac{\partial}{\partial \theta^{\prime}}+d^{N_{0}}\left(\theta^{\prime}, z\left(\theta^{\prime}, y\right), \alpha\right)=\frac{\partial}{\partial \theta^{\prime}}+\widetilde{d}\left(\theta^{\prime}, y, \alpha\right) .
$$

Now we note that

$$
\frac{\partial}{\partial \theta^{\prime}}\left(e^{\int_{0}^{\theta^{\prime}} \widetilde{d}(t, y, \alpha) d t} u(t, y, \alpha)\right)=e^{\int_{0}^{\theta^{\prime}} \widetilde{d}(t, y, \alpha) d t} L u(t, y, \alpha) .
$$

It follows that the problem

$$
L \widetilde{A}_{0}=0, \quad \widetilde{A}_{0}(0, y, \alpha)=1
$$

has the (unique) solution $\widetilde{A}_{0}\left(\theta^{\prime}, y, \alpha\right)=e^{-\int_{0}^{\theta^{\prime}} \widetilde{d}(t, y, \alpha) d t}$. By the same way the problems

$$
L \widetilde{A}_{\ell}=i \widetilde{Q} \widetilde{A}_{\ell-1}, \quad \widetilde{A}_{\ell}(0, y, \alpha)=0, \quad \ell=1, \ldots, N_{0}+1,
$$

are solved by

$$
\widetilde{A}_{\ell}\left(\theta^{\prime}, y, \alpha\right)=e^{-\int_{0}^{\theta^{\prime}} \widetilde{d}(t, y, \alpha) d t} \int_{0}^{\theta^{\prime}} i\left(\widetilde{Q} \widetilde{A}_{\ell-1}\right)(t, y, \alpha) e^{\int_{0}^{t} \widetilde{d}(s, y, \alpha) d s} d t .
$$

To end the proof of Lemma 5.2.2 we are left with the uniform estimates (ii). 
First of all, using the estimate in (5.2.14), (5.2.15), Lemma 5.2.3 (ii) and the Faa di Bruno formula we see that,

$$
\mid \partial_{y}^{\gamma}\left(d^{N_{0}}\left(\theta^{\prime}, z\left(\theta^{\prime}, y\right), \alpha\right) \mid \leqslant \frac{C_{\gamma}}{\left\langle\theta^{\prime}\right\rangle^{2}}\right.
$$

Denoting by $\kappa(\theta, z)$ the inverse map of $y \mapsto z(\theta, y)$, that is $\kappa(\theta, z(\theta, y))=y$ and using Lemma 5.2.3 we see that,

$$
\left|\partial_{z}^{\gamma} \kappa(\theta, z)\right| \leqslant C_{\gamma} \text { for } \theta \geqslant 0 \text { and }|z| \leqslant \delta .
$$

Then let us set for $\ell=0, \ldots, N_{0}+1$

$$
A_{\ell}(\theta, z, \alpha)=\widetilde{A}_{\ell}(\theta, \kappa(\theta, z), \alpha) .
$$

Using (5.2.34), (5.2.35), (5.2.16), (5.2.15) and the estimate in (5.2.12) we see that $\left(A_{\ell}\right)_{\ell=0, \ldots, N_{0}+1}$ satisfy all the requirements of Lemma 5.2.2. This ends the proof of Theorem 5.1.1 in the case of outgoing points.

We consider now the case of incoming points.

\subsection{The case of incoming points}

We assume here that $\alpha \in T^{*} \mathbb{R}^{n}$ is such that $\frac{1}{2} \leqslant\left|\alpha_{\varepsilon}\right| \leqslant 2$ and

$$
\alpha_{x} \cdot \alpha_{\xi} \leqslant-c_{0}\left\langle\alpha_{x}\right\rangle\left|\alpha_{\xi}\right|
$$

Since such points belong to $\mathcal{S}_{-}$(see Definition 3.2.2) the case where $\theta \leqslant 0$ is covered by the Section 5.2. We focus now on the case $\theta \geqslant 0$. Here the method used in Section 5.2 does not work for many technical reasons. For instance, when $\left|\alpha_{x}\right|$ is very large, $\alpha_{\xi}=-\alpha_{x} /\left|\alpha_{x}\right|$ and $\theta=\frac{1}{2}\left|\alpha_{x}\right|$ we can see that $\langle x(\theta, \alpha)\rangle$ is of magnitude one. Therefore we are far from the estimate $\langle x(\theta, \alpha)\rangle \geqslant \frac{1}{\sqrt{2}}\langle\theta\rangle$ used for instance to get (5.2.3). Here also we shall use the method which consists to straighten the vector field $X$, defined in (5.1.3). This is done by a change of variables in $(\theta, x)$ deduced from the flow of $X$. The problem here is that $X$ has non real coefficients (because of $\frac{\partial \varphi}{\partial x_{j}}$ ) which are merely $C^{\infty}$. Therefore we are led to push the problem in the complex domain by extending all the functions almost analytically as in [MS] for instance. So we begin our Section by a Lemma on almost analytic extensions adapted to our situation. In that follows we shall consider together two cases. Case 1: $\Omega=\mathbb{R}_{x}^{n}$, case 2: $\Omega=\Omega_{\delta}$ (see Definition 4.1.1). We shall denote by $X$ the variable in $\Omega$ that is $X=x$ in the first case, $X=(\theta, x)$ in the second one. 
Lemma 5.3.1. - Let $f$ be a function defined on $\Omega$ which is $C^{\infty}$ in $X$ and satisfies for all $X$ in $\Omega$, in case 1 (resp. case 2$)$,

$$
\left\{\begin{array}{l}
|f(X)| \leqslant \frac{M_{0}}{\langle x\rangle^{\sigma_{1}}}\left(\text { resp. } M_{0}\left(\frac{1}{\langle x\rangle^{\sigma_{1}}}+\frac{1}{\langle\theta\rangle^{\sigma_{2}}}\right)\right) \\
\sum_{|\gamma|=k}\left|\partial_{x}^{\gamma} f(X)\right| \leqslant \frac{M_{k}}{\langle x\rangle^{k+\sigma_{3}}}\left(\text { resp. } M_{k}\left(\frac{1}{\langle x\rangle^{k+\sigma_{3}}}+\frac{1}{\langle\theta\rangle^{k+\sigma_{3}}}\right), \quad k \geqslant 1\right.
\end{array}\right.
$$

where $\left(M_{k}\right)_{k \geqslant 0}$ is an increasing sequence in $] 0,+\infty\left[\right.$ and $0 \leqslant \sigma_{1} \leqslant \sigma_{3}, 0 \leqslant \sigma_{2} \leqslant \sigma_{3}$. Then there exists $F=F(X, y)$ defined on $\Omega \times \mathbb{R}_{y}^{n}$ which is $C^{\infty}$ in $(X, y)$ and satisfies for all $(X, y)$ in $\Omega \times \mathbb{R}_{y}^{n}$,

(i) $F(X, 0)=f(X)$.

(ii) $|F(X, y)| \leqslant \frac{C_{0}}{\langle x\rangle^{\sigma_{1}}}$ (resp. $C_{0}\left(\frac{1}{\langle x\rangle^{\sigma_{1}}}+\frac{1}{\langle\theta\rangle^{\sigma_{2}}}\right)$ ).

(iii) For every $A, B$ in $\mathbb{N}^{n}$ with $|A|+|B| \geqslant 1$ there exists $C_{A B}>0$ such that $\left|\partial_{x}^{A} \partial_{y}^{B} F(X, y)\right| \leqslant \frac{C_{A B}}{\langle x\rangle^{|A|+|B|+\sigma_{3}}}\left(\right.$ resp. $C_{A B}\left(\frac{1}{\langle x\rangle^{|A|+|B|+\sigma_{3}}}+\frac{1}{\langle\theta\rangle^{|A|+|B|+\sigma_{3}}}\right)$ ).

(iv) For every $N \in \mathbb{N}$ there exists $C_{N}>0$ such that for $j=1, \ldots, n$,

$\left|\bar{\partial}_{j} F(X, y)\right| \leqslant C_{N}\left(\frac{|y|}{\langle x\rangle}\right)^{N} \cdot \frac{1}{\langle x\rangle^{1+\sigma_{3}}} \quad$ (resp. $\quad C_{N}|y|^{N}\left[\left(\frac{1}{\langle x\rangle}+\frac{1}{\langle\theta\rangle}\right)^{N}\left(\frac{1}{\langle x\rangle^{1+\sigma_{3}}}+\right.\right.$ $\left.\left.\frac{1}{\langle\theta\rangle^{1+\sigma_{3}}}\right)\right]$ )

where $\bar{\partial}_{j}=\frac{1}{2}\left(\frac{\partial}{\partial x_{j}}+i \frac{\partial}{\partial y_{j}}\right)$.

Proof. - See Section A.4 in the Appendix.

Now recall that for $\alpha \in T^{*} \mathbb{R}^{n}$ such that $\alpha_{x} \cdot \alpha_{\xi} \leqslant-c_{0}\left\langle\alpha_{x}\right\rangle\left|\alpha_{\varepsilon}\right|$ and $\frac{1}{2} \leqslant\left|\alpha_{\varepsilon}\right| \leqslant 2$ we have constructed in Theorem 4.4 .10 a function $\Phi=\Phi(\theta, x, \alpha)$ uniformly bounded on the set $\Omega_{\delta}$. By Lemma 5.3.1 we can extend $\Phi$ almost analytically as a function, which we denote by $\Phi(\theta, z, \alpha)$, on the set

$$
\Omega_{\delta}^{\mathbb{C}}=\left\{(\theta, z) \in \mathbb{R} \times \mathbb{C}^{n}:|z-x(\theta, \alpha)| \leqslant \delta\langle\theta\rangle, \operatorname{Re} z \cdot \alpha_{\xi} \leqslant c_{0}\langle\operatorname{Re} z\rangle\left|\alpha_{\xi}\right|,|\operatorname{Im} z| \leqslant \delta\right\}
$$

and $\Phi(\theta, z, \alpha)$ is still uniformly bounded on this set.

Again by Lemma 5.3.1 one can extend almost analytically the coefficients of our symbol $p$, keeping the bounds of its coefficients. In that follows for $z \in \mathbb{C}^{n}$ we shall denote by $X(t, \theta, z)$ the solution, whenever it exists, of the following problem.

$$
\left\{\begin{array}{l}
\dot{X}(t, \theta, z)=\frac{\partial p}{\partial \xi}(X(t, \theta, z), \Phi(t, X(t, \theta, z), \alpha)), \\
X(\theta, \theta, z)=z .
\end{array}\right.
$$

Our aim is to prove the following result. 
TheOREM 5.3.2. - One can find positive constants $c_{1}, \delta_{1}, K, \widetilde{K}$, with $c_{1} \ll c_{0}$, $\delta_{1} \ll \delta$, such that for all $x \in \mathbb{R}^{n}$ such that

$$
|x-x(\theta, \alpha)| \leqslant \delta_{1}\langle\theta\rangle, \quad x \cdot \alpha_{\xi} \leqslant c_{1}\langle x\rangle\left|\alpha_{\xi}\right|,
$$

the solution of (5.3.4) exists on $[0, \theta]$ and satisfies the estimates,

$$
\begin{cases}\text { (i) } & |X(t, \theta, x)-x(t, \alpha)| \leqslant K|x-x(\theta, \alpha)| \frac{\langle t\rangle}{\langle\theta\rangle}, \\ \text { (ii) } & |\operatorname{Im} X(t, \theta, x)| \leqslant K \frac{|x-x(\theta, \alpha)|}{\langle\theta\rangle} \\ \text { (iii) } & \langle x\rangle+\langle\theta-t\rangle \leqslant K\langle\operatorname{Re} X(t, \theta, x)\rangle, \\ \text { (iv) } & \operatorname{Re} X(t, \theta, x) \cdot \alpha_{\xi} \leqslant \frac{1}{\widetilde{K}}\langle\operatorname{Re} X(t, \theta, x)\rangle\left|\alpha_{\xi}\right|,\end{cases}
$$

uniformly for $t \in[0, \theta]$.

Let us remark that the estimates (5.3.5) ensure in particular that if $\delta_{1}$ is small enough we have $(t, X(t, \theta, \alpha)) \in \Omega_{\delta}^{\mathbb{C}}$. With $0<c_{1} \ll c_{2} \ll c_{0}$ to be chosen, we divide the proof in three cases.

- Case 1: $x \cdot \alpha_{\xi} \leqslant c_{2}\langle x\rangle\left|\alpha_{\xi}\right|, x(\theta, \alpha) \cdot \alpha_{\xi} \leqslant c_{2}\langle x(\theta, \alpha)\rangle\left|\alpha_{\xi}\right|,|x-x(\theta, \alpha)| \leqslant|x(\theta, \alpha)|$.

- Case 2: $x \cdot \alpha_{\xi} \leqslant c_{2}\langle x\rangle\left|\alpha_{\xi}\right|, x(\theta, \alpha) \cdot \alpha_{\xi} \leqslant c_{2}\langle x(\theta, \alpha)\rangle\left|\alpha_{\xi}\right|,|x-x(\theta, \alpha)|>|x(\theta, \alpha)|$.

- Case 3: $x \cdot \alpha_{\xi} \leqslant c_{1}\langle x\rangle\left|\alpha_{\xi}\right|, x(\theta, \alpha) \cdot \alpha_{\xi}>c_{2}\langle x(\theta, \alpha)\rangle\left|\alpha_{\xi}\right|$.

Here is the geometrical interpretation of case 1 and 2 . We denote by $[a, b]$ the segment joining two points $a, b \in \mathbb{R}^{n}$.

LEMmA 5.3.3. - Let $c_{2}>0$ and assume that $x \in \mathbb{R}^{n}$ is such that $x \cdot \alpha_{\xi} \leqslant c_{2}\langle x\rangle\left|\alpha_{\xi}\right|$, $|x-x(\theta, \alpha)| \leqslant \delta\langle\theta\rangle$ and that $x(\theta, \alpha) \cdot \alpha_{\xi} \leqslant c_{2}\langle x(\theta, \alpha)\rangle\left|\alpha_{\xi}\right|$. Then we have:

(i) either $|x-x(\theta, \alpha)| \leqslant|x(\theta, \alpha)|$ and then,

$$
\forall y \in[x, x(\theta, \alpha)], \quad y \cdot \alpha_{\varepsilon} \leqslant 2 c_{2}\langle y\rangle\left|\alpha_{\xi}\right|,
$$

(ii) or $|x-x(\theta, \alpha)|>|x(\theta, \alpha)|$ and then,

$$
[0, x(\theta, \alpha)] \cup[0, x] \subset\left\{y \in \mathbb{R}^{n}:|y-x(\theta, \alpha)| \leqslant \delta_{0}\langle\theta\rangle \quad \text { and } \quad y \cdot \alpha_{\xi} \leqslant c_{2}\langle y\rangle\left|\alpha_{\xi}\right|\right\} .
$$

Moreover

$$
|x|+|x(\theta, \alpha)| \leqslant 3|x-x(\theta, \alpha)| \leqslant 3(|x|+|x(\theta, \alpha)|) .
$$

Proof. - In the first case applying Lemma 4.4 .16 we obtain for $t \in[0,1]$

$$
\begin{aligned}
(t x+(1-t) x(\theta, \alpha)) \cdot \alpha_{\xi} & \leqslant c_{2}(t\langle x\rangle+(1-t)\langle x(\theta, \alpha)\rangle)\left|\alpha_{\xi}\right| \\
& \leqslant c_{2}(1+t|x|+(1-t)|x(\theta, \alpha)|)\left|\alpha_{\xi}\right| \\
& \leqslant c_{2}(1+\sqrt{2}|t x+(1-t) x(\theta, \alpha)|)\left|\alpha_{\xi}\right| \\
& \leqslant 2 c_{2}\langle t x+(1-t) x(\theta, \alpha)\rangle\left|\alpha_{\xi}\right| .
\end{aligned}
$$


Assume now that $|x-x(\theta, \alpha)|>|x(\theta, \alpha)|$. Then $|x(\theta, \alpha)| \leqslant \delta_{0}\langle\theta\rangle$. Therefore $[0, x] \cup$ $[0, x(\theta, \alpha)] \subset B\left(x(\theta, \alpha), \delta_{0}\langle\theta\rangle\right)$. Now if $t \in[0,1]$ and $Z=x$ or $x(\theta, \alpha)$ we have $t Z \cdot \alpha_{\xi} \leqslant t c_{2}\langle Z\rangle\left|\alpha_{\xi}\right| \leqslant c_{2}\langle t Z\rangle\left|\alpha_{\xi}\right|$. Moreover

$$
\begin{aligned}
3|x-x(\theta, \alpha)| & =|x-x(\theta, \alpha)|+2|x-x(\theta, \alpha)| \geqslant|x|-|x(\theta, \alpha)|+2|x(\theta, \alpha)| \\
& =|x|+|x(\theta, \alpha)| .
\end{aligned}
$$

1) Proof of Theorem 5.3.2 in case 1 and 2. - Let us take $c_{2}, \delta_{2}$ such that $0<c_{2} \ll c_{0}, 0<\delta_{2} \ll \delta$. Let $A$ be the set of $T \in[0, \theta]$ such that for every $z \in \mathbb{C}^{n}$ such that $|z-x(\theta, \alpha)| \leqslant \delta_{2}\langle\theta\rangle, \operatorname{Re} z \cdot \alpha_{\xi} \leqslant c_{2}\langle\operatorname{Re} z\rangle\left|\alpha_{\xi}\right|,|\operatorname{Im} z| \leqslant \delta_{2}$, $x(\theta, \alpha) \cdot \alpha_{\xi} \leqslant c_{2}\langle x(\theta, \alpha)\rangle\left|\alpha_{\xi}\right|$ the problem (5.3.5) has a unique solution on $[T, \theta]$ which satisfies for $t \in[T, \theta]$, in case 1 :

$$
\begin{aligned}
& |X(t, \theta, z)-x(t, \alpha)| \leqslant M_{1}|z-x(\theta, \alpha)| \frac{\langle t\rangle}{\langle\theta\rangle} \\
& \langle\theta-t\rangle+\langle\operatorname{Re}(s z+(1-s) x(\theta, \alpha))\rangle \\
& \leqslant M_{1}\langle s \operatorname{Re} X(t, \theta, z)+(1-s) x(t, \alpha)\rangle, \quad s \in[0,1] \\
& (s \operatorname{Re} X(t, \theta, z)+(1-s) x(\theta, \alpha)) \cdot \alpha_{\xi} \\
& \leqslant M_{2}\langle s \operatorname{Re} X(t, \theta, z)+(1-s) x(\theta, \alpha)\rangle\left|\alpha_{\xi}\right| \\
& |\operatorname{Im} X(t, \theta, z)| \leqslant M_{3}\left(\frac{|z-x(\theta, \alpha)|}{\langle\theta\rangle}+|\operatorname{Im} z|\right)
\end{aligned}
$$

in case 2 :

$$
\begin{gathered}
|X(t, \theta, z)-X(t, \theta, 0)| \leqslant M_{1}|z| \frac{\langle t\rangle}{\langle\theta\rangle} \\
\langle\theta-t\rangle+\langle\operatorname{Re}(s z)\rangle \leqslant M_{1}\langle s \operatorname{Re} X(t, \theta, z)+(1-s) \operatorname{Re} X(t, \theta, 0)\rangle, \quad s \in[0,1] \\
(s \operatorname{Re} X(t, \theta, z)+(1-s) \operatorname{Re} X(t, \theta, 0)) \cdot \alpha_{\xi} \\
\leqslant M_{2}\langle s \operatorname{Re} X(t, \theta, z)+(1-s) \operatorname{Re} X(t, \theta, 0)\rangle
\end{gathered}
$$

and (5.3.9).

Our aim is to show that if $M_{1}, M_{2}, M_{3}$ are correctly chosen then $A=[0, \theta]$.

Let us show that the set $A$ is not empty. Indeed if $t=\theta$ the estimates (5.3.6) to (5.3.12) are satisfied with strict inequalities if $M_{1}>1, M_{2}>2 C_{2}, M_{3}>1$ (using Lemma 5.3.3). It follows that they still hold for $T=\theta-\varepsilon$, if $\varepsilon$ is small enough.

On the other hand $A$ is an interval. Let $T_{*}=\inf A$. If $T_{*}=0$ then the theorem 5.3.2 is proved. Assume then that $T_{*}>0$ and let $T \geqslant T_{*}$. Then on $[T, \theta],(5.3 .6)$ to (5.3.12) hold.

REMARK 5.3.4. - If the case 2 is not empty then the point $z_{0}=0$ satisfies all the requirements of case 1 . Indeed if there exists $z_{1}$ such that $\left|z_{1}-x(\theta, \alpha)\right|>|x(\theta, \alpha)|$ then $|x(\theta, \alpha)|<\delta_{2}\langle\theta\rangle$ so $|0-x(\theta, \alpha)|<\delta_{2}\langle\theta\rangle$ and the other requirements are trivial. Therefore if the case 2 is not empty then $X(t, \theta, 0)$ is well defined on $[T, \theta]$ and satisfies (5.3.6) to $(5.3 .9)$. 
Let us show that we have $(t, X(t, \theta, z)) \in \Omega_{\delta}^{\mathbb{C}}$ (see (5.3.3)). This is the case if $M_{1} \delta_{2} \leqslant \delta, M_{2} \leqslant c_{0}, 2 M_{3} \delta_{2} \leqslant \delta$. Indeed the only non trivial point is to prove that $|X(t, \theta, z)-x(t, \alpha)| \leqslant \delta\langle t\rangle$ in case 2 . We have

$$
|X(t, \theta, z)-x(t, \alpha)| \leqslant|X(t, \theta, z)-X(t, \theta, 0)|+|X(t, \theta, 0)-x(t, \alpha)|=(1)+(2) .
$$

It follows from (5.3.10) that $(1) \leqslant M_{1}|z| \frac{\langle t\rangle}{\langle\theta\rangle}$ and from (5.3.6) with $z=0$, that $(2) \leqslant M_{1}|x(\theta, \alpha)| \frac{\langle t\rangle}{\langle\theta\rangle}$. Now by Lemma 5.3 .3 (ii) we have $|\operatorname{Re} z|+|x(\theta, \alpha)|<3 \mid \operatorname{Re} z$ $-x(\theta, \alpha) \mid$; since $|\operatorname{Im} z| \leqslant \delta_{2}$ we will have $(1)+(2) \leqslant M_{1}\left(\delta_{2}+3|\operatorname{Re} z-x(\theta, \alpha)|\right) \frac{\langle t\rangle}{\langle\theta\rangle}$. Since $|\operatorname{Re} z-x(\theta, \alpha)| \leqslant \delta_{2}\langle\theta\rangle$ we obtain finally $(1)+(2) \leqslant 4 M_{1} \delta_{2}\langle t\rangle \leqslant \delta\langle t\rangle$.

In the sequel we shall denote by $C$ or $O(1)$ the constants which may depend on bounds of $p, \Phi$ but are independent of $M_{1}, M_{2}, M_{3}$. Moreover for the sake of simplicity we shall write

$$
\left\{\begin{array}{l}
X(t)=X(t, \theta, z) \\
\widetilde{X}(t)=x(t, \alpha) \text { in case } 1, X(t, \theta, 0) \text { in case } 2 .
\end{array}\right.
$$

In particular $\tilde{X}(\theta)=x(\theta, \alpha)$ in case 1 and $\tilde{X}(\theta)=0$ in case 2 . Our goal is to show that the estimates (5.3.6) to (5.3.12) hold on $[T, \theta]$ with better constants than $M_{1}, M_{2}, M_{3}$.

a) Improvement of (5.3.7) and (5.3.11). - By Theorem 4.4.10 (iii) we have $\Phi(\theta, x, \alpha)-\alpha_{\xi}=\mathcal{O}(\varepsilon+\delta)$ if $(\theta, x) \in \Omega_{\delta}$ and by Lemma 5.3.1 this estimate still hold on $\Omega_{\delta}^{\mathbb{C}}$; it follows that $\Phi(t, X(t), \alpha)-\alpha_{\xi}=\mathcal{O}(\varepsilon+\delta)$. On the other hand $\frac{\partial p}{\partial \xi}(x, \xi)-2 \xi=\mathcal{O}(\varepsilon)|\xi|$ which also extends for $z \in \mathbb{C}^{n},|\operatorname{Im} z| \leqslant \delta_{2}$. It follows then from (5.3.4) that $\dot{X}(t)=2 \alpha_{\varepsilon}+\mathcal{O}(\varepsilon+\delta)$. Therefore

$$
\left\{\begin{array}{l}
X(t)=z-2(\theta-t) \alpha_{\xi}+\mathcal{O}(\varepsilon+\sqrt{\delta})(\theta-t) \\
\widetilde{X}(t)=\widetilde{X}(\theta)-2(\theta-t) \alpha_{\xi}+\mathcal{O}(\varepsilon+\sqrt{\delta})(\theta-t) .
\end{array}\right.
$$

Now for $s \in[0,1]$

$$
\begin{aligned}
&(1)=\mid \operatorname{Re}(s X(t)+(1-s)\widetilde{X}(t))\left.\right|^{2}=|s \operatorname{Re} z+(1-s) \operatorname{Re} \widetilde{X}(\theta)|^{2}+4(\theta-t)^{2}\left|\alpha_{\xi}\right|^{2} \\
&-4(\theta-t)(s \operatorname{Re} z+(1-s) \operatorname{Re} \widetilde{X}(\theta)) \cdot \alpha_{\xi} \\
&+\mathcal{O}\left((\varepsilon+\delta)\left[(\theta-t)^{2}+|s \operatorname{Re} z+(1-s) \operatorname{Re} \widetilde{X}(\theta)|^{2}\right] .\right.
\end{aligned}
$$

It follows from the conditions on $z$ and the definition of $\widetilde{X}(\theta)$ that

$$
(s \operatorname{Re} z+(1-s) \operatorname{Re} \tilde{X}(\theta)) \cdot \alpha_{\varepsilon} \leqslant 2 c_{2}\langle s \operatorname{Re} z+(1-s) \operatorname{Re} \tilde{X}(\theta)\rangle\left|\alpha_{\xi}\right|
$$

so

$(1) \geqslant \frac{1}{2}|s \operatorname{Re} z+(1-s) \operatorname{Re} \widetilde{X}(\theta)|^{2}+3(\theta-t)^{2}\left|\alpha_{\xi}\right|^{2}-8 c_{2}\langle s \operatorname{Re} z+(1-s) \operatorname{Re} \widetilde{X}(\theta)\rangle(\theta-t)\left|\alpha_{\xi}\right|$ if $\varepsilon+\delta$ is small enough. It follows that

$$
(1) \geqslant\left(\frac{1}{2}-16 c_{2}\right)|s \operatorname{Re} z+(1-s) \operatorname{Re} \tilde{X}(\theta)|^{2}+\left(3-16 c_{2}\right)(\theta-t)^{2}\left|\alpha_{\xi}\right|^{2}-16 c_{2} .
$$


If $c_{2}$ has been chosen small enough we obtain in particular

$$
\left\{\begin{aligned}
&(\mathrm{i})|\operatorname{Re}(s X(t)+(1-s) \tilde{X}(t))|^{2} \geqslant \frac{1}{4} \mid \operatorname{Re}\left(s z+\left.(1-s) \tilde{X}(\theta)\right|^{2}\right. \\
&+2(\theta-t)^{2}\left|\alpha_{\xi}\right|^{2}-\frac{1}{2} \\
& \text { (ii) }\langle\operatorname{Re}(s X(t)+(1-s) \tilde{X}(t))\rangle^{2} \geqslant \frac{1}{10}\left[\langle\theta-t\rangle^{2}+\langle\operatorname{Re}(s z+(1-s) \tilde{X}(\theta))\rangle^{2}\right]
\end{aligned}\right.
$$

This improves (5.3.7) and (5.3.11) if $M_{1}>4$.

b) Improvement of (5.3.8), (5.3.12). - It follows from (5.3.14) that

$$
\begin{aligned}
(2) & =\operatorname{Re}(s X(t)+(1-s) \tilde{X}(t)) \cdot \alpha_{\xi} \\
& =\operatorname{Re}(s z+(1-s) \tilde{X}(\theta)) \cdot \alpha_{\xi}-2(\theta-t)\left|\alpha_{\xi}\right|^{2}+\mathcal{O}(\varepsilon+\delta)(\theta-t) .
\end{aligned}
$$

Applying Lemma 5.3.3 we obtain if $\varepsilon+\delta$ is small,

$$
(2) \leqslant 2 c_{2}\langle\operatorname{Re}(s z+(1-s) \tilde{X}(\theta))\rangle-(\theta-t)\left|\alpha_{\xi}\right|^{2} .
$$

Using (5.3.15) (i) we obtain, $(2) \leqslant 4 c_{2}\left\langle\operatorname{Re}(s X(t)+(1-s) \widetilde{X}(t)\rangle\right.$. Taking $16 c_{2} \leqslant M_{2}$ we deduce finally that

$$
\operatorname{Re}(s X(t)+(1-s) \tilde{X}(t)) \cdot \alpha_{\xi} \leqslant \frac{1}{2} M_{2}\langle\operatorname{Re}(s X(t)+(1-s) \tilde{X}(t))\rangle\left|\alpha_{\xi}\right| .
$$

This improves (5.3.8) and (5.3.12).

c) Improvement of (5.3.6) and (5.3.10). - We have

$$
\left\{\begin{array}{l}
\dot{X}(t)=\frac{\partial p}{\partial \xi}(X(t), \Phi(t, X(t), \alpha)) \\
\dot{\widetilde{X}}(t)=\frac{\partial p}{\partial \xi}(\widetilde{X}(t), \Phi(t, \widetilde{X}(t), \alpha))
\end{array}\right.
$$

the second equation being true in the case 1 according to the fact that the identity $\Phi(t, x(t, \alpha), \alpha)=\xi(t, \alpha)$. Let us set

$$
Z(t)=X(t)-\tilde{X}(t)
$$

Then

$$
\begin{aligned}
\dot{Z}(t)=2[\Phi(t, X(t), \alpha)-\Phi(t, \tilde{X}(t), \alpha)]+\frac{\partial q}{\partial \xi}(X(t), \Phi(t, X(t), \alpha)) & \\
& -\frac{\partial q}{\partial \xi}(\widetilde{X}(t), \Phi(t, \widetilde{X}(t), \alpha)),
\end{aligned}
$$

since $p=|\xi|^{2}+q$.

Now we use (4.4.39) and Theorem 4.4.2 (i). It follows after extending almost analytically $\widetilde{a}, \widetilde{b}$ and the coefficients of $q$ by Lemma 5.3.1,

$$
\Phi(t, z, \alpha)=\xi(t, \alpha)+\frac{z-x(t, \alpha)}{2 t-i}-\left(\widetilde{a}+\frac{i}{\langle t\rangle} \widetilde{b}\right)(t, z, \alpha) .
$$


It follows then that

(5.3.19)

$$
\left\{\begin{aligned}
\dot{Z}(t) & =\frac{2 Z(t)}{2 t-i}-(\widetilde{a}(t, X(t), \alpha)-\widetilde{a}(t, \widetilde{X}(t), \alpha))-\frac{i}{\langle t\rangle}(\widetilde{b}(t, X(t), \alpha)-\widetilde{b}(t, \widetilde{X}(t), \alpha)) \\
& +\frac{\partial q}{\partial \xi}(X(t), \Phi(t, X(t), \alpha))-\frac{\partial q}{\partial \xi}(\widetilde{X}(t), \Phi(t, X(t), \alpha))+\frac{\partial q}{\partial \xi}(\widetilde{X}(t), \Phi(t, X(t), \alpha)) \\
& -\frac{\partial q}{\partial \xi}(\widetilde{X}(t), \Phi(t, \widetilde{X}(t), \alpha)) .
\end{aligned}\right.
$$

We have the following lemma.

Lemma 5.3.5. - One can find a positive constant $C$ such that

$$
\left|\dot{Z}(t)-\frac{2 Z(t)}{2 t-i}\right| \leqslant C(\varepsilon+\delta)|Z(t)|\left(\frac{1}{\langle\theta-t\rangle^{2}}+\frac{1}{\langle t\rangle^{2}}\right) .
$$

Proof

(i) Estimation of $(1)=\widetilde{a}(t, X(t), \alpha)-\widetilde{a}(t, \widetilde{X}(t), \alpha)$. We have

$$
\begin{aligned}
(1)=\int_{0}^{1} \frac{\partial \widetilde{a}}{\partial z}(t, s X(t)+ & (1-s) \widetilde{X}(t), \alpha)(X(t)-\widetilde{X}(t)) d s \\
& +\int_{0}^{1} \frac{\partial \widetilde{a}}{\partial \bar{z}}(t, s X(t)+(1-s) \widetilde{X}(t), \alpha) \overline{(X(t)-\widetilde{X}(t))} d s .
\end{aligned}
$$

Using the estimates on $\widetilde{a}$ given in Theorem 4.4.2 and Lemma 5.3.1 with $\sigma_{3}=1$ we find

$$
\left(\left|\frac{\partial \widetilde{a}}{\partial z}\right|+\left|\frac{\partial \widetilde{a}}{\partial \bar{z}}\right|\right)(t, \cdots) \leqslant C(\varepsilon+\delta)\left(\frac{1}{\langle\operatorname{Re}(s X(t)+(1-s) \widetilde{X}(t))\rangle^{2}}+\frac{1}{\langle t\rangle^{2}}\right) .
$$

Using (5.3.7) and (5.3.12) we deduce that

$$
\left|\frac{\partial \widetilde{a}}{\partial z}\right|+\left|\frac{\partial \widetilde{a}}{\partial \bar{z}}\right|(t, \cdots) \leqslant C\left(M_{1}\right)(\varepsilon+\delta)\left(\frac{1}{\langle\theta-t\rangle^{2}}+\frac{1}{\langle t\rangle^{2}}\right) .
$$

It follows that,

$$
|(1)| \leqslant C\left(M_{1}\right)(\varepsilon+\delta)|Z(t)|\left(\frac{1}{\langle\theta-t\rangle^{2}}+\frac{1}{\langle t\rangle^{2}}\right) .
$$

Here $C\left(M_{1}\right)$ is a constant depending only on $M_{1}$.

(ii) Setting $(2)=\frac{1}{\langle t\rangle}(\widetilde{b}(t, X(t), \alpha)-\widetilde{b}(t, \widetilde{X}(t), \alpha))$ we have exactly by the same way

$$
|(2)| \leqslant C\left(M_{1}\right)(\varepsilon+\delta) \frac{|Z(t)|}{\langle t\rangle}\left(\frac{1}{\langle\theta-t\rangle^{2}}+\frac{1}{\langle t\rangle^{2}}\right) .
$$

(iii) Estimation of $(3)=\frac{\partial q}{\partial \xi}(X(t), \Phi(t, X(t), \alpha))-\frac{\partial q}{\partial \xi}(\widetilde{X}(t), \Phi(t, X(t), \alpha))$. We have

$$
\begin{aligned}
(3)=\int_{0}^{1} \frac{\partial^{2} q}{\partial z \partial \xi}(s X(t)+(1-s) \tilde{X}(t), \Phi(t, X(t), \alpha)) & (X(t)-\tilde{X}(t)) d s \\
& + \text { analogue term with } \frac{\partial^{2} q}{\partial \bar{z} \partial \xi} .
\end{aligned}
$$


Now the coefficients of $q$ say $b_{j k}$, extended by Lemma 5.3 .1 satisfy

$$
\left|\frac{\partial b_{j k}}{\partial z}(z)\right|+\left|\frac{\partial b_{j k}}{\partial \bar{z}}(z)\right| \leqslant \frac{C \varepsilon}{\langle z\rangle^{2+\sigma_{0}}} .
$$

Using again (5.3.7) and (5.3.13) we obtain

$$
|(3)| \leqslant \frac{C \varepsilon|Z(t)|}{\langle\theta-t\rangle^{2+\sigma_{0}}}
$$

(iv) Estimation of $(4)=\frac{\partial q}{\partial \xi}(\widetilde{X}(t), \Phi(t, X(t), \alpha))-\frac{\partial q}{\partial \xi}(\widetilde{X}(t), \Phi(t, \widetilde{X}(t), \alpha))$. We have, by (5.3.18),

$$
|\Phi(t, X(t), \alpha)-\Phi(t, \widetilde{X}(t), \alpha)| \leqslant C \frac{|Z(t)|}{\langle t\rangle}+|(1)|+|(2)| .
$$

On the other hand (5.3.7), (5.3.11) with $s=0$ imply that $M_{1}\langle\operatorname{Re} \tilde{X}(t)\rangle \geqslant\langle\theta-t\rangle$. Therefore using the decay of the coefficients $b_{j k}$ of $q$ and the estimates (5.3.20), (5.3.21) we obtain

$$
|(4)| \leqslant \frac{C \varepsilon}{\langle\theta-t\rangle^{1+\sigma_{0}}}|Z(t)|\left(\frac{1}{\langle t\rangle}+\frac{1}{\langle\theta-t\rangle^{2}}\right) .
$$

It follows then that,

$$
|(4)| \leqslant C \varepsilon|Z(t)|\left(\frac{1}{\langle t\rangle^{2}}+\frac{1}{\langle\theta-t\rangle^{2}}\right) .
$$

Gathering the estimates (5.3.20) to (5.3.23) we obtain the claim of the Lemma.

Next we state the following Lemma.

Lemma 5.3.6. - Let $0 \leqslant T<\theta$. Let $Y(t)=\left(Y_{1}(t), \ldots, Y_{n}(t)\right) \in \mathbb{C}^{n}$ be such that $Y \in C^{1}([T, \theta])$ and satisfies on $[T, \theta]$ the inequality

$$
\left|\dot{Y}(t)-\frac{2}{2 t-i} Y(t)\right| \leqslant|h(t)||Y(t)|+|g(t)|,
$$

for some continuous functions $h, g$. Then for all $t$ in $[T, \theta]$ we have

$$
|Y(t)| \leqslant\left(\frac{\langle 2 t\rangle}{\langle 2 \theta\rangle}|Y(\theta)|+\langle 2 t\rangle \int_{T}^{\theta} \frac{|g(s)|}{\langle 2 s\rangle} d s\right) \exp \left(\int_{T}^{\theta}|h(s)| d s\right) .
$$

Proof. - Let us set $W(t)=\frac{Y(t)}{2 t-i}$. Then $|W(t)|=\frac{|Y(t)|}{\langle 2 t\rangle}$,

$$
\dot{W}(t)=\frac{\dot{Y}(t)}{2 t-i}-\frac{2 Y(t)}{(2 t-i)^{2}}=\frac{1}{2 t-i}\left(\dot{Y}(t)-\frac{2 Y(t)}{2 t-i}\right) .
$$

It follows that $|\dot{W}(t)| \leqslant \frac{1}{\langle 2 t\rangle}(|h(t)||Y(t)|+|g(t)|) \leqslant|h(t)||W(t)|+\frac{|g(t)|}{\langle 2 t\rangle}$. Then, for $t \geqslant T$ and $\sigma \in[t, \theta]$,

$$
|W(\sigma)| \leqslant|W(\theta)|+\int_{\sigma}^{\theta}|h(s)||W(s)| d s+\int_{t}^{\theta} \frac{|g(s)|}{\langle 2 s\rangle} d s .
$$

MÉMOIRES DE LA SMF 101/102 
By the Gronwall Lemma we obtain

$$
|W(t)| \leqslant\left(|W(\theta)|+\int_{T}^{\theta} \frac{|g(s)|}{\langle 2 s\rangle} d s\right) \exp \left(\int_{T}^{\theta}|h(s)| d s\right) .
$$

Coming back to $Y(t)$ we obtain the claim of the Lemma.

Corollary 5.3.7. - Let $Z(t)$ be defined by (5.3.17). Then if $\varepsilon+\delta$ is small compared to $M_{1}$ we have

$$
|Z(t)| \leqslant 2 \frac{\langle 2 t\rangle}{\langle 2 \theta\rangle}|z-\widetilde{X}(\theta)| .
$$

Proof. - We apply Lemma 5.3.6 and Lemma 5.3.5 with

$$
\left\{\begin{array}{l}
g(t)=0 \\
h(t)=C(\varepsilon+\delta)\left(\frac{1}{\langle\theta-t\rangle^{2}}+\frac{1}{\langle t\rangle^{2}}\right) .
\end{array}\right.
$$

Then

$$
\int_{T}^{\theta}|h(s)| d s \leqslant C(\varepsilon+\delta) \int_{\mathbb{R}} \frac{d \sigma}{\langle\sigma\rangle^{2}} .
$$

It follows that

$$
|Z(t)| \leqslant e^{C^{\prime}(\varepsilon+\delta)} \cdot \frac{\langle 2 t\rangle}{\langle 2 \theta\rangle}|Z(\theta)| .
$$

Since $Z(\theta)=X(\theta)-\tilde{X}(\theta)=z-\widetilde{X}(\theta)$ our lemma follows.

We can now show the improvement of (5.3.6) and (5.3.10). In the case 1 we have $\widetilde{X}(\theta)=x(\theta, \alpha)$ and in the case $2, \widetilde{X}(\theta)=0$. Therefore in case 1 we find by Corollary 5.3.7,

$$
|X(t, \theta, z)-x(t, \alpha)| \leqslant 4 \frac{\langle t\rangle}{\langle\theta\rangle}|z-x(\theta, \alpha)|,
$$

and in case 2 ,

$$
|X(t, \theta, z)-X(t, \theta, 0)| \leqslant 4 \frac{\langle t\rangle}{\langle\theta\rangle}|z| .
$$

Taking $M_{1}>4$ this shows that (5.3.6) and (5.3.10) have been improved. 
d) Improvement of (5.3.9). - Let us set

$$
\begin{aligned}
& \left\{\begin{aligned}
U(t) & =\operatorname{Im}(X(t)-\tilde{X}(t)) \\
(1) & =\frac{4 t}{1+4 t^{2}} U(t) \\
(2) & =\frac{2 \operatorname{Re}(X(t)-\widetilde{X}(t))}{1+4 t^{2}}
\end{aligned}\right. \\
& (3)=-\operatorname{Im}[\widetilde{a}(t, X(t), \alpha)-\widetilde{a}(t, \widetilde{X}(t), \alpha)], \\
& (4)=-\frac{1}{\langle t\rangle} \operatorname{Re}[\widetilde{b}(t, X(t), \alpha)-\widetilde{b}(t, \widetilde{X}(t), \alpha)] \text {, } \\
& (5)=\operatorname{Im}\left[\frac{\partial q}{\partial \xi}(\operatorname{Re} X(t), \Phi(t, X(t), \alpha))-\frac{\partial q}{\partial \xi}(\operatorname{Re} X(t), \Phi(t, \tilde{X}(t), \alpha))\right] \text {, } \\
& (6)=\operatorname{Im}\left[\frac{\partial q}{\partial \xi}(\operatorname{Re} X(t), \Phi(t, \widetilde{X}(t), \alpha))-\frac{\partial q}{\partial \xi}(\operatorname{Re} \tilde{X}(t), \Phi(t, \widetilde{X}(t), \alpha))\right] \text {, } \\
& \text { (7) }=\operatorname{Im} \int_{0}^{1} \frac{\partial^{2} q}{\partial \xi \partial z}(\operatorname{Re} X(t)+i s \operatorname{Im} X(t), \Phi(t, X(t), \alpha)) d s(i \operatorname{Im} X(t)) \text {, } \\
& (8)=\operatorname{Im} \int_{0}^{1} \frac{\partial^{2} q}{\partial \xi \partial \bar{z}}(\operatorname{Re} X(t)+i s \operatorname{Im} X(t), \Phi(t, X(t), \alpha)) d s(-i \operatorname{Im} X(t)) \text {, } \\
& (9)=-\operatorname{Im} \int_{0}^{1} \frac{\partial^{2} q}{\partial \xi \partial z}(\operatorname{Re} \tilde{X}(t)+i s \operatorname{Im} \tilde{X}(t), \Phi(t, \tilde{X}(t), \alpha)) d s(i \operatorname{Im} \tilde{X}(t)) \text {, } \\
& (10)=-\operatorname{Im} \int_{0}^{1} \frac{\partial^{2} q}{\partial \xi \partial \bar{z}}(\operatorname{Re} \widetilde{X}(t)+i s \operatorname{Im} \widetilde{X}(t), \Phi(t, \widetilde{X}(t), \alpha)) d s(-i \operatorname{Im} \widetilde{X}(t)) .
\end{aligned}
$$

Then it follows from (5.3.17) and (5.3.19) that,

$$
\dot{U}(t)=\frac{4 t}{1+4 t^{2}} U(t)+\sum_{i=2}^{10}(i) .
$$

LEMMA 5.3.8. - With the above notations, if $\varepsilon+\delta$ is small enough we have

$$
\begin{array}{r}
\left|\dot{U}(t)-\frac{4 t}{1+4 t^{2}} U(t)\right| \leqslant \frac{3 M_{1}|z-x(\theta, \alpha)|}{\langle\theta\rangle\langle t\rangle}+\frac{|z-x(\theta, \alpha)|}{\langle\theta\rangle}\left(\frac{1}{\langle\theta-t\rangle^{1+\sigma_{0}}}+\frac{1}{\langle t\rangle^{2}}\right) \\
+\left(\frac{1}{\langle\theta-t\rangle^{2}}+\frac{1}{\langle t\rangle^{2}}\right)|U(t)| .
\end{array}
$$

Proof. - We use (5.3.25) and (5.3.24). We estimate the terms $(i)$ for $i=2, \ldots, 10$.

(i) Estimation of (2). It follows from (5.3.6) and (5.3.10), since $\widetilde{X}(\theta)=x(\theta, \alpha)$ in case 1 and $\widetilde{X}(\theta)=0$ in case 2 , that

$$
\frac{|X(t)-\tilde{X}(t)|}{1+4 t^{2}} \leqslant M_{1} \frac{\langle t\rangle}{\langle\theta\rangle\left(1+4 t^{2}\right)}|z-\widetilde{X}(\theta)| \leqslant \frac{M_{1}}{\langle\theta\rangle\langle t\rangle} \quad\left\{\begin{array}{ll}
|z-x(\theta, \alpha)|, & \text { case } 1 \\
|z|, & \text { case } 2
\end{array}\right. \text {. }
$$

But in case 2 according to Lemma 5.3 .3 (ii) we have $|z| \leqslant 3|z-x(\theta, \alpha)|$. It follows that in both cases we have

$$
|(2)| \leqslant \frac{3 M_{1}|z-x(\theta, \alpha)|}{\langle\theta\rangle\langle t\rangle}
$$


(ii) Estimation of (3) and (4). We note that $\widetilde{a}(t, \operatorname{Re} X(t), \alpha)$ and $\widetilde{a}(t, \operatorname{Re} \widetilde{X}(t), \alpha)$ are real. It follows that

$$
\begin{aligned}
&(3)=-\operatorname{Im} \int_{0}^{1} \frac{\partial \widetilde{a}}{\partial z}(t,\operatorname{Re} X(t)+s i \operatorname{Im} X(t), \alpha) d s(i \operatorname{Im} X(t)) \\
&-\operatorname{Im} \int_{0}^{1} \frac{\partial \widetilde{a}}{\partial \bar{z}} \quad(\operatorname{idem}) d s(-i \operatorname{Im} X(t)) \\
&+\operatorname{Im} \int_{0}^{1} \frac{\partial \widetilde{a}}{\partial z}(t, \operatorname{Re} \widetilde{X}(t)+s i \operatorname{Im} \widetilde{X}(t), \alpha) d s(i \operatorname{Im} \widetilde{X}(t)) \\
&+\operatorname{Im} \int_{0}^{1} \frac{\partial \widetilde{a}}{\partial \bar{z}} \quad(\mathrm{idem}) d s(-i \operatorname{Im} \widetilde{X}(t)) .
\end{aligned}
$$

Now, according to Theorem 4.4.2 and Lemma 5.3.1 we have

$$
\left|\frac{\partial \widetilde{a}}{\partial z}(t, w, \alpha)\right|+\left|\frac{\partial \widetilde{a}}{\partial \bar{z}}(t, w, \alpha)\right| \leqslant C(\varepsilon+\delta)\left(\frac{1}{\langle\operatorname{Re} w\rangle^{2}}+\frac{1}{\langle t\rangle^{2}}\right) .
$$

We use this estimate with $w=\operatorname{Re} X(t)+i s \operatorname{Im} X(t)$ and $w=\operatorname{Re} \widetilde{X}(t)+i s \operatorname{Im} \widetilde{X}(t)$. By (5.3.7) (with $s=1$ ) and (5.3.11) (with $s=0$ ) we have $\langle\operatorname{Re} w\rangle \geqslant \frac{1}{M_{1}}\langle\theta-t\rangle$.

Moreover in case $1, \operatorname{Im} \widetilde{X}(t)=\operatorname{Im} x(t, \alpha)=0$ and in case 2,

$$
|\operatorname{Im} \widetilde{X}(t)| \leqslant M_{3} \frac{|z|}{\langle\theta\rangle} \leqslant \frac{3 M_{5}|z-x(\theta, \alpha)|}{\langle\theta\rangle} .
$$

Summing up we obtain

$$
|(3)| \leqslant C(\varepsilon+\delta)\left(\frac{1}{\langle t\rangle^{2}}+\frac{M_{1}^{2+\sigma_{0}}}{\langle\theta-t\rangle^{2}}\right)\left(|U(t)|+M_{5} \frac{|z-x(\theta, \alpha)|}{\langle\theta\rangle}\right),
$$

since $|\operatorname{Im} X(t)| \leqslant|U(t)|+|\operatorname{Im} \tilde{X}(t)|$.

For the term (4), due to the factor $\frac{1}{\langle t\rangle}$ we have a better estimate. Indeed by (5.3.21), (5.3.17), (5.3.6) and (5.3.10) we have

$$
|(4)| \leqslant C\left(M_{1}\right)(\varepsilon+\delta) \frac{|z-x(\theta, \alpha)|}{\langle\theta\rangle}\left(\frac{1}{\langle\theta-t\rangle^{2}}+\frac{1}{\langle t\rangle^{2}}\right) .
$$

(iii) Estimation of (5). We note here that $\frac{\partial q}{\partial \xi}(x, \xi)$ is linear in $\xi$ and real if $(x, \xi) \in \mathbb{R}^{n} \times \mathbb{R}^{n}$. It follows that

$$
(5)=\frac{\partial q}{\partial \xi}(\operatorname{Re} X(t), \operatorname{Im}(\Phi(t, X(t), \alpha)-\Phi(t, \widetilde{X}(t), \alpha))) .
$$

Using (5.3.18) we obtain

$$
\begin{array}{r}
\operatorname{Im}(\Phi(t, X(t), \alpha)-\Phi(t, \widetilde{X}(t), \alpha))=\frac{\operatorname{Re}(X(t)-\widetilde{X}(t))}{1+4 t^{2}}+\frac{2 t}{1+4 t^{2}} \operatorname{Im}(X(t)-\widetilde{X}(t)) \\
-\operatorname{Im}[\widetilde{a}(t, X(t), \alpha)-\widetilde{a}(t, \widetilde{X}(t), \alpha)]-\frac{1}{\langle t\rangle} \operatorname{Re}[\widetilde{b}(t, X(t), \alpha)-\widetilde{b}(t, \widetilde{X}(t), \alpha)] .
\end{array}
$$


By (5.3.6) and (5.3.10) we have $|X(t)-\widetilde{X}(t)| \leqslant 3 M_{1}|z-x(\theta, \alpha)| \frac{\langle t\rangle}{\langle\theta\rangle}$. Moreover we can use (5.3.28) and (5.3.29). Finally we use the fact that the coefficients of $\frac{\partial q}{\partial \xi}(\operatorname{Re} X(t), \cdots)$ are bounded by $\frac{C \varepsilon}{\langle\operatorname{Re} X(t)\rangle^{1+\sigma_{0}}}$ which by (5.3.7) (with $s=1$ ) and (5.3.11) (with $s=1$ ) can be estimated by $\frac{C \varepsilon}{\langle\theta-t\rangle^{1+\sigma_{0}}}$. Gathering these informations we see that

$$
\begin{aligned}
|(5)| \leqslant \frac{C \varepsilon}{\langle\theta-t\rangle^{1+\sigma_{0}}} & \left(\frac{1}{\langle t\rangle^{2}}+\frac{1}{\langle\theta-t\rangle^{2}}\right)|U(t)|+\frac{C\left(M_{1} \varepsilon|z-x(\theta, \alpha)|\right.}{\langle\theta-t\rangle^{1+\sigma_{0}}\langle\theta\rangle} \\
& +C \varepsilon C\left(M_{1}, M_{3}\right)(\varepsilon+\delta)\left(\frac{1}{\langle t\rangle^{2}}+\frac{1}{\langle\theta-t\rangle^{2}}\right) \frac{|z-x(\theta, \alpha)|}{\langle\theta\rangle} .
\end{aligned}
$$

(iv) Estimation of (6). Since again $\frac{\partial q}{\partial \xi}(x, \xi)$ is linear in $\xi$ and real when $(x, \xi) \in \mathbb{R}^{n} \times \mathbb{R}^{n}$ we can write

$$
|(6)| \leqslant\left|\frac{\partial q}{\partial \xi}(\operatorname{Re} X(t), \operatorname{Im} \Phi(t, \widetilde{X}(t), \alpha))\right|+\left|\frac{\partial q}{\partial \xi}(\operatorname{Re} \widetilde{X}(t), \operatorname{Im} \Phi(t, \widetilde{X}(t), \alpha))\right| .
$$

Since the coefficients of $\frac{\partial q}{\partial \xi}$ are bounded by $\frac{C \varepsilon}{\langle\theta-t\rangle^{1+\sigma_{0}}}$ we obtain

$$
|(6)| \leqslant \frac{C \varepsilon}{\langle\theta-t\rangle^{1+\sigma_{0}}}|\operatorname{Im} \Phi(t, \widetilde{X}(t), \alpha)| .
$$

In the case $1, \tilde{X}(t)=x(t, \alpha)$ which implies that $\operatorname{Im} \Phi(t, \widetilde{X}(t), \alpha)=0$. In the case 2, $|\operatorname{Im} \Phi(t, \tilde{X}(t), \alpha)| \leqslant M_{3} \frac{|x(\theta, \alpha)|}{\langle\theta\rangle} \leqslant 3 M_{3} \frac{|z-x(\theta, \alpha)|}{\langle\theta\rangle}$. Therefore

$$
|(6)| \leqslant \frac{C M_{3} \varepsilon|z-x(\theta, \alpha)|}{\langle\theta-t\rangle^{1+\sigma_{0}}\langle\theta\rangle} .
$$

(v) Estimation of (7), (8), (9), (10). Using (5.3.7) and (5.3.11) and the estimates on the coefficients of $q$ we find that

$$
|(7)+(8)+(8)+(10)| \leqslant \frac{C \varepsilon}{\langle\theta-t\rangle^{2+\sigma_{0}}}(|U(t)|+|\operatorname{Im} \widetilde{X}(t)|) .
$$

Using (5.3.28) we obtain finally

$$
|(7)+(8)+(9)+(10)| \leqslant \frac{C \varepsilon}{\langle\theta-t\rangle^{2+\sigma_{0}}}\left(|U(t)|+\frac{3 M_{3}|z-x(\theta, \alpha)|}{\langle\theta\rangle}\right) .
$$

Gathering the estimates given by (5.3.26) to (5.3.32) and taking $\varepsilon+\delta$ small compared to $M_{1}, M_{3}$ we obtain the conclusion of Lemma 5.3.8.

Lemma 5.3.9. - Let $Y(t)=\left(Y,(t), \ldots, Y_{n}(t)\right)$ be a $C^{1}$ function from $[T, \theta]$ to $\mathbb{R}^{n}$ which satisfies

$$
\left|\dot{Y}(t)-\frac{4 t}{1+4 t^{2}} Y(t)\right| \leqslant|h(t)||Y(t)|+|g(t)|+\frac{K}{\langle 2 t\rangle}
$$

for some continuous functions $h, g$ and $K \geqslant 0$. Then

$$
|Y(t)| \leqslant\left(\frac{\langle 2 t\rangle}{\langle 2 \theta\rangle}|Y(\theta)|+\int_{T}^{\theta}|g(s)| d s+K\right) \exp \left(\int_{T}^{\theta}|h(s)| d s\right) .
$$


Proof. - Let us set $Z(t)=\frac{Y(t)}{\langle 2 t\rangle}$. Then $\dot{Z}(t)=\frac{\dot{Y}(t)}{\langle 2 t\rangle}-\frac{4 t}{\langle 2 t\rangle^{3}} Y(t)$. It follows that

$$
|\dot{Z}(t)| \leqslant|h(t)||Z(t)|+\frac{|g(t)|}{\langle 2 t\rangle}+\frac{K}{1+4 t^{2}} .
$$

Therefore for $\sigma \in[t, \theta], t \geqslant T$ we have

$$
|Z(\sigma)| \leqslant|Z(\theta)|+\int_{\sigma}^{\theta}|h(s)||Z(s)| d s+\int_{t}^{\theta} \frac{|g(s)|}{\langle 2 s\rangle} d s+K \int_{t}^{\theta} \frac{d s}{1+4 s^{2}} .
$$

Now we have,

$$
\int_{t}^{+\infty} \frac{d s}{1+4 s^{2}} \leqslant \frac{1}{\langle 2 t\rangle}, \quad \int_{t}^{\theta} \frac{|g(s)|}{\langle 2 s\rangle} d s \leqslant \frac{1}{\langle 2 t\rangle} \int_{t}^{\theta}|g(s)| d s .
$$

Using Gronwall's Lemma we obtain

$$
|Z(\sigma)| \leqslant\left(|Z(\theta)|+\frac{1}{\langle 2 t\rangle} \int_{t}^{\theta}|g(s)| d s+\frac{K}{\langle 2 t\rangle}\right) \exp \left(\int_{t}^{\theta}|h(s)| d s\right) .
$$

Taking $t=T$ and $\sigma=t$ we obtain, since $Y(t)=\langle 2 t\rangle|Z(t)|$, the claim of the Lemma.

Corollary 5.3.10. - With $U(t)=\operatorname{Im}(X(t)-\tilde{X}(t))$ introduced in (5.3.24) we have

$$
|U(t)| \leqslant C\left(|U(\theta)|+\left(6 M_{1}+C\right) \frac{|z-x(\theta, \alpha)|}{\langle\theta\rangle}\right) .
$$

Proof. - This follows from Lemmas 5.3.8 and 5.3.9.

We can now finish the proof of the improvement of (5.3.9).

Indeed we have $U(\theta)=\operatorname{Im}(X(\theta, \theta, z)-\widetilde{X}(\theta, \theta, 0))=\operatorname{Im} z$. Therefore Corollary 5.3.10 and Remark 5.3.4 show that if $C<M_{1}$ and $\left(6 M_{1}+C\right) \cdot C<M_{3}$ then (5.3.9) is improved.

End of the proof of Theorem 5.3.2 in the cases 1 and 2. - The estimates (5.3.5) to (5.3.12) improved are true for $t \in[T, \theta]$ for all $T>T_{*}$. By continuity they continue to hold on $\left[T_{*}, \theta\right]$. Now we consider problem (5.3.5) with data at $t=T_{*}$ equal to $X\left(T_{*}, \theta, \alpha\right)$. For this problem the estimates (5.3.6) to (5.3.12) hold on $\left[T_{*}-\varepsilon_{0}, T_{*}\right]$ which contradicts the fact that $T_{*}=\inf A$. Therefore $A=[0, \theta]$ which implies Theorem 5.3.2 in this case.

2) Proof of Theorem 5.3.2 in case 3. - Here we shall take $x \in \mathbb{R}^{n}$ such that $x \cdot \alpha_{\xi} \leqslant c_{1}\langle x\rangle\left|\alpha_{\xi}\right|$ and $|x-x(\theta, \alpha)| \leqslant \delta_{1}\langle\theta\rangle$, with $0<c_{1} \ll c_{2}, 0<\delta_{1} \ll \delta_{2}$.

Let us recall (see (4.4.49)) that there exists a unique $\theta^{*} \in[0, \theta]$ such that $x\left(\theta^{*}, \alpha\right)$. $\alpha_{\xi}=0$. We shall make use of Lemma 4.4.17. To prove the claim of Theorem 5.3.2 we shall use the same method as in the cases 1 and 2 . 
We introduce first the set $A$ of $T \geqslant \theta^{*}$ such that the problem (5.3.4) has a solution on $[T, \theta]$ which satisfies

$$
\begin{aligned}
|X(t, \theta, x)-x| & \leqslant M_{4}|t-\theta| \\
\operatorname{Re} X(t, \theta, x) \cdot \alpha_{\xi} & \leqslant M_{5}\langle\operatorname{Re} X(t, \theta, x)\rangle\left|\alpha_{\varepsilon}\right| \\
\langle x\rangle+\langle t-\theta\rangle & \leqslant M_{4}\langle\operatorname{Re} X(t, \theta, x)\rangle \\
|\operatorname{Im} X(t, \theta, x)| & \leqslant M_{6} \frac{|x-x(\theta, \alpha)|}{\langle\theta\rangle} .
\end{aligned}
$$

If $M_{4}$ is large enough, $M_{5}>c_{1}, M_{6}>0$ one can find $\varepsilon_{0}>0$ such that $\theta-\varepsilon_{0} \in A$. Let $T_{*}=\inf A$. We want to prove that $T_{*}=\theta^{*}$ if $M_{4}, M_{5}, M_{6}$ are correctly chosen; let us assume $T_{*}>\theta$ and let $t \geqslant T_{*}$. Then on $[T, \theta]$ we have a solution $X(t, \theta, z)$ which satisfies (5.3.33) to (5.3.36). Let us show that this implies that $(t, X(t, \theta, x)) \in \Omega_{\delta}^{\mathbb{C}}$ for $t \in[T, \theta]$ (see (5.3.3)) if $\delta_{1}$ is small enough.

From (5.3.36) we have $|\operatorname{Im} X(t, \theta, z)| \leqslant M_{6} \delta_{1} \leqslant \delta$ if $\delta_{1}$ is small enough. Moreover by (5.3.34) we have

$$
\operatorname{Re} X(t, \theta, x) \cdot \alpha_{\xi} \leqslant M_{5}\langle\operatorname{Re} X(t, \theta, x)\rangle\left|\alpha_{\varepsilon}\right| \leqslant c_{0}\langle\operatorname{Re} X(t, \theta, x)\rangle\left|\alpha_{\varepsilon}\right|
$$

if $M_{5} \leqslant c_{0}$. Finally,

$$
(1)=|X(t, \theta, x)-x(t, \alpha)| \leqslant|X(t, \theta, x)-x|+\left|x-x\left(\theta^{*}, \alpha\right)\right|+\left|x\left(\theta^{*}, \alpha\right)-x(t, \alpha)\right| .
$$

From (5.3.33) we have $|X(t, \theta, x)-x| \leqslant M_{4}|t-\theta| \leqslant M_{4}\left(\theta-\theta^{*}\right)$ since $t \geqslant \theta^{*}$. Now we use Lemma 4.4 .17 to write

$$
|X(t, \theta, x)-x| \leqslant 10 M_{4}|x-x(\theta, \alpha)| \leqslant 10 M_{4} \delta_{1}\langle\theta\rangle \leqslant \frac{10 M_{4}}{K_{1}} \delta_{1}\left\langle\theta^{*}\right\rangle \leqslant \frac{10 M_{4}}{K_{1}} \delta_{1}\langle t\rangle .
$$

Again by Lemma 4.4.17,

$$
\left|x-x\left(\theta^{*}, \alpha\right)\right| \leqslant 6|x-x(\theta, \alpha)| \leqslant 6 \delta_{1}\langle\theta\rangle \leqslant \frac{6 \delta_{1}}{K_{1}}\left\langle\theta^{*}\right\rangle \leqslant \frac{6 \delta_{1}}{K_{1}}\langle t\rangle .
$$

Finally $\left|x(t, \alpha)-x\left(\theta^{*}, \alpha\right)\right| \leqslant \int_{\theta^{*}}^{t}|\dot{x}(s, \alpha)| d s \leqslant 5\left(t-\theta^{*}\right)$ if $\varepsilon$ is small enough. It follows from Lemma 4.4.17 that

$$
\left|x(t, \alpha)-x\left(\theta^{*}, \alpha\right)\right| \leqslant 5\left(\theta-\theta^{*}\right) \leqslant 50|x-x(\theta, \alpha)| \leqslant 50 \delta_{1}\langle\theta\rangle \leqslant \frac{50 \delta_{1}}{K_{1}}\langle t\rangle .
$$

Summing up we find that if $\delta_{1}$ is small enough,

$$
(1) \leqslant \max \left(\frac{10 M_{4}}{K_{1}}, \frac{56}{K_{1}}\right) \delta_{1}\langle t\rangle \leqslant \delta\langle t\rangle .
$$

As in the cases 1 and 2 our goal is to prove that one can improve the estimates (5.3.33) to $(5.3 .36)$. 
(i) Improvement of (5.3.33). - We have by (5.3.5), $\dot{X}(t, \theta, x)=2 \alpha_{\xi}+\mathcal{O}(\varepsilon+\delta)$. Therefore

$$
X(t, \theta, x)=x-2(\theta-t) \alpha_{\xi}+\mathcal{O}((\varepsilon+\delta)(\theta-t)) .
$$

It follows that $|X(t, \theta, x)-x| \leqslant 5(\theta-t)$ if $\varepsilon+\delta$ is small enough. We shall take $M_{4}$ so that $5 \leqslant \frac{1}{2} M_{4}$ and then, (5.3.33) will be improved.

(ii) Improvement of (5.3.35). - We deduce from (5.3.38) that

$1+|\operatorname{Re} X(t, \theta, z)|^{2}=1+|x|^{2}+4(\theta-t)^{2}\left|\alpha_{\xi}\right|^{2}+\mathcal{O}\left((\varepsilon+\delta)\left(|x|^{2}+(\theta-t)^{2}\right)\right)-2(\theta-t) x \cdot \alpha_{\xi}$.

Since $x \cdot \alpha_{\varepsilon} \leqslant c_{1}\langle x\rangle\left|\alpha_{\varepsilon}\right|$, taking $\varepsilon+\delta$ small enough we obtain, $1+|\operatorname{Re} X(t, \theta, z)|^{2} \geqslant$ $1+\frac{1}{2}|x|^{2}+\frac{1}{2}(\theta-t)^{2}-4 c_{1}(\theta-t)\langle x\rangle$, so

$$
1+|\operatorname{Re} X(t, \theta, z)|^{2} \geqslant \frac{1}{4}\left(\langle x\rangle^{2}+(\theta-t)^{2}\right),
$$

if $c_{1} \leqslant \frac{1}{10}$. In particular $3\langle\operatorname{Re} X(t, \theta, x)\rangle \geqslant\langle\theta-t\rangle+\langle x\rangle$, so $\langle\theta-t\rangle+\langle x\rangle \leqslant$ $\frac{1}{2} M_{4}\langle\operatorname{Re} X(t, \theta, x)\rangle$ if $M_{4} \geqslant 6$.

(iii) Improvement of (5.3.34). - From (5.3.38) we have

$$
\operatorname{Re} X(t, \theta, x) \cdot \alpha_{\xi}=x \cdot \alpha_{\xi}-2(\theta-t)\left|\alpha_{\xi}\right|^{2}+\mathcal{O}((\varepsilon+\delta)(\theta-t)) .
$$

It follows that

$$
\operatorname{Re} X(t, \theta, x) \cdot \alpha_{\xi} \leqslant c_{1}\langle x\rangle\left|\alpha_{\xi}\right|-\frac{(\theta-t)}{4} \leqslant 10 c_{1}\langle\operatorname{Re} X(t, \theta, x)\rangle\left|\alpha_{\xi}\right|,
$$

by (5.3.39). We shall take $10 c_{1} \leqslant \frac{1}{2} M_{5}$ and (5.3.34) will be improved.

(iv) Improvement of (5.3.36). - Let us set $X(t, \theta, x)=X(t)=Y_{1}(t)+i Y_{2}(t)$ where $Y_{1}, Y_{2}$ are real.

Lemma 5.3.11. - There exists positive constants $C, K$ independent of $\varepsilon, \delta$ and $T$ such that for all $t \in[T, \theta]$ we have

$$
\left|\dot{Y}_{2}(t)\right| \leqslant C M_{6}\left(\frac{\delta_{1}}{\langle\theta\rangle}+(\varepsilon+\delta) \frac{|x-x(\theta, \alpha)|}{\langle\theta\rangle} g(t)\right)+\frac{\delta_{1}}{\langle\theta\rangle}
$$

where $g$ is a continuous positive function satisfying $\int_{T}^{\theta} g(s) d s \leqslant K$.

Proof. - From (5.3.36) and (5.3.37) we get

$$
|X(t)-x(t, \alpha)| \leqslant C\left(M_{4}\right) \delta_{1}\langle t\rangle, \quad\left|Y_{2}(t)\right| \leqslant M_{6} \delta_{1} .
$$

Now (5.3.18) shows that

$$
\operatorname{Im} \Phi(t, X(t), \alpha)=\operatorname{Im}\left[\frac{X(t)-x(t)}{2 t-i}-\widetilde{a}\left(t, X(t, X(t), \alpha)-\frac{i}{\langle t\rangle} \widetilde{b}(t, X(t), \alpha)\right]\right.
$$

where $x(t)=x(t, \alpha)$. First of all we have,

$$
\operatorname{Im} \frac{X(t)-x(t)}{2 t-i}=\frac{2 t Y_{2}(t)}{1+4 t^{2}}+\frac{Y_{1}(t)-x(t)}{1+4 t^{2}} .
$$


Using (5.3.40) we deduce, since $\langle\theta\rangle \sim\langle t\rangle$,

$$
\left|\operatorname{Im} \frac{X(t)-x(t)}{2 t-i}\right| \leqslant C M_{6} \frac{\delta_{1}}{\langle\theta\rangle}+C \frac{\delta_{1}}{\langle\theta\rangle} .
$$

On the other hand we can write with $f=\widetilde{a}$ or $\widetilde{b}$,

$$
\begin{aligned}
f(t, X(t), \alpha)=f\left(t, Y_{1}(t), \alpha\right)+\int_{0}^{1} \frac{\partial f}{\partial z}( & \left.t, Y_{1}(t)+i s Y_{2}(t), \alpha\right) d s\left(i Y_{2}(t)\right) \\
& +\int_{0}^{1} \frac{\partial f}{\partial \bar{z}}\left(t, Y_{1}(t)+i s Y_{2}(t), \alpha\right) d s\left(-i Y_{2}(t)\right) .
\end{aligned}
$$

Since $\widetilde{a}\left(t, Y_{1}(t), \alpha\right)$ is real, using the estimates on the derivatives of $\widetilde{a}$ and $\widetilde{b}$ given by Theorem 4.4.2 and their extensions to the complex domain proved in Lemma 5.3.1 we obtain

$$
|\operatorname{Im} \widetilde{a}(t, X(t), \alpha)| \leqslant C M_{6}(\varepsilon+\delta)\left(\frac{1}{\langle\theta-t\rangle^{2}}+\frac{1}{\langle t\rangle^{2}}\right) \frac{|x-x(\theta, \alpha)|}{\langle\theta\rangle} .
$$

Here we have used the estimate in (ii) and (5.3.36).

Moreover we have by Theorem 4.4.2 (ii) and (5.3.37),

$$
\left|\widetilde{b}\left(t, Y_{1}(t), \alpha\right)\right| \leqslant \sqrt{\delta} \frac{\left|Y_{1}(t)-x(t)\right|}{\langle t\rangle} \leqslant \sqrt{\delta} C\left(M_{4}\right) \delta_{1} \leqslant \delta_{1}
$$

if $\delta$ is small enough. Therefore

$$
\left|\operatorname{Im} \frac{i \widetilde{b}(t, X(t), \alpha)}{\langle t\rangle}\right| \leqslant C \frac{\delta_{1}}{\langle\theta\rangle}+C M_{6}\left(\frac{1}{\langle\theta-t\rangle^{2}}+\frac{1}{\langle t\rangle^{2}}\right) \frac{|x-x(\theta, \alpha)|}{\langle\theta\rangle} .
$$

We deduce from (5.3.41) to (5.3.43) that

$$
|\operatorname{Im} \Phi(t, X(t), \alpha)| \leqslant \frac{C \delta_{1}}{\langle\theta\rangle}+C M_{6} \frac{\delta_{1}}{\langle\theta\rangle}+M_{6} \frac{|x-x(\theta, \alpha)|}{\langle\theta\rangle} g(t)
$$

where $g(t)=C\left(\frac{1}{\langle\theta-t\rangle^{2}}+\frac{1}{\langle t\rangle^{2}}\right)$.

It follows from (5.3.4) that

$$
\begin{aligned}
\left|\dot{Y}_{2}(t)\right| \leqslant 2 & |\operatorname{Im} \Phi(t, X(t), \alpha)|+\left|\frac{\partial q}{\partial \xi}\left(Y_{1}(t), \operatorname{Im} \Phi(t, X(t), \alpha)\right)\right| \\
+ & \left|\int_{0}^{1} \frac{\partial^{2} q}{\partial \xi \partial z}\left(Y_{1}(t)+i s Y_{2}(t), \Phi(t, X(t), \alpha)\right) d s\right|\left|Y_{2}(t)\right| \\
+ & \mid \int_{0}^{1} \frac{\partial^{2} q}{\partial \xi \partial \bar{z}} \text { (idem) } d s|| Y_{2}(t) \mid \\
\leqslant C & \mid \operatorname{Im} \Phi\left(t, X(t), \alpha\left|+\frac{C \varepsilon}{\langle\theta-t\rangle^{2+\sigma_{0}}}\right| Y_{2}(t) \mid .\right.
\end{aligned}
$$

This estimate together with (5.3.36), (5.3.44) prove the Lemma. 
We can now improve (5.3.36). Indeed, by Lemma 5.3.11, we have, since $X(\theta, \theta, x)=x$ is real

$$
\left|Y_{2}(t)\right| \leqslant \int_{t}^{\theta}\left|\dot{Y}_{2}(s)\right| d s \leqslant C M_{6}\left[\frac{\delta_{1}(\theta-t)}{\langle\theta\rangle}+(\varepsilon+\delta) K \frac{|x-x(\theta, \alpha)|}{\langle\theta\rangle}\right]+\delta_{1} \frac{\theta-t}{\langle\theta\rangle} .
$$

Moreover by Lemma 4.4.17 we have

$$
\theta-t \leqslant \theta-\theta^{*} \leqslant C|x-x(\theta, \alpha)| .
$$

Taking $\delta_{1}, \delta, \varepsilon$ small enough we obtain $\left|Y_{2}(t)\right| \leqslant \frac{1}{2} M_{6} \frac{|x-x(\theta, \alpha)|}{\langle\theta\rangle}$ which improves (5.3.36).

The improvements (i) to (iv) show that the set $A$ where (5.3.33) to (5.3.36) are true is equal to $\left[\theta^{*}, \theta\right]$.

We can now give the proof of Theorem 5.3.2 in the case 3. Indeed (5.3.34) to (5.3.36) imply the estimates (ii) to (iv) in this Theorem. To prove (i) we just remark that

$$
\begin{aligned}
|X(t, \theta, x)-x(t, \alpha)| & \leqslant|X(t, \theta, x)-x|+|x-x(\theta, \alpha)| \\
& \leqslant\left(10 M_{4}+1\right)|x-x(\theta, \alpha)| \leqslant C|x-x(\theta, \alpha)| \frac{\langle t\rangle}{\langle\theta\rangle}
\end{aligned}
$$

since $\langle t\rangle \sim\langle\theta\rangle$ when $t \in\left[\theta^{*}, \theta\right]$. Therefore we are done for $t \in\left[\theta^{*}, \theta\right]$. For $t \in\left[0, \theta^{*}\right]$ we first remark that

$$
X(t, \theta, x)=X\left(t, \theta^{*}, X\left(\theta^{*}, \theta, x\right)\right) .
$$

We would like to apply the cases 1 and 2 already done, with $\theta=\theta^{*}$ and $z=X\left(\theta^{*}, \theta, x\right)$. So we have to prove that

(i) $x\left(\theta^{*}, \alpha\right) \cdot \alpha_{\xi} \leqslant c_{2}\left\langle x\left(\theta^{*}, \alpha\right)\right\rangle\left|\alpha_{\xi}\right|$,

(ii) $\left|z-x\left(\theta^{*}, \alpha\right)\right| \leqslant \delta_{2}\left\langle\theta^{*}\right\rangle$,

(iii) $\operatorname{Re} z \cdot \alpha_{\xi} \leqslant c_{2}\langle\operatorname{Re} z\rangle\left|\alpha_{\xi}\right|$,

(iv) $|\operatorname{Im} z| \leqslant \delta_{2}$.

First of all (i) is trivial since $x\left(\theta^{*}, \alpha\right) \cdot \alpha_{\varepsilon}=0$. Now we have,

$$
\left|X\left(\theta^{*}, \theta, x\right)-x\left(\theta^{*}, \alpha\right)\right| \leqslant\left|X\left(\theta^{*}, \theta, x\right)-x\right|+\left|x-x\left(\theta^{*}, \alpha\right)\right|=(1)+(2) .
$$

By (5.3.33) and Lemma 4.4.17 we have if $\delta_{1} \ll \delta_{2}$

$$
\begin{aligned}
& (1) \leqslant M_{4}\left(\theta-\theta^{*}\right) \leqslant 10 M_{4}|x-x(\theta, \alpha)| \leqslant 10 M_{4} \delta_{1}\langle\theta\rangle \leqslant C \delta_{1}\left\langle\theta^{*}\right\rangle \leqslant \frac{\delta_{2}}{2}\left\langle\theta^{*}\right\rangle \\
& (2) \leqslant 5|x-x(\theta, \alpha)| \leqslant C^{\prime} \delta_{1}\left\langle\theta^{*}\right\rangle \leqslant \frac{\delta_{2}}{2}\left\langle\theta^{*}\right\rangle
\end{aligned}
$$

since $\langle\theta\rangle \sim\left\langle\theta^{*}\right\rangle$. Thus (ii) is satisfied. Now (iii) is also satisfied if $M_{5} \leqslant c_{2}$. This is possible since the only constraint on $M_{5}$ (see (iii) improvement of (5.3.34)) was $M_{5} \geqslant 20 c_{1}$. Finally by (5.3.36), $\left|\operatorname{Im} X\left(\theta^{*}, \theta, x\right)\right| \leqslant \delta_{1} M_{6} \leqslant \delta_{2}$ if $\delta_{1} \ll \delta_{2}$. Therefore 
$X\left(t, \theta^{*}, X\left(\theta^{*}, \theta, x\right)\right)$ satisfies the estimates (5.3.5) to (5.3.9) in case 1 and (5.3.9) to (5.3.12) in case 2. Therefore we have the following estimate,

$$
\begin{aligned}
& (1)=\left|X\left(t, \theta^{*}, X\left(\theta^{*}, \theta, x\right)\right)-x(t, \alpha)\right| \leqslant 3 M_{1}\left|X\left(\theta^{*}, \theta, x\right)-x\left(\theta^{*}, \alpha\right)\right| \frac{\langle t\rangle}{\left\langle\theta^{*}\right\rangle} \\
& (1) \leqslant 3 M_{1} \frac{\langle t\rangle}{\left\langle\theta^{*}\right\rangle}\left(\left|X\left(\theta^{*}, \theta, x\right)-x\right|+\left|x-x\left(\theta^{*}, \alpha\right)\right|\right) \\
& (1) \leqslant 3 M_{1} \frac{\langle t\rangle}{\left\langle\theta^{*}\right\rangle}\left(M_{4}\left(\theta-\theta^{*}\right)+\left|x-x\left(\theta^{*}, \alpha\right)\right|\right) \\
& (1) \leqslant C M_{1}\left(1+M_{4}\right)|x-x(\theta, \alpha)| \frac{\langle t\rangle}{\langle\theta\rangle} .
\end{aligned}
$$

Here we have used (5.3.33), Lemma 4.4.17 and $\left\langle\theta^{*}\right\rangle \sim\langle\theta\rangle$. Therefore we obtain (i) of (5.3.5) if $K \geqslant C M_{1}\left(1+M_{4}\right)$. Now (5.3.9) implies that

$$
(2)=\left|\operatorname{Im} X\left(t, \theta^{*}, X\left(\theta^{*}, \theta, x\right)\right)\right| \leqslant M_{3}\left(\frac{\left|X\left(\theta^{*}, \theta, x\right)-x\left(\theta^{*}, \alpha\right)\right|}{\left\langle\theta^{*}\right\rangle}+\left|\operatorname{Im} X\left(\theta^{*}, \theta, x\right)\right|\right) .
$$

Using (5.3.36) and the same argument as in the term (1) we obtain

$$
(2) \leqslant C\left(M_{1}, M_{4}, M_{6}\right) \frac{|x-x(\theta, \alpha)|}{\langle\theta\rangle} .
$$

Thus (ii) in (5.3.5) is satisfied if $K \geqslant C\left(M_{1}, M_{4}, M_{6}\right)$.

From (5.3.15) (ii) with $s=1$ we have

$$
(3)=\left\langle\operatorname{Re} X\left(t, \theta^{*}, X\left(\theta^{*}, \theta, x\right)\right)\right\rangle \geqslant \frac{1}{5}\left[\left\langle\theta^{*}-t\right\rangle+\left\langle\operatorname{Re} X\left(\theta^{*}, \theta, x\right)\right\rangle\right] .
$$

So using (5.3.35) we obtain

$$
(3) \geqslant \frac{1}{5}\left[\left\langle\theta^{*}-t\right\rangle+\frac{1}{M_{4}}\left\langle\theta^{*}-\theta\right\rangle\right] \geqslant C\left(M_{4}\right)\langle\theta-t\rangle,
$$

and (iii) satisfied $K \cdot C\left(M_{4}\right) \geqslant 1$.

Finally let us set $(4)=\operatorname{Re} X\left(t, \theta^{*}, X\left(\theta^{*}, \theta, x\right)\right) \cdot \alpha_{\xi}$. Using (5.3.8) and (5.3.12) with $s=1$ we can write,

$$
(4) \leqslant M_{2}\left\langle\operatorname{Re} X\left(t, \theta^{*}, X\left(\theta^{*}, \theta, x\right)\right)\right\rangle .
$$

This shows that (5.3.5) (iv) holds if $\widetilde{K} \geqslant \frac{1}{M_{2}}$ and completes the proof of Theorem 5.3.2.

Having proved in Theorem 5.3.2 the existence of the solution $X(t, \theta, x)$ of (5.3.4) we want to give estimates on its derivatives with respect to $(\theta, x)$.

Proposition 5.3.12. - The solution given by Theorem 5.3.2 is $C^{\infty}$ with respect to $y=(\theta, x)$ and satisfies the following estimates,

$$
\left|\partial_{y}^{A} X(t, \theta, x)\right| \leqslant\left\{\begin{array}{ll}
C \frac{\langle t\rangle}{\langle\theta\rangle} & \text { if }|A|=1, \\
C_{A} \frac{\langle t\rangle}{\langle\theta\rangle}\left(\frac{1}{\langle x\rangle^{|A|+\sigma_{0}}}+\frac{1}{\langle\theta\rangle^{|A|-1}}\right) & \text { if }|A| \geqslant 2 .
\end{array} .\right.
$$

uniformly in $(t, \theta, x) \in[0, \theta] \times \Omega_{\delta}$. 
To prove this result we need a Lemma.

Lemma 5.3.13. - For $j=1, \ldots, n$ let us set $L_{j}(t, z)=\frac{\partial p}{\partial \xi_{j}}(z, \Phi(t, z, \alpha))$. Then for any integer $N>0$ one can find $C_{N}>0$ such that for $j=1, \ldots, n$ and all $(t, \theta, x)$ in $[0, \theta] \times \Omega_{\delta}$ we have

$$
\begin{gathered}
\left|\frac{\partial L_{j}}{\partial z_{k}}(t, X(t, \theta, x))-\frac{2 \delta_{j k}}{2 t-i}\right| \leqslant C_{1}\left(\frac{1}{(\langle x\rangle+\langle\theta-t\rangle)^{2+\sigma_{0}}}+\frac{1}{\langle t\rangle^{2}}\right) \\
\left|\frac{\partial L_{j}}{\partial \bar{z}_{k}}(t, X(t, \theta, x))\right| \leqslant C_{N}\left(\frac{1}{(\langle x\rangle+\langle\theta-t\rangle)^{2+\sigma_{0}}}+\frac{1}{\langle t\rangle^{2}}\right)\left(\frac{|x-x(\theta, \alpha)|}{\langle\theta\rangle}\right)^{N} .
\end{gathered}
$$

For any $\mu, \nu \in \mathbb{N}^{n}$, such that $|\mu|+|\nu|=N \geqslant 2, j=1, \ldots, n$,

$$
\left|\frac{\partial^{\mu+\nu} L_{j}}{\partial z^{\mu} \partial \bar{z}^{\nu}}(t, X(t, \theta, x))\right| \leqslant C_{\mu \nu}\left(\frac{1}{(\langle x\rangle+\langle\theta-t\rangle)^{|\mu|+|\nu|+1+\sigma_{0}}}+\frac{1}{\langle t\rangle^{|\mu|+|\nu|+1}}\right) .
$$

Proof of Lemma 5.3.13. - We have

$$
L_{j}(t, z)=2 \Phi_{j}(t, z, \alpha)+2 \varepsilon \sum_{\ell=1}^{n} b_{j \ell}(z) \Phi_{\ell}(t, z, \alpha) .
$$

Using (5.3.18) we obtain

$$
\frac{\partial \Phi_{j}}{\partial z_{k}}(t, z, \alpha)=\frac{\delta_{j k}}{2 t-i}-\left(\frac{\partial \widetilde{a}_{j}}{\partial z_{k}}+\frac{i}{\langle t\rangle} \frac{\partial \widetilde{b}_{j}}{\partial z_{k}}\right)(t, z, \alpha) .
$$

Then (5.3.46) follows easily from the estimates on $\widetilde{a}, \widetilde{b}$ given in Theorem 4.4.2, the estimates on the coefficients $b_{j \ell}$ and from the inequality (iii) in Theorem 5.3.2.

The estimate (5.3.47) follows from the same arguments and Lemma 5.3.1 (iv), Theorem 5.3.2 (ii), (iii). The same method can also be used to prove (5.3.48).

Proof of Proposition 5.3.12. - Let us set for $k=1, \ldots, n, q \geqslant 1$,

$$
Y_{k}^{q}(t)=\partial_{x}^{A} X_{k}(t, \theta, x)
$$

where $|A|=q$.

We begin by the case $q=1$. Differentiating one time (5.3.4) with respect to $y$ we obtain

$$
\dot{Y}_{k}^{1}(t)=\sum_{j=1}^{n}\left[\frac{\partial L_{k}}{\partial z_{j}}(t, X(t, \theta, x)) Y_{j}^{1}(t)+\frac{\partial L_{k}}{\partial \bar{z}_{j}}(t, X(t, \theta, x)) \overline{Y_{j}^{1}(t)}\right] .
$$

Using (5.3.46) and (5.3.47) we see that $Y^{1}(t)=\left(Y_{1}^{1}(t), \ldots, Y_{n}^{1}(t)\right)$ satisfies the hypotheses of Lemma 5.3.6 with $g \equiv 0$ and $h(t)=\frac{1}{(\langle x\rangle+\langle\theta-t\rangle)^{2+\sigma_{0}}}+\frac{1}{\langle t\rangle^{2}}$. Since $\frac{\partial X_{j}}{\partial x_{k}}(\theta, \theta, x)=\delta_{j k}$ and $\frac{\partial X_{j}}{\partial \theta}(\theta, \theta, x)$ is bounded we obtain (5.3.45) when $|A|=1$. Let us consider the case $|A|=2$. Differentiating (5.3.49) with respect to $y$ we see that $Y_{k}^{2}(t)$ satisfies the equation

$$
\dot{Y}_{k}^{2}(t)=\sum_{j=1}^{n}\left[\frac{\partial L_{k}}{\partial z_{j}}(t, X(t, \theta, x)) Y_{j}^{2}(t)+\frac{\partial L_{k}}{\partial \bar{z}_{j}}(t, X(t, \theta, x)) \overline{Y_{j}^{2}(t)}\right]+Z_{k}(t, \theta, x)
$$


where, by (5.3.48) and (5.3.45) for $|A|=1, Z_{k}(t)$ is estimated as follows

$$
\left|Z_{k}(t, \theta, x)\right| \leqslant C \frac{\langle t\rangle^{2}}{\langle\theta\rangle^{2}}\left(\frac{1}{(\langle x\rangle+\langle\theta-t\rangle)^{3+\sigma_{0}}}+\frac{1}{\langle t\rangle^{3}}\right) .
$$

We want to use Lemma 5.3.6 (with $T=0$ ) so we are led to estimate the quantity $(1)=\int_{0}^{\theta} \frac{\left|Z_{k}(s, \theta, x)\right|}{\langle 2 s\rangle} d s$. Using the above estimation we see that

$$
(1) \leqslant C^{\prime} \frac{1}{\langle\theta\rangle^{2}} \int_{0}^{\theta}\left(\frac{\langle\sigma\rangle}{(\langle x\rangle+\langle\theta-\sigma\rangle)^{3+\sigma_{0}}}+\frac{1}{\langle\sigma\rangle^{2}}\right) d \sigma .
$$

By a straightforward computation we see that we have

$$
\int_{0}^{\theta} \frac{\langle\sigma\rangle^{\ell}}{(\langle x\rangle+\langle\theta-t\rangle)^{k+\sigma_{0}}} d \sigma \leqslant C \frac{\langle\theta\rangle^{\ell}}{\langle x\rangle^{k-1+\sigma_{0}}}, \quad k, \ell \geqslant 1 .
$$

It follows that

$$
(1) \leqslant \frac{C}{\langle\theta\rangle}\left(\frac{1}{\langle x\rangle^{2+\sigma_{0}}}+\frac{1}{\langle\theta\rangle}\right) .
$$

Using Lemma 5.3.6 and the fact that $\partial_{x}^{A} X(\theta, \theta, x)=0$, since $|A| \geqslant 2$, we obtain (5.3.45) when $|A|=2$.

Now we proceed by induction on $q \geqslant 2$. Let $|A|=q+1$ and let us differentiate the equation $\dot{X}_{k}(t, \theta, x)=L_{k}(t, X(t, \theta, x))|A|$ times with respect to $x$. Using the Faa di Bruno formula and the notation $Y_{k}^{q+1}=\partial_{x}^{A} X_{k}$ we obtain the equation

$$
\dot{Y}_{k}^{q+1}(t)=\sum_{j=1}^{n}\left[\frac{\partial L_{k}}{\partial z_{j}}(t, X(t, \theta, x)) Y_{j}^{q+1}(t)+\frac{\partial L_{k}}{\partial \bar{z}_{j}}(t, X(t, \theta, x))\right] \overline{Y_{j}^{q+1}(t)}+Z_{k}(t)
$$

where $Z_{k}(t)$ is a finite linear combination of terms of the form

$$
(2)=\left(\partial_{(z, \bar{z})}^{\beta} L_{j}\right)(t, X(t, \theta, x)) \prod_{\ell=1}^{s}\left(\partial_{x}^{L_{\ell}} X(t, \theta, x)\right)^{K_{\ell}}
$$

where $2 \leqslant|\beta| \leqslant q+1,1 \leqslant s \leqslant q+1,\left|K_{\ell}\right| \geqslant 1,\left|L_{\ell}\right| \geqslant 1, \sum_{\ell=1}^{s} K_{\ell}=\beta, \sum_{\ell=1}^{s}\left|K_{\ell}\right| L_{\ell}=A$. It follows that $\left|L_{\ell}\right| \leqslant|A|-1=q$.

Since by (5.3.45) we have different estimates for $\left|L_{\ell}\right|=1$ and $\left|L_{\ell}\right| \geqslant 2$ we must separate these two cases. So let us write $\{1, \ldots, s\}=I_{1} \cup I_{2}, I_{1}=\left\{\ell:\left|L_{\ell}\right|=1\right\}$, $\left|I_{2}\right|=\left\{\ell:\left|L_{\ell}\right| \geqslant 2\right\}$.

Now let us use (5.3.48) and the induction. We obtain

$$
\begin{aligned}
&|(2)| \leqslant C\left(\frac{1}{(\langle x\rangle+\langle\theta-t\rangle)^{|\beta|+1+\sigma_{0}}}+\frac{1}{\langle t\rangle^{|\beta|+1}}\right) \prod_{\ell \in I_{1}}\left(\frac{\langle t\rangle}{\langle\theta\rangle}\right)^{\left|K_{\ell}\right|} \\
& \cdot \prod_{\ell \in I_{2}}\left[\frac{\langle t\rangle}{\langle\theta\rangle}\left(\frac{1}{\langle x\rangle^{\left|L_{\ell}\right|+\sigma_{0}}}+\frac{1}{\langle\theta\rangle^{\left|L_{\ell}\right|-1}}\right)\right]^{\left|K_{\ell}\right|} .
\end{aligned}
$$


Since $\sum_{i=1}^{s}\left|K_{i}\right|=|\beta|$ we have

$$
\prod_{\ell \in I_{1}}\left(\frac{\langle t\rangle}{\langle\theta\rangle}\right)^{\left|K_{\ell}\right|} \prod_{\ell \in I_{2}}\left(\frac{\langle t\rangle}{\langle\theta\rangle}\right)^{\left|K_{\ell}\right|}=\frac{\langle t\rangle^{|\beta|}}{\langle\theta\rangle^{|\beta|}} .
$$

It follows from (5.3.50) that

$$
\int_{0}^{\theta} \frac{|(2)|}{\langle\sigma\rangle} d \sigma \leqslant \frac{C}{\langle\theta\rangle}\left(\frac{1}{\langle x\rangle^{|\beta|+\sigma_{0}}}+\frac{1}{\langle\theta\rangle^{|\beta|-1}}\right) \prod_{\ell \in I_{2}}\left(\frac{1}{\langle x\rangle^{\left|L_{\ell}\right|+\sigma_{0}}}+\frac{1}{\langle\theta\rangle^{\left|L_{\ell}\right|-1}}\right)^{\left|K_{\ell}\right|} .
$$

Now we have

$$
\begin{aligned}
\prod_{\ell \in I_{2}}\left(\frac{1}{\langle x\rangle^{\left|L_{\ell}\right|+\sigma_{0}}}\right. & \left.+\frac{1}{\langle\theta\rangle^{\left|L_{\ell}\right|-1}}\right)^{\left|K_{\ell}\right|} \leqslant \prod_{\ell \in I_{2}}\left(\frac{1}{\langle x\rangle^{\left|L_{\ell}\right|-1}}+\frac{1}{\langle\theta\rangle^{\left|L_{\ell}\right|-1}}\right)^{\left|K_{\ell}\right|} \\
& \leqslant C \prod_{\ell \in I_{2}}\left(\frac{1}{\langle x\rangle^{\left|K_{\ell}\right|\left(\left|L_{\ell}\right|-1\right)}}+\frac{1}{\langle\theta\rangle^{\left|K_{\ell}\right|\left(\left|L_{\ell}\right|-1\right)}}\right) \\
& \leqslant C^{\prime}\left[\left(\frac{1}{\langle x\rangle}\right)^{\sum_{\ell \in I_{2}}\left|K_{\ell}\right|\left(\left|L_{\ell}\right|-1\right)}+\left(\frac{1}{\langle\theta\rangle}\right)^{\sum_{\ell \in I_{2}}\left|K_{\ell}\right|\left(\left|L_{\ell}\right|-1\right)}\right] \\
& \leqslant C^{\prime}\left[\left(\frac{1}{\langle x\rangle}\right)^{|A|-|\beta|}+\left(\frac{1}{\langle\theta\rangle}\right)^{|A|-|\beta|}\right] .
\end{aligned}
$$

Indeed

$$
\begin{aligned}
|A|-|\beta|=\sum_{\ell=1}^{s}\left|K_{\ell}\right|\left|L_{\ell}\right|-\sum_{\ell=1}^{s}\left|K_{\ell}\right| & =\sum_{\ell \in I_{1}}\left|K_{\ell}\right|+\sum_{\ell \in I_{2}}\left|K_{\ell}\right|\left|L_{\ell}\right|-\sum_{\ell \in I_{1}}\left|K_{\ell}\right|-\sum_{\ell \in I_{2}}\left|K_{\ell}\right| \\
& =\sum_{\ell \in I_{2}}\left|K_{\ell}\right|\left(\left|L_{\ell}\right|-1\right) .
\end{aligned}
$$

It follows that

$$
\begin{aligned}
\int_{0}^{\theta} \frac{|(2)|}{\langle\sigma\rangle} d \sigma & \leqslant \frac{C}{\langle\theta\rangle}\left(\frac{1}{\langle x\rangle^{|\beta|+\sigma_{0}}}+\frac{1}{\langle\theta\rangle^{|\beta|-1}}\right)\left(\frac{1}{\langle x\rangle^{|A|-|\beta|}}+\frac{1}{\langle\theta\rangle^{|A|-|\beta|}}\right) \\
& \leqslant \frac{C^{\prime}}{\langle\theta\rangle}\left(\frac{1}{\langle x\rangle^{|A|+\sigma_{0}}}+\frac{1}{\langle\theta\rangle^{|A|-1}}\right) .
\end{aligned}
$$

Then using Lemma 5.3.6 and the fact that $\partial_{x}^{A} X(\theta, \theta, x)=0$ since $|A| \geqslant 2$ we obtain (5.3.45) for $|A|=q+1$.

We need another lemma. Let us recall that we have set $L_{j}(\theta, x)=\frac{\partial p}{\partial \xi_{j}}(x, \Phi(\theta, x, \alpha))$.

Lemma 5.3.14. - Let $u_{j}(t)=\frac{\partial X_{j}}{\partial \theta}(t, \theta, x)+\sum_{k=1}^{n} \frac{\partial p}{\partial \xi_{k}}(x, \Phi(\theta, x, \alpha)) \frac{\partial X_{j}}{\partial x_{k}}(t, \theta, x)$. Then for every integer $N>0$ one can find a constant $C_{N}>0$ such that for all $t \in[0, \theta]$ and all $(\theta, x)$ in $\Omega_{\delta}$ we have

$$
\left|u_{j}(t)\right| \leqslant C_{N}\left(\frac{|x-x(\theta, \alpha)|}{\langle\theta\rangle}\right)^{N} .
$$


Proof. - First of all we claim that $u_{j}(\theta)=0$. Indeed since $\dot{X}_{j}(\theta, \theta, x)+\frac{\partial X_{j}}{\partial \theta}(\theta, \theta, x)=0$ we have $\frac{\partial X_{j}}{\partial \theta}(\theta, \theta, x)=-L_{j}(\theta, x)$. Then our claim follows from the fact that $\frac{\partial X_{j}}{\partial x_{k}}(\theta, \theta, x)=\delta_{j k}$. Now

$$
\dot{u}_{j}(t)=\frac{\partial \dot{X}_{j}}{\partial \theta}(t, \theta, x)+\sum_{k=1}^{n} L_{k}(\theta, x) \frac{\partial \dot{X}_{j}}{\partial x_{k}}(t, \theta, x) .
$$

Using (5.3.4) we obtain

$$
\begin{aligned}
\dot{u}_{j}(t)= & \sum_{\mu=1}^{n}\left[\frac{\partial L_{j}}{\partial z_{\mu}}(t, X(t, \theta, x)) \frac{\partial X_{\mu}}{\partial \theta}(t, \theta, x)+\frac{\partial L_{j}}{\partial \bar{z}_{\mu}}(t, X(t, \theta, x)) \frac{\overline{\partial X_{\mu}}}{\partial \theta}(t, \theta, x)\right] \\
& +\sum_{k=1}^{n} L_{k}(\theta, x) \sum_{\mu=1}^{n}\left[\frac{\partial L_{j}}{\partial z_{\mu}}(t, X(t, \theta, x)) \frac{\partial X_{\mu}}{\partial x_{k}}(t, \theta, x)\right. \\
& \left.+\frac{\partial L_{j}}{\partial \bar{z}_{\mu}}(t, X(t, \theta, x)) \frac{\overline{\partial X_{\mu}}}{\partial x_{k}}(t, \theta, x)\right] \\
\dot{u}_{j}(t)= & \sum_{\mu=1}^{n} \frac{\partial L_{j}}{\partial z_{\mu}}(t, X(t, \theta, x)) u_{\mu}(t) \quad\left[\frac{\partial X_{\mu}}{\partial \theta}(t, \theta, x)+\sum_{k=1}^{n} L_{k}(\theta, x) \frac{\partial X_{\mu}}{\partial x_{k}}(t, \theta, x)\right] .
\end{aligned}
$$

It follows then from (5.3.45) that with $u(t)=\left(u_{1}(t), \ldots, u_{n}(t)\right)$,

$$
\left|\dot{u}_{j}(t)-\frac{2 u_{j}(t)}{2 t-i}\right| \leqslant|h(t)||u(t)|+|g(t)|,
$$

where

$$
\left\{\begin{array}{l}
h(t)=\sum_{\mu, j=1}^{n}\left|\frac{\partial L_{j}}{\partial z_{\mu}}(t, X(t, \theta, x))-\frac{2 \delta_{j k}}{2 t-i}\right|, \\
g(t)=C \sum_{\mu, j=1}^{n}\left|\frac{\partial L_{j}}{\partial \bar{z}_{\mu}}(t, X(t, \theta, x))\right| .
\end{array}\right.
$$

Now using (5.3.46) and (5.3.47) we have $\int_{0}^{\theta} h(t) d t \leqslant C$ and

$$
\int_{0}^{\theta} g(t) d t \leqslant C C_{N} \frac{|x-x(\theta, \alpha)|^{N}}{\langle\theta\rangle^{N}}
$$

so Lemma 5.3.14 follows from Lemma 5.3.6 since $u_{j}(\theta)=0$.

To solve the transport equations we need to introduce some notations. First of all we shall set,

$D=\left\{(\theta, z) \in \mathbb{R} \times \mathbb{C}^{n}:|z-x(\theta, \alpha)| \leqslant \frac{\delta_{1}}{K}\langle\theta\rangle,|\operatorname{Im} z| \leqslant \frac{\delta_{1}}{K}, \operatorname{Re} z \cdot \alpha_{\varepsilon} \leqslant c_{1}\langle\operatorname{Re} z\rangle\left|\alpha_{\varepsilon}\right|\right\}$ where $\delta_{1}, c_{1}, K$ have been introduce in the statement of Theorem 5.3.2. 
Let $a \in C^{\infty}(D)$. We introduce some possible estimates.

$$
\begin{gathered}
\left\{\begin{array}{l}
\forall \mu, \nu \in \mathbb{N}^{n}, \exists C_{\mu, \nu, \ell} \geqslant 0 \text { such that } \\
\text { for all }(\theta, z) \in D,\left|\partial_{z}^{\mu} \partial \bar{z} a(\theta, z)\right| \leqslant C_{\mu, \nu}
\end{array}\right. \\
\left\{\begin{array}{l}
\forall N \in \mathbb{N}, \exists C_{N} \geqslant 0: \forall j=1, \ldots, n, \forall(\theta, z) \in D \\
\left|\frac{\partial a}{\partial \bar{z}_{j}}(\theta, z)\right| \leqslant C_{N}|\operatorname{Im} z|^{N}
\end{array}\right. \\
\left\{\begin{array}{l}
\exists \sigma_{0}>0: \forall \mu, \nu \in \mathbb{N}^{n}, \exists C_{\mu, \nu} \geqslant 0: \forall(\theta, z) \in D \\
\left|\partial_{z}^{\mu} \partial \bar{\nu}_{z} a(\theta, z)\right| \leqslant \frac{C_{\mu, \nu}}{\langle\operatorname{Re} z\rangle^{1+\sigma_{0}}} .
\end{array}\right.
\end{gathered}
$$

We first state the following result.

Proposition 5.3.15. - Let $u_{0}=u_{0}(z)$ be a $C^{\infty}$ function in a neighborhood of $D_{0}=\left\{z \in \mathbb{C}^{n}:\left|z-\alpha_{x}\right| \leqslant \delta_{1}\right\}$ such that for any $N \in \mathbb{N}$ one can find $C_{N} \geqslant 0$ such that for every $j=1, \ldots, n$ and $z \in D_{0}$,

$$
\left|\frac{\partial u_{0}}{\partial \bar{z}_{j}}(z)\right| \leqslant C_{N}|\operatorname{Im} z|^{N} .
$$

For $(\theta, x) \in \mathbb{R} \times \mathbb{R}^{n},(\theta, x) \in D$ we set $u(\theta, x)=u_{0}(X(0, \theta, x))$. Then for any $N \geqslant 0$ we can find $C_{N}^{\prime} \geqslant 0$ such that

$$
\begin{gathered}
\left|\frac{\partial u}{\partial \theta}(\theta, x)+\sum_{k=1}^{n} \frac{\partial p}{\partial \xi_{k}}(x, \Phi(\theta, x, \alpha)) \frac{\partial u}{\partial x_{k}}(\theta, x)\right| \leqslant C_{N}^{\prime}\left(\frac{|x-x(\theta, \alpha)|}{\langle\theta\rangle}\right)^{N} \\
u(0, x)=u_{0}(x)
\end{gathered}
$$

$$
\left\{\begin{array}{l}
\text { For any } \gamma \in \mathbb{N}^{n} \quad \text { one can find } C_{\gamma} \geqslant 0 \text { such that } \\
\left|\partial_{x}^{\gamma} u(\theta, x)\right| \leqslant C_{\gamma} \text { for every }(\theta, x) \text { in } D \cap \mathbb{R} \times \mathbb{R}^{n} .
\end{array}\right.
$$

Proof. - First of all by (5.3.5) (i) we have for $(\theta, x) \in D \cap \mathbb{R} \times \mathbb{R}^{n}$,

$$
\left|X(0, \theta, x)-\alpha_{x}\right| \leqslant K \frac{|x-x(\theta, \alpha)|}{\langle\theta\rangle} \leqslant K \frac{\delta_{1}}{K}=\delta_{1} .
$$

Therefore $u(\theta, x)=u_{0}(X(0, \theta, x))$ is well defined and satisfies (5.3.57) by Proposition 5.3.12, the fact that $u_{0}$ is $C^{\infty}$ in a neighborhood of $D_{0}$ and the Faa di Bruno formula (Chapter 7.2). Now since $X(0,0, x)=x,(5.3 .56)$ is obvious. Let us check (5.3.55). We set

$$
(1)=\frac{\partial u}{\partial \theta}(\theta, x)+\sum_{k=1}^{n} \frac{\partial p}{\partial \xi_{k}}(x, \Phi(\theta, x, \alpha)) \frac{\partial u}{\partial x_{k}}(\theta, x) .
$$


Then

$$
\begin{aligned}
(1)= & \sum_{j=1}^{n} \frac{\partial u_{0}}{\partial z_{j}}(X(0, \theta, x))\left[\frac{\partial X_{j}}{\partial \theta}(0, \theta, x)+\sum_{k=1}^{n} \frac{\partial p}{\partial \xi_{k}}(x, \Phi(\theta, x, \alpha)) \frac{\partial X_{j}}{\partial x_{k}}(0, \theta, x)\right] \\
& +\sum_{j=1}^{n} \frac{\partial u_{0}}{\partial \bar{z}_{j}}(X(0, \theta, x))\left[\overline{\frac{\partial X_{j}}{\partial \theta}(0, \theta, x)}+\sum_{k=1}^{n} \frac{\partial p}{\partial \xi_{k}}(x, \Phi(\theta, x, \alpha)) \overline{\frac{\partial X_{j}}{\partial x_{k}}(0, \theta, x)}\right]
\end{aligned}
$$

and we write $(1)=(A)+(B)$.

By Lemma 5.3.14 with $t=0$, the term $(A)$ satisfies (5.3.55). By the hypothesis made on $u_{0}$ and (5.3.5) (ii) we have

$$
\left|\frac{\partial u_{0}}{\partial \bar{z}_{j}}(X(0, \theta, x))\right| \leqslant C_{N}|\operatorname{Im} X(0, \theta, x)|^{N} \leqslant C_{N}^{\prime}\left(\frac{|x-x(\theta, \alpha)|}{\langle\theta\rangle}\right)^{N} .
$$

Using (5.3.45) and the fact that $\Phi$ and the coefficients of $p$ are bounded we deduce that the term $(B)$ satisfies also (5.3.55).

Proposition 5.3.16. - Let $a \in C^{\infty}$ on $D$ which satisfies (5.3.53), (5.3.54). Let us set

$$
A(s, \theta, x)=\int_{\theta}^{s} a(\sigma, X(\sigma, \theta, x)) d \sigma
$$

for $s \in[0, \theta]$ and $(\theta, x) \in D \cap \mathbb{R} \times \mathbb{R}^{n}$. Then

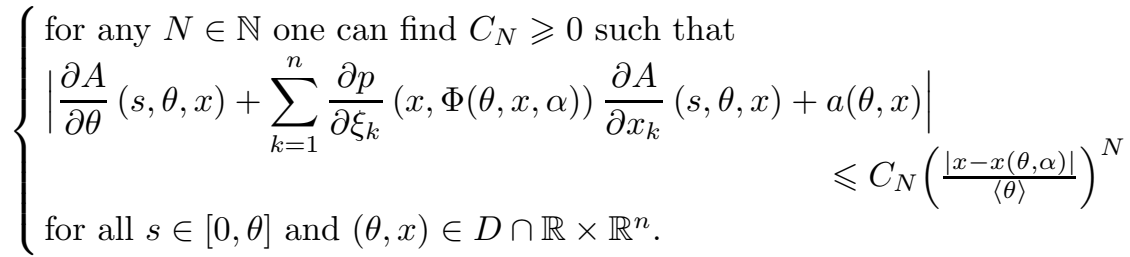

$$
\begin{aligned}
& A(\theta, \theta, x)=0 . \\
& \left\{\text { For every } \gamma \in \mathbb{N}^{n} \text {, there exists } C_{\gamma} \geqslant 0\right. \text { such that } \\
& \left\{\left|\partial_{x}^{\gamma} A(s, \theta, x)\right| \leqslant C_{\gamma} \text { on }[0, \theta] \times D \cap \mathbb{R} \times \mathbb{R}^{n} .\right.
\end{aligned}
$$

Proof. - The claim (5.3.59) is trivial, (5.3.60) follows from Proposition 5.3.12, (5.3.54) and (5.3.5) (iii). Let us show (5.3.58). We set

$$
(1)=\frac{\partial A}{\partial \theta}(s, \theta, x)+\sum_{k=1}^{n} \frac{\partial p}{\partial \xi_{k}}(x, \Phi(\theta, x, \alpha)) \frac{\partial A}{\partial x_{k}}(s, \theta, x)+a(\theta, x) .
$$

Then

$$
\begin{aligned}
(1) & =\int_{\theta}^{s} \sum_{j=1}^{n} \frac{\partial a}{\partial z_{j}}(\sigma, X(\sigma, \theta, x))\left[\frac{\partial X_{j}}{\partial \theta}(\sigma, \theta, x)+\sum_{k=1}^{n} \frac{\partial p}{\partial \xi_{k}}(x, \Phi(\theta, x, \alpha)) \frac{\partial X_{j}}{\partial x_{k}}(\sigma, \theta, x)\right] d \sigma \\
& +\int_{\theta}^{s} \sum_{j=1}^{n} \frac{\partial a}{\partial \bar{z}_{j}}(\sigma, X(\sigma, \theta, x))\left[\overline{\frac{\partial X_{j}}{\partial \theta}(\sigma, \theta, x)}+\sum_{k=1}^{n} \frac{\partial p}{\partial \xi_{k}}(x, \Phi(\theta, x, \alpha)) \frac{\partial X_{j}}{\partial x_{k}}(\sigma, \theta, x)\right] d \sigma \\
& =(A)+(B) .
\end{aligned}
$$


By (5.3.54) and (5.3.5)(iii) we have $\left|\frac{\partial a}{\partial z_{j}}(\sigma, X(\sigma, \theta, x))\right| \leqslant C /\langle\theta-\sigma\rangle^{1+\sigma_{0}}$. Therefore using Lemma 5.3.14 we obtain (5.3.58) for the term $(A)$.

Now it follows from (5.3.53) and (5.3.54) by interpolation that

$$
\left|\frac{\partial a}{\partial \bar{z}_{j}}(\theta, z)\right| \leqslant C_{N, \sigma_{0}}^{\prime \prime} \frac{|\operatorname{Im} z|^{N}}{\langle\operatorname{Re} z\rangle^{1+\sigma_{0} / 2}} .
$$

Thus using (5.3.45), (5.3.5) (ii), (iii), we obtain (5.3.58) for the term $(B)$ since $\Phi$ and the coefficients of $p$ are uniformly bounded.

This is the last result before the final one solving the transport equations.

Proposition 5.3.17. - Let b be $C^{\infty}$ on $D$ satisfying (5.3.52) and (5.3.53). Let us set $B(s, \theta, x)=b(s, X(s, \theta, x)), s \in[0, \theta],(\theta, x) \in D \cap \mathbb{R} \times \mathbb{R}^{n}$. Then,

$$
\begin{gathered}
\left\{\begin{array}{r}
\text { for every } N \geqslant 0 \text { there exists } C_{N} \geqslant 0 \text { such that } \\
\left|\frac{\partial B}{\partial \theta}(s, \theta, x)+\sum_{k=1}^{n} \frac{\partial p}{\partial \xi_{k}}(x, \Phi(\theta, x, \alpha)) \frac{\partial B}{\partial x_{k}}(s, \theta, x)\right| \\
\leqslant C_{N}\left(\frac{|x-x(\theta, \alpha)|}{\langle\theta\rangle}\right)^{N}, \\
B(\theta, \theta, x)=b(\theta, x),
\end{array}\right. \\
\left\{\begin{array}{l}
\text { for every } \gamma \in \mathbb{N}^{n}, \quad \ell, m \in \mathbb{N} \text { there exists } C_{\gamma} \geqslant 0 \text { such that } \\
\left|\partial_{x}^{\gamma} B(s, \theta, x)\right| \leqslant C_{\gamma}, \quad \forall(s, \theta, x) \in[0, \theta] \times D \cap \mathbb{R} \times \mathbb{R}^{n} .
\end{array}\right.
\end{gathered}
$$

Proof. - The claim (5.3.62) is obvious and (5.3.63) follows from Proposition 5.3.12 and (5.3.52). Let us show (5.3.61). The left hand side of (5.3.61) can be written,

$$
\begin{aligned}
(1) & =\sum_{j=1}^{n} \frac{\partial b}{\partial z_{j}}(s, X(s, \theta, x))\left[\frac{\partial X_{j}}{\partial \theta}(s, \theta, x)+\sum_{k=1}^{n} \frac{\partial p}{\partial \xi_{k}}(x, \Phi(\theta, x, \alpha)) \frac{\partial X_{j}}{\partial x_{k}}(s, \theta, x)\right] \\
& +\sum_{j=1}^{n} \frac{\partial b}{\partial \bar{z}_{j}}(s, X(s, \theta, x))\left[\overline{\frac{\partial X_{j}}{\partial \theta}(s, \theta, x)}+\sum_{k=1}^{n} \frac{\partial p}{\partial \xi_{k}}(x, \Phi(\theta, x, \alpha)) \frac{\partial X_{j}}{\partial x_{k}}(s, \theta, x)\right] \\
= & (A)+(B) .
\end{aligned}
$$

The estimation of $(A)$ follows from Lemma 5.3.14 and (5.3.52). Now from (5.3.53), (5.3.5) (ii) and Proposition 5.3.12 we deduce the estimation of $(B)$ since $\Phi$ and the coefficients of $p$ are uniformly bounded.

TheOREm 5.3.18. - Let $a=a(\theta, z)$ be $a C^{\infty}$ function on $D$ satisfying (5.3.53), (5.3.54). Let $b=b(\theta, z)$ be a $C^{\infty}$ function on $D$ satisfying (5.3.52), (5.3.53). Let $u_{0}=u_{0}(z)$ be a $C^{\infty}$ function on $D_{0}$ satisfying the hypothesis of Proposition 5.3.15. With the notations of Propositions 5.3.15, 5.3.16 and 5.3.17 we set

$$
v(\theta, x)=\int_{0}^{\theta} e^{A(s, \theta, x)} B(s, \theta, x) d s+e^{A(0, \theta, x)} u(\theta, x) .
$$


Then

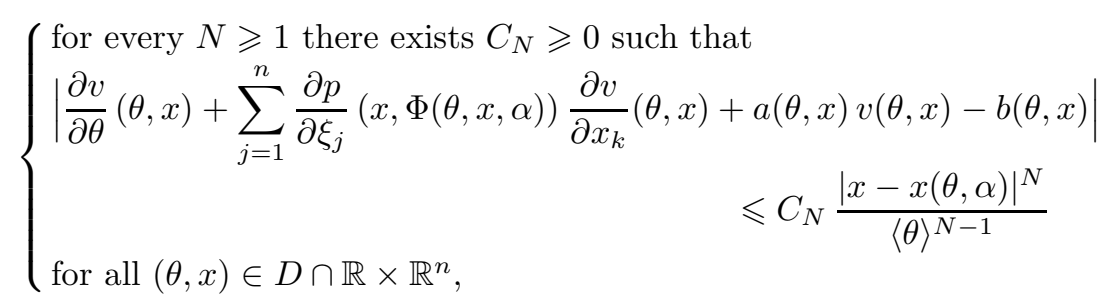

$$
v(0, x)=u_{0}(x)
$$

$\left\{\right.$ for all $\gamma \in \mathbb{N}^{n}$, there exists $C_{\gamma} \geqslant 0$ such that $\left\{\left|\partial_{x}^{\nu} v(\theta, x)\right| \leqslant C_{\gamma}\langle\theta\rangle\right.$ for all $(\theta, x) \in D \cap \mathbb{R} \times \mathbb{R}^{n}$.

Proof. - (5.3.65) is obvious, (5.3.66) follows from (5.3.57), (5.3.60) and (5.3.63). Let us show (5.3.64). We set

$$
\mathcal{L}=\frac{\partial}{\partial \theta}+\sum_{k=1}^{n} \frac{\partial p}{\partial \xi_{k}}(x, \Phi(\theta, x, \alpha)) \frac{\partial}{\partial x_{k}}
$$

Then

$$
\begin{aligned}
\mathcal{L} v(\theta, x)+ & a(\theta, x) v(\theta, x)-b(\theta, x)=b(\theta, x) \\
& +\int_{0}^{\theta} e^{A(s, \theta, x)}[\mathcal{L} B(s, \theta, x)+\mathcal{L} A(s, \theta, x) B(s, \theta, x)] d s \\
& +e^{A(0, \theta, x)}(u(\theta, x) \mathcal{L} A(0, \theta, x)+\mathcal{L} u(\theta, x))+a(\theta, x) e^{A(0, \theta, x)} u(\theta, x) \\
& +a(\theta, x) \int_{0}^{\theta} e^{A(s, \theta, x)} B(s, \theta, x) d s-b(\theta, x) .
\end{aligned}
$$

So

$$
\begin{aligned}
\mathcal{L} v(\theta, x)+a(\theta, x) v(\theta, x)-b(\theta, x) & \\
=\int_{0}^{\theta} e^{A(s, \theta, x)} & {[\mathcal{L} B(s, \theta, x)+(\mathcal{L} A(s, \theta, x)+a(\theta, x)) B(s, \theta, x)] d s } \\
+ & e^{A(0, \theta, x)}[\mathcal{L} A(0, \theta, x)+a(\theta, x)] u(\theta, x)+e^{A(0, \theta, x)} \mathcal{L} u(\theta, x) .
\end{aligned}
$$

The Propositions 5.3.15, 5.3.16 and 5.3.17 show that

$$
|\mathcal{L} B(s, \theta, x)|+|\mathcal{L} A(s, \theta, x)+a(\theta, x)|+|\mathcal{L} u(\theta, x)| \leqslant C_{N} \frac{|x-x(\theta, \alpha)|^{N}}{\langle\theta\rangle^{N}}
$$

and $|A(s, \theta, x)| \leqslant C$. Then (5.3.64) follows. 
Proof of Theorem 5.1 .1 (continued). Case of incoming points. — Let us set

$$
\left\{\begin{array}{l}
\mathcal{L}=\frac{\partial}{\partial \theta}+\sum_{j=1}^{n} \frac{\partial p}{\partial \xi_{j}}(x, \Phi(\theta, x, \alpha)) \frac{\partial}{\partial x_{j}} \\
a(\theta, x)=\sum_{j, k=1}^{n} g^{j k}(x) \frac{\partial \Phi_{j}}{\partial x_{k}}(\theta, x, \alpha)-\frac{n}{2} \frac{\theta}{1+\theta^{2}}+i \sum_{j=1}^{n} g_{j}(x) \Phi_{j}(\theta, x, \alpha) .
\end{array}\right.
$$

By Proposition 4.4.14 (ii) we have

$$
\left|\frac{\partial \varphi}{\partial x}(\theta, x, \alpha)-\Phi(\theta, x, \alpha)\right| \leqslant C_{N}\left(\frac{|x-x(\theta, \alpha)|}{\langle\theta\rangle}\right)^{N} .
$$

Therefore using (5.1.3), (5.1.4) we see that to prove Theorem 5.1.1 it will be sufficient to construct a smooth symbol $f=f(\theta, x, \lambda)$ (with all derivatives in $x$ bounded) such that

$$
\left|\mathcal{L} f+a f+\frac{1}{\lambda}{ }^{t} P f\right| \leqslant C_{N} \lambda^{-N} .
$$

We shall take $f$ on the form

$$
f(\theta, x, \lambda, \alpha)=\sum_{k=0}^{N} \lambda^{-k} f_{k}(\theta, x, \alpha),
$$

where the $f_{k}^{\prime} s$ are the solutions of the problems,

$$
\left\{\begin{array}{l}
\mathcal{L} f_{0}+a f_{0}=0,\left.\quad f_{0}\right|_{\theta=0}=1, \\
\mathcal{L} f_{k}+a f_{k}=-{ }^{t} P f_{k-1},\left.f_{k}\right|_{\theta=0}=0, \quad k \geqslant 1 .
\end{array}\right.
$$

Since $\alpha$ is fixed, we shall skip it in writing the $f_{j}$ 's. By Theorem 5.3.18 we have,

$$
\left\{\begin{array}{l}
f_{0}(\theta, x)=e^{A(0, \theta, x)}, \\
f_{k}(\theta, x)=\int_{0}^{\theta} e^{A(s, \theta, x)} B_{k}(s, \theta, x) d s \text { where, } \\
A(s, \theta, x)=-\int_{s}^{\theta} a(\sigma, X(\sigma, \theta, x)) d \sigma, \\
B_{k}(s, \theta, x)=-{ }^{t} P f_{k-1}(s, X(s, \theta, x)), \quad k \geqslant 1 .
\end{array}\right.
$$

Our aim is to prove, by induction on $k \geqslant 0$ that,

$$
\left|\partial_{x}^{\gamma} f_{k}(\theta, x)\right| \leqslant C_{k, \gamma}\left(\frac{1}{\langle x\rangle}+\frac{1}{\langle\theta\rangle}\right)^{|\gamma|} .
$$

We claim that for all $\ell \in \mathbb{N}^{n}$,

$$
\left|\partial_{x}^{\ell} A(s, \theta, x)\right| \leqslant C_{\ell}\left(\frac{1}{\langle x\rangle}+\frac{1}{\langle\theta\rangle}\right)^{|\ell|},
$$

uniformly with respect to $s \in[0, \theta]$ and $(\theta, x) \in \Omega_{\delta}$. 
Indeed using (5.3.18) and the estimates on $\widetilde{a}, \widetilde{b}$ given in Theorem 4.4 .2 we see easily that

$$
\left|\partial_{(z, \bar{z})}^{\beta} a(\sigma, z)\right| \leqslant C_{\beta}\left(\frac{1}{\langle\operatorname{Re} z\rangle^{|\beta|+2+\sigma_{0}}}+\frac{1}{\langle\sigma\rangle^{|\beta|+2}}\right) .
$$

Moreover by Theorem 5.3.2 (iii) we have

$$
\langle\operatorname{Re} X(\sigma, \theta, x)\rangle \geqslant \frac{1}{K}(\langle x\rangle+\langle\theta-\sigma\rangle) .
$$

Using the Faa di Bruno formula we see that $\partial_{x}^{\ell} A(s, \theta, x)$ is bounded by a finite sum of terms of the following form.

$$
(1)=\int_{0}^{\theta}\left|\partial_{(z, \bar{z})}^{\beta} a(\sigma, X(\sigma, \theta, x)) \prod_{j=1}^{s}\left(\partial_{x}^{\ell_{j}} X(\sigma, \theta, x)\right)^{k_{j}}\right| d \sigma
$$

where $1 \leqslant|\beta| \leqslant|\ell|, 1 \leqslant s \leqslant|\beta|, \sum_{j=1}^{s} k_{j}=\beta, \sum_{j=1}^{s}\left|k_{j}\right| \ell_{j}=\ell$.

Setting $I_{1}=\left\{j \in\{1,2, \ldots, s\}:\left|\ell_{j}\right|=1\right\}, I_{2}=\left\{j \in\{1,2, \ldots, s\}:\left|\ell_{j}\right| \geqslant 2\right\}$ and using (5.3.72), (5.3.73) and (5.3.45) we can write

$$
(1) \leqslant C \int_{0}^{\theta}\left[\frac{1}{(\langle x\rangle+\langle\theta-\sigma\rangle)^{|\beta|+2+\sigma_{0}}}+\frac{1}{\langle\sigma\rangle^{|\beta|+2}}\right] \frac{\langle\sigma\rangle^{|\beta|}}{\langle\theta\rangle^{|\beta|}} d \sigma \prod_{j \in I_{2}}\left(\frac{1}{\langle x\rangle^{\left|\ell_{j}\right|-1}}+\frac{1}{\langle\theta\rangle^{\left|\ell_{j}\right|-1}}\right)^{\left|k_{j}\right|}
$$

since $\sum_{j=1}^{s} k_{j}=\beta$.

Using (5.3.50) and the fact that $\sum_{j \in I_{2}}\left|k_{j}\right|\left|\ell_{j}\right|=|\ell|-|\beta|$ we obtain

$$
(1) \leqslant C\left(\frac{1}{\langle x\rangle}+\frac{1}{\langle\theta\rangle}\right)^{|\beta|}\left(\frac{1}{\langle x\rangle}+\frac{1}{\langle\theta\rangle}\right)^{|\ell|-|\beta|}
$$

which proves (5.3.71).

As a consequence of (5.3.71) we claim that

$$
\left|\partial_{x}^{\gamma}\left(e^{A(s, \theta, x)}\right)\right| \leqslant C_{\gamma}\left(\frac{1}{\langle x\rangle}+\frac{1}{\langle\theta\rangle}\right)^{|\gamma|} .
$$

Indeed by the Faa di Bruno formula the left hand side of (5.3.74) can be bounded by a finite sum of terms of the form

$$
(2)=\left|e^{A(s, \theta, x)} \prod_{j=1}^{s}\left(\partial_{x}^{\ell_{j}} A(s, \theta, x)\right)^{k_{j}}\right|
$$

where $1 \leqslant s \leqslant|\gamma|, 1 \leqslant \sum\left|k_{j}\right| \leqslant \ell, \sum_{j=1}^{s}\left|k_{j}\right| \ell_{j}=|\gamma|$.

Then (5.3.74) follows easily from (5.3.71).

Now (5.3.70) for $k=0$ follows from (5.3.74) (take $s=0$ ). On the other hand by the Faa di Bruno formula $\partial_{x}^{\gamma} f_{k}(\theta, x, \alpha)$ can be bounded by a finite sum of terms of the form

$$
(3)=\int_{0}^{\theta}\left|\partial_{x}^{\gamma_{1}}\left(e^{A(s, \theta, x)}\right)\right| \partial_{x}^{\gamma_{2}}\left[{ }^{t} P f_{k-1}(s, X(s, \theta, x))\right] d s .
$$


Setting for convenience $b_{k}={ }^{t} P f_{k-1}$ it follows from the induction and Lemma 5.3.1 that

$$
\left|\partial_{(z, \bar{z})}^{\beta} b_{k}(s, z)\right| \leqslant C_{\beta}\left(\frac{1}{\langle\operatorname{Re} z\rangle}+\frac{1}{\langle s\rangle}\right)^{|\beta|+2}
$$

Then the Faa di Bruno formula shows that the term $\partial_{x}^{\gamma_{2}}\left[{ }^{t} P f_{k-1}(s, X(s, \theta, x))\right]$ can be estimated by a finite sum of terms of the form

$$
(4)=\left|\left(\partial_{(z, \bar{z})}^{\beta} b_{k}\right)(s, X(s, \theta, x)) \prod_{\ell=1}^{s}\left(\partial_{x}^{\ell_{j}} X\right)^{k_{j}}\right|
$$

where $1 \leqslant|\beta| \leqslant\left|\gamma_{2}\right|, 1 \leqslant s \leqslant\left|\gamma_{2}\right|, \sum_{j=1}^{s} k_{j}=\beta, \sum_{j=1}^{s}\left|k_{j}\right| \ell_{j}=\gamma_{2}$. Then using (5.3.75), (5.3.73), (5.3.45) we see that

$$
(4) \leqslant C\left(\frac{1}{(\langle x\rangle+\langle\theta-s\rangle)^{|\beta|+2}}+\frac{1}{\langle s\rangle^{|\beta|+2}}\right) \frac{\langle s\rangle^{|\beta|}}{\langle\theta\rangle^{|\beta|}} \prod_{j \in I_{2}}\left(\frac{1}{\langle x\rangle}+\frac{1}{\langle\theta\rangle}\right)^{\left|k_{j}\right|\left(\left|\ell_{j}\right|-1\right)} .
$$

Now by (5.3.71) we can write

$$
(3) \leqslant C \int_{0}^{\theta}\left[\frac{1}{(\langle x\rangle+\langle\theta-s\rangle)^{|\beta|+2}}+\frac{1}{\langle s\rangle^{|\beta|+2}}\right] \frac{\langle s\rangle^{|\beta|}}{\langle\theta\rangle^{|\beta|}} d s\left(\frac{1}{\langle x\rangle}+\frac{1}{\langle\theta\rangle}\right)^{\left|\gamma_{1}\right|+\left|\gamma_{2}\right|-|\beta|}
$$

since $\sum_{j \in I_{2}}\left|k_{j}\right|\left(\left|\ell_{j}\right|-1\right)=\left|\gamma_{2}\right|-|\beta|$.

It follows from (5.3.50) that

$$
(3) \leqslant C\left(\frac{1}{\langle x\rangle^{|\beta|}}+\frac{1}{\langle\theta\rangle^{|\beta|}}\right)\left(\frac{1}{\langle x\rangle}+\frac{1}{\langle\theta\rangle}\right)^{\left|\gamma_{1}\right|+\left|\gamma_{2}\right|-|\beta|} \leqslant C^{\prime}\left(\frac{1}{\langle x\rangle}+\frac{1}{\langle\theta\rangle}\right)^{|\gamma|}
$$

since $\left|\gamma_{1}\right|+\left|\gamma_{2}\right|=|\gamma|$. This proves (5.3.70) for all $k$.

\subsection{The amplitude for short time}

We shall need the following precision on the amplitude when $|\theta| \leqslant 1$.

Proposition 5.4.1. - Let $a_{N}$ be the amplitude defined in Corollary 5.1.2. Then for every $\gamma \in \mathbb{N}^{2 n}$ one can find a constant $C_{\gamma} \geqslant 0$ such that

$$
\left|\partial_{\alpha}^{\gamma}\left[a_{N}(\theta, x, \alpha, \lambda)\right]\right| \leqslant C_{\gamma}
$$

for all $|\theta| \leqslant 1,|x-x(\theta, \alpha)| \leqslant \delta\langle\theta\rangle, \lambda \geqslant 1$ and $\alpha \in T^{*} \mathbb{R}^{n}$ such that $\frac{1}{2} \leqslant\left|\alpha_{\varepsilon}\right| \leqslant 2$. 
Proof. - When $\theta \leqslant 1$ we can use the method of Section 5.2 no matter is $\alpha$, provided that $\frac{1}{2} \leqslant\left|\alpha_{\xi}\right| \leqslant 2$. Let us recall how the amplitude $a_{N}$ is produced. We have

$$
\left\{\begin{array}{l}
a_{N}(\theta, x, \alpha, \lambda)=\langle\theta\rangle^{-n / 2} e_{N}(\theta, x-x(\theta, \alpha), \alpha, \lambda), \\
e_{N}(\theta, x-x(\theta, \alpha), \alpha, \lambda)=f_{N}\left(\theta, \frac{x-x(\theta, \alpha)}{\langle\theta\rangle}, \alpha, \lambda\right), \\
f_{N}(\theta, z, \alpha)=\sum_{\ell=0}^{N+1} \lambda^{-\ell} A_{\ell}(\theta, z, \alpha), \\
L A_{0}=0, L A_{\ell}=i Q A_{\ell-1}, \quad \ell=1, \ldots, N+1, \\
A_{0}(0, z, \alpha)=1, \quad A_{\ell}(0, z, \alpha)=0, \\
\left|\partial_{z}^{\beta} A_{\ell}(\theta, z, \alpha)\right| \leqslant C_{\beta}, \quad \forall \beta \in \mathbb{N}^{n},|\theta| \leqslant 1,|z| \leqslant \delta, \quad \frac{1}{2} \leqslant\left|\alpha_{\xi}\right| \leqslant 2 .
\end{array}\right.
$$

Now, according to Proposition 3.2.1 for every $\gamma \in \mathbb{N}^{2 n}$ such that $|\gamma| \geqslant 1$ one can find $C_{\gamma}^{\prime} \geqslant 0$ such that

$$
\left|\partial_{\alpha}^{\gamma} x(\theta, \alpha)\right|+\left|\partial_{\alpha}^{\gamma} \xi(\theta, \alpha)\right| \leqslant C_{\gamma}^{\prime}
$$

if $|\theta| \leqslant 1, \alpha \in T^{*} \mathbb{R}^{n}, \frac{1}{2} \leqslant\left|\alpha_{\xi}\right| \leqslant 2$.

Assume that we show that for all $\beta \in \mathbb{N}^{n}, \gamma \in \mathbb{N}^{2 n}$ one can find $C_{\beta \gamma} \geqslant 0$ such that

$$
\left|\partial_{z}^{\beta} \partial_{\alpha}^{\gamma} A_{\ell}(\theta, z, \alpha)\right| \leqslant C_{\beta \gamma}
$$

if $|\theta| \leqslant 1$ and $\frac{1}{2} \leqslant\left|\alpha_{\xi}\right| \leqslant 2$. It will follow from (5.4.1) to (5.4.3) and the Faa di Bruno formula that

$$
\left|\partial_{\alpha}^{\gamma} a_{N}(\theta, x, \alpha, \lambda)\right| \leqslant C_{\gamma}
$$

if $|\theta| \leqslant 1,|x-x(\theta, \alpha)| \leqslant \delta\langle\theta\rangle, \frac{1}{2} \leqslant\left|\alpha_{\xi}\right| \leqslant 2$, which is the claim of Proposition 5.4.1.

So we are left with the proof of (5.4.3). By (5.2.8), (5.2.9) and (5.4.2) for all $\mu \in \mathbb{N}^{n}$, $\gamma \in \mathbb{N}^{2 n}$ there exists $C_{\mu \gamma} \geqslant 0$ such that

$$
\left|\partial_{y}^{\mu} \partial_{\alpha}^{\gamma} E_{j}(s, y, \alpha)\right| \leqslant C_{\mu \gamma},
$$

for all $|s| \leqslant 1,|y| \leqslant \delta\langle s\rangle$ and $\frac{1}{2} \leqslant\left|\alpha_{\xi}\right| \leqslant 2$. It follows from (5.2.10), (5.2.11) that for all $\beta \in \mathbb{N}^{n}, \gamma \in \mathbb{N}^{2 n}$ there exists $C_{\beta \gamma} \geqslant 0$ such that

$$
\left|\partial_{z}^{\beta} \partial_{\alpha}^{\gamma} h_{j}(\theta, z, \alpha)\right| \leqslant C_{\beta \gamma},
$$

for all $|\theta| \leqslant 1,|z| \leqslant \delta, \frac{1}{2} \leqslant\left|\alpha_{\xi}\right| \leqslant 2$. And we see easily from (5.2.5), (5.4.2), (5.2.15), (5.2.16) that $h_{j}^{N_{0}}, d^{N_{0}}, k_{\nu}^{N_{0}}$ satisfy also the bound (5.4.5). By induction on the size of derivation, using the Faa di Bruno formula and the Gronwall Lemma we see easily that the solution $z=z(\theta, y, \alpha)$ of (5.2.26) satisfies the bound

$$
\left|\partial_{y}^{\mu} \partial_{\alpha}^{\gamma} z(\theta, y, \alpha)\right| \leqslant C_{\mu \gamma}
$$

uniformly for $|\theta| \leqslant 1,|y| \leqslant \eta, \frac{1}{2} \leqslant\left|\alpha_{\xi}\right| \leqslant 2$. Moreover by Lemma 5.2.3, if we denote by $\kappa(\theta, z)$ the inverse map of $z(\theta, y)$ we have also by (5.4.6),

$$
\left|\partial_{z}^{\beta} \partial_{\alpha}^{\gamma} \kappa(\theta, z, \alpha)\right| \leqslant C_{\beta \gamma}
$$


uniformly for $|\theta| \leqslant 1,|z| \leqslant \delta, \frac{1}{2} \leqslant\left|\alpha_{\xi}\right| \leqslant 2$. Finally we have set for $\ell=0,1, \ldots, N_{0}+1$,

$$
A_{\ell}(\theta, z, \alpha)=\widetilde{A}_{\ell}(\theta, \kappa(\theta, z, \alpha), \alpha)
$$

where

$$
\begin{aligned}
& \widetilde{A}_{0}(\theta, y, \alpha)=\exp \left[-\int_{0}^{\theta} d^{N_{0}}(t, z(t, y, \alpha), \alpha) d t\right] \\
& \widetilde{A}_{\ell}(\theta, y, \alpha)=\exp \left[-\int_{0}^{\theta} d^{N_{0}}(t, z(t, y, \alpha), \alpha) d t\right] \int_{0}^{\theta} i\left(\widetilde{Q} A_{\ell-1}\right)(t, y, \alpha) d t
\end{aligned}
$$

so using (5.4.6), (5.4.7), (5.4.8) and the estimates (5.4.5) for $d^{N_{0}}, k_{\nu}^{N_{0}}$ we see easily that (5.4.3) holds, which completes the proof of Proposition 5.4.1. 



\section{CHAPTER 6}

\section{MICROLOCAL LOCALIZATIONS AND THE USE OF THE FBI TRANSFORM}

In this Chapter using the phase and the amplitude constructed in Chapters 4 and 5 we shall define general FBI transforms which will lead to a parametrix for the Schrödinger equation. These constructions will be microlocal so we will need several microlocal localizations.

\subsection{Preliminaries}

6.1.1. The semi-classical calculus. - We shall work with semi-classical pseudodifferential operators (p.d.o) and we shall use the Weyl calculus described by Hörmander. We refer to $[\mathbf{H}]$ for notations and details.

Let $p \in S_{1,0}^{m}\left(\mathbb{R}^{n}\right)$ (the usual class of symbol of order $m$ ) and let us set $a(x, \xi)=p\left(x, \frac{\xi}{\lambda}\right), \lambda \geqslant 1$. It is easy to see that $a \in S(M, g)$ where,

$$
g=d x^{2}+\frac{d \xi^{2}}{\lambda^{2}+|\xi|^{2}}, \quad M=\lambda^{-m}\left(\lambda^{2}+|\xi|^{2}\right)^{m / 2} .
$$

The p.d.o associated to the symbol $a$ is denoted by $p\left(x, \frac{D}{\lambda}\right)$. Then we have the following symbolic calculus.

i) Let $p \in S_{1,0}^{m}, q \in S_{1,0}^{m^{\prime}}$. Then one can find $\ell_{\lambda} \in S_{1,0}^{m+m^{\prime}}$ such that

$$
p\left(x, \frac{D}{\lambda}\right) \circ q\left(x, \frac{D}{\lambda}\right)=\ell_{\lambda}\left(x, \frac{D}{\lambda}\right) .
$$

The semi norms of $\ell_{\lambda}$ are uniformly bounded when $\lambda \geqslant 1$ and for any $N \in \mathbb{N}^{*}$ we have

$$
\ell_{\lambda}(x, \xi)=\sum_{|\alpha| \leqslant N-1} \frac{1}{\alpha !} \frac{1}{i^{|\alpha|}} \frac{1}{\lambda^{|\alpha|}} \partial_{\xi}^{\alpha} p(x, \xi) \partial_{x}^{\alpha} q(x, \xi)+\frac{1}{\lambda^{N}} r_{N}(\lambda, x, \xi)
$$

where $r_{N} \in S_{1,0}^{m+m^{\prime}-N}$ uniformly for $\lambda \geqslant 1$. 
ii) Let $p \in S_{1,0}^{0}$. Then there exists $C>0$ such that

$$
\left\|p\left(x, \frac{D}{\lambda}\right) u\right\|_{L^{2}} \leqslant C\|u\|_{L^{2}}
$$

for every $u \in L^{2}\left(\mathbb{R}^{n}\right)$ and $\lambda \geqslant 1$. As a consequence, for all $s \in \mathbb{R}$ and all $p \in S_{1,0}^{m}$ one can find a constant $C>0$ such that for every $u \in \mathcal{S}\left(\mathbb{R}^{n}\right)$,

$$
\left\|\left(I-\frac{\Delta}{\lambda^{2}}\right)^{s} p\left(x, \frac{D}{\lambda}\right) u\right\|_{L^{2}} \leqslant C\left\|\left(I-\frac{\Delta}{\lambda^{2}}\right)^{s+m} u\right\|_{L^{2}}
$$

for all $\lambda \geqslant 1$, where $\Delta=\sum_{j=1}^{n} \partial_{j}^{2}$.

6.1.2. The FBI transform. - We recall here the definition of the classical FBI transform as described in Sjöstrand $[\mathbf{S j}]$. We set for $\alpha=\left(\alpha_{x}, \alpha_{\xi}\right) \in T^{*} \mathbb{R}^{n}, \lambda \geqslant 1$, $u \in L^{2}\left(\mathbb{R}^{n}\right)$ and $c_{n}=2^{-n / 2} \pi^{-3 n / 4}$,

$$
T u(\alpha, \lambda)=c_{n} \lambda^{3 n / 4} \int_{\mathbb{R}^{n}} e^{i \lambda\left(y-\alpha_{x}\right) \cdot \alpha_{\varepsilon}-\frac{\lambda}{2}\left|y-\alpha_{x}\right|^{2}+\frac{\lambda}{2}\left|\alpha_{\xi}\right|^{2}} u(y) d y .
$$

Then $T$ maps continuously the space $L^{2}\left(\mathbb{R}^{n}\right)$ to the space of functions $v=v(\alpha)$ such that $e^{-\frac{\lambda}{2}\left|\alpha_{\xi}\right|^{2}} v \in L^{2}\left(\mathbb{R}^{2 n}\right)$.

The adjoint of $T$ is then given by the formula

$$
T^{*} v(x, \lambda)=c_{n} \lambda^{3 n / 4} \int e^{-i \lambda\left(x-\alpha_{x}\right) \cdot \alpha_{\xi}-\frac{\lambda}{2}\left|x-\alpha_{x}\right|^{2}-\frac{\lambda}{2}\left|\alpha_{\xi}\right|^{2}} v(x) d \alpha .
$$

Then we have

$$
T^{*} T \text { is the identity operator of } L^{2}\left(\mathbb{R}^{n}\right) \text {. }
$$

We shall need also the expressions of $T$ and $T^{*}$ by means of the Fourier transform. We have,

$$
\left\{\begin{array}{l}
T u(\alpha, \lambda)=\left(\frac{\lambda}{\pi}\right)^{n / 4} \int e^{i \sigma \cdot \alpha_{x}-\frac{\lambda}{2}\left|\alpha_{\xi}+\frac{\sigma}{\lambda}\right|^{2}+\frac{\lambda}{2}\left|\alpha_{\xi}\right|^{2}} \widehat{u}(\sigma) d \sigma \\
\widehat{T^{*} v}(\xi, \lambda)=c_{n}^{\prime} \lambda^{n / 4} \int e^{-i \xi \cdot \alpha_{x}-\frac{\lambda}{2}\left|\alpha_{\xi}+\frac{\xi}{\lambda}\right|^{2}-\frac{\lambda}{2}\left|\alpha_{\xi}\right|^{2}} v(\alpha) d \alpha .
\end{array}\right.
$$

Let us consider now a self adjoint operator,

$$
P=\sum_{j, k=1}^{n} D_{j}\left(g^{j k} D_{k}\right)+\sum_{j=1}^{n}\left(D_{j} b_{j}+b_{j} D_{j}\right)+b_{0}
$$

with $g^{j k}=\delta_{j k}+\varepsilon b_{j k}$, where $\varepsilon$ is a small positive constant, $\delta_{j k}$ is the Kronecker symbol and,

$$
\sum_{|\alpha|=k}\left|\partial_{x}^{\alpha} b_{j k}(x)\right| \leqslant \frac{A_{k}}{\langle x\rangle^{k+1+\sigma_{0}}}, \quad k=0,1, \ldots, x \in \mathbb{R}^{n}, \quad \sigma_{0}>0 .
$$

Then by interpolation and duality we can prove the following estimates. For all $s \in \mathbb{R}$ there exists $C \geqslant 1$ such that for all $u \in C_{0}^{\infty}\left(\mathbb{R}^{n}\right)$

$$
\frac{1}{C}\|u\|_{H^{s}} \leqslant\left\|(I+P)^{s / 2} u\right\|_{L^{2}} \leqslant C\|u\|_{H^{s}}
$$


For all $s \geqslant 0$ there exists $C \geqslant 1$ such that for all $\lambda \geqslant 1$ and $u \in C_{0}^{\infty}\left(\mathbb{R}^{n}\right)$,

$$
\begin{aligned}
\frac{1}{C} \lambda^{-s}\|u\|_{H^{s}} & \leqslant\left\|\left(I+P\left(x, \frac{D}{\lambda}\right)\right)^{s / 2} u\right\|_{L^{2}} \leqslant C\|u\|_{H^{s}} \\
\frac{1}{C}\|u\|_{H^{-s}} & \leqslant\left\|\left(I+P\left(x, \frac{D}{\lambda}\right)\right)^{-s / 2} u\right\|_{L^{2}} \leqslant C \lambda^{s}\|u\|_{H^{-s}} .
\end{aligned}
$$

\subsection{The microlocalization procedure}

We begin by introducing several cut-off functions. Generally speaking we shall denote by $\chi$ (resp. $\psi$ ) cut-off functions in the space (resp. frequency) variables.

Let $\xi_{0} \in \mathbb{R}^{n},\left|\xi_{0}\right|=1$ be fixed.

Let $\chi_{0} \in C^{\infty}(\mathbb{R})$ be such that,

$$
\chi_{0}(s)=1 \text { if } s \leqslant \frac{3}{4}, \quad \chi_{0}(s)=0 \text { if } s \geqslant 1, \quad 0 \leqslant \chi_{0} \leqslant 1 .
$$

With $\delta_{1}>0$ to be chosen later on we set

$$
\left\{\begin{array}{l}
\chi_{1}^{+}(x)=\chi_{0}\left(-\frac{x \cdot \xi_{0}}{\delta_{1}}\right), \quad \chi_{2}^{+}(x)=\chi_{0}\left(-\frac{x \cdot \xi_{0}}{2 \delta_{1}}\right), \chi_{3}^{+}(x)=\chi_{0}\left(-\frac{x \cdot \xi_{0}}{3 \delta_{1}}\right) \\
\chi_{1}^{-}(x)=\chi_{0}\left(\frac{x \cdot \xi_{0}}{\delta_{1}}\right), \quad \chi_{2}^{+}(x)=\chi_{0}\left(\frac{x \cdot \xi_{0}}{2 \delta_{1}}\right), \quad \chi_{3}^{+}(x)=\chi_{0}\left(\frac{x \cdot \xi_{0}}{3 \delta_{1}}\right) .
\end{array}\right.
$$

These cut-off functions will correspond to outgoing and incoming points. Now for convenience we shall set,

$$
a=\frac{6}{10}, \quad b=\frac{19}{10},
$$

and with $\delta_{2} \leqslant 1 / 100$ (chosen later on) we introduce the following cut-off functions.

Let $\psi_{0} \in C^{\infty}\left(\mathbb{R}^{n}\right)$ be such that $0 \leqslant \psi_{0} \leqslant 1$ and

$$
\left\{\begin{array}{l}
\psi_{0}(\xi)=1 \text { if }\left|\frac{\xi}{|\xi|}-\xi_{0}\right| \leqslant \delta_{2} \text { and }|\xi| \geqslant 2 \delta_{2} \\
\operatorname{supp} \psi_{0} \subset\left\{\xi:\left|\frac{\xi}{|\xi|}-\xi_{0}\right| \leqslant 2 \delta_{2} \text { and }|\xi| \geqslant \delta_{2}\right\}
\end{array}\right.
$$

Let $\psi_{1} \in C_{0}^{\infty}\left(\mathbb{R}^{n}\right)$ be such that, $0 \leqslant \psi_{1} \leqslant 1$ and

$$
\left\{\begin{array}{l}
\psi_{1}(\xi)=1 \text { if } a-\delta_{2} \leqslant|\xi| \leqslant b+\delta_{2} \\
\operatorname{supp} \psi_{1} \subset\left\{\xi: a-2 \delta_{2} \leqslant|\xi| \leqslant b+2 \delta_{2}\right\} .
\end{array}\right.
$$

We shall set

$$
\psi_{2}(\xi)=\psi_{0}(\xi) \psi_{1}(\xi)
$$

Now we introduce for $t \in \mathbb{R}$ the operators,

$$
\left\{\begin{array}{l}
U_{+}(t)=\chi_{1}^{+}(x) \psi_{2}\left(\frac{D}{\lambda}\right) e^{-i t P} \\
U_{-}(t)=\chi_{1}^{-}(x) \psi_{2}\left(\frac{D}{\lambda}\right) e^{-i t P}
\end{array}\right.
$$


It follows that if we denote by $U^{*}$ the adjoint of $U$ then we have

$$
\left\{\begin{array}{l}
U_{ \pm}\left(t_{1}\right) U_{ \pm}\left(t_{2}\right)^{*}=K_{ \pm}\left(t_{1}-t_{2}\right), \text { where } \\
K_{+}(t)=\chi_{1}^{+}(x) \psi_{2}\left(\frac{D}{\lambda}\right) e^{-i t P} \psi_{2}\left(\frac{D}{\lambda}\right) \chi_{1}^{+}(x), \\
K_{-}(t)=\chi_{1}^{-}(x) \psi_{2}\left(\frac{D}{\lambda}\right) e^{-i t P} \psi_{2}\left(\frac{D}{\lambda}\right) \chi_{1}^{-}(x) .
\end{array}\right.
$$

Let now $\psi_{3} \in C_{0}^{\infty}\left(\mathbb{R}^{n}\right)$ be such that $0 \leqslant \psi_{3} \leqslant 1$ and

$$
\left\{\begin{array}{l}
\psi_{3}(\xi)=1 \text { if }\left|\frac{\xi}{|\xi|}+\xi_{0}\right| \leqslant 3 \delta_{2} \text { and } a-3 \delta_{2} \leqslant|\xi| \leqslant b+3 \delta_{2} \\
\operatorname{supp} \psi_{3} \subset\left\{\xi:\left|\frac{\xi}{|\xi|}+\xi_{0}\right| \leqslant 4 \delta_{2} \text { and } a-4 \delta_{2} \leqslant|\xi| \leqslant b+4 \delta_{2}\right\} .
\end{array}\right.
$$

The first localization result requires to introduce the following Definition.

Definition 6.2.1. - We shall call $\mathcal{R}$ the set of families of operators $R=\left(R_{\lambda}(t)\right)$ depending on $\lambda \in[1,+\infty[$ and $t \in[-T, T]$ such that for every $N$ in $\mathbb{N}$ one can find a constant $C_{N} \geqslant 0$ such that for every $u \in \mathcal{S}\left(\mathbb{R}^{n}\right)$,

$$
\left\|R_{\lambda}(t) u\right\|_{H^{2 N}\left(\mathbb{R}^{n}\right)} \leqslant C_{N}\|u\|_{H^{-2 N}\left(\mathbb{R}^{n}\right)}
$$

uniformly with respect to $(\lambda, t) \in[1,+\infty[\times[-T, T]$.

Then we can state the following result.

Theorem 6.2.2. - Let $T>0$. For every $t \in[-T, T]$ and $\lambda \geqslant 1$ one can write

$$
\begin{array}{r}
K_{+}(t)=\chi_{1}^{+} \psi_{2}\left(\frac{D}{\lambda}\right) \chi_{2}^{+} T_{\alpha \rightarrow x}^{*} \chi_{3}^{+}\left(\alpha_{x}\right) \psi_{3}\left(\alpha_{\xi}\right) T_{y \rightarrow \alpha}\left[e^{-i t P} \chi_{2}^{+} \psi_{2}\left(\frac{D}{\lambda}\right) \chi_{1}^{+}\right] \\
+R_{\lambda}^{+}(t)
\end{array}
$$

where $\left(R_{\lambda}^{+}(t)\right) \in \mathcal{R}$. The same formula is true with the sign - instead of + .

The proof of this Theorem requires several steps.

Lemma 6.2.3. - There exist a constant $C>0$ such that

$$
\left\|\psi_{2}\left(\frac{D}{\lambda}\right) T^{*}\left[\left(1-\psi_{3}\left(\alpha_{\xi}\right)\right) v\right]\right\|_{L^{2}\left(\mathbb{R}^{n}\right)} \leqslant C e^{-\frac{\lambda}{8} \delta_{2}^{2}}\left\|e^{-\frac{\lambda}{2}\left|\alpha_{\xi}\right|^{2}} v\right\|_{L^{2}\left(\mathbb{R}^{2 n}\right)}
$$

for every $v$ such that $e^{-\frac{\lambda}{2}\left|\alpha_{\xi}\right|^{2}} v \in L^{2}\left(\mathbb{R}^{2 n}\right)$.

Proof. - We claim that on the support of $\psi_{2}(\xi)\left(1-\psi_{3}\left(\alpha_{\xi}\right)\right)$ we have

$$
\left|\xi+\alpha_{\xi}\right| \geqslant \frac{1}{2} \delta_{2}\left|\alpha_{\xi}\right|
$$

Indeed, according to (6.2.4) to (6.2.6) and (6.2.9) we have on this support $\left|\frac{\xi}{|\xi|}-\xi_{0}\right| \leqslant 2 \delta_{2}, a-2 \delta_{2} \leqslant|\xi| \leqslant b+2 \delta_{2}$ and either $\left|\alpha_{\xi}\right| \leqslant a-3 \delta_{2}$ or $\left|\alpha_{\xi}\right| \geqslant b+3 \delta_{2}$ or 
$\left|\frac{\alpha_{\xi}}{\alpha_{\xi} \mid}+\xi_{0}\right| \geqslant 3 \delta_{2}$. In the first case we have $\left|\xi+\alpha_{\xi}\right| \geqslant|\xi|-\left|\alpha_{\xi}\right| \geqslant \delta_{2}$. In the second case we have $\left|\xi+\alpha_{\xi}\right| \geqslant\left|\alpha_{\xi}\right|-|\xi| \geqslant \delta_{2}$ and in the third one we have

$$
\left|\frac{\alpha_{\xi}}{\left|\alpha_{\xi}\right|}+\frac{\xi}{|\xi|}\right| \geqslant\left|\frac{\alpha_{\xi}}{\left|\alpha_{\xi}\right|}+\xi_{0}\right|-\left|\frac{\xi}{|\xi|}-\xi_{0}\right| \geqslant \delta_{2} \text {. }
$$

Therefore $\left|\alpha_{\xi}+\frac{\left|\alpha_{\xi}\right|}{|\xi|} \xi\right| \geqslant \delta_{2}\left|\alpha_{\xi}\right|$. It follows that

$$
\left|\alpha_{\xi}+\xi\right| \geqslant\left|\alpha_{\xi}+\frac{\left|\alpha_{\xi}\right|}{|\xi|} \xi\right|-\left|\xi-\frac{\left|\alpha_{\xi}\right|}{|\xi|} \xi\right| \geqslant \delta_{2}\left|\alpha_{\xi}\right|-|| \xi|-| \alpha_{\xi}|| \geqslant \delta_{2}\left|\alpha_{\xi}\right|-\left|\alpha_{\xi}+\xi\right|
$$

so $\left|\alpha_{\xi}+\xi\right| \geqslant \frac{1}{2} \delta_{2}\left|\alpha_{\xi}\right|$ and our claim is proved.

Now using (6.1.4) we can write

$$
\begin{aligned}
(1) & =: \mathcal{F}\left(\psi_{2}\left(\frac{D}{\lambda}\right) T^{*}\left[\left(1-\psi_{3}\left(\alpha_{\xi}\right)\right)\right] v\right)(\xi) \\
& =c_{n} \lambda^{\frac{n}{4}} \psi_{2}\left(\frac{\xi}{\lambda}\right) \int_{\mathbb{R}^{2 n}} e^{-i \xi \cdot \alpha_{x}-\frac{\lambda}{2}\left|\alpha_{\xi}+\frac{\xi}{\lambda}\right|^{2}-\frac{\lambda}{2}\left|\alpha_{\xi}\right|^{2}}\left(1-\psi_{3}\left(\alpha_{\xi}\right)\right) v(\alpha) d \alpha .
\end{aligned}
$$

Let us set

$$
w\left(\xi, \alpha_{\xi}\right)=\int_{\mathbb{R}^{n}} e^{-i \xi \cdot \alpha_{x}-\frac{\lambda}{2}\left|\alpha_{\xi}\right|^{2}} v(\alpha) d \alpha_{x}=\mathcal{F}_{\alpha_{x}}\left(e^{-\frac{\lambda}{2}\left|\alpha_{\xi}\right|^{2}} v\right)\left(\xi, \alpha_{\xi}\right) .
$$

Using Cauchy-Schwartz inequality we obtain

$$
|(1)|^{2} \leqslant C \lambda^{n / 2}\left(\int_{\mathbb{R}^{n}} e^{-\lambda\left|\alpha_{\xi}+\frac{\xi}{\lambda}\right|^{2}} \psi_{2}^{2}\left(\frac{\xi}{\lambda}\right)\left(1-\psi_{3}\left(\alpha_{\xi}\right)\right)^{2} d \alpha_{\xi}\right)\left(\int\left|w\left(\xi, \alpha_{\xi}\right)\right|^{2} d \alpha_{\xi}\right)
$$

so by $(6.2 .12)$

$$
|(1)|^{2} \leqslant C \lambda^{\lambda / n} e^{-\frac{\lambda}{8} \delta_{2}^{2}}\left(\int e^{-\frac{\lambda}{2}\left|\alpha_{\xi}+\frac{\xi}{\lambda}\right|^{2}} d \alpha_{\xi}\right)\left(\int\left|w\left(\xi, \alpha_{\xi}\right)\right|^{2} d \alpha_{\xi}\right) .
$$

It follows that

$$
|(1)|^{2} \leqslant C^{\prime} e^{-\frac{\lambda}{8} \delta_{2}^{2}} \int\left|w\left(\xi, \alpha_{\xi}\right)\right|^{2} d \alpha_{\xi}
$$

Integrating with respect to $\xi$ and using Parseval identity we obtain

$$
\left\|\psi_{2}\left(\frac{D}{\lambda}\right) T^{*}\left[\left(1-\psi_{3}\left(\alpha_{\xi}\right)\right) v\right]\right\|_{L^{2}} \leqslant C e^{-\frac{\lambda}{8} \delta_{2}^{2}} \int_{\mathbb{R}^{2 n}} e^{-\frac{\lambda}{2}\left|\alpha_{\xi}\right|^{2}}|v(\alpha)|^{2} d \alpha .
$$

Corollary 6.2.4. - We have for $t \in[-T, T]$ and $\lambda \geqslant 1$,

$$
K_{+}(t)=\chi_{1}^{+} \psi_{2}\left(\frac{D}{\lambda}\right) T^{*} \psi_{3}\left(\alpha_{\xi}\right) T e^{-i t P} \psi_{2}\left(\frac{D}{\lambda}\right) \chi_{1}^{+}+R_{\lambda}^{+}(t)
$$

where $\left(R_{\lambda}^{+}(t)\right) \in \mathcal{R}$. The same is true for the sign -.

Proof. - Using (6.1.3) we write Id $=T^{*} T=T^{*} \psi_{3}\left(\alpha_{\xi}\right) T+T^{*}\left(1-\psi_{3}\left(\alpha_{\xi}\right)\right) T$. So we have to prove that the family of operators

belong to $\mathcal{R}$.

$$
R_{\lambda}^{+}(t)=\chi_{1}^{+} \psi_{2}\left(\frac{D}{\lambda}\right) T^{*}\left(1-\psi_{3}\left(\alpha_{\xi}\right)\right) T e^{-i t P} \psi_{2}\left(\frac{D}{\lambda}\right) \chi_{1}^{+}
$$


Let $\widetilde{\psi} \in C_{0}^{\infty}\left(\mathbb{R}^{n} \backslash 0\right)$ be such that $\widetilde{\psi}(\xi) \psi_{2}(\xi)=\psi_{2}(\xi)$. Then writing $\psi_{2}\left(\frac{D}{\lambda}\right)=\widetilde{\psi}\left(\frac{D}{\lambda}\right) \psi_{2}\left(\frac{D}{\lambda}\right)$, using Lemma 6.2 .3 and the fact that $\left\|\widetilde{\psi}\left(\frac{D}{\lambda}\right) v\right\|_{H^{2 N}} \leqslant C \lambda^{2 N}\|v\|_{L^{2}}$ we obtain

$$
\left\|R_{\lambda}^{+}(t) v\right\|_{H^{2 N}} \leqslant C \lambda^{2 N} e^{-\frac{1}{8} \lambda \delta_{2}^{2}}\left\|e^{-\frac{\lambda}{2}\left|\alpha_{\xi}\right|^{2}} T e^{-i t P} \psi_{2}\left(\frac{D}{\lambda}\right) \chi_{1}^{+} u\right\|_{L^{2}} .
$$

Since $T$ is continuous from $L^{2}$ to the space of $v$ such that $e^{-\frac{\lambda}{2}\left|\alpha_{\xi}\right|^{2}} v \in L^{2}$ and using the conservation of $L^{2}$ norm we obtain

$$
\left\|R_{\lambda}^{+}(t) v\right\|_{H^{2 N}} \leqslant C \lambda^{2 N} e^{-\frac{1}{8} \lambda \delta_{2}^{2}}\left\|\psi_{2}\left(\frac{D}{\lambda}\right) \chi_{1}^{+} u\right\|_{L^{2}} \leqslant C^{\prime} \lambda^{4 n} e^{-\frac{1}{8} \lambda \delta_{2}^{2}}\|u\|_{H^{-2 N}}
$$

so our claim is proved.

Lemma 6.2.5. - Let $\chi_{2}^{+}$be defined in (6.2.2). Then we have for $t \in[-T, T]$ and $\lambda \geqslant 1$,

$$
K_{+}(t)=\chi_{1}^{+} \psi_{2}\left(\frac{D}{\lambda}\right) \chi_{2}^{+} T^{*} \psi_{3}\left(\alpha_{\xi}\right) T e^{-i t P} \chi_{2}^{+} \psi_{2}\left(\frac{D}{\lambda}\right) \chi_{1}^{+}+R_{\lambda}^{+}(t)
$$

where $\left(R_{\lambda}^{+}(t)\right) \in \mathcal{R}$.

Proof. - Since by (6.2.2) the support of $\chi_{1}^{+}$and $1-\chi_{2}^{+}$are disjoint, the symbolic calculus shows that the operators $\chi_{1}^{+} \psi_{2}\left(\frac{D}{\lambda}\right)\left(1-\chi_{2}^{+}\right)$and $\left(1-\chi_{2}^{+}\right) \psi_{2}\left(\frac{D}{\lambda}\right) \chi_{1}^{+}$belong to $\frac{1}{\lambda^{M}} S_{1,0}^{-M}$ for any $M \in \mathbb{N}$. It follows from (VI.1.7) that if $M \geqslant 2 N$,

$$
\begin{aligned}
\| \chi_{1}^{+} \psi_{2}\left(\frac{D}{\lambda}\right)(1- & \left.\chi_{2}^{+}\right) T^{*} \psi_{3}\left(\alpha_{\xi}\right) T e^{-i t P} \psi_{2}\left(\frac{D}{\lambda}\right) \chi_{1}^{+} u \|_{H^{2 N}} \\
& \leqslant C \lambda^{2 N}\left\|\left(I-\frac{\Delta}{\lambda^{2}}\right)^{N} \chi_{1}^{+} \psi_{2}\left(\frac{D}{\lambda}\right)\left(1-\chi_{2}^{+}\right) T^{*} \ldots\right\|_{L^{2}} \\
& \leqslant C \lambda^{2 N-M}\left\|T^{*} \psi_{3}\left(\alpha_{\xi}\right) T e^{-i t P} \psi_{2}\left(\frac{D}{\lambda}\right) \chi_{1}^{+} u\right\|_{L^{2}} \\
& \leqslant C \lambda^{2 N-M}\left\|e^{-\frac{\lambda}{2}\left|\alpha_{\xi}\right|^{2}} \psi_{3}\left(\alpha_{\xi}\right) T e^{-i t P} \psi_{2}\left(\frac{D}{\lambda}\right) \chi_{1}^{+} u\right\|_{L^{2}\left(\mathbb{R}^{2 n}\right)} \\
& \leqslant C \lambda^{2 N-M}\left\|e^{-i t P} \psi_{2}\left(\frac{D}{\lambda}\right) \chi_{1}^{+} u\right\|_{L^{2}} \leqslant C \lambda^{2 N-M}\left\|\psi_{2}\left(\frac{D}{\lambda}\right) \chi_{1}^{+} u\right\|_{L^{2}} \\
& \leqslant C \lambda^{4 N-M}\|u\|_{H^{-2 N}}
\end{aligned}
$$

Taking $M \geqslant 4 N$ we conclude that the remainder under consideration belong to $\mathcal{R}$. By the same way

$$
\begin{aligned}
\| \chi_{1}^{+} \psi_{2}\left(\frac{D}{\lambda}\right) & \chi_{2}^{+} T^{*} \psi_{3}\left(\alpha_{\xi}\right) T e^{-i t P}\left(1-\chi_{2}^{+}\right) \psi_{2}\left(\frac{D}{\lambda}\right) \chi_{1}^{+} u \|_{H^{2 N}} \\
& \leqslant C \lambda^{2 N}\left\|\left(I-\frac{\Delta}{\lambda^{2}}\right)^{N} \psi_{2}\left(\frac{D}{\lambda}\right) \chi_{2}^{+} T^{*} \cdots u\right\|_{L^{2}} \\
& \leqslant C \lambda^{2 N}\left\|\left(1-\chi_{2}^{+}\right) \psi_{2}\left(\frac{D}{\lambda}\right) \chi_{1}^{+}\left(I-\frac{\Delta}{\lambda^{2}}\right)^{N}\left(I-\frac{\Delta}{\lambda^{2}}\right)^{-N} u\right\|_{L^{2}} \\
& \leqslant C^{\prime} \lambda^{2 N-M}\left\|\left(I-\frac{\Delta}{\lambda^{2}}\right)^{-N} u\right\|_{L^{2}} \leqslant C^{\prime \prime} \lambda^{4 N-M}\|u\|_{H^{-2 N}} .
\end{aligned}
$$

The proof is complete. 
Lemma 6.2.6. - Let $\psi_{a} \in C_{0}^{\infty}(\mathbb{R})$ and $\chi_{a}(x), \chi_{b}\left(\alpha_{x}\right)$ be $C^{\infty}$ functions such that one can find $\mu>0$ such that $\left|x-\alpha_{x}\right| \geqslant \mu$ if $\left(x, \alpha_{x}\right)$ belongs to $\operatorname{supp}\left[\chi_{a}(x)\left(1-\chi_{b}\left(\alpha_{x}\right)\right)\right]$. Then one can find $\varepsilon>0, C>0$ such that

$$
\left\|\chi_{a} T^{*}\left[\psi_{a}\left(\alpha_{\xi}\right)\left(1-\chi_{b}\left(\alpha_{x}\right)\right) v\right]\right\|_{L^{2}\left(\mathbb{R}^{n}\right)} \leqslant C e^{-\varepsilon \lambda}\left\|e^{-\frac{\lambda}{2}\left|\alpha_{\xi}\right|^{2}} v\right\|_{L^{2}\left(\mathbb{R}_{\alpha}^{2 n}\right)}
$$

for all $v$ such that the right hand side norm is finite.

Proof. - It follows from (6.1.2) that

$$
\chi_{a}(x) T^{*}\left(\psi_{a}\left(\alpha_{\xi}\right)\left(1-\chi_{b}\left(\alpha_{x}\right)\right) v\right)(x)=\int K(x, \alpha) e^{-\frac{\lambda}{2}\left|\alpha_{\xi}\right|^{2}} v(\alpha) d \alpha
$$

where

$$
K(x, \alpha)=c_{n} \lambda^{3 n / 4} e^{-i \lambda\left(x-\alpha_{x}\right) \cdot \alpha_{\xi}-\frac{\lambda}{2}\left|x-\alpha_{x}\right|^{2}} \psi_{a}\left(\alpha_{\xi}\right) \chi_{a}(x)\left(1-\chi_{b}\left(\alpha_{x}\right)\right) .
$$

Using our assumption we can write

$$
|K(x, \alpha)| \leqslant C \lambda^{3 n / 4} e^{-\frac{\lambda}{4} \mu^{2}} e^{-\frac{\lambda}{4}\left|x-\alpha_{x}\right|^{2}} \psi_{a}\left(\alpha_{\xi}\right) .
$$

Therefore one can find $\varepsilon>0$ such that,

$$
\sup _{x} \int|K(x, \alpha)| d \alpha \leqslant C e^{-\varepsilon \lambda}, \quad \sup _{\alpha} \int|K(x, \alpha)| d x \leqslant C e^{-\varepsilon \lambda},
$$

so the Lemma follows from the well known Schur Lemma.

Corollary 6.2.7. - We have

$$
K_{+}(t)=\chi_{1}^{+} \psi\left(\frac{D}{\lambda}\right) \chi_{2}^{+} T^{*} \psi_{3}\left(\alpha_{\xi}\right) \chi_{3}^{+}\left(\alpha_{x}\right) T e^{-i t P} \chi_{2}^{+} \psi_{2}\left(\frac{D}{\lambda}\right) \chi_{1}^{+}+R_{\lambda}^{+}(t)
$$

where $R \in \mathcal{R}$ and the same is true for the sign -.

Proof. - We have to show that the operator

$$
R_{\lambda}^{+}(t)=\chi_{1}^{+} \psi_{2}\left(\frac{D}{\lambda}\right) \chi_{2}^{+} T^{*} \psi_{3}\left(\alpha_{\xi}\right)\left(1-\chi_{3}^{+}\left(\alpha_{x}\right)\right) T e^{-i t P} \chi_{2}^{+} \psi_{2}\left(\frac{D}{\lambda}\right) \chi_{1}^{+}
$$

belongs to $\mathcal{R}$.

We apply Lemma 6.2.6 with $\chi_{a}=\chi_{2}^{+}, \psi_{a}=\psi_{3}, \chi_{b}=\chi_{3}^{+}$. Then according to (6.2.2) we have on the support of $\chi_{2}^{+}(x)\left(1-\chi_{3}^{+}\left(\alpha_{x}\right)\right),-x \cdot \xi_{0} \leqslant 2 \delta_{1},-\alpha_{x} \cdot \xi_{0} \geqslant \frac{9}{4} \delta_{1}$. Therefore we have, $\frac{9}{4} \delta_{1} \leqslant-\alpha_{x} \cdot \xi_{0} \leqslant\left(x-\alpha_{x}\right) \cdot \xi_{0}-x \cdot \xi_{0} \leqslant\left|x-\alpha_{x}\right| \cdot\left|\xi_{0}\right|+2 \delta_{1}$, so $\left|x-\alpha_{x}\right| \geqslant \mu>0$. Then using Lemma 6.2.6 we can write

$$
\begin{aligned}
\left\|R_{\lambda}(t) u\right\|_{H^{2 N}} & \leqslant C \lambda^{2 N} e^{-\varepsilon \lambda}\left\|e^{-\frac{\lambda}{2}\left|\alpha_{\xi}\right|^{2}} T e^{-i t P} \chi_{2}^{+} \psi_{2}\left(\frac{D}{\lambda}\right) \chi_{1}^{+} u\right\|_{L^{2}} \\
& \leqslant C^{\prime} \lambda^{2 N} e^{-\varepsilon \lambda}\left\|\psi_{2}\left(\frac{D}{\lambda}\right) \chi_{1}^{+} u\right\|_{L^{2}} \leqslant C^{\prime \prime} \lambda^{4 N} e^{-\varepsilon \lambda}\|u\|_{H^{-2 N}}
\end{aligned}
$$

Proof of Theorem 6.2.2. - It follows immediately from Corollary 6.2.7. 


\subsection{The one sided parametrix}

The purpose of this Section is to use the results of Chapters 4,5 and 6.2 to show that the operators $K_{+}(t)$ and $K_{-}(t)$ introduced in (6.2.5) can be written as Fourier integral operators with complex phase functions. We shall take the expression of $K_{ \pm}(t)$ given by Theorem 6.2 .2 and we begin by considering the expression

$$
T\left[e^{-i t P} \chi_{2}^{+} \psi_{2}\left(\frac{D}{\lambda}\right) \chi_{1}^{+} u\right](\alpha, \lambda) \quad(\text { see }(6.1 .1)) .
$$

Let us first introduce some other cut-off functions.

$$
\left\{\begin{array}{l}
\text { If }\left|\alpha_{x} \cdot \alpha_{\xi}\right| \leqslant \frac{c_{0}}{2}\left\langle\alpha_{x}\right\rangle\left|\alpha_{\xi}\right| \text { we set } \chi_{4}^{ \pm}(y) \equiv 1 . \\
\text { If }\left|\alpha_{x} \cdot \alpha_{\xi}\right|>\frac{c_{0}}{2}\left\langle\alpha_{x}\right\rangle\left|\alpha_{\xi}\right| \text { we set, } \\
\text { (i) in the }+ \text { case, } \chi_{4}^{+}(y)=\chi_{0}\left(-\frac{y \cdot \xi_{0}}{5 \delta_{1}}\right), \\
\text { (ii) in the }- \text { case, } \chi_{4}^{-}(y)=\chi_{0}\left(\frac{y \cdot \xi_{0}}{5 \delta_{1}}\right), \\
\text { where } \chi_{0} \text { has been defined (in }(6.2 .1) \text { ). }
\end{array}\right.
$$

In all cases let $\chi_{5} \in C_{0}^{\infty}\left(\mathbb{R}^{n}\right)$ be such $0 \leqslant \chi_{5} \leqslant 1$ and

$$
\chi_{5}(y)=1 \quad \text { if }|y| \leqslant \frac{\delta}{2}, \quad \operatorname{supp} \chi_{5} \subset\{y:|y| \leqslant \delta\}
$$

where $\delta$ is the small constant introduced in Theorem 4.1.2. For the convenience of the reader let us recall the main properties of the phase and the symbol constructed in Corollary 5.1.2, and Theorem 4.1.2. First of all the phase $\varphi$ is defined on the set $\Omega_{\delta}$ where,

(i) if $\left|\alpha_{x} \cdot \alpha_{\xi}\right| \leqslant c_{0}\left\langle\alpha_{x}\right\rangle\left|\alpha_{\xi}\right|$ then

$$
\Omega_{\delta}=\left\{(\theta, y) \in \mathbb{R} \times \mathbb{R}^{n}:|y-x(\theta, \alpha)|<\delta\langle\theta\rangle\right\} .
$$

(ii) if $\alpha_{x} \cdot \alpha_{\xi}>c_{0}\left\langle\alpha_{x}\right\rangle\left|\alpha_{\xi}\right|$ then,

$$
\begin{array}{r}
\Omega_{\delta}=\left\{(\theta, y) \in(0,+\infty) \times \mathbb{R}^{n}:|y-x(\theta, \alpha)|<\delta\langle\theta\rangle\right\} \cup\left\{(\theta, y) \in(-\infty, 0) \times \mathbb{R}^{n}:\right. \\
\left.|y-x(\theta, \alpha)|<\delta\langle\theta\rangle \text { and } y \cdot \alpha_{\xi} \geqslant-c_{1}\langle y\rangle\left|\alpha_{\xi}\right|\right\}
\end{array}
$$

(iii) if $\alpha_{x} \cdot \alpha_{\xi}<-c_{0}\left\langle\alpha_{x}\right\rangle\left|\alpha_{\xi}\right|$ then,

$$
\begin{array}{r}
\Omega_{\delta}=\left\{(\theta, y) \in(-\infty, 0) \times \mathbb{R}^{n}:|y-x(\theta, \alpha)|<\delta\langle\theta\rangle\right\} \cup\left\{(\theta, y) \in(0,+\infty) \times \mathbb{R}^{n}:\right. \\
\left.|y-x(\theta, \alpha)|<\delta\langle\theta\rangle \text { and } y \cdot \alpha_{\xi} \leqslant c_{1}\langle y\rangle\left|\alpha_{\xi}\right|\right\} .
\end{array}
$$

Moreover on this domain

$$
\operatorname{Im} \varphi(\theta, y, \alpha) \geqslant \frac{1}{4} \frac{|y-x(\theta, \alpha)|^{2}}{1+4 \theta^{2}}-\frac{1}{2}\left|\alpha_{\xi}\right|^{2} .
$$

Now if we set, with the notations of Theorem 5.1.1, for $N \in \mathbb{N}$,

$$
a(\theta, y, \alpha, \lambda)=\left(1+\theta^{2}\right)^{-n / 4} e_{N}(\theta, y-x(\theta, \alpha), \alpha, \lambda),
$$


then $a$ is defined on $\Omega_{\delta}$ for $\lambda \geqslant 1$ and satisfies,

$$
\left\{\begin{array}{l}
a(0, y, \alpha, \lambda)=1, \\
|a(\theta, y, \alpha, \lambda)| \leqslant c\left(1+\theta^{2}\right)^{-n / 4}, \\
\left(i \lambda \frac{\partial}{\partial \theta}+{ }^{t} P\right)\left(e^{i \lambda \varphi(\theta, y, \alpha)} a(\theta, y, \alpha, \lambda)\right)=b_{N}(\theta, y, \alpha, \lambda) e^{i \lambda \varphi(\theta, y, \alpha)}, \\
\text { with }\left|b_{N}(\theta, y, \alpha)\right| \leqslant c_{N}\left(1+\theta^{2}\right)^{-n / 4}\left(\lambda^{-N}+\lambda^{2}\left(\frac{|y-x(\theta, \alpha)|}{\langle\theta\rangle}\right)^{N}\right) .
\end{array}\right.
$$

Let us introduce now the following set.

$$
\begin{aligned}
& W^{ \pm}=\left\{\alpha \in T^{*} \mathbb{R}^{n}: \frac{1}{2} \leqslant\left|\alpha_{\xi}\right| \leqslant 2,\left|\alpha_{x} \cdot \alpha_{\xi}\right| \leqslant c_{0}\left\langle\alpha_{x}\right\rangle\left|\alpha_{\xi}\right|\right\} \\
& \cup\left\{\alpha \in T^{*} \mathbb{R}^{n}:\left|\alpha_{x} \cdot \alpha_{\xi}\right|>c_{0}\left\langle\alpha_{x}\right\rangle\left|\alpha_{\xi}\right|, \quad\left(\alpha_{x}, \alpha_{\xi}\right) \in \operatorname{supp}\left(\chi_{3}^{ \pm}\left(\alpha_{x}\right) \cdot \psi_{3}\left(\alpha_{\xi}\right)\right)\right\}
\end{aligned}
$$

where $\chi_{3}^{ \pm}$and $\psi_{3}$ have been defined in (6.2.2) and (6.2.9).

Then we can state the main result of this Section.

Theorem 6.3.1. - We have for $t \in[-T, T]$, and $\alpha \in W^{+}$,

$$
\begin{aligned}
& T\left[e^{-i t P} \chi_{2}^{+} v\right](\alpha, \lambda) \\
& =\lambda^{3 n / 4} \int e^{i \lambda \varphi(-\lambda t, y, \alpha)} a(-\lambda t, y, \alpha, \lambda) \chi_{4}^{+}(y) \chi_{5}\left(\frac{y-x(-\lambda t, \alpha)}{\langle\lambda t\rangle}\right)\left[\chi_{2}^{+} v\right](y) d y \\
& +J_{\lambda}^{+}(t) v(\alpha)
\end{aligned}
$$

where the operator $J_{\lambda}^{+}$is such that, for any $M \in \mathbb{N}$ one can find a constant $C_{M}>0$ such that for all $\lambda \geqslant 1$ and $t \in[-T, T]$

$$
\left\|e^{-\frac{\lambda}{2}\left|\alpha_{\xi}\right|^{2}} J_{\lambda}^{+}(t) v\right\|_{L^{2}\left(W^{+}\right)} \leqslant \frac{C_{M}}{\lambda^{M}}\|v\|_{L^{2}\left(\mathbb{R}^{n}\right)},
$$

and the same is true with the minus sign.

Proof. - Let us introduce the following family of operators. We set for $\alpha \in W^{+}$

$$
\begin{aligned}
& S v(\theta, t, \alpha, \lambda) \\
= & \lambda^{3 n / 4} \int_{\mathbb{R}^{n}} e^{i \lambda \varphi(\theta, y, \alpha)} a(\theta, y, \alpha, \lambda) \chi_{4}^{+}(y) \chi_{5}\left(\frac{y-x(\theta, \alpha)}{\langle\theta\rangle}\right)\left[e^{-i t P} \chi_{2}^{+} v\right](y) d y .
\end{aligned}
$$

We must verify that the right hand side is indeed well defined.

On the support of $\chi_{5}$ we have $|y-x(\theta, \alpha)|<\delta\langle\theta\rangle$ (which is one of the conditions for $(\theta, y)$ to be in $\left.\Omega_{\delta}\right)$. If $\alpha \in W^{+}$then either $\left|\alpha_{x} \cdot \alpha_{\varepsilon}\right| \leqslant c_{0}\left\langle\alpha_{x}\right\rangle\left|\alpha_{\varepsilon}\right|$ or $\left|\alpha_{x} \cdot \alpha_{\varepsilon}\right|>c_{0}\left\langle\alpha_{x}\right\rangle\left|\alpha_{\varepsilon}\right|$ and $\left(\alpha_{x}, \alpha_{\varepsilon}\right) \in \operatorname{supp}\left(\chi_{2}^{+} \cdot \psi_{3}\right)$. In the first case by (6.3.1) $\chi_{4}^{+}(y)=1$ but $(\theta, y) \in \Omega_{\delta}$ for $\theta \in \mathbb{R}$. In the second case since $\alpha_{x} \in \operatorname{supp} \chi_{3}^{+}$and $\alpha_{\xi} \in \operatorname{supp} \psi_{3}$ we have, by (6.2.1), (6.2.2) and (6.2.9), $\alpha_{x} \cdot \xi_{0} \geqslant-3 \delta_{1}$ and $\left|\frac{\alpha_{\xi}}{\left|\alpha_{\xi}\right|}+\xi_{0}\right| \leqslant 4 \delta_{2}$. It follows that

$$
\alpha_{x} \cdot \frac{\alpha_{\xi}}{\left|\alpha_{\xi}\right|}=-\alpha_{x} \cdot \xi_{0}+\alpha_{x} \cdot\left(\frac{\alpha_{\xi}}{\left|\alpha_{\xi}\right|}+\xi_{0}\right) \leqslant 7 \delta_{1}\left\langle\alpha_{x}\right\rangle .
$$


On the other hand we have $\left|\alpha_{x} \cdot \alpha_{\xi}\right|>c_{0}\left\langle\alpha_{x}\right\rangle\left|\alpha_{\xi}\right|$. According to (6.3.8) we cannot have $\alpha_{x} \cdot \alpha_{\xi}>c_{0}\left\langle\alpha_{x}\right\rangle\left|\alpha_{\xi}\right|$ if $\delta_{1} \ll c_{0}$; thus we must have $\alpha_{x} \cdot \alpha_{\xi}<-c_{0}\left\langle\alpha_{x}\right\rangle\left|\alpha_{\xi}\right|$. So we are in the case (iii) for the definition of $\Omega_{\delta}$. Since in the integral, on the support of $\chi_{4}^{+}(y)$ we have by (6.3.1), $y \cdot \xi_{0} \geqslant-5 \delta_{1} \geqslant-5 \delta_{1}\langle y\rangle$, we deduce that

$$
y \cdot \frac{\alpha_{\xi}}{\left|\alpha_{\xi}\right|}=-y \cdot \xi_{0}+y \cdot\left(\frac{\alpha_{\xi}}{\left|\alpha_{\xi}\right|}+\xi_{0}\right) \leqslant 9 \delta_{1}\langle y\rangle<c_{0}\langle y\rangle .
$$

Therefore $(\theta, y) \in \Omega_{\delta}$ and the right hand side of (6.3.7) is well defined. Of course the same argument is valid in the case of the minus sign.

Now let us set for $s \in[0, t]$ if $t>0$ (resp. $s \in[t, 0]$ if $t<0$ )

$$
g(s)=S v(\lambda(s-t), s, \alpha, \lambda) .
$$

Since $g^{\prime}(s)=\left(\lambda \partial_{\theta}+\partial_{t}\right)(S v)(\lambda(s-t), s, \alpha, \lambda)$ we obtain

$$
\left\{\begin{aligned}
S v(0, t, \alpha, \lambda)=S v & -\lambda t, 0, \alpha, \lambda) \\
& +\int_{0}^{t}\left(\lambda \partial_{\theta}+\partial_{t}\right)(S v)(\lambda(s-t), s, \alpha, \lambda) d s \quad \text { if } t>0 \\
S v(0, t, \alpha, \lambda)=S v & -\lambda t, 0, \alpha, \lambda) \\
& \quad-\int_{0}^{t}\left(\lambda \partial_{\theta}+\partial_{t}\right)(S v)(\lambda(s-t), s, \alpha, \lambda) d s \quad \text { if } t<0 .
\end{aligned}\right.
$$

Let us set

$$
U(t, y, \lambda)=\left[e^{-i t P} \chi_{2}^{+} v\right](y) .
$$

Then according to (6.3.7) we can write

$$
\left(\lambda \partial_{\theta}+\partial_{t}\right)(S v)(\theta, t, \alpha, \lambda)=\lambda^{3 n / 4}\left(A_{1}+A_{2}+A_{3}\right)
$$

where

$$
\left\{\begin{array}{c}
A_{1}=\int \lambda \partial_{\theta}\left(e^{i \lambda \varphi(\theta, y, \alpha)} a(\theta, y, \alpha, \lambda)\right) \chi_{4}^{+}(y) \chi_{5}\left(\frac{y-x(\theta, \alpha)}{\langle\theta\rangle}\right) U(t, y, \lambda) d y \\
A_{2}=\int e^{i \lambda \varphi(\theta, y, \alpha)} a(\theta, y, \alpha, \lambda) \chi_{4}^{+}(y) \chi_{5}\left(\frac{y-x(\theta, \alpha)}{\langle\theta\rangle}\right)(-i P) U(t, y, \lambda) d y \\
A_{3}=\sum_{j=1}^{n} \int e^{i \lambda \varphi(\theta, y, \alpha)} a(\theta, y, \alpha, \lambda) \chi_{4}^{+}(y)\left(\partial_{j} \chi_{5}\right)\left(\frac{y-x(\theta, \alpha)}{\langle\theta\rangle}\right) \\
\cdot \partial_{\theta}\left(\frac{y_{j}-x_{j}(\theta, \alpha)}{\langle\theta\rangle}\right) U(t, y, \lambda) d y
\end{array}\right.
$$

Integrating by parts in the integral giving $A_{2}$ we obtain

$$
\begin{array}{r}
A_{2}=\int\left(-i^{t} P\right)\left[e^{i \lambda \varphi(\theta, y, \alpha)} a(\theta, y, \alpha, \lambda)\right] \chi_{4}^{+}(y) \chi_{5}\left(\frac{y-x(\theta, \alpha)}{\langle\theta\rangle}\right) U(t, y, \lambda) d y \\
+\sum_{\substack{1 \leqslant|\beta| \leqslant 2 \\
\beta=\beta_{1}+\beta_{2}}} \int e^{i \lambda \varphi(\theta, y, \alpha)} b_{\beta}(\theta, y, \alpha, \lambda)\left(\partial_{y}^{\beta_{1}} \chi_{4}^{+}\right)(y)\langle\theta\rangle^{-\left|\beta_{2}\right|} \\
\cdot\left(\partial_{y}^{\beta_{2}} \chi_{5}\right)\left(\frac{y-x(\theta, \alpha)}{\langle\theta\rangle}\right) U(t, y, \lambda) d y
\end{array}
$$

where $b_{\beta}$ is a symbol satisfying the same estimates as $a(\theta, y, \alpha, \lambda)$ (see (6.3.5)). 
Combining the term $A_{1}$ with the first part of $A_{2}$ and using (6.3.5) we can write

$$
\left(\lambda \partial_{\theta}+\partial_{t}\right)(S v)(\theta, t, \alpha, \lambda)=\lambda^{3 n / 4}\left(B_{1}+B_{2}+B_{3}\right)
$$

where

$$
\left\{\begin{array}{c}
B_{1}(\theta, t, \alpha, \lambda)=\int e^{i \lambda \varphi(\theta, y, \alpha)} b_{N}(\theta, y, \alpha, \lambda) \chi_{4}^{+}(y) \\
\chi_{5}\left(\frac{y-x(\theta, \alpha)}{\langle\theta\rangle}\right) U(t, y, \lambda) d y \\
B_{2}(\theta, t, \alpha, \lambda)=\sum_{\substack{\left|\beta_{1}+\beta_{2}\right| \leqslant 2 \\
\beta_{2} \neq 0}} \int e^{i \lambda \varphi(\theta, y, \alpha)} C_{\beta_{1} \beta_{2}}(\theta, y, \alpha, \lambda)\left(\partial_{y}^{\beta_{1}} \chi_{4}^{+}\right)(y)\langle\theta\rangle^{-\left|\beta_{2}\right|} \\
\left(\partial_{y}^{\beta_{2}} \chi_{5}\right)\left(\frac{y-x(\theta, \alpha)}{\langle\theta\rangle}\right) U(t, y, \lambda) d y \\
B_{3}(\theta, t, \alpha, \lambda)=\sum_{1 \leqslant|\gamma| \leqslant 2} \int e^{i \lambda \varphi(\theta, y, \alpha)} d_{\gamma}(\theta, y, \alpha, \lambda)\left(\partial_{y}^{\gamma} \chi_{4}^{+}\right)(y) \\
\chi_{5}\left(\frac{y-x(\theta, \alpha)}{\langle\theta\rangle}\right) U(t, y, \lambda) d y
\end{array}\right.
$$

where $C_{\beta_{1}, \beta_{2}}$ and $d_{\gamma}$ are bounded symbols. Here we used the estimate $\left|\partial_{\theta}\left(\frac{y_{j}-x_{j}(\theta, \alpha)}{\langle\theta\rangle}\right)\right| \leqslant$ $C /\langle\theta\rangle$. Now we state a Lemma.

Lemma 6.3.2. - One can find constants $C>0, \eta>0$ and for every $M \geqslant 0$ a constant $C_{M}>0$ such that for all $\theta, t, \lambda$ such that $\lambda \geqslant 1,|\theta| \leqslant|\lambda t|,|t| \leqslant T$ we have

$$
\begin{aligned}
& \left\|e^{-\frac{\lambda}{2}\left|\alpha_{\xi}\right|^{2}} B_{1}(\theta, t, \cdot, \lambda)\right\|_{L^{2}\left(W^{+}\right)} \leqslant C_{M} \lambda^{-M}\|v\|_{L^{2}\left(\mathbb{R}^{n}\right)} \\
& \left\|e^{-\frac{\lambda}{2}\left|\alpha_{\xi}\right|^{2}} B_{2}(\theta, t, \cdot, \lambda)\right\|_{L^{2}\left(W^{+}\right)} \leqslant C e^{-\eta \lambda}\|v\|_{L^{2}\left(\mathbb{R}^{n}\right)}
\end{aligned}
$$

for all $v$ in $L^{2}\left(\mathbb{R}^{n}\right)$.

Proof. - Let us consider the term $B_{1}$. Using (6.3.5) and (6.3.3) we obtain

$$
\begin{aligned}
&\left|e^{i \lambda \varphi(\theta, y, \alpha)-\frac{\lambda}{2}\left|\alpha_{\xi}\right|^{2}} b_{N}(\theta, y, \alpha, \lambda) \chi_{5}\left(\frac{y-x(\theta, \alpha)}{\langle\theta\rangle}\right) \chi_{4}^{+}(y) \mathbf{1}_{W^{+}}(\alpha)\right| \\
& \leqslant C_{N} e^{-\frac{\lambda}{16} \frac{|y-x(\theta, \alpha)|^{2}}{\langle\theta\rangle^{2}}}\langle\theta\rangle^{-n / 2}\left(\lambda^{-N}+\lambda^{2}\left(\frac{|y-x(\theta, \alpha)|}{\langle\theta\rangle}\right)^{N}\right) \mathbf{1}_{W^{+}(\alpha)} \\
&=: K(\theta, y, \alpha, \lambda) .
\end{aligned}
$$

On the other hand we have

$$
e^{-\frac{\lambda}{2}\left|\alpha_{\xi}\right|^{2}} B_{1}=\int K(\theta, y, \alpha, \lambda) U(t, y, \lambda) d y .
$$

We want to apply Schur Lemma to this integral operator. First of all making the change of variables $y-x(\theta, \alpha)=\langle\theta\rangle z / \sqrt{\lambda}$ we can write

$$
\int K(\theta, y, \alpha, \lambda) d y \leqslant C_{N}\langle\theta\rangle^{n / 2} \int e^{-\frac{|z|^{2}}{16}} \lambda^{-n / 2}\left(\lambda^{-N}+\lambda^{2} \lambda^{-N / 2}|z|^{N}\right) d z .
$$

Since $\langle\theta\rangle \leqslant 1+\lambda T$ we obtain finally for all $M \geqslant 0$ and $\lambda \geqslant 1, \int K(\theta, y, \alpha, \lambda) d y \leqslant$ $C_{M} \lambda^{-M}$. 
In order to estimate $(1)=\int K(\theta, y, \alpha, \lambda) d \alpha$ we make the change of variable $\beta=$ $(x(\theta, \alpha), \xi(\theta, \alpha))$ Since this transformation is symplectic we have $d \alpha=d \beta$. Therefore we obtain,

$$
(1) \leqslant C_{N} \int e^{-\frac{\lambda}{16} \frac{\left|y-\beta_{x}\right|^{2}}{\langle\theta\rangle^{2}}}\langle\theta\rangle^{-n / 2} \mathbf{1}_{\frac{1}{2} \leqslant|\xi(-\theta, \beta)| \leqslant 2}(\beta)\left(\lambda^{-N}+\lambda^{2}\left(\frac{\left|y-\beta_{x}\right|}{\langle\theta\rangle}\right)^{N}\right) d \beta .
$$

Since $\xi(-\theta, \beta)=\beta_{\xi}+\mathcal{O}(\varepsilon)$ we have $\frac{1}{3} \leqslant\left|\beta_{\xi}\right| \leqslant 3$. Setting as before $y-\beta_{x}=\frac{\langle\theta\rangle}{\sqrt{\lambda}}$ we deduce easily that $(1) \leqslant C_{M} \lambda^{-M}$ for every $M \geqslant 0$. It follows from these estimates, the Schur Lemma and (6.3.11) that,

$$
\left\|e^{-\frac{\lambda}{2}\left|\alpha_{\xi}\right|^{2}} B_{1}\right\|_{L^{2}\left(W^{+}\right)} \leqslant C_{M} \lambda^{-M}\left\|e^{-i t P} \psi^{2}\left(\frac{P}{\lambda^{2}}\right) \chi_{2}^{+} v\right\|_{L^{2}\left(\mathbb{R}^{n}\right)} \leqslant C_{M}^{\prime} \lambda^{-M}\|v\|_{L^{2}\left(\mathbb{R}^{n}\right)} .
$$

To deal with the term $B_{2}$ we use exactly the same computations and the fact that on the support of $\left(\partial_{y}^{\beta_{2}} \chi_{5}\right)\left(\frac{y-x(\theta, \alpha)}{\langle\theta\rangle}\right)$, for $\left|\beta_{2}\right| \geqslant 1$ we have $\frac{\delta}{2} \leqslant \frac{|y-x(\theta, \alpha)|}{\langle\theta\rangle} \leqslant \delta$.

Proof of Theorem 6.3.1 in the following cases

$$
\left\{\begin{array}{l}
\text { (i) }\left|\alpha_{x} \cdot \alpha_{\xi}\right| \leqslant c_{0}\left\langle\alpha_{x}\right\rangle\left|\alpha_{\xi}\right| \text { and } t \in[-T, T], \\
\text { (ii) }\left|\alpha_{x} \cdot \alpha_{\xi}\right|>c_{0}\left\langle\alpha_{x}\right\rangle\left|\alpha_{\xi}\right| \text { and } t \in[0, T] .
\end{array}\right.
$$

In both these cases we are going to show, with the notations of (6.3.15), that $B_{3} \equiv 0$.

In the case (i) this is obvious since by (6.3.1) we took $\chi_{4}^{+}(y) \equiv 1$. Now if $t>0$, by (6.3.9) we have $\theta=\lambda(s-t) \leqslant 0$. In the case (ii) we have seen that in the case + we must have $\alpha_{x} \cdot \alpha_{\varepsilon} \leqslant-c_{0}\left\langle\alpha_{x}\right\rangle\left|\alpha_{\varepsilon}\right|$. Now on the support of $\chi_{5}\left(\frac{y-y(\theta, \alpha)}{\langle\theta\rangle}\right)$ we have $|y-x(\theta, \alpha)| \leqslant \delta\langle\theta\rangle$ and it follows from Proposition 3.4.1 that $x(\theta, \alpha)=\alpha_{x}+2 \theta \alpha_{\xi}+\mathcal{O}(\varepsilon\langle\theta\rangle)$. Then we write

$$
y \cdot \xi_{0}=(y-x(\theta, \alpha)) \cdot \xi_{0}+\left(\alpha_{x}+2 \theta \alpha_{\xi}\right) \cdot \xi_{0}+\mathcal{O}(\varepsilon\langle\theta\rangle) .
$$

On the support of $\psi_{3}\left(\alpha_{\xi}\right)$ we have $\left|\frac{\alpha_{\xi}}{\left|\alpha_{\xi}\right|}+\xi_{0}\right| \leqslant 4 \delta_{1}$ so

$$
\begin{gathered}
y \cdot \xi_{0}=\left(\alpha_{x}+2 \theta \alpha_{\xi}\right) \cdot\left(\xi_{0}+\frac{\alpha_{\xi}}{\left|\alpha_{\xi}\right|}\right)-\left(\alpha_{x}+2 \theta \alpha_{\xi}\right) \cdot \frac{\alpha_{\xi}}{\left|\alpha_{\xi}\right|}+\mathcal{O}((\varepsilon+\delta)\langle\theta\rangle) \\
y \cdot \xi_{0} \geqslant c_{0}\left\langle\alpha_{x}\right\rangle+2|\theta|\left|\alpha_{\xi}\right|-4 \delta_{1}\left\langle\alpha_{x}\right\rangle-C\left(\delta+\varepsilon+\delta_{1}\right)\langle\theta\rangle .
\end{gathered}
$$

Since $\delta, \varepsilon, \delta_{1}$ are small compared to $c_{0}$ we deduce that $y \cdot \xi_{0} \geqslant \frac{c_{0}}{2}\langle\theta\rangle$ in the integral defining $B_{3}$ in (6.3.15). Since the support of $\partial_{y}^{\gamma} \chi_{4}^{+}(y)$, for $\gamma \neq 0$, is contained in $\frac{3}{4} \leqslant \frac{-y \cdot \xi_{0}}{5 \delta_{1}} \leqslant 1$ we deduce that $B_{3} \equiv 0$ in this case, (see (6.3.1) and (6.2.2)). It follows from (6.3.10) and (6.3.14) that

$$
S v(0, t, \alpha, \lambda)=S v(-\lambda t, 0, \alpha, \lambda)+\int_{0}^{t}\left(B_{1}+B_{2}\right)(s) d s .
$$

It follows from Lemma 6.3.2 that,

$$
\left\|e^{-\frac{\lambda}{2}\left|\alpha_{\xi}\right|^{2}} \int_{0}^{t}\left(B_{1}+B_{2}\right)(s) d s\right\|_{L^{2}\left(W^{+}\right)} \leqslant \frac{C}{\lambda^{M}}\|v\|_{L^{2}\left(\mathbb{R}^{n}\right)} .
$$


Now we have

$$
\left\{\begin{array}{l}
\varphi(0, y, \alpha)=\varphi_{0}(y, \alpha)+g\left(y-\alpha_{x}\right), \text { where } \\
\varphi_{0}(y, \alpha)=\left(y-\alpha_{x}\right) \cdot \alpha_{\xi}+\frac{i}{2}\left|y-\alpha_{x}\right|^{2}+\frac{1}{2 i}\left|\alpha_{\xi}\right|^{2}, \\
|g(x)| \leqslant C_{N}|x|^{N} \text { for every } N \in \mathbb{N} .
\end{array}\right.
$$

Let $\chi \in C_{0}^{\infty}\left(\mathbb{R}^{n}\right)$ be such that $\chi(x)=1$ if $|x| \leqslant 1$, supp $\chi \subset\{x:|x| \leqslant 2\}$ and let us fix $N \geqslant 3$. We can write

$$
S v(0, t, \alpha, \lambda)=A_{1}-A_{2}-A_{3}+A_{4}
$$

where

$$
\left\{\begin{array}{r}
A_{1}=c_{n} \lambda^{3 n / 4} \int e^{i \lambda \varphi_{0}(y, \alpha)} \chi_{4}^{+}(y) \chi_{5}\left(y-\alpha_{x}\right) U(t, y, \lambda) d y \\
A_{2}=c_{n} \lambda^{3 n / 4} \int e^{i \lambda \varphi_{0}(y, \alpha)}\left(1-\chi\left(C_{N} \lambda\left|y-\alpha_{x}\right|^{N}\right)\right) \\
\cdot \chi_{4}^{+}(y) \chi_{5}\left(y-\alpha_{x}\right) U(t, y, \lambda) d y \\
A_{3}=c_{n} \lambda^{3 n / 4} \int e^{i \lambda \varphi_{0}(y, \alpha)}\left(1-e^{i \lambda g\left(y-\alpha_{x}\right)}\right) \chi\left(C_{N} \lambda\left|y-\alpha_{x}\right|^{N}\right) \chi_{4}^{+}(y) \\
\cdot \chi_{5}\left(y-\alpha_{x}\right) U(t, y, \lambda) d y \\
A_{4}=c_{n} \lambda^{3 n / 4} \int e^{i \lambda\left(\varphi_{0}(y, \alpha)+g\left(y-\alpha_{x}\right)\right)}\left(1-\chi\left(C_{N} \lambda\left|y-\alpha_{x}\right|^{N}\right)\right) \chi_{4}^{+}(y) \\
\cdot \chi_{5}\left(y-\alpha_{x}\right) U(t, y, \lambda) d y .
\end{array}\right.
$$

We claim that we have for $j=2,3,4$,

$$
\left\|e^{-\frac{\lambda}{2}\left|\alpha_{\xi}\right|^{2}} A_{j}\right\|_{L^{2}\left(W^{+}\right)} \leqslant \frac{C_{M_{N}}}{\lambda^{M_{N}}}\|v\|_{L^{2}\left(\mathbb{R}^{n}\right)}, M_{N} \longrightarrow+\infty \text { if } N \longrightarrow+\infty .
$$

(i) Term $A_{2}$

On the support $1-\chi\left(C_{N} \lambda\left|y-\alpha_{x}\right|^{N}\right)$ we have $\left|y-\alpha_{x}\right| \geqslant C_{N}^{\prime} \lambda^{-1 / N}$. So,

$$
\left|e^{-\frac{\lambda}{2}\left|\alpha_{\xi}\right|^{2}} e^{i \varphi_{0}(y, \alpha)}\left(1-\chi\left(C_{N} \lambda\left|y-\alpha_{x}\right|^{N}\right)\right)\right| \leqslant C e^{-\frac{\lambda}{4}\left|y-\alpha_{x}\right|^{2}} e^{-C_{N}^{\prime \prime} \lambda^{1-\frac{2}{N}}} .
$$

Using the Schur Lemma and the inequality $\|U(t, \cdot, \alpha)\|_{L^{2}} \leqslant C\|v\|_{L^{2}}$ (see (6.3.11)) we obtain (6.3.21) for $A_{2}$.

(ii) Term $A_{3}$

On the support of $\chi\left(C_{N} \lambda\left|y-\alpha_{x}\right|^{N}\right)$ we have $\lambda\left|g\left(y-\alpha_{x}\right)\right| \leqslant C_{N} \lambda\left|y-\alpha_{x}\right|^{N} \leqslant 2$. Therefore we have $\left|1-e^{i \lambda g\left(y-\alpha_{x}\right)}\right| \leqslant C \lambda\left|g\left(y-\alpha_{x}\right)\right| \leqslant C_{N}^{\prime} \lambda\left|y-\alpha_{x}\right|^{N}$. It follows that

$$
\begin{aligned}
\mid e^{-\frac{\lambda}{2}\left|\alpha_{\xi}\right|^{2}} e^{i \lambda \varphi_{0}(y, \alpha)} & \left(1-e^{i \lambda g\left(y-\alpha_{x}\right.}\right) \chi\left(C_{N} \lambda\left|y-\alpha_{x}\right|^{N}\right)\left|\leqslant C_{N}^{\prime} e^{-\frac{\lambda}{2}\left|y-\alpha_{x}\right|^{2}} \cdot \lambda\right| y-\left.\alpha_{x}\right|^{N} \\
& \leqslant \frac{C_{N}^{\prime}}{\lambda^{\frac{N}{2}}-1}\left(\lambda^{1 / 2}\left|y-\alpha_{x}\right|\right)^{N} e^{-\frac{\lambda}{4}\left|y-\alpha_{x}\right|^{2}} e^{-\frac{\lambda}{4}\left|y-\alpha_{x}\right|^{2}} \\
& \leqslant \frac{C_{N}^{\prime}}{\lambda^{\frac{N}{2}}-1} e^{-\frac{\lambda}{4}\left|y-\alpha_{x}\right|^{2}} .
\end{aligned}
$$

The Schur Lemma shows again that $A_{3}$ satisfies (6.3.21).

(iii) Term $A_{4}$ 
On the support of $\chi_{5}\left(y-\alpha_{x}\right)$ we have, according to (6.3.2), $\left|y-\alpha_{x}\right| \leqslant \delta$. It follows that $\left|g\left(\alpha_{x}-y\right)\right| \leqslant \delta C_{3}\left|y-\alpha_{x}\right|^{2}$ so if $\delta$ is small enough,

$$
-\frac{1}{2}\left|\alpha_{\xi}\right|^{2}-\operatorname{Im} \varphi_{0}(y, \alpha)-\operatorname{Im} g\left(y-\alpha_{x}\right) \leqslant-\frac{1}{4}\left|y-\alpha_{x}\right|^{2}
$$

and, as for $A_{2}$, the Schur Lemma implies that $A_{4}$ satisfies (6.3.21).

Using (6.3.19) and (6.3.21) we see that

$$
\begin{gathered}
S v(0, t, \alpha, \lambda)=c_{n} \lambda^{3 n / 4} \int e^{i \lambda \varphi_{0}(y, \alpha)} \chi_{4}^{+}(y) \chi_{5}\left(y-\alpha_{x}\right) U(t, y, \lambda) d y \\
+J_{\lambda}^{+}(t) v(\alpha) . \\
\left\|e^{-\frac{\lambda}{2}\left|\alpha_{\xi}\right|^{2}} J_{\lambda}^{+}(t) v\right\| \leqslant C_{M} \lambda^{-M}\|v\|_{L^{2}\left(\mathbb{R}^{n}\right) .}
\end{gathered}
$$

Now, on the support of $\chi_{5}\left(y-\alpha_{x}\right)-1$ we have $\left|y-\alpha_{x}\right| \geqslant \frac{\delta}{2}$ so modulo a term which satisfies (6.3.23) we can remove $\chi_{5}\left(y-\alpha_{x}\right)$ in the right hand side of (6.3.22). Let us remove $\chi_{4}^{+}$. When $\alpha \in W^{+}$we have $\left|\alpha_{x} \cdot \alpha_{\varepsilon}\right| \leqslant c_{0}\left\langle\alpha_{x}\right\rangle\left|\alpha_{\xi}\right|$ and $\chi_{4}^{+}(y) \equiv 1$ (see (6.3.1)) (so there is nothing to remove) or $\alpha_{x} \in \operatorname{supp} \chi_{3}^{+}$(see (6.3.6)) that is $-\alpha_{x} \cdot \xi_{0} \leqslant 3 \delta_{1}$. In the later case on the support of $1-\chi_{4}^{+}(y)$ we have $\frac{-y \cdot \xi_{0}}{5 \delta_{1}} \geqslant \frac{3}{4}$ (see $(6.2 .1),(6.3 .1)$ ) so $\left|y-\alpha_{x}\right| \geqslant \alpha_{x} \cdot \xi_{0}-y \cdot \xi_{0} \geqslant \frac{3}{4} \delta_{1}$. The corresponding term, again by the Schur Lemma, satisfies (6.3.23).

Using (6.1.1) we see therefore that

$$
S v(0, t, \alpha, \lambda)=T\left[e^{-i t P} \chi_{2}^{+} v\right](\alpha, \lambda)+J_{\lambda}^{+}(t) v(\alpha),
$$

where $J_{\lambda}^{+}(t)$ satisfies (6.3.23).

Gathering the informations given by (6.3.17), (6.3.18), (6.3.24) and (6.3.23) we obtain the claim of Theorem 6.3.1 in the case (6.3.16).

Proof of Theorem 6.3.1 in the following case

$$
\left|\alpha_{x} \cdot \alpha_{\xi}\right|>c_{0}\left\langle\alpha_{x}\right\rangle\left|\alpha_{\xi}\right| \text { and } t \in[-T, 0] .
$$

According to (6.3.14), (6.3.15) and Lemma 6.3.2, we must prove that for all $N \in \mathbb{N}$ one can find $C_{N}>0$ such that for $\lambda \geqslant 1$,

$$
\left\|e^{-\frac{\lambda}{2}\left|\alpha_{\xi}\right|^{2}} B_{3}(\theta, t, \cdot, \lambda)\right\|_{L^{2}\left(W^{+}\right)} \leqslant C_{N} \lambda^{-N}\|v\|_{L^{2}\left(\mathbb{R}^{n}\right)} .
$$

Here $\theta=\lambda(s-t)>0$ since $s \in[t, 0]$.

Let us introduce a new cut-off function. Let $\psi_{4} \in C_{0}^{\infty}\left(\mathbb{R}^{n}\right)$ be such that $0 \leqslant \psi_{4} \leqslant 1$ and,

$$
\left\{\begin{array}{l}
\psi_{4}(\xi)=1 \text { if }\left|\frac{\xi}{|\xi|}+\xi_{0}\right| \leqslant 5 \delta_{2}, a-5 \delta_{2} \leqslant|\xi| \leqslant b+5 \delta_{2} \\
\operatorname{supp} \psi_{4} \subset\left\{\xi:\left|\frac{\xi}{|\xi|}+\xi_{0}\right| \leqslant 6 \delta_{2}, a-6 \delta_{2} \leqslant|\xi| \leqslant b+6 \delta_{2}\right\} .
\end{array}\right.
$$

We state a Lemma. 
Lemma 6.3.3. - Let us set

(6.3.28)

$\widetilde{W}^{+}=\left\{\alpha: \frac{1}{2} \leqslant\left|\alpha_{\xi}\right| \leqslant 2,\left|\alpha_{x} \cdot \alpha_{\xi}\right|>c_{0}\left\langle\alpha_{x}\right\rangle\left|\alpha_{\xi}\right|, \quad\left(\alpha_{x}, \alpha_{\xi}\right) \in \operatorname{supp}\left(\chi_{3}^{+}\left(\alpha_{x}\right) \psi_{3}\left(\alpha_{\xi}\right)\right)\right\}$.

Let $k(\theta, y, \alpha, \lambda)$ be a symbol and let us set

$$
\begin{array}{r}
F(\theta, \alpha, \lambda)=\mathbf{1}_{\widetilde{W}^{+}}(\alpha) e^{-\frac{\lambda}{2}\left|\alpha_{\xi}\right|^{2}} \int e^{i \lambda \varphi(\theta, y, \alpha)} k(\theta, y, \alpha, \lambda) \chi_{5}\left(\frac{y-x(\theta, \alpha)}{\langle\theta\rangle}\right) \\
\cdot \partial_{y}^{\gamma} \chi_{4}^{+}(y)\left[I-\psi_{4}\left(\frac{D}{\lambda}\right)\right] v(y) d y
\end{array}
$$

Then for every $N \in \mathbb{N}$ one can find $C_{N}>0$ such that for $\lambda \geqslant 1$ and $|\theta| \leqslant \lambda T$ we have

$$
\|F(\theta, \cdot, \lambda)\|_{L^{2}} \leqslant C_{N} \lambda^{-N}\|v\|_{L^{2}\left(\mathbb{R}^{n}\right)} .
$$

Proof. - By (6.2.12) we have $\left|\xi+\alpha_{\xi}\right| \geqslant \mu>0$ on the support of $\psi_{3}\left(\alpha_{\xi}\right)\left(1-\psi_{4}(\xi)\right)$. Now recall that Theorem 4.1.2 shows that the phase $\varphi$ satisfies

$$
\left\{\begin{array}{l}
\left|\frac{\partial \varphi}{\partial y}(\theta, y, \alpha)-\alpha_{\xi}\right| \leqslant C(\varepsilon+\sqrt{\delta}) \\
\left|\partial_{y}^{\beta} \varphi(\theta, y, \alpha)\right| \leqslant C_{\beta} \quad \text { if }|\beta| \geqslant 1
\end{array}\right.
$$

on the support of $\chi_{5}\left(\frac{y-x(\theta, \alpha)}{\langle\theta\rangle}\right) \partial_{y}^{\gamma} \chi_{4}^{+}(y) \mathbf{1}_{\widetilde{W}^{+}}(\alpha)$.

Let $g \in C_{0}^{\infty}\left(\mathbb{R}^{n}\right)$ be such that $g(\xi)=1$ if $|\xi| \leqslant 1$. Then

$$
\left(I-\psi_{4}\left(\frac{D}{\lambda}\right)\right) v(y)=\lim _{\varepsilon \rightarrow 0}\left(\frac{\lambda}{2 \pi}\right)^{n} \iint e^{i \lambda(y-z) \cdot \xi}\left(1-\psi_{4}(\xi)\right) g(\varepsilon \xi) v(z) d z d \xi .
$$

It follows that

$$
\left\{\begin{aligned}
& F(\theta, \alpha, \lambda)=\lim _{\varepsilon \rightarrow 0} \int K_{\varepsilon}(\alpha, z) v(z) d z \text { with } \\
& K_{\varepsilon}(\alpha, z)=\left(\frac{\lambda}{2 \pi}\right) \cdot \iint e^{-\frac{\lambda}{2}\left|\alpha_{\xi}\right|^{2}} \mathbf{1}_{\widetilde{W}^{+}}(\alpha) e^{i \lambda[\varphi(\theta, y, \alpha)+(y-z) \cdot \xi]} k(\theta, y, \alpha, \lambda) \\
& \cdot \chi_{5}\left(\frac{y-x(\theta, \alpha)}{\langle\theta\rangle}\right) \partial_{y}^{\gamma} \chi_{4}^{+}(y)\left(1-\psi_{4}(\xi)\right) g(\varepsilon \xi) d \xi d y
\end{aligned}\right.
$$

Let us consider the vector field

$$
X=\frac{1}{1+\lambda|y-z|^{2}}\left(1+\frac{1}{i} \sum_{j=1}^{n}\left(y_{j}-z_{j}\right) \frac{\partial}{\partial \xi_{j}}\right) .
$$

Then it is easy to see that

$$
\left\{\begin{array}{l}
X e^{i \lambda(y-z) \cdot \xi}=e^{i \lambda(y-z) \cdot \xi} \\
\left({ }^{t} X\right)^{N}=\sum_{|A| \leqslant N} \frac{C_{A}(y-z)^{A} \partial_{\xi}^{\alpha}}{\left(1+\lambda|y-z|^{2}\right)^{N}} .
\end{array}\right.
$$


Then we can write

$$
\begin{aligned}
& \int e^{i(y-z) \cdot \xi}\left(1-\psi_{4}(\xi)\right) g(\varepsilon \xi) d \xi=\int e^{i \lambda(y-z) \cdot \xi}\left({ }^{t} X\right)^{N}\left[\left(1-\psi_{4}(\xi)\right) g(\varepsilon \xi)\right] d \xi \\
&= \sum_{\substack{A=A_{1}+A_{2} \\
|A| \leqslant N}} \int e^{i \lambda(y-z) \cdot \xi} C_{A_{1} A_{2}} \frac{(y-z)^{A}}{\left(1+\lambda|y-z|^{2}\right)^{N}} \partial_{\xi}^{A_{1}}\left(1-\psi_{4}(\xi)\right) \varepsilon^{\left|A_{2}\right|} \\
& \cdot\left(\partial_{\xi}^{A_{2}} g\right)(\varepsilon \xi) d \xi .
\end{aligned}
$$

Now on the support of $\psi_{3}\left(\alpha_{\xi}\right)\left(1-\psi_{4}(\xi)\right)$ we have $\left|\xi+\alpha_{\xi}\right| \geqslant \mu>0$, it follows from (6.3.29) that

$$
\left|\frac{\partial \varphi}{\partial y}(\theta, y, \alpha)+\xi\right| \geqslant\left|\alpha_{\xi}+\xi\right|-\left|\frac{\partial \varphi}{\partial y}(\theta, y, \alpha)-\alpha_{\xi}\right| \geqslant \mu-C(\varepsilon+\sqrt{\delta}) \geqslant \frac{\mu}{2}
$$

if $\varepsilon$ and $\delta$ are small enough.

If $|\xi| \geqslant 2 \sup \left|\frac{\partial \varphi}{\partial y}(\theta, y, \alpha)\right|$ we have $\left|\frac{\partial \varphi}{\partial y}(\theta, y, \alpha)+\xi\right| \geqslant \frac{|\xi|}{2}$. Therefore in all cases we have, with $\eta_{0}>0$,

$$
\left|\frac{\partial \varphi}{\partial y}(\theta, y, \alpha)+\xi\right| \geqslant \eta_{0}\langle\xi\rangle
$$

Let us set then

$$
Y=\frac{1}{i \lambda\left|\xi+\frac{\partial \varphi}{\partial y}(\theta, y, \alpha)\right|^{2}} \sum_{j=1}^{n}\left(\frac{\partial \varphi}{\partial y_{j}}(\theta, y, \alpha)+\xi_{j}\right) \frac{\partial}{\partial y_{j}} .
$$

and $T=\frac{\partial \varphi}{\partial y}(\theta, y, \alpha)+\xi$.

Then

$$
\left\{\begin{array}{l}
Y e^{i \lambda(\varphi(\theta, y, \alpha)+(y-z) \cdot \xi)}=e^{i \lambda(\varphi(\theta, y, \alpha)+(y-z) \cdot \xi)} \\
\left({ }^{t} Y\right)^{N}=\frac{1}{(i \lambda)^{N}}\left\{\sum_{|\nu|=N}\left(\frac{T}{|T|^{2}}\right)^{\nu} \partial_{y}^{\nu}+\sum_{|\nu| \leqslant N-1} \frac{P_{3 N-2|\nu|-1}(\theta, y, \alpha, T, \bar{T})}{|T|^{4 N-2|\nu|}} \partial_{y}^{\nu}\right\}
\end{array}\right.
$$

where $P_{k}(\theta, y, \alpha, T, \bar{T})$ is a polynomial in $T, \bar{T}$ of order $\leqslant k$ with $C^{\infty}$-bounded coefficients.

It follows from (6.3.32) that on the support of $\psi_{3}\left(\alpha_{\xi}\right)\left(1-\psi_{4}(\xi)\right)$ we have,

$$
\sum_{|\nu|=N}\left|\left(\frac{T}{|T|^{2}}\right)^{\nu}\right|+\sum_{|\nu| \leqslant N-1} \frac{\left|P_{3 N-2|\nu|-1}(\cdots)\right|}{|T|^{4 N-2|\nu|}} \leqslant \frac{C_{N}}{\langle\xi\rangle^{N}} .
$$

On the other hand we check by induction that

$$
\partial_{y}^{\nu}\left[\frac{(y-z)^{A}}{\left(1+\lambda|y-z|^{2}\right)^{N}}\right]=\sum_{\substack{j \leqslant|\nu| \\|\beta| \leqslant|A|+j \\ 2 j+|A| \leqslant|\beta|+|\nu|}} b_{N, j, A, \beta} \frac{(y-z)^{\beta} \lambda^{j}}{\left(1+\lambda|y-z|^{2}\right)^{N+j}} .
$$

Now if we insert (6.3.31) into (6.3.30) and if we make integration by parts with respect to $Y$ using (6.3.33) we see using (6.3.34) and (6.3.3) that $K_{\varepsilon}(\alpha, z)$ is bounded by a 
finite sum of integrals of the following type

$$
\begin{array}{r}
\int \frac{\lambda^{n}|y-z|^{|\beta|} \lambda^{j}}{\lambda^{N}\langle\xi\rangle^{N}\left(1+\lambda|y-z|^{2}\right)^{N+j}} \mathbf{1}_{\widetilde{W}^{+}}(\alpha)\left|\partial_{\xi}^{A_{1}}\left(1-\psi_{4}(\xi)\right)\right| \varepsilon^{\left|A_{2}\right|}\left(\partial_{\xi}^{A_{2}} g\right)(\varepsilon \xi)\left|\partial_{y}^{\nu_{1}} k\right| \\
\left|\left(\partial_{y}^{\nu_{2}} \chi_{5}\right)\left(\frac{y-x(\theta, \alpha)}{\langle\theta\rangle}\right)\right|\langle\theta\rangle^{-\left|\nu_{2}\right|}\left|\partial_{y}^{\gamma+\nu_{3}} \chi_{4}^{+}(y)\right| e^{-\frac{\lambda}{16} \frac{|y-x(\theta, \alpha)|^{2}}{\langle\theta\rangle^{2}}} d y d \xi
\end{array}
$$

where $|\beta| \leqslant|A|+j, \nu_{1}+\nu_{2}+\nu_{3}=\nu,|\nu| \leqslant N, A=A_{1}+A_{2},|A| \leqslant N, j \leqslant|\nu|$, $2 j+|A| \leqslant|\beta|+|\nu|$.

Claim. - We have

$$
\left\{\begin{array}{l}
(1)=\sup _{\alpha} \int\left|K_{\varepsilon}(\alpha, z)\right| d z \leqslant C_{N} \frac{\langle\theta\rangle^{n}}{\lambda^{N / 2}} \\
(2)=\sup _{z} \int\left|K_{\varepsilon}(\alpha, z)\right| d \alpha \leqslant C_{N}^{\prime} \frac{\langle\theta\rangle^{n}}{\lambda^{N / 2}} .
\end{array}\right.
$$

Let us first remark that

$$
\int \frac{\lambda^{j}|x|^{|\beta|}}{\left(1+\lambda|x|^{2}\right)^{N+j}} d x=C \lambda^{j-\frac{|\beta|}{2}-\frac{n}{2}} \leqslant C \lambda^{\frac{N}{2}-\frac{n}{2}} .
$$

Indeed $-|\beta| \leqslant|\nu|-2 j-|A|$ so $j-\frac{|\beta|}{2} \leqslant \frac{|\nu|}{2}-\frac{|A|}{2} \leqslant \frac{N}{2}$.

To estimate (1) we use the above estimate of $K_{\varepsilon}(\alpha, z)$ which we integrate with respect to $z$. For the integral in $z$ we use (6.3.36). The integral in $\xi$ is estimated thanks to the term $\frac{1}{\langle\xi\rangle^{N}}$ where $|\nu| \leqslant N$, finally the integral in $y$ is bounded by

$$
\int e^{-\frac{\lambda}{16} \frac{|y-x(\theta, \alpha)|^{2}}{\langle\theta\rangle^{2}}} d y \leqslant C \frac{\langle\theta\rangle^{n}}{\lambda^{n / 2}} \text {. }
$$

Therefore we obtain

$$
(1) \leqslant C \frac{\lambda^{n}}{\lambda^{N}} \lambda^{\frac{N}{2}-\frac{n}{2}} \frac{\langle\theta\rangle^{n}}{\lambda^{n / 2}}=C \frac{\langle\theta\rangle^{n}}{\lambda^{N / 2}} .
$$

To estimate the term (2) we use the change of variables $\widetilde{\alpha}=(x(\theta, \alpha), \xi(\theta, \alpha))$ as in the proof of Lemma 6.3.2 and (6.3.36). This gives, $(2) \leqslant C \frac{\langle\theta\rangle^{n}}{\lambda^{N / 2}}$. Since $|\theta| \leqslant \lambda T$ we obtain

$$
(1)+(2) \leqslant C \lambda^{-\frac{N}{2}+n} \text {. }
$$

We can therefore use the Schur Lemma and (6.3.30) to achieve the proof of Lemma 6.3.3.

Corollary 6.3.4. - With the notations of (6.3.15) and (6.3.11) we have

$$
\begin{gathered}
B_{3}=\sum_{1 \leqslant|\gamma| \leqslant 2} \int e^{i \lambda \varphi(\theta, y, \alpha)} d_{\gamma}(\theta, y, \alpha, \lambda)\left(\partial_{y}^{\gamma} \chi_{4}^{+}\right)(y) \chi_{5}\left(\frac{y-x(\theta, \alpha)}{\langle\theta\rangle}\right) \\
\cdot \psi_{4}\left(\frac{D}{\lambda}\right) U(t, y, \lambda) d y+J_{\lambda}^{+}(t) v(\alpha) \\
\left\|J_{\lambda}^{+}(t) v\right\|_{L^{2}\left(W^{+}\right)} \leqslant C_{N} \lambda^{-N}\|v\|_{L^{2}\left(\mathbb{R}^{n}\right)} .
\end{gathered}
$$

Now we state the following result. 
Lemma 6.3.5. - Let $b=b(\theta, y, \alpha, \lambda)$ be a bounded symbol. Let us set

$$
G(\theta, \alpha)=\int e^{i \lambda \varphi(\theta, y, \alpha)} \chi_{5}\left(\frac{y-x(\theta, \alpha)}{\langle\theta\rangle}\right) b(\theta, y, \alpha, \lambda) v(y) d y .
$$

Then one can find $C>0$ such that for all $|\theta| \leqslant \lambda T$ and $v \in L^{2}\left(\mathbb{R}^{n}\right)$,

$$
\left\|e^{-\frac{\lambda}{2}\left|\alpha_{\xi}\right|^{2}} G(\theta, \cdot)\right\|_{L^{2}\left(W^{+}\right)} \leqslant C \frac{\langle\theta\rangle^{n}}{\lambda^{n / 2}}\|v\|_{L^{2}\left(\mathbb{R}^{n}\right)} .
$$

Proof. - Let us write

$$
G(\theta, \alpha)=\int K(\theta, \alpha, y, \lambda) v(y) d y .
$$

Then using the estimate (6.3.3) we see that

$$
e^{-\frac{\lambda}{2}\left|\alpha_{\xi}\right|^{2}} \mathbf{1}_{W^{+}}(\alpha)|K(\theta, \alpha, y, \lambda)| \leqslant C e^{-\frac{\lambda}{16} \frac{|y-x(\theta, \alpha)|^{2}}{\langle\theta\rangle^{2}}} \mathbf{1}_{\frac{1}{2} \leqslant\left|\alpha_{\xi}\right| \leqslant 2}
$$

From this estimate we can use the Schur Lemma (making the change of variables $\widetilde{\alpha}=(x(\theta, \alpha), \xi(\theta, \alpha)))$ to conclude.

Let now $\psi_{5} \in C_{0}^{\infty}\left(\mathbb{R}^{n}\right)$ be such that $0 \leqslant \psi_{5} \leqslant 1$ and

$$
\left\{\begin{array}{l}
\psi_{5}(\xi)=1 \text { if }\left|\frac{\xi}{|\xi|}+\xi_{0}\right| \leqslant 7 \delta_{2}, a-7 \delta_{2} \leqslant|\xi| \leqslant b+7 \delta_{2} \\
\operatorname{supp} \psi_{5} \subset\left\{\left|\frac{\xi}{|\xi|}+\xi_{0}\right| \leqslant 8 \delta_{2}\right\}, a-8 \delta_{2} \leqslant|\xi| \leqslant b+8 \delta_{2}
\end{array}\right.
$$

The analogue of Lemma 6.2.3 proves that one can find $C>0, \varepsilon_{0}>0$ such that

$$
\left\|\psi_{4}\left(\frac{D}{\lambda}\right) T_{\beta \rightarrow y}^{*}\left[\left(1-\psi_{5}\left(\beta_{\xi}\right)\right) v\right]\right\|_{L^{2}\left(\mathbb{R}_{y}^{n}\right)} \leqslant C e^{-\varepsilon_{0} \lambda}\left\|e^{-\frac{\lambda}{2}\left|\beta_{\xi}\right|^{2}} v\right\|_{L^{2}\left(\mathbb{R}_{\beta}^{n}\right)} .
$$

Corollary 6.3.6. - We have

$$
\begin{aligned}
B_{3}=\sum_{1 \leqslant|\gamma| \leqslant 2} \int e^{i \lambda \varphi(\theta, y, \alpha)} d_{\gamma}(\theta, y, \alpha, \lambda)\left(\partial_{y}^{\gamma} \chi_{4}^{+}\right)(y) & \\
\cdot \chi_{5}\left(\frac{y-x(\theta, \alpha)}{\langle\theta\rangle}\right) T_{\beta \rightarrow y}^{*}\left[\psi_{5}\left(\beta_{\xi}\right) T_{z \rightarrow \beta} U(t, z, \lambda)\right] d y & \\
& +J_{\lambda}^{+}(t) v(\alpha)
\end{aligned}
$$

where $J_{\lambda}^{+}(t)$ satisfies (6.3.41).

Proof. - We use Corollary 6.3.4, (6.3.39) and Lemma 6.3.3 to remove $\psi_{4}\left(\frac{D}{\lambda}\right)$.

Let now $\chi_{6}^{+}=\chi_{6}^{+}\left(\beta_{x}\right) \in C_{0}^{\infty}\left(\mathbb{R}^{n}\right)$ be such that $0 \leqslant \chi_{6}^{+} \leqslant 1$ and

$$
\left\{\begin{array}{l}
\chi_{6}^{+}\left(\beta_{x}\right)=1 \text { if } \frac{7}{2} \delta_{1} \leqslant-\beta_{x} \cdot \xi_{0} \leqslant 6 \delta_{1} \\
\operatorname{supp} \chi_{6}^{+} \subset\left\{\beta_{x}: \frac{17}{5} \delta_{1} \leqslant-\beta_{x} \cdot \xi_{0} \leqslant 7 \delta_{1}\right\} .
\end{array}\right.
$$


Then we can apply Lemma 6.2.6 with $\chi_{a}=\partial_{y}^{\gamma} \chi_{4}^{+},|\gamma| \geqslant 1, \chi_{b}=\chi_{6}^{+}$. Indeed on the support of $\partial_{y}^{\gamma} \chi_{4}^{+}(y)\left(1-\chi_{6}^{+}\left(\beta_{x}\right)\right)$ we have $\frac{15}{4} \delta_{1} \leqslant-y \cdot \xi_{0} \leqslant 5 \delta_{1}$ and $-\beta_{x} \cdot \xi_{0} \leqslant \frac{7}{2} \delta_{1}$ or $-\beta_{x} \cdot \xi_{0} \geqslant 6 \delta_{1}$. In the first case we write

$$
\left|y-\beta_{x}\right| \geqslant \beta_{x} \cdot \xi_{0}-y \cdot \xi_{0} \geqslant \frac{15}{4} \delta_{1}-\frac{7}{2} \delta_{1}=\frac{1}{4} \delta_{1},
$$

and in the second case we have,

$$
\left|y-\beta_{x}\right| \geqslant y \cdot \xi_{0}-\beta_{x} \cdot \xi_{0} \geqslant 6 \delta_{1}-5 \delta_{1}=\delta_{1} .
$$

Therefore we obtain

$$
\left\|\partial_{y}^{\gamma} \chi_{4}^{+}(y) T_{\beta \rightarrow y}^{*} \psi_{5}\left(\beta_{\xi}\right)\left(1-\chi_{6}\left(\beta_{x}\right)\right) W\right\|_{L^{2}} \leqslant C e^{-\varepsilon \lambda}\left\|e^{-\frac{\lambda}{2}\left|\beta_{\xi}\right|^{2}} W\right\|_{L^{2}} .
$$

Using Corollary 6.3.6 we deduce the following Lemma.

LEMMA 6.3.7. - We have

$$
\begin{aligned}
B_{3}=\sum_{1 \leqslant|\gamma| \leqslant 2} \int e^{i \lambda \varphi(\theta, y, \alpha)} d_{\gamma}(\theta, y, \alpha, \lambda)\left(\partial_{y}^{\gamma} \chi_{4}^{+}\right)(y) \\
\cdot \chi_{5}\left(\frac{y-x(\theta, \alpha)}{\langle\theta\rangle}\right) T_{\beta \rightarrow y}^{*}\left[\psi_{5}\left(\beta_{\xi}\right) \chi_{6}^{+}\left(\beta_{x}\right) T_{z \rightarrow \beta} U(t, \cdot, \lambda)\right](y) d y \\
+J_{\lambda}^{+}(t) v(\alpha),
\end{aligned}
$$

where $J_{\lambda}^{+}(t)$ satisfies (6.3.37).

Now on the support of $\psi_{5}\left(\beta_{\xi}\right) \chi_{6}^{+}\left(\beta_{x}\right)$ we have by (6.3.38), (6.3.40),

$$
\begin{aligned}
\left|\beta_{x} \cdot \beta_{\xi}\right| & \leqslant\left(\left|\beta_{x} \cdot \xi_{0}\right|+\left|\beta_{x}\right|\left|\frac{\beta_{\xi}}{\left|\beta_{\xi}\right|}+\xi_{0}\right|\right)\left|\beta_{\xi}\right| \\
& \leqslant\left(16 \delta_{1}+8 \delta_{2}\left|\beta_{x}\right|\right)\left|\beta_{\xi}\right| \leqslant c_{0}\left\langle\beta_{x}\right\rangle\left|\beta_{\xi}\right|
\end{aligned}
$$

if $16 \delta_{1}+8 \delta_{2} \leqslant c_{0}$. Therefore we are in the case (i) of (6.3.17) and since Theorem 6.3.1 is already proved in this case for $t \in[-T, T]$ we can write,

$$
\begin{aligned}
T_{z \rightarrow \beta} U(t, \cdot, \lambda)=\lambda^{3 n / 4} \int & e^{i \lambda \varphi(-\lambda t, z, \beta)} a(-\lambda t, z, \beta, \lambda) \chi_{4}^{+}(z) \\
& \cdot \chi_{5}\left(\frac{z-x(-\lambda t, z)}{\langle\lambda t\rangle}\right)\left(\chi_{2}^{+} v\right)(z) d z+J_{\lambda}^{+}(t) v(\beta),
\end{aligned}
$$

where $J_{\lambda}^{+}(t)$ satisfies

$$
\left\{\begin{array}{l}
\text { for every } N \in \mathbb{N} \text { one can find } C_{N}>0 \text { such that } \\
\left\|e^{-\frac{\lambda}{2}\left|\beta_{\xi}\right|^{2}} \psi_{5}\left(\beta_{\xi}\right) \chi_{6}^{+}\left(\beta_{x}\right) J_{\lambda}^{+}(t) v\right\|_{L^{2}} \leqslant C_{N} \lambda^{-N}\|v\|_{L^{2}\left(\mathbb{R}^{n}\right)} .
\end{array}\right.
$$

From this we can deduce the following result. 
Corollary 6.3.8. - We have

$$
\begin{aligned}
& T_{z \rightarrow \beta} U(t, \cdot, \lambda)=\lambda^{3 n / 4} \int e^{i \varphi(-\lambda t, z, \beta)} a(-\lambda t, z, \beta, \lambda) \\
& \cdot \chi_{5}\left(\frac{z-x(-\lambda t, \beta)}{\langle\lambda t\rangle}\right) \chi_{3}^{+}(z)\left(\chi_{2}^{+} v\right)(z) d z+J_{\lambda}^{+}(t) v(\beta)
\end{aligned}
$$

where $J_{\lambda}^{+}(t)$ satisfies (6.3.43).

Proof. - We have just to show that we can replace $\chi_{4}^{+}$by $\chi_{3}^{+}$in (VI.3.42). But this is obvious since (see $(6.2 .1),(6.3 .1))$ we have $\chi_{4}^{+} \chi_{2}^{+}=\chi_{4}^{+}\left(1-\chi_{3}^{+}\right) \chi_{2}^{+}+\chi_{4}^{+} \chi_{3}^{+} \chi_{2}^{+}$and $\left(1-\chi_{3}^{+}\right) \chi_{2}^{+} \equiv 0, \chi_{4}^{+} \chi_{3}^{+}=\chi_{3}^{+}$.

We are ready now to prove (6.3.26).

Lemma 6.3.9. - For all $N \in \mathbb{N}$ one can find $C_{N}>0$ such that

$$
\left\|e^{-\frac{\lambda}{2}\left|\alpha_{\xi}\right|^{2}} B_{3}(\theta, t, \cdot, \lambda)\right\|_{L^{2}\left(W^{+}\right)} \leqslant C_{N} \lambda^{-N}\|v\|_{L^{2}\left(\mathbb{R}^{n}\right)}
$$

for all $\lambda \geqslant 1, \theta=\lambda(s-t) \in[0, \lambda T]$ and all $v \in L^{2}\left(\mathbb{R}^{n}\right)$.

Proof. - We use first Corollary 6.3.8 and Lemma 6.3.7. On the support of $\psi_{5}\left(\beta_{\xi}\right) \chi_{6}^{+}\left(\beta_{x}\right) \chi_{5}\left(\frac{z-x(-\lambda t, \beta)}{\langle\lambda t\rangle}\right)$ we have by $(6.3 .38),(6.3 .40),(6.3 .2)$, since $x(-\lambda t, \beta)=$ $\beta_{x}-2 \lambda t \beta_{\xi}+\mathcal{O}(\varepsilon\langle t\rangle)$,

$$
\begin{aligned}
z \cdot \xi_{0} & \leqslant(z-x(-\lambda t, \beta)) \cdot \xi_{0}+\left(\beta_{x}-2 \lambda t \beta_{\xi}\right) \cdot \xi_{0}+C \varepsilon\langle\lambda t\rangle \\
& \leqslant \beta_{x} \cdot \xi_{0}-2 \lambda t \beta_{\xi} \cdot\left(\xi_{0}+\frac{\beta_{\xi}}{\left|\beta_{\xi}\right|}\right)+2 \lambda t\left|\beta_{\xi}\right|+C(\varepsilon+\delta)\langle\lambda t\rangle \\
& \leqslant \frac{7}{2} \delta_{1}+2 \lambda t\left|\beta_{\xi}\right|+C\left(\varepsilon+\delta+\delta_{2}\right)\langle\lambda t\rangle .
\end{aligned}
$$

Since $\left|\beta_{\xi}\right| \geqslant a-\delta_{2}$ we obtain

$$
z \cdot \xi_{0} \leqslant-\frac{17}{5} \delta_{1}-2\left(a-\delta_{2}\right) \lambda|t|+C\left(\varepsilon+\delta+\delta_{2}\right)\langle\lambda t\rangle .
$$

Taking $\varepsilon, \delta, \delta_{2}$ small with respect to $\delta_{1}$ and $a$ we obtain $z \cdot \xi_{0} \leqslant-\frac{10}{3} \delta_{1}$. Now on the support of $\chi_{3}^{+}(z)$ we have by $(6.2 .1), z \cdot \xi_{0} \geqslant-3 \delta_{1}$.

It follows from Corollary 6.3 .8 that $T_{z \rightarrow \beta} U(t, \cdot, \lambda)=R^{+} v$ where $R^{+}$satisfies (6.3.37). Now we use Lemma 6.3.5 and we obtain since $|\theta| \leqslant \lambda T$,

$$
\begin{aligned}
\left\|e^{-\frac{\lambda}{2}\left|\alpha_{\xi}\right|^{2}} B_{3}(\theta, \cdot, \lambda)\right\|_{L^{2}\left(W^{+}\right)} & \leqslant C \frac{\langle\theta\rangle^{n / 2}}{\lambda^{n / 2}}\left\|T_{\beta \rightarrow y}\left[\psi_{5}\left(\beta_{\xi}\right) \chi_{6}^{+}\left(\beta_{x}\right) T_{z \rightarrow \beta} U(t, \cdot, \lambda)\right]\right\|_{L^{2}\left(\mathbb{R}^{n}\right)} \\
& \leqslant\left\|e^{-\frac{\lambda}{2}\left|\beta_{\xi}\right|^{2}} \psi_{5}\left(\beta_{\xi}\right) \chi_{6}^{+}\left(\beta_{x}\right) T_{z \rightarrow \beta} U(t, \cdot)\right\|_{L^{2}} .
\end{aligned}
$$

Since $T_{z \rightarrow \beta} U(t, \cdot)=R^{+} v$, where $R^{+}$satisfies (6.3.37), we obtain the conclusion of Lemma 6.3.9.

To complete the proof of Theorem 6.3.1 in the case (6.3.25) we use (6.3.11), (6.3.14), Lemmas 6.3.2, 6.3.9 and the same argument as in the end of the proof of the case (6.3.17) to remove the cut-off functions $\chi_{4}^{+}(y)$ and $\chi_{5}\left(y-\alpha_{x}\right)$. 


\subsection{Conclusion of Chapter 6}

Here we state a result which combines the conclusions of Theorems 6.2.2, 6.3.1 and (6.3.5).

Theorem 6.4.1. - Let $K_{ \pm}$the operators defined in (6.2.8). Then we can write

$$
K_{+}(t) u(x)=I+I I+I I I
$$

where

$$
\begin{aligned}
I=\lambda^{3 n / 2} \chi_{1}^{+}(x) \psi_{2}\left(\frac{D_{x}}{\lambda}\right) \chi_{2}^{+}(x)\left[\iint e^{i \lambda F(-\lambda t, x, y, \alpha)} a(-\lambda t, y, \alpha) \chi_{2}^{+}(y) \chi_{3}^{+}\left(\alpha_{x}\right)\right. \\
\left.\cdot \psi_{3}\left(\alpha_{\xi}\right) \chi_{5}\left(\frac{y-x(-\lambda t, \alpha)}{\langle\lambda t\rangle}\right)\left(\psi_{2}\left(\frac{D}{\lambda}\right) \chi_{1}^{+} u\right)(y) d y d \alpha\right] \\
I I=\chi_{1}^{+}(x) \psi_{2}\left(\frac{D_{x}}{\lambda}\right) \chi_{2}^{+}(x) T_{\alpha \rightarrow x}^{*}\left[\chi_{3}^{+}\left(\alpha_{x}\right)\left(\alpha_{x}\right) \psi_{3}\left(\alpha_{\xi}\right) J_{\lambda}^{+}(t)\left(\chi_{2}^{+} \psi_{2}\left(\frac{D_{x}}{\lambda}\right) \chi_{1}^{+} u\right)\right] \\
I I I=R_{\lambda}^{+}(t) u
\end{aligned}
$$

where

$$
\left\{\begin{array}{l}
F(-\lambda t, x, y, \alpha)=\varphi(-\lambda t, y, \alpha)-\left(x-\alpha_{x}\right) \cdot \alpha_{\xi}+\frac{i}{2}\left|x-\alpha_{x}\right|^{2}+\frac{i}{2}\left|\alpha_{\xi}\right|^{2}, \\
|a(-\lambda t, y, \alpha, \lambda)| \leqslant C\langle\lambda t\rangle^{-n / 2}, \\
\left\|\chi_{3}^{+}\left(\alpha_{x}\right) \psi_{3}\left(\alpha_{\xi}\right) e^{-\frac{\lambda}{2}\left|\alpha_{\xi}\right|^{2}} J_{\lambda}^{+}(t) v\right\|_{L^{2}} \leqslant C \lambda^{-N}\|v\|_{L^{2}}, \quad \forall N \in \mathbb{N}, \\
\left\|R_{\lambda}^{+}(t) u\right\|_{H^{2 N}} \leqslant C_{N}\|u\|_{H^{-2 N}}, \quad \forall N \in \mathbb{N},
\end{array}\right.
$$

and $\chi_{i}^{+}, \psi_{j}$ have been defined in (6.2.1) to (6.2.6), (6.2.9), (6.3.1) and (6.3.2). Moreover the same result holds with the minus sign. 



\section{CHAPTER 7}

\section{THE DISPERSION ESTIMATE AND THE END OF THE PROOF OF THEOREM 1.0.1}

\subsection{The dispersion estimate for the operators $K_{ \pm}(t)$}

Let us recall that $K_{ \pm}(t)$ have been introduced in (6.2.8). The purpose of this paragraph is to prove the following result.

TheOrem 7.1.1. - Let $T>0$. Then there exists a constant $C \geqslant 0$ such that

$$
\left\|K_{ \pm}(t) u\right\|_{L^{\infty}} \leqslant \frac{C}{|t|^{n / 2}}\|u\|_{L^{1}}
$$

for all $0<|t| \leqslant T$ and all $u \in L^{1}\left(\mathbb{R}^{n}\right)$.

Proof. - We shall use Theorem 6.4.1 and its notation and we shall consider only $K_{+}(t)$. Then we can write

$$
\left\|K_{+}(t) u\right\|_{L^{\infty}} \leqslant\|I\|_{L^{\infty}}+\|I I\|_{L^{\infty}}+\|I I I\|_{L^{\infty}} .
$$

Let $N_{0} \in \mathbb{N}$ be such that $2 N_{0}>\frac{n}{2}$. By the Sobolev embedding and (6.4.1) we have

$$
\|I I I\|_{L^{\infty}} \leqslant C\left\|R_{\lambda}^{+}(t) u\right\|_{H^{2 N_{0}}} \leqslant C_{N_{0}}^{\prime}\|u\|_{H^{-2 N_{0}}} \leqslant C^{\prime \prime}\|u\|_{L^{1}} \leqslant \frac{C(T)}{|t|^{n / 2}}\|u\|_{L^{1}} .
$$

Let us consider the term $I I$. We have

$$
\begin{aligned}
\|I I\|_{L^{\infty}} & \leqslant C\left\|\chi_{1}^{+} \psi_{2}\left(\frac{D}{\lambda}\right) \chi_{2}^{+} T^{*}\left(\chi_{3}^{+} \psi_{3} J_{\lambda}^{+}\left(\chi_{2}^{+} \psi_{2}\left(\frac{D}{\lambda}\right) \chi_{1}^{+} u\right)\right)\right\|_{H^{2 N_{0}}} \\
& \leqslant C^{\prime} \lambda^{2 N_{0}}\left\|T^{*}(\cdots)\right\|_{L^{2}\left(\mathbb{R}^{n}\right)} \\
& \leqslant C^{\prime \prime} \lambda^{2 N_{0}}\left\|e^{-\frac{\lambda}{2}\left|\alpha_{\xi}\right|^{2}} \chi_{3}^{+}\left(\alpha_{x}\right) \psi_{3}\left(\alpha_{\xi}\right) J_{\lambda}^{+}(t)\left(\chi_{2}^{+} \psi_{2}\left(\frac{D}{\lambda}\right) \chi_{1}^{+} u\right)\right\|_{L^{2}\left(\mathbb{R}_{\alpha}^{2 n}\right)} \\
& \leqslant C_{N} \lambda^{2 N_{0}-N}\left\|\chi_{2}^{+} \psi_{2}\left(\frac{D}{\lambda}\right) \chi_{1}^{+} u\right\|_{L^{2}\left(\mathbb{R}^{n}\right)} \\
& \leqslant C_{N}^{\prime} \lambda^{2 N_{0}-N}\left\|\psi_{2}\left(\frac{D}{\lambda}\right)(I-\Delta)^{-N_{0}} \chi_{1}^{+} u\right\|_{L^{2}\left(\mathbb{R}^{n}\right)} \\
& \leqslant C_{N}^{\prime \prime} \lambda^{4 N_{0}-N}\|u\|_{H^{-2 N_{0}}}
\end{aligned}
$$


Taking $N=4 N_{0}$ we obtain finally,

$$
\|I I\|_{L^{\infty}} \leqslant C\|u\|_{L^{1}} \leqslant \frac{C(T)}{|t|^{n / 2}}\|u\|_{L^{1}}
$$

So we are left with the estimation of $\|I\|_{L^{\infty}}$. Let us set

$$
\begin{array}{r}
k_{+}(t, x, y, \lambda)=\lambda^{3 n / 2} \int e^{i \lambda F(-\lambda t, x, y, \alpha)} a(-\lambda t, y, \alpha, \lambda) \chi_{2}^{+}(y) \chi_{3}^{+}\left(\alpha_{x}\right) \psi_{3}\left(\alpha_{\xi}\right) \\
\cdot \chi_{5}\left(\frac{y-x(-\lambda t, \alpha)}{\langle\lambda t\rangle}\right) d \alpha
\end{array}
$$

and

$$
\widetilde{K}_{+}(t) v(x)=\int k_{+}(t, x, y, \lambda)\left[\psi_{2}\left(\frac{D}{\lambda}\right) \chi_{1}^{+} v\right](y) d y
$$

Then

$$
I=\chi_{1}^{+} \psi_{2}\left(\frac{D}{\lambda}\right) \chi_{2}^{+} \widetilde{K}_{+}(t) v .
$$

Since the operator $\chi_{1}^{+} \psi_{2}\left(\frac{D}{\lambda}\right) \chi_{2}^{+}$is bounded from $L^{\infty}$ to $L^{\infty}$ with bound independent of $\lambda$ we have,

$$
\|I\|_{L^{\infty}} \leqslant C\left\|\widetilde{K}_{+}(t) v\right\|_{L^{\infty}}
$$

Assume that the kernel $k_{+}$has the following bound,

$$
\left|k_{+}(t, x, y, \lambda)\right| \leqslant \frac{C}{|t|^{n / 2}}
$$

with $C$ independent of $\lambda$. It will follow from (7.1.7), (7.1.5) and (7.1.8) that

$$
\|I\|_{L^{\infty}} \leqslant \frac{C}{|t|^{n / 2}}\left\|\psi_{2}\left(\frac{D}{\lambda}\right) \chi_{1}^{+} v\right\|_{L^{1}} .
$$

Since the operator $\psi_{2}\left(\frac{D}{\lambda}\right) \chi_{1}^{+}$is uniformly bounded on $L^{1}$ we will have

$$
\|I\|_{L^{\infty}} \leqslant \frac{C}{|t|^{n / 2}}\|v\|_{L^{1}} .
$$

Then Theorem 7.1.1 follows from (7.1.1), (7.1.2), (7.1.3) and (7.1.9).

Proof of (7.1.8). - We divide the proof in three cases: $\lambda t \geqslant 1, \lambda t \leqslant-1,|\lambda t| \leqslant 1$.

Let us remark first that in the integral in the right hand side of (6.1.4), on the support of $\chi_{3}^{+}\left(\alpha_{x}\right) \cdot \psi_{3}\left(\alpha_{\xi}\right)$ we have $-\alpha_{x} \cdot \xi_{0} \leqslant 3 \delta_{1}$ and $\left|\frac{\alpha_{\xi}}{\left|\alpha_{\xi}\right|}+\xi_{0}\right| \leqslant 4 \delta_{2}$. Therefore

SO

$$
\alpha_{x} \cdot \frac{\alpha_{\xi}}{\left|\alpha_{\xi}\right|}=\alpha_{x} \cdot\left(\frac{\alpha_{\xi}}{\left|\alpha_{\xi}\right|}+\xi_{0}\right)-\alpha_{x} \cdot \xi_{0} \leqslant\left(4 \delta_{2}+3 \delta_{1}\right)\left\langle\alpha_{x}\right\rangle
$$

$$
\alpha_{x} \cdot \alpha_{\xi} \leqslant c_{0}\left\langle\alpha_{x}\right\rangle\left|\alpha_{\xi}\right|,
$$

if $\delta_{1}$ and $\delta_{2}$ are small compared to $c_{0}$. 
Case A: proof of (7.1.8) when $\lambda t \geqslant 1$. - In this case $\theta=-\lambda t<0$ and it follows from (7.1.10) and Definition 3.2.2 that all the points $\alpha$ in the integral giving $k_{+}$are outgoing for $\theta<0$. It follows from Corollary 3.3.3 that

$$
\frac{\partial x_{j}}{\partial \alpha_{\xi}^{k}}(\theta, \alpha)=2 \theta \delta_{j k}+\mathcal{O}(\varepsilon\langle\theta\rangle), \quad 1 \leqslant j, k \leqslant n .
$$

Now using Theorem 6.4.1 and (7.1.4) we obtain

$$
\left|k^{+}(t, x, y, \lambda)\right| \leqslant c \lambda^{3 n / 2} \int e^{-\frac{\lambda}{16} \frac{|y-x(-\lambda t, \alpha)|^{2}}{\langle\lambda t\rangle^{2}}-\frac{\lambda}{2}\left|x-\alpha_{x}\right|^{2}} \psi_{3}\left(\alpha_{\xi}\right)\langle\lambda t\rangle^{-n / 2} d \alpha .
$$

By (7.1.11) we can make the change of variables

$$
\widetilde{\alpha}_{x}=\alpha_{x}, \quad \widetilde{\alpha}_{\xi}=x(-\lambda t, \alpha)
$$

and $\left|\operatorname{det} \frac{\partial \widetilde{\alpha}}{\partial \alpha}\right| \geqslant(\lambda t)^{n}$ if $\varepsilon$ is small enough (since $\left.\langle\lambda t\rangle \leqslant \sqrt{2}|\lambda t|\right)$. It follows from (7.1.12) that

$$
\left|k^{+}(t, x, y, \lambda)\right| \leqslant \lambda^{3 n / 2}\langle\lambda t\rangle^{-n / 2}|\lambda t|^{-n} \iint e^{-\frac{\lambda}{16} \frac{\left|y-\widetilde{\alpha}_{\xi}\right|^{2}}{\langle\lambda t\rangle^{2}}} e^{-\frac{\lambda}{2}\left|x-\widetilde{\alpha}_{x}\right|^{2}} d \widetilde{\alpha} .
$$

Setting $\widetilde{\alpha}_{\xi}-y=\frac{4\langle\lambda t\rangle}{\sqrt{\lambda}} z_{1}, \widetilde{\alpha}_{x}-x=\frac{\sqrt{2}}{\sqrt{\lambda}} z_{2}, Z=\left(z_{1}, z_{2}\right)$ we obtain

$$
\left|k^{+}(t, x, y, \lambda)\right| \leqslant C \lambda^{3 n / 2}\langle\lambda t\rangle^{-n / 2}(\lambda t)^{-n}\langle\lambda t\rangle^{n} \lambda^{-n} \int_{\mathbb{R}^{2 n}} e^{-|Z|^{2}} d Z
$$

so

$$
\left|k^{+}(t, x, y, \lambda)\right| \leqslant \frac{C}{t^{n / 2}},
$$

since $\langle\lambda t\rangle \leqslant \sqrt{2} \lambda t$. This proves (7.1.8) in this case.

Case B: proof of (7.1.4) when $\lambda t \leqslant-1$. - In the right hand side of (7.1.4) we integrate on the support of $\chi_{3}^{+}\left(\alpha_{x}\right) \cdot \psi_{3}\left(\alpha_{\xi}\right)$ on which we have (7.1.10). We divide this support in two subsets $U_{1}$ and $U_{2}$ where

$$
\begin{aligned}
& U_{1}=\left\{\alpha=\left(\alpha_{x}, \alpha_{\xi}\right) \in \operatorname{supp}\left(\chi_{3}^{+}\left(\alpha_{x}\right) \psi_{3}\left(\alpha_{\xi}\right)\right):-c_{0}\left\langle\alpha_{x}\right\rangle\left|\alpha_{\xi}\right| \leqslant \alpha_{x} \cdot \alpha_{\xi} \leqslant c_{0}\left\langle\alpha_{x}\right\rangle\left|\alpha_{\xi}\right|\right\} \\
& U_{2}=\left\{\alpha=\left(\alpha_{x}, \alpha_{\xi}\right) \in \operatorname{supp}\left(\chi_{3}^{+}\left(\alpha_{x}\right) \psi_{3}\left(\alpha_{\xi}\right)\right): \alpha_{x} \cdot \alpha_{\xi} \leqslant-c_{0}\left\langle\alpha_{x}\right\rangle\left|\alpha_{\xi}\right|\right\}
\end{aligned}
$$

According to Definition 3.2.2 we have $U_{1} \subset \mathcal{S}_{+} \cap \mathcal{S}_{-}$(which means that the points in $U_{1}$ are outgoing both for $\theta \geqslant 0$ and $\left.\theta \leqslant 0\right)$.

According to Corollary 3.3.3 we have (7.1.11) for $\theta \in \mathbb{R}$ so in particular for $\theta=-\lambda t \geqslant 1$. Therefore the same arguments as those used in case 1 work. It follows that the part of the integral giving $k_{+}$which concerns $U_{1}$ is bounded by $C|t|^{-n / 2}$. We consider now the integral on $U_{2}$. Here $\theta=-\lambda t \geqslant 1$ and the points in $U_{2}$ are incoming for $\theta \geqslant 0$. The needed estimate on $k_{+}$will follow from the following result. 
Proposition 7.1.2. - One can find a function $g=g\left(\theta, y, \alpha_{x}\right)$ such that for all $\theta \geqslant 1$, all $\alpha \in U_{2}$ and all $y \in \operatorname{supp}\left(\chi_{2}^{+}(y) \chi_{5}\left(\frac{y-x(\theta, \alpha)}{\langle\theta\rangle}\right)\right)$ we have,

$$
\left|\alpha_{\xi}-g\left(\theta, y, \alpha_{x}\right)\right| \leqslant \frac{C}{\theta}|y-x(\theta, \alpha)| .
$$

For the proof we need the following Lemma.

Lemma 7.1.3. - Let $\alpha \in U_{2}$ and $\theta \geqslant 1$. Then for all $Y \in \mathbb{R}^{n}$ such that,

$$
\left\{\begin{array}{l}
\text { (i) } Y \cdot \alpha_{\xi} \leqslant 20 c_{0}\langle Y\rangle\left|\alpha_{\xi}\right|, \\
\text { (ii) }\left|\frac{Y-\alpha_{x}}{2 \theta}-\alpha_{\xi}\right| \leqslant c_{0},
\end{array}\right.
$$

there exists a unique $\beta_{\xi}\left(\theta, Y, \alpha_{x}\right) \in \mathbb{R}^{n}$ such that

$$
\left\{\begin{array}{l}
\left|\beta_{\xi}\left(\theta, Y, \alpha_{x}\right)-\alpha_{\xi}\right| \leqslant 2 c_{0}, \\
x\left(-\theta, Y, \beta_{\xi}\left(\theta, z, \alpha_{x}\right)\right)=\alpha_{x} .
\end{array}\right.
$$

Proof. - Let $E=\left\{\beta_{\xi} \in \mathbb{R}^{n}:\left|\beta_{\xi}-\alpha_{\xi}\right| \leqslant 2 c_{0}\right\}$. Then we have

$$
Y \cdot \beta_{\xi}=Y \cdot \alpha_{\xi}+Y \cdot\left(\beta_{\xi}-\alpha_{\xi}\right) \leqslant 20 c_{0}\langle Y\rangle\left|\alpha_{\xi}\right|+2 c_{0}\langle Y\rangle<\frac{1}{4}\langle Y\rangle\left|\alpha_{\xi}\right| .
$$

It follows that the point $\left(Y, \beta_{\xi}\right)$ belongs to $\mathcal{S}_{-}$(see Definition 3.2.2). Since $-\theta<0$ it follows from Proposition 3.3.1 that the equation $x\left(-\theta, Y, \beta_{\xi}\right)=\alpha_{x}$ is equivalent to,

$$
Y-2 \theta \xi\left(-\theta, Y, \beta_{\xi}\right)+z\left(-\theta, Y, \beta_{\xi}\right)=\alpha_{x}
$$

that is to the equation, $Y-2 \theta \beta_{\xi}-2 \theta \zeta\left(-\theta, Y, \beta_{\xi}\right)+z\left(-\theta, Y, \beta_{\xi}\right)=\alpha_{x}$. This equation can be written,

$$
\beta_{\xi}=\frac{Y-\alpha_{x}}{2 \theta}-\zeta\left(-\theta, Y, \beta_{\xi}\right)+\frac{1}{2 \theta} z\left(-\theta, Y, \beta_{\xi}\right)=: F\left(\beta_{\xi}\right) .
$$

We show now that we can apply the fixed point theorem to $F$ in the set $E$. First of all if $\beta_{\xi} \in E$ we have,

$$
\left|F\left(\beta_{\xi}\right)-\alpha_{\xi}\right| \leqslant\left|\frac{Y-\alpha_{x}}{2 \theta}-\alpha_{\xi}\right|+\left|\zeta\left(-\theta, Y, \beta_{\xi}\right)\right|+\frac{1}{2 \theta}\left|z\left(-\theta, Y, \beta_{\xi}\right)\right| .
$$

Using our assumption and Proposition 3.3.2 we obtain,

$$
\left|F\left(\beta_{\xi}\right)-\alpha_{\xi}\right| \leqslant c_{0}+2 \varepsilon \leqslant 2 c_{0} \text { if } 2 \varepsilon \leqslant c_{0} .
$$

Now if $\beta_{\xi} \in E, \beta_{\xi}^{\prime} \in E$ we have again by Proposition 3.3.2,

$$
\left|F\left(\beta_{\xi}\right)-F\left(\beta_{\xi}^{\prime}\right)\right| \leqslant C \varepsilon\left|\beta_{\xi}-\beta_{\xi}^{\prime}\right| .
$$

Taking $\varepsilon$ so small that $C \varepsilon<1$ we obtain the conclusion of the Lemma.

Remark 7.1.4. - Let $(\theta, \alpha, y)$ as in Proposition 7.1.2. Then they satisfy the conditions of Lemma 7.1.3. Indeed we have $y \in \operatorname{supp} \chi_{4}^{+}$that is $-y \cdot \xi_{0} \leqslant 5 \delta_{1}$ and $\left|\frac{\alpha_{\xi}}{\left|\alpha_{\xi}\right|}+\xi_{0}\right| \leqslant 4 \delta_{2}$. It follows that

$$
y \cdot \frac{\alpha_{\xi}}{\left|\alpha_{\xi}\right|} \leqslant y \cdot\left(\frac{\alpha_{\xi}}{\left|\alpha_{\xi}\right|}+\xi_{0}\right)-y \cdot \xi_{0} \leqslant 4 \delta_{2}|y|+5 \delta_{1}<10 c_{0}\langle y\rangle
$$

since $\delta_{1}$ and $\delta_{2}$ are small compared to $c_{0}$.

MÉMOIRES DE LA SMF 101/102 
Moreover we have $|y-x(\theta, \alpha)| \leqslant \delta\langle\theta\rangle \leqslant \sqrt{2} \delta \theta$. By Proposition 3.4.1 we have $x(\theta, \alpha)=\alpha_{x}+2 \theta \alpha_{\xi}+\mathcal{O}(\varepsilon\langle\theta\rangle)$ so

$$
\left|y-\alpha_{x}-2 \theta \alpha_{\xi}+\mathcal{O}(\varepsilon\langle\theta\rangle)\right| \leqslant \sqrt{2} \delta \theta .
$$

It follows that

$$
\left|\frac{y-\alpha_{x}}{2 \theta}-\alpha_{\xi}\right| \leqslant C(\delta+\varepsilon) \leqslant c_{0}
$$

if $\varepsilon+\delta$ is small enough.

Now for fixed $\theta \geqslant 1$ and $\alpha \in U_{2}$ let us set

$$
A=\left\{Y \in \mathbb{R}^{n}: Y \cdot \alpha_{\xi}<20 c_{0}\langle Y\rangle\left|\alpha_{\xi}\right|, \quad\left|\frac{Y-\alpha_{x}}{2 \theta}-\alpha_{\xi}\right|<c_{0}\right\},
$$

and for $Y \in A$ let us set

$$
H(Y)=\xi\left(-\theta, Y, \beta_{\xi}\left(\theta, Y, \alpha_{x}\right)\right) .
$$

Lemma 7.1.5. - There exists a constant $C>0$ independent of $\theta, \alpha$ such that

$$
\left\|\frac{\partial H}{\partial Y}(Y)\right\| \leqslant \frac{C}{\theta}
$$

for every $Y$ in $A$.

Proof. - By Lemma 7.1.3 we have for $j=1, \ldots, n$,

$$
x_{j}\left(-\theta, Y, \beta_{\xi}\left(\theta, Y, \alpha_{x}\right)\right)=\alpha_{x}^{j} .
$$

Let us differentiate this equality with respect to $Y_{k}$. We obtain

$$
\frac{\partial x_{j}}{\partial x_{k}}\left(-\theta, Y, \beta_{\xi}\left(\theta, Y, \alpha_{x}\right)\right)+\sum_{\ell} \frac{\partial x_{j}}{\partial \xi_{\ell}}\left(-\theta, Y, \beta_{\xi}\left(\theta, Y, \alpha_{x}\right)\right) \frac{\partial \beta_{\xi}^{\ell}}{\partial Y_{k}}\left(\theta, Y, \alpha_{x}\right)=0 .
$$

Since the point $\left(Y, \beta_{\xi}\right)$ belongs to $\mathcal{S}_{-}$we have by Corollary 3.3 .3

$$
\frac{\partial x_{j}}{\partial x_{k}}\left(-\theta, Y, \beta_{\xi}(\cdots)\right)=\delta_{j k}+\mathcal{O}(\varepsilon \theta), \quad \frac{\partial x_{j}}{\partial \xi_{\ell}}\left(-\theta, Y, \beta_{\xi}(\cdots)\right)=-2 \theta \delta_{j k}+\mathcal{O}(\varepsilon \theta) .
$$

It follows that

$$
\left\|\frac{\partial \beta_{\xi}}{\partial Y}\left(\theta, Y, \alpha_{x}\right)\right\| \leqslant C\left(\frac{1}{\theta}+\varepsilon\right) .
$$

Now thanks again to the fact that $\left(Y, \beta_{\xi}\right) \in \mathcal{O}_{-}$we deduce from Proposition 3.3.1 that

$$
\alpha_{x}^{j}=x_{j}\left(-\theta, Y, \beta_{\xi}\left(\theta, Y, \alpha_{x}\right)\right)=Y_{j}-2 \theta H_{j}(Y)+z_{j}\left(-\theta, Y, \beta_{\xi}(\cdots)\right) .
$$

Differentiating with respect to $Y_{k}$ yields

$$
\delta_{j k}-2 \theta \frac{\partial H_{j}}{\partial Y_{k}}(Y)+\frac{\partial z_{j}}{\partial x_{k}}\left(-\theta, Y, \beta_{\xi}(\cdots)\right)+\sum_{\ell=1}^{n} \frac{\partial z_{j}}{\partial \xi_{\ell}}\left(-\theta, Y, \beta_{\xi}(\cdots)\right) \frac{\partial \beta_{\xi}}{\partial Y_{k}}\left(\theta, Y, \alpha_{x}\right)=0 .
$$

Using Proposition 3.3.2 and (7.1.15) we obtain the claim of the Lemma. 
Proof of Proposition 7.1.2. - We shall prove that the function $g$ defined by,

$$
g\left(\theta, y, \alpha_{x}\right)=H(y)=\xi\left(-\theta, y, \beta_{\xi}\left(\theta, y, \alpha_{x}\right)\right),
$$

satisfies the claim of the Proposition.

To do so we must consider several cases.

Case 1. - Here we assume that

$$
\left\{\begin{array}{l}
x(\theta, \alpha) \cdot \alpha_{\xi} \leqslant 10 c_{0}\langle x(\theta, \alpha)\rangle\left|\alpha_{\xi}\right|, \\
|y-x(\theta, \alpha)|<|x(\theta, \alpha)| .
\end{array}\right.
$$

Let us show that this implies that

$$
t y+(1-t) x(\theta, \alpha) \in A \text { for all } t \in[0,1]
$$

where $A$ has been defined in (7.1.13).

First of all using the Remark 7.1.4 and (7.1.17) we can write,

$$
\begin{aligned}
(t y+(1-t) x(\theta, \alpha)) \cdot \alpha_{\xi} & <10 c_{0}(t\langle y\rangle+(1-t)\langle x(\theta, \alpha)\rangle)\left|\alpha_{\xi}\right| \\
& \leqslant 10 c_{0}[t(1+|y|)+(1-t)(1+|x(\theta, \alpha)|)]\left|\alpha_{\xi}\right| .
\end{aligned}
$$

Now we use Lemma 4.4.16 and we obtain

$$
\begin{aligned}
(t y+(1-t) x(\theta, \alpha)) \cdot \alpha_{\xi} & <10 c_{0}(1+\sqrt{2} \cdot|t y+(1-t) x(\theta, \alpha)|)\left|\alpha_{\xi}\right| \\
& \leqslant 20 c_{0}\langle t y+(1-t) x(\theta, \alpha)\rangle\left|\alpha_{\xi}\right| .
\end{aligned}
$$

On the other hand we have

$$
\begin{aligned}
\left|t y+(1-t) x(\theta, \alpha)-\alpha_{x}-2 \theta \alpha_{\xi}\right| & \leqslant t|y-x(\theta, \alpha)|+\left|x(\theta, \alpha)-\alpha_{x}-20 \alpha_{\xi}\right| \\
& \leqslant 2 t \delta \theta+C \varepsilon \theta<2 c_{0} \theta,
\end{aligned}
$$

since $y \in \operatorname{supp} \chi_{5}\left(\frac{y-x(\theta, \alpha)}{\langle\theta\rangle}\right)$ and $\varepsilon, \delta$ are small compared to $c_{0}$.

In particular (7.1.18) for $t=0$ and $t=1$ shows that we can apply Lemma 7.1.3 to $Y=x(\theta, \alpha), Y=y$. Therefore we have

$$
x\left(-\theta, x(\theta, \alpha), \beta_{\xi}\left(\theta, x(\theta, \alpha), \alpha_{x}\right)\right)=\alpha_{x} .
$$

But we have also

$$
x(-\theta, x(\theta, \alpha), \xi(\theta, \alpha))=\alpha_{x}
$$

and since $\left|\xi(\theta, \alpha)-\alpha_{\xi}\right| \leqslant C \varepsilon \leqslant 2 c_{0}$ it follows from the uniqueness of $\beta_{\xi}$ in the set $E$ (see the proof of Lemma 7.1.3) that $\beta_{\xi}\left(\theta, x(\theta, \alpha), \alpha_{x}\right)=\xi(\theta, \alpha)$. Therefore we have by (7.1.16),

$$
\alpha_{\xi}=\xi\left(-\theta, x(\theta, \alpha), \beta_{\xi}\left(\theta, x(\theta, \alpha), \alpha_{x}\right)\right)=H(x(\theta, \alpha)) .
$$

Finally we write

$$
\begin{aligned}
\left|\alpha_{\xi}^{j}-g_{j}\left(\theta, y, \alpha_{x}\right)\right| & =\left|H_{j}(x(\theta, \alpha))-H_{j}(y)\right| \\
& \leqslant|y-x(\theta, \alpha)| \int_{0}^{1} \sum_{k=1}^{n}\left|\frac{\partial H_{j}}{\partial Y_{k}}(t y+(1-t) x(\theta, \alpha))\right| d t
\end{aligned}
$$


so using (7.1.18) and Lemma 7.1.5 we obtain

$$
\left|\alpha_{\xi}-g\left(\theta, y, \alpha_{x}\right)\right| \leqslant \frac{C}{\theta}|y-x(\theta, \alpha)| .
$$

Case 2. - Here we assume that

$$
\left\{\begin{array}{l}
x(\theta, \alpha) \cdot \alpha_{\xi} \leqslant 10 c_{0}\langle x(\theta, \alpha)\rangle\left|\alpha_{\xi}\right| \\
|y-x(\theta, \alpha)|>|x(\theta, \alpha)|
\end{array}\right.
$$

It follows that

$$
|y| \leqslant 2|y-x(\theta, \alpha)|
$$

We claim that in this case we have

$$
\text { ty } \in A, \quad t x(\theta, \alpha) \in A \text { for } t \in[0,1] .
$$

Indeed we have by Remark 7.1.4

$$
\begin{aligned}
t y \cdot \alpha_{\xi} & \leqslant t \cdot 10 c_{0}\langle y\rangle\left|\alpha_{\xi}\right| \leqslant 10 c_{0}\langle t y\rangle\left|\alpha_{\xi}\right| \\
\left|t y-\alpha_{x}-2 \theta \alpha_{\xi}\right| & \leqslant t|y|+|x(\theta, \alpha)|+\left|x(\theta, \alpha)-\alpha_{x}-2 \theta \alpha_{\xi}\right| \\
& \leqslant 3|y-x(\theta, \alpha)|+C \varepsilon\langle\theta\rangle \leqslant 2(C \varepsilon+3 \delta) \theta \leqslant 2 c_{0} \theta . \\
t x(\theta, \alpha) \cdot \alpha_{\xi} & \leqslant t \cdot 10 c_{0}\langle x(\theta, \alpha)\rangle\left|\alpha_{\xi}\right| \leqslant 10 c_{0}\langle t x(\theta, \alpha)\rangle\left|\alpha_{\xi}\right| \\
\left|t x(\theta, \alpha)-\alpha_{x}-2 \theta \alpha_{\xi}\right| & \leqslant(1-t)|x(\theta, \alpha)|+\left|x(\theta, \alpha)-\alpha_{x}-2 \theta \alpha_{\xi}\right| \leqslant 2 c_{0} \theta .
\end{aligned}
$$

As before we have

$$
(1)=\left|\alpha_{\xi}-g\left(\theta, y, \alpha_{x}\right)\right|=|H(x(\theta, \alpha))-H(y)|
$$

and we write

$$
\begin{aligned}
(1) & \leqslant|H(x(\theta, \alpha))-H(0)|+|H(0)-H(y)| \\
& \leqslant|x(\theta, \alpha)| \int_{0}^{1}\left\|\frac{\partial H}{\partial Y}(t x(\theta, \alpha))\right\| d t+|y| \int_{0}^{1}\left\|\frac{\partial H}{\partial Y}(t, y)\right\| d t .
\end{aligned}
$$

Then we use (7.1.21), Lemma 7.1.5 and (7.1.19), (7.1.20) to conclude that

$$
(1) \leqslant \frac{C}{\theta}|y-x(\theta, \alpha)| \text {. }
$$

The last case is the following.

Case 3. - We assume that

$$
x(\theta, \alpha) \cdot \alpha_{\xi}>10 c_{0}\langle x(\theta, \alpha)\rangle\left|\alpha_{\xi}\right| .
$$

Recall that $\alpha \in U_{2}$ that is in particular $\alpha_{x} \cdot \alpha_{\xi} \leqslant-c_{0}\left\langle\alpha_{x}\right\rangle\left|\alpha_{\xi}\right|$. By (4.4.49) there exists $\left.\theta^{*} \in\right] 0, \theta\left[\right.$ depending only on $\alpha$ such that $x\left(\theta^{*}, \alpha\right) \cdot \alpha_{\xi}=0$. Then by Lemma 4.4.17 we have

$$
\left\{\begin{array}{l}
\frac{3}{2}\left|\theta-\theta^{*}\right|\left|\alpha_{\xi}\right| \leqslant\left|x(\theta, \alpha)-x\left(\theta^{*}, \alpha\right)\right| \leqslant 3\left|\theta-\theta^{*}\right|\left|\alpha_{\xi}\right| \\
\left|\theta-\theta^{*}\right| \geqslant \frac{c_{0}}{40} \\
|y-x(\theta, \alpha)| \leqslant\left|y-x\left(\theta^{*}, \alpha\right)\right|+\left|x\left(\theta^{*}, \alpha\right)-x(\theta, \alpha)\right| \leqslant 5|y-x(\theta, \alpha)| .
\end{array}\right.
$$


Here again we consider two subcases.

Case 3.1: $x(\theta, \alpha) \cdot \alpha_{\xi}>10 c_{0}\langle x(\theta, \alpha)\rangle\left|\alpha_{\xi}\right|,\left|y-x\left(\theta^{*}, \alpha\right)\right|<\left|x\left(\theta^{*}, \alpha\right)\right|$. - In this case, (7.1.23) ensures that

$$
t y+(1-t) x\left(\theta^{*}, \alpha\right) \in A .
$$

This follows from Lemma 4.4.16, since $t|y| \leqslant \sqrt{2}\left|t y+(1-t) x\left(\theta^{*}, \alpha\right)\right|$ and from the following estimates,

$$
\begin{array}{r}
\left|t y+(1-t) x\left(\theta^{*}, \alpha\right)-\alpha_{x}-2 \theta \alpha_{\xi}\right| \leqslant t\left|y-x\left(\theta^{*}, \alpha\right)\right|+\left|x\left(\theta^{*}, \alpha\right)-\alpha_{x}-2 \theta \alpha_{\xi}\right| \\
\leqslant 5 t|y-x(\theta, \alpha)|+2\left|\theta-\theta^{*}\right|\left|\alpha_{\xi}\right|+\mathcal{O}\left(\varepsilon \theta^{*}\right) \leqslant C(\delta+\varepsilon) \theta \leqslant 2 c_{0} \theta .
\end{array}
$$

Then we write

$$
\left|\alpha_{\xi}-g\left(y, \theta, \alpha_{x}\right)\right|=\left|H(y)-\alpha_{\xi}\right| \leqslant \underbrace{\left|H(y)-H\left(x\left(\theta^{*}, \alpha\right)\right)\right|}_{(1)}+\underbrace{\left|H\left(x\left(\theta^{*}, \alpha\right)\right)-\alpha_{\xi}\right|}_{(2)} .
$$

By (7.1.24), Lemma 7.1.5 and (7.1.23) we have

$$
(1) \leqslant \frac{C}{\theta}|y-x(\theta, \alpha)| \text {. }
$$

Now we have

$$
x\left(-\theta, x\left(\theta^{*}, \alpha\right), \frac{\theta^{*}}{\theta} \xi\left(\theta^{*}, \alpha\right)\right)=x\left(-\theta^{*}, x\left(\theta^{*}, \alpha\right), \xi\left(\theta^{*}, \alpha\right)\right)=\alpha_{x} .
$$

Therefore

$$
\beta_{\xi}\left(\theta, x\left(\theta^{*}, \alpha\right), \alpha_{x}\right)=\frac{\theta^{*}}{\theta} \xi\left(\theta^{*}, \alpha\right)
$$

It follows that

$$
H\left(x\left(\theta^{*}, \alpha\right)\right)=\xi\left(-\theta, x\left(\theta^{*}, \alpha\right), \frac{\theta^{*}}{\theta} \xi\left(\theta^{*}, \alpha\right)\right)=\frac{\theta^{*}}{\theta} \xi\left(-\theta^{*}, x\left(\theta^{*}, \alpha\right), \xi\left(\theta^{*}, \alpha\right)\right)=\frac{\theta^{*}}{\theta} \alpha_{\xi} .
$$

Therefore

$$
(2)=\left|\alpha_{\xi}\right| \frac{\left|\theta-\theta^{*}\right|}{\theta} \leqslant \frac{C}{\theta}|y-x(\theta, \alpha)|
$$

by (7.1.23). The estimates obtained on (1) and (2) show that

$$
\left|\alpha_{\xi}-g\left(\theta, y, \alpha_{x}\right)\right| \leqslant \frac{C}{\theta}|y-x(\theta, \alpha)|
$$

which is the claim of Proposition 7.1.2.

The last step concerns the following case. 
Case 3.2: $x(\theta, \alpha) \cdot \alpha_{\xi}>10 c_{0}\langle x(\theta, \alpha)\rangle\left|\alpha_{\xi}\right|,\left|x\left(\theta^{*}, \alpha\right)\right| \leqslant\left|y-x\left(\theta^{*}, \alpha\right)\right|$. - It follows then from (7.1.23) that we have

$$
|y|+\left|x\left(\theta^{*}, \alpha\right)\right| \leqslant C|y-x(\theta, \alpha)| .
$$

Moreover we have

$$
t y \in A, \quad t x\left(\theta^{*}, \alpha\right) \in A \text { for } t \in[0,1]
$$

Indeed we can write,

$$
\begin{aligned}
t y \cdot \alpha_{\xi} & \leqslant t \cdot 10 c_{0}\langle y\rangle\left|\alpha_{\xi}\right| \leqslant 10 c_{0}\langle t y\rangle\left|\alpha_{\xi}\right| \\
\left|t y-\alpha_{x}-2 \theta \alpha_{\xi}\right| & \leqslant t|y|+\left|x\left(\theta^{*}, \alpha\right)\right|+\left|x\left(\theta^{*}, \alpha\right)-\alpha_{x}-2 \theta \alpha_{\xi}\right| \\
& \leqslant C\left(|y-x(\theta, \alpha)|+\left|\theta-\theta^{*}\right|+C \varepsilon \theta^{*}\right) \leqslant 2 c_{0} \theta \\
t x\left(\theta^{*}, \alpha\right) \cdot \alpha_{\xi} & =0 \\
\left|t x\left(\theta^{*}, \alpha\right)-\alpha_{x}-2 \theta \alpha_{\xi}\right| & \leqslant(1-t)\left|x\left(\theta^{*}, \alpha\right)\right|+\left|x\left(\theta^{*}, \alpha\right)-\alpha_{x}-2 \theta \alpha_{\xi}\right| \\
& \leqslant C(1-t)|y-x(\theta, \alpha)|+C\left|\theta-\theta^{*}\right|+C \varepsilon \theta^{*} \\
& \leqslant 2 c_{0} \theta
\end{aligned}
$$

by (7.1.23). Then we can write

$$
\begin{aligned}
\left|\alpha_{\xi}-g\left(\theta, y, \alpha_{x}\right)\right| & \leqslant \mid H(y)-H\left(x ( \theta ^ { * } , \alpha ) | + | H \left(x\left(\theta^{*}, \alpha\right)-\alpha_{\xi} \mid\right.\right. \\
& \leqslant|H(y)-H(0)|+\left|H(0)-H\left(x\left(\theta^{*}, \alpha\right)\right)\right|+\mid H\left(x\left(\theta^{*}, \alpha\right)-\alpha_{\xi} \mid .\right.
\end{aligned}
$$

Using (7.1.28), Lemma 7.1.5, (7.1.26) we obtain

$$
\left|\alpha_{\xi}-g\left(\theta, y, \alpha_{x}\right)\right| \leqslant \frac{C}{\theta}|y-x(\theta, \alpha)| .
$$

This completes the proof of Proposition 7.1.2.

End of the proof of (7.1.8) in case $B,(\lambda t \leqslant-1)$. - For the part of the integral in (7.1.4) (giving $k_{+}$) where $\alpha \in U_{2}$ we use Proposition 7.1.2. Let us call it (1). As in (7.1.12) we have

$$
\begin{aligned}
& |(1)| \leqslant C \lambda^{3 n / 2} \int e^{-\frac{1}{16} \frac{|y-x(-\lambda t, \alpha)|^{2}}{\langle\lambda t\rangle^{2}}-\frac{\lambda}{2}\left|x-\alpha_{x}\right|^{2}}\langle\lambda t\rangle^{-n / 2} d \alpha \\
& |(1)| \leqslant C \lambda^{3 n / 2}\langle\lambda t\rangle^{-n / 2} \iint e^{-\varepsilon_{0} \frac{|\lambda t|^{2} \lambda}{\langle\lambda t\rangle^{2}}\left|\alpha_{\xi}-g\left(-\lambda t, y, \alpha_{x}\right)\right|^{2}} e^{-\frac{\lambda}{2}\left|x-\alpha_{x}\right|^{2}} d \alpha_{x} d \alpha_{\xi} \\
& |(1)| \leqslant C^{\prime} \frac{\lambda^{3 n / 2}\langle\lambda t\rangle^{-n / 2}\langle\lambda t\rangle^{n}}{\lambda^{n}|\lambda t|^{n}} \leqslant \frac{C^{\prime \prime}}{|t|^{n / 2}} .
\end{aligned}
$$

Case C: proof of (7.1.8) when $|\lambda t| \leqslant 1$. - In this case the proof made above does not give the needed result since $\langle\lambda t\rangle \approx 1$. We will use instead a stationary phase method. Let us set $\theta=-\lambda t$ and let us recall (see (7.1.4)) that we have to bound the following 
kernel

$$
\begin{array}{r}
k_{+}(t, x, y, \lambda)=\lambda^{3 n / 2} \int e^{i \lambda F(\theta, x, y, \alpha)} a(\theta, y, \alpha) \chi_{2}^{+}(y) \chi_{3}^{+}\left(\alpha_{x}\right) \psi_{3}\left(\alpha_{\xi}\right) \\
\chi_{5}\left(\frac{y-x(\theta, \alpha)}{\langle\theta\rangle}\right) d \alpha .
\end{array}
$$

Let us recall also that, according to the Theorems 6.4.1 and 4.1.2 we have,

$$
\operatorname{Im} F(\theta, x, y, \alpha) \geqslant \frac{1}{2}\left|x-\alpha_{x}\right|^{2} .
$$

Let $\chi_{6} \in C_{0}^{\infty} \mathbb{R}^{n}$ ) be such that,

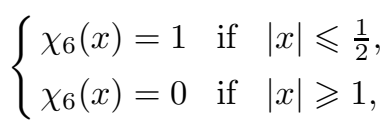

We write in the integral in the right hand side of (7.1.29) $1=\chi_{6}\left(x-\alpha_{x}\right)+1-\chi_{6}\left(x-\alpha_{x}\right)$.

The part of the integral containing $1-\chi_{6}\left(x-\alpha_{x}\right)$ can be bounded, using (7.1.30), by the quantity

$$
C \lambda^{3 n / 2} e^{-\frac{\lambda}{16}} \iint e^{-\frac{\lambda}{4}\left|x-\alpha_{x}\right|^{2}}\left|\psi_{3}\left(\alpha_{\xi}\right)\right| d \alpha
$$

which is $0(1)$ uniformly in $(t, x, y, \lambda)$. Setting $a_{1}(\theta, y, \alpha)=a(\theta, y, \alpha) \chi_{2}^{+}(y) \chi_{3}^{+}\left(\alpha_{x}\right)$ we see therefore that we are left with the bound of the following kernel.

$$
\begin{aligned}
& \widetilde{k}_{+}(t, x, y, \lambda) \\
& =\lambda^{3 n / 2} \int e^{i \lambda F(\theta, x, y, \alpha)} a_{1}(\theta, y, \alpha) \chi_{6}\left(x-\alpha_{x}\right) \psi_{3}\left(\alpha_{\xi}\right) \chi_{5}\left(\frac{y-x(\theta, \alpha)}{\langle\theta\rangle}\right) d \alpha .
\end{aligned}
$$

Now, according to Theorem 6.4.1 we have,

$$
F(\theta, x, y, \alpha)=\varphi(\theta, y, \alpha)-\left(x-\alpha_{x}\right) \cdot \alpha_{\xi}+\frac{i}{2}\left|x-\alpha_{x}\right|^{2}-\frac{1}{2 i}\left|\alpha_{\xi}\right|^{2} .
$$

Using Theorem 4.5.1 we obtain

$$
\text { (7.1.33) } \quad F(\theta, x, y, \alpha)
$$

$$
\begin{array}{r}
=\frac{(y-x) \cdot \alpha_{\xi}-2 i \theta\left(x-\alpha_{x}\right) \cdot \alpha_{\xi}-\theta\left|\alpha_{\xi}\right|^{2}-\theta\left|x-\alpha_{x}\right|^{2}+\frac{i}{2}\left|x-\alpha_{x}\right|^{2}+\frac{i}{2}\left|y-\alpha_{x}\right|^{2}}{1+2 i \theta}+R(\theta, y, \alpha) \\
+R(\theta)
\end{array}
$$

where

$$
\left\{\begin{aligned}
\left|\frac{\partial R}{\partial \alpha_{x}}\right|+\left|\frac{\partial^{2} R}{\partial \alpha_{x}^{2}}\right| & \leqslant C(\varepsilon+\delta)\left(\left|y-\alpha_{x}\right|+|\theta|\right), \\
\left|\frac{\partial R}{\partial \alpha_{\xi}}\right|+\left|\frac{\partial^{2} R}{\partial \alpha_{\xi}^{2}}\right|+\left|\frac{\partial^{2} R}{\partial \alpha_{x} \partial \alpha_{\xi}}\right| & \leqslant C(\varepsilon+\delta)|\theta|, \\
\left|\partial_{\alpha_{x}}^{A_{1}} \partial_{\alpha_{\xi}}^{A_{2}} R\right| & \leqslant\left\{\begin{array}{lll}
C_{A_{1}} & \text { if } A_{2}=0, \\
C_{A_{1}, A_{2}}|\theta| & \text { if }\left|A_{2}\right| \geqslant 1 .
\end{array}\right.
\end{aligned}\right.
$$


It follows that we have,

$$
\begin{cases}\frac{\partial F}{\partial \alpha_{\xi}} & =\frac{1}{1+2 i \theta}\left[(y-x)-2 i \theta\left(x-\alpha_{x}\right)-2 \theta \alpha_{\xi}\right]+\frac{\partial R}{\partial \alpha_{\xi}} \\ \frac{\partial F}{\partial \alpha_{x}} & =\frac{1}{1+2 i \theta}\left[2 i \theta \alpha_{\xi}+2 \theta\left(x-\alpha_{x}\right)-2 i\left(x-\alpha_{x}\right)-i(y-x)\right]+\frac{\partial R}{\partial \alpha_{x}} \\ \frac{\partial^{2} F}{\partial \alpha_{\xi}^{j} \partial \alpha_{\xi}^{k}} & =\frac{-2 \theta \delta_{j k}}{1+2 i \theta}+\frac{\partial^{2} R}{\partial \alpha_{\xi}^{j} \partial \alpha_{\xi}^{k}} \\ \frac{\partial^{2} F}{\partial \alpha_{\xi}^{j} \partial \alpha_{x}^{k}} & =\frac{2 i \theta \delta_{j k}}{1+2 i \theta}+\frac{\partial^{2} R}{\partial \alpha_{\xi}^{j} \partial \alpha_{x}^{k}} \\ \frac{\partial^{2} F}{\partial \alpha_{x}^{j} \partial \alpha_{x}^{k}} & =\frac{(2 i-2 \theta) \delta_{j k}}{1+2 i \theta}+\frac{\partial^{2} R}{\partial \alpha_{x}^{j} \partial \alpha_{x}^{k}} \\ \frac{\partial^{|A|} F}{\partial \alpha^{A}} & =\frac{\partial^{|A|} R}{\partial \alpha^{A}} \text { if }|A| \geqslant 3 .\end{cases}
$$

Let us recall that (6.2.9) shows that

$$
\operatorname{supp} \psi_{3}\left(\alpha_{\xi}\right) \subset\left\{\alpha_{\xi}: a-4 \delta_{2} \leqslant\left|\alpha_{\xi}\right| \leqslant b+4 \delta_{2}\right\} .
$$

We shall divide the proof into three cases

$$
\begin{cases}\text { case } 1: & \frac{|x-y|}{2|\theta|} \leqslant a-5 \delta_{2} \\ \text { case } 2: & \frac{|x-y|}{2|\theta|} \geqslant b+5 \delta_{2} \\ \text { case 3: } & a-5 \delta_{2} \leqslant \frac{|x-y|}{2|\theta|} \leqslant b+5 \delta_{2}\end{cases}
$$

Case 1. - We have the following result.

Lemma 7.1.6. - When $x-\alpha_{x} \in \operatorname{supp} \chi_{6}, y-x(\theta, \alpha) \in \operatorname{supp} \chi_{5}, \alpha_{\xi} \in \operatorname{supp} \psi_{3}$ we have

$$
\begin{aligned}
Q & =:\left|\frac{\partial F}{\partial \alpha_{x}}\right|^{2}+\frac{1}{|\theta|}\left|\frac{\partial F}{\partial \alpha_{\xi}}\right|^{2} \geqslant C\left(\left|x-\alpha_{x}\right|^{2}+|\theta|\right), \\
\left|\partial_{\alpha_{x}}^{A_{1}} \partial_{\alpha_{\xi}}^{A_{2}} F\right| & \leqslant \begin{cases}C_{A_{1}} & \text { if }\left|A_{1}\right| \geqslant 1, A_{2}=0, \\
C_{A_{1}, A_{2}}|\theta| & \text { if }\left|A_{2}\right| \geqslant 1 .\end{cases}
\end{aligned}
$$

uniformly in $(\theta, x, y, \alpha)$.

Proof. - Let us set $X=x-\alpha_{x}, Y=\alpha_{\xi}-\frac{y-x}{2 \theta}$. Using (7.1.35) we see that

$$
\frac{\partial F}{\partial \alpha_{\xi}}=\frac{-2 \theta}{1+2 i \theta}(Y+i X)+\frac{\partial R}{\partial \alpha_{\xi}} \text {. }
$$


It follows from (7.1.34) that

$$
\frac{2|\theta|}{|1+2 i \theta|}|Y+i X| \leqslant\left|\frac{\partial F}{\partial \alpha_{\xi}}\right|+C(\varepsilon+\delta)|\theta| .
$$

Therefore

$$
\frac{4|\theta|}{1+4 \theta^{2}}\left(|X|^{2}+|Y|^{2}\right) \leqslant \frac{2}{|\theta|}\left|\frac{\partial F}{\partial \alpha_{\xi}}\right|^{2}+C_{1}(\varepsilon+\delta)^{2}|\theta| .
$$

By the same way we have

$$
\frac{\partial F}{\partial \alpha_{x}}=\frac{1}{1+2 i \theta}(2 i \theta Y-2 i X+2 \theta X)+\frac{\partial R}{\partial \alpha_{x}} .
$$

Since $\left|y-\alpha_{x}\right| \leqslant|y-x|+\left|x-\alpha_{x}\right| \leqslant\left|x-\alpha_{x}\right|+C|\theta|$, we obtain

$$
\frac{4}{1+4 \theta^{2}}\left(|\theta Y-X|^{2}+\theta^{2}|X|^{2}\right) \leqslant 2\left|\frac{\partial F}{\partial \alpha_{x}}\right|^{2}+C_{2}(\varepsilon+\delta)^{2}\left(|\theta|+|X|^{2}\right) .
$$

It follows that

$$
\begin{aligned}
2\left(\left|\frac{\partial F}{\partial \alpha_{x}}\right|^{2}+\frac{1}{|\theta|}\left(\left|\frac{\partial F}{\partial \alpha_{\xi}}\right|^{2}\right)+C_{3}(\varepsilon+\delta)^{2}(|\theta|\right. & \left.+|X|^{2}\right) \\
& \geqslant \frac{4}{1+4 \theta^{2}}\left(|\theta Y-X|^{2}+|\theta||Y|^{2}\right) .
\end{aligned}
$$

Since $2|\theta||X||Y| \leqslant \frac{2}{3}|X|^{2}+\frac{3}{2} \theta^{2}|Y|^{2}$ we can write

$$
|\theta Y-X|^{2}+|\theta||Y|^{2} \geqslant \frac{1}{3}|X|^{2}-\frac{1}{2} \theta^{2}|Y|^{2}+|\theta||Y|^{2} \geqslant \frac{1}{3}|X|^{2}+\frac{1}{2}|\theta||Y|^{2}
$$

since $|\theta| \leqslant 1$. We deduce from (7.1.39) that

$$
Q \geqslant \frac{2}{15}\left(|X|^{2}+|\theta||Y|^{2}\right)-C_{4}(\varepsilon+\delta)^{2}\left(|\theta|+|X|^{2}\right) .
$$

Now in case 1 we have, according to (7.1.37)

$$
|Y|=\left|\alpha_{\xi}-\frac{y-x}{2 \theta}\right| \geqslant\left|\alpha_{\xi}\right|-\frac{|y-x|}{2|\theta|} \geqslant \delta_{2} .
$$

Taking $\varepsilon$ and $\delta$ small compared to $\delta_{2}$ we deduce that $Q \geqslant c\left(|X|^{2}+|\theta|\right)$ which is the first claim of the Lemma. The second claim follows easily from the fact that $|Y|,|X|$ are uniformly bounded and from (7.1.35), (7.1.34).

Case 2. - We have the following result.

Lemma 7.1.7. - When $x-\alpha_{x} \in \operatorname{supp} \chi_{6}, y-x(\theta, \alpha) \in \operatorname{supp} \chi_{5}, \alpha_{\xi} \in \operatorname{supp} \psi_{3}$ we have

$$
\begin{aligned}
Q & =:\left|\frac{\partial F}{\partial \alpha_{x}}\right|^{2}+\frac{1}{|y-x|}\left|\frac{\partial F}{\partial \alpha_{\xi}}\right|^{2} \geqslant c\left(\left|x-\alpha_{x}\right|^{2}+|\theta|\right), \\
\left|\partial_{\alpha_{x}}^{A} F\right| & \leqslant C_{A} \text { if }|A| \geqslant 1, \\
\left|\partial_{\alpha_{x}}^{A_{1}} \partial_{\alpha_{\xi}}^{A_{2}} F\right| & \leqslant C_{A_{1} A_{2}}|y-x| \text { if }\left|A_{2}\right| \geqslant 1 .
\end{aligned}
$$

MÉMOIRES DE LA SMF 101/102 
Proof. - Here we set $X=x-\alpha_{x}, Y=\frac{y-x}{|y-x|}-2 \theta \frac{\alpha_{\xi}}{|y-x|}$. Then we can write,

$$
\begin{aligned}
\frac{\partial F}{\partial \alpha_{\xi}} & =\frac{1}{1+2 i \theta}(|y-x| Y-2 i \theta X)+\frac{\partial R}{\partial \alpha_{\xi}}, \\
\frac{\partial F}{\partial \alpha_{x}} & =\frac{1}{1+2 i \theta}(2 \theta X-i(2 X+|y-x| Y))+\frac{\partial R}{\partial \alpha_{x}} .
\end{aligned}
$$

It follows that

$$
\frac{1}{1+4 \theta^{2}}\left(|y-x|^{2}|Y|^{2}+4 \theta^{2}|X|^{2}\right) \leqslant 2\left(\left|\frac{\partial F}{\partial \alpha_{\xi}}\right|^{2}+C_{1}(\varepsilon+\delta)^{2}|\theta|^{2}\right) .
$$

Therefore we have the estimate

$$
|y-x||Y|^{2} \leqslant 10 \frac{1}{|y-x|}\left|\frac{\partial F}{\partial \alpha_{\xi}}\right|^{2}+C_{2}(\varepsilon+\delta)^{2}|\theta| .
$$

On the other hand since $\left|y-\alpha_{x}\right| \leqslant|y-x|+\left|x-\alpha_{x}\right|$

$$
|2 X+| y-x|Y|^{2} \leqslant 10\left|\frac{\partial F}{\partial \alpha_{x}}\right|^{2}+C_{3}(\varepsilon+\delta)^{2}\left(|\theta|^{2}+|y-x|^{2}+\left|x-\alpha_{x}\right|^{2}\right) .
$$

Summing up we see that

$$
10 Q+C_{4}(\varepsilon+\delta)^{2}\left(|\theta|^{2}+|y-x|^{2}+|X|^{2}\right) \geqslant|2 X+| y-x|Y|^{2}+|y-x||Y|^{2} .
$$

Writing $y-x=y-x(\theta, \alpha)+x(\theta, \alpha)-\alpha_{x}+\alpha_{x}-x$, we see that one can find a constant $K_{0}$ such that $|y-x| \leqslant K_{0}$. Let $\eta_{0}>0$ be such that $\frac{\eta_{0}}{4-\eta_{0}} K_{0} \leqslant \frac{1}{2}$. Then we have

$$
4|y-x||X| \cdot|Y| \leqslant\left(4-\eta_{0}\right)|X|^{2}+\frac{4}{4-\eta_{0}}|y-x|^{2}|Y|^{2} .
$$

It follows that

$$
|2 X+| y-x|Y|^{2} \geqslant \eta_{0}|X|^{2}-\frac{\eta_{0}}{4-\eta_{0}}|y-x|^{2}|Y|^{2} .
$$

This implies that

$$
\begin{aligned}
10 Q+C_{4}(\varepsilon+\delta)^{2}\left(|\theta|^{2}+|y-x|^{2}+|X|^{2}\right) & \geqslant \eta_{0}|X|^{2}+|y-x||Y|^{2}\left(1-\frac{\eta_{0}}{4-\eta_{0}} K_{0}\right) \\
& \geqslant \min \left(\eta_{0}, \frac{1}{2}\right)\left(|X|^{2}+|y-x||Y|^{2}\right) .
\end{aligned}
$$

On the other hand we have

$$
|Y| \geqslant 1-2|\theta| \frac{\left|\alpha_{\xi}\right|}{|y-x|} \geqslant 1-\frac{b+4 \delta_{2}}{b+5 \delta_{2}}=\frac{\delta_{2}}{b+5 \delta_{2}}
$$

because $|y-x| \geqslant 2\left(b+5 \delta_{2}\right)|\theta|$, since we are in case 2 . Therefore

$$
10 Q+C_{5}(\varepsilon+\delta)\left(|\theta|+|y-x|+|X|^{2}\right) \geqslant C_{6}\left(|X|^{2}+|y-x|\right) .
$$

Taking $\varepsilon+\delta$ small with respect to $C_{6}$ we obtain the first part of the Lemma. The bounds on the derivatives of $F$ can be easily obtained since $|\theta| \leqslant\left(b+5 \delta_{2}\right)^{-1}|y-x|$. 
Case 3. - Recall that we have $a-5 \delta_{2} \leqslant \frac{|x-y|}{2|\theta|} \leqslant b+5 \delta_{2}$. Then we have the following result.

LEMMA 7.1.8. - One can find $\rho_{0}>0$ such that the equation $x\left(\theta, x, \alpha_{\xi}\right)=y$ has a unique solution in the set $E=\left\{\alpha_{\xi}:\left|\alpha_{\xi}-\frac{y-x}{2 \theta}\right| \leqslant \rho_{0}\right\}$.

Proof. - First of all one can find $\rho_{0}$ such that if $\alpha_{\xi} \in E$ then $\frac{1}{2} \leqslant\left|\alpha_{\xi}\right| \leqslant 2$. It follows from Proposition 3.2.1 that $x\left(\theta, x, \alpha_{\xi}\right)=x+2 \theta \alpha_{\xi}+r\left(\theta, x, \alpha_{\xi}\right)$ where $|r| \leqslant C \varepsilon|\theta|$. The equation to be solved is in $E$ equivalent to the equation $\alpha_{\xi}=\frac{y-x}{2 \theta}+\frac{1}{2 \theta} r\left(\theta, x, \alpha_{\xi}\right)$. If we set $\Phi\left(\alpha_{\xi}\right)=\frac{y-x}{2 \theta}+\frac{1}{2 \theta} u\left(\theta, x, \alpha_{\xi}\right)$ then $\Phi$ maps $E$ in itself if $C \varepsilon<\rho_{0}$. Moreover, again by Proposition 3.2.1 we have $\left|\Phi\left(\alpha_{\xi}\right)-\Phi\left(\alpha_{\xi}^{\prime}\right)\right| \leqslant C^{\prime} \varepsilon\left|\alpha_{\xi}-\alpha_{\xi}^{\prime}\right|$. Taking $\varepsilon$ small enough the Lemma follows from the fixed point theorem.

We shall set

$$
\alpha_{c}=\left(x, \alpha_{\xi}^{c}\right)
$$

where $\alpha_{\xi}^{c}$ is the unique solution of $x\left(\theta, x, \alpha_{\xi}\right)=y$ given by Lemma 7.1.8. Then we have the following result.

LEMMA 7.1.9. - We have $\frac{\partial F}{\partial \alpha_{x}}\left(\theta, x, y, \alpha^{c}\right)=\frac{\partial F}{\partial \alpha_{\xi}}\left(\theta, x, y, \alpha^{c}\right)=0$.

Proof. - Let us recall that

$$
\left\{\begin{array}{l}
\frac{\partial F}{\partial \alpha_{x}}=\alpha_{\xi}-i\left(x-\alpha_{x}\right)+\frac{\partial \varphi}{\partial \alpha_{x}} \\
\frac{\partial F}{\partial \alpha_{\xi}}=-\left(x-\alpha_{x}\right)+i \alpha_{\xi}+\frac{\partial \varphi}{\partial \alpha_{\xi}} .
\end{array}\right.
$$

On the other hand we have (see (4.5.17))

$$
\varphi(\theta, x(\theta, \alpha), \alpha)=\theta p(\alpha)+\frac{1}{2 i}\left|\alpha_{\xi}\right|^{2} .
$$

Therefore

$$
\frac{\partial \varphi}{\partial \alpha_{x}^{j}}(\theta, x(\theta, \alpha), \alpha)=\theta \frac{\partial p}{\partial x_{j}}(\alpha)-\sum_{k=1}^{n} \frac{\partial \varphi}{\partial x_{k}}(\theta, x(\theta, \alpha), \alpha) \frac{\partial x_{k}}{\partial x_{j}}(\theta, \alpha) .
$$

Moreover we have

$$
\frac{\partial \varphi}{\partial x_{k}}(\theta, x(\theta, \alpha), \alpha)=\Phi(\theta, x(\theta, \alpha), \alpha)=\xi(\theta, \alpha)
$$

SO

$$
\frac{\partial \varphi}{\partial \alpha_{x}^{j}}(\theta, x(\theta, \alpha), \alpha)=\theta \frac{\partial p}{\partial x_{j}}(\alpha)-\sum_{k=1}^{n} \xi_{k}(\theta, \alpha) \frac{\partial x_{j}}{\partial x_{j}}(\theta, \alpha)
$$


Now by the definition of the flow and the Euler relation we have

$$
\left\{\begin{array}{l}
\sum_{k=1}^{n} \dot{x}_{k}(\theta, \alpha) \xi_{k}(\theta, \alpha)=\sum_{k=1}^{n} \xi_{k}(\theta, \alpha) \frac{\partial p}{\partial \xi_{k}}(x(\theta, \alpha), \xi(\theta, \alpha))=2 p(\alpha) \\
p(x(\theta, \alpha), \xi(\theta, \alpha))=p(\alpha) .
\end{array}\right.
$$

Differentiating these two relations with respect to $\alpha_{x}^{j}$ we obtain

$$
\begin{gathered}
\frac{\partial \dot{x}}{\partial x_{j}}(\theta, \alpha) \cdot \xi(\theta, \alpha)+\dot{x}(\theta, \alpha) \cdot \frac{\partial \xi}{\partial x_{j}}(\theta, \alpha)=2 \frac{\partial p}{\partial x_{j}}(\alpha) \\
\frac{\partial p}{\partial x}(x(\theta, \alpha), \xi(\theta, \alpha)) \cdot \frac{\partial x}{\partial x_{j}}(\theta, \alpha)+\frac{\partial p}{\partial \xi}(x(\theta, \alpha), \xi(\theta, \alpha)) \frac{\partial \xi}{\partial x_{j}}(\theta, \alpha) \\
=\frac{\partial p}{\partial x_{j}}(\alpha) .
\end{gathered}
$$

Using the equations of the flow the last equality can be written as

$$
-\dot{\xi}(\theta, \alpha) \cdot \frac{\partial x}{\partial x_{j}}(\theta, \alpha)+\dot{x}(\theta, \alpha) \frac{\partial \xi}{\partial x_{j}}(\theta, \alpha)=\frac{\partial p}{\partial x_{j}}(\alpha) .
$$

Combining with (7.1.44) we obtain

$$
\frac{\partial \dot{x}}{\partial x_{j}}(\theta, \alpha) \cdot \xi(\theta, \alpha)+\dot{\xi}(\theta, \alpha) \cdot \frac{\partial x}{\partial x_{j}}(\theta, \alpha)=\frac{\partial p}{\partial x_{j}}(\alpha)
$$

which can be written as

$$
\frac{d}{d \theta}\left[\frac{\partial x}{\partial x_{j}}(\theta, \alpha) \cdot \xi(\theta, \alpha)\right]=\frac{\partial p}{\partial x_{j}}(\alpha)
$$

Integrating both side we obtain

$$
\frac{\partial x}{\partial x_{j}}(\theta, \alpha) \cdot \xi(\theta, \alpha)=\theta \frac{\partial p}{\partial x_{j}}(\alpha)+\alpha_{\xi}^{j} .
$$

Using (7.1.43) we deduce

$$
\frac{\partial \varphi}{\partial \alpha_{x}^{j}}(\theta, x(\theta, \alpha), \alpha)=-\alpha_{\xi}^{j} .
$$

Since $\alpha^{c}=\left(x, \alpha_{\xi}^{c}\right)$ where $x\left(\theta, x, \alpha_{\xi}^{c}\right)=y$ we deduce from (7.1.41) that

$$
\frac{\partial F}{\partial \alpha_{x}^{j}}\left(\theta, x, y, \alpha^{c}\right)=\alpha_{\xi}^{j}+\frac{\partial \varphi}{\partial \alpha_{x}^{j}}\left(\theta, x\left(\theta, \alpha^{c}\right), \alpha^{c}\right)=\alpha_{\xi}^{j}-\alpha_{\xi}^{j}=0 .
$$

Now differentiating (7.1.42) with respect to $\alpha_{\xi}^{j}$ yields

$$
\frac{\partial \varphi}{\partial \alpha_{\xi}^{j}}(\theta, x(\theta, \alpha), \alpha)+\frac{\partial \varphi}{\partial x}(\theta, x(\theta, \alpha), \alpha) \cdot \frac{\partial x}{\partial \alpha_{\xi}^{j}}(\theta, \alpha)=\theta \frac{\partial p}{\partial \xi_{j}}(\alpha)+\frac{1}{i} \alpha_{\xi}^{j} .
$$

As above we see easily that,

$$
\frac{d}{d \theta}\left[\frac{\partial x}{\partial \alpha_{\xi}^{j}}(\theta, \alpha) \cdot \xi(\theta, \alpha)\right]=\frac{\partial p}{\partial \xi_{j}}(\alpha)
$$


from which we deduce that $\frac{\partial x}{\partial \alpha_{\xi}^{J}}(\theta, \alpha) \cdot \xi(\theta, \alpha)=\theta \frac{\partial p}{\partial \xi_{j}}(\alpha)$. Since $\frac{\partial \varphi}{\partial x}(\theta, x(\theta, \alpha), \xi(\theta, \alpha))=$ $\xi(\theta, \alpha)$, we obtain

$$
\frac{\partial \varphi}{\partial \alpha_{\xi}^{j}}(\theta, x(\theta, \alpha), \alpha)=\frac{1}{i} \alpha_{\xi}^{j}
$$

which implies that $\frac{\partial F}{\partial \alpha_{\xi}^{j}}\left(\theta, x, y, \alpha^{c}\right)=0$.

Lemma 7.1.10. - Let us set

$$
\left\{\begin{array}{l}
Q=\left[\left|\frac{\partial F}{\partial \alpha_{x}}\right|^{2}+\frac{1}{|\theta|}\left|\frac{\partial F}{\partial \alpha_{\xi}}\right|^{2}\right](\theta, x, y, \alpha) \\
X=\alpha_{x}-x \\
Y=\alpha_{\xi}-\alpha_{\xi}^{c}
\end{array}\right.
$$

where $\alpha_{\xi}^{c}$ has been introduced in (7.1.40). Then we have

$$
\left\{\begin{array}{l}
Q \geqslant C\left(|X|^{2}+|\theta||Y|^{2}\right), \\
\left|\partial_{\alpha_{x}}^{A} F\right| \leqslant C_{A} \text { if }|A| \geqslant 1, \\
\left|\partial_{\alpha_{x}}^{A_{1}} \partial_{\alpha_{\xi}}^{A_{2}} F\right| \leqslant C_{A_{1} A_{2}}|\theta| \text { if }\left|A_{2}\right| \geqslant 1,
\end{array}\right.
$$

uniformly in $(\theta, x, y, \alpha)$ when $|\theta| \leqslant 1, x-\alpha_{x} \in \operatorname{supp} \chi_{6}, y-x(\theta, \alpha) \in \operatorname{supp} \chi_{6}$, $\alpha_{\xi} \in \operatorname{supp} \psi_{3}$.

Proof. - For $t \in(0,1)$ let us set $m_{t}=\left(\theta, x, y, t \alpha+(1-t) \alpha^{c}\right)$. Then using Lemma 7.1.9 we can write for $j=1, \ldots, n$,

$$
\begin{aligned}
& \left.\frac{\partial F}{\partial \alpha_{x}^{j}}(\theta, x, y, \alpha)=\sum_{k=1}^{n}\left(\int_{0}^{1}\left[\frac{\partial^{2} F}{\partial \alpha_{x}^{j} \partial \alpha_{x}^{k}}\left(m_{t}\right)\left(\alpha_{x}^{k}-x_{k}\right)+\frac{\partial^{2} F}{\partial \alpha_{x}^{j} \partial \alpha_{\xi}^{k}}\left(m_{t}\right)\left(\alpha_{\xi}^{k}-\left(\alpha_{\xi}^{c}\right)^{k}\right)^{k}\right)\right] d t\right) \\
& \frac{\partial F}{\partial \alpha_{\xi}^{j}}(\theta, x, y, \alpha)=\sum_{k=1}^{n}\left(\int_{0}^{1}\left[\frac{\partial^{2} F}{\partial \alpha_{\xi}^{j} \partial \alpha_{x}^{k}}\left(m_{t}\right)\left(\alpha_{x}^{k}-x_{k}\right)+\frac{\partial^{2} F}{\partial \alpha_{\xi}^{j} \partial \alpha_{\xi}^{k}}\left(m_{t}\right)\left(\alpha_{\xi}^{k}-\left(\alpha_{\xi}^{c}\right)^{k}\right)\right] d t\right) .
\end{aligned}
$$

It follows from (7.1.35) and (7.1.34) that

$$
\begin{aligned}
& \frac{\partial F}{\partial \alpha_{x}^{j}}=\frac{2 i-2 \theta}{1+2 i \theta} X_{j}+\frac{2 i \theta}{1+2 i \theta} Y_{j}+\mathcal{O}[(\varepsilon+\delta)(|X|+|\theta||Y|] \\
& \frac{\partial F}{\partial \alpha_{\xi}^{j}}=\frac{2 i \theta}{1+2 i \theta} X_{j}-\frac{2 \theta}{1+2 i \theta} Y_{j}+\mathcal{O}[(\varepsilon+\delta)|\theta|(|X|+|Y|)] .
\end{aligned}
$$

Therefore we have

$$
\theta^{2}|X|^{2}+|X-\theta Y|^{2}+|\theta||X|^{2}+|\theta||Y|^{2} \leqslant \frac{5}{2} Q+C(\varepsilon+\delta)^{2}\left(|X|^{2}+|\theta|^{2}|Y|^{2}\right) .
$$

Taking $\varepsilon+\delta$ small enough we obtain $Q \geqslant C\left(|X|^{2}+|\theta||Y|^{2}\right)$ which the first claim of the Lemma. The other claims follows from (7.1.35) and the fact that $|x-y| \leqslant 2\left(b+5 \delta_{2}\right)|\theta|$ since we are in the case 3 . 
We shall set in what follows,

$$
\left\{\begin{array}{l}
L=\frac{1}{i} \sum_{j=1}^{n}\left(\frac{\partial \bar{F}}{\partial \alpha_{x}^{j}}(\theta, x, y, \alpha) \frac{\partial}{\partial \alpha_{x}^{j}}+\frac{1}{D} \frac{\partial \bar{F}}{\partial \alpha_{\xi}^{j}}(\theta, x, y, \alpha) \frac{\partial}{\partial \alpha_{\xi}^{j}}\right) \\
Q=\left(\left|\frac{\partial F}{\partial \alpha_{x}}\right|^{2}+\frac{1}{D}\left|\frac{\partial F}{\partial \alpha_{\xi}}\right|^{2}\right)(\theta, x, y, \alpha) \\
\text { where } D=|\theta| \text { in the cases } 1 \text { and } 3, D=|x-y| \text { in the case } 2 .
\end{array}\right.
$$

Let us note that, according to Lemmas 7.1.6, 7.1.7 and 7.1.10 $\mathrm{L}$ is a vector field whose coefficients are uniformly bounded together with their derivatives with respect to $\alpha$. Moreover we have

$$
L e^{i \lambda F}=\lambda Q e^{i \lambda F} .
$$

Our first goal is to prove the following result.

Proposition 7.1.11. - For any $N$ in $\mathbb{N}^{n}$ we can write

$$
L^{N} e^{i \lambda F(\theta, x, y, \alpha)}=\left(\lambda^{N} Q^{N}+\sum_{k=1}^{N-1} h_{k, N}(\theta, x, y, \alpha) \lambda^{k}\right) e^{i \lambda F(\theta, x, y, \alpha)}
$$

where $h_{k, N}$ are smooth functions satisfying

$$
\left|L^{j} h_{k, N}(\theta, x, y, \alpha)\right| \leqslant C_{j, N} Q^{k}, \quad 0 \leqslant k \leqslant N-1, j \in \mathbb{N},
$$

uniformly when $0<|\theta| \leqslant 1,\left|x-\alpha_{x}\right| \leqslant 1,|y-x(\theta, \alpha)| \leqslant 2 \delta,\left|\alpha_{\xi}\right| \leqslant 2$.

Proof

Step 1. - Let $\mathcal{H}$ be the set of $C^{\infty}$ functions $c=c(\theta, x, y, \alpha)$ such that for any $\gamma \in \mathbb{N}^{2 n}, \partial_{\alpha}^{\gamma} c$ is uniformly bounded when $0<|\theta| \leqslant 1,\left|x-\alpha_{x}\right| \leqslant 1,|y-x(\theta, \alpha)| \leqslant 2 \delta$, $\left|\alpha_{\xi}\right| \leqslant 2$. For instance $\frac{\partial F}{\partial \alpha_{x}^{j}}$ and $\frac{1}{D} \frac{\partial F}{\partial \alpha_{\xi}^{j}}$ belong to $\mathcal{H}$.

Let us set $T=\frac{\partial F}{\partial \alpha_{x}}, \bar{T}=\frac{\partial \bar{F}}{\partial \alpha_{x}}, S=\frac{1}{\sqrt{D}} \frac{\partial F}{\partial \alpha_{\xi}}, \bar{S}=\frac{1}{\sqrt{D}} \frac{\partial \bar{F}}{\partial \alpha_{\xi}}$.

Let $\mathcal{P}$ be the set of homogeneous polynomials of order 2 in $T, \bar{T}, S, \bar{S}$ with coefficients in $\mathcal{H}$. For instance we have $Q \in \mathcal{P}$. We claim that $L$ sends $\mathcal{P}$ into $\mathcal{P}$. First of all if $P \in \mathcal{P}$ then $\frac{\partial \bar{F}}{\partial \alpha_{x}^{j}} \frac{\partial P}{\partial \alpha_{x}^{3}} \in \mathcal{P}$ since $\frac{\partial \bar{F}}{\partial \alpha_{x}^{3}} \in \mathcal{H}, \frac{\partial^{2} F}{\partial \alpha^{2}} \in \mathcal{H}$ and $\frac{\partial \bar{F}}{\partial \alpha_{x}^{3}}=\bar{T}_{j}$. On the other hand $\frac{1}{D} \frac{\partial \bar{F}}{\partial \alpha_{\xi}^{j}} \frac{\partial P}{\partial \alpha_{\xi}^{j}} \in \mathcal{P}$ since $\frac{1}{D} \frac{\partial \bar{F}}{\partial \alpha_{\xi}^{j}} \in \mathcal{H}, \frac{1}{\sqrt{D}} \frac{\partial \bar{F}}{\partial \alpha_{\xi}^{j}}=\bar{T}_{j}$ and $\frac{1}{D} \frac{\partial^{2} F}{\partial \alpha_{\xi} \partial \alpha} \in \mathcal{H}$. It follows that $L$ maps $\mathcal{P}$ into $\mathcal{P}$.

Now if $P \in \mathcal{P}$ then $|P| \leqslant C Q$. It follows that for all $j \in \mathbb{N}$,

$$
\left|L^{j} Q\right| \leqslant C_{j} Q
$$

uniformly in $(\theta, x, y, \alpha)$.

Step 2. - We claim that

$$
\left\{\begin{array}{l}
\text { for all } N \in \mathbb{N}, \quad j \in \mathbb{N} \text { we have }\left|L^{j} Q^{N}\right| \leqslant C_{j N} Q^{N} \text { uniformly when } \\
0<|\theta|<1,\left|x-\alpha_{x}\right| \leqslant 1, \quad|y-x(\theta, \alpha)| \leqslant 2 \delta, \quad\left|\alpha_{\xi}\right| \leqslant 2
\end{array}\right.
$$


Indeed by the Faa di Bruno formula (since $L$ is a homogeneous vector field) $L^{j} Q^{N}$ is a finite linear combination of terms of the form

$$
Q^{N-M} \prod_{i=1}^{s}\left(L^{\ell_{i}} Q\right)^{k_{i}}
$$

where $1 \leqslant M \leqslant N, 1 \leqslant s \leqslant M, \sum_{i=1}^{s} k_{i}=M, \sum_{i=1}^{s} k_{i} \ell_{i}=j$. Each of such terms is bounded, according to (7.1.47) by $C Q^{N-M} Q^{\sum_{i}^{s} k_{i}}=C Q^{N-M} Q^{M}=C Q^{N}$ which proves our claim.

Step 3. - Proof of Proposition 7.1.11

We use an induction on $N$. For $N=1$ the result follows from (7.1.46). Let us assume it is true up to the order $N$. Then

$$
\begin{gathered}
L^{N+1}\left(e^{i \lambda F}\right)=e^{i \lambda F}\left(\lambda^{N} N Q^{N-1} L Q+\sum_{k=0}^{N-1}\left(L h_{k, N}\right) \lambda^{k}+\lambda^{N+1} Q^{N+1}+\sum_{k=0}^{N-1} Q h_{k, N} \lambda^{k+1}\right) \\
=e^{i \lambda F}\left(\lambda^{N+1} Q^{N+1}+\sum_{k=0}^{N} h_{k, N+1} \lambda^{k}\right)
\end{gathered}
$$

where

$$
\left\{\begin{array}{l}
h_{N, N+1}=N Q^{N-1} L Q+Q h_{N-1, N} \\
h_{k, N+1}=L h_{k, N}+Q h_{k-1, N}, \quad 1 \leqslant k \leqslant N-1 \\
h_{0, N+1}=L h_{0, N}
\end{array}\right.
$$

Now we have

$$
\left|L^{j} h_{N, N+1}\right| \leqslant N \sum_{i=0}^{j}\left(\begin{array}{l}
j \\
i
\end{array}\right)\left|L^{i} Q^{N-1}\right|\left|L^{j-i} Q\right|+\sum_{i=0}^{j}\left(\begin{array}{l}
j \\
i
\end{array}\right)\left|L^{i} Q\right|\left|L^{j-i} h_{N-1, N}\right| .
$$

Using (7.1.47), (7.1.48) and the induction hypothesis we deduce that $\left|L^{j} h_{N, N+1}\right| \leqslant$ $C_{j, N} Q^{N}$. The estimates for the other terms are completely analogous.

We need another Lemma.

LEMMA 7.1.12

(i) For any $N \in \mathbb{N}^{*}$ we can write

$$
L^{N}\left(e^{i \lambda F}\right)=G_{N}(\theta, x, y, \alpha, \lambda) e^{i \lambda F} .
$$

(ii) There exists a constant $K_{N}>0$ such that

$$
\left|K_{N}+G_{N}(\theta, x, y, \alpha, \lambda)\right| \geqslant \frac{1}{2}\left(\lambda^{N} Q^{N}+1\right) .
$$

(iii) For any $j \in \mathbb{N}, N \in \mathbb{N}^{*}$ we can find a constant $C_{j, N}>0$ such that

$$
\left|L^{j} G_{N}(\theta, x, y, \alpha, \lambda)\right| \leqslant C_{j, N}\left|G_{N}(\theta, x, y, \alpha, \lambda)+K_{N}\right| .
$$


(iv) For any $j \in \mathbb{N}, N \in \mathbb{N}^{*}$ we can find a constant $C_{j N}^{\prime}>0$ such that

$$
\left|\left({ }^{t} L\right)^{j}\left(\frac{1}{K_{N}+G_{N}}\right)\right| \leqslant \frac{C^{\prime}}{\left|K_{N}+G_{N}\right|}
$$

where ${ }^{t} L$ denotes the transpose of $L$.

Proof. - (i) is a consequence of Proposition 7.1.11 with

$$
G_{N}(\theta, x, y, \alpha, \lambda)=\lambda^{N} Q^{N}+\sum_{k=0}^{N-1} h_{k, N}(\theta, x, y, \alpha) \lambda^{k}
$$

and $\left|h_{k, N}\right| \leqslant C_{N} Q^{k}, 0 \leqslant k \leqslant N-1, C_{N} \geqslant 1$.

(ii) If $\lambda Q \leqslant 2 N C_{N}$ then

$$
\sum_{k=0}^{N-1}\left|h_{k, N}\right| \lambda^{k} \leqslant C_{N} \sum_{k=0}^{N-1}(\lambda Q)^{k} \leqslant C_{N} \sum_{k=0}^{N-1}\left(2 N C_{N}\right)^{k} .
$$

If $\lambda Q \geqslant 2 N C_{N}$ then

$$
(\lambda Q)^{N}=(\lambda Q)^{N-k}(\lambda Q)^{k} \geqslant 2 N C_{N}(\lambda Q)^{k} \quad \text { if } 0 \leqslant k \leqslant N-1 .
$$

Then

Therefore

$$
\sum_{k=0}^{N-1}\left|h_{k, N}\right| \lambda^{k} \leqslant \frac{C_{N}}{2 N C_{N}}\left(\sum_{k=0}^{N-1} 1\right)(\lambda Q)^{N}=\frac{1}{2}(\lambda Q)^{N} .
$$

This implies

$$
\sum_{k=0}^{N-1}\left|h_{k, N}\right| \lambda^{k} \leqslant \frac{1}{2}(\lambda Q)^{N}+K_{N}^{\prime}
$$

$$
\left|K_{N}^{\prime}+\frac{1}{2}+G_{N}\right| \geqslant K_{N}^{\prime}+\frac{1}{2}+\lambda^{N} Q^{N}-\frac{1}{2} \lambda^{N} Q^{N}-K_{N}^{\prime} \geqslant \frac{1}{2}\left(\lambda^{N} Q^{N}+1\right) .
$$

(iii) We have, by (7.1.48) and Proposition 7.1.11

$$
\begin{aligned}
\left|L^{j} G_{N}\right| & =\left|\lambda^{N} L^{j} Q^{N}+\sum_{k=0}^{N-1} L^{j} h_{k, N} \lambda^{k}\right| \leqslant C_{j, N} \lambda^{N} Q^{N}+\sum_{k=0}^{N-1} C_{j N}^{\prime} \lambda^{k} Q^{k} \\
& \leqslant C_{j N}^{\prime \prime}\left(1+\lambda^{N} Q^{N}\right) \leqslant \widetilde{C}_{j, N}\left|K_{N}+G_{N}\right| .
\end{aligned}
$$

(iv) Let us recall that $L=\frac{\partial \bar{F}}{\partial \alpha_{x}} \cdot \frac{\partial}{\partial \alpha_{x}}+\frac{1}{D} \frac{\partial \bar{F}}{\partial \alpha_{\xi}} \cdot \frac{\partial}{\partial \alpha_{\xi}}$. According to the Lemmas 7.1.6, 7.1.7 and 7.1.10, we see that ${ }^{t} L=-L+c(\theta, x, y, \alpha)$ where

$$
\left|\partial_{\alpha}^{\gamma} c(\theta, x, y, \alpha)\right| \leqslant C_{\gamma}
$$

uniformly in $(\theta, x, y, \alpha)$. We claim that for $j \in \mathbb{N}$

$$
\left\{\begin{array}{l}
\left({ }^{t} L\right)^{j}=\sum_{k=0}^{j} c_{j, k}(\theta, x, y, \alpha) L^{k}, \text { with } \\
\left|\partial_{\alpha}^{\gamma} c_{j k}(\theta, x, y, \alpha)\right| \leqslant c_{j, k, \gamma}
\end{array}\right.
$$


We saw above that this is true when $j=1$. Let us assume that (7.1.49) is true up to the order $j$. Then

$$
\left({ }^{t} L\right)^{j+1}=(-L+c) \sum_{k=0}^{j} c_{j k}(\theta, x, y, \alpha) L^{k}=\sum_{k=0}^{j+1} c_{j+1, k}(\theta, x, y, \alpha) L^{k}
$$

where

$$
\left\{\begin{array}{l}
c_{j+1,0}=c c_{j, 0}-L c_{j, 0} \\
c_{j+1, k}=c c_{j k}-L c_{j k}-c_{j, k-1}, \quad 1 \leqslant k \leqslant j \\
c_{j+1, j+1}=-c_{j, k} .
\end{array}\right.
$$

Then the estimate on $\left|\partial_{\alpha}^{\gamma} c_{j+1, k}\right|$ follows from the induction and the fact that the coefficients of $L$ have all their derivatives with respect to $\alpha$ bounded uniformly. It follows that

$$
\left|\left({ }^{t} L\right)^{j}\left(\frac{1}{K_{N}+G_{N}}\right)\right| \leqslant C_{j, N} \sum_{k=0}^{j}\left|L^{k}\left(\frac{1}{K_{N}+G_{N}}\right)\right| .
$$

Now by the Faa di Bruno formula, $L^{k}\left(\frac{1}{K_{N}+G_{N}}\right)$ is a finite linear combination of terms of the form

$$
\frac{1}{\left(K_{N}+G_{N}\right)^{1+\beta}} \prod_{i=1}^{s}\left(L^{\ell_{i}} G_{N}\right)^{k_{i}}
$$

where $1 \leqslant \beta \leqslant k, 1 \leqslant s \leqslant k, \sum_{i=1}^{s} k_{i}=\beta, \sum_{i=1}^{s} k_{i} \ell_{i}=k$. It follows from (iii) that

$$
\left|L^{k}\left(\frac{1}{K_{N}+G_{N}}\right)\right| \leqslant C_{j, N} \frac{1}{\left|K_{N}+G_{N}\right|^{1+\beta}}\left|K_{N}+G_{N}\right|^{\sum_{i=1}^{s} k_{i}} \leqslant \frac{C_{j, N}}{\left|K_{N}+G_{N}\right|} .
$$

Then (iv) follows from (7.1.50).

We can now state the estimate on the kernel for $|\theta| \leqslant 1$.

Lemma 7.1.13. - Let $\widetilde{k}_{+}=\widetilde{k}_{+}(t, x, y, \lambda)$ be the kernel defined by (7.1.32). Then one can find a positive constant $C$ such that

$$
\left|\widetilde{k}_{+}(t, x, y, \lambda)\right| \leqslant \frac{C}{|t|^{n / 2}}
$$

for all $(t, x, y, \lambda)$ such that $\lambda \geqslant 1,|\lambda t| \leqslant 1, x \in \mathbb{R}^{n}, y \in \mathbb{R}^{n}$.

Proof. - The kernel $\widetilde{k}_{+}$can be written as

$$
\widetilde{k}_{+}(t, x, y, \lambda)=\int e^{i \lambda F(-\lambda t, x, y, \alpha)} \widetilde{a}(\lambda t, x, y, \alpha) d \alpha
$$

where

$$
\widetilde{a}(\lambda t, x, y, \alpha)=a_{1}(-\lambda t, y, \alpha) \chi_{6}\left(x-\alpha_{x}\right) \psi_{3}\left(\alpha_{\xi}\right) \chi_{5}\left(\frac{y-x(-\lambda t, \alpha)}{\langle\lambda t\rangle}\right) .
$$

Let us note that all the derivatives of $\widetilde{a}$ with respect to $\alpha$ are uniformly bounded. 
We use Lemma 7.1.12 to write

$$
\begin{aligned}
\widetilde{k}_{+}(t, x, y, \lambda) & =\int\left(K_{N}+L^{N}\right) e^{i \lambda F(-\lambda t, x, y, \alpha)} \frac{\widetilde{a}(\lambda t, x, y, \alpha)}{K_{N}+G_{N}(-\lambda t, x, y, \alpha, \lambda)} d \alpha \\
& =\int e^{i \lambda F(\cdots)}\left(K_{N}+\left({ }^{t} L\right)^{N}\right)\left[\frac{\widetilde{a}(\cdots)}{K_{+} G_{N}(\cdots)}\right] d \alpha .
\end{aligned}
$$

Now using (ii), (iv) of Lemma 7.1.12, (7.1.49) and the fact that $\operatorname{Im} F \geqslant 0$ we obtain

$$
\left|\widetilde{k}_{+}(t, x, y, \lambda)\right| \leqslant C_{N} \lambda^{3 n / 2} \int \frac{\left|\widetilde{\psi}\left(\alpha_{\xi}\right)\right|}{1+\lambda^{N} Q^{N}} d \alpha=C_{N} I
$$

where $Q$ has been introduced in (7.1.45) and $\widetilde{\psi}\left(\alpha_{\xi}\right)$ is a smooth function with compact support. Now according to the Lemmas 7.1.6, 7.1.7 and 7.1.10 we have

$$
\left\{\begin{array}{l}
Q \geqslant C\left(\left|x-\alpha_{x}\right|^{2}+|\lambda t|\right) \quad(\text { case } 1 \text { and 2) } \\
Q \geqslant C\left(\left|x-\alpha_{x}\right|^{2}+|\lambda t|\left|\alpha_{\xi}-\alpha_{\xi}^{c}\right|^{2}\right) \quad(\text { case 3). }
\end{array}\right.
$$

Let us fix the integer $N$ such that $N>n$. Since $\widetilde{\psi}$ has compact support we have in case 1 and 2

$$
I \leqslant \lambda^{3 n / 2} \int_{\mathbb{R}^{n}} \frac{d X}{1+\lambda^{N}|X|^{2 N}+\lambda^{N}|\lambda t|^{N}} .
$$

Let us set $X=\frac{\left(1+\lambda^{N}|\lambda t|^{N}\right)^{1 / 2 N}}{\sqrt{\lambda}} z$. Then

$$
I \leqslant \lambda^{3 n / 2} \frac{\left(1+\lambda^{N}|\lambda t|^{N}\right)^{n / 2 N}}{\lambda^{n / 2}} \frac{1}{1+\lambda^{N}|\lambda t|^{N}} \int_{\mathbb{R}^{n}} \frac{d z}{1+|z|^{2 N}} .
$$

If $\lambda|\lambda t| \leqslant 1$, that is $\lambda^{2} \leqslant \frac{1}{|t|}$, we have,

$$
I \leqslant C_{N} \lambda^{n} \leqslant \frac{C_{N}}{|t|^{n / 2}}
$$

If $\lambda|\lambda t| \geqslant 1$, that is $\frac{1}{\lambda^{2}} \leqslant|t|$, we can write for $N>n$,

$$
I \leqslant C_{N} \lambda^{n} \frac{(\lambda|\lambda t|)^{n / 2}}{(\lambda|\lambda t|)^{N}} \leqslant \frac{C_{N}}{\lambda^{2(N-n)}}|t|^{\frac{n}{2}-N} \leqslant C_{N}|t|^{N-n+\frac{n}{2}-N} \leqslant \frac{C_{N}}{|t|^{n / 2}} .
$$

It follows from (7.1.51) that $\left|\widetilde{k}_{+}(t, x, y, \lambda)\right| \leqslant \frac{C}{|t|^{n / 2}}$.

In case 3 we have

$$
I \leqslant C \lambda^{3 n / 2} \int_{\mathbb{R}^{2 n}} \frac{d \alpha}{1+\left(\lambda\left|x-\alpha_{x}\right|^{2}+\lambda|\lambda t|\left|\alpha_{\xi}-\alpha_{\xi}^{c}\right|^{2}\right)^{N}} .
$$

Setting $X=\sqrt{\lambda}\left(x-\alpha_{x}\right), Y=\sqrt{\lambda} \sqrt{|\lambda t|}\left(\alpha_{\xi}-\alpha_{\xi}^{c}\right)$ we obtain

$$
I \leqslant C \lambda^{3 n / 2} \lambda^{-n / 2} \lambda^{-n / 2}|\lambda t|^{-n / 2} \int_{\mathbb{R}^{2 n}} \frac{d X d Y}{1+\left(|X|^{2}+|Y|^{2}\right)^{N}} .
$$

It follows that

$$
|\widetilde{k}(t, x, y, \lambda)| \leqslant \frac{C}{|t|^{n / 2}} .
$$

This completes the proof of Lemma 7.1.13 thus the proof of Theorem 7.1.1. 


\subsection{End of the proof of Theorem 2.2.1}

Let us recall that we have set in (6.2.7),

$$
U_{ \pm}(t)=\chi_{1}^{+} \psi_{2}\left(\frac{D}{\lambda}\right) e^{-i t P}
$$

It follows from Theorem 7.1.1 that there exists $C \geqslant 0$ such that

$$
\left\|U_{ \pm}\left(t_{1}\right) U_{ \pm}\left(t_{2}\right)^{*} f\right\|_{L^{\infty}\left(\mathbb{R}^{n}\right)} \leqslant \frac{C}{\left|t_{1}-t_{2}\right|^{n / 2}}\|f\|_{L^{1}\left(\mathbb{R}^{n}\right)}
$$

for all $t_{1}, t_{2}$ in $[-T, T]$ with $t_{1} \neq t_{2}$ and all $f \in L^{1}\left(\mathbb{R}^{n}\right)$. Moreover there is a conservation of the $L^{2}$ norm for $e^{-i t P}$ which implies,

$$
\left\|U_{ \pm}(t) u_{0}\right\|_{L^{2}\left(\mathbb{R}^{n}\right)} \leqslant C\left\|u_{0}\right\|_{L^{2}\left(\mathbb{R}^{n}\right)}
$$

for all $|t| \leqslant T$ and $u_{0} \in L^{2}\left(\mathbb{R}^{n}\right)$.

We can therefore apply Lemma 2.1 .3 with $X=\mathbb{R}^{n}, H=L^{2}\left(\mathbb{R}^{n}\right)$ and we obtain

$$
\left\|U_{ \pm}(\cdot) u_{0}\right\|_{L^{q}\left([-T, T], L^{r}\left(\mathbb{R}^{n}\right)\right)} \leqslant C\left\|u_{0}\right\|_{L^{2}\left(\mathbb{R}^{n}\right)}
$$

for all $u_{0} \in L^{2}\left(\mathbb{R}^{n}\right)$, where $q \geqslant 2$ and $\frac{2}{q}=\frac{n}{2}-\frac{n}{r}$. Now it follows from (6.2.2) that we have

$$
\chi_{1}^{+}(x)+\chi_{1}^{-}(x) \geqslant 1 \text { for all } x \in \mathbb{R}^{n} .
$$

Then (7.2.1) and (7.2.2) show that

$$
\left\|\psi_{2}\left(\frac{D}{\lambda}\right) e^{-i t P} u_{0}\right\|_{L^{q}\left(I, L^{r}\left(\mathbb{R}^{n}\right)\right)} \leqslant C\left\|u_{0}\right\|_{L^{2}\left(\mathbb{R}^{n}\right)}
$$

for all $u_{0} \in L^{2}\left(\mathbb{R}^{n}\right)$, where $I=[-T, T]$.

Now let us recall $($ see $(6.2 .6))$ that $\psi_{2}(\xi)=\psi_{0}(\xi) \psi_{1}(\xi)$ where

$$
\begin{gathered}
\left\{\begin{array}{l}
\psi_{0}(\xi)=1 \quad \text { if }\left|\frac{\xi}{|\xi|}-\xi_{0}\right| \leqslant \delta_{2},|\xi| \geqslant 2 \delta_{2}, \quad\left|\xi_{0}\right|=1, \\
\operatorname{supp} \psi_{0} \subset\left\{\xi: \frac{\xi}{|\xi|}-\xi_{0} \mid \leqslant 2 \delta_{2}\right\} \quad \text { and }|\xi| \geqslant \delta_{2}
\end{array}\right. \\
\left\{\begin{array}{l}
\psi_{1}(\xi)=1 \text { if } a-\delta_{2} \leqslant|\xi| \leqslant b+\delta_{2}, \quad a=\frac{6}{10}, \quad b=\frac{19}{10}, \\
\operatorname{supp} \psi_{1} \subset\left\{\xi: a-2 \delta_{2} \leqslant|\xi| \leqslant b+2 \delta_{2}\right\} .
\end{array}\right.
\end{gathered}
$$

By a finite partition of unity we deduce easily from (7.2.3) that

$$
\left\|\psi_{1}\left(\frac{D}{\lambda}\right) e^{-i t P} u_{0}\right\|_{L^{q}\left(I, L^{r}\left(\mathbb{R}^{n}\right)\right)} \leqslant C\left\|u_{0}\right\|_{L^{2}\left(\mathbb{R}^{n}\right)} .
$$

Now let us recall that $p(x, \xi)=|\xi|^{2}+\varepsilon \sum_{j, k=1}^{n} b_{j k}(x) \xi_{j} \xi_{k}$. Therefore if $\varepsilon$ is small enough we have

$$
\frac{9}{10}|\xi|^{2} \leqslant p(x, \xi) \leqslant \frac{11}{10}|\xi|^{2} .
$$

Let $\varphi_{0}(t) \in C_{0}^{\infty}(\mathbb{R})$ be such that

$$
\varphi_{0}(t)=1 \text { if }|t| \leqslant \frac{9}{5} \varphi(t)=0 \text { if }|t| \geqslant 3
$$


and set

$$
\varphi(t)=\varphi_{0}(t)-\varphi_{0}(4 t) .
$$

It follows that $\operatorname{supp} \varphi \subset\left\{t \in \mathbb{R}: \frac{9}{20} \leqslant|t| \leqslant 3\right\}$.

Let $\widetilde{\varphi} \in C^{\infty}(\mathbb{R})$ be such that

$$
\left\{\begin{array}{l}
\operatorname{supp} \widetilde{\varphi} \subset\left\{t \in \mathbb{R}: \frac{4}{10} \leqslant|t| \leqslant \frac{31}{10}\right\} \\
\widetilde{\varphi}=1 \text { on a neighborhood of } \operatorname{supp} \varphi, \text { so } \\
\varphi(t) \widetilde{\varphi}(t)=\varphi(t)
\end{array}\right.
$$

We claim that for every $(x, \xi) \in T^{*} \mathbb{R}^{n}$,

$$
\left(1-\psi_{1}(\xi)\right) \widetilde{\varphi}(p(x, \xi)) \equiv 0 .
$$

Indeed on the support of $1-\psi_{1}(\xi)$ we have

$$
\text { (i) }|\xi| \leqslant \frac{6}{10}-\delta_{2} \quad \text { or } \quad \text { (ii) }|\xi| \geqslant \frac{19}{10}+\delta_{2} .
$$

In the case (i), (7.2.5) shows that

$$
0 \leqslant p(x, \xi) \leqslant \frac{11}{10}|\xi|^{2} \leqslant \frac{11}{10}\left(\frac{6}{10}\right)^{2}<\frac{4}{10} .
$$

In the case (ii) we have

$$
p(x, \xi) \geqslant \frac{9}{10}\left(\frac{19}{10}\right)^{2}>\frac{31}{10} .
$$

Thus $\widetilde{\varphi}(p(x, \xi))=0$ by $(7.2 .7)$.

Now, with $\varphi$ introduced in (7.2.6) we claim that

$$
(1)=\left\|\varphi\left(\frac{P}{\lambda^{2}}\right) e^{-i t P} u_{0}\right\|_{L^{q}\left(I, L^{r}\left(\mathbb{R}^{n}\right)\right)} \leqslant C\left\|\varphi\left(\frac{P}{\lambda^{2}}\right) u_{0}\right\|_{L^{2}\left(\mathbb{R}^{n}\right)}
$$

for all $u_{0} \in L^{2}\left(\mathbb{R}^{n}\right)$. Indeed we can write

$$
(1) \leqslant\|\psi_{1} \underbrace{\left(\frac{D}{\lambda}\right) \varphi\left(\frac{P}{\lambda^{2}}\right)}_{(2)} e^{-i t P} u_{0}\|_{L^{q}\left(I, L^{r}\right)}+\|(I-\psi_{1} \underbrace{\left.\left(\frac{D}{\lambda}\right)\right) \varphi\left(\frac{P}{\lambda^{2}}\right)}_{(3)} e^{-i t P} u_{0} \|_{L^{q}\left(I, L^{r}\right)} \text {. }
$$

Since $\varphi\left(\frac{P}{\lambda^{2}}\right)$ commutes with $e^{-i t P}$, we deduce from (7.2.4) that

$$
(2) \leqslant\left\|\varphi\left(\frac{P}{\lambda^{2}}\right) u_{0}\right\|_{L^{2}\left(\mathbb{R}^{n}\right)} .
$$

Using (7.2.7) we can write

$$
(3)=\left\|\left(I-\psi_{1}\left(\frac{D}{\lambda}\right)\right) \widetilde{\varphi}\left(\frac{P}{\lambda^{2}}\right) e^{-i t P} \varphi\left(\frac{P}{\lambda^{2}}\right) u_{0}\right\|_{L^{q}\left(I, L^{r}\right)} .
$$

Now, since $r \geqslant 2$, there exists, by the Sobolev embedding, $s \geqslant 0$ such that $H^{s}\left(\mathbb{R}^{n}\right) \hookrightarrow L^{r}\left(\mathbb{R}^{n}\right)$. We fix such an $s$. Then

$$
(3) \leqslant\left\|\left(I-\psi_{1}\left(\frac{D}{\lambda}\right)\right) \widetilde{\varphi}\left(\frac{P}{\lambda^{2}}\right) e^{-i t P} \varphi\left(\frac{P}{\lambda^{2}}\right) u_{0}\right\|_{L^{\infty}\left(I, H^{s}\left(\mathbb{R}^{n}\right)\right.} .
$$


Now we use (7.2.8) and Proposition A.1 in [BGT]. It follows that

$$
(3) \leqslant C^{\prime}\left\|e^{-i t P} \varphi\left(\frac{P}{\lambda^{2}}\right) u_{0}\right\|_{L^{\infty}\left(I, L^{2}\left(\mathbb{R}^{n}\right)\right)} \leqslant C^{\prime \prime}\left\|\varphi\left(\frac{P}{\lambda^{2}}\right) u_{0}\right\|_{L^{2}\left(\mathbb{R}^{n}\right)},
$$

which, together with (7.2.10) proves our claim (7.2.9). By (7.2.6) we have

$$
\varphi_{0}(t)+\sum_{k=1}^{+\infty} \varphi\left(2^{-2 k} t\right)=1
$$

Then using Corollary 2.3 in $[\mathbf{B G T}]$ we can write

$$
\left\|e^{-i t P} u_{0}\right\|_{L^{q}\left(I, L^{r}\left(\mathbb{R}^{n}\right)\right)} \leqslant C\left\|u_{0}\right\|_{L^{2}\left(\mathbb{R}^{n}\right)}+\left\|\left(\sum_{k=1}^{+\infty}\left\|e^{-i t P} \varphi\left(2^{-2 k} P\right) u_{0}\right\|_{L^{r}\left(\mathbb{R}^{n}\right)}^{2}\right)^{1 / 2}\right\|_{L^{q}(I)} .
$$

By the Minkowski inequality and (7.2.9) we obtain

$$
\left\|e^{-i t P} u_{0}\right\|_{L^{q}\left(I, L^{r}\left(\mathbb{R}^{n}\right)\right)} \leqslant C\left\|u_{0}\right\|_{L^{2}\left(\mathbb{R}^{n}\right)}+\left(\sum_{k=1}^{+\infty}\left\|\varphi\left(2^{-2 k} P\right) u_{0}\right\|_{L^{2}\left(\mathbb{R}^{n}\right)}^{2}\right)^{1 / 2} .
$$

This implies that

$$
\left\|e^{-i t P} u_{0}\right\|_{L^{q}\left(I, L^{r}\left(\mathbb{R}^{n}\right)\right)} \leqslant C^{\prime}\left\|u_{0}\right\|_{L^{2}}
$$

which is the estimate claimed in Theorem 2.2.1. 


\section{APPENDIX}

\section{A.1. The Faa di Bruno Formula}

We shall make a repeat use of the following formula which can be found in even a more precise form in the paper of Constantin and Savits [CS].

Let $m \in \mathbb{N}^{*}, F \in C^{m}\left(\mathbb{R}^{N}, \mathbb{C}\right), U_{k} \in C^{m}\left(\mathbb{R}^{p}, \mathbb{R}\right), k=1, \ldots, N$. Then for $|\Lambda| \leqslant m$ we have

$$
\partial_{Y}^{\Lambda}[F(U(Y))]=\sum_{k=1}^{N} \frac{\partial F}{\partial U_{k}}(U(Y)) \partial_{Y}^{\Lambda} U_{k}(Y)+(1)
$$

where (1) is a finite linear combination of terms of the form

$$
\left(\partial_{U}^{\beta} F\right)(U(Y)) \prod_{j=1}^{s}\left(\partial_{Y}^{L_{j}} U(Y)\right)^{K_{j}}
$$

where $2 \leqslant|\beta| \leqslant|\Lambda|, 1 \leqslant s \leqslant|\Lambda|,\left|K_{j}\right| \geqslant 1,\left|L_{j}\right| \geqslant 1$ and

$$
\sum_{j=1}^{s} K_{i}=\beta, \quad \sum_{j=1}^{s}\left|K_{j}\right| L_{j}=\Lambda .
$$

The precise coefficients of this sum can be found in $[\mathbf{C S}]$.

\section{A.2. Proof of Proposition 3.2.1}

The system satisfied by $r$ and $\zeta$ is the following.

$$
\begin{cases}\dot{r}_{j}(t)=2 \zeta_{j}(t)+2 \varepsilon \sum_{k=1}^{n} b_{j k}(x(t)) \xi_{k}(t), & r_{j}(0)=0 \\ \dot{\zeta}_{j}(t)=-\varepsilon \sum_{p, q=1}^{n} \frac{\partial b_{p q}}{\partial x_{j}}(x(t)) \xi_{p}(t) \xi_{q}(t), & \zeta_{j}(0)=0 .\end{cases}
$$

We prove our claim by induction on $|A|+|B|=k$. Moreover it is clear that (ii) implies (i) since $r(0)=\zeta(0)=0$. Setting $\Phi(t)=|\dot{r}(t)|+|\dot{\zeta}(t)|$ the above equations 
show that $\Phi(t) \leqslant C_{0} \varepsilon+C_{1} \int_{0}^{t} \Phi(\sigma) d \sigma$. Thus by Gronwall inequality, $\Phi(t) \leqslant C_{2}(T) \varepsilon$. This shows that (i) and (ii) are true for $k=0$. Let us assume they are true up to the order $k-1$. Let us set $X=(x, \xi)$ and if $\alpha=(A, B) \in \mathbb{N}^{n} \times \mathbb{N}^{n}, \partial_{X}^{\alpha}=\partial_{x}^{A} \partial_{\xi}^{B}$. It follows from the induction that

$$
\left\{\begin{array}{l}
|\xi(t, x, \xi)| \leqslant C(T) \\
\left|\partial_{X}^{\alpha} x(t, x, \xi)\right|+\left|\partial_{X}^{\alpha} \xi(t, x, \xi)\right| \leqslant C_{\alpha}(T)
\end{array}\right.
$$

if $1 \leqslant|\alpha| \leqslant k-1$ and $k \geqslant 2$.

It follows that if $1 \leqslant|\alpha| \leqslant k-1$ we have $\left|\partial_{X}^{\alpha}[F(x(t))]\right| \leqslant C_{\alpha}(T)$ if $F=b_{j k}$ or $\frac{\partial b_{p q}}{\partial x_{j}}$.

Let us now take $|\alpha|=k \geqslant 1$ and let us set $\Phi(t)=\left|\partial_{X}^{\alpha} \dot{r}(t)\right|+\left|\partial_{X}^{\alpha} \dot{\zeta}(t)\right|$. Then using the Leibniz and Faa di Bruno formulas (see Section A.1) and differentiating the above differential system we find

$$
\Phi(t) \leqslant C_{\alpha} \varepsilon+C_{\alpha}^{\prime} \int_{0}^{t} \Phi(\sigma) d \sigma
$$

after using the induction. We conclude again by the Gronwall inequality.

\section{A.3. Proof of Proposition 3.3.2}

The system satisfied by $z$ and $\zeta$ is the following.

$$
\left\{\begin{array}{l}
\dot{z}_{j}(t)=2 \varepsilon \sum_{k=1}^{n} b_{j k}(x(t)) \xi_{k}(t)-2 \varepsilon t \sum_{p, q=1}^{n} \frac{\partial b_{p q}}{\partial x_{j}}(x(t)) \xi_{p}(t) \xi_{q}(t) \\
\dot{\zeta}_{j}(t)=-\varepsilon \sum_{p, q=1}^{n} \frac{\partial b_{p q}}{\partial x_{j}}(x(t)) \xi_{p}(t) \xi_{q}(t) \\
z_{j}(0)=\zeta_{j}(0)=0
\end{array}\right.
$$

By Proposition 3.3.1 we have $\langle x(t, x, \xi)\rangle \geqslant C(1+|x|+t)$. This implies that for $\ell \geqslant 1$,

$$
\int_{0}^{t} \frac{d s}{\langle x(s)\rangle^{\ell+\sigma_{0}}} \leqslant \frac{C_{\ell}}{\langle x\rangle^{\ell-1+\sigma_{0}}} \text {. }
$$

We proceed by induction on $k$. Let us begin by the case $k=0$. We deduce from (A.3.1) that

$$
\begin{aligned}
& \left|\dot{z}_{j}(t)\right| \leqslant C\left(A_{0}, A_{1}\right) \varepsilon\left(\frac{1}{\langle x(t)\rangle^{1+\sigma_{0}}}+\frac{t}{\langle x(t)\rangle^{2+\sigma_{0}}}\right) \leqslant \frac{C^{\prime}\left(A_{0}, A_{1}\right) \varepsilon}{\langle x(t)\rangle^{1+\sigma_{0}}} \\
& \left|\dot{\zeta}_{j}(t)\right| \leqslant \frac{C\left(A_{1}\right) \varepsilon}{\langle x(t)\rangle^{2+\sigma_{0}}}
\end{aligned}
$$

since $|\xi(t, x, \xi)| \leqslant 2|\xi| \leqslant 4$. Then the estimates in Proposition 3.3.2 when $k=0$ follow from (A.3.2). Assume now that these estimates are true when $|A|+|B| \leqslant k-1$ and let us deduce several facts.

For $\ell \in \mathbb{N}$ let us introduce the space,

$$
\mathcal{B}_{\sigma_{0}}^{\ell}=\left\{F \in C^{\infty}\left(\mathbb{R}^{n}\right):\left|\partial^{\beta} F(y)\right| \leqslant \frac{C_{\beta}}{\langle y\rangle^{\ell+|\beta|+\sigma_{0}}}, \text { for all } y \in \mathbb{R}^{n}\right\} .
$$


Let us set $X=(x, \xi)$ and if $\alpha=(A, B) \in \mathbb{N}^{n} \times \mathbb{N}^{n}, \partial_{X}^{\alpha}=\partial_{x}^{A} \partial_{\xi}^{B}$. Then a straightforward computation shows that for $\alpha \in \mathbb{N}^{n} \times \mathbb{N}^{n}$,

$$
\left|\partial_{X}^{\alpha}\left(\xi_{j}\right)\right| \leqslant \frac{1}{\langle x\rangle^{|A|}}, \quad\left|\partial_{X}^{\alpha}\left(x_{j}\right)\right| \leqslant \frac{1}{\langle x\rangle^{|A|-1}} .
$$

It follows that for $|\alpha| \leqslant k-1$,

$$
\left|\partial_{X}^{\alpha} \xi_{j}(t, x, \xi)\right| \leqslant \frac{2 M_{k-1}}{\langle x\rangle^{|A|}} .
$$

Indeed

$$
\mid \partial_{X}^{\alpha}\left(\xi_{j}(t, x, \xi)|\leqslant| \partial_{X}^{\alpha}\left(\xi_{j}\right)|+| \partial_{X}^{\alpha} \zeta_{j}(t, x, \xi) \mid \leqslant \frac{1}{\langle x\rangle^{|A|}}+\frac{\varepsilon M_{k-1}}{\langle x\rangle^{|A|+1+\sigma_{0}}} .\right.
$$

Therefore if $|\alpha| \leqslant k-1$

$$
\left|\partial_{X}^{\alpha}\left(\xi_{p}(t) \xi_{q}(t)\right)\right| \leqslant \frac{C_{k} M_{k-1}^{2}}{\langle x\rangle^{|A|}} .
$$

Now if $|\alpha|=k$ we have from (A.3.3)

$$
\begin{aligned}
& \partial_{X}^{\alpha} \xi_{j}(t, x, \xi)=\partial_{X}^{\alpha} \zeta_{j}(t, x, \xi)+R_{j, \alpha}, \quad\left|R_{j, \alpha}\right| \leqslant \frac{1}{\langle x\rangle^{|A|}} \\
& \left\{\begin{array}{l}
\partial_{X}^{\alpha}\left(\xi_{p}(t) \cdot \xi_{q}(t)\right)=\xi_{p}(t) \partial_{X}^{\alpha} \xi_{q}(t)+\xi_{q}(t) \partial_{X}^{\alpha} \xi_{p}(t)+R_{p, q, \alpha} \\
\left|R_{p, q, \alpha}\right| \leqslant \frac{C_{k} M_{k-1}^{2}}{\langle x\rangle^{|A|}} .
\end{array}\right.
\end{aligned}
$$

Now we claim that if $F \in \mathcal{B}_{\sigma_{0}}^{\ell+1}$ and $|\alpha| \leqslant k-1$ we have

$$
\left|\partial_{X}^{\alpha}[F(x(t))]\right| \leqslant \frac{C_{k} M_{k-1}^{|\alpha|}}{\langle x(t)\rangle^{\ell+1+\sigma_{0}}\langle x\rangle^{|A|}} .
$$

This estimate is easy if $k=1$ and if $k \geqslant 2$ we use the Faa di Bruno formula (see Section A.1). It follows that $\partial_{X}^{\alpha}[F(x(t))]$ is a finite sum of terms of the following form

$$
\left(\partial^{\beta} F\right)(x(t)) \prod_{j=1}^{s}\left(\partial_{X}^{\ell_{j}} x(t)\right)^{k_{j}}, \quad \ell_{j} \in \mathbb{N}^{n} \times \mathbb{N}^{n}, \quad k_{j} \in \mathbb{N}^{n}
$$

where $1 \leqslant|\beta| \leqslant|\alpha|, 1 \leqslant s \leqslant|\alpha|,\left|k_{j}\right| \geqslant 1$ and

$$
\sum_{j=1}^{s} k_{j}=\beta, \quad \sum_{j=1}^{n}\left|k_{j}\right| \ell_{j}=\alpha
$$

If we write $\ell_{j}=\left(a_{j}, b_{j}\right), \alpha=(A, B)$ we have in particular

$$
\sum_{j=1}^{s}\left|k_{j}\right| a_{j}=A .
$$

Now we write $\{1,2, \ldots, s\}=I_{1} \cup I_{2} \cup I_{3}$ where

$$
I_{1}=\left\{j:\left|\ell_{j}\right| \geqslant 2\right\}, I_{2}=\left\{j:\left|\ell_{j}\right|=1, \ell_{j}=\left(a_{j}, 0\right)\right\}, I_{3}=\left\{j:\left|\ell_{j}\right|=1, \ell_{j}=\left(0, b_{j}\right)\right\}
$$


and we denote by $\Sigma_{i}$ the sum $\sum_{j \in I_{i}}, i=1,2,3$. When $j \in I_{1}$ we have

$$
\partial_{X}^{\ell_{j}}\left(x_{j}(t)\right)=2 t \partial_{X}^{\alpha}\left(\zeta_{j}(t)\right)+\partial_{X}^{\alpha}\left(z_{j}(t)\right) .
$$

Since $\left|\ell_{j}\right| \leqslant|\alpha| \leqslant k-1$ it follows from the induction that

$$
\left|\partial_{X}^{\alpha}(x(t))\right| \leqslant \frac{2 t \varepsilon M_{k-1}}{\langle x\rangle^{\left|a_{j}\right|+\sigma_{0}+1}}+\frac{\varepsilon M_{k-1}}{\langle x\rangle^{\left|a_{j}\right|+\sigma_{0}}}=\frac{\varepsilon M_{k-1}}{\langle x\rangle^{\left|a_{j}\right|+\sigma_{0}}}\left(1+\frac{2 t}{\langle x\rangle}\right) .
$$

It follows that

$$
\left|\prod_{j \in I_{1}}\left(\partial_{X}^{\ell_{j}}(x(t))\right)^{k_{j}}\right| \leqslant \frac{C_{k}\left(\varepsilon M_{k-1}\right)^{\Sigma_{1}\left|k_{j}\right|}}{\langle x\rangle^{\Sigma_{1}\left|k_{j}\right|\left|a_{j}\right|}}\left(1+\frac{t^{\Sigma_{1}\left|k_{j}\right|}}{\langle x\rangle^{\Sigma_{1}\left|k_{j}\right|}}\right) .
$$

Now when $j \in I_{2}$ we have $\ell_{j}=\left(a_{j}, 0\right),\left|a_{j}\right|=1$. Then

$$
\left|\partial_{x}^{a_{j}}\left(x_{p}(t)\right)\right| \leqslant 1+\frac{2 \varepsilon t M_{1}}{\langle x\rangle^{2+\sigma_{0}}}+\frac{\varepsilon M_{1}}{\langle x\rangle^{1+\sigma_{0}}} \leqslant 2 M_{1}\left(1+\frac{t}{\langle x\rangle^{2+\sigma_{0}}}\right) .
$$

Therefore we have

$$
\left|\prod_{j \in I_{2}}\left(\partial_{X}^{\ell_{1}}(x(t))\right)^{k_{j}}\right| \leqslant C\left(2 M_{1}\right)^{\Sigma_{2}\left|k_{j}\right|}\left(1+\frac{t^{\Sigma_{2}\left|k_{j}\right|}}{\langle x\rangle^{\Sigma_{2}\left|k_{j}\right|\left(2+\sigma_{0}\right)}}\right) .
$$

Finally for $j \in I_{3}$ we have $\ell_{j}=\left(0, b_{j}\right),\left|b_{j}\right|=1$. Then

$$
\left|\partial_{\xi}^{b_{j}}\left(x_{p}(t)\right)\right| \leqslant 2 M_{1}\langle t\rangle \text {. }
$$

It follows that

$$
\left|\prod_{j \in I_{3}}\left(\partial_{X}^{\ell_{1}}(x(t))\right)^{k_{j}}\right| \leqslant\left(2 M_{1}\right)^{\Sigma_{3}\left|k_{j}\right|}\langle t\rangle^{\Sigma_{3}\left|k_{j}\right|}, .
$$

Using (A.3.10), (A.3.11) and (A.3.12) we obtain

$$
\begin{aligned}
(1) & =\left|\left(\partial^{\beta} F\right)(x(t)) \prod_{j=1}^{s}\left(\partial_{X}^{\ell_{j}}(x(t))\right)^{k_{j}}\right| \\
& \leqslant \frac{C_{\beta} M_{k-1}^{k-1}}{\langle x(t)\rangle^{|\beta|+\ell+1+\sigma_{0}}} \cdot \frac{1}{\langle x\rangle^{\Sigma_{1}\left|k_{j}\right|\left|a_{j}\right|}}\left(1+\frac{t^{\Sigma_{1}\left|k_{j}\right|}}{\langle x\rangle^{\Sigma_{1}\left|k_{j}\right|}}\right)\left(1+\frac{t^{\Sigma_{2}\left|k_{j}\right|}}{\langle x\rangle^{\Sigma_{2}\left|k_{j}\right|}}\right)\langle t\rangle^{\Sigma_{3}\left|k_{j}\right|} .
\end{aligned}
$$

Now we have

$$
\Sigma_{1}\left|k_{j}\right|+\Sigma_{2}\left|k_{j}\right|+\Sigma_{3}\left|k_{j}\right|=|\beta|, \quad\langle x(t)\rangle \geqslant C(t) \text { and }\langle x(t)\rangle \geqslant C\langle x\rangle .
$$

It follows that

$$
(1) \leqslant \frac{C_{k} M_{k-1}^{k-1}}{\langle x(t)\rangle^{\ell+1+\sigma_{0}}} \frac{1}{\langle x\rangle^{\Sigma_{1}\left|k_{j}\right|\left|a_{j}\right|+\Sigma_{2}\left|k_{j}\right|}}
$$

On the other hand,

$$
|A|=\sum_{j=1}^{s}\left|k_{j}\right|\left|a_{j}\right|=\Sigma_{1}\left|k_{j}\right|\left|a_{j}\right|+\Sigma_{2}\left|k_{j}\right| \text { since }\left|a_{j}\right|=1 \text { for } t \in I_{2}
$$

and $a_{j}=0$ if $j \in I_{3}$. Therefore (A.3.13) implies (A.3.8) and our claim is proved. 
Moreover if $|\alpha|=k$ we can write

$$
\left\{\begin{array}{l}
\partial_{X}^{\alpha}[F(x(t))]=\sum_{\ell=1}^{n} \frac{\partial F}{\partial y_{\ell}}(x(t)) \partial_{X}^{\alpha} x_{\ell}(t)+R \text { where } \\
|R| \leqslant \frac{C_{k} M_{k-1}^{k-1}}{\langle x(t)\rangle^{\ell+1+\sigma_{0}}} \cdot \frac{1}{\langle x\rangle^{|A|}} .
\end{array}\right.
$$

Indeed $R$ is a finite sum of terms of the form $\left(\partial^{\beta} F\right)(x(t)) \prod_{j=1}^{s}\left(\partial_{X}^{\ell_{j}} x\right)^{k_{j}}$ where $2 \leqslant|\beta| \leqslant|\alpha|$. It follows then that $\left|\ell_{j}\right| \leqslant|\alpha|-1=k-1$ and the above computations are valid.

Let us now prove Proposition 3.3.2 for $|\alpha|=k$. Let us set

$$
Z(t)=\partial_{X}^{\alpha} z(t), \quad \Xi(t)=\langle x\rangle \partial_{X}^{\alpha} \zeta(t) .
$$

We can write

$$
\begin{aligned}
& \dot{Z}_{j}(t)=2 \varepsilon \sum_{j, k=1}^{n}\{\underbrace{b_{j k}(x(t)) \partial_{X}^{\alpha} \xi_{k}(t)}_{(1)}+\underbrace{\partial_{X}^{\alpha}\left[b_{j k}(x(t))\right] \xi_{k}(t)}_{(2)} \\
& +\sum_{\substack{\alpha=\alpha_{1}+\alpha_{2} \\
\alpha_{j} \neq 0}}\left(\begin{array}{c}
\alpha \\
\alpha_{1}
\end{array}\right) \underbrace{\partial_{X}^{\alpha_{1}}\left[b_{j k}(x(t))\right] \partial_{X}^{\alpha_{2}} \xi_{k}(t)}_{(3)}\}-2 \varepsilon t \sum_{p, q=1}^{n}\{\underbrace{\partial_{X}^{\alpha}\left[\frac{\partial b_{p q}}{\partial x_{j}}(x(t))\right] \xi_{p}(t) \xi_{q}(t)}_{(4)} \\
& +\underbrace{\frac{\partial b_{p q}}{\partial x_{j}}(x(t)) \partial_{X}^{\alpha}\left(\xi_{p}(t) \xi_{q}(t)\right)}_{(5)}+\sum_{\substack{\alpha=\alpha_{1}+\alpha_{2} \\
\alpha_{j} \neq 0}}\left(\begin{array}{c}
\alpha \\
\alpha_{1}
\end{array}\right) \underbrace{\partial_{X}^{\alpha_{1}}\left[\frac{\partial b_{p q}}{\partial x_{j}}(x(t))\right] \partial_{X}^{\alpha_{2}}\left(\xi_{p}(t) \xi_{q}(t)\right)}_{(6)}\} \\
& \dot{\Xi}_{j}(t)=-\varepsilon\langle x\rangle \sum_{p, q=1}^{n}\{\underbrace{\partial_{X}^{\alpha}\left[\frac{\partial b_{p q}}{\partial x_{j}}(x(t))\right] \xi_{p}(t) \xi_{q}(t)}_{(7)}+\underbrace{\frac{\partial b_{p q}}{\partial x_{j}}(x(t)) \partial_{X}^{\alpha}\left(\xi_{p}(t) \xi_{q}(t)\right)}_{(8)} \\
& +\sum_{\substack{\alpha=\alpha_{1}+\alpha_{2} \\
\alpha_{j} \neq 0}}\left(\begin{array}{c}
\alpha \\
\alpha_{1}
\end{array}\right) \underbrace{\partial_{X}^{\alpha_{1}}\left[\frac{\partial b_{p q}}{\partial x_{j}}(x(t))\right] \partial_{X}^{\alpha_{2}}\left(\xi_{p}(t) \xi_{q}(t)\right)}_{(9)}\} .
\end{aligned}
$$

We shall use the fact that $b_{j k} \in \mathcal{B}_{\sigma_{0}}^{1}$ and $\frac{\partial b_{p q}}{\partial x_{j}} \in \mathcal{B}_{\sigma_{0}}^{2}$. We deduce from (A.3.3) that

$$
|(1)| \leqslant \frac{C \varepsilon}{\langle x(t)\rangle^{1+\sigma_{0}}\langle x\rangle^{|A|}}+\frac{C \varepsilon}{\langle x(t)\rangle^{1+\sigma_{0}}} \frac{|\Xi(t)|}{\langle x\rangle} .
$$

To estimate the term (2) we use (A.3.14) with $F=b_{j k}$ and the equality

$$
\partial_{X}^{\alpha} x_{j}(t)=\partial_{X}^{\alpha}\left(x_{j}+2 t \xi_{j}\right)+2 t \frac{\Xi_{j}(t)}{\langle x\rangle}+Z_{j}(t) .
$$

We obtain

$$
|(2)| \leqslant \frac{C \varepsilon}{\langle x(t)\rangle^{2+\sigma_{0}}}|Z(t)|+\frac{C \varepsilon}{\langle x(t)\rangle^{1+\sigma_{0}}} \frac{|\Xi(t)|}{\langle x\rangle}+\frac{C \varepsilon}{\langle x(t)\rangle^{1+\sigma_{0}}\langle x\rangle^{|A|}} .
$$


To estimate (3) we use (A.3.4) and (A.3.8) ; we obtain,

$$
|(3)| \leqslant \frac{C_{k} \varepsilon M_{k-1}^{k-1}}{\langle x(t)\rangle^{1+\sigma_{0}}\langle x\rangle^{|A|}} .
$$

By the same way we have,

$$
|(6)| \leqslant \frac{C_{k} \varepsilon M_{k-1}^{k-1}}{\langle x(t)\rangle^{1+\sigma_{0}}\langle x\rangle^{|A|}} .
$$

Then (4) has the same estimate as (2) and (5) as (1) since $\langle x(t)\rangle \geqslant C\langle t\rangle$.

To take care of (7) we use (A.3.14) with $F=\frac{\partial b_{p q}}{\partial x_{j}} \in \mathcal{B}_{\sigma_{0}}^{2}$. We obtain

$$
|(7)| \leqslant \frac{C \varepsilon}{\langle x(t)\rangle^{2+\sigma_{0}}}|Z(t)|+\frac{C \varepsilon}{\langle x(t)\rangle^{2+\sigma_{0}}}|\Xi(t)|+\frac{C \varepsilon}{\langle x(t)\rangle^{1+\sigma_{0}}\langle x\rangle^{|A|}}
$$

since $\langle x(t)\rangle \geqslant C\langle x\rangle$ and $\langle x(t)\rangle \geqslant C\langle t\rangle$. Finally

$$
\left\{\begin{array}{l}
|(8)| \leqslant \frac{C \varepsilon}{\langle x(t)\rangle^{1+\sigma_{0}}}|\Xi(t)|+\frac{C \varepsilon}{\langle x(t)\rangle^{1+\sigma_{0}}\langle x\rangle^{|A|}}, \\
|(9)| \leqslant \frac{C \varepsilon M_{k-1}^{k-1}}{\langle x(t)\rangle^{1+\sigma_{0}}\langle x\rangle^{|A|}} .
\end{array}\right.
$$

Gathering the estimates obtained in (A.3.15) to (A.3.20) we obtain

$$
|\dot{Z}(t)|+|\dot{\Xi}(t)| \leqslant \frac{C \varepsilon}{\langle x(t)\rangle^{1+\sigma_{0}}}(|Z(t)|+|\Xi(t)|)+\frac{C_{k} \varepsilon M_{k-1}^{k-1}}{\langle x(t)\rangle^{1+\sigma_{0}}\langle x\rangle^{|A|}} .
$$

It follows from Gronwall's Lemma, (A.3.2) and the estimate $\langle x(t)\rangle \geqslant C\langle t\rangle$ that

$$
|Z(t)|+|\Xi(t)| \leqslant \frac{C\left(M_{k-1}\right) \varepsilon}{\langle x\rangle^{|A|+\sigma_{0}}}
$$

which, according to the definition of $Z$ and $\Xi$ proves Proposition 3.3.2 when $|\alpha|=k$.

\section{A.4. Proof of Lemma 5.3.1}

The proof is the same for the two cases so we shall consider the more general case where $f=f(x, \theta)$.

Let $\chi \in C_{0}^{\infty}\left(\mathbb{R}^{n}\right), \chi(\xi)=1$ if $|\xi| \leqslant \frac{1}{2}, \chi(\xi)=0$ if $|\xi| \geqslant 1$. We set

$$
\sum_{|\beta| \leqslant \ell} \sup _{\mathbb{R}^{n}}\left|\partial^{\beta} \chi\right|=D_{\ell}, \quad \ell \in \mathbb{N} .
$$

We want to show that one can find an increasing sequence $\left(L_{k}\right)_{k \geqslant 1}$ in $] 1,+\infty[$ such that if we set for $(\theta, x, y)$ in $\Omega \times \mathbb{R}_{y}^{n}$

$$
\left\{\begin{array}{l}
F_{\gamma}(\theta, x, y)=\partial_{x}^{\gamma} f(\theta, x) \frac{(i y)^{\gamma}}{\gamma !} \chi\left(L_{|\gamma|} y\left(\frac{1}{\langle x\rangle}+\frac{1}{\langle\theta\rangle}\right)\right), \quad|\gamma| \geqslant 1 \\
F(\theta, x, y)=f(\theta, x)+\sum_{\gamma \neq 0} F_{\gamma}(x, \theta, y)
\end{array}\right.
$$

then $F$ is well defined in $\Omega \times \mathbb{R}_{y}^{n}$ and satisfies all the requirements of Lemma 5.3.1. 
First of all in the expression of $F_{\gamma}$, on the support of $\chi$ we have $|y| \leqslant \frac{1}{L_{|\gamma|}} \frac{\langle\theta\rangle\langle x\rangle}{\langle\theta\rangle+\langle x\rangle}$. It follows that

$$
\frac{|y|}{\langle x\rangle} \leqslant \frac{1}{L_{|\gamma|}}, \quad \frac{|y|}{\langle\theta\rangle} \leqslant \frac{1}{L_{|\gamma|}} .
$$

Using (5.3.2) we can write,

$$
\left|F_{\gamma}(\theta, x, y)\right| \leqslant M_{|\gamma|}\left(\frac{1}{\langle x\rangle|\gamma|+\sigma_{3}}+\frac{1}{\langle\theta\rangle|\gamma|+\sigma_{3}}\right) \frac{|y||\gamma|}{\gamma !}\left|\chi\left(L_{|\gamma|} y(\cdots)\right)\right|
$$

so

$$
\left|F_{\gamma}(\theta, x, y)\right| \leqslant \frac{D_{0} M_{|\gamma|}}{\gamma ! L_{|\gamma|}^{|\gamma|}}\left(\frac{1}{\langle x\rangle^{\sigma_{3}}}+\frac{1}{\langle\theta\rangle^{\sigma_{3}}}\right)
$$

Taking

$$
L_{|\gamma|}^{|\gamma|} \geqslant D_{0} M_{|\gamma|}
$$

we deduce that $F$ defined in (A.4.2) is well defined and satisfies

$$
|F(\theta, x, y)| \leqslant C_{0}\left(\frac{1}{\langle x\rangle^{\sigma_{1}}}+\frac{1}{\langle\theta\rangle^{\sigma_{2}}}\right)
$$

since $\sigma_{3} \geqslant \sigma_{1}$ and $\sigma_{3} \geqslant \sigma_{2}$.

Therefore (ii) in Lemma 5.3.1 is satisfied and (i) follows immediately from (A.4.2). We shall strengthen the condition (A.4.4) on $L_{|\gamma|}$ to obtain a $C^{\infty}$ function $F$. First all there exists absolute constants $C_{i, \ell},(i \in \mathbb{N}, \ell \in \mathbb{N})$ such that

$$
\sum_{|\gamma|=i}\left|\partial_{x}^{\gamma}\left[\left(\frac{1}{\langle x\rangle}+\frac{1}{\langle\theta\rangle}\right)^{\ell}\right]\right| \leqslant C_{i, \ell}\left(\frac{1}{\langle x\rangle}+\frac{1}{\langle\theta\rangle}\right)^{\ell+i} .
$$

Let $\beta \in \mathbb{N}^{n}$. For any $\mu \in \mathbb{N}^{n}$ one can find an absolute constant $K_{|\mu|}$ independent of $\left.\left(L_{k}\right)\right)$ such that for all $(\theta, x, y)$ in $\mathbb{R} \times \mathbb{R}_{x}^{n} \times \mathbb{R}_{y}^{n}$ we have

$$
\left|\partial_{x}^{\mu}\left[\left(\partial_{\xi}^{\beta} \chi\right)\left(L_{|\gamma|} y\left(\frac{1}{\langle x\rangle}+\frac{1}{\langle\theta\rangle}\right)\right)\right]\right| \leqslant K_{|\mu|} D_{|\beta|+|\mu|}\left(\frac{1}{\langle x\rangle}+\frac{1}{\langle\theta\rangle}\right)^{|\mu|} .
$$

Indeed let us set $h(\theta, x, y)=L_{|\gamma|} y\left(\frac{1}{\langle x\rangle}+\frac{1}{\langle\theta\rangle}\right)$. By the Faa di Bruno formula, $\partial_{x}^{\mu}\left[\left(\partial^{\beta} \chi\right)(h(\theta, x, y))\right]$ is a finite linear combination with absolute coefficients of terms of the form

$$
\left(\partial_{\xi}^{(\beta+\nu)} \chi\right)(h(\theta, x, y)) \prod_{j=1}^{s}\left(\partial_{x}^{\ell_{j}} h(\theta, x, y)\right)^{k_{j}}
$$

where $1 \leqslant|\nu| \leqslant|\mu|, 1 \leqslant s \leqslant|\mu|,\left|k_{j}\right| \geqslant 1,\left|\ell_{j}\right| \geqslant 1$ and

$$
\sum_{j=1}^{s} k_{j}=\nu, \quad \sum_{j=1}^{s}\left|k_{j}\right| \ell_{j}=\mu
$$

Since $|\nu|+|\beta| \leqslant|\mu|+|\beta|$ we have

$$
\left|\partial_{\xi}^{(\beta+\nu)} \chi(h(\theta, x, y))\right| \leqslant D_{|\mu|+|\beta|} .
$$


On the other hand it follows from (A.4.6) that

$$
(1)=:\left|\prod_{j=1}^{s}\left(\partial_{x}^{\ell_{j}} h(\theta, x, y)\right)^{k_{j}}\right| \leqslant \prod_{j=1}^{s} C_{\left|\ell_{j}\right|, 1}^{\left|k_{j}\right|}\left(|y| L_{|\gamma|}\right)^{\sum_{1}^{s}\left|k_{j}\right|}\left(\frac{1}{\langle x\rangle}+\frac{1}{\langle\theta\rangle}\right)^{\sum_{1}^{s}\left|k_{j}\right|\left(1+\left|\ell_{j}\right|\right)} .
$$

On the support of $\chi$ we use the estimates (A.4.3). Moreover we have $\sum_{1}^{s}\left|k_{j}\right|=|\nu|$, $\sum_{1}^{s}\left|k_{j}\right|\left|\ell_{j}\right|=|\mu|$. It follows then that,

$$
(1) \leqslant C_{|\mu|}^{\prime}\left(\frac{1}{\langle x\rangle}+\frac{1}{\langle\theta\rangle}\right)^{|\mu|} .
$$

Then (A.4.7) follows from (A.4.8) and (A.4.9).

Now with $F_{\gamma}$ defined in (A.4.2) we can write

$$
\begin{array}{r}
\partial_{y}^{B} F_{\gamma}(\theta, x, y)=i^{|\gamma|} \frac{\partial_{x}^{\gamma} f(\theta, x)}{\gamma !} \sum_{\substack{B_{1} \leqslant B \\
B_{1} \leqslant \gamma}}\left(\begin{array}{c}
B \\
B_{1}
\end{array}\right) \frac{y^{\gamma-B_{1}}}{\left(\gamma-B_{1}\right) !}\left[L_{|\gamma|}\left(\frac{1}{\langle x\rangle}+\frac{1}{\langle\theta\rangle}\right)\right]^{|B|-\left|B_{1}\right|} \\
\left(\partial_{\xi}^{B-B_{1}} \chi\right)\left(L_{|\gamma|} y\left(\frac{1}{\langle x\rangle}+\frac{1}{\langle\theta\rangle}\right)\right)
\end{array}
$$

Then

$$
\begin{aligned}
& \partial_{x}^{A} \partial_{y}^{B} F_{\gamma}(\theta, x, y) \\
& =i^{|\gamma|} \sum_{\substack{B_{1} \leqslant B \\
B_{1} \leqslant \gamma}} \sum_{A_{1} \leqslant A} \sum_{A_{2} \leqslant A-A_{1}}\left(\begin{array}{c}
B \\
B_{1}
\end{array}\right)\left(\begin{array}{c}
A \\
A_{1}
\end{array}\right)\left(\begin{array}{c}
A-A_{1} \\
A_{2}
\end{array}\right) \frac{1}{\left(\gamma-B_{1}\right) !} y^{\gamma-B_{1}} \\
& L_{|\gamma|}^{|B|-\left|B_{1}\right|} \partial_{x}^{\gamma+A_{1}} f(\theta, x) \partial_{x}^{A_{2}}\left[\left(\frac{1}{\langle x\rangle}+\frac{1}{\langle\theta\rangle}\right)^{|B|-\left|B_{1}\right|}\right] \\
& \partial_{x}^{A-A_{1}-A_{2}}\left[\left(\partial_{\xi}^{B-B_{1}} \chi\right)\left(y L_{|\gamma|}\left(\frac{1}{\langle x\rangle}+\frac{1}{\langle\theta\rangle}\right)\right)\right] .
\end{aligned}
$$

Now we use (A.4.3), (A.4.6) and (A.4.7). Since $|\gamma|+\left|A_{1}\right| \leqslant|\gamma|+|A|,\left|A-A_{1}-A_{2}\right| \leqslant|A|$, $\left|B-B_{1}\right| \leqslant|B|$, we obtain

$$
\begin{aligned}
& \left|\partial_{x}^{A} \partial_{y}^{B} F_{\gamma}(\theta, x, y)\right| \\
& \begin{array}{c}
\leqslant \sum_{\substack{B_{1} \leqslant B \\
B_{1} \leqslant \gamma}} \sum_{\substack{A_{1} \leqslant A \\
A_{2} \leqslant A-A_{1}}}\left(\begin{array}{c}
B \\
B_{1}
\end{array}\right)\left(\begin{array}{c}
A \\
A_{1}
\end{array}\right)\left(\begin{array}{c}
A-A_{1} \\
A_{2}
\end{array}\right) 2^{|\gamma|-\left|B_{1}\right|} L_{|\gamma|}^{\left|B_{1}\right|-|\gamma|}\left(\frac{1}{\langle x\rangle}+\frac{1}{\langle\theta\rangle}\right)^{\left|B_{1}\right|-|\gamma|} \\
L_{|\gamma|}^{|B|-\left|B_{1}\right|} M_{|\gamma|+|A|}\left(\frac{1}{\langle x\rangle^{|\gamma|+\left|A_{1}\right|+\sigma_{3}}}+\frac{1}{\langle\theta\rangle|\gamma|+\left|A_{1}\right|+\sigma_{3}}\right) C_{\left|A_{2}\right|,\left|B-B_{1}\right|}
\end{array} \\
& \left(\frac{1}{\langle x\rangle}+\frac{1}{\langle\theta\rangle}\right)^{\left|A_{2}\right|+|B|-\left|B_{1}\right|} K_{|A|} D_{|A|+|B|}\left(\frac{1}{\langle x\rangle}+\frac{1}{\langle\theta\rangle}\right)^{|A|-\left|A_{1}\right|-\left|A_{2}\right|}
\end{aligned}
$$

where $|y|$ satisfies (A.4.3). 
It follows that we can find a constant $\widetilde{C}_{|A|,|B|} \leqslant 1$, depending only on $|A|,|B|$ and the dimension such that

$$
\begin{aligned}
& \left|\partial_{x}^{A} \partial_{y}^{B} F_{\gamma}(\theta, x, y)\right| \\
& \quad \leqslant \frac{\widetilde{C}_{|A|,|B|} D_{|A|+|B|} M_{|\gamma|+|A|}}{L_{|\gamma|}^{|\gamma|-B \mid}}\left(\frac{1}{\langle x\rangle^{|A|+|B|+\sigma_{3}}}+\frac{1}{\langle\theta|| A|+| B|+\sigma_{3}}\right) .
\end{aligned}
$$

Let us set

$$
\widetilde{C}_{k}=\max _{|A|+|B| \leqslant k} \widetilde{C}_{|A|,|B|}
$$

We shall take the increasing sequence $\left(L_{k}\right)_{k \geqslant 0}$ such that

$$
L_{k} \geqslant \max \left(1,2^{k} \widetilde{C}_{k} D_{k} M_{2 k}\right) .
$$

Then we write, according to (A.4.2)

$$
F(\theta, x, y)=f(\theta, x)+\sum_{|\gamma| \leqslant|A|+|B|} F_{\gamma}(\theta, x, y)+\sum_{|\gamma|>|A|+|B|} F_{\gamma}(\theta, x, y) .
$$

The first two terms in the right hand side of (A.4.13) define a $C^{\infty}$ function. For the third one we deduce from (A.4.10), (A.4.11) and (A.4.12) that

$$
\left|\partial_{x}^{A} \partial_{y}^{B} f_{\gamma}(\theta, x, y)\right| \leqslant \frac{\widetilde{C}_{|\gamma|} D_{|\gamma|} M_{2|\gamma|}}{L_{|\gamma|}} \leqslant \frac{1}{2^{|\gamma|}} .
$$

This shows that the third term define also a $C^{\infty}$ function. Thus $F$ is $C^{\infty}$ in $(x, y)$. Let us prove (iii). According to (A.4.2) if $|A|+|B| \geqslant 1$ we have

$$
(1)=: \partial_{x}^{A} \partial_{y}^{B} F(\theta, x, y)=\partial_{x}^{A} \partial_{y}^{B} f(\theta, x)+\sum_{\gamma \neq 0} \partial_{x}^{A} \partial_{y}^{B} F_{\gamma}(\theta, x, y) .
$$

If $|B| \geqslant 1$ we use (A.4.10), (A.4.13) and (A.4.12). We get

$$
|(1)| \leqslant C_{A B}\left(\frac{1}{\langle x\rangle^{|A|+|B|+\sigma_{3}}}+\frac{1}{\langle\theta\rangle^{|A|+|B|+\sigma_{3}}}\right) .
$$

If $B=0$ we use furthermore (5.3.2). We obtain

$$
|(1)| \leqslant M_{|A|}\left(\frac{1}{\langle x\rangle^{|A|+\sigma_{3}}}+\frac{1}{\langle\theta\rangle^{|A|+\sigma_{3}}}\right)+C_{A B}^{\prime}\left(\frac{1}{\langle x\rangle^{|A|+\sigma_{3}}}+\frac{1}{\langle\theta\rangle^{|A|+\sigma_{3}}}\right) .
$$

This shows that (iii) holds.

Finally let us prove (iv). Let us set again

$$
h_{|\gamma|}(\theta, x, y)=L_{|\gamma|} y\left(\frac{1}{\langle x\rangle}+\frac{1}{\langle\theta\rangle}\right) .
$$


According to (A.4.2) we have for $j \in\{1,2, \ldots, n\}$,

$$
\begin{aligned}
& \partial_{x_{j}} F=\partial_{x_{j}} f(\theta, x)+ \sum_{\gamma \neq 0}\left(\partial_{x_{j}} \partial_{x}^{\gamma} f(\theta, x) \chi\left(h_{|\gamma|}(\theta, x, y)\right)\right. \\
&\left.-\sum_{\ell=1}^{n} L_{|\gamma|} \frac{x_{j} y_{\ell}}{\langle x\rangle^{3}} \partial_{x}^{\gamma} f(\theta, x) \cdot \frac{\partial \chi}{\partial \xi_{\ell}}\left(h_{|\gamma|}(\theta, x, y)\right)\right) \frac{(i y)^{\gamma}}{\gamma !} \\
& \partial_{y_{j}} F=\sum_{\gamma \neq 0} \partial_{x}^{\gamma} f(\theta, x) \frac{(i y)^{\gamma}}{\gamma !} L_{|\gamma|}\left(\frac{1}{\langle x\rangle}+\frac{1}{\langle\theta\rangle}\right) \frac{\partial \chi}{\partial \xi_{j}}\left(h_{|\gamma|}(\theta, x, y)\right) \\
&+i \sum_{\gamma_{j} \geqslant 1} \partial_{x}^{\gamma} f(\theta, x) \frac{(i y)^{\gamma-e_{j}}}{\left(\gamma-e_{j}\right) !} \chi\left(h_{|\gamma|}(\theta, x, y)\right),
\end{aligned}
$$

where $e_{j}=(0,0, \ldots, 1, \ldots, 0)$. Setting $\gamma^{\prime}=\gamma-e_{j}$ the sum above can be written

$$
i \partial_{x_{j}} f(\theta, x) \chi\left(L_{1} y\left(\frac{1}{\langle x\rangle}+\frac{1}{\langle\theta\rangle}\right)\right)+i \sum_{\gamma \neq 0} \partial_{x_{j}} \partial_{x}^{\gamma} f(\theta, x) \frac{(i y)^{\gamma}}{\gamma !} \chi\left(h_{|\gamma|+1}(\theta, x, y)\right) .
$$

It follows that

$$
\left(\partial_{x_{j}} F+i \partial_{y_{j}} F\right)(\theta, x, y)=(1)+(2)+(3)
$$

where

$$
\left\{\begin{aligned}
(1)= & \left(1-\chi\left(L_{1} y\left(\frac{1}{\langle x\rangle}+\frac{1}{\langle\theta\rangle}\right)\right) \partial_{x_{j}} f(\theta, x)\right. \\
(2)=\sum_{\gamma \neq 0} \partial_{x_{j}} \partial_{x}^{\gamma} f(\theta, x) \frac{(i y)^{\gamma}}{\gamma !}\left[\chi\left(h_{|\gamma|}(\theta, x, y)\right)-\chi\left(h_{|\gamma|+1}(\theta, x, y)\right)\right] & \\
(3)=\sum_{\gamma \neq 0} \partial_{x}^{\gamma} f(\theta, x) \frac{(i y)^{\gamma}}{\gamma !} L_{|\gamma|}( & -\sum_{\ell=1}^{n} \frac{x_{j} y_{\ell}}{\langle x\rangle^{3}} \frac{\partial \chi}{\partial \xi_{\ell}}\left(h_{|\gamma|}(\theta, x, y)\right) \\
& \left.+i\left(\frac{1}{\langle x\rangle}+\frac{1}{\langle\theta\rangle}\right) \frac{\partial \chi}{\partial \xi_{j}}\left(h_{|\gamma|}(\theta, x, y)\right)\right) .
\end{aligned}\right.
$$

Let us set for convenience

$$
R=|y|\left(\frac{1}{\langle x\rangle}+\frac{1}{\langle\theta\rangle}\right) .
$$

On the support of $1-\chi\left(L_{1} y\left(\frac{1}{\langle x\rangle}+\frac{1}{\langle\theta\rangle}\right)\right)$ in the term (1) above, we have $L_{1} R \geqslant \frac{1}{2}$. Therefore

$$
\frac{(1)}{R^{N}} \leqslant\left(2 L_{1}\right)^{N} M_{1}\left(\frac{1}{\langle x\rangle^{1+\sigma_{3}}}+\frac{1}{\langle\theta\rangle^{1+\sigma_{3}}}\right) .
$$

On the support of $\chi\left(h_{|\gamma|}(\theta, x, y)\right)-\chi\left(h_{|\gamma|+1}(\theta, x, y)\right)$ we have $\frac{1}{2 L_{|\gamma|+1}} \leqslant R \leqslant \frac{1}{L_{|\gamma|}}$. Now we write with $N \geqslant 2$,

$$
(2)=\sum_{1 \leqslant|\gamma| \leqslant N-1} G_{\gamma}(\theta, x, y)+\sum_{|\gamma| \geqslant N} G_{\gamma}(\theta, x, y) .
$$


When $|\gamma| \leqslant N-1$ we have $L_{|\gamma|+1} \leqslant L_{N}$ so $R \geqslant \frac{1}{2 L_{N}}$. It follows that

$$
\sum_{1 \leqslant|\gamma| \leqslant N-1}\left|G_{\gamma}(\theta, x, y)\right| \cdot \frac{1}{R^{N}} \leqslant\left(2 L_{N}\right)^{N} \sum_{1 \leqslant|\gamma| \leqslant N-1} M_{|\gamma|+1} \cdot \frac{1}{L_{|\gamma|}^{|\gamma|}}\left(\frac{1}{\langle x\rangle^{1+\sigma_{3}}}+\frac{1}{\langle\theta\rangle^{1+\sigma_{3}}}\right) .
$$

Therefore we obtain

$$
\sum_{1 \leqslant|\gamma| \leqslant N-1}\left|G_{\gamma}(\theta, x, y)\right| \leqslant C_{N} R^{N}\left(\frac{1}{\langle x\rangle^{1+\sigma_{3}}}+\frac{1}{\langle\theta\rangle^{1+\sigma_{3}}}\right) .
$$

On the other hand we have

$$
\begin{aligned}
& \sum_{|\gamma| \geqslant N}\left|G_{\gamma}(\theta, x, y)\right| \\
& \quad \leqslant\left(\frac{1}{\langle x\rangle^{1+\sigma_{3}}}+\frac{1}{\langle\theta\rangle^{1+\sigma_{3}}}\right) R^{N} \sum_{|\gamma| \geqslant N} M_{|\gamma|} R^{|\gamma|-N}\left[\chi\left(h_{|\gamma|}\right)-\chi\left(h_{|\gamma|+1}\right)\right] \\
& \sum_{|\gamma| \geqslant N}\left|G_{\gamma}(\theta, x, y)\right| \\
& \quad \leqslant\left(\frac{1}{\langle x\rangle^{1+\sigma_{3}}}+\frac{1}{\langle\theta\rangle^{1+\sigma_{3}}}\right) R^{N} \sum_{\beta} M_{|\beta|+N} R^{|\beta|}\left[\chi\left(h_{|\beta|+N}\right)-\chi\left(h_{|\beta|+N+1}\right)\right]
\end{aligned}
$$

On the support of $\chi\left(h_{|\beta|+N}\right)-\chi\left(h_{|\beta|+N+1}\right)$, we have $L_{|\beta|+N} R \leqslant 1$. It follows that

$$
\sum_{|\gamma| \geqslant N}\left|G_{\gamma}(\theta, x, y)\right| \leqslant C_{N} R^{N}\left(\frac{1}{\langle x\rangle^{1+\sigma_{3}}}+\frac{1}{\langle\theta\rangle^{1+\sigma_{3}}}\right) .
$$

Combining (A.4.18) and (A.4.19) we obtain

$$
|(2)| \leqslant C_{N}^{\prime} R^{N}\left(\frac{1}{\langle x\rangle^{1+\sigma_{3}}}+\frac{1}{\langle\theta\rangle^{1+\sigma_{3}}}\right) .
$$

Finally we consider the term (3) in (A.4.15). We have $\frac{\left|x_{j}\right|}{\langle x\rangle} \leqslant 1$ and on the support of $\frac{\partial \chi}{\partial \xi_{j}}\left(h_{|\gamma|}(\theta, x, y)\right)$ we have $L_{|\gamma|} \frac{|y|}{\langle x\rangle} \leqslant 1$ it follows that (3) is bounded by a finite sum of terms of the form

$$
(3)^{\prime}=\left(\frac{1}{\langle x\rangle^{1+\sigma_{3}}}+\frac{1}{\langle\theta\rangle^{1+\sigma_{3}}}\right) \sum_{\gamma \neq 0} M_{|\gamma|} R^{|\gamma|} L_{|\gamma|}\left|\frac{\partial \chi}{\partial \xi_{j}}\left(h_{|\gamma|}(\theta, x, y)\right)\right| .
$$

As before we write the above sum as $\sum_{1 \leqslant|\gamma| \leqslant N-1}+\sum_{|\gamma| \geqslant N}$. If $|\gamma| \leqslant N-1$ then $L_{|\gamma|} \leqslant L_{N-1}$ so on the support of $\frac{\partial \chi}{\partial \xi_{j}}$ we have $R \geqslant \frac{1}{2 L_{|\gamma|}} \geqslant \frac{1}{2 L_{N-1}}$ and $R \leqslant \frac{1}{L_{|\gamma|}}$. It follows that

$$
\frac{1}{R^{N}}\left|\sum_{1 \leqslant|\gamma| \leqslant N-1}\right| \leqslant\left(2 L_{N-1}\right)^{N} \sum_{1 \leqslant|\gamma| \leqslant N-1} M_{|\gamma|} \frac{1}{L_{|\gamma|}^{|\gamma|-1}} D_{1}=C_{N} .
$$


For the second sum we write

$$
\begin{aligned}
\left|\sum_{|\gamma| \geqslant N}\right| & \leqslant R^{N} \sum_{|\gamma| \geqslant N} M_{|\gamma|} R^{|\gamma|-N} L_{|\gamma|}\left|\frac{\partial \chi}{\partial \xi_{j}}\left(h_{|\gamma|}\right)\right| \\
& \leqslant R^{N} \sum_{\beta} M_{|\beta|+N} \frac{L_{|\beta|+N}}{L_{|\beta|+N}^{|\beta|}} D_{1}=C_{N}^{\prime} R^{N} .
\end{aligned}
$$

It follows that

$$
|(3)| \leqslant C_{N} R^{N}\left(\frac{1}{\langle x\rangle^{1+\sigma_{3}}}+\frac{1}{\langle\theta\rangle^{1+\sigma_{3}}}\right) .
$$

Using (A.4.14) to (A.4.21) we obtain the part (iv) of Lemma 5.3.1. The proof is complete. 


\section{BIBLIOGRAPHY}

[B] N. BurQ - Estimations de Strichartz pour des perturbations à longue portée de l'opérateur de Schrödinger, in Séminaire Equations aux Dérivées Partielles, 2001-2002, École polytechnique, exp. $\mathrm{n}^{\circ} 11$.

[BGT] N. Burq, P. GÉrard \& N. Tzvetkov - Strichartz inequalities and the non linear Schrödinger equation on compact manifold, Amer. J. Math. 126 (2004), p. 569-605.

[CK] M. Christ \& A. Kiselev - Maximal functions associatef to filtrations, J. Funct. Anal. 179 (2001), no. 2, p. 409-425.

[CS] G.M. Constantin \& T.H. Savits - A multivariate Faa di Bruno formula with applications, Trans. Amer. Math. Soc. 348 (1996), no. 2, p. 503-520.

[D] S.I. DoI - Smoothing effects of Schrödinger evolution groups on Riemannian manifolds, Duke Math. J. 82 (1996), p. 679-706.

[GV] J. Ginibre \& G. Velo - Smoothing properties and retarded estimates for some dispersive evolutions, Comm. Math. Phys. 144 (1992), p. 163-188.

[HTW1] A. Hassell, T. TAO \& J. Wunsch - A Strichartz inequality for the Schrödinger equation on non trapping asymptotically conic manifold, preprint.

[HTW2] _ Sharp Strichartz estimates on non trapping asymptotically conic manifolds, preprint.

[H] L. HÖRMANDER - The analysis of linear partial differential operators, vol. I \& IV, Grundhehren, Springer.

[KT] M. Kell \& T. TAO - End point Strichartz estimate, Amer. J. Math. 120 (1998), p. 955-980.

[MS] A. Melin \& J. SJÖstrand - Fourier integral operators with complex valued phase function, Lect. Notes in Math., vol. 459, Springer, p. 121-223. 
[RZ2] L. RobBiano \& C. ZUILY - Analytic theory for the quadratic scattering wave front set and application to the Schrödinger equation, Astérisque, vol. 283, Société Mathématique de France, 2002.

[Sj] J. SJÖSTRAND - Singularités analytiques microlocales, Astérisque, vol. 95, Société Mathématique de France, 1982.

[SS] H. Smith \& C. Sogge - Global Strichartz estimates for non trapping perturbations of the Laplacian, Comm. Partial Differential Equations $\mathbf{2 5}$ (2000), no. 11 \& 12, p. 2171-2183.

[ST] G. Staffilani \& D. Tataru - Strichartz estimates for a Schrödinger operator with non smooth coefficients, Comm. Partial Differential Equations (2002), no. 5 \& 6, p. 1337-1372.

[Str] R. Strichartz - Restriction of Fourier transform to quadratic surfaces and decay of solutions to the wave equation, Duke Math. J. 44 (1977), p. 705714 .

[Y] K. YAJima - Existence of solutions for Schrödinger evolution equations, Comm. Math. Phys. 110 (1987), p. 415-426. 